\title{
Accurate computational thermodynamics using anharmonic DFT calculations: the case study of B-H species
}

Robert Maillard, ${ }^{\dagger}$ Daniel Sethio, ${ }^{\ddagger}, \dagger$ Hans Hagemann,$^{\dagger}$ and Latévi M. Lawson

$$
\mathrm{Daku}^{*, \dagger}
$$

$\dagger$ †epartment of physical chemistry - University of Geneva, Quai Ernest Ansermet 30,

$$
1211 \text { Geneva, Switzerland }
$$

$\ddagger$ Southern Methodist University, 3215 Daniel Avenue, Dallas, Texas 75275-0314, United

$$
\text { States }
$$

E-mail: max.lawson@unige.ch 


\section{Table of contents}

\begin{tabular}{|c|c|c|c|c|}
\hline \multicolumn{2}{|c|}{ Introduction } & S.3 & $23 \mathbf{B}_{4} \mathbf{H}_{2}$ & S.184 \\
\hline 1 & $\mathbf{B H}_{3}$ & $\mathrm{~S} .4$ & $24 \mathrm{~B}_{4} \mathrm{H}_{3}$ & S.192 \\
\hline 2 & $\mathrm{BH}_{3}^{-}$ & S.12 & $25 \mathbf{B}_{4} \mathbf{H}_{4}$ & $\mathrm{~S} .200$ \\
\hline 3 & $\mathrm{BH}_{4}$ & $\mathrm{S.20}$ & $26 \mathbf{B}_{4} \mathbf{H}_{5}$ - Isomer $\mathbf{A}$ & $\mathrm{S} .208$ \\
\hline 4 & $\mathrm{BH}_{4}^{-}$ & S.28 & $27 \mathbf{B}_{4} \mathbf{H}_{5}$ - Isomer B & $\mathrm{S} .216$ \\
\hline 5 & $\mathbf{B}_{2} \mathbf{H}_{3}$ & S.36 & $28 \mathbf{B}_{4} \mathbf{H}_{5}$ - Isomer $\mathbf{C}$ & S.224 \\
\hline 6 & $\mathbf{B}_{2} \mathbf{H}_{4}$ - Isomer $\mathbf{A}$ & S.44 & $29 \mathbf{B}_{4} \mathbf{H}_{7}$ - Isomer $\mathbf{A}$ & S.232 \\
\hline 7 & $\mathbf{B}_{2} \mathbf{H}_{4}$ - Isomer B & $\mathrm{S} .52$ & $30 \mathbf{B}_{4} \mathbf{H}_{7}$ - Isomer B & $\mathrm{S} .241$ \\
\hline 8 & $\mathbf{B}_{2} \mathbf{H}_{5}$ & $\mathrm{~S} .60$ & $31 \mathbf{B}_{4} \mathbf{H}_{7}$ - Isomer $\mathbf{C}$ & S.250 \\
\hline 9 & $\mathbf{B}_{2} \mathbf{H}_{5}^{-}$ & S.68 & $32 \mathbf{B}_{4} \mathbf{H}_{8}$ & $\mathrm{~S} .259$ \\
\hline 10 & $\mathbf{B}_{2} \mathbf{H}_{6}$ & S.76 & $33 \mathbf{B}_{4} \mathbf{H}_{9}$ & $\mathrm{~S} .268$ \\
\hline 11 & $\mathbf{B}_{2} \mathbf{H}_{7}$ & S.84 & $34 \mathbf{B}_{4} \mathbf{H}_{10}$ & $\mathrm{~S} .277$ \\
\hline 12 & $\mathbf{B}_{3} \mathbf{H}$ & S.92 & $35 \mathbf{B}_{5} \mathbf{H}_{9}$ & $\mathrm{~S} .286$ \\
\hline 13 & $\mathbf{B}_{3} \mathbf{H}_{2}$ & S.100 & $36 \mathbf{B}_{5} \mathbf{H}_{11}$ & $\mathrm{~S} .295$ \\
\hline 14 & $\mathbf{B}_{3} \mathbf{H}_{3}$ & S.108 & $37 \mathbf{B}_{6} \mathbf{H}_{10}$ & $\mathrm{~S} .304$ \\
\hline 15 & $\mathbf{B}_{3} \mathbf{H}_{5}$ & S.116 & $38 \mathbf{B}_{6} \mathbf{H}_{12}$ & S.313 \\
\hline 16 & $\begin{array}{l}\mathbf{B}_{3} \mathbf{H}_{6} \text { - Isomer } \mathbf{A} \\
\mathbf{B}_{3} \mathbf{H}_{6} \text { - Isomer B }\end{array}$ & S.124 & $39 \mathrm{~B}_{8} \mathrm{H}_{8}{ }^{2-}$ & S.323 \\
\hline 18 & $\mathbf{B}_{3} \mathbf{H}_{7}$ & $\mathrm{~S} .140$ & $40 \mathbf{B}_{8} \mathbf{H}_{14}$ & S.332 \\
\hline 19 & $\mathbf{B}_{3} \mathbf{H}_{8}^{-}$ & $\mathrm{S} .149$ & $41 \mathrm{~B}_{9} \mathrm{H}_{9}{ }^{2-}$ & S.342 \\
\hline 20 & $\mathbf{B}_{3} \mathbf{H}_{9}$ & $\mathrm{~S} .158$ & $42 \mathbf{B}_{10} \mathbf{H}_{10}^{2-}$ & $\mathrm{S} .352$ \\
\hline 21 & $\mathbf{B}_{3} \mathbf{H}_{10}$ & S.167 & $43 \mathbf{B}_{11} \mathbf{H}_{14}^{-}$ & S.362 \\
\hline 22 & $\mathbf{B}_{4} \mathbf{H}$ & S.176 & $44 \mathbf{B}_{12} \mathbf{H}_{12}{ }^{2-}$ & S.372 \\
\hline
\end{tabular}




\section{Introduction}

We have proceeded to the accurate determination of the key thermodynamic functions $\left(\Delta H(\mathrm{~T}), S(\mathrm{~T}) \mathrm{v}, C_{P}(\mathrm{~T})\right)$ of 43 isolated B-H molecular species involved in the decomposition of B-H solids, with the inclusion of anharmonic effects. We report the analytic expressions of these functions obtained by fitting them with NASA functions ${ }^{1,2}$ (Equations (1) - (3)) in the 200-900 K temperature range.

$$
\begin{aligned}
\frac{C_{P}}{R} & =a_{1}+a_{2} \mathrm{~T}+a_{3} \mathrm{~T}^{2}+a_{4} \mathrm{~T}^{3}+a_{5} \mathrm{~T}^{4} \\
\frac{S}{R} & =a_{1} \ln \mathrm{T}+a_{2} \mathrm{~T}+\frac{a_{3} \mathrm{~T}^{2}}{2}+\frac{a_{4} \mathrm{~T}^{3}}{3}+\frac{a_{5} \mathrm{~T}^{4}}{4}+a_{7} \\
\frac{\Delta H}{R \mathrm{~T}} & =a_{1}+\frac{a_{2} \mathrm{~T}}{2}+\frac{a_{3} \mathrm{~T}^{2}}{2}+\frac{a_{4} \mathrm{~T}^{3}}{3}+\frac{a_{5} \mathrm{~T}^{4}}{4}+\frac{a_{6}}{\mathrm{~T}}
\end{aligned}
$$

With $\Delta H=H(\mathrm{~T})-H(0 \mathrm{~K})$. Because the vibrational spectra of these species are their fingerprints, we also report the predicted IR and Raman spectra (with the contributions of fundamentals, combinations and overtones).

(1) Gordon, S.; Mcbride, B. J. Computer Program for Calculation of Complex Chemical Equilibrium Compositions, Rocket Performance, Incident and Reflected Shocks, and Chapman-Jouguet Detonations. Interim Revision. 1976.

(2) Gardiner, W. C. Combustion Chemistry; Springer US, 1984. 


\section{$1 \quad \mathrm{BH}_{3}$}

Figure S.1.1. Structure of $\mathrm{BH}_{3}$

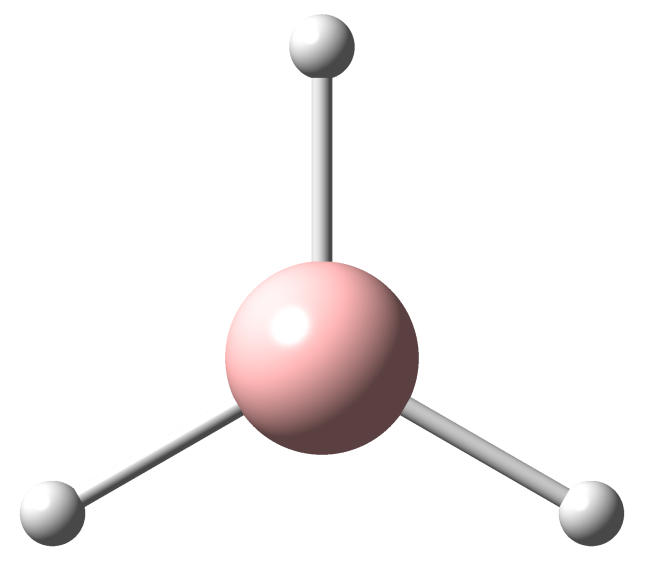

\begin{aligned} & \hline Charge 0 \\ & Spin multiplicity 1 \\ & Framework Group $\mathrm{D}_{3 \mathrm{~h}} \\ &$ Rotor Type Oblate Symmetric Top \\ & Symmetry number 6 \\ & \hline\end{aligned}

Table S.1.1. $\mathrm{BH}_{3}$ : Selected anharmonic results obtained at the B3LYP-D2/cc-pVTZ level

\begin{tabular}{rll}
\hline $\mathrm{ZPE}_{\mathrm{v}}$ & 5703 & $\mathrm{~cm}^{-1}$ \\
$\mathrm{~A}$ & 7.89169 & $\mathrm{~cm}^{-1}$ \\
$\mathrm{~B}$ & 7.89169 & $\mathrm{~cm}^{-1}$ \\
$\mathrm{C}$ & 3.94584 & $\mathrm{~cm}^{-1}$ \\
\hline$C_{P}(300 \mathrm{~K})$ & 36.202 & $\mathrm{~J} \mathrm{~mol}^{-1} \mathrm{~K}-1$ \\
$S(300 \mathrm{~K})$ & 188.592 & $\mathrm{~J} \mathrm{~mol}^{-1} \mathrm{~K}-1$ \\
$H(300 \mathrm{~K})-H(0 \mathrm{~K})$ & 10.134 & $\mathrm{~kJ} \mathrm{~mol}^{-1}$ \\
\hline
\end{tabular}

$\mathrm{ZPE}_{\mathrm{v}}$ : vibrational contribution to the zero-point energy. A, B, C: Rotational constants. $C_{P}$ : Heat capacity at constant pressure. $S$ : Entropy. $H$ : Enthalpy. Ideal-gas calculations (1 bar). 
Table S.1.2. $\mathrm{BH}_{3}$ : Cartesian coordinates $(\AA)$ of the B3LYP-D2/cc-pVTZ optimized structure

\begin{tabular}{cccc} 
atom & $\mathrm{x}$ & $\mathrm{y}$ & $\mathrm{z}$ \\
\hline B1 & 0.0000 & 0.0000 & 0.0000 \\
H2 & 0.0000 & 1.1887 & 0.0000 \\
H3 & 1.0295 & -0.5944 & -0.0000 \\
H4 & -1.0295 & -0.5944 & -0.0000 \\
\hline
\end{tabular}


Table S.1.3. $\mathrm{BH}_{3}$ : Vibrational frequencies, infrared integrated intensities and Raman activities (B3LYP-D2/cc-pVTZ results)

\begin{tabular}{cccccccc}
\hline & & \multicolumn{3}{c}{ Harmonic } & \multicolumn{3}{c}{ Anharmonic } \\
mode & symm. & $\omega$ & IR & Raman & $\omega$ & IR & Raman \\
& & {$\left[\mathrm{cm}^{-1}\right]$} & {$\left[\mathrm{km} \mathrm{mol}^{-1}\right]$} & {$\left[\AA^{6}\right]$} & {$\left[\mathrm{cm}^{-1}\right]$} & {$\left[\mathrm{km} \mathrm{mol}^{-1}\right]$} & {$\left[\AA^{6}\right]$} \\
\hline 1 & $\mathrm{~A}_{2}^{\prime \prime}$ & 1161 & 82.837 & 0.000 & 1142 & 80.361 & 0.000 \\
2 & $\mathrm{E}^{\prime}$ & 1230 & 14.336 & 0.013 & 1179 & 13.422 & 0.015 \\
3 & $\mathrm{E}^{\prime}$ & 1230 & 14.336 & 0.013 & 1179 & 13.422 & 0.015 \\
4 & $\mathrm{~A}_{1}^{\prime}$ & 2604 & 0.000 & 1.280 & 2489 & 6.242 & 1.387 \\
5 & $\mathrm{E}^{\prime}$ & 2697 & 125.266 & 0.222 & 2533 & 126.581 & 0.343 \\
6 & $\mathrm{E}^{\prime}$ & 2697 & 125.266 & 0.222 & 2533 & 126.581 & 0.343 \\
\hline
\end{tabular}


Figure S.1.2. Anharmonic IR spectrum of $\mathrm{BH}_{3}$ obtained by convoluting the calculated intensities with Lorentzians having a FWHM of $4 \mathrm{~cm}^{-1}$ (B3LYP-D2/cc-pVTZ results): (top) full spectrum, (middle) contributions from fundamentals and combination bands, (bottom) contributions from overtones
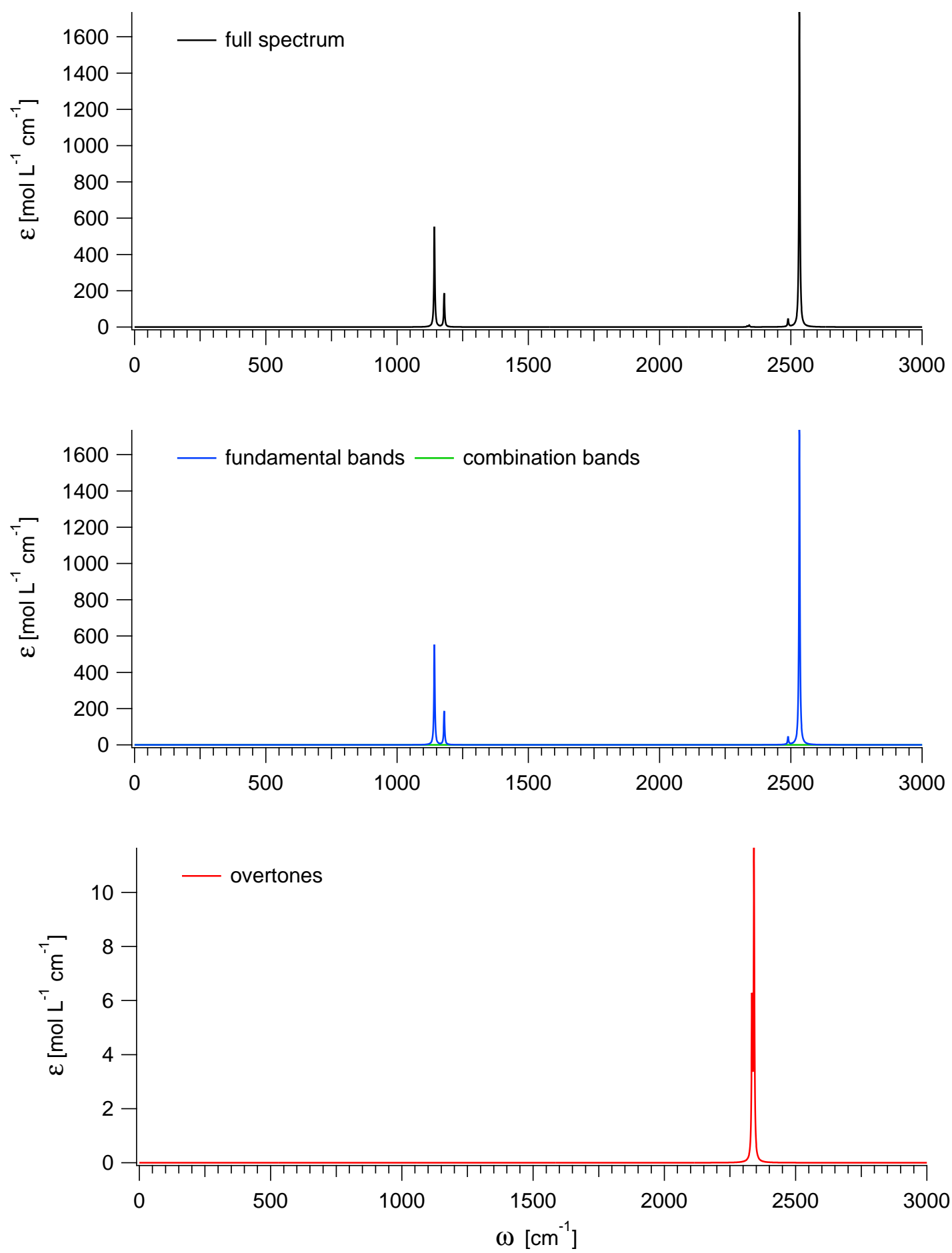
Figure S.1.3. Anharmonic Raman spectrum of $\mathrm{BH}_{3}$ obtained by convoluting the calculated activities with Lorentzians having a FWHM of $4 \mathrm{~cm}^{-1}$ (B3LYP-D2/cc-pVTZ results): (top) full spectrum, (middle) contributions from fundamentals and combination bands, (bottom) contributions from overtones
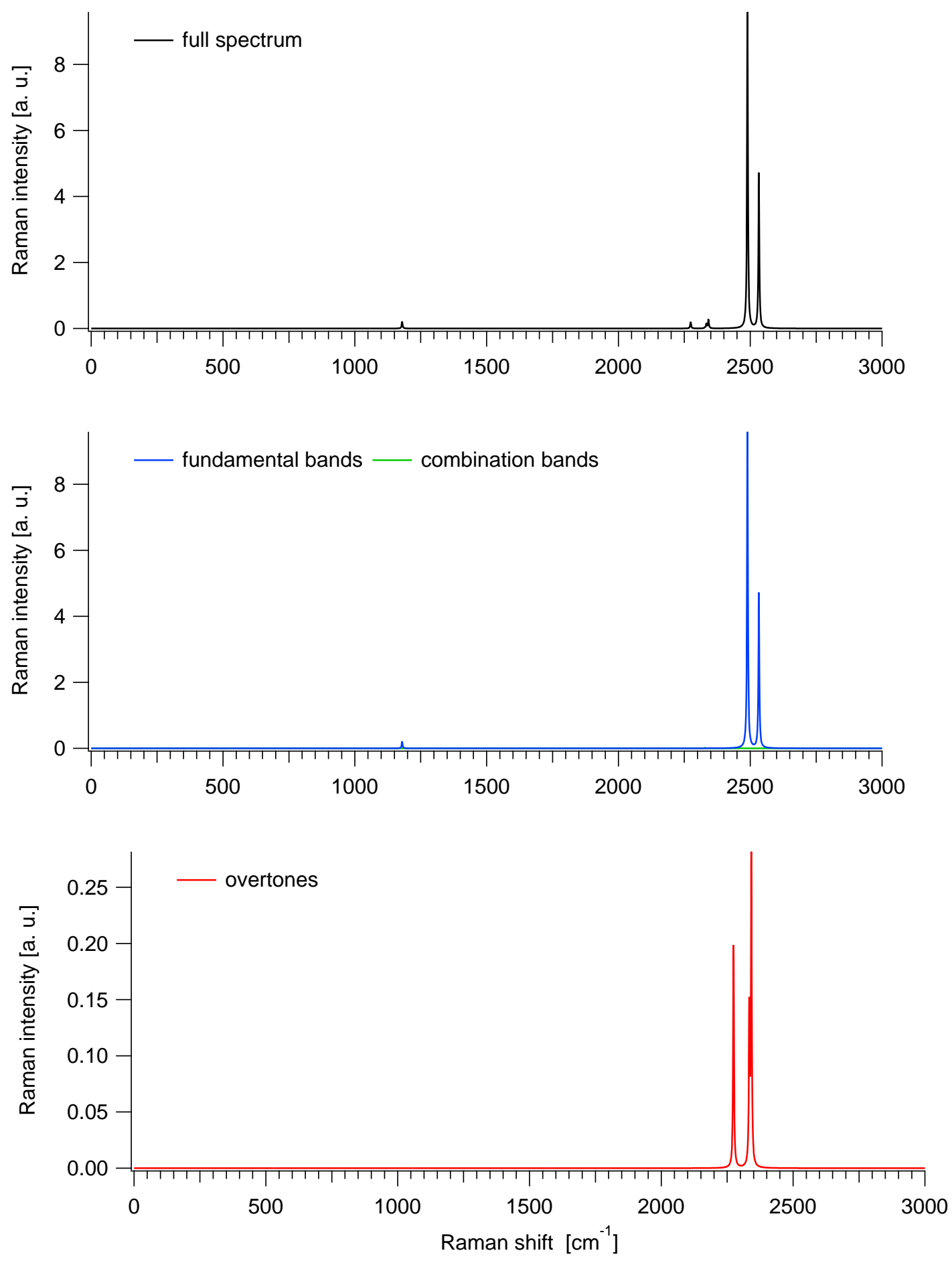
Table S.1.4. $\mathrm{BH}_{3}$ : Ideal-gas thermodynamic functions at 1 bar (anharmonic B3LYP-D2/ccpVTZ results)

\begin{tabular}{cccc}
\hline$T$ & $C_{P}$ & $S$ & $H(T)-H(0 K)$ \\
{$[\mathrm{K}]$} & {$\left[\mathrm{J} \mathrm{mol}^{-1} \mathrm{~K}^{-1}\right]$} & {$\left[\mathrm{J} \mathrm{mol}^{-1} \mathrm{~K}^{-1}\right]$} & {$\left[\mathrm{kJ} \mathrm{mol}^{-1}\right]$} \\
\hline 100 & 33.258 & 151.438 & 3.326 \\
200 & 33.657 & 174.544 & 6.661 \\
300 & 36.202 & 188.592 & 10.134 \\
400 & 40.309 & 199.551 & 13.953 \\
500 & 44.702 & 209.018 & 18.204 \\
600 & 48.929 & 217.546 & 22.888 \\
700 & 52.850 & 225.387 & 27.980 \\
800 & 56.399 & 232.680 & 33.446 \\
900 & 59.551 & 239.509 & 39.246 \\
1000 & 62.311 & 245.930 & 45.343 \\
1100 & 64.706 & 251.983 & 51.697 \\
1200 & 66.775 & 257.705 & 58.273 \\
1300 & 68.558 & 263.122 & 65.042 \\
1400 & 70.097 & 268.260 & 71.977 \\
1500 & 71.427 & 273.143 & 79.054 \\
1600 & 72.579 & 277.790 & 86.256 \\
1700 & 73.581 & 282.221 & 93.565 \\
1800 & 74.456 & 286.452 & 100.968 \\
1900 & 75.222 & 290.499 & 108.453 \\
2000 & 75.896 & 294.375 & 116.009 \\
\hline$T$ & & & $S: E$ \\
\hline
\end{tabular}

$T$ : Temperature. $C_{P}$ : Heat capacity at constant pressure. $S$ : Entropy. $H$ : Enthalpy. 
Figure S.1.4. $\mathrm{BH}_{3}$ : Ideal-gas thermodynamic functions at 1 bar (anharmonic B3LYPD2/cc-pVTZ results)
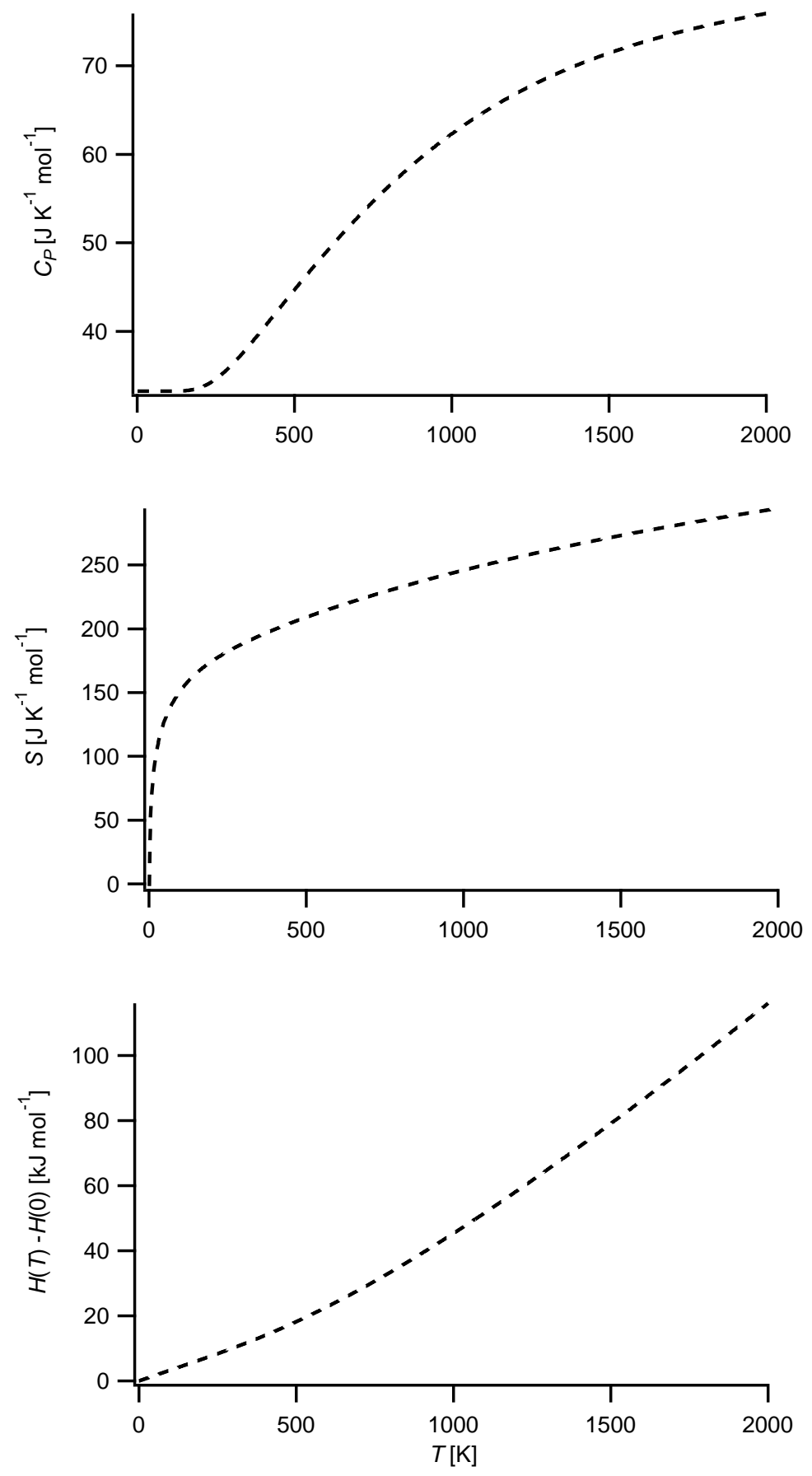

$T$ : Temperature. $C_{P}$ : Heat capacity at constant pressure. $S$ : Entropy. $H$ : Enthalpy. 
Table S.1.5. $\mathrm{BH}_{3}$ : Fits (red solid lines) of the computed thermodynamic functions (blue dashed lines) with NASA type functions (Equations (1) - (3)) in the 200 - $900 \mathrm{~K}$ temperature range (anharmonic B3LYP-D2/cc-pVTZ results). In each case, the difference curve between the thermodynamic and NASA functions is plotted in the upper graph

Fit parameters

\begin{tabular}{llllll}
\hline $\mathrm{a}_{1}$ & $4.43646625 \mathrm{e}+00$ & $\mathrm{a}_{2}\left[\mathrm{~K}^{-1}\right]$ & $-7.34715737 \mathrm{e}-03$ & $\mathrm{a}_{3}\left[\mathrm{~K}^{-2}\right]$ & $3.36216205 \mathrm{e}-05$ \\
$\mathrm{a}_{4}\left[\mathrm{~K}^{-3}\right]$ & $-3.76287308 \mathrm{e}-08$ & $\mathrm{a}_{5}\left[\mathrm{~K}^{-4}\right]$ & $1.45622595 \mathrm{e}-11$ & $\mathrm{a}_{6}[\mathrm{~K}]$ & $-1.47281413 \mathrm{e}+01$ \\
$\mathrm{a}_{7}$ & $-1.62137247 \mathrm{e}+00$ & & & &
\end{tabular}
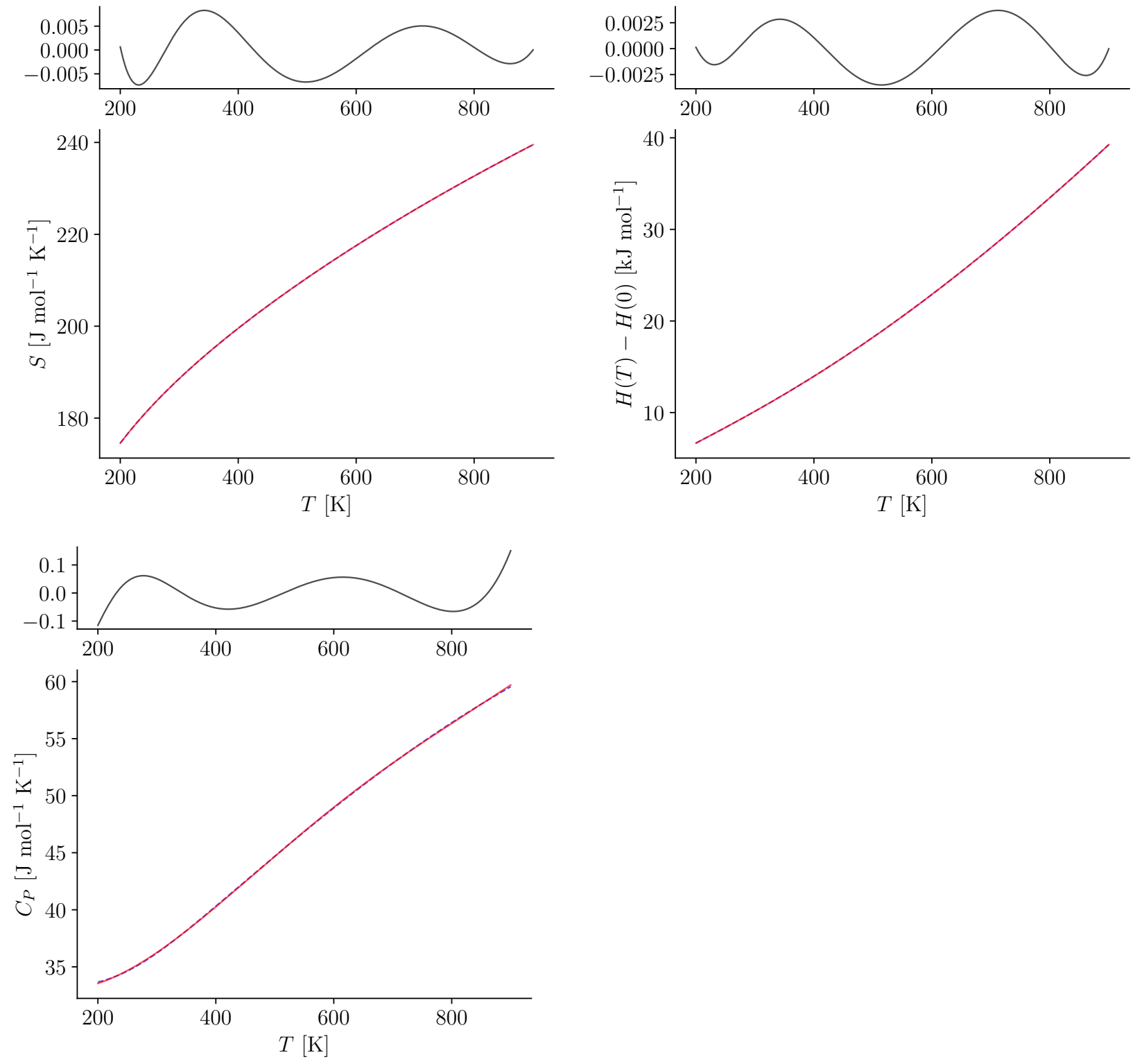


\section{$2 \mathrm{BH}_{3}$}

Figure S.2.1. Structure of $\mathrm{BH}_{3}{ }^{-}$

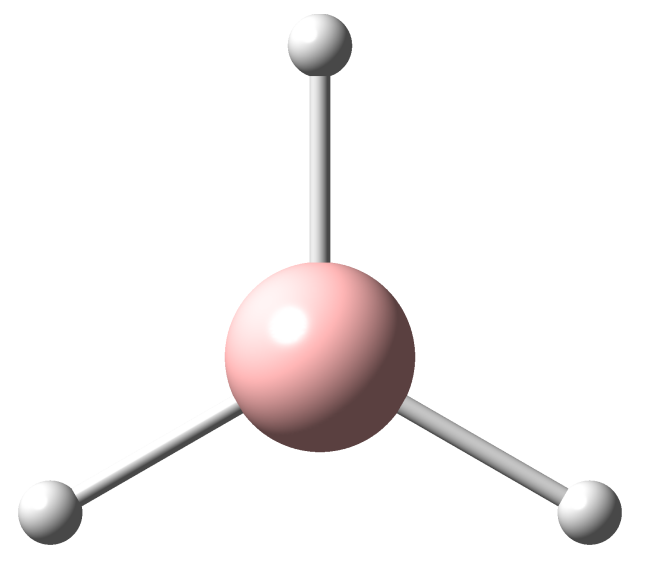

\begin{aligned} & \hline Charge -1 \\ & Spin multiplicity 1 \\ & Framework Group $\mathrm{D}_{3 h} \\ &$ Rotor Type Oblate Symmetric Top \\ & Symmetry number 6 \\ & \hline\end{aligned}

Table S.2.1. $\mathrm{BH}_{3}{ }^{-}$: Selected anharmonic results obtained at the B3LYP-D2/cc-pVTZ level

\begin{tabular}{rll}
\hline $\mathrm{ZPE}_{\mathrm{v}}$ & 4948 & $\mathrm{~cm}^{-1}$ \\
$\mathrm{~A}$ & 7.60828 & $\mathrm{~cm}^{-1}$ \\
$\mathrm{~B}$ & 7.60828 & $\mathrm{~cm}^{-1}$ \\
$\mathrm{C}$ & 3.80414 & $\mathrm{~cm}^{-1}$ \\
\hline$C_{P}(300 \mathrm{~K})$ & 40.581 & $\mathrm{~J} \mathrm{~mol}^{-1} \mathrm{~K}-1$ \\
$S(300 \mathrm{~K})$ & 191.446 & $\mathrm{~J} \mathrm{~mol}^{-1} \mathrm{~K}-1$ \\
$H(300 \mathrm{~K})-H(0 \mathrm{~K})$ & 10.651 & $\mathrm{~kJ} \mathrm{~mol}^{-1}$ \\
\hline
\end{tabular}

$\mathrm{ZPE}_{\mathrm{v}}$ : vibrational contribution to the zero-point energy. A, B, C: Rotational constants. $C_{P}$ : Heat capacity at constant pressure. $S$ : Entropy. $H$ : Enthalpy. Ideal-gas calculations (1 bar). 
Table S.2.2. $\mathrm{BH}_{3}{ }^{-}$: Cartesian coordinates $(\AA)$ of the B3LYP-D2/cc-pVTZ optimized structure

\begin{tabular}{cccc} 
atom & $\mathrm{x}$ & $\mathrm{y}$ & $\mathrm{z}$ \\
\hline B1 & 0.0000 & 0.0000 & 0.0000 \\
H2 & 0.0000 & 1.2106 & 0.0000 \\
H3 & 1.0484 & -0.6053 & -0.0000 \\
H4 & -1.0484 & -0.6053 & -0.0000 \\
\hline
\end{tabular}


Table S.2.3. $\mathrm{BH}_{3}{ }^{-}$: Vibrational frequencies, infrared integrated intensities and Raman activities (B3LYP-D2/cc-pVTZ results)

\begin{tabular}{cccccccc}
\hline & & \multicolumn{3}{c}{ Harmonic } & \multicolumn{3}{c}{ Anharmonic } \\
mode & symm. & $\omega$ & IR & Raman & $\omega$ & IR & Raman \\
& & {$\left[\mathrm{cm}^{-1}\right]$} & {$\left[\mathrm{km} \mathrm{mol}^{-1}\right]$} & {$\left[\AA^{6}\right]$} & {$\left[\mathrm{cm}^{-1}\right]$} & {$\left[\mathrm{km} \mathrm{mol}^{-1}\right]$} & {$\left[\AA^{6}\right]$} \\
\hline 1 & $\mathrm{~A}_{2}^{\prime \prime}$ & 276 & 62.783 & 0.000 & 528 & 1.104 & 0.003 \\
2 & $\mathrm{E}^{\prime}$ & 1152 & 27.008 & 0.009 & 1118 & 24.530 & 0.030 \\
3 & $\mathrm{E}^{\prime}$ & 1152 & 27.008 & 0.009 & 1118 & 24.530 & 0.030 \\
4 & $\mathrm{~A}_{1}^{\prime}$ & 2449 & 0.000 & 1.554 & 2309 & 130.341 & 1.926 \\
5 & $\mathrm{E}^{\prime}$ & 2483 & 335.289 & 0.390 & 2275 & 358.911 & 1.433 \\
6 & $\mathrm{E}^{\prime}$ & 2483 & 335.289 & 0.390 & 2275 & 358.911 & 1.433 \\
\hline
\end{tabular}


Figure S.2.2. Anharmonic IR spectrum of $\mathrm{BH}_{3}{ }^{-}$obtained by convoluting the calculated intensities with Lorentzians having a FWHM of $4 \mathrm{~cm}^{-1}$ (B3LYP-D2/cc-pVTZ results): (top) full spectrum, (middle) contributions from fundamentals and combination bands, (bottom) contributions from overtones
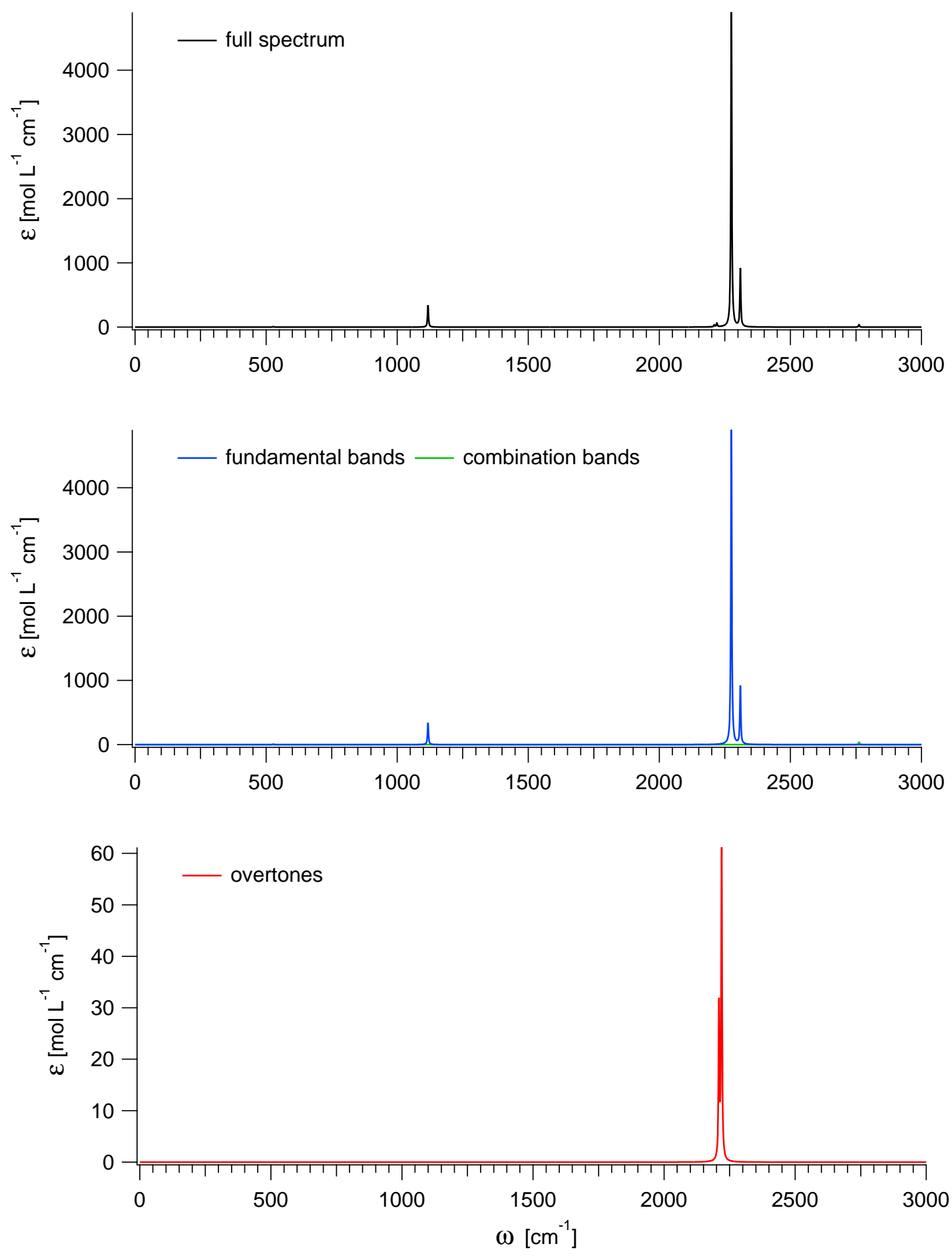
Figure S.2.3. Anharmonic Raman spectrum of $\mathrm{BH}_{3}{ }^{-}$obtained by convoluting the calculated activities with Lorentzians having a FWHM of $4 \mathrm{~cm}^{-1}$ (B3LYP-D2/cc-pVTZ results): (top) full spectrum, (middle) contributions from fundamentals and combination bands, (bottom) contributions from overtones
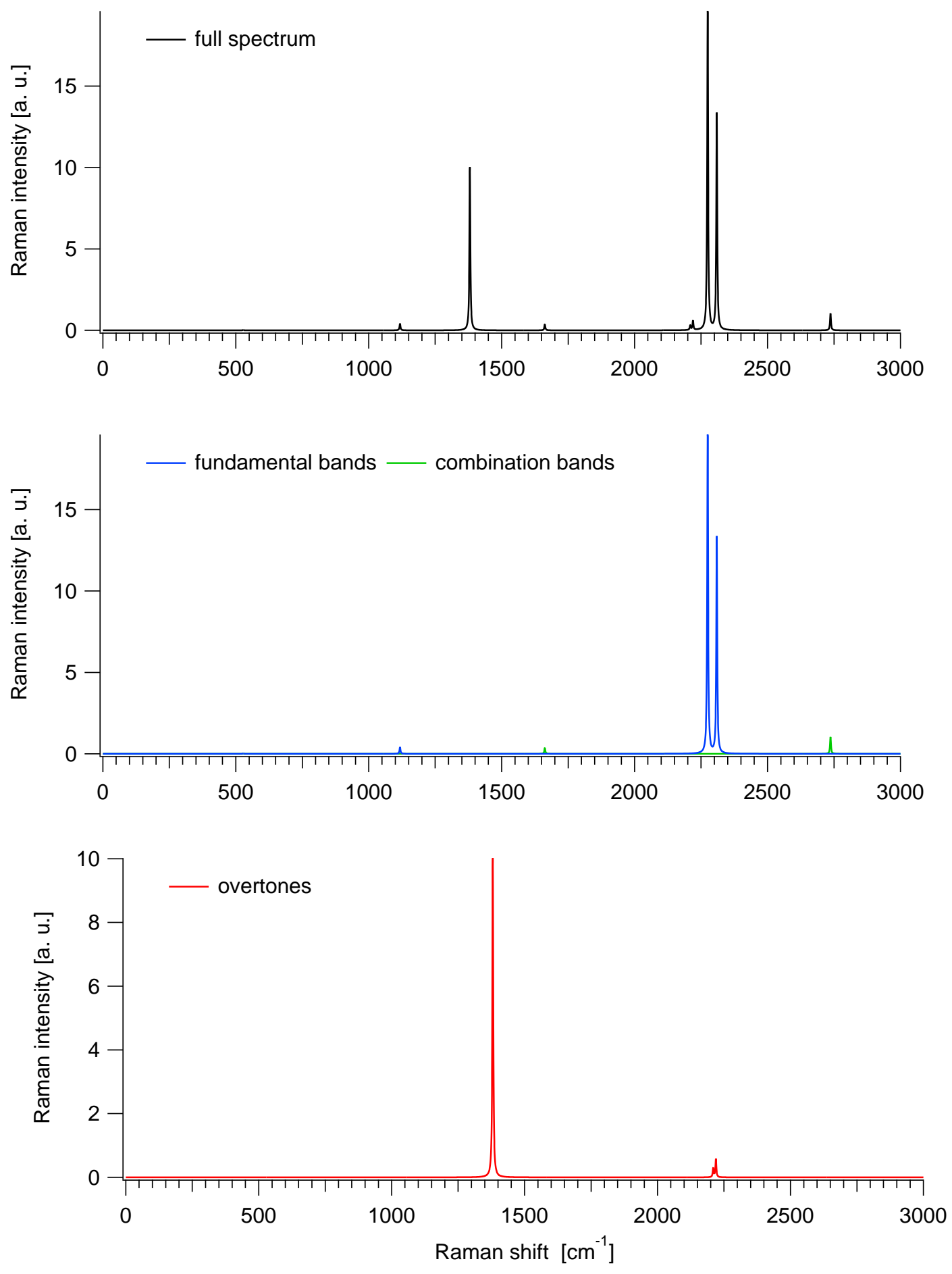
Table S.2.4. $\mathrm{BH}_{3}{ }^{-}$: Ideal-gas thermodynamic functions at 1 bar (anharmonic B3LYP$\mathrm{D} 2 /$ cc-pVTZ results)

\begin{tabular}{cccc}
\hline$T$ & $C_{P}$ & $S$ & $H(T)-H(0 K)$ \\
{$[\mathrm{K}]$} & {$\left[\mathrm{J} \mathrm{mol}^{-1} \mathrm{~K}^{-1}\right]$} & {$\left[\mathrm{J} \mathrm{mol}^{-1} \mathrm{~K}^{-1}\right]$} & {$\left[\mathrm{kJ} \mathrm{mol}^{-1}\right]$} \\
\hline 100 & 33.500 & 151.930 & 3.329 \\
200 & 36.421 & 175.909 & 6.805 \\
300 & 40.581 & 191.446 & 10.651 \\
400 & 44.925 & 203.716 & 14.927 \\
500 & 49.137 & 214.197 & 19.631 \\
600 & 53.131 & 223.513 & 24.747 \\
700 & 56.817 & 231.984 & 30.247 \\
800 & 60.119 & 239.791 & 36.097 \\
900 & 63.010 & 247.043 & 42.257 \\
1000 & 65.506 & 253.814 & 48.686 \\
1100 & 67.641 & 260.160 & 55.346 \\
1200 & 69.463 & 266.126 & 62.204 \\
1300 & 71.016 & 271.749 & 69.230 \\
1400 & 72.343 & 277.062 & 76.400 \\
1500 & 73.481 & 282.093 & 83.692 \\
1600 & 74.459 & 286.867 & 91.090 \\
1700 & 75.305 & 291.407 & 98.580 \\
1800 & 76.040 & 295.733 & 106.148 \\
1900 & 76.680 & 299.862 & 113.784 \\
2000 & 77.241 & 303.809 & 121.481 \\
\hline
\end{tabular}

$T$ : Temperature. $C_{P}$ : Heat capacity at constant pressure. $S$ : Entropy. $H$ : Enthalpy. 
Figure S.2.4. $\mathrm{BH}_{3}{ }^{-}$: Ideal-gas thermodynamic functions at 1 bar (anharmonic B3LYPD2/cc-pVTZ results)
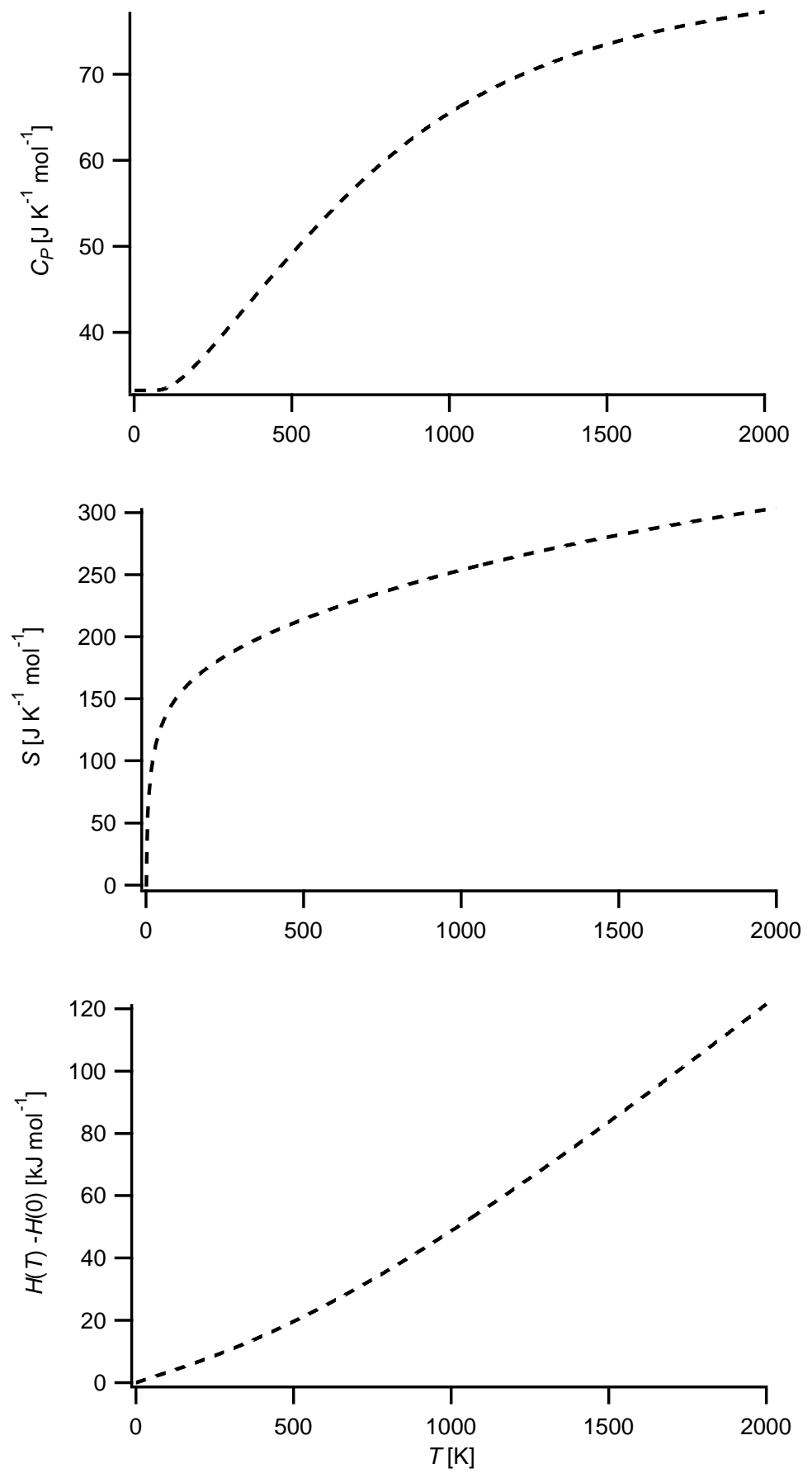

$T$ : Temperature. $C_{P}$ : Heat capacity at constant pressure. $S$ : Entropy. $H$ : Enthalpy. 
Table S.2.5. $\mathrm{BH}_{3}{ }^{-}$: Fits (red solid lines) of the computed thermodynamic functions (blue dashed lines) with NASA type functions (Equations (1) - (3)) in the 200 - $900 \mathrm{~K}$ temperature range (anharmonic B3LYP-D2/cc-pVTZ results). In each case, the difference curve between the thermodynamic and NASA functions is plotted in the upper graph

Fit parameters

\begin{tabular}{llllll}
\hline $\mathrm{a}_{1}$ & $3.51626969 \mathrm{e}+00$ & $\mathrm{a}_{2}\left[\mathrm{~K}^{-1}\right]$ & $3.41787957 \mathrm{e}-03$ & $\mathrm{a}_{3}\left[\mathrm{~K}^{-2}\right]$ & $5.69329038 \mathrm{e}-06$ \\
$\mathrm{a}_{4}\left[\mathrm{~K}^{-3}\right]$ & $-7.07535773 \mathrm{e}-09$ & $\mathrm{a}_{5}\left[\mathrm{~K}^{-4}\right]$ & $2.33876733 \mathrm{e}-12$ & $\mathrm{a}_{6}[\mathrm{~K}]$ & $3.43660996 \mathrm{e}+01$ \\
$\mathrm{a}_{7}$ & $1.74721584 \mathrm{e}+00$ & & & &
\end{tabular}
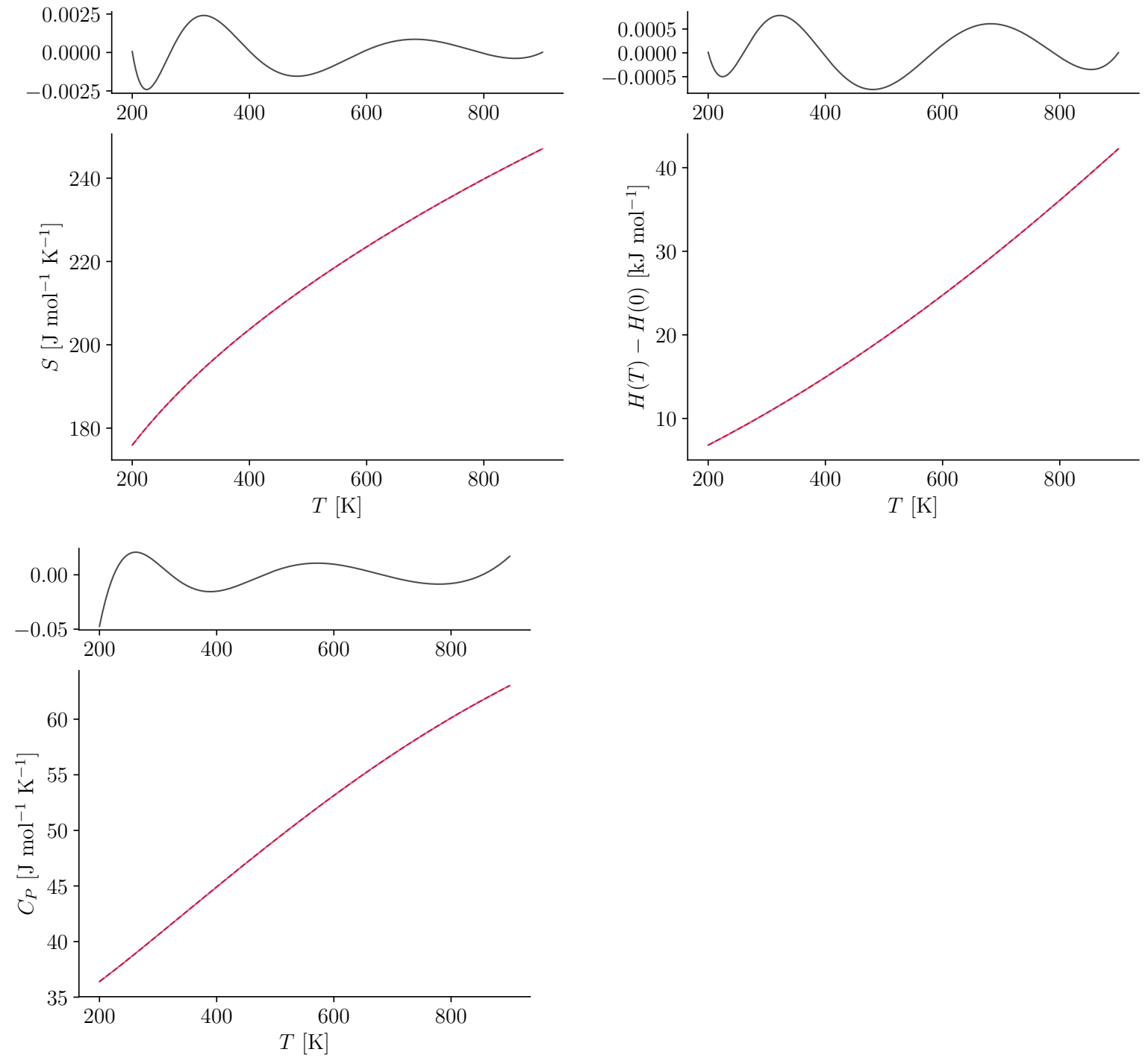


\section{$3 \mathrm{BH}_{4}$}

Figure S.3.1. Structure of $\mathrm{BH}_{4}$

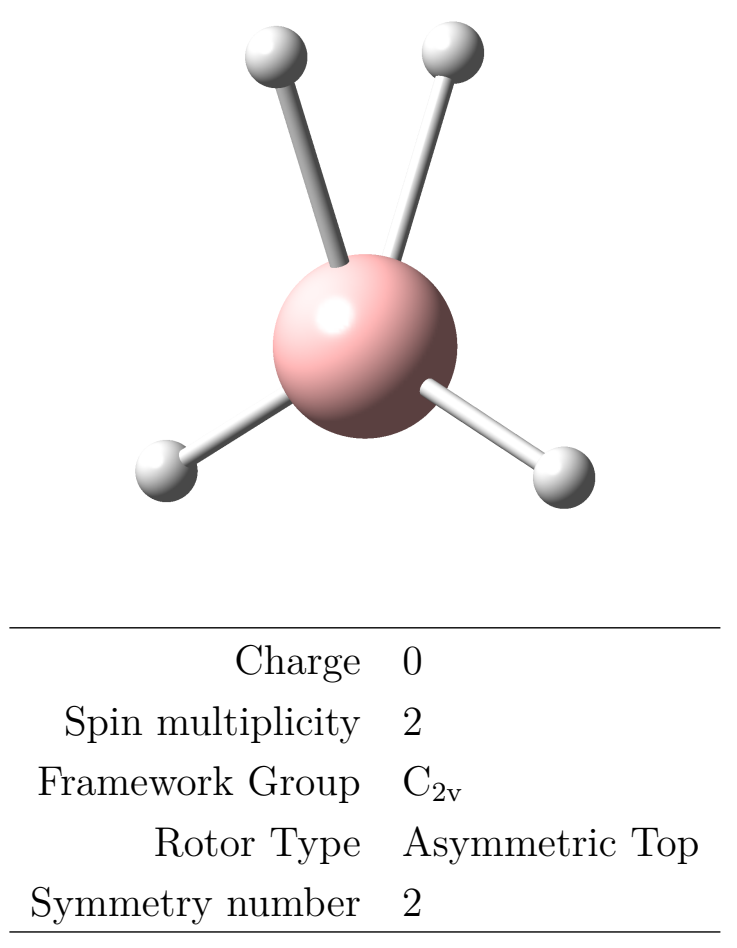

Table S.3.1. $\mathrm{BH}_{4}$ : Selected anharmonic results obtained at the B3LYP-D2/cc-pVTZ level

\begin{tabular}{rll}
\hline $\mathrm{ZPE}_{\mathrm{v}}$ & 7067 & $\mathrm{~cm}^{-1}$ \\
$\mathrm{~A}$ & 5.89803 & $\mathrm{~cm}^{-1}$ \\
$\mathrm{~B}$ & 4.49348 & $\mathrm{~cm}^{-1}$ \\
$\mathrm{C}$ & 3.09134 & $\mathrm{~cm}^{-1}$ \\
\hline$C_{P}(300 \mathrm{~K})$ & 45.286 & $\mathrm{~J} \mathrm{~mol}^{-1} \mathrm{~K}-1$ \\
$S(300 \mathrm{~K})$ & 206.649 & $\mathrm{~J} \mathrm{~mol}^{-1} \mathrm{~K}-1$ \\
$H(300 \mathrm{~K})-H(0 \mathrm{~K})$ & 10.938 & $\mathrm{~kJ} \mathrm{~mol}^{-1}$ \\
\hline
\end{tabular}

$\mathrm{ZPE}_{\mathrm{v}}$ : vibrational contribution to the zero-point energy. A, B, C: Rotational constants. $C_{P}$ : Heat capacity at constant pressure. $S$ : Entropy. $H$ : Enthalpy. Ideal-gas calculations (1 bar). 
Table S.3.2. $\mathrm{BH}_{4}$ : Cartesian coordinates $(\AA)$ of the B3LYP-D2/cc-pVTZ optimized structure

\begin{tabular}{cccc} 
atom & $\mathrm{x}$ & $\mathrm{y}$ & $\mathrm{z}$ \\
\hline B1 & 0.0000 & 0.0000 & 0.1458 \\
H2 & 0.0000 & 1.0635 & 0.6595 \\
H3 & -0.5356 & 0.0000 & -1.0239 \\
H4 & 0.5356 & -0.0000 & -1.0239 \\
H5 & -0.0000 & -1.0635 & 0.6595 \\
\hline
\end{tabular}


Table S.3.3. $\mathrm{BH}_{4}$ : Vibrational frequencies, infrared integrated intensities and Raman activities (B3LYP-D2/cc-pVTZ results)

\begin{tabular}{|c|c|c|c|c|c|c|c|}
\hline \multirow[b]{2}{*}{ mode } & \multirow[b]{2}{*}{ symm. } & \multicolumn{3}{|c|}{ Harmonic } & \multicolumn{3}{|c|}{ Anharmonic } \\
\hline & & $\begin{array}{c}\omega \\
{\left[\mathrm{cm}^{-1}\right]}\end{array}$ & $\begin{array}{c}\mathrm{IR} \\
{\left[\mathrm{km} \mathrm{mol}^{-1}\right]}\end{array}$ & $\begin{array}{c}\text { Raman } \\
{\left[\AA^{6}\right]}\end{array}$ & $\begin{array}{c}\omega \\
{\left[\mathrm{cm}^{-1}\right]}\end{array}$ & $\begin{array}{c}\mathrm{IR} \\
{\left[\mathrm{km} \mathrm{mol}^{-1}\right]}\end{array}$ & $\begin{array}{c}\text { Raman } \\
{\left[\AA^{6}\right]}\end{array}$ \\
\hline 1 & $\mathrm{~A}_{2}$ & 733 & 0.000 & 0.204 & 616 & 0.000 & 0.309 \\
\hline 2 & $\mathrm{~B}_{1}$ & 748 & 0.038 & 0.073 & 716 & 0.019 & 0.063 \\
\hline 3 & $\mathrm{~A}_{1}$ & 999 & 1.307 & 0.096 & 925 & 2.640 & 0.060 \\
\hline 4 & $\mathrm{~B}_{2}$ & 1095 & 0.852 & 0.072 & 1010 & 0.168 & 0.085 \\
\hline 5 & $\mathrm{~A}_{1}$ & 1372 & 48.065 & 0.216 & 1261 & 31.839 & 0.204 \\
\hline 6 & $\mathrm{~B}_{1}$ & 2004 & 0.165 & 0.633 & 1807 & 0.586 & 0.650 \\
\hline 7 & $\mathrm{~A}_{1}$ & 2143 & 87.224 & 0.936 & 1994 & 31.556 & 0.426 \\
\hline 8 & $\mathrm{~A}_{1}$ & 2672 & 30.359 & 0.746 & 2538 & 23.587 & 0.802 \\
\hline 9 & $\mathrm{~B}_{2}$ & 2771 & 80.449 & 0.166 & 2637 & 80.229 & 0.194 \\
\hline
\end{tabular}


Figure S.3.2. Anharmonic IR spectrum of $\mathrm{BH}_{4}$ obtained by convoluting the calculated intensities with Lorentzians having a FWHM of $4 \mathrm{~cm}^{-1}$ (B3LYP-D2/cc-pVTZ results): (top) full spectrum, (middle) contributions from fundamentals and combination bands, (bottom) contributions from overtones
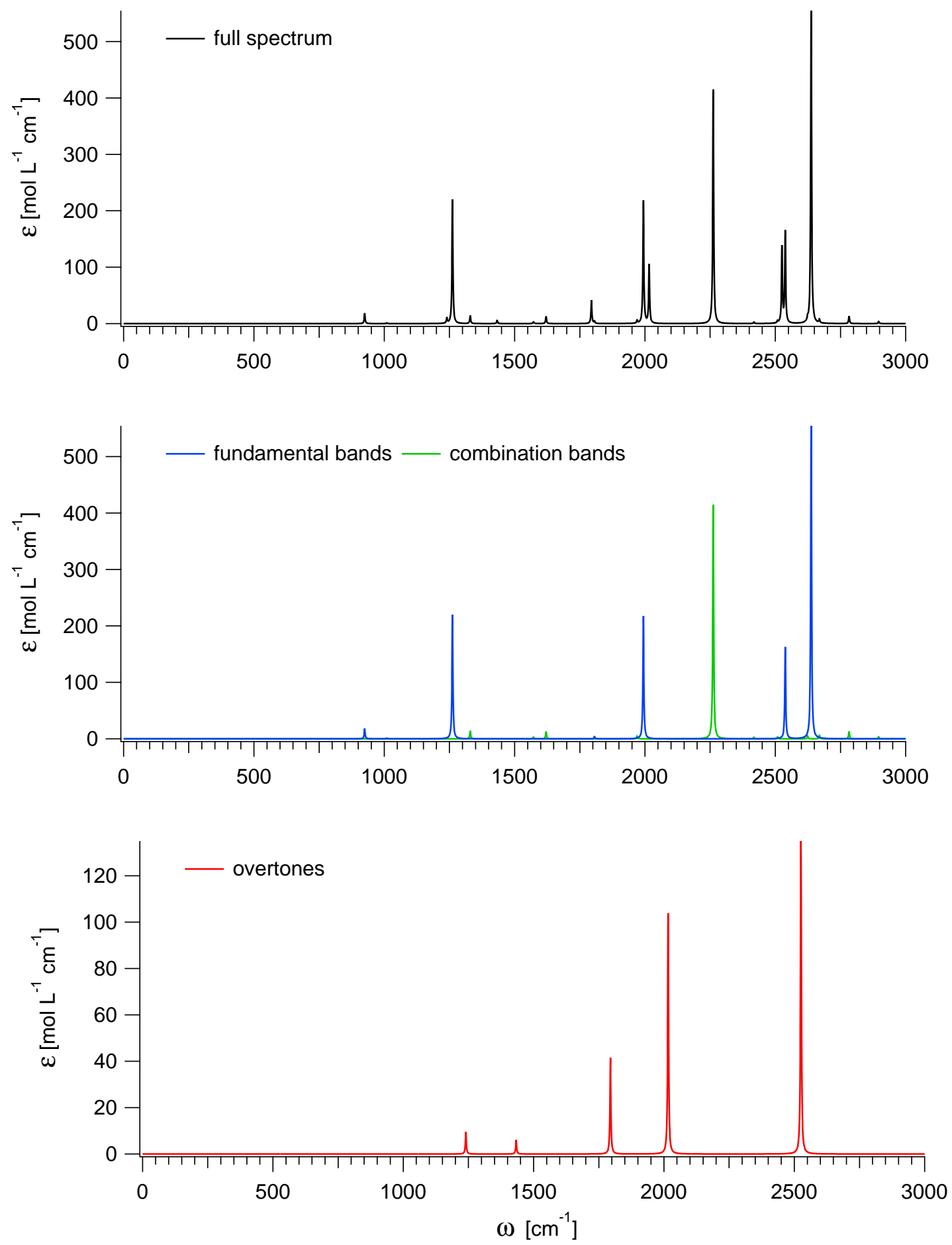
Figure S.3.3. Anharmonic Raman spectrum of $\mathrm{BH}_{4}$ obtained by convoluting the calculated activities with Lorentzians having a FWHM of $4 \mathrm{~cm}^{-1}$ (B3LYP-D2/cc-pVTZ results): (top) full spectrum, (middle) contributions from fundamentals and combination bands, (bottom) contributions from overtones
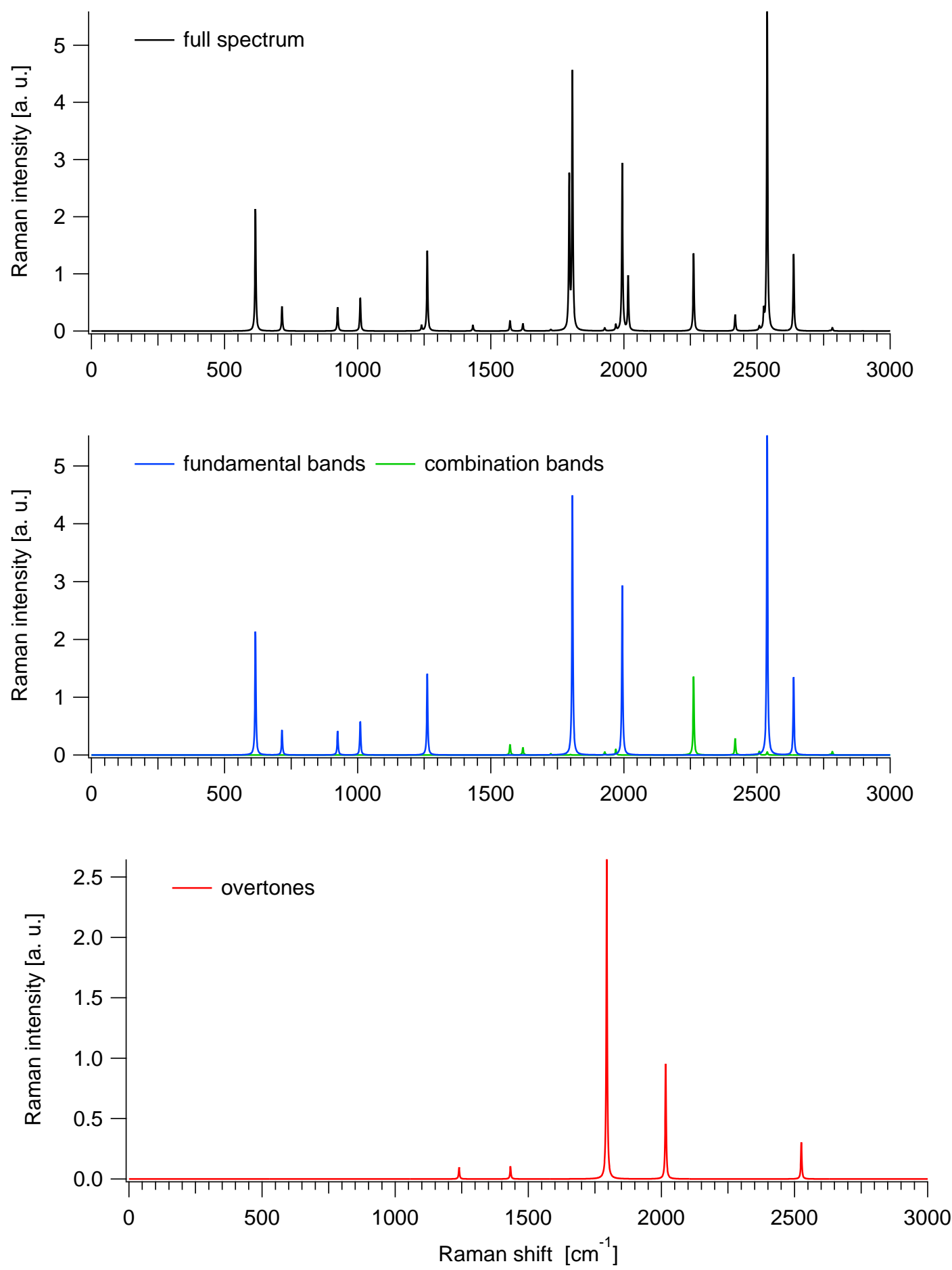
Table S.3.4. $\mathrm{BH}_{4}$ : Ideal-gas thermodynamic functions at 1 bar (anharmonic B3LYP-D2/ccpVTZ results)

\begin{tabular}{cccc}
\hline$T$ & $C_{P}$ & $S$ & $H(T)-H(0 K)$ \\
{$[\mathrm{K}]$} & {$\left[\mathrm{J} \mathrm{mol}^{-1} \mathrm{~K}^{-1}\right]$} & {$\left[\mathrm{J} \mathrm{mol}^{-1} \mathrm{~K}^{-1}\right]$} & {$\left[\mathrm{kJ} \mathrm{mol}^{-1}\right]$} \\
\hline 100 & 33.383 & 166.019 & 3.327 \\
200 & 37.408 & 190.038 & 6.815 \\
300 & 45.286 & 206.649 & 10.938 \\
400 & 53.268 & 220.786 & 15.872 \\
500 & 60.410 & 233.457 & 21.563 \\
600 & 66.683 & 245.038 & 27.925 \\
700 & 72.145 & 255.737 & 34.873 \\
800 & 76.854 & 265.686 & 42.329 \\
900 & 80.880 & 274.977 & 50.221 \\
1000 & 84.302 & 283.681 & 58.484 \\
1100 & 87.205 & 291.856 & 67.064 \\
1200 & 89.667 & 299.552 & 75.911 \\
1300 & 91.761 & 306.814 & 84.985 \\
1400 & 93.546 & 313.681 & 94.253 \\
1500 & 95.075 & 320.189 & 103.686 \\
1600 & 96.390 & 326.368 & 113.260 \\
1700 & 97.526 & 332.246 & 122.958 \\
1800 & 98.513 & 337.849 & 132.761 \\
1900 & 99.373 & 343.199 & 142.656 \\
2000 & 100.127 & 348.316 & 152.632 \\
\hline$T$ & & & $S$ \\
\hline
\end{tabular}

$T$ : Temperature. $C_{P}$ : Heat capacity at constant pressure. $S$ : Entropy. $H$ : Enthalpy. 
Figure S.3.4. $\mathrm{BH}_{4}$ : Ideal-gas thermodynamic functions at 1 bar (anharmonic B3LYPD2/cc-pVTZ results)
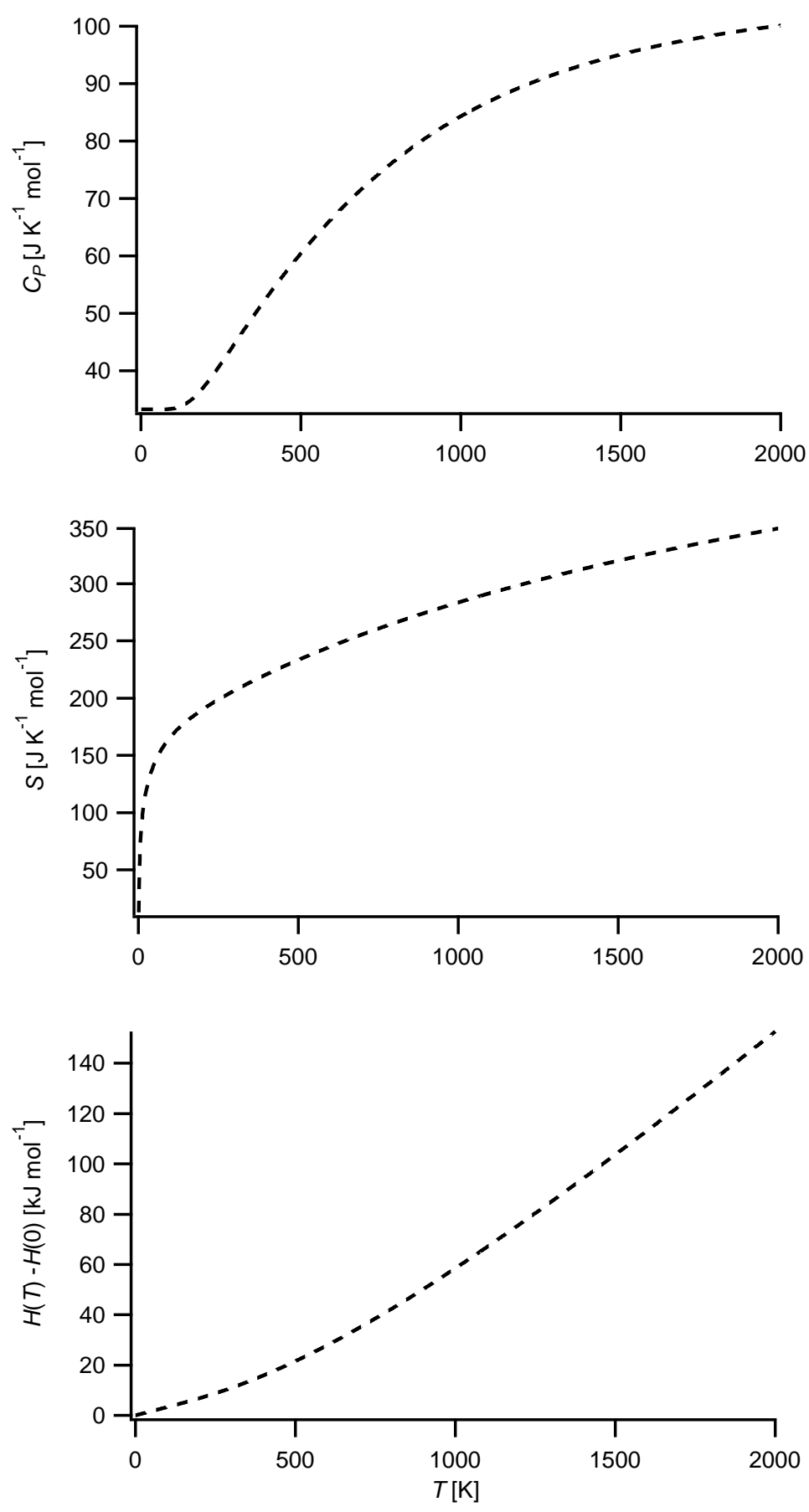

$T$ : Temperature. $C_{P}$ : Heat capacity at constant pressure. $S$ : Entropy. $H$ : Enthalpy. 
Table S.3.5. $\mathrm{BH}_{4}$ : Fits (red solid lines) of the computed thermodynamic functions (blue dashed lines) with NASA type functions (Equations (1) - (3)) in the 200 - $900 \mathrm{~K}$ temperature range (anharmonic B3LYP-D2/cc-pVTZ results). In each case, the difference curve between the thermodynamic and NASA functions is plotted in the upper graph

Fit parameters

\begin{tabular}{llllll}
\hline $\mathrm{a}_{1}$ & $2.75690268 \mathrm{e}+00$ & $\mathrm{a}_{2}\left[\mathrm{~K}^{-1}\right]$ & $6.77530806 \mathrm{e}-03$ & $\mathrm{a}_{3}\left[\mathrm{~K}^{-2}\right]$ & $1.31144691 \mathrm{e}-05$ \\
$\mathrm{a}_{4}\left[\mathrm{~K}^{-3}\right]$ & $-2.21348044 \mathrm{e}-08$ & $\mathrm{a}_{5}\left[\mathrm{~K}^{-4}\right]$ & $9.76117263 \mathrm{e}-12$ & $\mathrm{a}_{6}[\mathrm{~K}]$ & $1.06003734 \mathrm{e}+02$
\end{tabular}

$\mathrm{a}_{7}$

$6.68717786 \mathrm{e}+00$
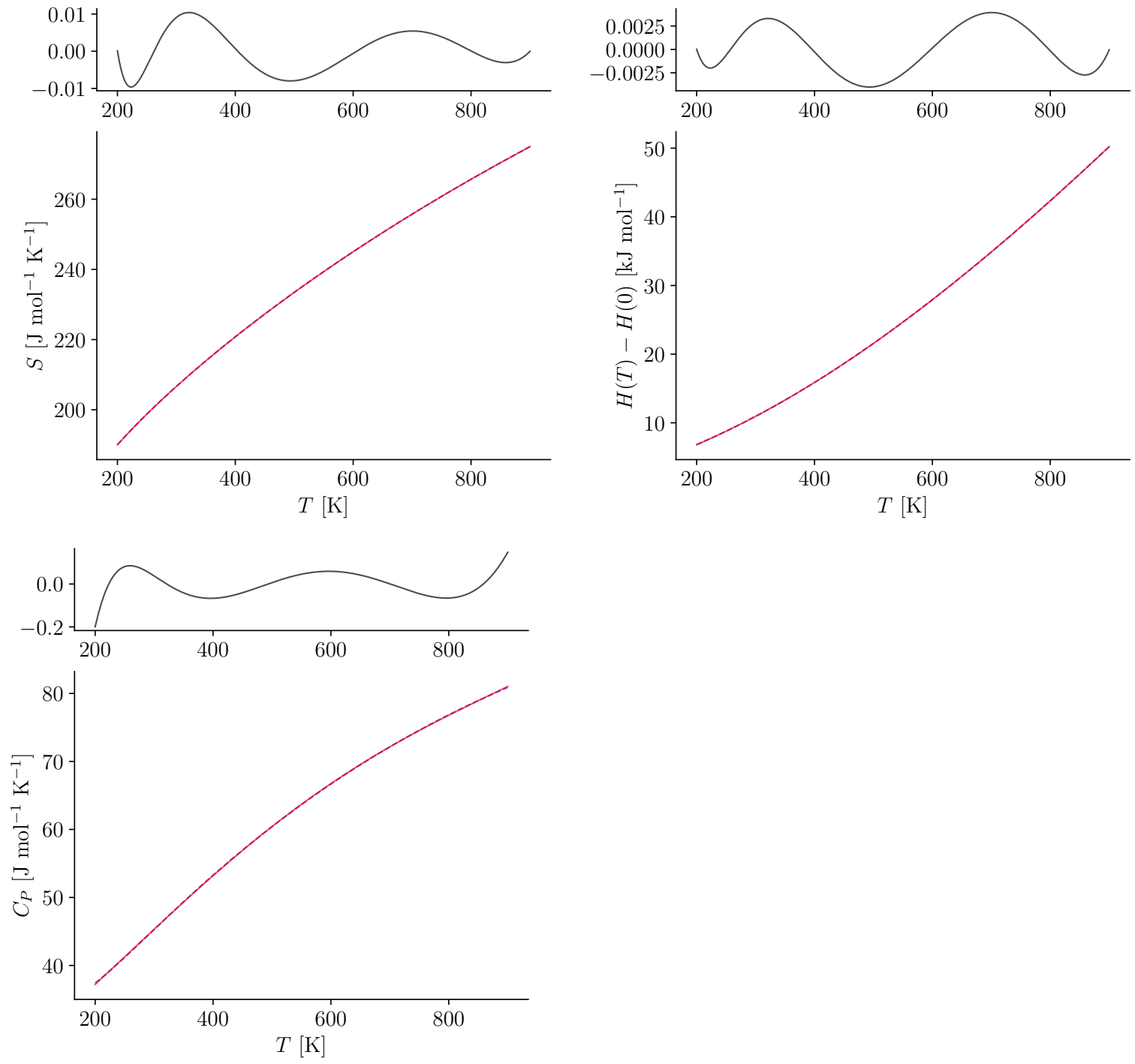


\section{$4 \mathrm{BH}_{4}$}

Figure S.4.1. Structure of $\mathrm{BH}_{4}{ }^{-}$

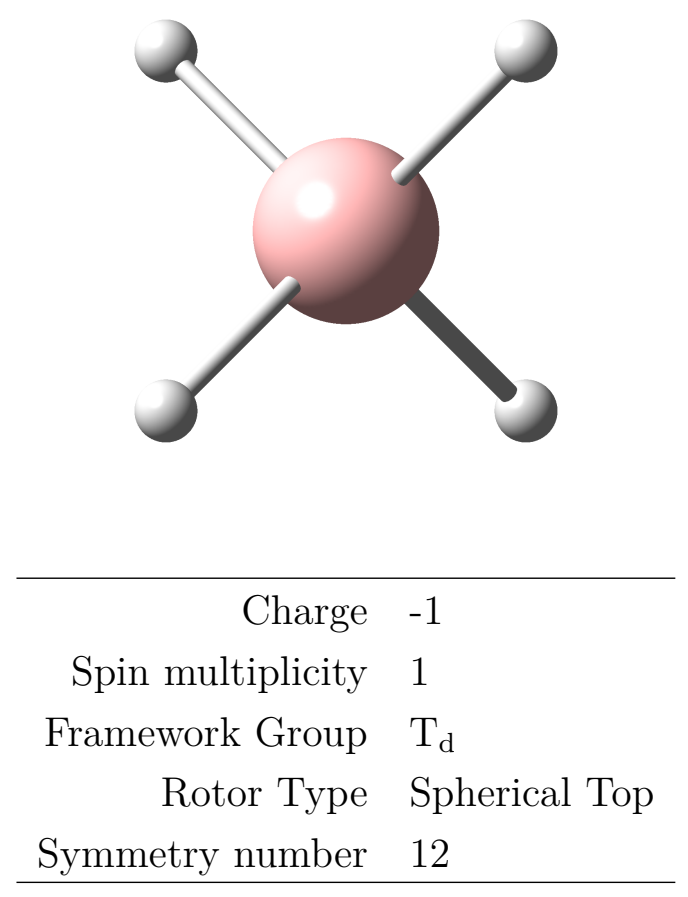

Table S.4.1. $\mathrm{BH}_{4}{ }^{-}$: Selected anharmonic results obtained at the B3LYP-D2/cc-pVTZ level

\begin{tabular}{rll}
\hline $\mathrm{ZPE}_{\mathrm{v}}$ & 7298 & $\mathrm{~cm}^{-1}$ \\
$\mathrm{~A}$ & 4.09844 & $\mathrm{~cm}^{-1}$ \\
$\mathrm{~B}$ & 4.09844 & $\mathrm{~cm}^{-1}$ \\
$\mathrm{C}$ & 4.09844 & $\mathrm{~cm}^{-1}$ \\
\hline$C_{P}(300 \mathrm{~K})$ & 39.238 & $\mathrm{~J} \mathrm{~mol}^{-1} \mathrm{~K}^{-1}$ \\
$S(300 \mathrm{~K})$ & 189.694 & $\mathrm{~J} \mathrm{~mol}^{-1} \mathrm{~K}-1$ \\
$H(300 \mathrm{~K})-H(0 \mathrm{~K})$ & 10.312 & $\mathrm{~kJ} \mathrm{~mol}^{-1}$ \\
\hline
\end{tabular}

$\mathrm{ZPE}_{\mathrm{v}}$ : vibrational contribution to the zero-point energy. A, B, C: Rotational constants. $C_{P}$ : Heat capacity at constant pressure. $S$ : Entropy. $H$ : Enthalpy. Ideal-gas calculations (1 bar). 
Table S.4.2. $\mathrm{BH}_{4}{ }^{-}$: Cartesian coordinates $(\AA)$ of the B3LYP-D2/cc-pVTZ optimized structure

\begin{tabular}{cccc} 
atom & $\mathrm{x}$ & $\mathrm{y}$ & $\mathrm{z}$ \\
\hline B1 & 0.0000 & 0.0000 & 0.0000 \\
H2 & 0.7143 & 0.7143 & 0.7143 \\
H3 & -0.7143 & -0.7143 & 0.7143 \\
H4 & -0.7143 & 0.7143 & -0.7143 \\
H5 & 0.7143 & -0.7143 & -0.7143 \\
\hline
\end{tabular}


Table S.4.3. $\mathrm{BH}_{4}{ }^{-}$: Vibrational frequencies, infrared integrated intensities and Raman activities (B3LYP-D2/cc-pVTZ results)

\begin{tabular}{cccccccc}
\hline & & \multicolumn{3}{c}{ Harmonic } & \multicolumn{3}{c}{ Anharmonic } \\
mode & symm. & $\omega$ & IR & Raman & $\omega$ & IR & Raman \\
& & {$\left[\mathrm{cm}^{-1}\right]$} & {$\left[\mathrm{km} \mathrm{mol}^{-1}\right]$} & {$\left[\AA^{6}\right]$} & {$\left[\mathrm{cm}^{-1}\right]$} & {$\left[\mathrm{km} \mathrm{mol}^{-1}\right]$} & {$\left[\AA^{6}\right]$} \\
\hline 1 & $\mathrm{~T}_{2}$ & 1113 & 44.423 & 0.002 & 1071 & 44.017 & 0.000 \\
2 & $\mathrm{~T}_{2}$ & 1113 & 44.423 & 0.002 & 1066 & 43.819 & 0.000 \\
3 & $\mathrm{~T}_{2}$ & 1113 & 44.423 & 0.002 & 1061 & 43.625 & 0.000 \\
4 & $\mathrm{E}$ & 1233 & 0.000 & 0.658 & 1178 & 0.000 & 0.733 \\
5 & $\mathrm{E}$ & 1233 & 0.000 & 0.658 & 1178 & 0.000 & 0.733 \\
6 & $\mathrm{~T}_{2}$ & 2259 & 515.023 & 0.869 & 2102 & 235.897 & 0.557 \\
7 & $\mathrm{~T}_{2}$ & 2259 & 515.023 & 0.869 & 2086 & 237.703 & 0.595 \\
8 & $\mathrm{~T}_{2}$ & 2259 & 515.023 & 0.869 & 2124 & 70.771 & 0.146 \\
9 & $\mathrm{~A}_{1}$ & 2328 & 0.000 & 1.715 & 2214 & 18.107 & 1.623 \\
\hline
\end{tabular}


Figure S.4.2. Anharmonic IR spectrum of $\mathrm{BH}_{4}{ }^{-}$obtained by convoluting the calculated intensities with Lorentzians having a FWHM of $4 \mathrm{~cm}^{-1}$ (B3LYP-D2/cc-pVTZ results): (top) full spectrum, (middle) contributions from fundamentals and combination bands, (bottom) contributions from overtones
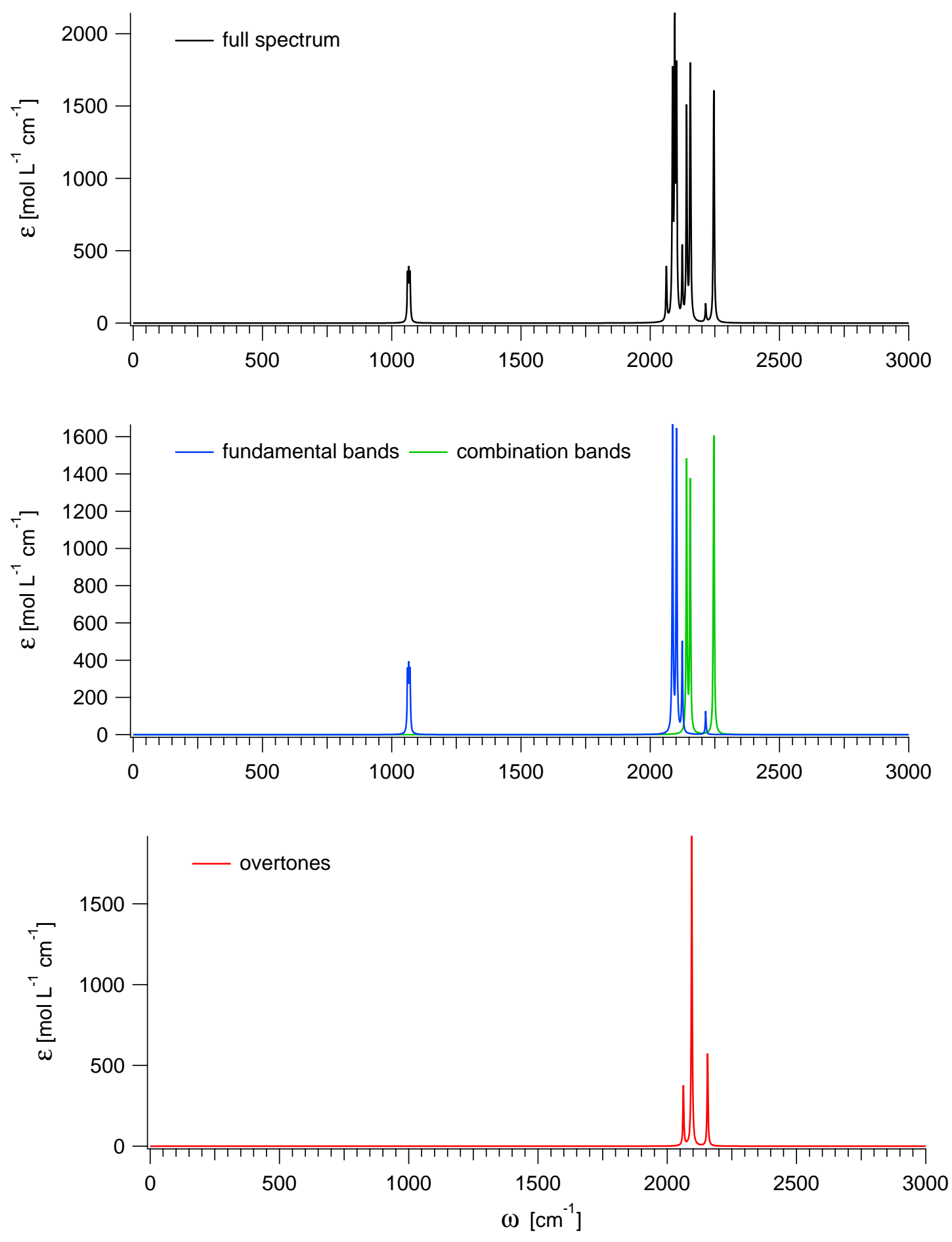
Figure S.4.3. Anharmonic Raman spectrum of $\mathrm{BH}_{4}{ }^{-}$obtained by convoluting the calculated activities with Lorentzians having a FWHM of $4 \mathrm{~cm}^{-1}$ (B3LYP-D2/cc-pVTZ results): (top) full spectrum, (middle) contributions from fundamentals and combination bands, (bottom) contributions from overtones
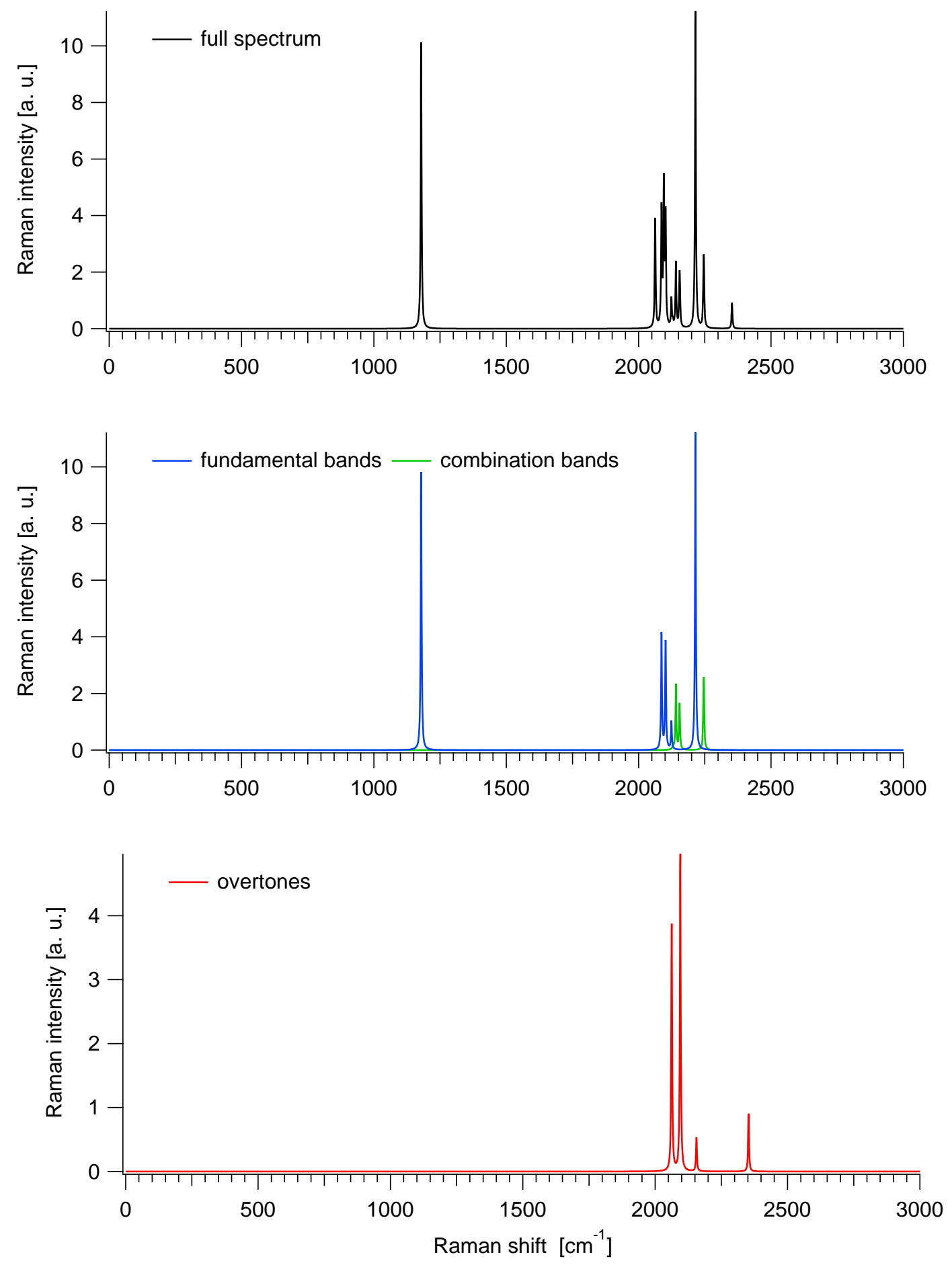
Table S.4.4. $\mathrm{BH}_{4}{ }^{-}$: Ideal-gas thermodynamic functions at 1 bar (anharmonic B3LYP$\mathrm{D} 2 / \mathrm{cc}-\mathrm{pVTZ}$ results)

\begin{tabular}{cccc}
\hline$T$ & $C_{P}$ & $S$ & $H(T)-H(0 K)$ \\
{$[\mathrm{K}]$} & {$\left[\mathrm{J} \mathrm{mol}^{-1} \mathrm{~K}^{-1}\right]$} & {$\left[\mathrm{J} \mathrm{mol}^{-1} \mathrm{~K}^{-1}\right]$} & {$\left[\mathrm{kJ} \mathrm{mol}^{-1}\right]$} \\
\hline 100 & 33.259 & 151.830 & 3.326 \\
200 & 34.195 & 175.017 & 6.675 \\
300 & 39.238 & 189.694 & 10.312 \\
400 & 46.899 & 202.003 & 14.609 \\
500 & 54.905 & 213.334 & 19.702 \\
600 & 62.327 & 224.012 & 25.570 \\
700 & 68.852 & 234.122 & 32.137 \\
800 & 74.425 & 243.690 & 39.309 \\
900 & 79.111 & 252.735 & 46.992 \\
1000 & 83.023 & 261.279 & 55.105 \\
1100 & 86.285 & 269.349 & 63.575 \\
1200 & 89.008 & 276.978 & 72.344 \\
1300 & 91.292 & 284.195 & 81.362 \\
1400 & 93.216 & 291.033 & 90.590 \\
1500 & 94.846 & 297.521 & 99.996 \\
1600 & 96.235 & 303.688 & 109.552 \\
1700 & 97.427 & 309.559 & 119.236 \\
1800 & 98.454 & 315.157 & 129.031 \\
1900 & 99.345 & 320.505 & 138.922 \\
2000 & 100.121 & 325.621 & 148.897 \\
\hline
\end{tabular}

$T$ : Temperature. $C_{P}$ : Heat capacity at constant pressure. $S$ : Entropy. $H$ : Enthalpy. 
Figure S.4.4. $\mathrm{BH}_{4}{ }^{-}$: Ideal-gas thermodynamic functions at 1 bar (anharmonic B3LYPD2/cc-pVTZ results)
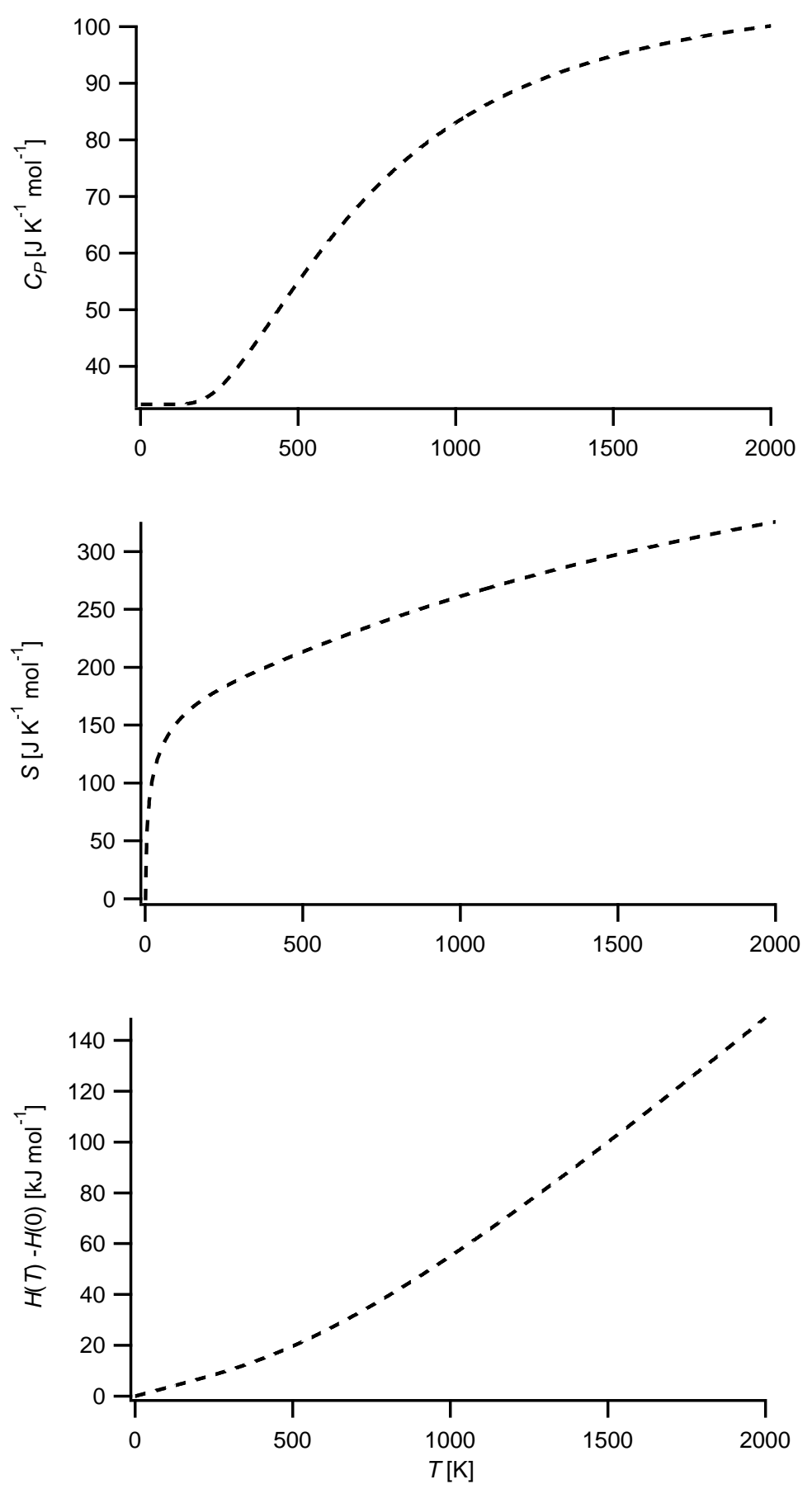

$T$ : Temperature. $C_{P}$ : Heat capacity at constant pressure. $S$ : Entropy. $H$ : Enthalpy. 
Table S.4.5. $\mathrm{BH}_{4}{ }^{-}$: Fits (red solid lines) of the computed thermodynamic functions (blue dashed lines) with NASA type functions (Equations (1) - (3)) in the 200 - $900 \mathrm{~K}$ temperature range (anharmonic B3LYP-D2/cc-pVTZ results). In each case, the difference curve between the thermodynamic and NASA functions is plotted in the upper graph

Fit parameters

\begin{tabular}{llllll}
\hline $\mathrm{a}_{1}$ & $4.80878961 \mathrm{e}+00$ & $\mathrm{a}_{2}\left[\mathrm{~K}^{-1}\right]$ & $-1.38063288 \mathrm{e}-02$ & $\mathrm{a}_{3}\left[\mathrm{~K}^{-2}\right]$ & $6.50008596 \mathrm{e}-05$ \\
$\mathrm{a}_{4}\left[\mathrm{~K}^{-3}\right]$ & $-7.51198500 \mathrm{e}-08$ & $\mathrm{a}_{5}\left[\mathrm{~K}^{-4}\right]$ & $2.93805256 \mathrm{e}-11$ & $\mathrm{a}_{6}[\mathrm{~K}]$ & $-2.79220100 \mathrm{e}+01$ \\
$\mathrm{a}_{7}$ & $-2.77891582 \mathrm{e}+00$ & & & &
\end{tabular}
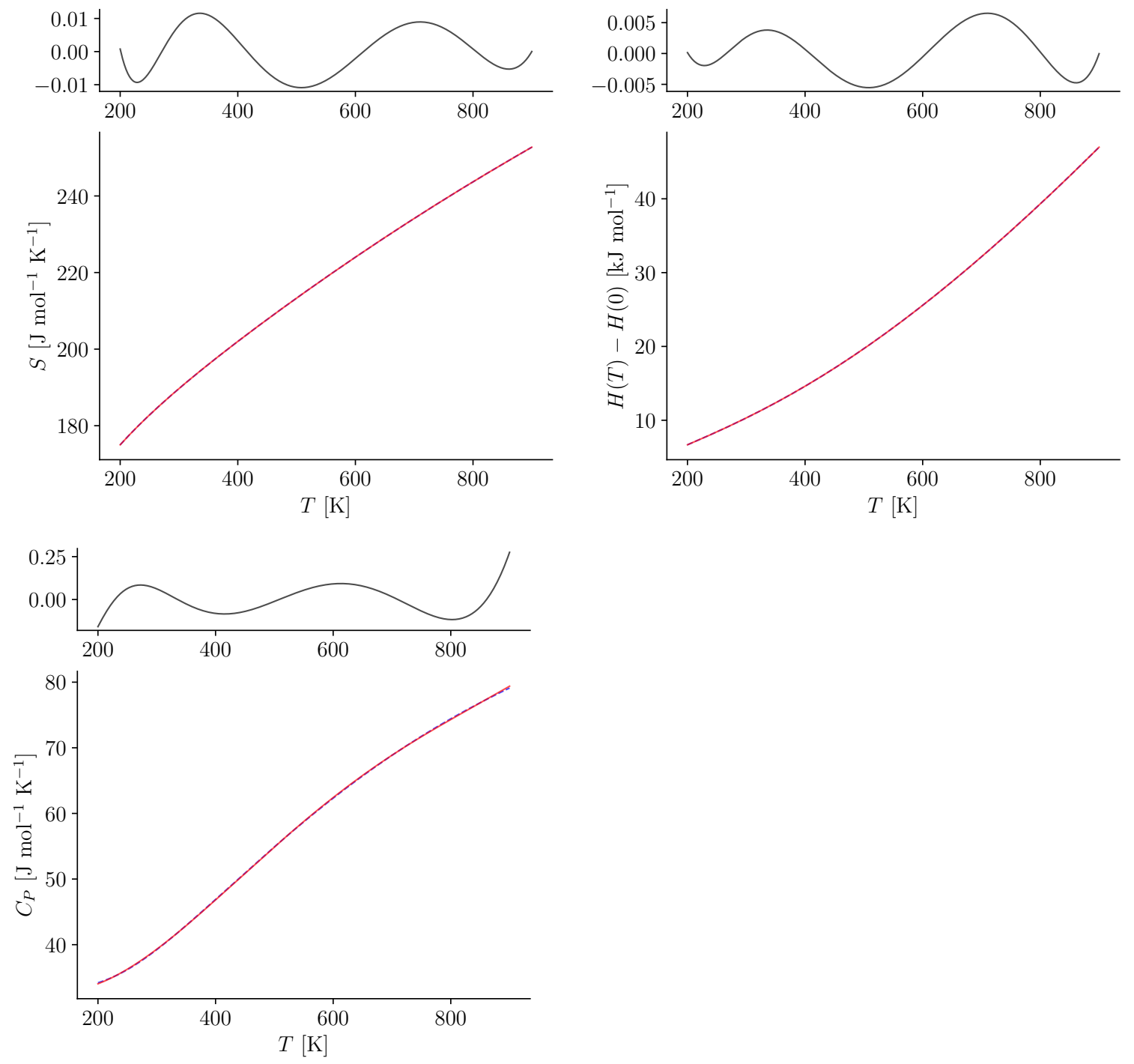


\section{$5 \quad \mathbf{B}_{2} \mathbf{H}_{3}$}

Figure S.5.1. Structure of $\mathrm{B}_{2} \mathrm{H}_{3}$

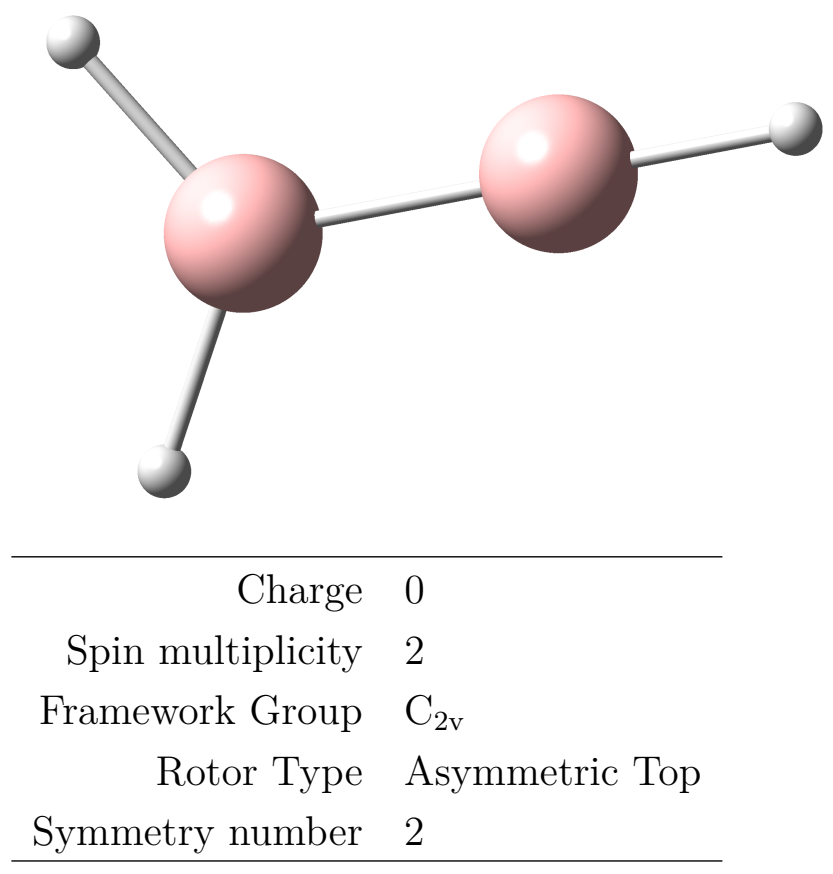

Table S.5.1. $\mathrm{B}_{2} \mathrm{H}_{3}$ : Selected anharmonic results obtained at the B3LYP-D2/cc-pVTZ level

\begin{tabular}{rll}
\hline $\mathrm{ZPE}_{\mathrm{v}}$ & 6258 & $\mathrm{~cm}^{-1}$ \\
$\mathrm{~A}$ & 7.96659 & $\mathrm{~cm}^{-1}$ \\
$\mathrm{~B}$ & 0.79581 & $\mathrm{~cm}^{-1}$ \\
$\mathrm{C}$ & 0.72353 & $\mathrm{~cm}^{-1}$ \\
\hline$C_{P}(300 \mathrm{~K})$ & 53.343 & $\mathrm{~J} \mathrm{~mol}^{-1} \mathrm{~K}-1$ \\
$S(300 \mathrm{~K})$ & 231.764 & $\mathrm{~J} \mathrm{~mol}^{-1} \mathrm{~K}-1$ \\
$H(300 \mathrm{~K})-H(0 \mathrm{~K})$ & 12.216 & $\mathrm{~kJ} \mathrm{~mol}^{-1}$ \\
\hline
\end{tabular}

$\mathrm{ZPE}_{\mathrm{v}}$ : vibrational contribution to the zero-point energy. A, B, C: Rotational constants. $C_{P}$ : Heat capacity at constant pressure. $S$ : Entropy. $H$ : Enthalpy. Ideal-gas calculations (1 bar). 
Table S.5.2. $\mathrm{B}_{2} \mathrm{H}_{3}$ : Cartesian coordinates $(\AA)$ of the B3LYP-D2/cc-pVTZ optimized structure

\begin{tabular}{cccc} 
atom & $\mathrm{x}$ & $\mathrm{y}$ & $\mathrm{z}$ \\
\hline B1 & 0.0000 & -0.0000 & -0.7163 \\
B2 & -0.0000 & 0.0000 & 0.8453 \\
H3 & -0.0000 & 0.0000 & 2.0199 \\
H4 & 0.0000 & 1.0246 & -1.3327 \\
H5 & -0.0000 & -1.0246 & -1.3327 \\
\hline
\end{tabular}


Table S.5.3. $\mathrm{B}_{2} \mathrm{H}_{3}$ : Vibrational frequencies, infrared integrated intensities and Raman activities (B3LYP-D2/cc-pVTZ results)

\begin{tabular}{|c|c|c|c|c|c|c|c|}
\hline \multirow[b]{2}{*}{ mode } & \multirow[b]{2}{*}{ symm. } & \multicolumn{3}{|c|}{ Harmonic } & \multicolumn{3}{|c|}{ Anharmonic } \\
\hline & & $\begin{array}{c}\omega \\
{\left[\mathrm{cm}^{-1}\right]}\end{array}$ & $\begin{array}{c}\mathrm{IR} \\
{\left[\mathrm{km} \mathrm{mol}^{-1}\right]}\end{array}$ & $\begin{array}{c}\text { Raman } \\
{\left[\AA^{6}\right]}\end{array}$ & $\begin{array}{c}\omega \\
{\left[\mathrm{cm}^{-1}\right]}\end{array}$ & $\begin{array}{c}\mathrm{IR} \\
{\left[\mathrm{km} \mathrm{mol}^{-1}\right]}\end{array}$ & $\begin{array}{c}\text { Raman } \\
{\left[\AA^{6}\right]}\end{array}$ \\
\hline 1 & $\mathrm{~B}_{2}$ & 334 & 0.000 & 0.012 & 302 & 0.047 & 0.007 \\
\hline 2 & $\mathrm{~B}_{1}$ & 515 & 1.418 & 0.465 & 510 & 1.353 & 0.365 \\
\hline 3 & $\mathrm{~B}_{1}$ & 792 & 6.958 & 0.065 & 773 & 6.290 & 0.065 \\
\hline 4 & $\mathrm{~B}_{2}$ & 894 & 16.546 & 0.000 & 881 & 14.521 & 0.000 \\
\hline 5 & $\mathrm{~A}_{1}$ & 1020 & 0.308 & 0.971 & 1000 & 0.040 & 0.910 \\
\hline 6 & $\mathrm{~A}_{1}$ & 1174 & 0.888 & 0.162 & 1126 & 1.631 & 0.231 \\
\hline 7 & $\mathrm{~A}_{1}$ & 2577 & 18.103 & 1.037 & 2464 & 13.499 & 1.163 \\
\hline 8 & $\mathrm{~B}_{2}$ & 2613 & 45.842 & 0.598 & 2501 & 48.425 & 0.619 \\
\hline 9 & $\mathrm{~A}_{1}$ & 2787 & 26.223 & 0.520 & 2690 & 30.719 & 0.570 \\
\hline
\end{tabular}


Figure S.5.2. Anharmonic IR spectrum of $\mathrm{B}_{2} \mathrm{H}_{3}$ obtained by convoluting the calculated intensities with Lorentzians having a FWHM of $4 \mathrm{~cm}^{-1}$ (B3LYP-D2/cc-pVTZ results): (top) full spectrum, (middle) contributions from fundamentals and combination bands, (bottom) contributions from overtones
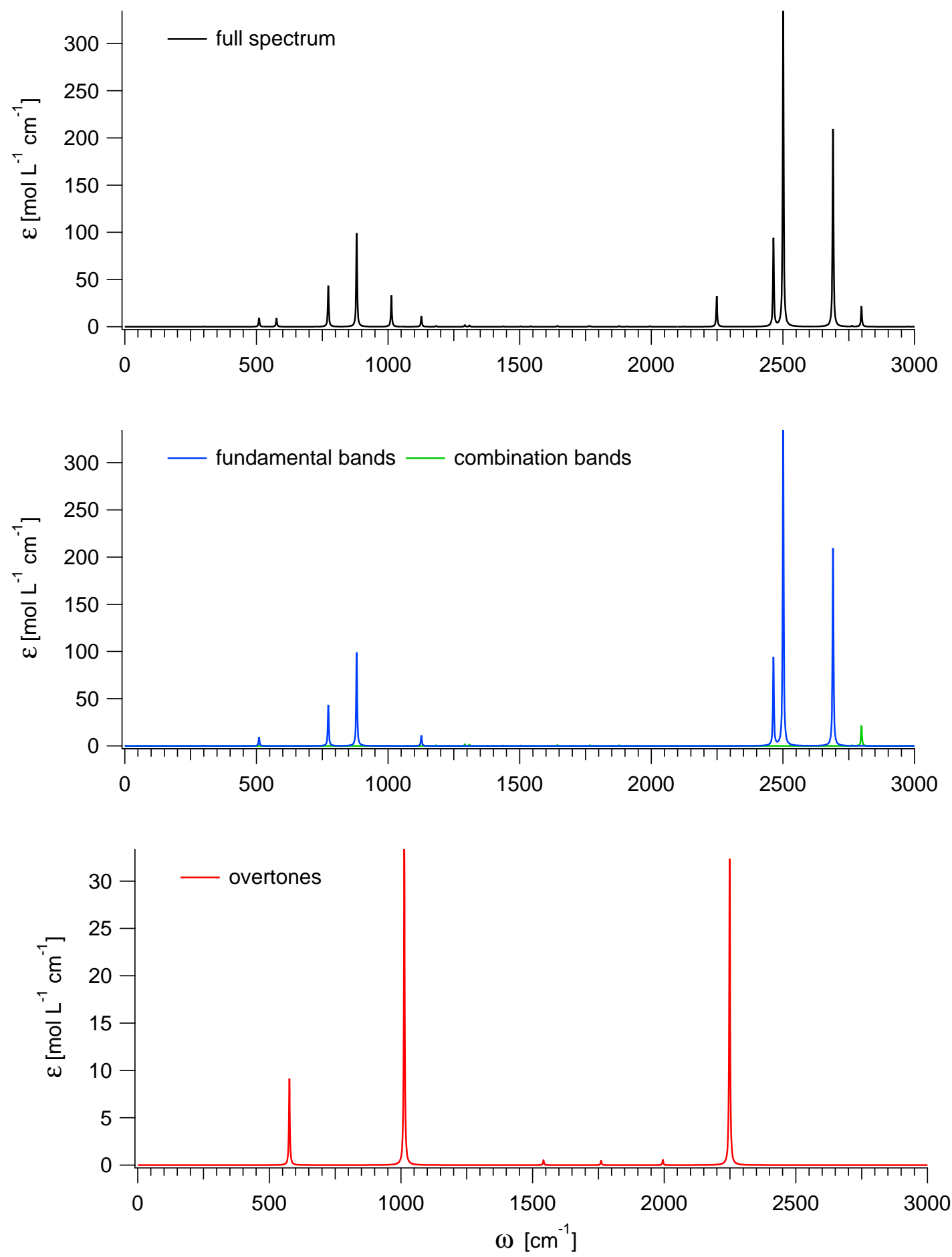
Figure S.5.3. Anharmonic Raman spectrum of $\mathrm{B}_{2} \mathrm{H}_{3}$ obtained by convoluting the calculated activities with Lorentzians having a FWHM of $4 \mathrm{~cm}^{-1}$ (B3LYP-D2/cc-pVTZ results): (top) full spectrum, (middle) contributions from fundamentals and combination bands, (bottom) contributions from overtones
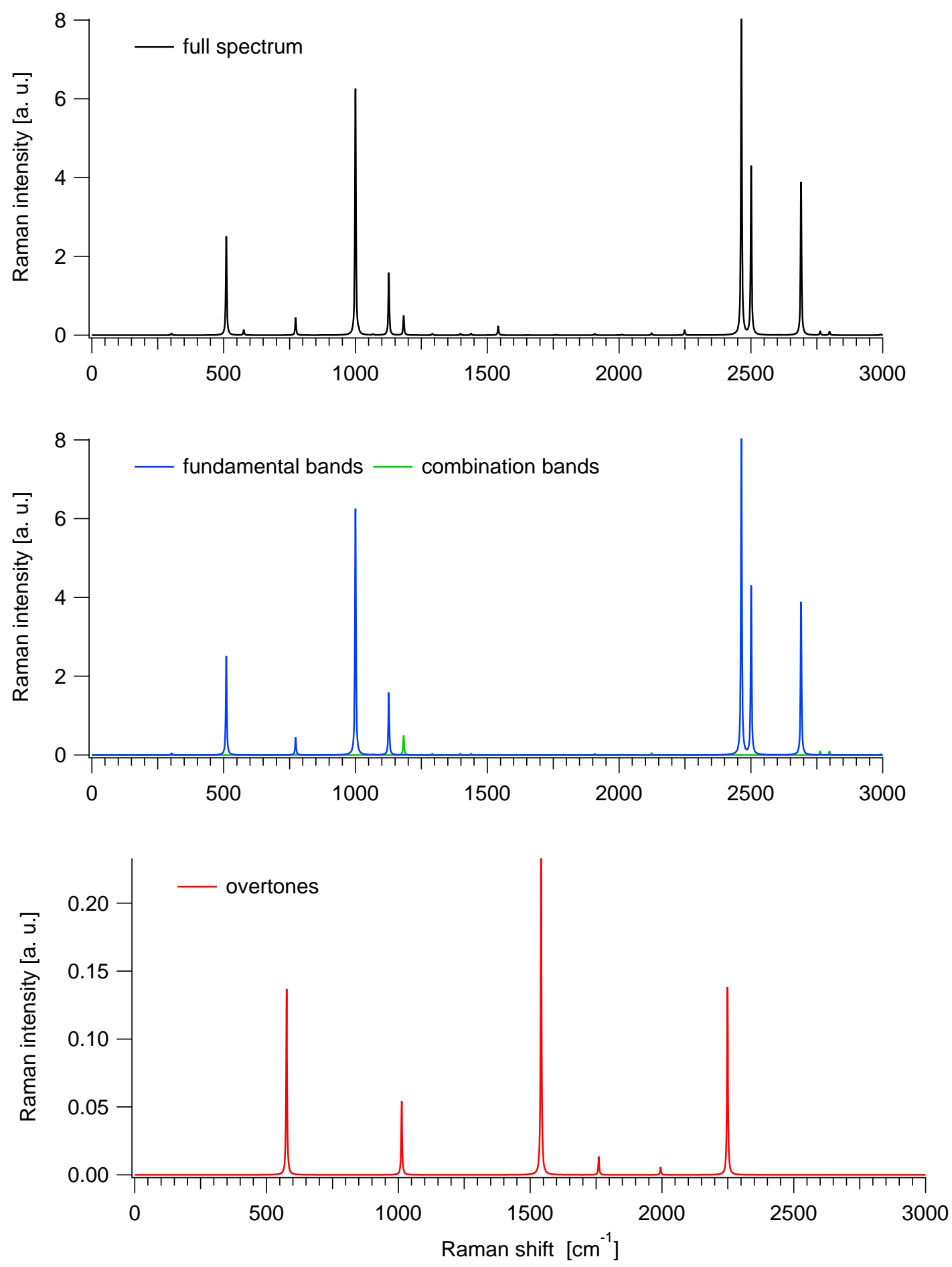

S.40 
Table S.5.4. $\mathrm{B}_{2} \mathrm{H}_{3}$ : Ideal-gas thermodynamic functions at 1 bar (anharmonic B3LYP$\mathrm{D} 2 / \mathrm{cc}-\mathrm{pVTZ}$ results)

\begin{tabular}{|c|c|c|c|}
\hline $\begin{array}{c}T \\
{[\mathrm{~K}]}\end{array}$ & $\begin{array}{c}C_{P} \\
{\left[\mathrm{~J} \mathrm{~mol}^{-1} \mathrm{~K}^{-1}\right]}\end{array}$ & $\begin{array}{c}S \\
{\left[\mathrm{~J} \mathrm{~mol}^{-1} \mathrm{~K}^{-1}\right]}\end{array}$ & $\begin{array}{c}H(T)-H(0 K) \\
{\left[\mathrm{kJ} \mathrm{mol}^{-1}\right]}\end{array}$ \\
\hline 100 & 35.661 & 184.976 & 3.377 \\
\hline 200 & 44.037 & 212.133 & 7.343 \\
\hline 300 & 53.343 & 231.764 & 12.216 \\
\hline 400 & 61.290 & 248.242 & 17.963 \\
\hline 500 & 67.569 & 262.620 & 24.418 \\
\hline 600 & 72.698 & 275.406 & 31.439 \\
\hline 700 & 77.059 & 286.949 & 38.932 \\
\hline 800 & 80.833 & 297.491 & 46.831 \\
\hline 900 & 84.107 & 307.205 & 55.082 \\
\hline 1000 & 86.938 & 316.217 & 63.638 \\
\hline 1100 & 89.379 & 324.620 & 72.457 \\
\hline 1200 & 91.480 & 332.490 & 81.502 \\
\hline 1300 & 93.288 & 339.885 & 90.743 \\
\hline 1400 & 94.847 & 346.857 & 100.152 \\
\hline 1500 & 96.193 & 353.448 & 109.705 \\
\hline 1600 & 97.360 & 359.694 & 119.384 \\
\hline 1700 & 98.375 & 365.627 & 129.172 \\
\hline 1800 & 99.261 & 371.276 & 139.055 \\
\hline 1900 & 100.038 & 376.664 & 149.021 \\
\hline 2000 & 100.722 & 381.813 & 159.060 \\
\hline
\end{tabular}

$T$ : Temperature. $C_{P}$ : Heat capacity at constant pressure. $S$ : Entropy. $H$ : Enthalpy. 
Figure S.5.4. $\mathrm{B}_{2} \mathrm{H}_{3}$ : Ideal-gas thermodynamic functions at 1 bar (anharmonic B3LYP$\mathrm{D} 2 / \mathrm{cc}-\mathrm{pVTZ}$ results)
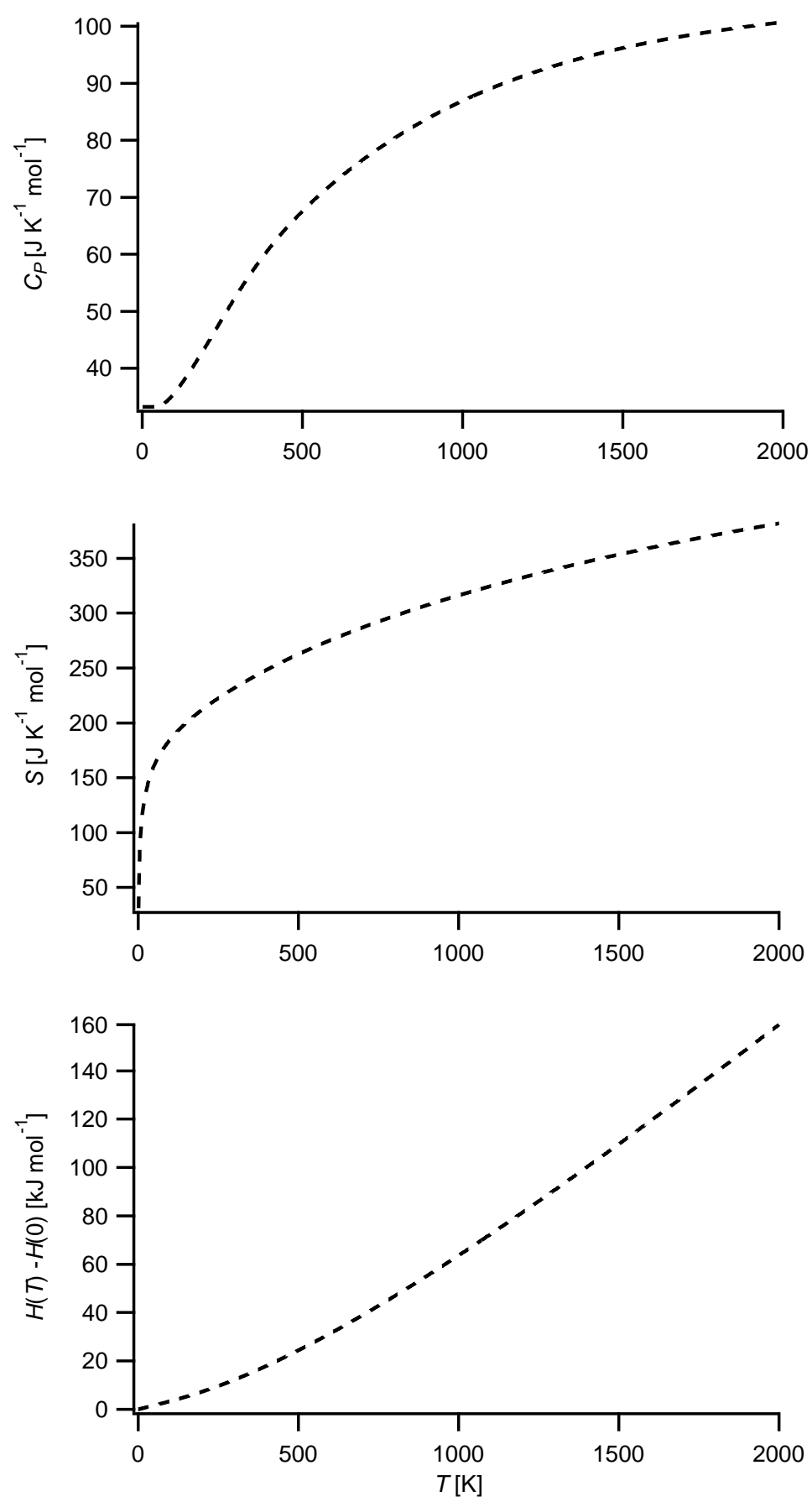

T: Temperature. $C_{P}$ : Heat capacity at constant pressure. $S$ : Entropy. $H$ : Enthalpy. 
Table S.5.5. $\mathrm{B}_{2} \mathrm{H}_{3}$ : Fits (red solid lines) of the computed thermodynamic functions (blue dashed lines) with NASA type functions (Equations (1) - (3)) in the 200 - $900 \mathrm{~K}$ temperature range (anharmonic B3LYP-D2/cc-pVTZ results). In each case, the difference curve between the thermodynamic and NASA functions is plotted in the upper graph

Fit parameters

\begin{tabular}{llllll}
\hline $\mathrm{a}_{1}$ & $2.21669916 \mathrm{e}+00$ & $\mathrm{a}_{2}\left[\mathrm{~K}^{-1}\right]$ & $1.80909315 \mathrm{e}-02$ & $\mathrm{a}_{3}\left[\mathrm{~K}^{-2}\right]$ & $-1.50820137 \mathrm{e}-05$ \\
$\mathrm{a}_{4}\left[\mathrm{~K}^{-3}\right]$ & $4.84636137 \mathrm{e}-09$ & $\mathrm{a}_{5}\left[\mathrm{~K}^{-4}\right]$ & $4.80517193 \mathrm{e}-13$ & $\mathrm{a}_{6}[\mathrm{~K}]$ & $1.16283966 \mathrm{e}+02$ \\
$\mathrm{a}_{7}$ & $1.04393087 \mathrm{e}+01$ & & & &
\end{tabular}
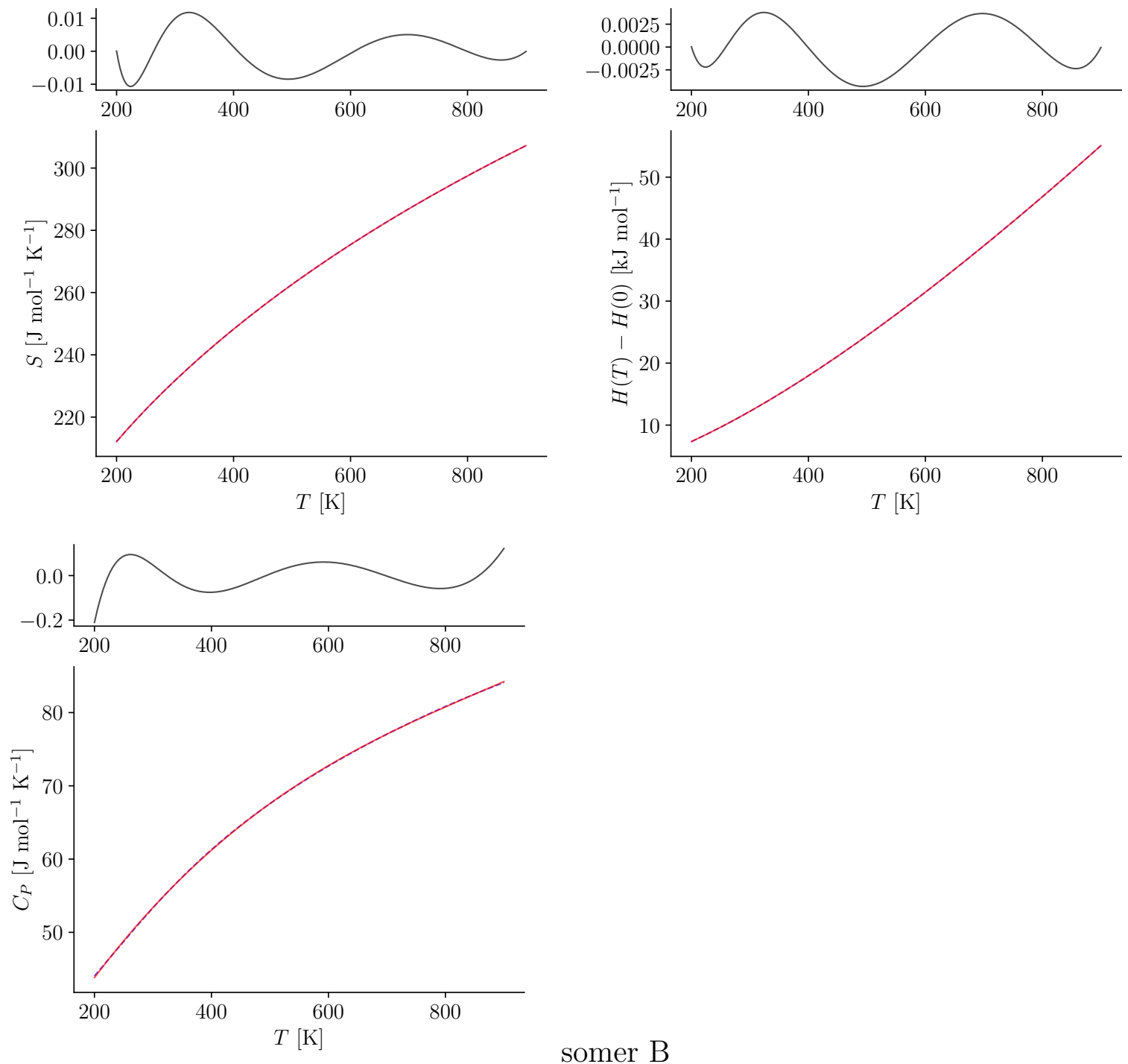

somer B 


\section{$6 \quad \mathrm{~B}_{2} \mathrm{H}_{4}$ - Isomer $\mathrm{A}$}

Figure S.6.1. Structure of $\mathrm{B}_{2} \mathrm{H}_{4}$ - Isomer A

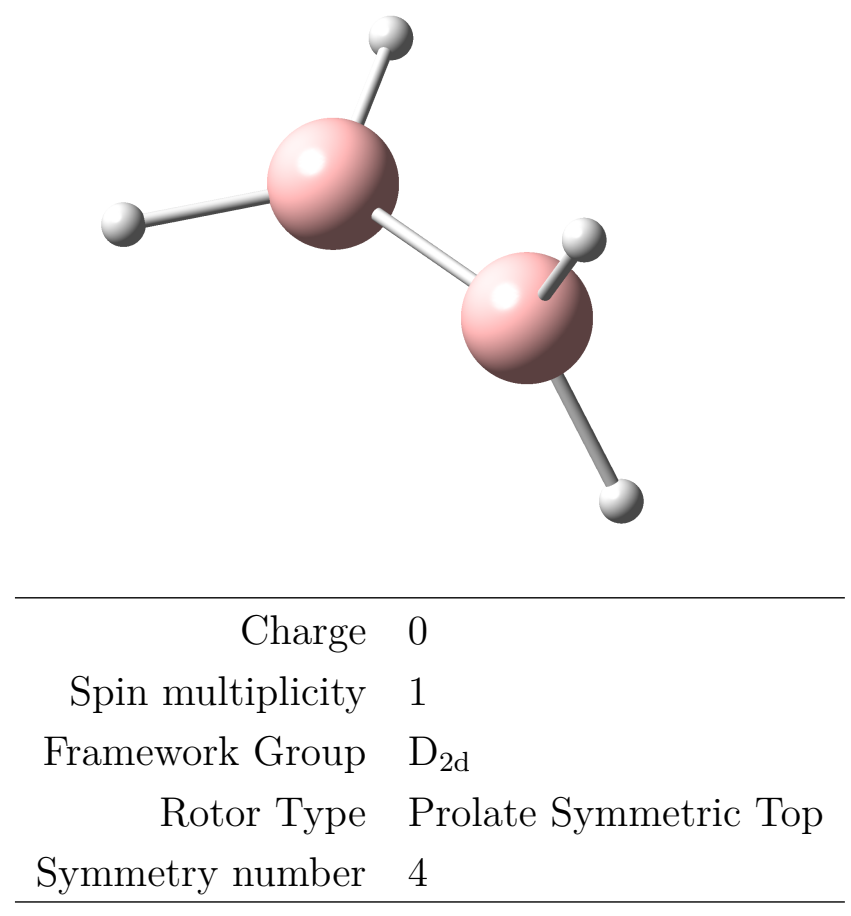

Table S.6.1. $\mathrm{B}_{2} \mathrm{H}_{4}$ - Isomer A: Selected anharmonic results obtained at the B3LYP-D2/ccpVTZ level

\begin{tabular}{rll}
\hline $\mathrm{ZPE}_{\mathrm{v}}$ & 8381 & $\mathrm{~cm}^{-1}$ \\
$\mathrm{~A}$ & 4.07483 & $\mathrm{~cm}^{-1}$ \\
$\mathrm{~B}$ & 0.66837 & $\mathrm{~cm}^{-1}$ \\
$\mathrm{C}$ & 0.66837 & $\mathrm{~cm}^{-1}$ \\
\hline$C_{P}(300 \mathrm{~K})$ & 58.277 & $\mathrm{~J} \mathrm{~mol}^{-1} \mathrm{~K}-1$ \\
$S(300 \mathrm{~K})$ & 232.266 & $\mathrm{~J} \mathrm{~mol}^{-1} \mathrm{~K}^{-1}$ \\
$H(300 \mathrm{~K})-H(0 \mathrm{~K})$ & 12.679 & $\mathrm{~kJ} \mathrm{~mol}^{-1}$ \\
\hline
\end{tabular}

$\mathrm{ZPE}_{\mathrm{v}}$ : vibrational contribution to the zero-point energy. A, B, C: Rotational constants. $C_{P}$ : Heat capacity at constant pressure. $S$ : Entropy. $H$ : Enthalpy. Ideal-gas calculations (1 bar). 
Table S.6.2. $\mathrm{B}_{2} \mathrm{H}_{4}$ - Isomer A: Cartesian coordinates $(\AA)$ of the B3LYP-D2/cc-pVTZ optimized structure

\begin{tabular}{cccc} 
atom & $\mathrm{x}$ & $\mathrm{y}$ & $\mathrm{z}$ \\
\hline B1 & 0.0000 & 0.0000 & 0.8156 \\
B2 & 0.0000 & 0.0000 & -0.8156 \\
H3 & -1.0130 & 0.0000 & -1.4527 \\
H4 & 0.0000 & 1.0130 & 1.4527 \\
H5 & -0.0000 & -1.0130 & 1.4527 \\
H6 & 1.0130 & 0.0000 & -1.4527 \\
\hline
\end{tabular}


Table S.6.3. $\mathrm{B}_{2} \mathrm{H}_{4}$ - Isomer A: Vibrational frequencies, infrared integrated intensities and Raman activities (B3LYP-D2/cc-pVTZ results)

\begin{tabular}{cccccccc}
\hline & & \multicolumn{3}{c}{ Harmonic } & \multicolumn{3}{c}{ Anharmonic } \\
mode & symm. & $\omega$ & $\mathrm{IR}$ & Raman & $\omega$ & $\mathrm{IR}$ & Raman \\
& & {$\left[\mathrm{cm}^{-1}\right]$} & {$\left[\mathrm{km} \mathrm{mol}^{-1}\right]$} & {$\left[\AA^{6}\right]$} & {$\left[\mathrm{cm}^{-1}\right]$} & {$\left[\mathrm{km} \mathrm{mol}^{-1}\right]$} & {$\left[\AA^{6}\right]$} \\
\hline 1 & $\mathrm{E}$ & 415 & 3.960 & 0.003 & 383 & 3.288 & 0.004 \\
2 & $\mathrm{E}$ & 415 & 3.960 & 0.003 & 383 & 3.288 & 0.004 \\
3 & $\mathrm{~B}_{1}$ & 562 & 0.000 & 0.516 & 563 & 0.000 & 0.550 \\
4 & $\mathrm{~A}_{1}$ & 882 & 0.000 & 0.149 & 898 & 0.143 & 0.142 \\
5 & $\mathrm{E}$ & 999 & 25.486 & 0.055 & 981 & 18.629 & 0.049 \\
6 & $\mathrm{E}$ & 999 & 25.486 & 0.055 & 981 & 18.630 & 0.049 \\
7 & $\mathrm{~B}_{2}$ & 1166 & 2.429 & 0.003 & 1115 & 2.368 & 0.006 \\
8 & $\mathrm{~A}_{1}$ & 1217 & 0.000 & 0.209 & 1167 & 0.000 & 0.228 \\
9 & $\mathrm{~B}_{2}$ & 2573 & 60.362 & 0.341 & 2465 & 47.276 & 0.362 \\
10 & $\mathrm{~A}_{1}$ & 2589 & 0.000 & 2.349 & 2483 & 0.000 & 2.555 \\
11 & $\mathrm{E}$ & 2613 & 78.034 & 0.573 & 2503 & 79.166 & 0.606 \\
12 & $\mathrm{E}$ & 2613 & 78.034 & 0.573 & 2503 & 79.166 & 0.606 \\
\hline
\end{tabular}


Figure S.6.2. Anharmonic IR spectrum of $\mathrm{B}_{2} \mathrm{H}_{4}$ - Isomer A obtained by convoluting the calculated intensities with Lorentzians having a FWHM of $4 \mathrm{~cm}^{-1}$ (B3LYP-D2/cc-pVTZ results): (top) full spectrum, (middle) contributions from fundamentals and combination bands, (bottom) contributions from overtones
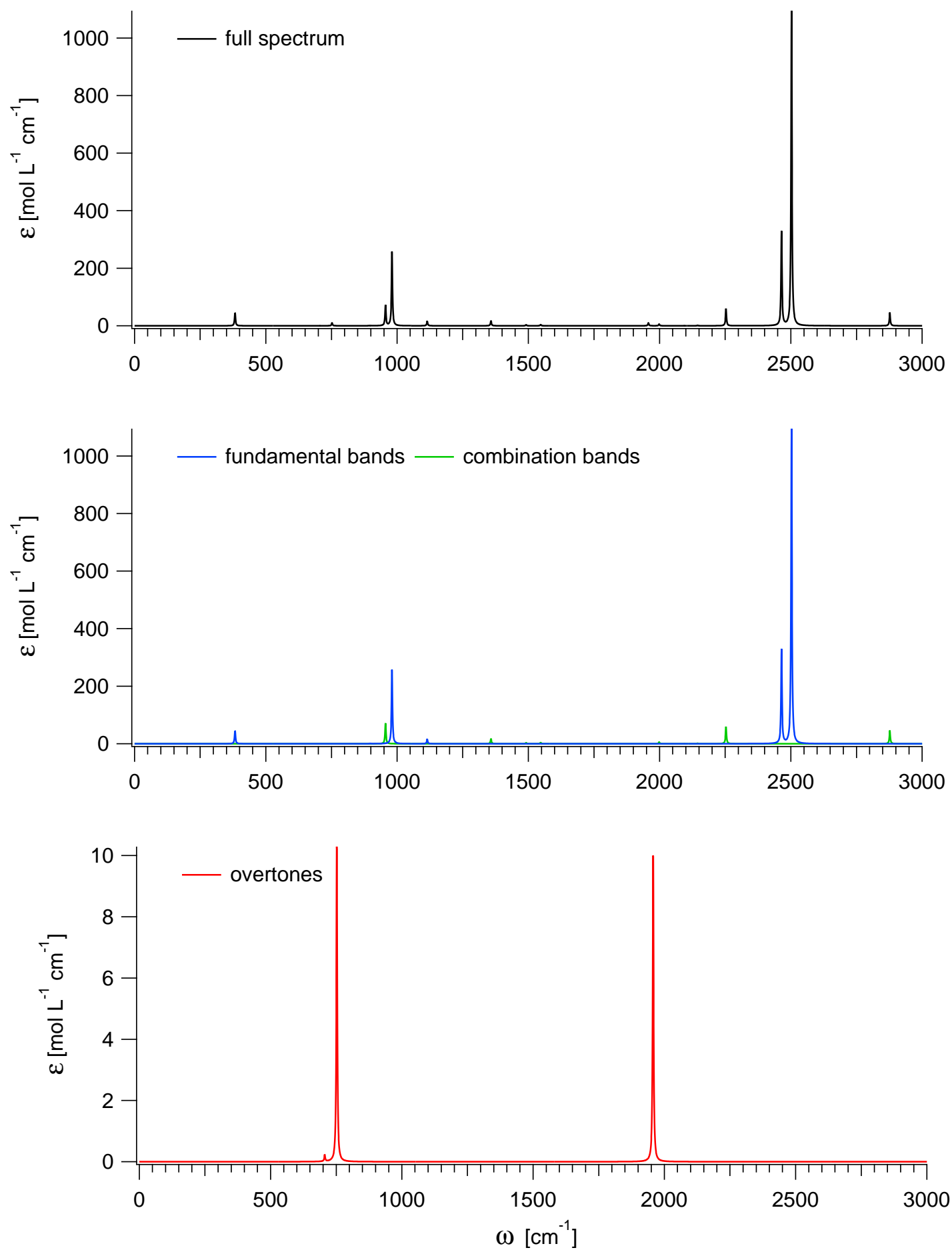
Figure S.6.3. Anharmonic Raman spectrum of $\mathrm{B}_{2} \mathrm{H}_{4}$ - Isomer A obtained by convoluting the calculated activities with Lorentzians having a FWHM of $4 \mathrm{~cm}^{-1}$ (B3LYP-D2/cc-pVTZ results): (top) full spectrum, (middle) contributions from fundamentals and combination bands, (bottom) contributions from overtones
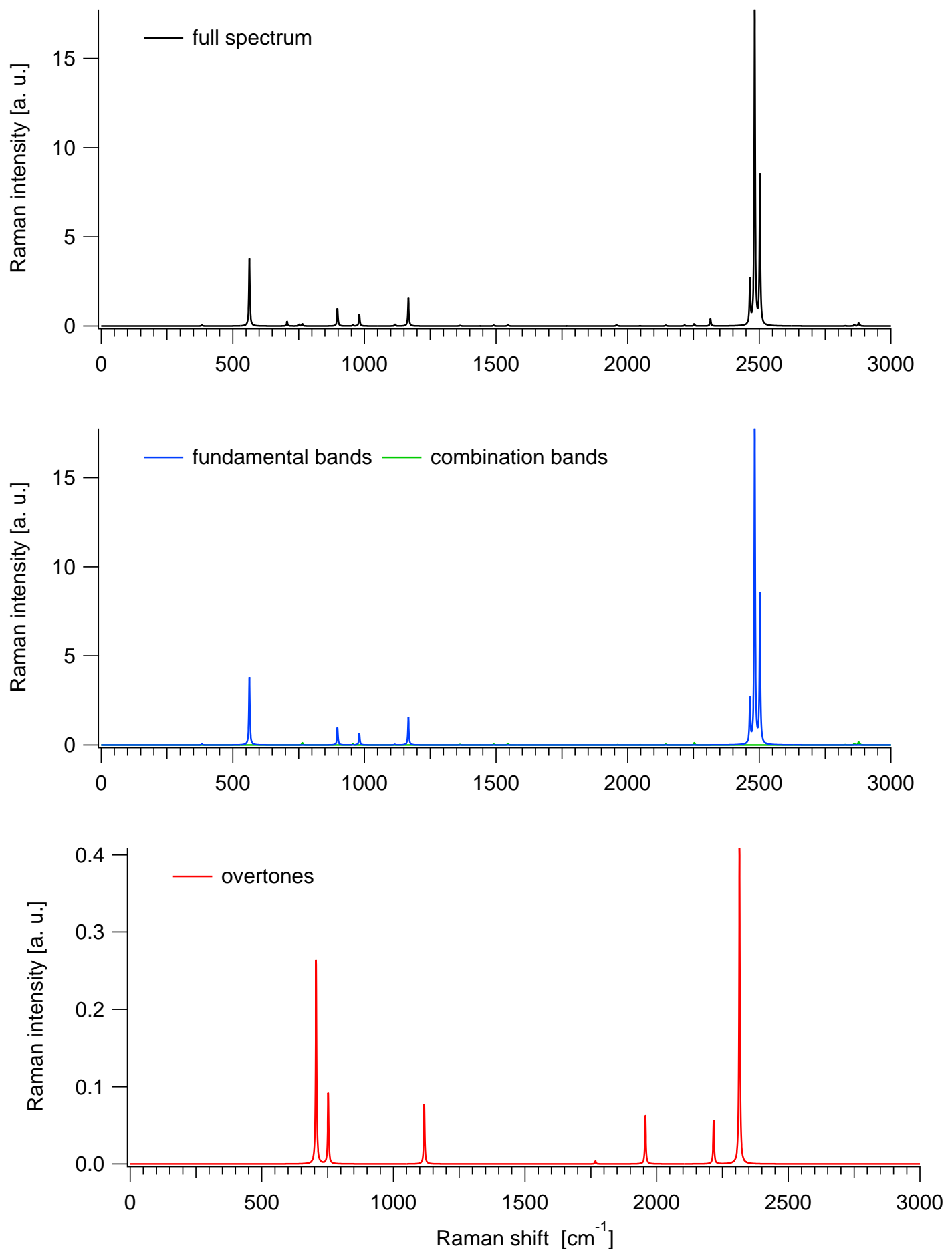
Table S.6.4. $\mathrm{B}_{2} \mathrm{H}_{4}$ - Isomer A: Ideal-gas thermodynamic functions at 1 bar (anharmonic B3LYP-D2/cc-pVTZ results)

\begin{tabular}{|c|c|c|c|}
\hline $\begin{array}{c}T \\
{[\mathrm{~K}]}\end{array}$ & $\begin{array}{c}C_{P} \\
{\left[\mathrm{~J} \mathrm{~mol}^{-1} \mathrm{~K}^{-1}\right]}\end{array}$ & $\begin{array}{c}S \\
{\left[\mathrm{~J} \mathrm{~mol}^{-1} \mathrm{~K}^{-1}\right]}\end{array}$ & $\begin{array}{c}H(T)-H(0 K) \\
{\left[\mathrm{kJ} \mathrm{mol}{ }^{-1}\right]}\end{array}$ \\
\hline 100 & 35.495 & 183.382 & 3.365 \\
\hline 200 & 46.454 & 211.180 & 7.439 \\
\hline 300 & 58.277 & 232.266 & 12.679 \\
\hline 400 & 68.773 & 250.514 & 19.048 \\
\hline 500 & 77.416 & 266.820 & 26.371 \\
\hline 600 & 84.635 & 281.591 & 34.484 \\
\hline 700 & 90.808 & 295.113 & 43.264 \\
\hline 800 & 96.130 & 307.595 & 52.617 \\
\hline 900 & 100.713 & 319.189 & 62.465 \\
\hline 1000 & 104.645 & 330.009 & 72.738 \\
\hline 1100 & 108.010 & 340.144 & 83.375 \\
\hline 1200 & 110.888 & 349.669 & 94.324 \\
\hline 1300 & 113.350 & 358.645 & 105.539 \\
\hline 1400 & 115.462 & 367.124 & 116.982 \\
\hline 1500 & 117.278 & 375.154 & 128.621 \\
\hline 1600 & 118.847 & 382.774 & 140.429 \\
\hline 1700 & 120.207 & 390.021 & 152.384 \\
\hline 1800 & 121.391 & 396.926 & 164.465 \\
\hline 1900 & 122.427 & 403.517 & 176.657 \\
\hline 2000 & 123.336 & 409.821 & 188.946 \\
\hline
\end{tabular}

$T$ : Temperature. $C_{P}$ : Heat capacity at constant pressure. $S$ : Entropy. $H$ : Enthalpy. 
Figure S.6.4. $\mathrm{B}_{2} \mathrm{H}_{4}$ - Isomer A: Ideal-gas thermodynamic functions at 1 bar (anharmonic B3LYP-D2/cc-pVTZ results)
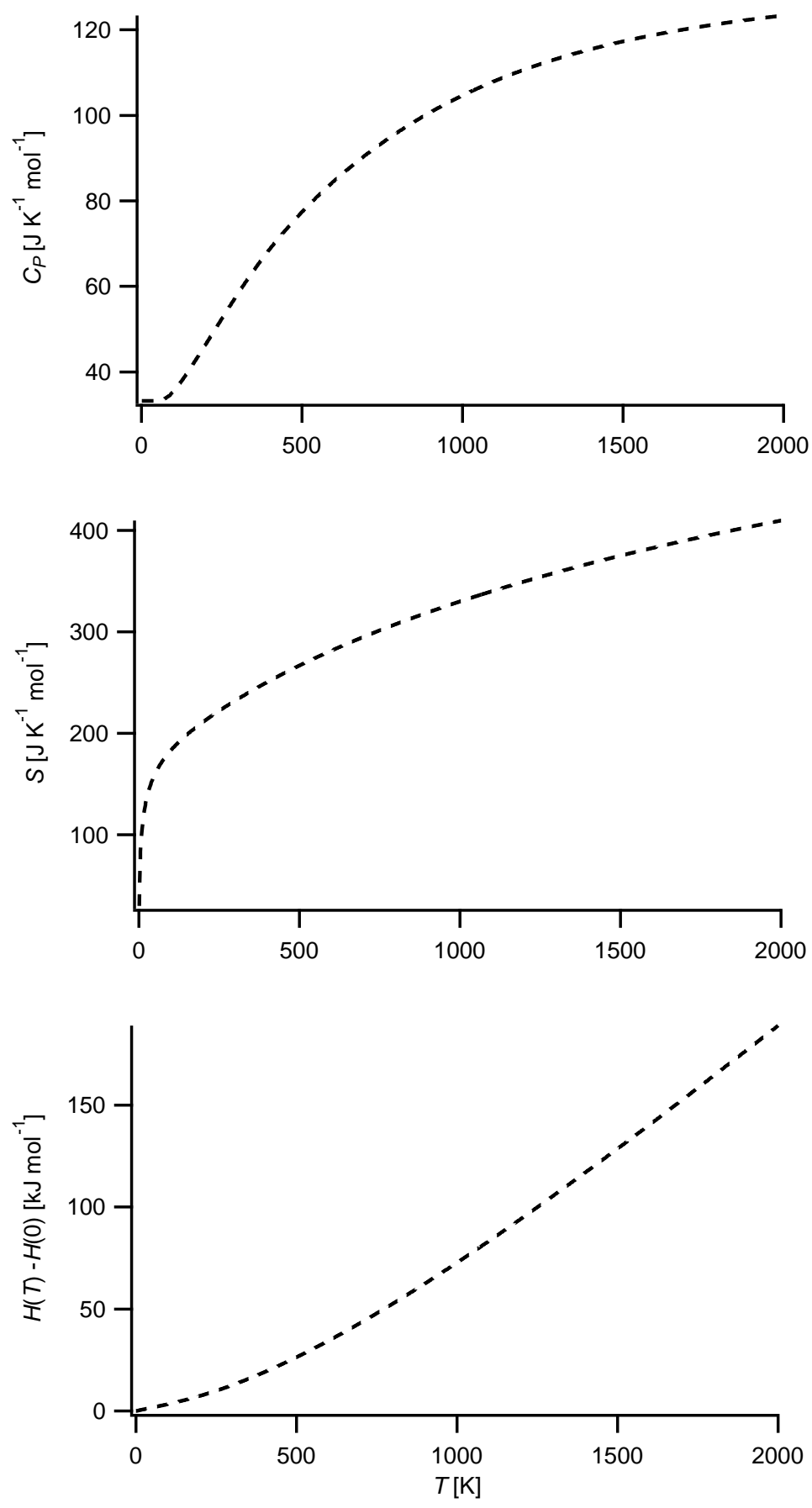

$T$ : Temperature. $C_{P}$ : Heat capacity at constant pressure. $S$ : Entropy. $H$ : Enthalpy. 
Table S.6.5. $\mathrm{B}_{2} \mathrm{H}_{4}$ - Isomer A: Fits (red solid lines) of the computed thermodynamic functions (blue dashed lines) with NASA type functions (Equations (1) - (3)) in the 200 - $900 \mathrm{~K}$ temperature range (anharmonic B3LYP-D2/cc-pVTZ results). In each case, the difference curve between the thermodynamic and NASA functions is plotted in the upper graph

Fit parameters

\begin{tabular}{llllll}
\hline $\mathrm{a}_{1}$ & $2.04240931 \mathrm{e}+00$ & $\mathrm{a}_{2}\left[\mathrm{~K}^{-1}\right]$ & $1.95462075 \mathrm{e}-02$ & $\mathrm{a}_{3}\left[\mathrm{~K}^{-2}\right]$ & $-9.10474574 \mathrm{e}-06$ \\
$\mathrm{a}_{4}\left[\mathrm{~K}^{-3}\right]$ & $-3.87862898 \mathrm{e}-09$ & $\mathrm{a}_{5}\left[\mathrm{~K}^{-4}\right]$ & $4.11675726 \mathrm{e}-12$ & $\mathrm{a}_{6}[\mathrm{~K}]$ & $1.20873375 \mathrm{e}+02$ \\
$\mathrm{a}_{7}$ & $1.08593909 \mathrm{e}+01$ & & & &
\end{tabular}
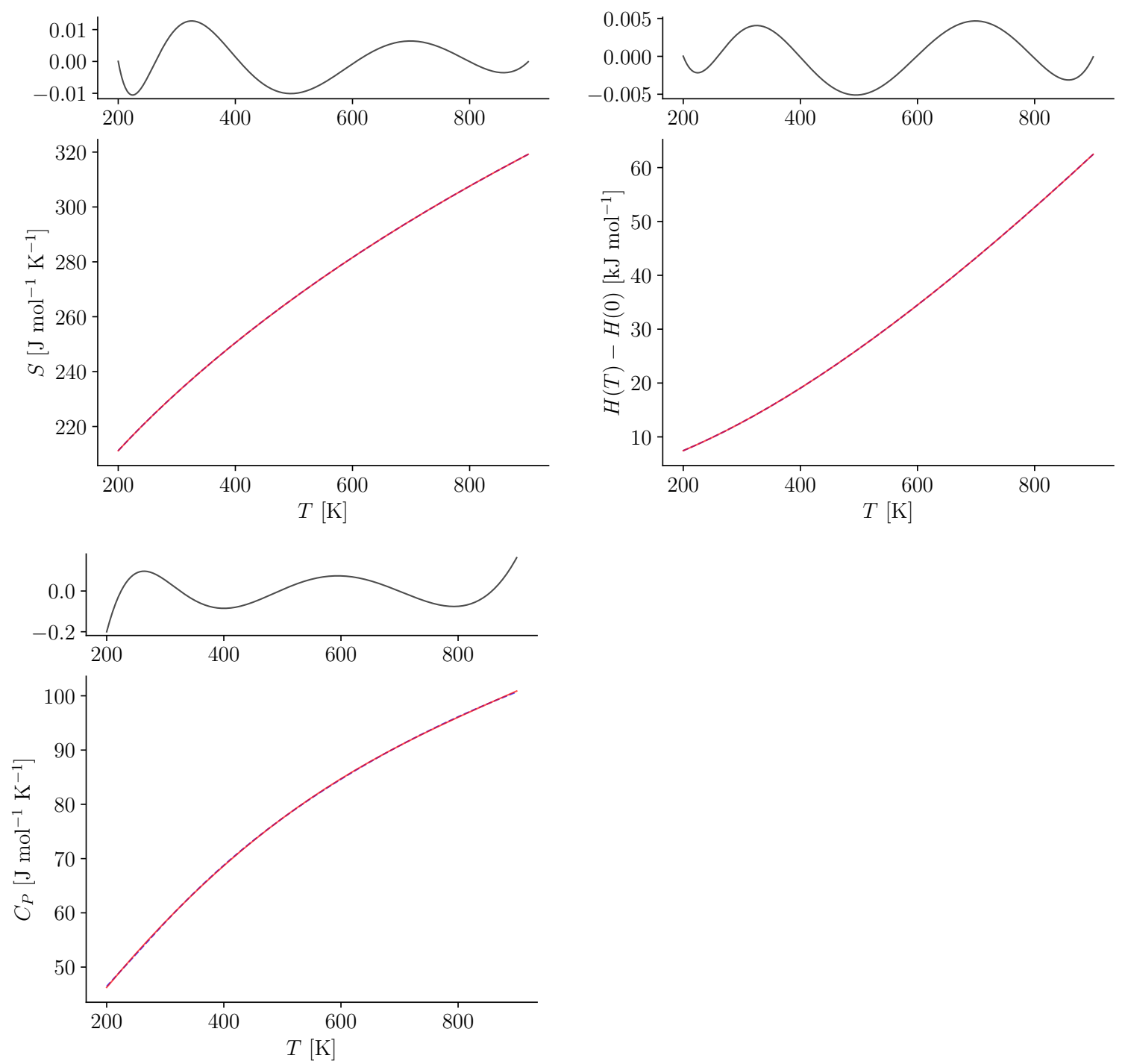


\section{$7 \quad \mathbf{B}_{2} \mathbf{H}_{4}$ - Isomer B}

Figure S.7.1. Structure of $\mathrm{B}_{2} \mathrm{H}_{4}$ - Isomer B

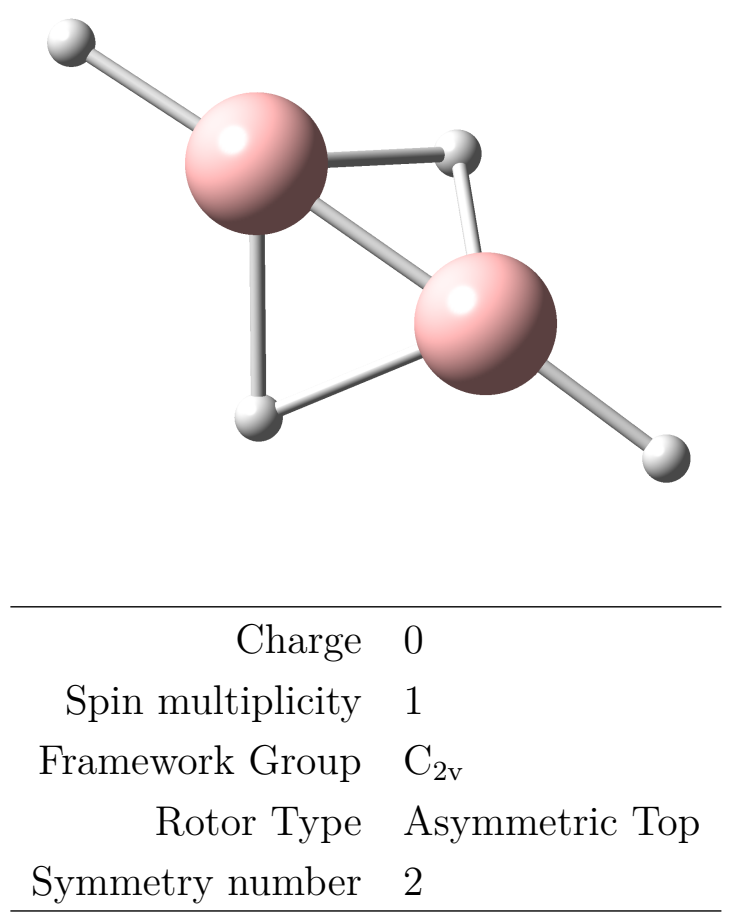

Table S.7.1. $\mathrm{B}_{2} \mathrm{H}_{4}$ - Isomer B: Selected anharmonic results obtained at the B3LYP-D2/ccpVTZ level

\begin{tabular}{rll}
\hline $\mathrm{ZPE}_{\mathrm{v}}$ & 8714 & $\mathrm{~cm}^{-1}$ \\
$\mathrm{~A}$ & 6.72211 & $\mathrm{~cm}^{-1}$ \\
$\mathrm{~B}$ & 0.85387 & $\mathrm{~cm}^{-1}$ \\
$\mathrm{C}$ & 0.82199 & $\mathrm{~cm}^{-1}$ \\
\hline$C_{P}(300 \mathrm{~K})$ & 52.836 & $\mathrm{~J} \mathrm{~mol}^{-1} \mathrm{~K}-1$ \\
$S(300 \mathrm{~K})$ & 228.694 & $\mathrm{~J} \mathrm{~mol}^{-1} \mathrm{~K}-1$ \\
$H(300 \mathrm{~K})-H(0 \mathrm{~K})$ & 11.676 & $\mathrm{~kJ} \mathrm{~mol}^{-1}$ \\
\hline
\end{tabular}

$\mathrm{ZPE}_{\mathrm{v}}$ : vibrational contribution to the zero-point energy. A, B, C: Rotational constants. $C_{P}$ : Heat capacity at constant pressure. $S$ : Entropy. $H$ : Enthalpy. Ideal-gas calculations (1 bar). 
Table S.7.2. $\mathrm{B}_{2} \mathrm{H}_{4}$ - Isomer B: Cartesian coordinates $(\AA)$ of the B3LYP-D2/cc-pVTZ optimized structure

\begin{tabular}{cccc} 
atom & $\mathrm{x}$ & $\mathrm{y}$ & $\mathrm{z}$ \\
\hline B1 & 0.0000 & 0.7279 & -0.1141 \\
B2 & -0.0000 & -0.7279 & -0.1141 \\
H3 & 0.0000 & 1.8907 & 0.0004 \\
H4 & 0.9011 & -0.0000 & 0.5703 \\
H5 & -0.0000 & -1.8907 & 0.0004 \\
H6 & -0.9011 & 0.0000 & 0.5703 \\
\hline
\end{tabular}


Table S.7.3. $\mathrm{B}_{2} \mathrm{H}_{4}$ - Isomer B: Vibrational frequencies, infrared integrated intensities and Raman activities (B3LYP-D2/cc-pVTZ results)

\begin{tabular}{cccccccc}
\hline & & \multicolumn{3}{c}{ Harmonic } & \multicolumn{3}{c}{ Anharmonic } \\
mode & symm. & $\omega$ & IR & Raman & $\omega$ & IR & Raman \\
& & {$\left[\mathrm{cm}^{-1}\right]$} & {$\left[\mathrm{km} \mathrm{mol}^{-1}\right]$} & {$\left[\AA^{6}\right]$} & {$\left[\mathrm{cm}^{-1}\right]$} & {$\left[\mathrm{km} \mathrm{mol}^{-1}\right]$} & {$\left[\AA^{6}\right]$} \\
\hline 1 & $\mathrm{~B}_{2}$ & 556 & 35.282 & 0.526 & 530 & 51.759 & 0.508 \\
2 & $\mathrm{~A}_{2}$ & 657 & 0.000 & 0.024 & 586 & 0.000 & 0.047 \\
3 & $\mathrm{~A}_{1}$ & 740 & 3.795 & 0.019 & 730 & 4.983 & 0.017 \\
4 & $\mathrm{~B}_{1}$ & 808 & 1.642 & 0.002 & 790 & 0.458 & 0.004 \\
5 & $\mathrm{~A}_{1}$ & 1119 & 2.329 & 0.141 & 1081 & 6.156 & 0.147 \\
6 & $\mathrm{~A}_{2}$ & 1283 & 0.000 & 0.088 & 1101 & 0.000 & 0.095 \\
7 & $\mathrm{~B}_{2}$ & 1371 & 186.782 & 0.043 & 1191 & 126.937 & 0.029 \\
8 & $\mathrm{~A}_{1}$ & 1376 & 2.726 & 0.900 & 1335 & 1.920 & 0.812 \\
9 & $\mathrm{~A}_{1}$ & 2085 & 16.719 & 0.789 & 2026 & 10.991 & 0.761 \\
10 & $\mathrm{~B}_{1}$ & 2096 & 46.990 & 0.345 & 2064 & 24.716 & 0.325 \\
11 & $\mathrm{~B}_{2}$ & 2821 & 33.387 & 0.001 & 2717 & 40.099 & 0.000 \\
12 & $\mathrm{~A}_{1}$ & 2855 & 0.043 & 0.589 & 2745 & 0.110 & 0.577 \\
\hline
\end{tabular}


Figure S.7.2. Anharmonic IR spectrum of $\mathrm{B}_{2} \mathrm{H}_{4}$ - Isomer B obtained by convoluting the calculated intensities with Lorentzians having a FWHM of $4 \mathrm{~cm}^{-1}$ (B3LYP-D2/cc-pVTZ results): (top) full spectrum, (middle) contributions from fundamentals and combination bands, (bottom) contributions from overtones
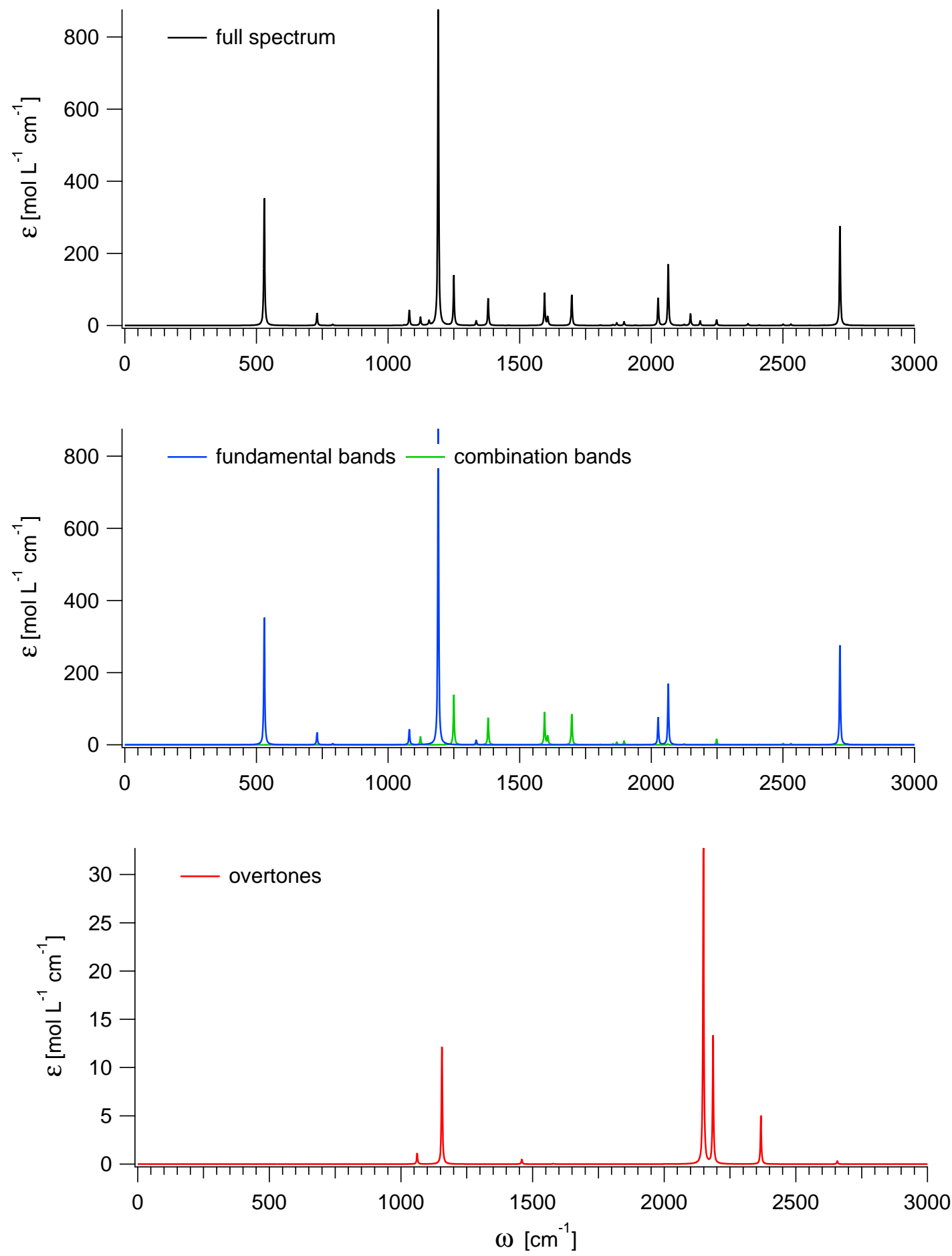
Figure S.7.3. Anharmonic Raman spectrum of $\mathrm{B}_{2} \mathrm{H}_{4}$ - Isomer B obtained by convoluting the calculated activities with Lorentzians having a FWHM of $4 \mathrm{~cm}^{-1}$ (B3LYP-D2/cc-pVTZ results): (top) full spectrum, (middle) contributions from fundamentals and combination bands, (bottom) contributions from overtones
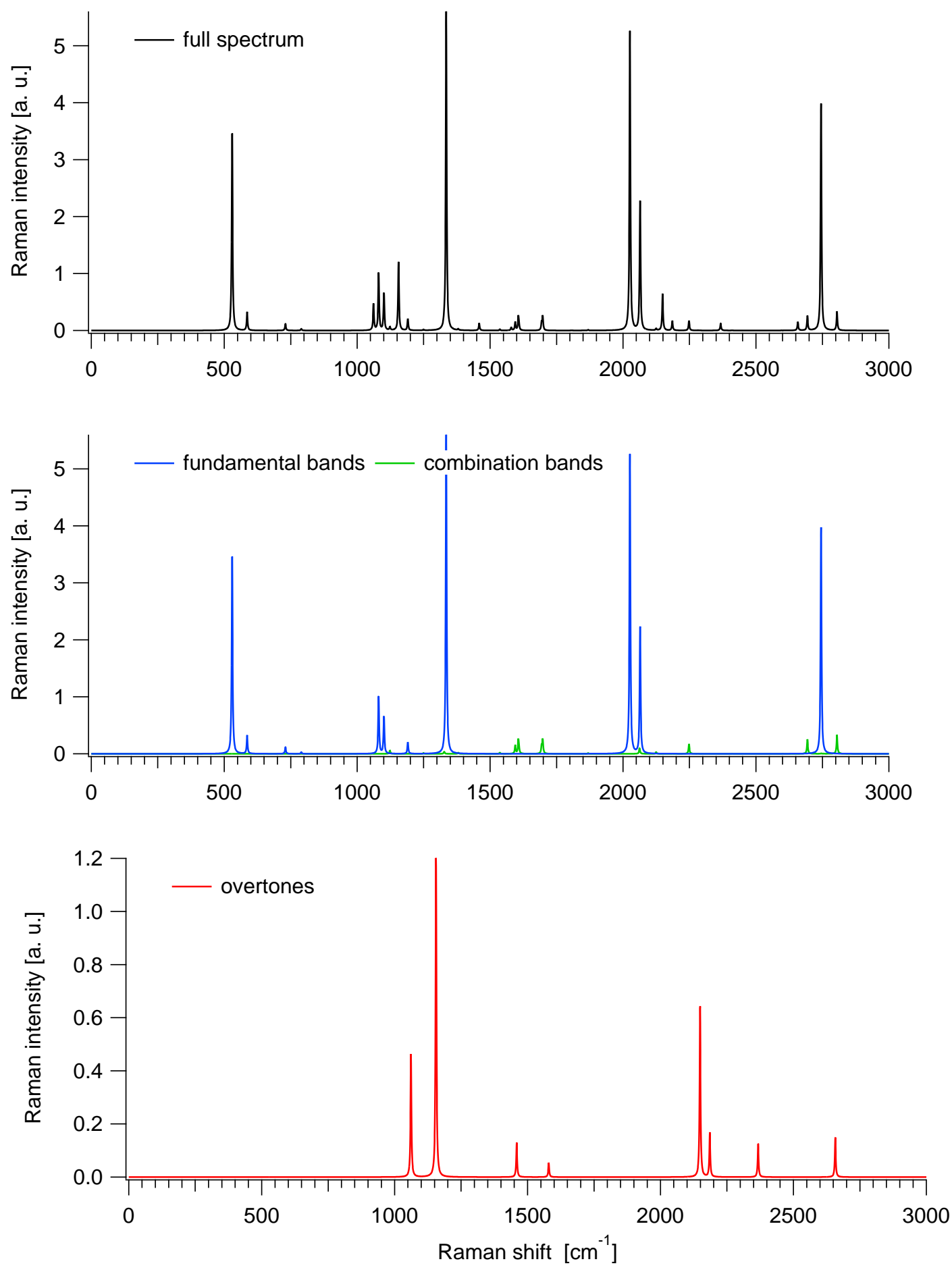
Table S.7.4. $\mathrm{B}_{2} \mathrm{H}_{4}$ - Isomer B: Ideal-gas thermodynamic functions at 1 bar (anharmonic B3LYP-D2/cc-pVTZ results)

\begin{tabular}{|c|c|c|c|}
\hline $\begin{array}{c}T \\
{[\mathrm{~K}]}\end{array}$ & $\begin{array}{c}C_{P} \\
{\left[\mathrm{~J} \mathrm{~mol}^{-1} \mathrm{~K}^{-1}\right]}\end{array}$ & $\begin{array}{c}S \\
{\left[\mathrm{~J} \mathrm{~mol}^{-1} \mathrm{~K}^{-1}\right]}\end{array}$ & $\begin{array}{c}H(T)-H(0 K) \\
{\left[\mathrm{kJ} \mathrm{mol}^{-1}\right]}\end{array}$ \\
\hline 100 & 33.662 & 184.777 & 3.331 \\
\hline 200 & 41.003 & 209.867 & 6.992 \\
\hline 300 & 52.836 & 228.694 & 11.676 \\
\hline 400 & 64.206 & 245.485 & 17.540 \\
\hline 500 & 73.963 & 260.891 & 24.462 \\
\hline 600 & 82.189 & 275.125 & 32.281 \\
\hline 700 & 89.129 & 288.330 & 40.857 \\
\hline 800 & 94.990 & 300.626 & 50.071 \\
\hline 900 & 99.938 & 312.108 & 59.825 \\
\hline 1000 & 104.114 & 322.860 & 70.033 \\
\hline 1100 & 107.642 & 332.953 & 80.626 \\
\hline 1200 & 110.629 & 342.451 & 91.544 \\
\hline 1300 & 113.167 & 351.409 & 102.737 \\
\hline 1400 & 115.330 & 359.877 & 114.164 \\
\hline 1500 & 117.183 & 367.898 & 125.792 \\
\hline 1600 & 118.778 & 375.513 & 137.592 \\
\hline 1700 & 120.156 & 382.757 & 149.541 \\
\hline 1800 & 121.354 & 389.659 & 161.618 \\
\hline 1900 & 122.400 & 396.249 & 173.806 \\
\hline 2000 & 123.316 & 402.551 & 186.093 \\
\hline
\end{tabular}

$T$ : Temperature. $C_{P}$ : Heat capacity at constant pressure. $S$ : Entropy. $H$ : Enthalpy. 
Figure S.7.4. $\mathrm{B}_{2} \mathrm{H}_{4}$ - Isomer B: Ideal-gas thermodynamic functions at 1 bar (anharmonic B3LYP-D2/cc-pVTZ results)
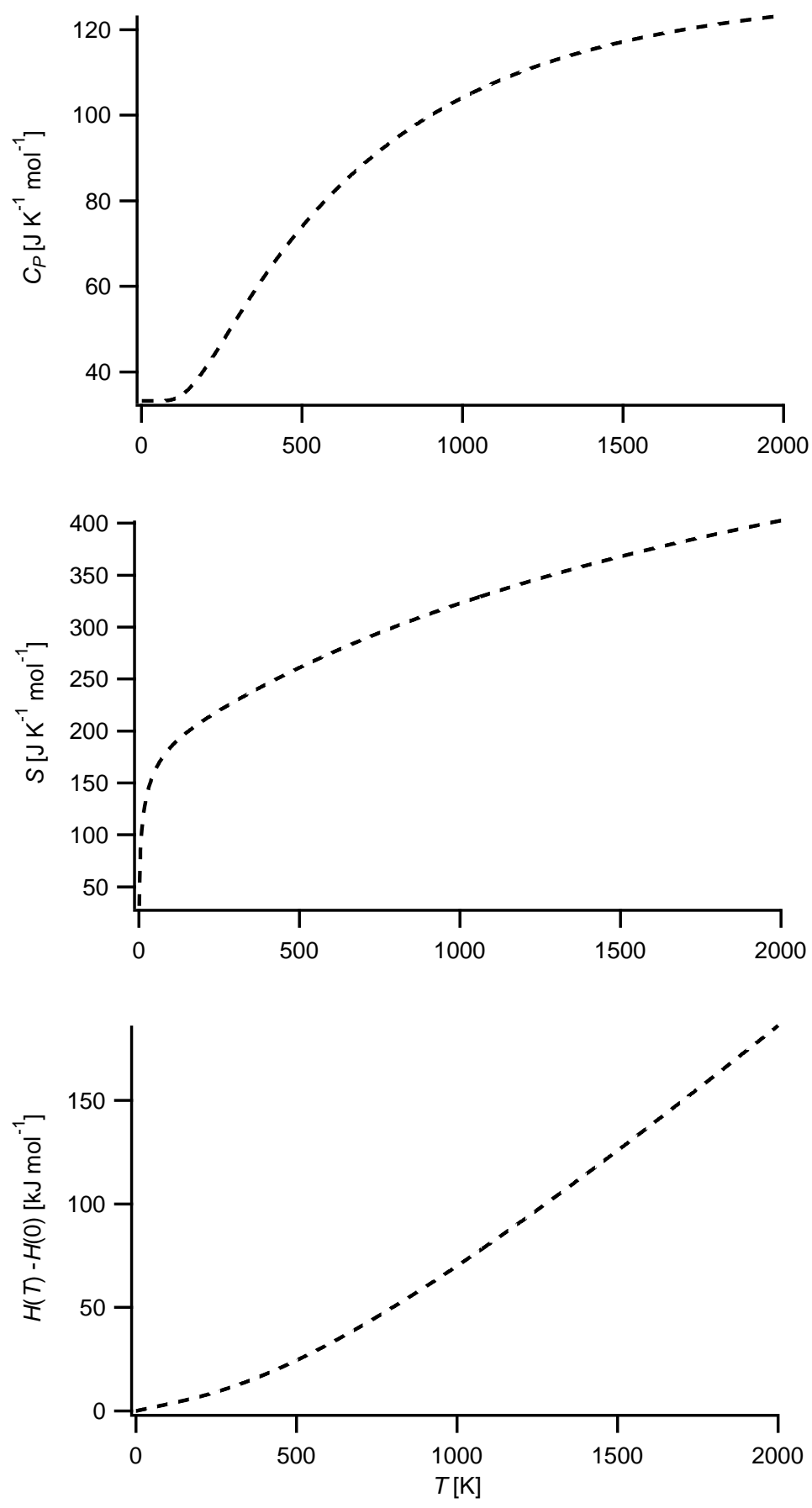

$T$ : Temperature. $C_{P}$ : Heat capacity at constant pressure. $S$ : Entropy. $H$ : Enthalpy. 
Table S.7.5. $\mathrm{B}_{2} \mathrm{H}_{4}$ - Isomer B: Fits (red solid lines) of the computed thermodynamic functions (blue dashed lines) with NASA type functions (Equations (1) - (3)) in the 200 - $900 \mathrm{~K}$ temperature range (anharmonic B3LYP-D2/cc-pVTZ results). In each case, the difference curve between the thermodynamic and NASA functions is plotted in the upper graph

Fit parameters

\begin{tabular}{llllll}
\hline $\mathrm{a}_{1}$ & $2.04379152 \mathrm{e}+00$ & $\mathrm{a}_{2}\left[\mathrm{~K}^{-1}\right]$ & $1.28067728 \mathrm{e}-02$ & $\mathrm{a}_{3}\left[\mathrm{~K}^{-2}\right]$ & $1.25545362 \mathrm{e}-05$ \\
$\mathrm{a}_{4}\left[\mathrm{~K}^{-3}\right]$ & $-2.83612361 \mathrm{e}-08$ & $\mathrm{a}_{5}\left[\mathrm{~K}^{-4}\right]$ & $1.36938361 \mathrm{e}-11$ & $\mathrm{a}_{6}[\mathrm{~K}]$ & $1.53064633 \mathrm{e}+02$ \\
$\mathrm{a}_{7}$ & $1.16702445 \mathrm{e}+01$ & & & &
\end{tabular}
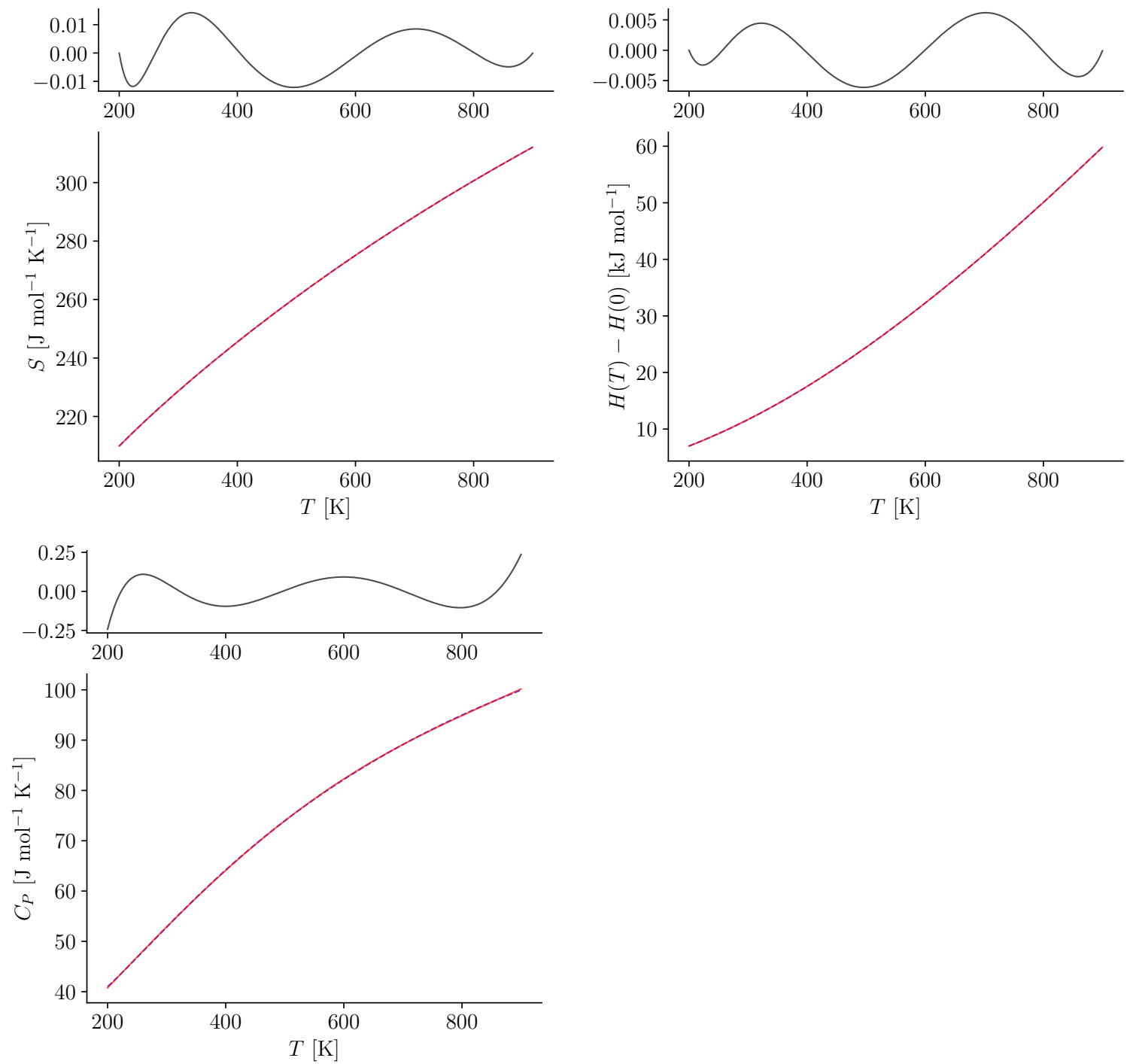


\section{$\begin{array}{lll}8 & \mathbf{B}_{2} \mathbf{H}_{5}\end{array}$}

Figure S.8.1. Structure of $\mathrm{B}_{2} \mathrm{H}_{5}$

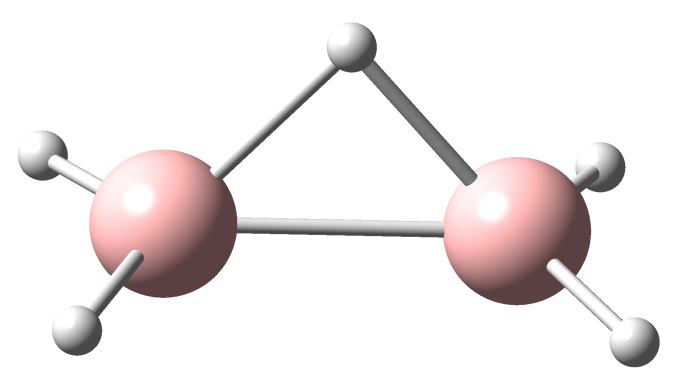

\begin{aligned} & \hline Charge 0 \\ & Spin multiplicity 2 \\ & Framework Group $\mathrm{C}_{2 \mathrm{v}} \\ &$ Rotor Type Asymmetric Top \\ & Symmetry number 2 \\ & \hline\end{aligned}

Table S.8.1. $\mathrm{B}_{2} \mathrm{H}_{5}$ : Selected anharmonic results obtained at the B3LYP-D2/cc-pVTZ level

\begin{tabular}{rll}
\hline $\mathrm{ZPE}_{\mathrm{v}}$ & 10664 & $\mathrm{~cm}^{-1}$ \\
$\mathrm{~A}$ & 3.03289 & $\mathrm{~cm}^{-1}$ \\
$\mathrm{~B}$ & 0.64144 & $\mathrm{~cm}^{-1}$ \\
$\mathrm{C}$ & 0.56604 & $\mathrm{~cm}^{-1}$ \\
\hline$C_{P}(300 \mathrm{~K})$ & 62.899 & $\mathrm{~J} \mathrm{~mol}^{-1} \mathrm{~K}-1$ \\
$S(300 \mathrm{~K})$ & 244.840 & $\mathrm{~J} \mathrm{~mol}^{-1} \mathrm{~K}-1$ \\
$H(300 \mathrm{~K})-H(0 \mathrm{~K})$ & 13.342 & $\mathrm{~kJ} \mathrm{~mol}^{-1}$ \\
\hline
\end{tabular}

$\mathrm{ZPE}_{\mathrm{v}}$ : vibrational contribution to the zero-point energy. A, B, C: Rotational constants. $C_{P}$ : Heat capacity at constant pressure. $S$ : Entropy. $H$ : Enthalpy. Ideal-gas calculations (1 bar). 
Table S.8.2. $\mathrm{B}_{2} \mathrm{H}_{5}$ : Cartesian coordinates $(\AA)$ of the B3LYP-D2/cc-pVTZ optimized structure

\begin{tabular}{cccc} 
atom & $\mathrm{x}$ & $\mathrm{y}$ & $\mathrm{z}$ \\
\hline B1 & 0.0000 & 0.8878 & -0.0343 \\
B2 & -0.0000 & -0.8878 & -0.0343 \\
H3 & -1.0600 & -1.3997 & -0.1525 \\
H4 & 0.0000 & 0.0000 & 0.9529 \\
H5 & 1.0600 & 1.3997 & -0.1525 \\
H6 & -1.0600 & 1.3997 & -0.1525 \\
H7 & 1.0600 & -1.3997 & -0.1525 \\
\hline
\end{tabular}


Table S.8.3. $\mathrm{B}_{2} \mathrm{H}_{5}$ : Vibrational frequencies, infrared integrated intensities and Raman activities (B3LYP-D2/cc-pVTZ results)

\begin{tabular}{cccccccc}
\hline & & \multicolumn{3}{c}{ Harmonic } & \multicolumn{3}{c}{ Anharmonic } \\
mode & symm. & $\omega$ & IR & Raman & $\omega$ & IR & Raman \\
& & {$\left[\mathrm{cm}^{-1}\right]$} & {$\left[\mathrm{km} \mathrm{mol}^{-1}\right]$} & {$\left[\AA^{6}\right]$} & {$\left[\mathrm{cm}^{-1}\right]$} & {$\left[\mathrm{km} \mathrm{mol}^{-1}\right]$} & {$\left[\AA^{6}\right]$} \\
\hline 1 & $\mathrm{~A}_{2}$ & 214 & 0.000 & 0.000 & 213 & 0.000 & 0.001 \\
2 & $\mathrm{~B}_{1}$ & 454 & 2.913 & 0.100 & 433 & 2.165 & 0.106 \\
3 & $\mathrm{~A}_{1}$ & 680 & 4.575 & 0.580 & 665 & 3.892 & 0.594 \\
4 & $\mathrm{~B}_{2}$ & 798 & 5.514 & 0.057 & 768 & 10.289 & 0.041 \\
5 & $\mathrm{~A}_{2}$ & 917 & 0.000 & 0.004 & 822 & 0.000 & 0.000 \\
6 & $\mathrm{~A}_{1}$ & 919 & 6.728 & 0.484 & 894 & 3.781 & 0.334 \\
7 & $\mathrm{~B}_{1}$ & 1048 & 5.578 & 0.093 & 955 & 4.779 & 0.089 \\
8 & $\mathrm{~B}_{2}$ & 1150 & 108.764 & 0.016 & 1095 & 93.048 & 0.020 \\
9 & $\mathrm{~A}_{1}$ & 1181 & 8.419 & 0.027 & 1129 & 8.328 & 0.028 \\
10 & $\mathrm{~B}_{2}$ & 1765 & 385.714 & 0.017 & 1508 & 273.608 & 0.024 \\
11 & $\mathrm{~A}_{1}$ & 1891 & 9.687 & 0.287 & 1769 & 8.916 & 0.266 \\
12 & $\mathrm{~B}_{2}$ & 2647 & 73.835 & 0.016 & 2517 & 53.157 & 0.022 \\
13 & $\mathrm{~A}_{1}$ & 2654 & 3.329 & 1.712 & 2529 & 3.627 & 1.963 \\
14 & $\mathrm{~A}_{2}$ & 2744 & 0.000 & 0.640 & 2606 & 0.000 & 0.698 \\
15 & $\mathrm{~B}_{1}$ & 2763 & 140.550 & 0.015 & 2626 & 124.782 & 0.031 \\
\hline
\end{tabular}


Figure S.8.2. Anharmonic IR spectrum of $\mathrm{B}_{2} \mathrm{H}_{5}$ obtained by convoluting the calculated intensities with Lorentzians having a FWHM of $4 \mathrm{~cm}^{-1}$ (B3LYP-D2/cc-pVTZ results): (top) full spectrum, (middle) contributions from fundamentals and combination bands, (bottom) contributions from overtones
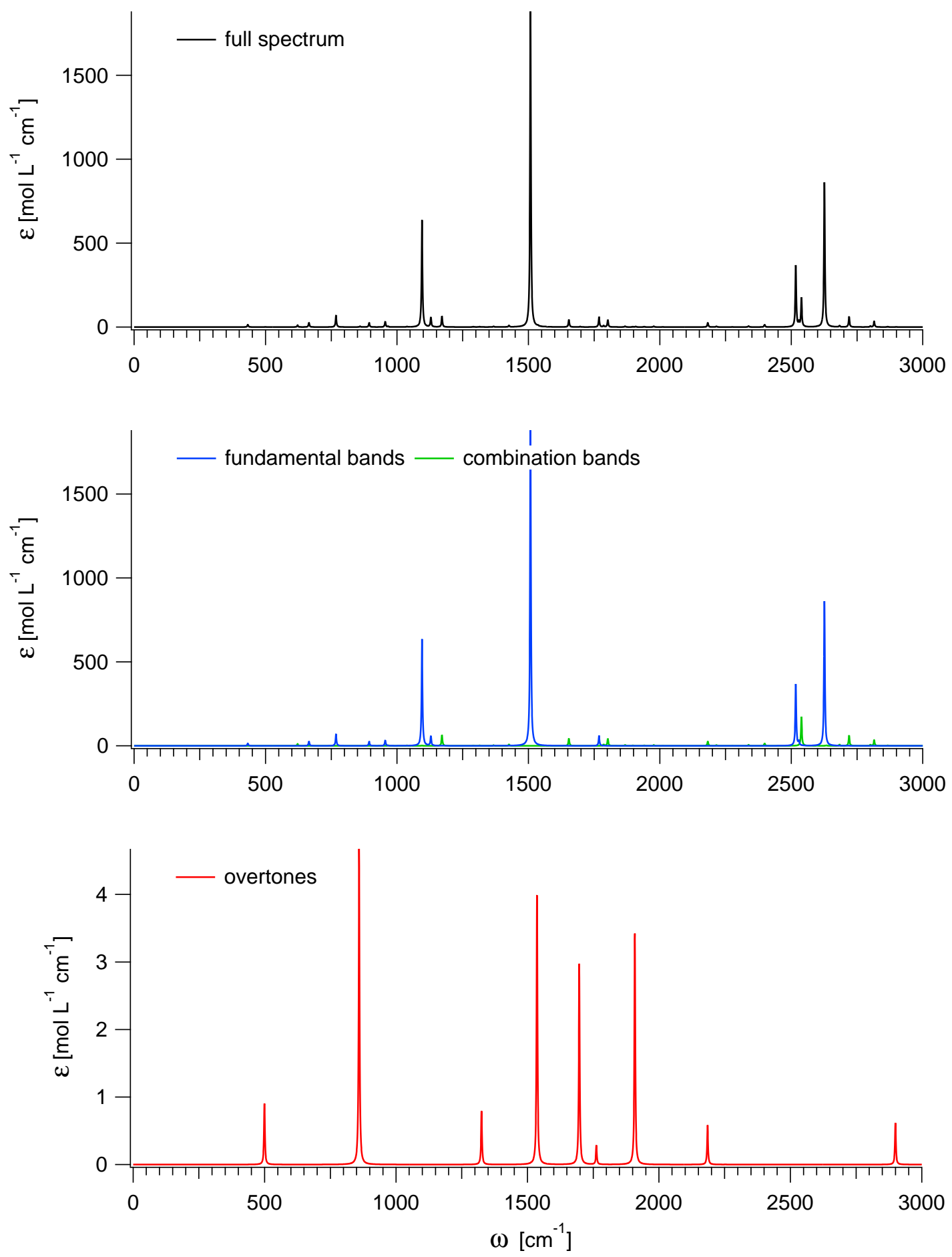
Figure S.8.3. Anharmonic Raman spectrum of $\mathrm{B}_{2} \mathrm{H}_{5}$ obtained by convoluting the calculated activities with Lorentzians having a FWHM of $4 \mathrm{~cm}^{-1}$ (B3LYP-D2/cc-pVTZ results): (top) full spectrum, (middle) contributions from fundamentals and combination bands, (bottom) contributions from overtones
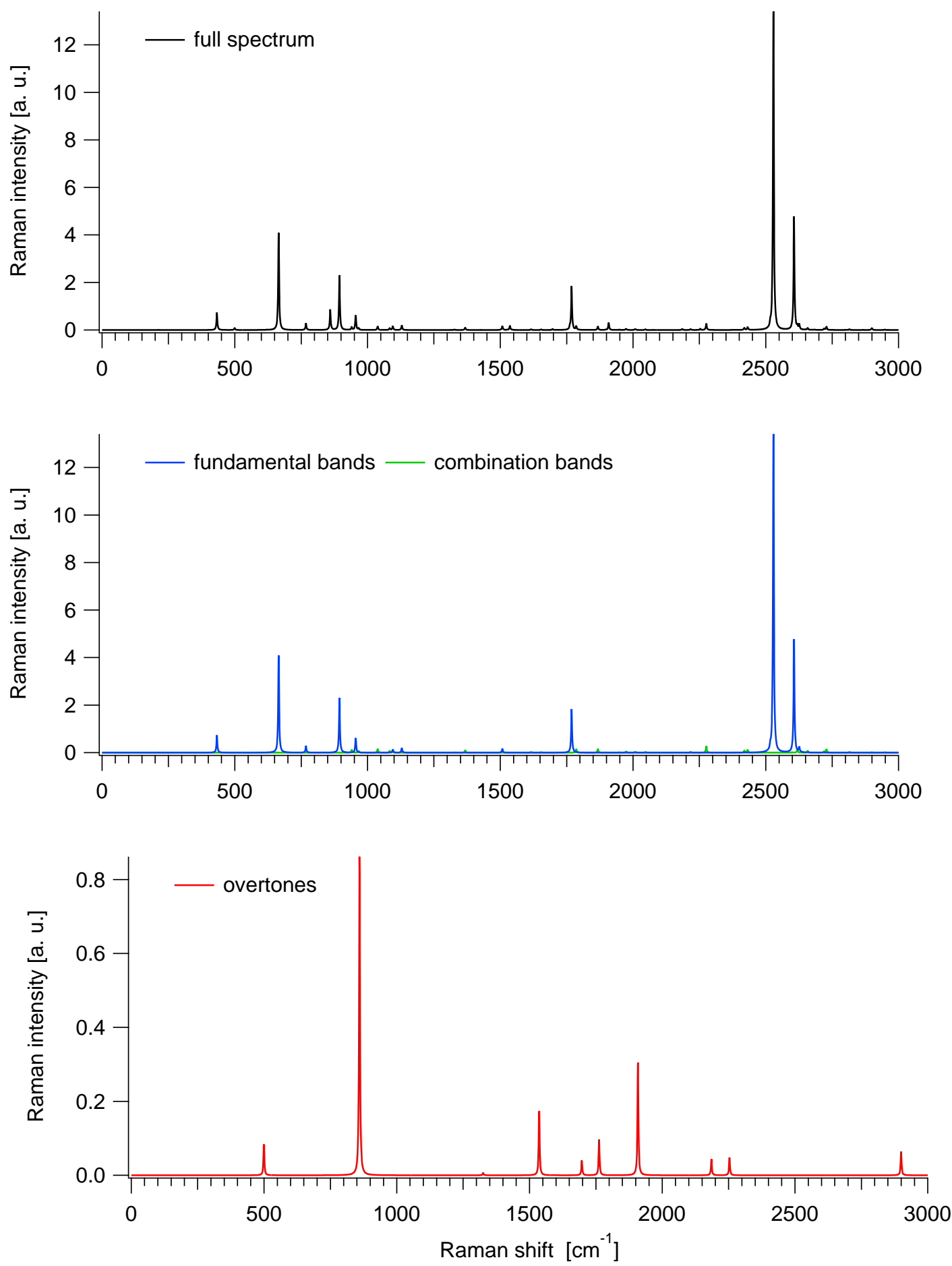
Table S.8.4. $\mathrm{B}_{2} \mathrm{H}_{5}$ : Ideal-gas thermodynamic functions at 1 bar (anharmonic B3LYPD2/cc-pVTZ results)

\begin{tabular}{cccc}
\hline$T$ & $C_{P}$ & $S$ & $H(T)-H(0 K)$ \\
{$[\mathrm{K}]$} & {$\left[\mathrm{J} \mathrm{mol}^{-1} \mathrm{~K}^{-1}\right]$} & {$\left[\mathrm{J} \mathrm{mol}^{-1} \mathrm{~K}^{-1}\right]$} & {$\left[\mathrm{kJ} \mathrm{mol}^{-1}\right]$} \\
\hline 100 & 37.995 & 193.018 & 3.462 \\
200 & 48.820 & 222.416 & 7.764 \\
300 & 62.899 & 244.840 & 13.342 \\
400 & 76.163 & 264.798 & 20.312 \\
500 & 87.382 & 283.036 & 28.505 \\
600 & 96.832 & 299.826 & 37.729 \\
700 & 104.874 & 315.373 & 47.825 \\
800 & 111.743 & 329.837 & 58.665 \\
900 & 117.605 & 343.346 & 70.140 \\
1000 & 122.596 & 356.003 & 82.157 \\
1100 & 126.843 & 367.892 & 94.635 \\
1200 & 130.458 & 379.088 & 107.504 \\
1300 & 133.542 & 389.655 & 120.709 \\
1400 & 136.180 & 399.651 & 134.198 \\
1500 & 138.445 & 409.125 & 147.932 \\
1600 & 140.398 & 418.124 & 161.876 \\
1700 & 142.089 & 426.688 & 176.003 \\
1800 & 143.560 & 434.852 & 190.287 \\
1900 & 144.846 & 442.649 & 204.709 \\
2000 & 145.974 & 450.108 & 219.251 \\
\hline$T:$ & & $S$ \\
\hline
\end{tabular}

$T$ : Temperature. $C_{P}$ : Heat capacity at constant pressure. $S$ : Entropy. $H$ : Enthalpy. 
Figure S.8.4. $\mathrm{B}_{2} \mathrm{H}_{5}$ : Ideal-gas thermodynamic functions at 1 bar (anharmonic B3LYPD2/cc-pVTZ results)
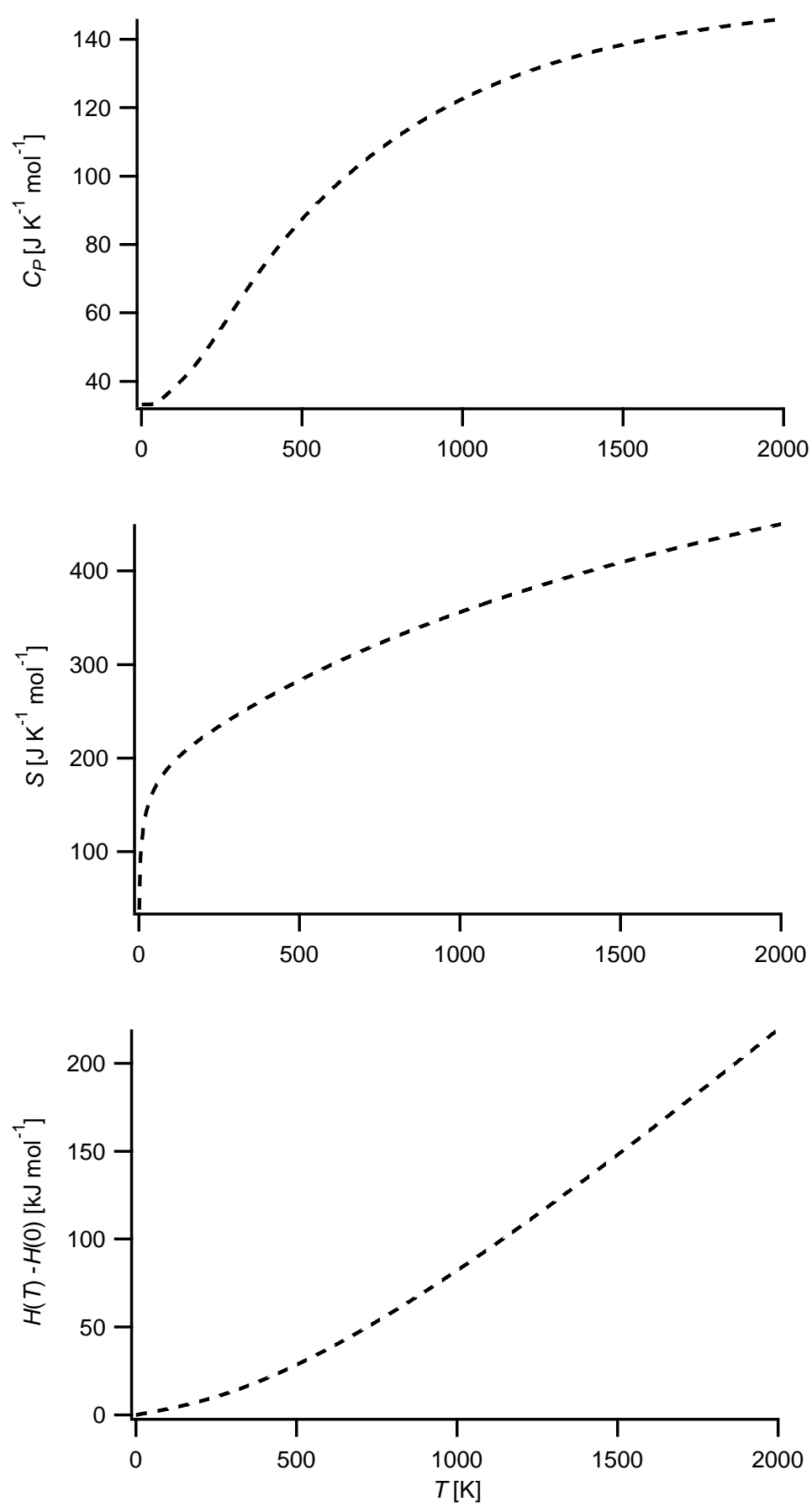

$T$ : Temperature. $C_{P}$ : Heat capacity at constant pressure. $S$ : Entropy. $H$ : Enthalpy. 
Table S.8.5. $\mathrm{B}_{2} \mathrm{H}_{5}$ : Fits (red solid lines) of the computed thermodynamic functions (blue dashed lines) with NASA type functions (Equations (1) - (3)) in the 200 - $900 \mathrm{~K}$ temperature range (anharmonic B3LYP-D2/cc-pVTZ results). In each case, the difference curve between the thermodynamic and NASA functions is plotted in the upper graph

Fit parameters

\begin{tabular}{llllll}
\hline $\mathrm{a}_{1}$ & $2.18504002 \mathrm{e}+00$ & $\mathrm{a}_{2}\left[\mathrm{~K}^{-1}\right]$ & $1.77067045 \mathrm{e}-02$ & $\mathrm{a}_{3}\left[\mathrm{~K}^{-2}\right]$ & $7.23971437 \mathrm{e}-06$ \\
$\mathrm{a}_{4}\left[\mathrm{~K}^{-3}\right]$ & $-2.52536177 \mathrm{e}-08$ & $\mathrm{a}_{5}\left[\mathrm{~K}^{-4}\right]$ & $1.31120001 \mathrm{e}-11$ & $\mathrm{a}_{6}[\mathrm{~K}]$ & $1.32626703 \mathrm{e}+02$
\end{tabular}

$\mathrm{a}_{7}$

$1.15494013 \mathrm{e}+01$
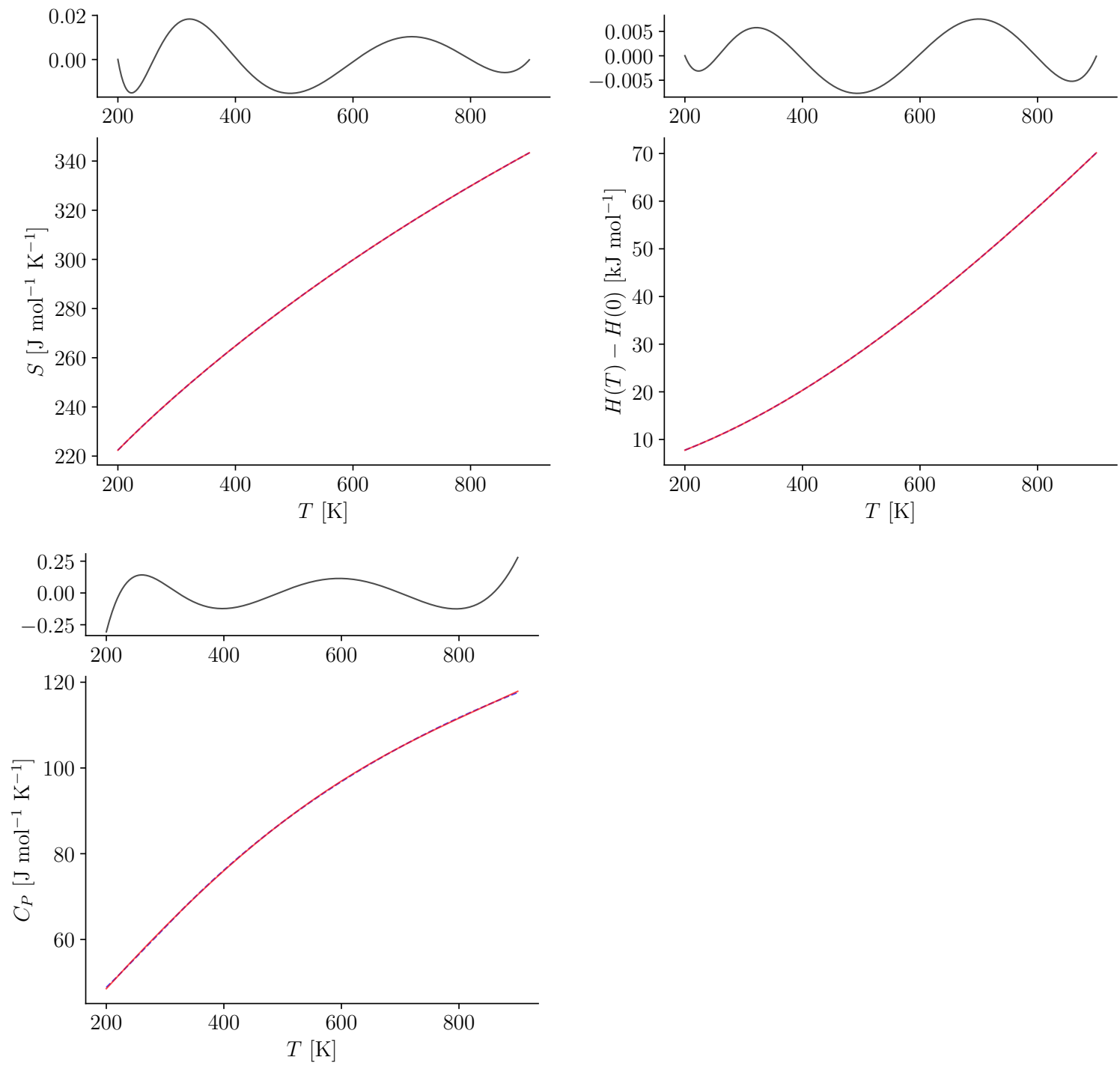


\section{$9 \quad \mathbf{B}_{2} \mathbf{H}_{5}$}

Figure S.9.1. Structure of $\mathrm{B}_{2} \mathrm{H}_{5}{ }^{-}$

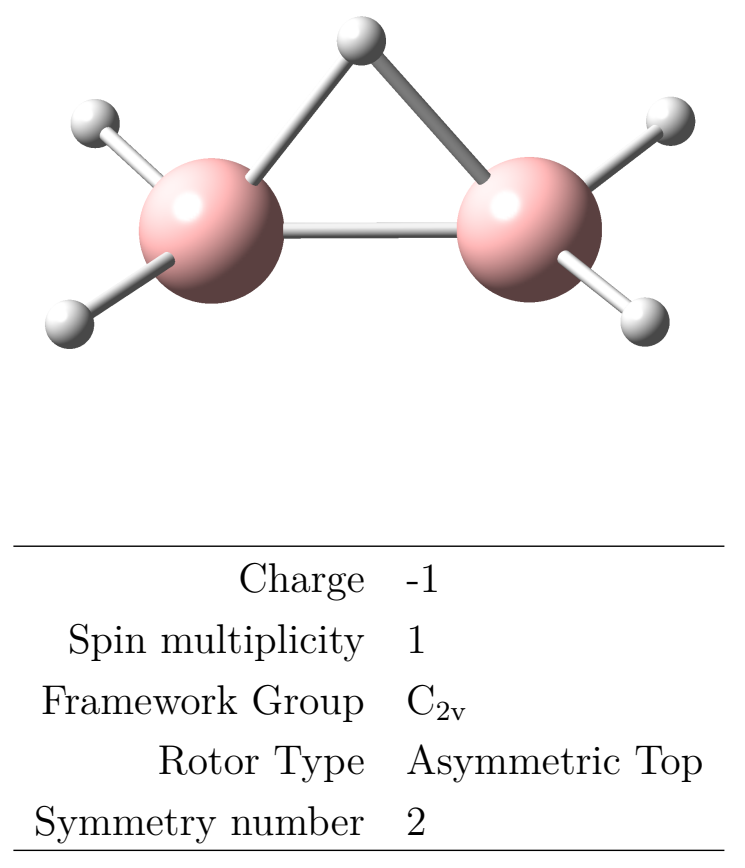

Table S.9.1. $\mathrm{B}_{2} \mathrm{H}_{5}{ }^{-}$: Selected anharmonic results obtained at the B3LYP-D2/cc-pVTZ level

\begin{tabular}{rll}
\hline $\mathrm{ZPE}_{\mathrm{v}}$ & 10397 & $\mathrm{~cm}^{-1}$ \\
$\mathrm{~A}$ & 3.11560 & $\mathrm{~cm}^{-1}$ \\
$\mathrm{~B}$ & 0.69286 & $\mathrm{~cm}^{-1}$ \\
$\mathrm{C}$ & 0.61619 & $\mathrm{~cm}^{-1}$ \\
\hline$C_{P}(300 \mathrm{~K})$ & 55.749 & $\mathrm{~J} \mathrm{~mol}^{-1} \mathrm{~K}-1$ \\
$S(300 \mathrm{~K})$ & 234.439 & $\mathrm{~J} \mathrm{~mol}^{-1} \mathrm{~K}-1$ \\
$H(300 \mathrm{~K})-H(0 \mathrm{~K})$ & 11.723 & $\mathrm{~kJ} \mathrm{~mol}^{-1}$ \\
\hline
\end{tabular}

$\mathrm{ZPE}_{\mathrm{v}}$ : vibrational contribution to the zero-point energy. A, B, C: Rotational constants. $C_{P}$ : Heat capacity at constant pressure. $S$ : Entropy. $H$ : Enthalpy. Ideal-gas calculations (1 bar). 
Table S.9.2. $\mathrm{B}_{2} \mathrm{H}_{5}{ }^{-}$: Cartesian coordinates $(\AA)$ of the B3LYP-D2/cc-pVTZ optimized structure

\begin{tabular}{cccc} 
atom & $\mathrm{x}$ & $\mathrm{y}$ & $\mathrm{z}$ \\
\hline B1 & 0.0000 & 0.8103 & -0.0859 \\
B2 & -0.0000 & -0.8103 & -0.0859 \\
H3 & -1.0230 & -1.4676 & -0.0418 \\
H4 & 0.0000 & 0.0000 & 1.0261 \\
H5 & 1.0230 & 1.4676 & -0.0418 \\
H6 & -1.0230 & 1.4676 & -0.0418 \\
H7 & 1.0230 & -1.4676 & -0.0418 \\
\hline
\end{tabular}


Table S.9.3. $\mathrm{B}_{2} \mathrm{H}_{5}{ }^{-}$: Vibrational frequencies, infrared integrated intensities and Raman activities (B3LYP-D2/cc-pVTZ results)

\begin{tabular}{cccccccc}
\hline & & \multicolumn{3}{c}{ Harmonic } & \multicolumn{3}{c}{ Anharmonic } \\
mode & symm. & $\omega$ & IR & Raman & $\omega$ & IR & Raman \\
& & {$\left[\mathrm{cm}^{-1}\right]$} & {$\left[\mathrm{km} \mathrm{mol}^{-1}\right]$} & {$\left[\AA^{6}\right]$} & {$\left[\mathrm{cm}^{-1}\right]$} & {$\left[\mathrm{km} \mathrm{mol}^{-1}\right]$} & {$\left[\AA^{6}\right]$} \\
\hline 1 & $\mathrm{~B}_{1}$ & 545 & 9.921 & 0.015 & 537 & 10.237 & 0.016 \\
2 & $\mathrm{~B}_{2}$ & 784 & 68.876 & 0.136 & 733 & 98.675 & 0.154 \\
3 & $\mathrm{~A}_{2}$ & 826 & 0.000 & 0.000 & 792 & 0.000 & 0.000 \\
4 & $\mathrm{~A}_{1}$ & 872 & 0.035 & 0.096 & 843 & 0.012 & 0.034 \\
5 & $\mathrm{~A}_{2}$ & 933 & 0.000 & 0.000 & 895 & 0.000 & 0.000 \\
6 & $\mathrm{~A}_{1}$ & 993 & 2.466 & 1.068 & 957 & 1.319 & 1.111 \\
7 & $\mathrm{~B}_{1}$ & 1078 & 6.798 & 0.871 & 983 & 4.421 & 0.747 \\
8 & $\mathrm{~B}_{2}$ & 1199 & 92.333 & 0.016 & 1147 & 91.890 & 0.015 \\
9 & $\mathrm{~A}_{1}$ & 1225 & 0.677 & 0.085 & 1174 & 0.095 & 0.134 \\
10 & $\mathrm{~B}_{2}$ & 1366 & 250.523 & 0.008 & 1119 & 162.958 & 0.005 \\
11 & $\mathrm{~A}_{1}$ & 1783 & 163.818 & 0.865 & 1628 & 112.188 & 0.445 \\
12 & $\mathrm{~A}_{2}$ & 2409 & 0.000 & 1.477 & 2277 & 0.000 & 1.401 \\
13 & $\mathrm{~B}_{2}$ & 2416 & 498.274 & 0.004 & 2242 & 356.155 & 0.006 \\
14 & $\mathrm{~B}_{1}$ & 2434 & 708.110 & 0.002 & 2305 & 716.576 & 0.001 \\
15 & $\mathrm{~A}_{1}$ & 2446 & 0.477 & 2.072 & 2260 & 1.112 & 0.865 \\
\hline
\end{tabular}


Figure S.9.2. Anharmonic IR spectrum of $\mathrm{B}_{2} \mathrm{H}_{5}{ }^{-}$obtained by convoluting the calculated intensities with Lorentzians having a FWHM of $4 \mathrm{~cm}^{-1}$ (B3LYP-D2/cc-pVTZ results): (top) full spectrum, (middle) contributions from fundamentals and combination bands, (bottom) contributions from overtones
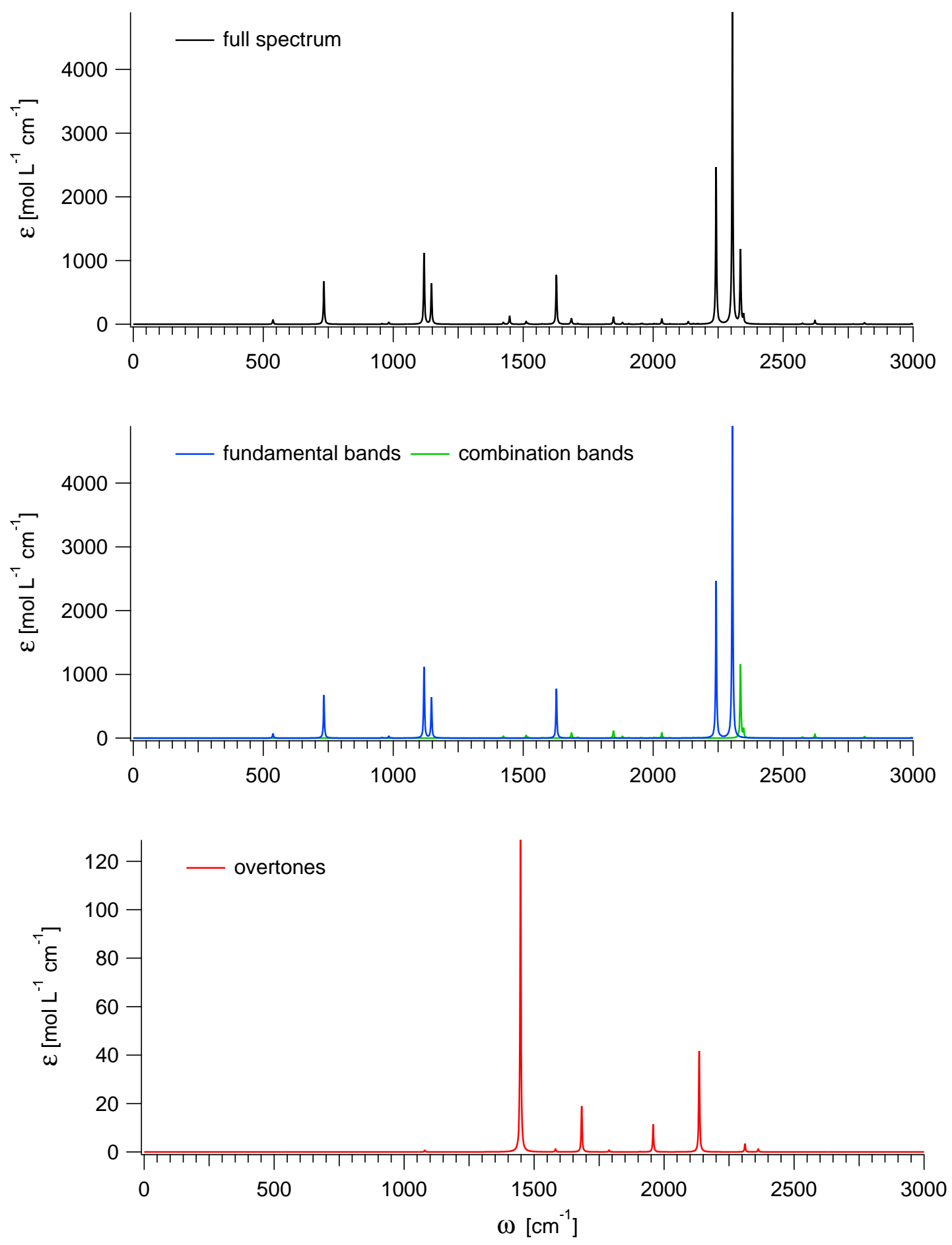
Figure S.9.3. Anharmonic Raman spectrum of $\mathrm{B}_{2} \mathrm{H}_{5}{ }^{-}$obtained by convoluting the calculated activities with Lorentzians having a FWHM of $4 \mathrm{~cm}^{-1}$ (B3LYP-D2/cc-pVTZ results): (top) full spectrum, (middle) contributions from fundamentals and combination bands, (bottom) contributions from overtones
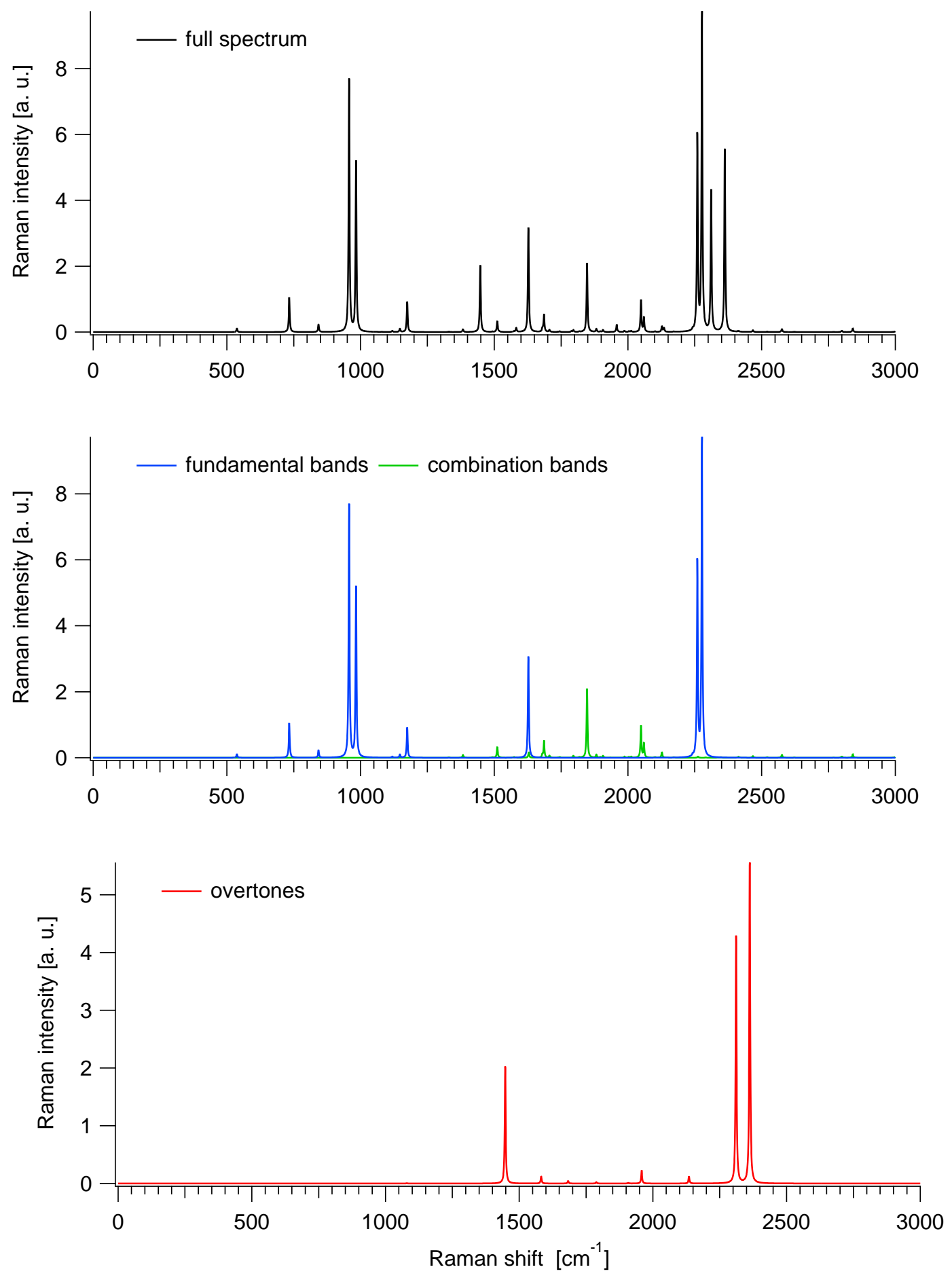

S.72 
Table S.9.4. $\mathrm{B}_{2} \mathrm{H}_{5}{ }^{-}$: Ideal-gas thermodynamic functions at 1 bar (anharmonic B3LYP$\mathrm{D} 2 / \mathrm{cc}-\mathrm{pVTZ}$ results)

\begin{tabular}{cccc}
\hline$T$ & $C_{P}$ & $S$ & $H(T)-H(0 K)$ \\
{$[\mathrm{K}]$} & {$\left[\mathrm{J} \mathrm{mol}^{-1} \mathrm{~K}^{-1}\right]$} & {$\left[\mathrm{J} \mathrm{mol}^{-1} \mathrm{~K}^{-1}\right]$} & {$\left[\mathrm{kJ} \mathrm{mol}^{-1}\right]$} \\
\hline 100 & 33.526 & 190.494 & 3.329 \\
200 & 40.561 & 215.251 & 6.939 \\
300 & 55.749 & 234.439 & 11.723 \\
400 & 71.534 & 252.678 & 18.101 \\
500 & 85.131 & 270.146 & 25.955 \\
600 & 96.425 & 286.698 & 35.050 \\
700 & 105.749 & 302.285 & 45.174 \\
800 & 113.434 & 316.924 & 56.146 \\
900 & 119.765 & 330.662 & 67.816 \\
1000 & 124.989 & 343.560 & 80.062 \\
1100 & 129.312 & 355.682 & 92.783 \\
1200 & 132.908 & 367.092 & 105.900 \\
1300 & 135.914 & 377.853 & 119.345 \\
1400 & 138.444 & 388.021 & 133.067 \\
1500 & 140.585 & 397.647 & 147.021 \\
1600 & 142.409 & 406.780 & 161.173 \\
1700 & 143.972 & 415.462 & 175.494 \\
1800 & 145.321 & 423.730 & 189.961 \\
1900 & 146.489 & 431.619 & 204.553 \\
2000 & 147.508 & 439.159 & 219.254 \\
\hline
\end{tabular}

$T$ : Temperature. $C_{P}$ : Heat capacity at constant pressure. $S$ : Entropy. $H$ : Enthalpy. 
Figure S.9.4. $\mathrm{B}_{2} \mathrm{H}_{5}{ }^{-}$: Ideal-gas thermodynamic functions at 1 bar (anharmonic B3LYPD2/cc-pVTZ results)
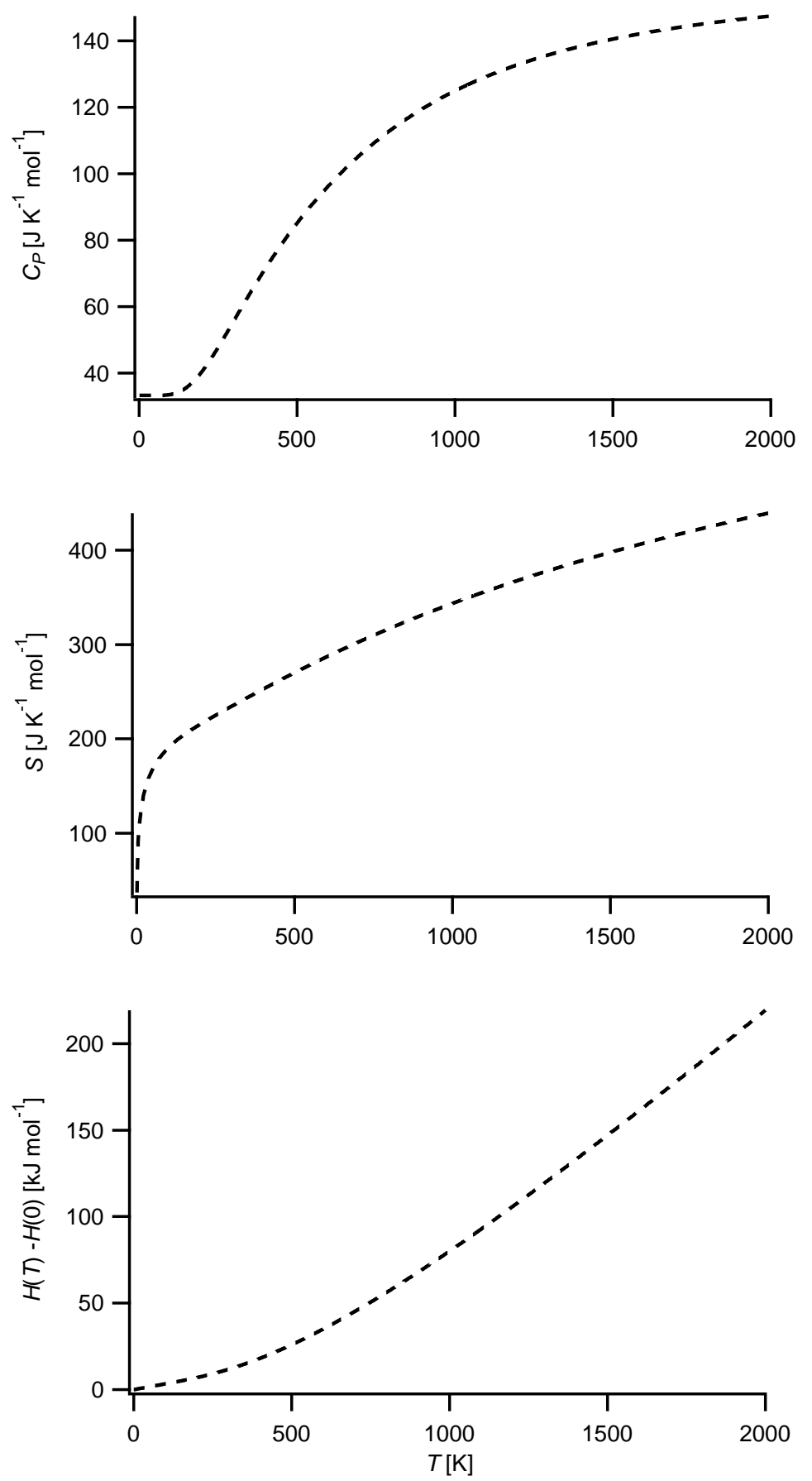

$T$ : Temperature. $C_{P}$ : Heat capacity at constant pressure. $S$ : Entropy. $H$ : Enthalpy. 
Table S.9.5. $\mathrm{B}_{2} \mathrm{H}_{5}{ }^{-}$: Fits (red solid lines) of the computed thermodynamic functions (blue dashed lines) with NASA type functions (Equations (1) - (3)) in the 200 - $900 \mathrm{~K}$ temperature range (anharmonic B3LYP-D2/cc-pVTZ results). In each case, the difference curve between the thermodynamic and NASA functions is plotted in the upper graph

Fit parameters

\begin{tabular}{llllll}
\hline $\mathrm{a}_{1}$ & $2.04605446 \mathrm{e}+00$ & $\mathrm{a}_{2}\left[\mathrm{~K}^{-1}\right]$ & $6.95773848 \mathrm{e}-03$ & $\mathrm{a}_{3}\left[\mathrm{~K}^{-2}\right]$ & $4.86872153 \mathrm{e}-05$ \\
$\mathrm{a}_{4}\left[\mathrm{~K}^{-3}\right]$ & $-7.71256114 \mathrm{e}-08$ & $\mathrm{a}_{5}\left[\mathrm{~K}^{-4}\right]$ & $3.49837356 \mathrm{e}-11$ & $\mathrm{a}_{6}[\mathrm{~K}]$ & $1.85026750 \mathrm{e}+02$
\end{tabular}

$a_{7}$
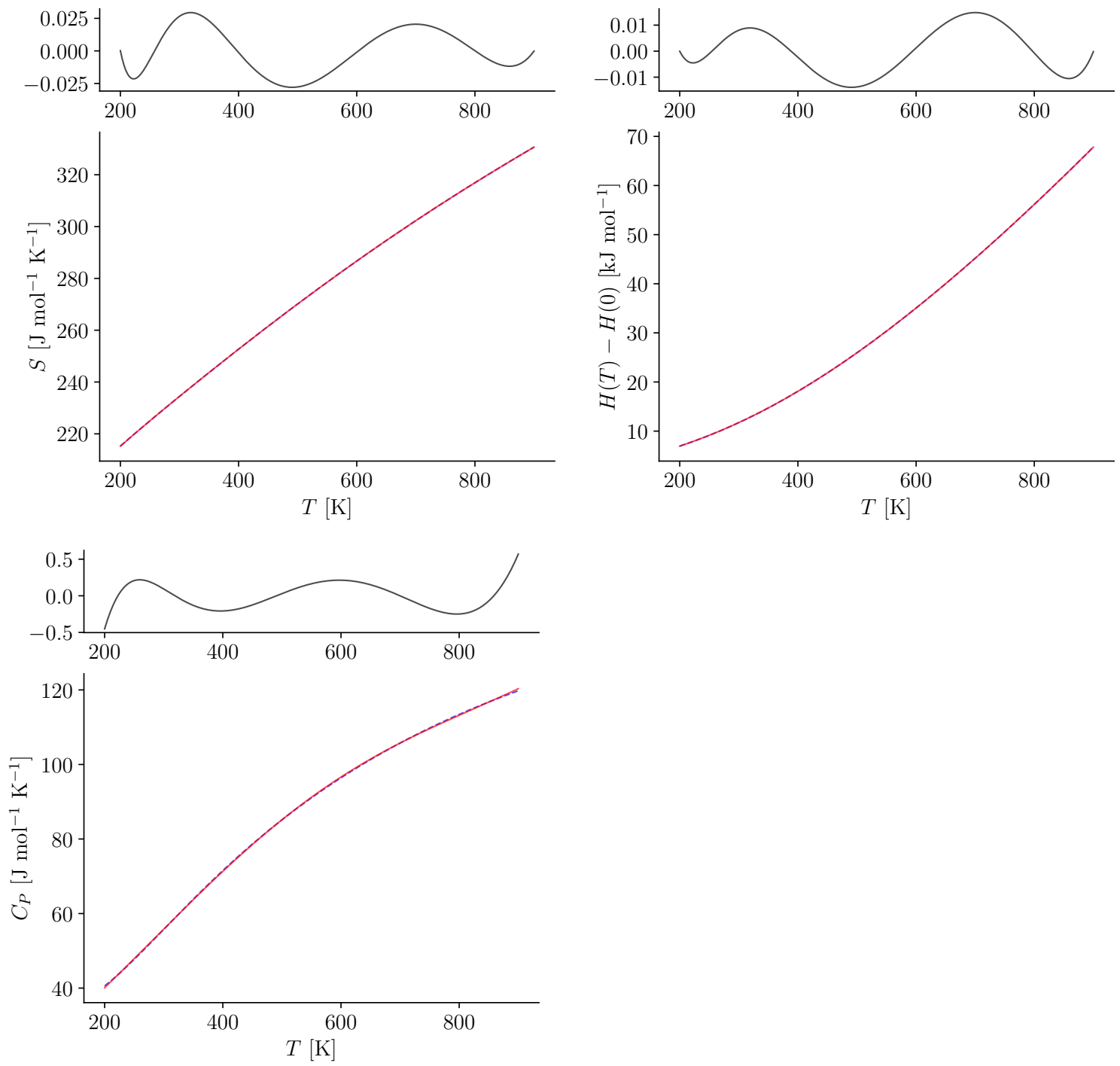


\section{$10 \mathbf{B}_{2} \mathbf{H}_{6}$}

Figure S.10.1. Structure of $\mathrm{B}_{2} \mathrm{H}_{6}$

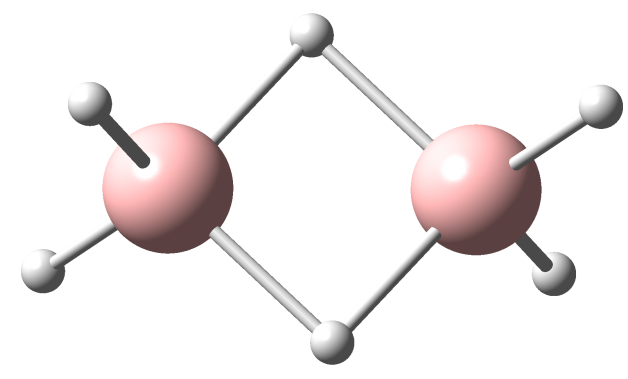

\begin{aligned} & \hline Charge 0 \\ & Spin multiplicity 1 \\ & Framework Group $\mathrm{D}_{2 \mathrm{~h}} \\ &$ Rotor Type Asymmetric Top \\ & Symmetry number 4 \\ & \hline\end{aligned}

Table S.10.1. $\mathrm{B}_{2} \mathrm{H}_{6}$ : Selected anharmonic results obtained at the B3LYP-D2/cc-pVTZ level

\begin{tabular}{rll}
\hline $\mathrm{ZPE}_{\mathrm{v}}$ & 13694 & $\mathrm{~cm}^{-1}$ \\
$\mathrm{~A}$ & 2.69951 & $\mathrm{~cm}^{-1}$ \\
$\mathrm{~B}$ & 0.61115 & $\mathrm{~cm}^{-1}$ \\
$\mathrm{C}$ & 0.56231 & $\mathrm{~cm}^{-1}$ \\
\hline$C_{P}(300 \mathrm{~K})$ & 57.690 & $\mathrm{~J} \mathrm{~mol}^{-1} \mathrm{~K}-1$ \\
$S(300 \mathrm{~K})$ & 232.832 & $\mathrm{~J} \mathrm{~mol}^{-1} \mathrm{~K}-1$ \\
$H(300 \mathrm{~K})-H(0 \mathrm{~K})$ & 12.112 & $\mathrm{~kJ} \mathrm{~mol}^{-1}$ \\
\hline
\end{tabular}

$\mathrm{ZPE}_{\mathrm{v}}$ : vibrational contribution to the zero-point energy. A, B, C: Rotational constants. $C_{P}$ : Heat capacity at constant pressure. $S$ : Entropy. $H$ : Enthalpy. Ideal-gas calculations (1 bar). 
Table S.10.2. $\mathrm{B}_{2} \mathrm{H}_{6}$ : Cartesian coordinates $(\AA)$ of the B3LYP-D2/cc-pVTZ optimized structure

\begin{tabular}{cccc} 
atom & $\mathrm{x}$ & $\mathrm{y}$ & $\mathrm{z}$ \\
\hline B1 & -0.8804 & 0.0000 & 0.0000 \\
B2 & 0.8804 & -0.0000 & 0.0000 \\
H3 & 0.0000 & 0.0000 & 0.9771 \\
H4 & -1.4599 & 1.0352 & 0.0000 \\
H5 & 1.4599 & 1.0352 & 0.0000 \\
H6 & 0.0000 & 0.0000 & -0.9771 \\
H7 & -1.4599 & -1.0352 & -0.0000 \\
H8 & 1.4599 & -1.0352 & 0.0000 \\
\hline
\end{tabular}


Table S.10.3. $\mathrm{B}_{2} \mathrm{H}_{6}$ : Vibrational frequencies, infrared integrated intensities and Raman activities (B3LYP-D2/cc-pVTZ results)

\begin{tabular}{|c|c|c|c|c|c|c|c|}
\hline \multirow[b]{2}{*}{ mode } & \multirow[b]{2}{*}{ symm. } & \multicolumn{3}{|c|}{ Harmonic } & \multicolumn{3}{|c|}{ Anharmonic } \\
\hline & & $\begin{array}{c}\omega \\
{\left[\mathrm{cm}^{-1}\right]}\end{array}$ & $\begin{array}{c}\mathrm{IR} \\
{\left[\mathrm{km} \mathrm{mol}^{-1}\right]}\end{array}$ & $\begin{array}{c}\text { Raman } \\
{\left[\AA^{6}\right]}\end{array}$ & $\begin{array}{c}\omega \\
{\left[\mathrm{cm}^{-1}\right]}\end{array}$ & $\begin{array}{c}\mathrm{IR} \\
{\left[\mathrm{km} \mathrm{mol}^{-1}\right]}\end{array}$ & $\begin{array}{c}\text { Raman } \\
{\left[\AA^{6}\right]}\end{array}$ \\
\hline 1 & $\mathrm{~B}_{2 \mathrm{u}}$ & 379 & 16.813 & 0.000 & 365 & 15.778 & 0.000 \\
\hline 2 & $\mathrm{~A}_{\mathrm{g}}$ & 819 & 0.000 & 0.346 & 775 & 0.000 & 0.388 \\
\hline 3 & $\mathrm{~A}_{\mathrm{u}}$ & 869 & 0.000 & 0.000 & 819 & 0.000 & 0.000 \\
\hline 4 & $\mathrm{~B}_{2 \mathrm{~g}}$ & 900 & 0.000 & 0.007 & 858 & 0.000 & 0.003 \\
\hline 5 & $\mathrm{~B}_{1 \mathrm{~g}}$ & 972 & 0.000 & 0.010 & 923 & 0.000 & 0.015 \\
\hline 6 & $\mathrm{~B}_{2 \mathrm{u}}$ & 1010 & 0.492 & 0.000 & 914 & 0.211 & 0.000 \\
\hline 7 & $\mathrm{~B}_{1 \mathrm{u}}$ & 1017 & 23.330 & 0.000 & 966 & 19.911 & 0.000 \\
\hline 8 & $\mathrm{~B}_{3 \mathrm{~g}}$ & 1072 & 0.000 & 0.250 & 972 & 0.000 & 0.252 \\
\hline 9 & $\mathrm{~B}_{3 \mathrm{u}}$ & 1228 & 86.278 & 0.000 & 1174 & 77.720 & 0.000 \\
\hline 10 & $\mathrm{~A}_{\mathrm{g}}$ & 1231 & 0.000 & 0.115 & 1178 & 0.000 & 0.142 \\
\hline 11 & $\mathrm{~B}_{3 \mathrm{u}}$ & 1746 & 460.425 & 0.000 & 1541 & 370.566 & 0.000 \\
\hline 12 & $\mathrm{~B}_{2 \mathrm{~g}}$ & 1889 & 0.000 & 0.036 & 1699 & 0.000 & 0.029 \\
\hline 13 & $\mathrm{~B}_{1 \mathrm{u}}$ & 1996 & 8.368 & 0.000 & 1944 & 4.997 & 0.000 \\
\hline 14 & $\mathrm{~A}_{\mathrm{g}}$ & 2198 & 0.000 & 0.532 & 2116 & 0.000 & 0.640 \\
\hline 15 & $\mathrm{~B}_{3 \mathrm{u}}$ & 2646 & 129.548 & 0.000 & 2517 & 130.844 & 0.000 \\
\hline 16 & $\mathrm{~A}_{\mathrm{g}}$ & 2653 & 0.000 & 1.826 & 2526 & 0.000 & 1.997 \\
\hline 17 & $\mathrm{~B}_{1 \mathrm{~g}}$ & 2706 & 0.000 & 0.712 & 2579 & 0.000 & 0.764 \\
\hline 18 & $\mathrm{~B}_{2 \mathrm{u}}$ & 2723 & 171.562 & 0.000 & 2598 & 168.079 & 0.000 \\
\hline
\end{tabular}


Figure S.10.2. Anharmonic IR spectrum of $\mathrm{B}_{2} \mathrm{H}_{6}$ obtained by convoluting the calculated intensities with Lorentzians having a FWHM of $4 \mathrm{~cm}^{-1}$ (B3LYP-D2/cc-pVTZ results): (top) full spectrum, (bottom) contributions from fundamentals and combination bands
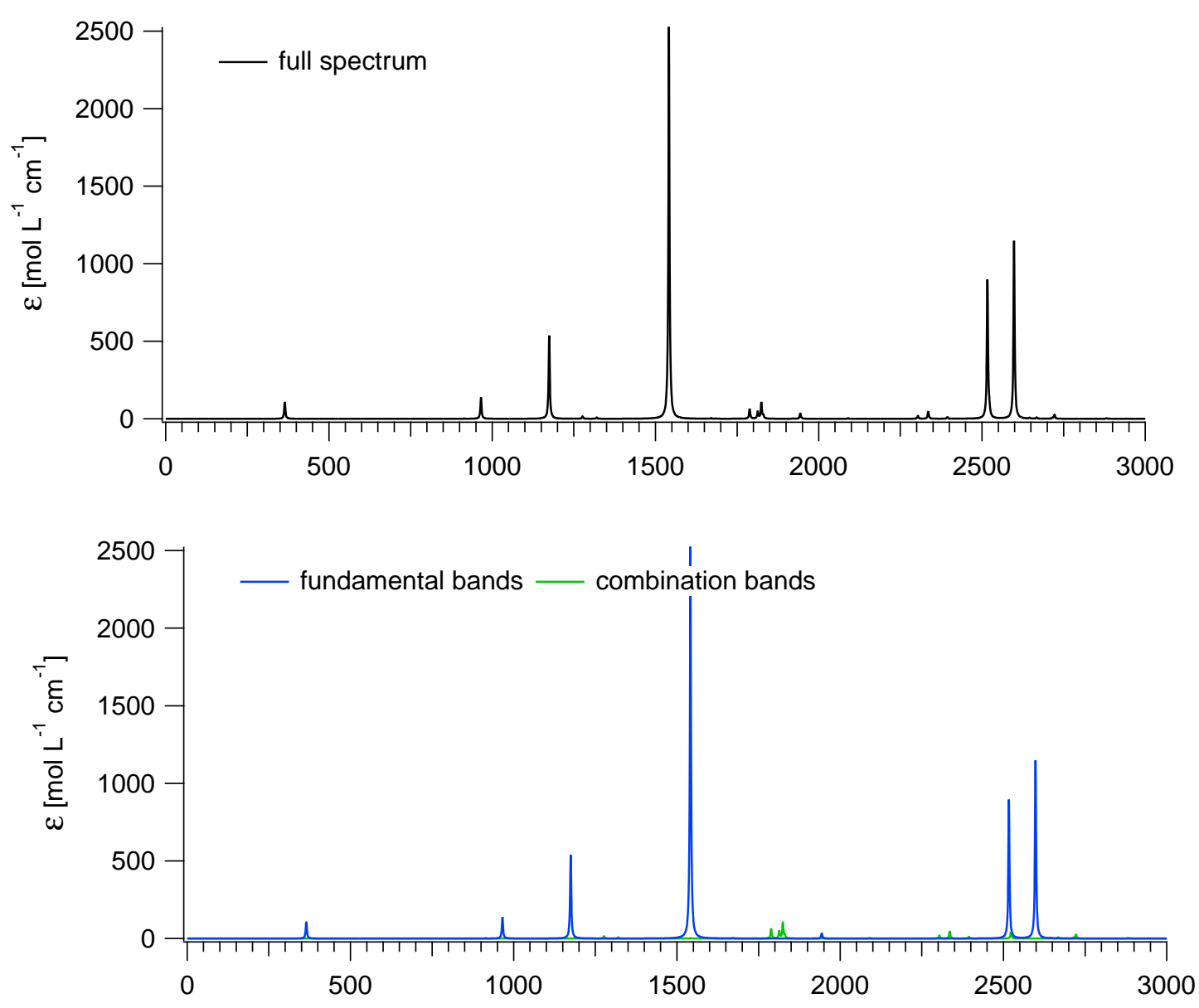
Figure S.10.3. Anharmonic Raman spectrum of $\mathrm{B}_{2} \mathrm{H}_{6}$ obtained by convoluting the calculated activities with Lorentzians having a FWHM of $4 \mathrm{~cm}^{-1}$ (B3LYP-D2/cc-pVTZ results): (top) full spectrum, (middle) contributions from fundamentals and combination bands, (bottom) contributions from overtones
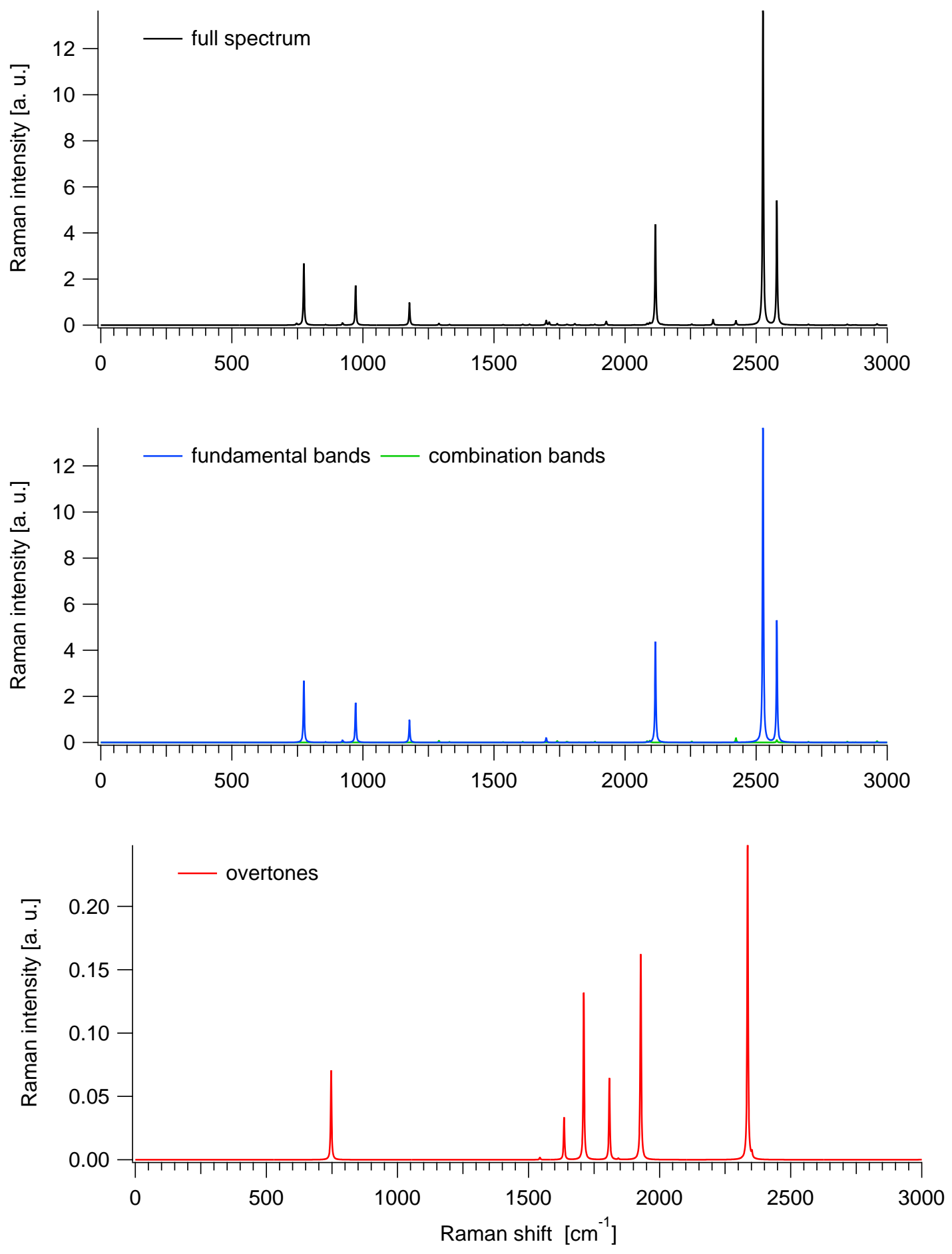
Table S.10.4. $\mathrm{B}_{2} \mathrm{H}_{6}$ : Ideal-gas thermodynamic functions at 1 bar (anharmonic B3LYPD2/cc-pVTZ results)

\begin{tabular}{cccc}
\hline$T$ & $C_{P}$ & $S$ & $H(T)-H(0 K)$ \\
{$[\mathrm{K}]$} & {$\left[\mathrm{J} \mathrm{mol}^{-1} \mathrm{~K}^{-1}\right]$} & {$\left[\mathrm{J} \mathrm{mol}^{-1} \mathrm{~K}^{-1}\right]$} & {$\left[\mathrm{kJ} \mathrm{mol}^{-1}\right]$} \\
\hline 100 & 34.513 & 186.927 & 3.349 \\
200 & 42.560 & 212.857 & 7.135 \\
300 & 57.690 & 232.832 & 12.112 \\
400 & 74.030 & 251.689 & 18.706 \\
500 & 88.766 & 269.827 & 26.862 \\
600 & 101.569 & 287.172 & 36.395 \\
700 & 112.580 & 303.677 & 47.116 \\
800 & 121.979 & 319.341 & 58.857 \\
900 & 129.955 & 334.181 & 71.465 \\
1000 & 136.699 & 348.232 & 84.807 \\
1100 & 142.396 & 361.536 & 98.770 \\
1200 & 147.215 & 374.139 & 113.257 \\
1300 & 151.301 & 386.088 & 128.188 \\
1400 & 154.779 & 397.431 & 143.497 \\
1500 & 157.753 & 408.214 & 159.127 \\
1600 & 160.308 & 418.479 & 175.034 \\
1700 & 162.513 & 428.265 & 191.177 \\
1800 & 164.426 & 437.609 & 207.526 \\
1900 & 166.094 & 446.545 & 224.054 \\
2000 & 167.554 & 455.103 & 240.738 \\
\hline$T$ & & & $S: E$ \\
\hline
\end{tabular}

$T$ : Temperature. $C_{P}$ : Heat capacity at constant pressure. $S$ : Entropy. $H$ : Enthalpy. 
Figure S.10.4. $\mathrm{B}_{2} \mathrm{H}_{6}$ : Ideal-gas thermodynamic functions at 1 bar (anharmonic B3LYPD2/cc-pVTZ results)
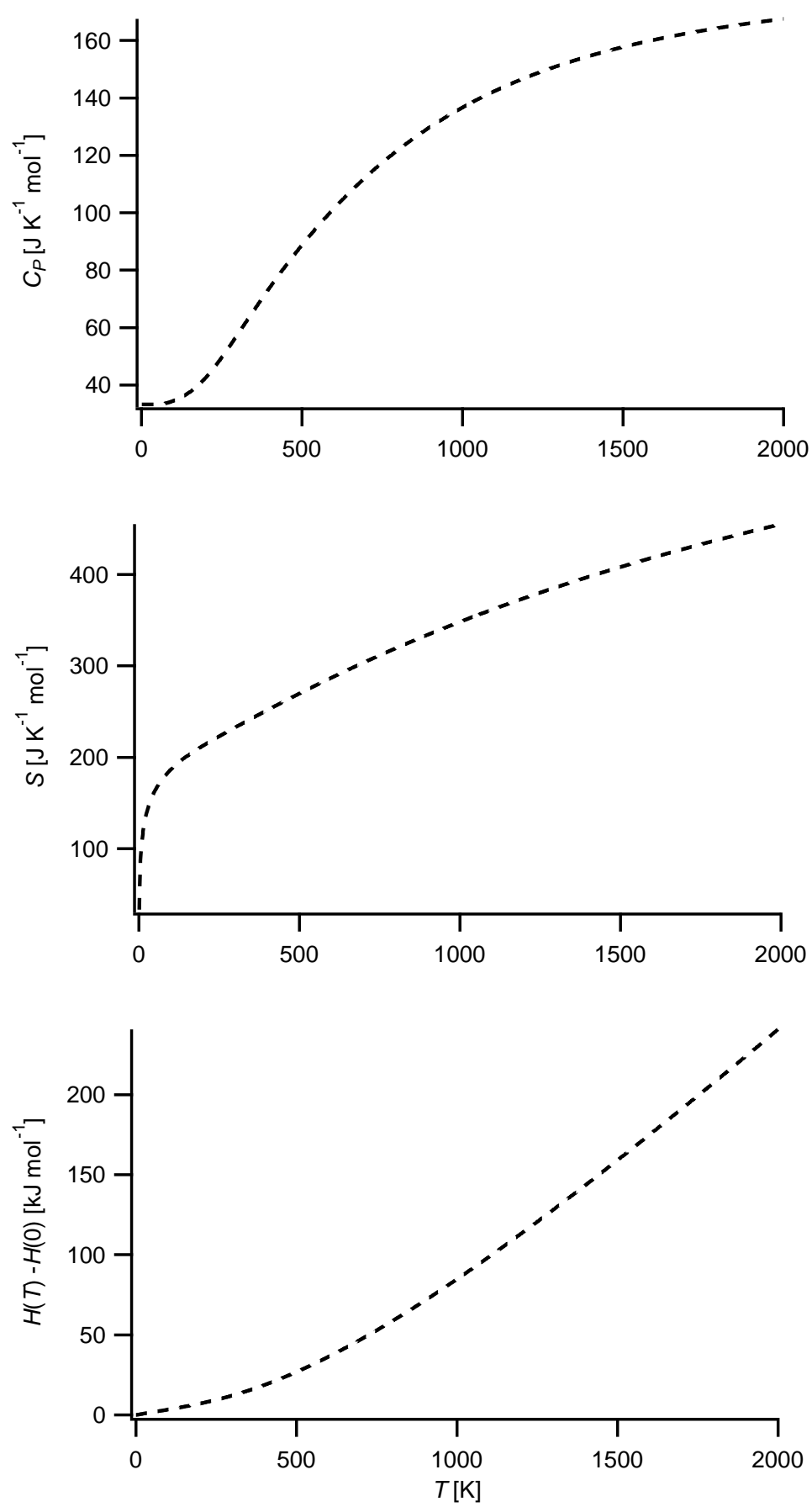

$T$ : Temperature. $C_{P}$ : Heat capacity at constant pressure. $S$ : Entropy. $H$ : Enthalpy. 
Table S.10.5. $\mathrm{B}_{2} \mathrm{H}_{6}$ : Fits (red solid lines) of the computed thermodynamic functions (blue dashed lines) with NASA type functions (Equations (1) - (3)) in the 200 - $900 \mathrm{~K}$ temperature range (anharmonic B3LYP-D2/cc-pVTZ results). In each case, the difference curve between the thermodynamic and NASA functions is plotted in the upper graph

Fit parameters

\begin{tabular}{llllll}
\hline $\mathrm{a}_{1}$ & $2.64851285 \mathrm{e}+00$ & $\mathrm{a}_{2}\left[\mathrm{~K}^{-1}\right]$ & $3.90242088 \mathrm{e}-03$ & $\mathrm{a}_{3}\left[\mathrm{~K}^{-2}\right]$ & $5.56787255 \mathrm{e}-05$ \\
$\mathrm{a}_{4}\left[\mathrm{~K}^{-3}\right]$ & $-8.01318548 \mathrm{e}-08$ & $\mathrm{a}_{5}\left[\mathrm{~K}^{-4}\right]$ & $3.48276191 \mathrm{e}-11$ & $\mathrm{a}_{6}[\mathrm{~K}]$ & $1.31708809 \mathrm{e}+02$
\end{tabular}

$\mathrm{a}_{7}$

$9.87391602 \mathrm{e}+00$
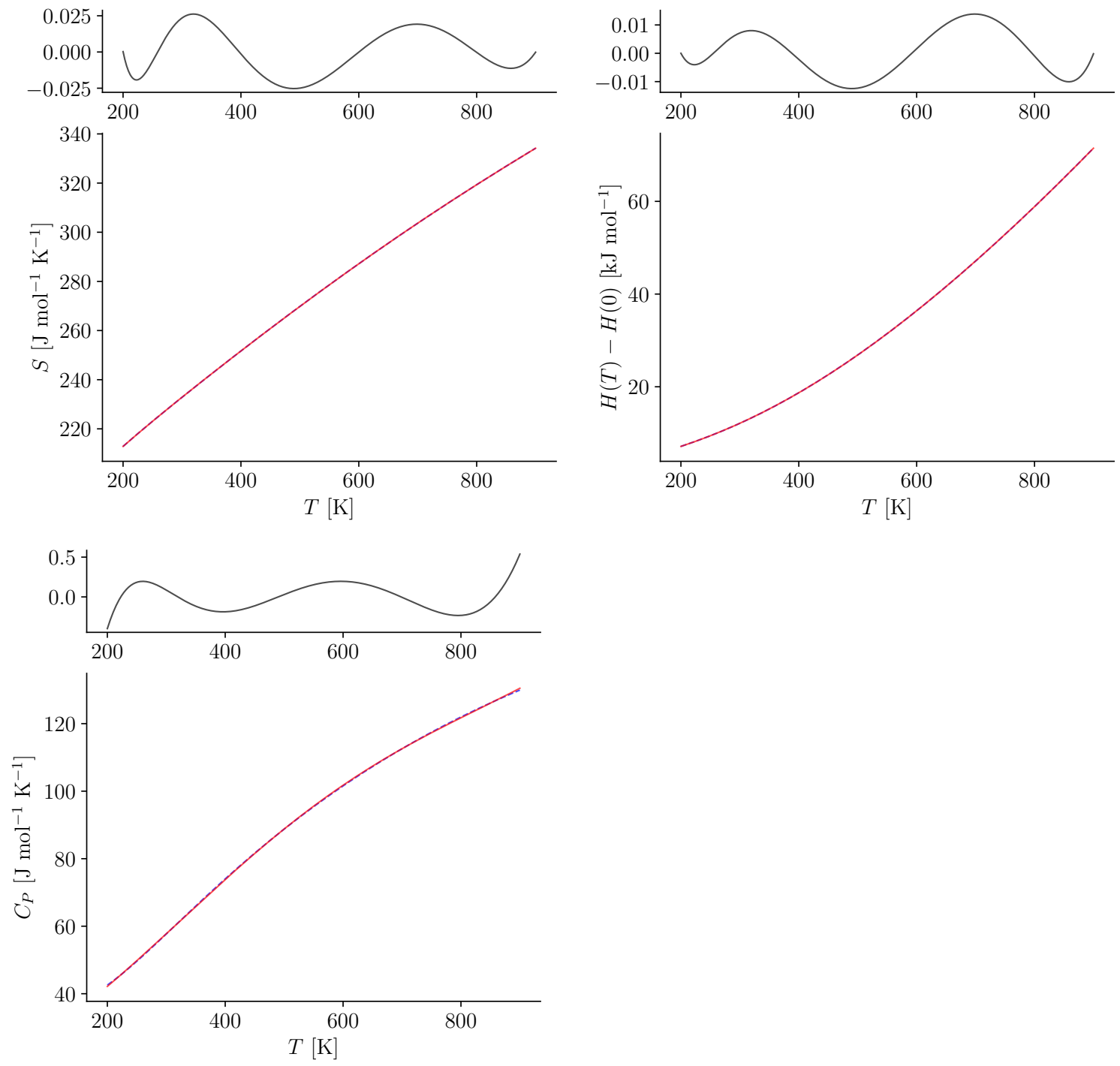


\section{$11 \mathrm{~B}_{2} \mathrm{H}_{7}$}

Figure S.11.1. Structure of $\mathrm{B}_{2} \mathrm{H}_{7}$

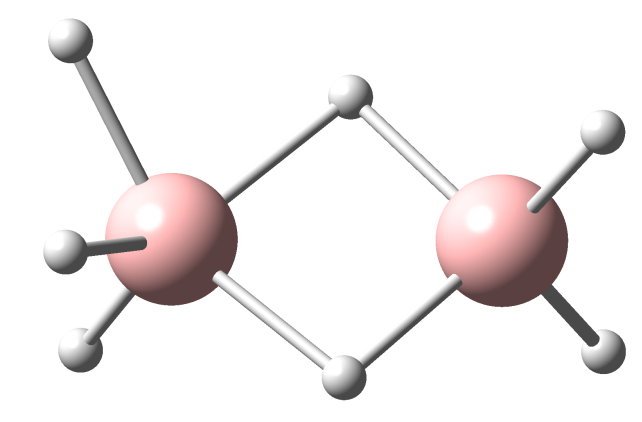

\begin{aligned} & \hline Charge 0 \\ & Spin multiplicity 2 \\ & Framework Group $\mathrm{C}_{\mathrm{s}} \\ &$ Rotor Type Asymmetric Top \\ & Symmetry number 1 \\ & \hline\end{aligned}

Table S.11.1. $\mathrm{B}_{2} \mathrm{H}_{7}$ : Selected anharmonic results obtained at the B3LYP-D2/cc-pVTZ level

\begin{tabular}{rll}
\hline $\mathrm{ZPE}_{\mathrm{v}}$ & 14655 & $\mathrm{~cm}^{-1}$ \\
$\mathrm{~A}$ & 2.16795 & $\mathrm{~cm}^{-1}$ \\
$\mathrm{~B}$ & 0.51520 & $\mathrm{~cm}^{-1}$ \\
$\mathrm{C}$ & 0.48764 & $\mathrm{~cm}^{-1}$ \\
\hline$C_{P}(300 \mathrm{~K})$ & 71.849 & $\mathrm{~J} \mathrm{~mol}^{-1} \mathrm{~K}-1$ \\
$S(300 \mathrm{~K})$ & 254.297 & $\mathrm{~J} \mathrm{~mol}^{-1} \mathrm{~K}-1$ \\
$H(300 \mathrm{~K})-H(0 \mathrm{~K})$ & 13.659 & $\mathrm{~kJ} \mathrm{~mol}^{-1}$ \\
\hline
\end{tabular}

$\mathrm{ZPE}_{\mathrm{v}}$ : vibrational contribution to the zero-point energy. A, B, C: Rotational constants. $C_{P}$ : Heat capacity at constant pressure. $S$ : Entropy. $H$ : Enthalpy. Ideal-gas calculations (1 bar). 
Table S.11.2. $\mathrm{B}_{2} \mathrm{H}_{7}$ : Cartesian coordinates $(\AA)$ of the B3LYP-D2/cc-pVTZ optimized structure

\begin{tabular}{cccc} 
atom & $\mathrm{x}$ & $\mathrm{y}$ & $\mathrm{z}$ \\
\hline B1 & 0.0429 & -0.8451 & 0.0000 \\
B2 & 0.0429 & 1.0003 & 0.0000 \\
H3 & 0.0179 & 0.1379 & 0.9688 \\
H4 & 0.0179 & 0.1379 & -0.9688 \\
H5 & -0.8342 & -1.4061 & -0.7291 \\
H6 & 1.1016 & -1.3717 & 0.0000 \\
H7 & 1.0936 & 1.5492 & 0.0000 \\
H8 & -0.8342 & -1.4061 & 0.7291 \\
H9 & -0.9920 & 1.5832 & 0.0000 \\
\hline
\end{tabular}


Table S.11.3. $\mathrm{B}_{2} \mathrm{H}_{7}$ : Vibrational frequencies, infrared integrated intensities and Raman activities (B3LYP-D2/cc-pVTZ results)

\begin{tabular}{|c|c|c|c|c|c|c|c|}
\hline \multirow[b]{2}{*}{ mode } & \multirow[b]{2}{*}{ symm. } & \multicolumn{3}{|c|}{ Harmonic } & \multicolumn{3}{|c|}{ Anharmonic } \\
\hline & & $\begin{array}{c}\omega \\
{\left[\mathrm{cm}^{-1}\right]}\end{array}$ & $\begin{array}{c}\mathrm{IR} \\
{\left[\mathrm{km} \mathrm{mol}^{-1}\right]}\end{array}$ & $\begin{array}{c}\text { Raman } \\
{\left[\AA^{6}\right]}\end{array}$ & $\begin{array}{c}\omega \\
{\left[\mathrm{cm}^{-1}\right]}\end{array}$ & $\begin{array}{c}\mathrm{IR} \\
{\left[\mathrm{km} \mathrm{mol}^{-1}\right]}\end{array}$ & $\begin{array}{c}\text { Raman } \\
{\left[\AA^{6}\right]}\end{array}$ \\
\hline 1 & A" & 46 & 4.040 & 6.717 & 3018 & 549.289 & 5.223 \\
\hline 2 & $A^{\prime}$ & 366 & 9.694 & 0.117 & 325 & 1.093 & 0.143 \\
\hline 3 & A" & 599 & 4.473 & 0.069 & 465 & 2.268 & 0.053 \\
\hline 4 & $A^{\prime}$ & 689 & 2.767 & 0.455 & 608 & 4.654 & 0.496 \\
\hline 5 & $A^{\prime \prime}$ & 737 & 0.061 & 0.120 & 664 & 0.421 & 0.059 \\
\hline 6 & $A^{\prime}$ & 862 & 28.552 & 0.094 & 728 & 30.842 & 0.057 \\
\hline 7 & $A^{\prime}$ & 883 & 11.668 & 0.246 & 884 & 2.264 & 0.178 \\
\hline 8 & $A^{\prime \prime}$ & 923 & 0.176 & 0.148 & 854 & 0.162 & 0.132 \\
\hline 9 & $A^{\prime}$ & 999 & 2.271 & 0.043 & 902 & 0.435 & 0.027 \\
\hline 10 & A" & 1059 & 2.802 & 0.152 & 942 & 1.827 & 0.132 \\
\hline 11 & $A^{\prime}$ & 1178 & 82.265 & 0.097 & 1036 & 40.067 & 0.107 \\
\hline 12 & $A^{\prime}$ & 1197 & 33.026 & 0.043 & 1143 & 54.840 & 0.056 \\
\hline 13 & A" & 1404 & 5.034 & 0.210 & 1011 & 3.628 & 0.017 \\
\hline 14 & $A^{\prime}$ & 1618 & 163.847 & 0.184 & 1268 & 114.901 & 0.101 \\
\hline 15 & A" & 1835 & 0.103 & 0.939 & 1139 & 16.063 & 0.721 \\
\hline 16 & A" & 1977 & 2.335 & 0.208 & 1655 & 0.311 & 4.859 \\
\hline 17 & $A^{\prime}$ & 2078 & 88.730 & 1.189 & 1739 & 18.466 & 0.582 \\
\hline 18 & $A^{\prime}$ & 2164 & 32.985 & 0.400 & 2070 & 1.195 & 0.345 \\
\hline 19 & $A^{\prime}$ & 2637 & 66.187 & 0.862 & 2435 & 73.852 & 0.777 \\
\hline 20 & $A^{\prime}$ & 2707 & 18.045 & 0.643 & 2567 & 38.224 & 0.871 \\
\hline 21 & $A^{\prime}$ & 2718 & 129.489 & 0.313 & 2589 & 88.881 & 0.470 \\
\hline
\end{tabular}


Figure S.11.2. Anharmonic IR spectrum of $\mathrm{B}_{2} \mathrm{H}_{7}$ obtained by convoluting the calculated intensities with Lorentzians having a FWHM of $4 \mathrm{~cm}^{-1}$ (B3LYP-D2/cc-pVTZ results): (top) full spectrum, (middle) contributions from fundamentals and combination bands, (bottom) contributions from overtones
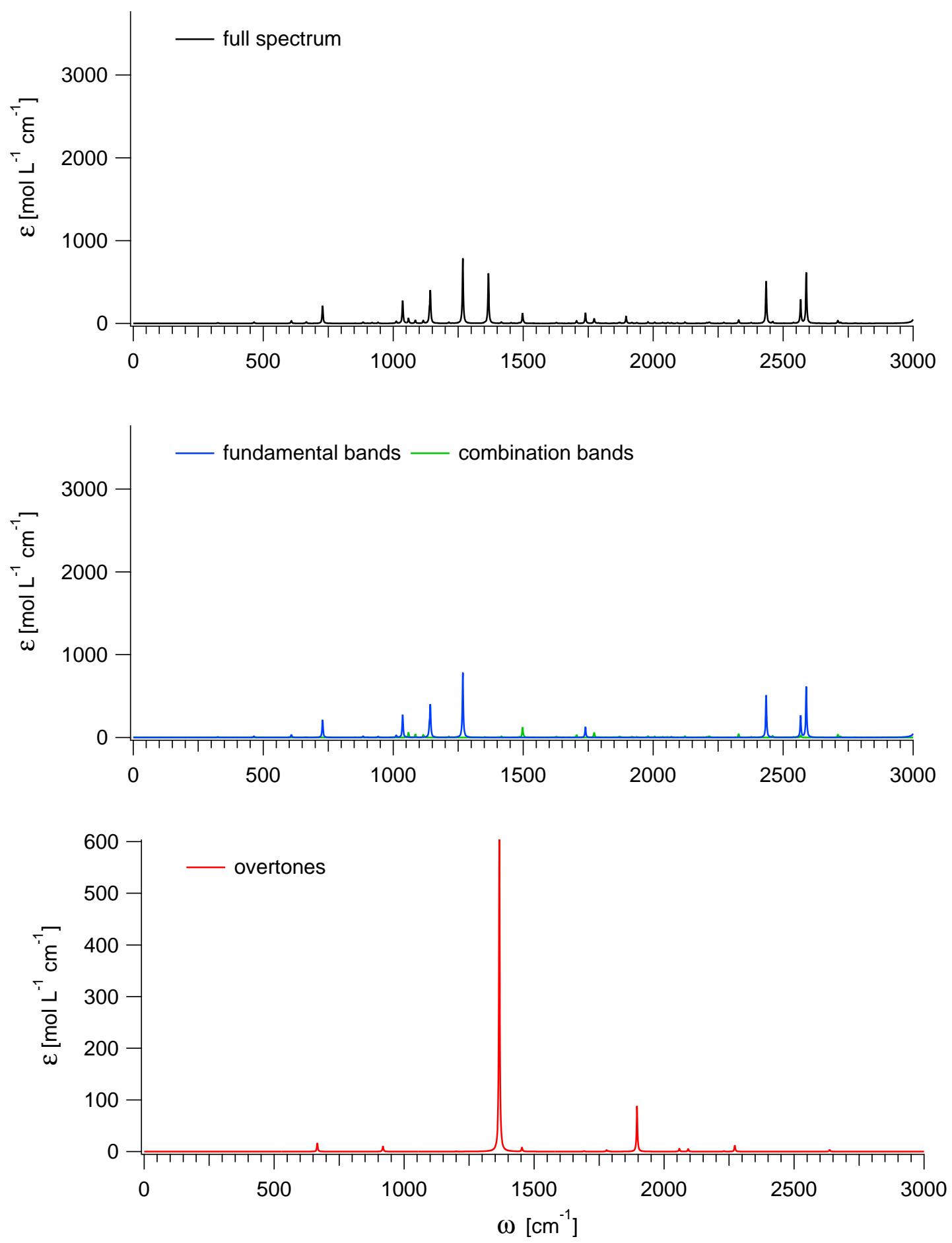
Figure S.11.3. Anharmonic Raman spectrum of $\mathrm{B}_{2} \mathrm{H}_{7}$ obtained by convoluting the calculated activities with Lorentzians having a FWHM of $4 \mathrm{~cm}^{-1}$ (B3LYP-D2/cc-pVTZ results): (top) full spectrum, (middle) contributions from fundamentals and combination bands, (bottom) contributions from overtones
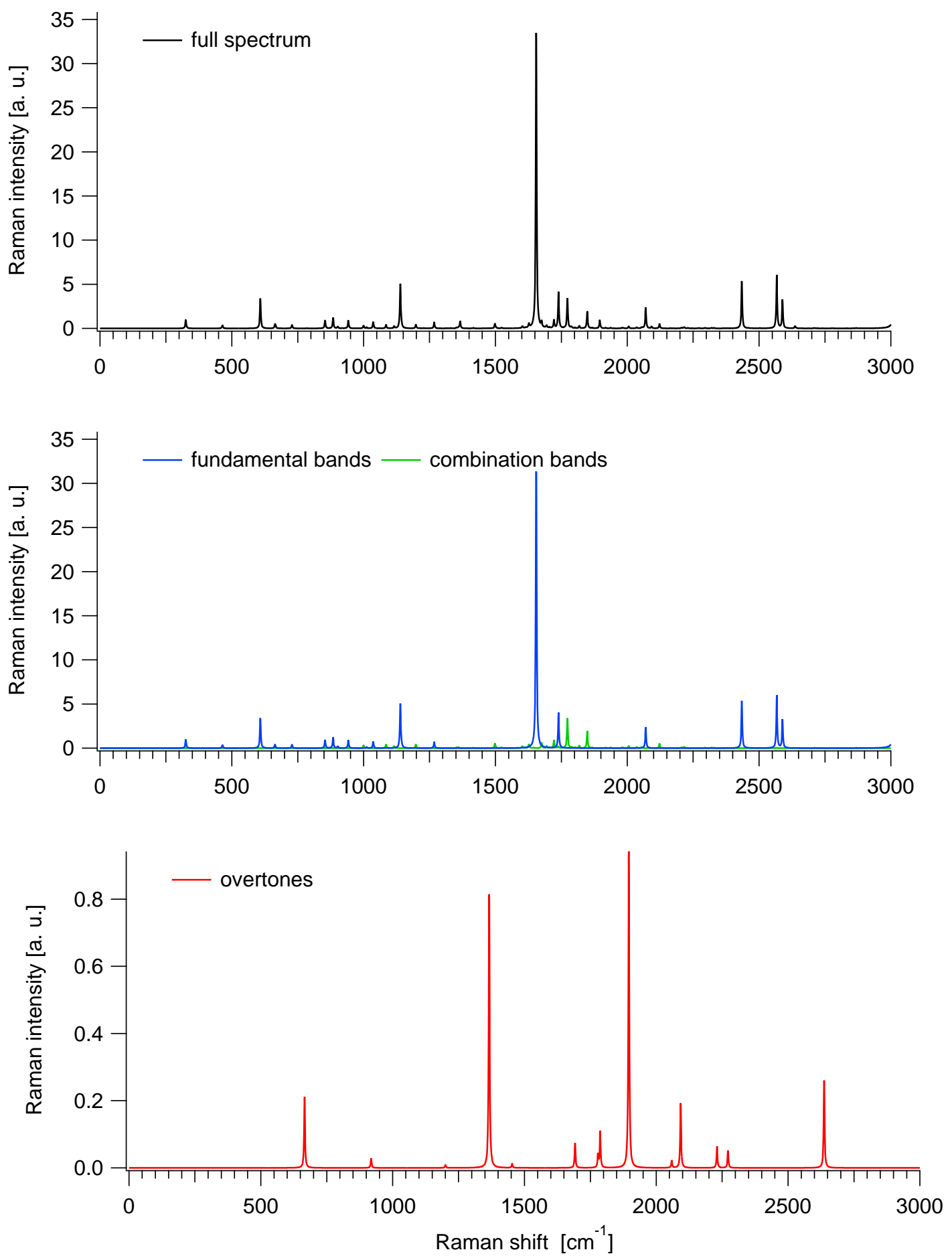
Table S.11.4. $\mathrm{B}_{2} \mathrm{H}_{7}$ : Ideal-gas thermodynamic functions at 1 bar (anharmonic B3LYPD2/cc-pVTZ results)

\begin{tabular}{cccc}
\hline$T$ & $C_{P}$ & $S$ & $H(T)-H(0 K)$ \\
{$[\mathrm{K}]$} & {$\left[\mathrm{J} \mathrm{mol}^{-1} \mathrm{~K}^{-1}\right]$} & {$\left[\mathrm{J} \mathrm{mol}^{-1} \mathrm{~K}^{-1}\right]$} & {$\left[\mathrm{kJ} \mathrm{mol}^{-1}\right]$} \\
\hline 100 & 35.641 & 201.374 & 3.371 \\
200 & 50.260 & 229.911 & 7.571 \\
300 & 71.849 & 254.297 & 13.659 \\
400 & 92.555 & 277.873 & 21.904 \\
500 & 109.940 & 300.457 & 32.057 \\
600 & 124.234 & 321.807 & 43.788 \\
700 & 136.045 & 341.873 & 56.821 \\
800 & 145.866 & 360.700 & 70.931 \\
900 & 154.066 & 378.369 & 85.940 \\
1000 & 160.936 & 394.967 & 101.700 \\
1100 & 166.709 & 410.585 & 118.091 \\
1200 & 171.580 & 425.305 & 135.012 \\
1300 & 175.707 & 439.206 & 152.382 \\
1400 & 179.220 & 452.360 & 170.133 \\
1500 & 182.225 & 464.829 & 188.209 \\
1600 & 184.808 & 476.675 & 206.564 \\
1700 & 187.041 & 487.947 & 225.159 \\
1800 & 188.979 & 498.694 & 243.962 \\
1900 & 190.671 & 508.958 & 262.947 \\
2000 & 192.154 & 518.777 & 282.089 \\
\hline & & & $S:$ \\
\hline
\end{tabular}

$T$ : Temperature. $C_{P}$ : Heat capacity at constant pressure. $S$ : Entropy. $H$ : Enthalpy. 
Figure S.11.4. $\mathrm{B}_{2} \mathrm{H}_{7}$ : Ideal-gas thermodynamic functions at 1 bar (anharmonic B3LYPD2/cc-pVTZ results)
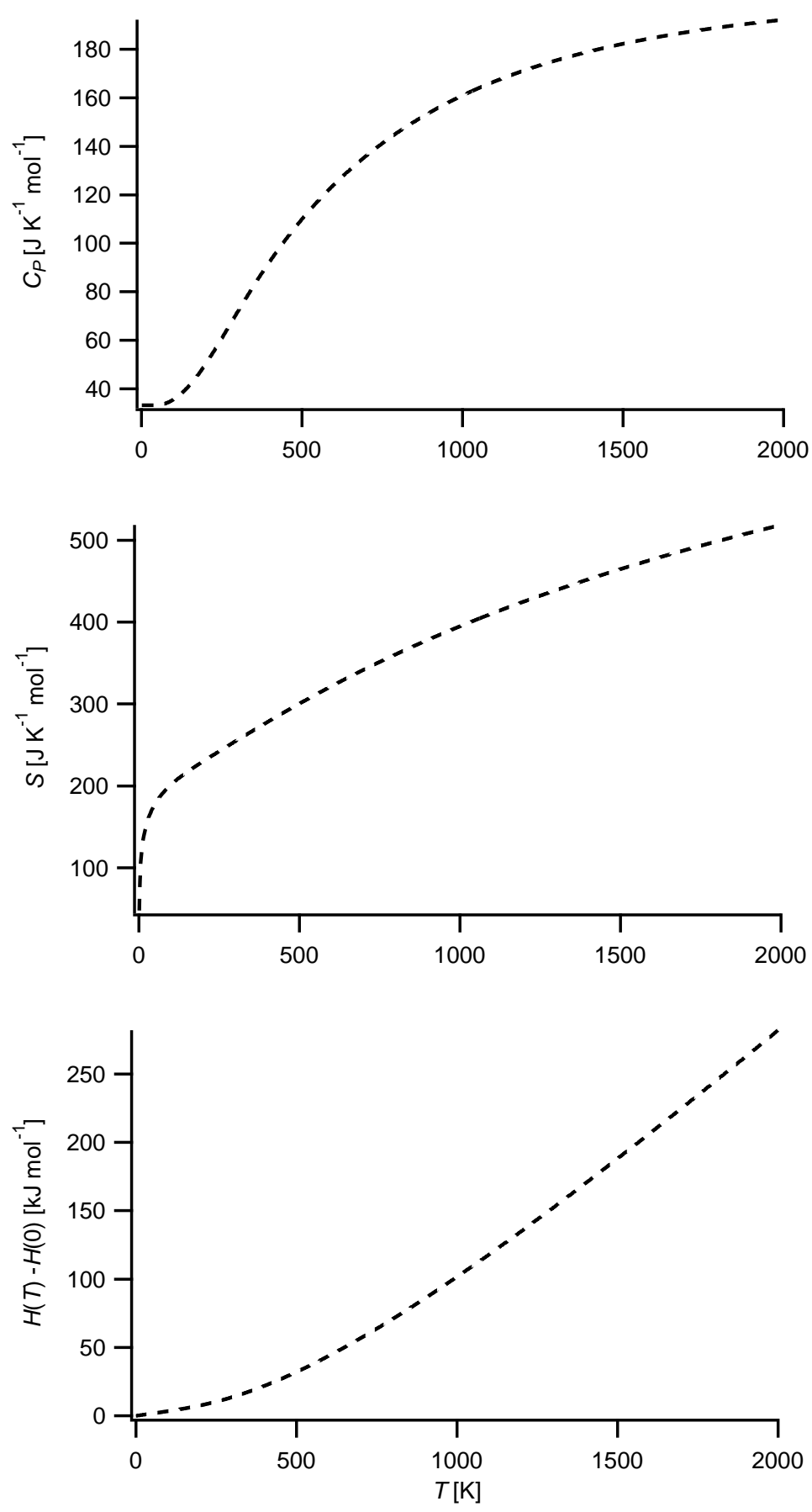

$T$ : Temperature. $C_{P}$ : Heat capacity at constant pressure. $S$ : Entropy. $H$ : Enthalpy. 
Table S.11.5. $\mathrm{B}_{2} \mathrm{H}_{7}$ : Fits (red solid lines) of the computed thermodynamic functions (blue dashed lines) with NASA type functions (Equations (1) - (3)) in the 200 - $900 \mathrm{~K}$ temperature range (anharmonic B3LYP-D2/cc-pVTZ results). In each case, the difference curve between the thermodynamic and NASA functions is plotted in the upper graph

Fit parameters

\begin{tabular}{llllll}
\hline $\mathrm{a}_{1}$ & $9.07279464 \mathrm{e}-01$ & $\mathrm{a}_{2}\left[\mathrm{~K}^{-1}\right]$ & $2.16705961 \mathrm{e}-02$ & $\mathrm{a}_{3}\left[\mathrm{~K}^{-2}\right]$ & $3.04145966 \mathrm{e}-05$ \\
$\mathrm{a}_{4}\left[\mathrm{~K}^{-3}\right]$ & $-6.48078244 \mathrm{e}-08$ & $\mathrm{a}_{5}\left[\mathrm{~K}^{-4}\right]$ & $3.17015472 \mathrm{e}-11$ & $\mathrm{a}_{6}[\mathrm{~K}]$ & $2.38513981 \mathrm{e}+02$
\end{tabular}

$\mathrm{a}_{7}$

$1.80626105 \mathrm{e}+01$
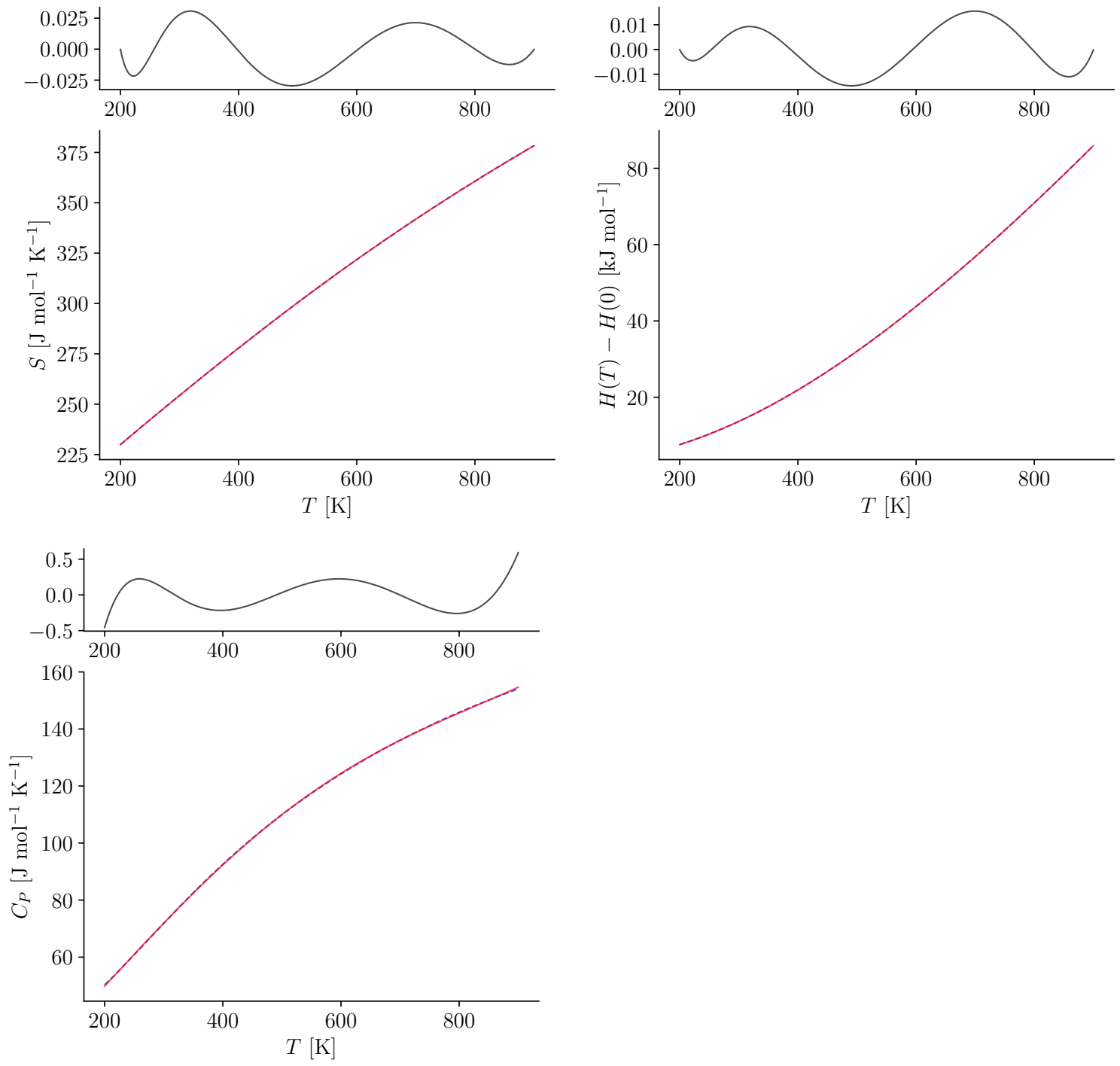


\section{$12 \quad \mathrm{~B}_{3} \mathrm{H}$}

Figure S.12.1. Structure of $\mathrm{B}_{3} \mathrm{H}$

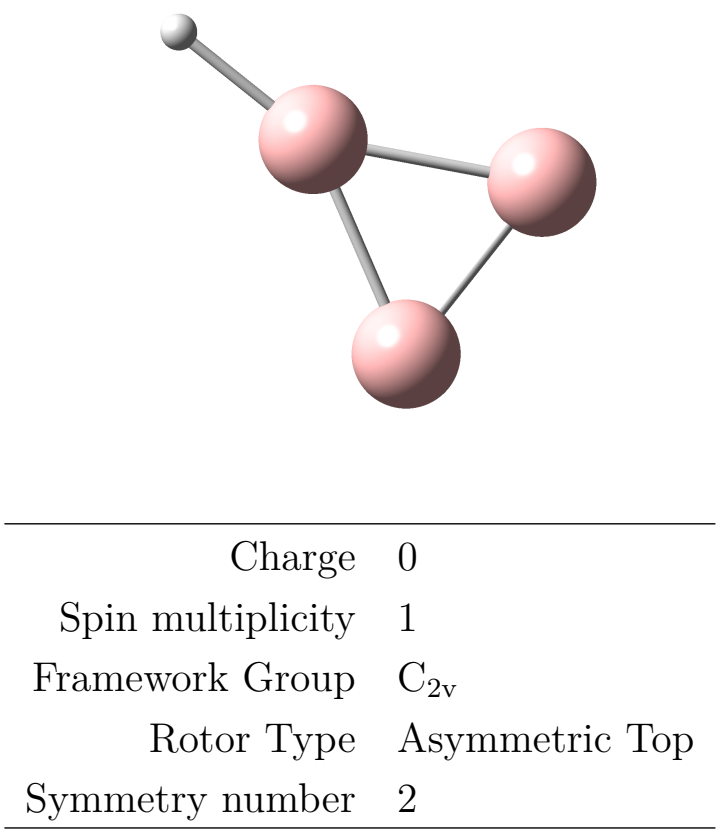

Table S.12.1. $\mathrm{B}_{3} \mathrm{H}$ : Selected anharmonic results obtained at the B3LYP-D2/cc-pVTZ level

\begin{tabular}{rll}
\hline $\mathrm{ZPE}_{\mathrm{v}}$ & 3590 & $\mathrm{~cm}^{-1}$ \\
$\mathrm{~A}$ & 1.38534 & $\mathrm{~cm}^{-1}$ \\
$\mathrm{~B}$ & 0.88975 & $\mathrm{~cm}^{-1}$ \\
$\mathrm{C}$ & 0.54178 & $\mathrm{~cm}^{-1}$ \\
\hline$C_{P}(300 \mathrm{~K})$ & 45.653 & $\mathrm{~J} \mathrm{~mol}^{-1} \mathrm{~K}-1$ \\
$S(300 \mathrm{~K})$ & 236.932 & $\mathrm{~J} \mathrm{~mol}^{-1} \mathrm{~K}-1$ \\
$H(300 \mathrm{~K})-H(0 \mathrm{~K})$ & 10.967 & $\mathrm{~kJ} \mathrm{~mol}^{-1}$ \\
\hline
\end{tabular}

$\mathrm{ZPE}_{\mathrm{v}}$ : vibrational contribution to the zero-point energy. A, B, C: Rotational constants. $C_{P}$ : Heat capacity at constant pressure. $S$ : Entropy. $H$ : Enthalpy. Ideal-gas calculations (1 bar). 
Table S.12.2. $\mathrm{B}_{3} \mathrm{H}$ : Cartesian coordinates $(\AA)$ of the B3LYP-D2/cc-pVTZ optimized structure

\begin{tabular}{cccc} 
atom & $\mathrm{x}$ & $\mathrm{y}$ & $\mathrm{z}$ \\
\hline B1 & 0.0000 & 0.0000 & 0.8070 \\
B2 & 0.0000 & 0.7434 & -0.6019 \\
B3 & -0.0000 & -0.7434 & -0.6019 \\
H4 & 0.0000 & 0.0000 & 1.9834 \\
\hline
\end{tabular}


Table S.12.3. $\mathrm{B}_{3} \mathrm{H}$ : Vibrational frequencies, infrared integrated intensities and Raman activities (B3LYP-D2/cc-pVTZ results)

\begin{tabular}{|c|c|c|c|c|c|c|c|}
\hline \multirow[b]{2}{*}{ mode } & \multirow[b]{2}{*}{ symm. } & \multicolumn{3}{|c|}{ Harmonic } & \multicolumn{3}{|c|}{ Anharmonic } \\
\hline & & $\begin{array}{c}\omega \\
{\left[\mathrm{cm}^{-1}\right]}\end{array}$ & $\begin{array}{c}\mathrm{IR} \\
{\left[\mathrm{km} \mathrm{mol}^{-1}\right]}\end{array}$ & $\begin{array}{c}\text { Raman } \\
{\left[\AA^{6}\right]}\end{array}$ & $\begin{array}{c}\omega \\
{\left[\mathrm{cm}^{-1}\right]}\end{array}$ & $\begin{array}{c}\mathrm{IR} \\
{\left[\mathrm{km} \mathrm{mol}^{-1}\right]}\end{array}$ & $\begin{array}{c}\text { Raman } \\
{\left[\AA^{6}\right]}\end{array}$ \\
\hline 1 & $\mathrm{~B}_{1}$ & 669 & 32.290 & 0.002 & 658 & 32.908 & 0.000 \\
\hline 2 & $\mathrm{~B}_{2}$ & 710 & 14.408 & 0.182 & 701 & 12.002 & 0.221 \\
\hline 3 & $\mathrm{~B}_{2}$ & 834 & 94.469 & 0.073 & 819 & 91.491 & 0.071 \\
\hline 4 & $\mathrm{~A}_{1}$ & 1024 & 0.337 & 1.075 & 997 & 0.118 & 1.080 \\
\hline 5 & $\mathrm{~A}_{1}$ & 1254 & 10.693 & 0.976 & 1228 & 9.841 & 0.987 \\
\hline 6 & $\mathrm{~A}_{1}$ & 2758 & 23.929 & 0.963 & 2653 & 24.266 & 1.076 \\
\hline
\end{tabular}


Figure S.12.2. Anharmonic IR spectrum of $\mathrm{B}_{3} \mathrm{H}$ obtained by convoluting the calculated intensities with Lorentzians having a FWHM of $4 \mathrm{~cm}^{-1}$ (B3LYP-D2/cc-pVTZ results): (top) full spectrum, (middle) contributions from fundamentals and combination bands, (bottom) contributions from overtones
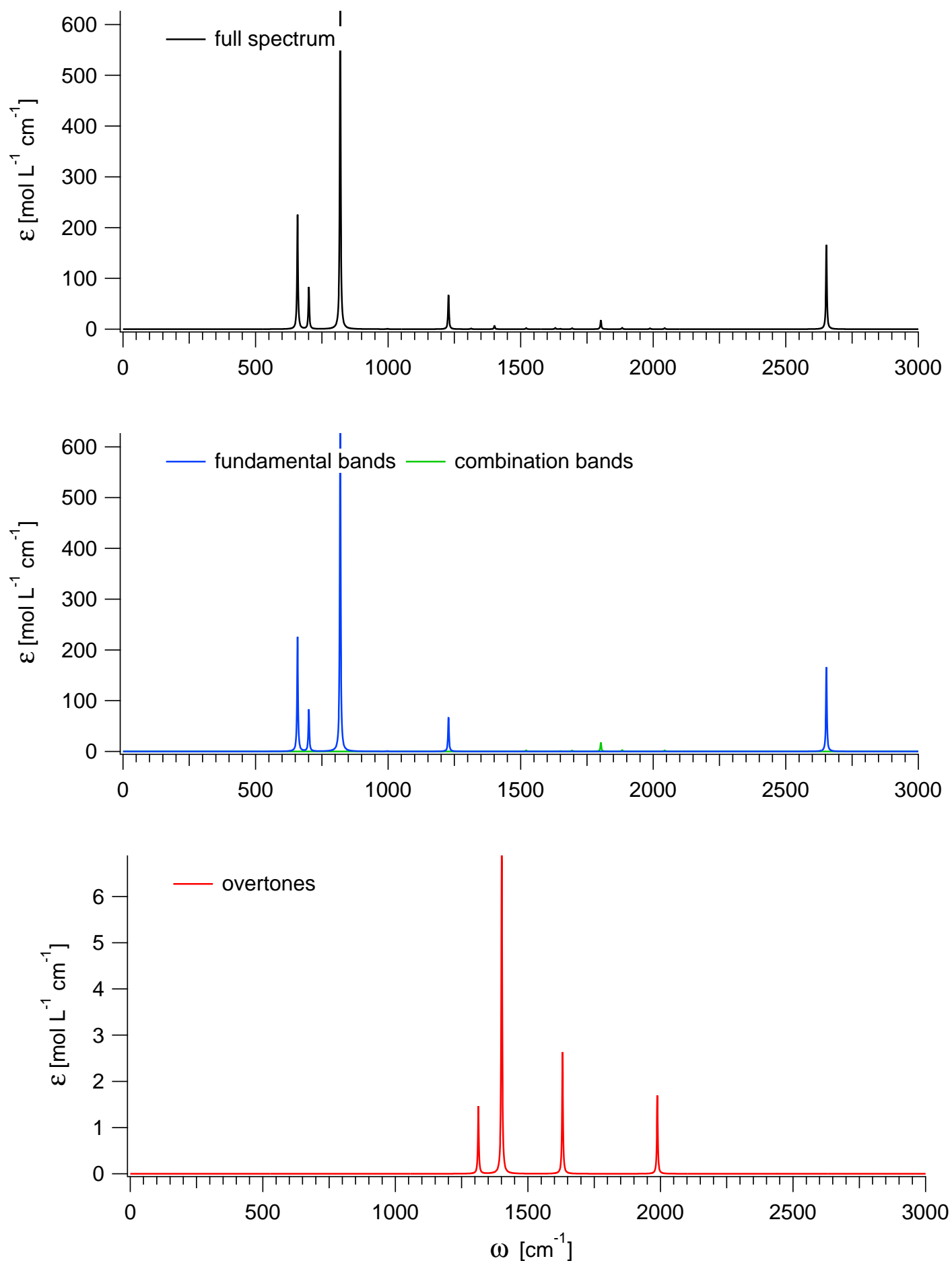
Figure S.12.3. Anharmonic Raman spectrum of $\mathrm{B}_{3} \mathrm{H}$ obtained by convoluting the calculated activities with Lorentzians having a FWHM of $4 \mathrm{~cm}^{-1}$ (B3LYP-D2/cc-pVTZ results): (top) full spectrum, (middle) contributions from fundamentals and combination bands, (bottom) contributions from overtones
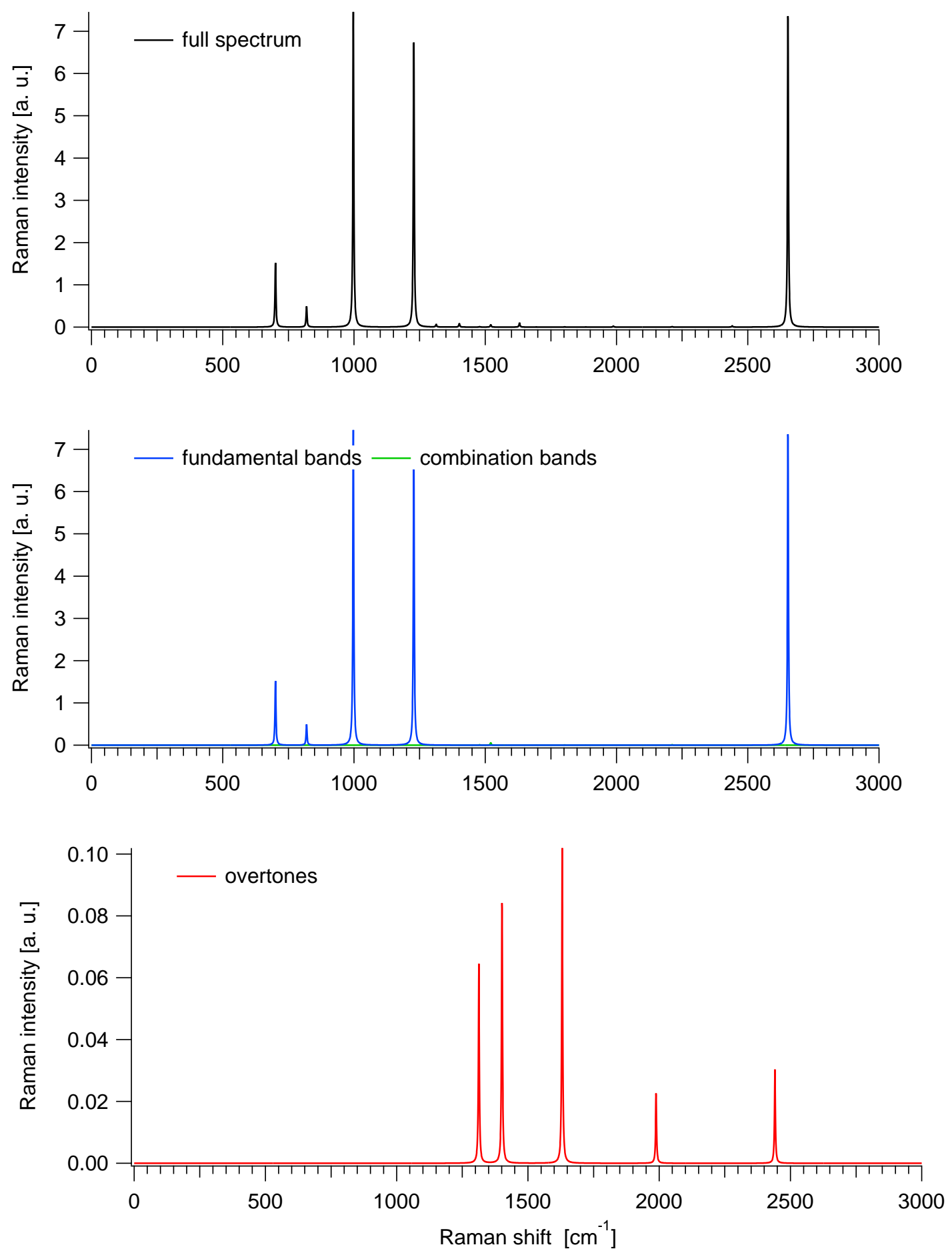
Table S.12.4. $\mathrm{B}_{3} \mathrm{H}$ : Ideal-gas thermodynamic functions at 1 bar (anharmonic B3LYPD2/cc-pVTZ results)

\begin{tabular}{|c|c|c|c|}
\hline $\begin{array}{c}T \\
{[\mathrm{~K}]}\end{array}$ & $\begin{array}{c}C_{P} \\
{\left[\mathrm{~J} \mathrm{~mol}^{-1} \mathrm{~K}^{-1}\right]}\end{array}$ & $\begin{array}{c}S \\
{\left[\mathrm{~J} \mathrm{~mol}^{-1} \mathrm{~K}^{-1}\right]}\end{array}$ & $\begin{array}{c}H(T)-H(0 K) \\
{\left[\mathrm{kJ} \mathrm{mol}^{-1}\right]}\end{array}$ \\
\hline 100 & 33.360 & 196.195 & 3.327 \\
\hline 200 & 37.530 & 220.207 & 6.814 \\
\hline 300 & 45.653 & 236.932 & 10.967 \\
\hline 400 & 53.012 & 251.115 & 15.914 \\
\hline 500 & 58.616 & 263.577 & 21.509 \\
\hline 600 & 62.814 & 274.652 & 27.591 \\
\hline 700 & 66.037 & 284.587 & 34.040 \\
\hline 800 & 68.578 & 293.578 & 40.775 \\
\hline 900 & 70.625 & 301.777 & 47.739 \\
\hline 1000 & 72.297 & 309.308 & 54.888 \\
\hline 1100 & 73.679 & 316.265 & 62.189 \\
\hline 1200 & 74.830 & 322.727 & 69.616 \\
\hline 1300 & 75.797 & 328.756 & 77.149 \\
\hline 1400 & 76.615 & 334.404 & 84.771 \\
\hline 1500 & 77.311 & 339.714 & 92.468 \\
\hline 1600 & 77.906 & 344.723 & 100.229 \\
\hline 1700 & 78.419 & 349.462 & 108.046 \\
\hline 1800 & 78.864 & 353.957 & 115.911 \\
\hline 1900 & 79.251 & 358.232 & 123.817 \\
\hline 2000 & 79.589 & 362.306 & 131.760 \\
\hline
\end{tabular}

$T$ : Temperature. $C_{P}$ : Heat capacity at constant pressure. $S$ : Entropy. $H$ : Enthalpy. 
Figure S.12.4. $\mathrm{B}_{3} \mathrm{H}$ : Ideal-gas thermodynamic functions at 1 bar (anharmonic B3LYPD2/cc-pVTZ results)
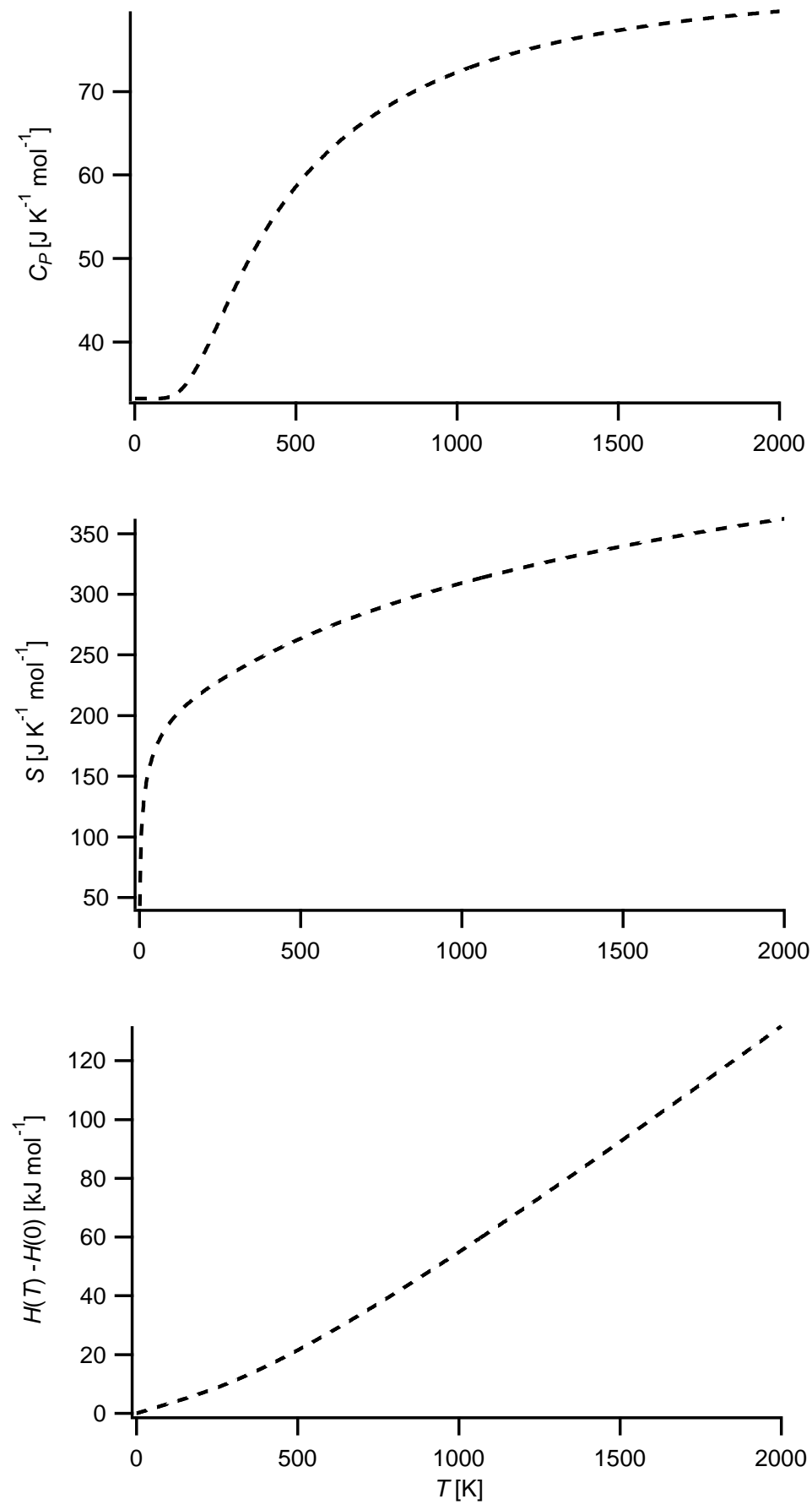

$T$ : Temperature. $C_{P}$ : Heat capacity at constant pressure. $S$ : Entropy. $H$ : Enthalpy. 
Table S.12.5. $\mathrm{B}_{3} \mathrm{H}$ : Fits (red solid lines) of the computed thermodynamic functions (blue dashed lines) with NASA type functions (Equations (1) - (3)) in the 200 - $900 \mathrm{~K}$ temperature range (anharmonic B3LYP-D2/cc-pVTZ results). In each case, the difference curve between the thermodynamic and NASA functions is plotted in the upper graph

Fit parameters

\begin{tabular}{llllll}
\hline $\mathrm{a}_{1}$ & $2.14017355 \mathrm{e}+00$ & $\mathrm{a}_{2}\left[\mathrm{~K}^{-1}\right]$ & $1.19420229 \mathrm{e}-02$ & $\mathrm{a}_{3}\left[\mathrm{~K}^{-2}\right]$ & $1.65174428 \mathrm{e}-06$ \\
$\mathrm{a}_{4}\left[\mathrm{~K}^{-3}\right]$ & $-1.67602595 \mathrm{e}-08$ & $\mathrm{a}_{5}\left[\mathrm{~K}^{-4}\right]$ & $9.92725495 \mathrm{e}-12$ & $\mathrm{a}_{6}[\mathrm{~K}]$ & $1.54349310 \mathrm{e}+02$
\end{tabular}

$a_{7}$

$1.27648239 \mathrm{e}+01$
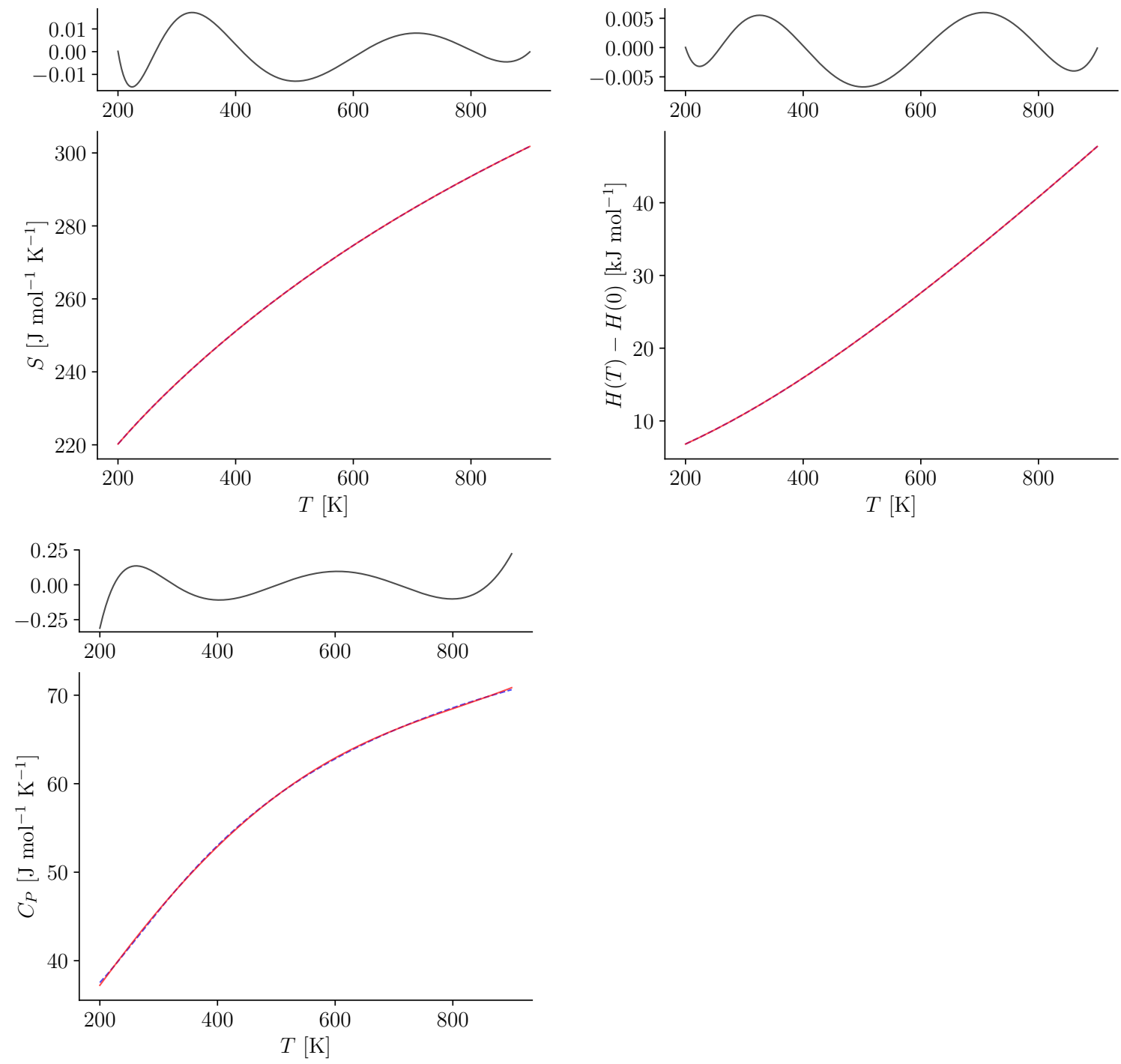


\section{$13 \mathbf{B}_{3} \mathbf{H}_{2}$}

Figure S.13.1. Structure of $\mathrm{B}_{3} \mathrm{H}_{2}$

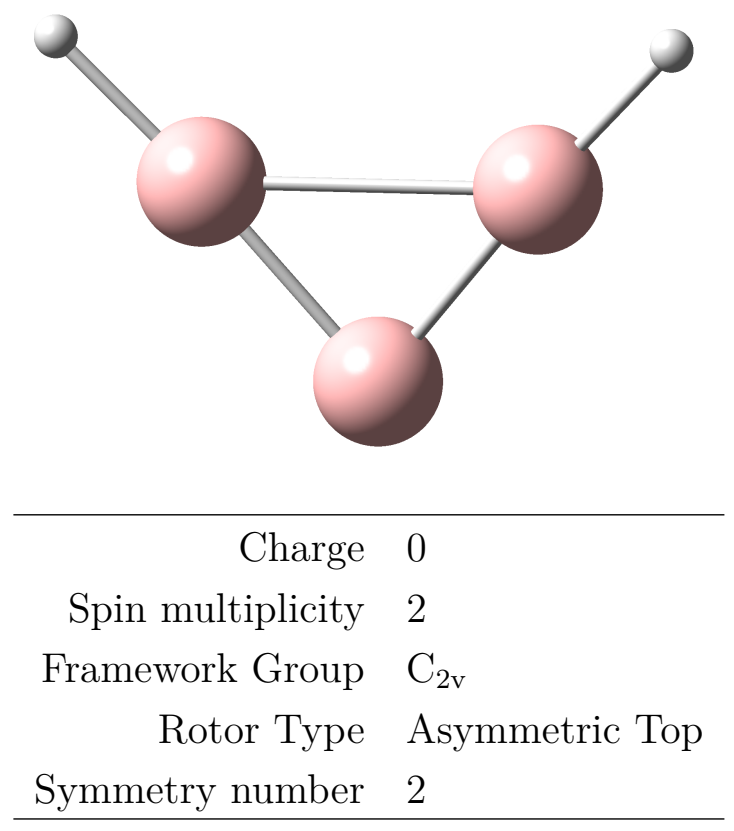

Table S.13.1. $\mathrm{B}_{3} \mathrm{H}_{2}$ : Selected anharmonic results obtained at the B3LYP-D2/cc-pVTZ level

\begin{tabular}{rll}
\hline $\mathrm{ZPE}_{\mathrm{v}}$ & 5417 & $\mathrm{~cm}^{-1}$ \\
$\mathrm{~A}$ & 1.31480 & $\mathrm{~cm}^{-1}$ \\
$\mathrm{~B}$ & 0.60810 & $\mathrm{~cm}^{-1}$ \\
$\mathrm{C}$ & 0.41579 & $\mathrm{~cm}^{-1}$ \\
\hline$C_{P}(300 \mathrm{~K})$ & 57.826 & $\mathrm{~J} \mathrm{~mol}^{-1} \mathrm{~K}-1$ \\
$S(300 \mathrm{~K})$ & 248.621 & $\mathrm{~J} \mathrm{~mol}^{-1} \mathrm{~K}-1$ \\
$H(300 \mathrm{~K})-H(0 \mathrm{~K})$ & 12.642 & $\mathrm{~kJ} \mathrm{~mol}^{-1}$ \\
\hline
\end{tabular}

$\mathrm{ZPE}_{\mathrm{v}}$ : vibrational contribution to the zero-point energy. A, B, C: Rotational constants. $C_{P}$ : Heat capacity at constant pressure. $S$ : Entropy. $H$ : Enthalpy. Ideal-gas calculations (1 bar). 
Table S.13.2. $\mathrm{B}_{3} \mathrm{H}_{2}$ : Cartesian coordinates $(\AA)$ of the B3LYP-D2/cc-pVTZ optimized structure

\begin{tabular}{cccc} 
atom & $\mathrm{x}$ & $\mathrm{y}$ & $\mathrm{z}$ \\
\hline B1 & -0.0000 & 0.9816 & -0.2429 \\
B2 & 0.0000 & 0.0000 & 0.9207 \\
B3 & -0.0000 & -0.9816 & -0.2429 \\
H4 & 0.0000 & -1.7969 & -1.0873 \\
H5 & 0.0000 & 1.7969 & -1.0873 \\
\hline
\end{tabular}


Table S.13.3. $\mathrm{B}_{3} \mathrm{H}_{2}$ : Vibrational frequencies, infrared integrated intensities and Raman activities (B3LYP-D2/cc-pVTZ results)

\begin{tabular}{cccccccc}
\hline & & \multicolumn{3}{c}{ Harmonic } & \multicolumn{3}{c}{ Anharmonic } \\
mode & symm. & $\omega$ & IR & Raman & $\omega$ & IR & Raman \\
& & {$\left[\mathrm{cm}^{-1}\right]$} & {$\left[\mathrm{km} \mathrm{mol}^{-1}\right]$} & {$\left[\AA^{6}\right]$} & {$\left[\mathrm{cm}^{-1}\right]$} & {$\left[\mathrm{km} \mathrm{mol}^{-1}\right]$} & {$\left[\AA^{6}\right]$} \\
\hline 1 & $\mathrm{~A}_{1}$ & 342 & 3.522 & 1.567 & 320 & 3.360 & 1.535 \\
2 & $\mathrm{~B}_{1}$ & 572 & 74.880 & 0.862 & 567 & 62.031 & 0.421 \\
3 & $\mathrm{~A}_{2}$ & 577 & 0.000 & 0.037 & 548 & 0.000 & 0.008 \\
4 & $\mathrm{~A}_{1}$ & 730 & 23.589 & 1.963 & 711 & 15.794 & 1.354 \\
5 & $\mathrm{~B}_{2}$ & 786 & 4.981 & 0.000 & 764 & 2.505 & 0.006 \\
6 & $\mathrm{~A}_{1}$ & 1190 & 4.681 & 1.752 & 1179 & 0.915 & 1.897 \\
7 & $\mathrm{~B}_{2}$ & 1203 & 12.556 & 0.381 & 1197 & 5.301 & 0.304 \\
8 & $\mathrm{~B}_{2}$ & 2781 & 63.802 & 0.427 & 2678 & 71.859 & 0.495 \\
9 & $\mathrm{~A}_{1}$ & 2786 & 31.713 & 0.658 & 2684 & 34.985 & 0.779 \\
\hline
\end{tabular}


Figure S.13.2. Anharmonic IR spectrum of $\mathrm{B}_{3} \mathrm{H}_{2}$ obtained by convoluting the calculated intensities with Lorentzians having a FWHM of $4 \mathrm{~cm}^{-1}$ (B3LYP-D2/cc-pVTZ results): (top) full spectrum, (middle) contributions from fundamentals and combination bands, (bottom) contributions from overtones
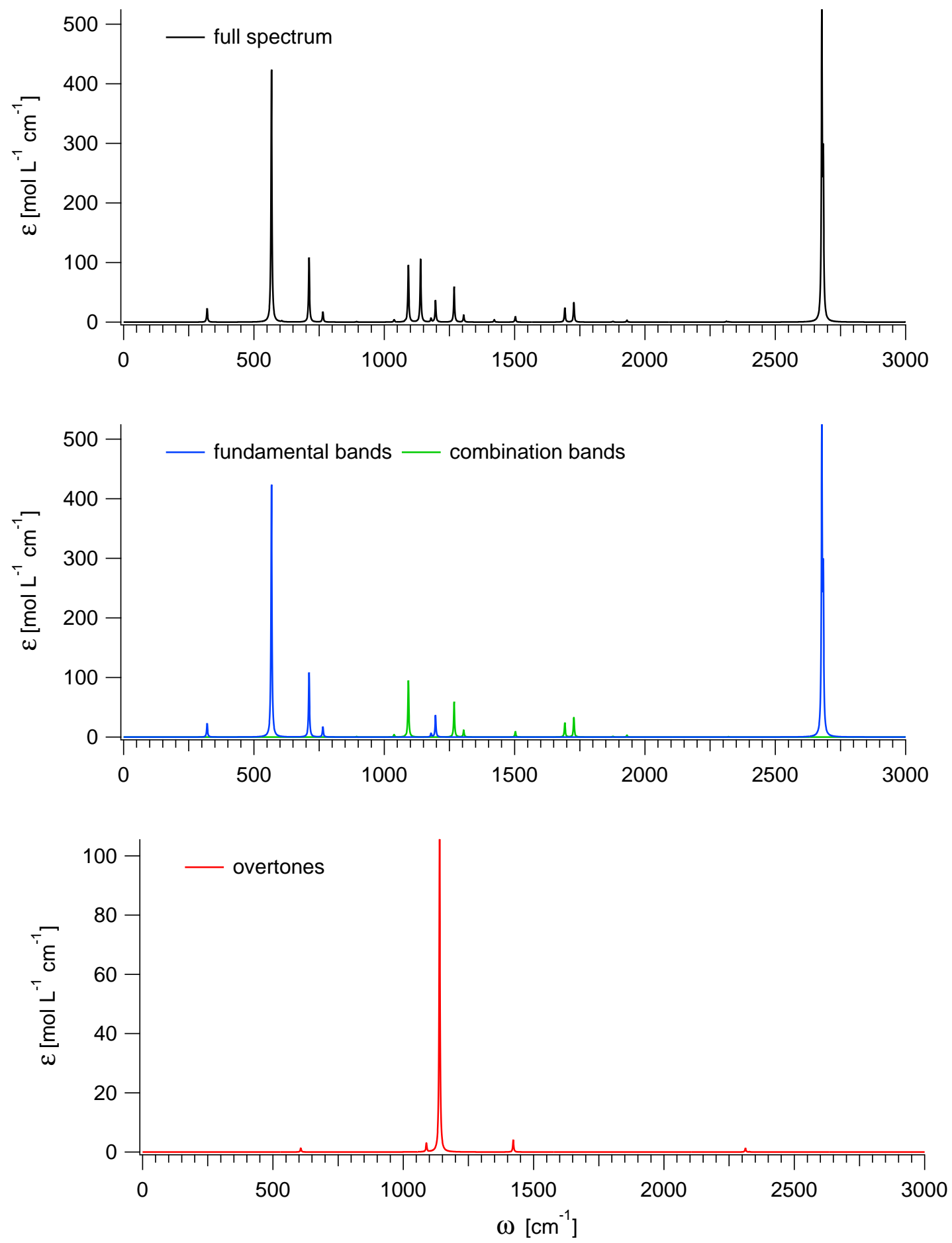

S.103 
Figure S.13.3. Anharmonic Raman spectrum of $\mathrm{B}_{3} \mathrm{H}_{2}$ obtained by convoluting the calculated activities with Lorentzians having a FWHM of $4 \mathrm{~cm}^{-1}$ (B3LYP-D2/cc-pVTZ results): (top) full spectrum, (middle) contributions from fundamentals and combination bands, (bottom) contributions from overtones
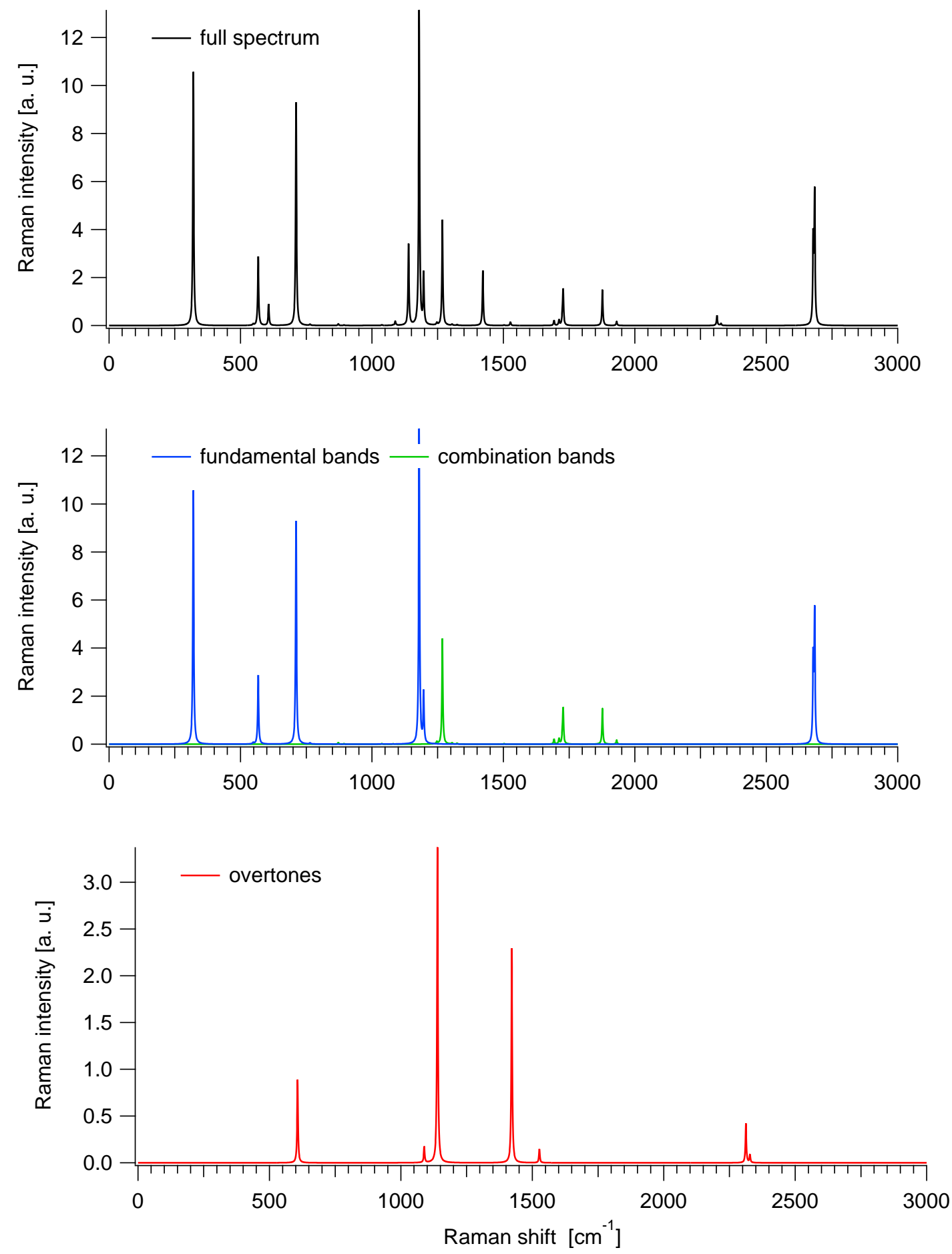
Table S.13.4. $\mathrm{B}_{3} \mathrm{H}_{2}$ : Ideal-gas thermodynamic functions at 1 bar (anharmonic B3LYPD2/cc-pVTZ results)

\begin{tabular}{|c|c|c|c|}
\hline $\begin{array}{c}T \\
{[\mathrm{~K}]}\end{array}$ & $\begin{array}{c}C_{P} \\
{\left[\mathrm{~J} \mathrm{~mol}^{-1} \mathrm{~K}^{-1}\right]}\end{array}$ & $\begin{array}{c}S \\
{\left[\mathrm{~J} \mathrm{~mol}^{-1} \mathrm{~K}^{-1}\right]}\end{array}$ & $\begin{array}{c}H(T)-H(0 K) \\
{\left[\mathrm{kJ} \mathrm{mol}^{-1}\right]}\end{array}$ \\
\hline 100 & 35.453 & 199.971 & 3.369 \\
\hline 200 & 46.316 & 227.593 & 7.417 \\
\hline 300 & 57.826 & 248.621 & 12.642 \\
\hline 400 & 66.719 & 266.539 & 18.892 \\
\hline 500 & 73.302 & 282.170 & 25.909 \\
\hline 600 & 78.343 & 295.998 & 33.501 \\
\hline 700 & 82.381 & 308.388 & 41.544 \\
\hline 800 & 85.720 & 319.614 & 49.954 \\
\hline 900 & 88.527 & 329.877 & 58.670 \\
\hline 1000 & 90.904 & 339.330 & 67.645 \\
\hline 1100 & 92.926 & 348.092 & 76.839 \\
\hline 1200 & 94.651 & 356.253 & 86.221 \\
\hline 1300 & 96.127 & 363.889 & 95.761 \\
\hline 1400 & 97.395 & 371.061 & 105.439 \\
\hline 1500 & 98.487 & 377.818 & 115.234 \\
\hline 1600 & 99.431 & 384.205 & 125.132 \\
\hline 1700 & 100.252 & 390.259 & 135.117 \\
\hline 1800 & 100.968 & 396.010 & 145.179 \\
\hline 1900 & 101.595 & 401.486 & 155.307 \\
\hline 2000 & 102.146 & 406.711 & 165.495 \\
\hline
\end{tabular}

$T$ : Temperature. $C_{P}$ : Heat capacity at constant pressure. $S$ : Entropy. $H$ : Enthalpy. 
Figure S.13.4. $\mathrm{B}_{3} \mathrm{H}_{2}$ : Ideal-gas thermodynamic functions at 1 bar (anharmonic B3LYPD2/cc-pVTZ results)
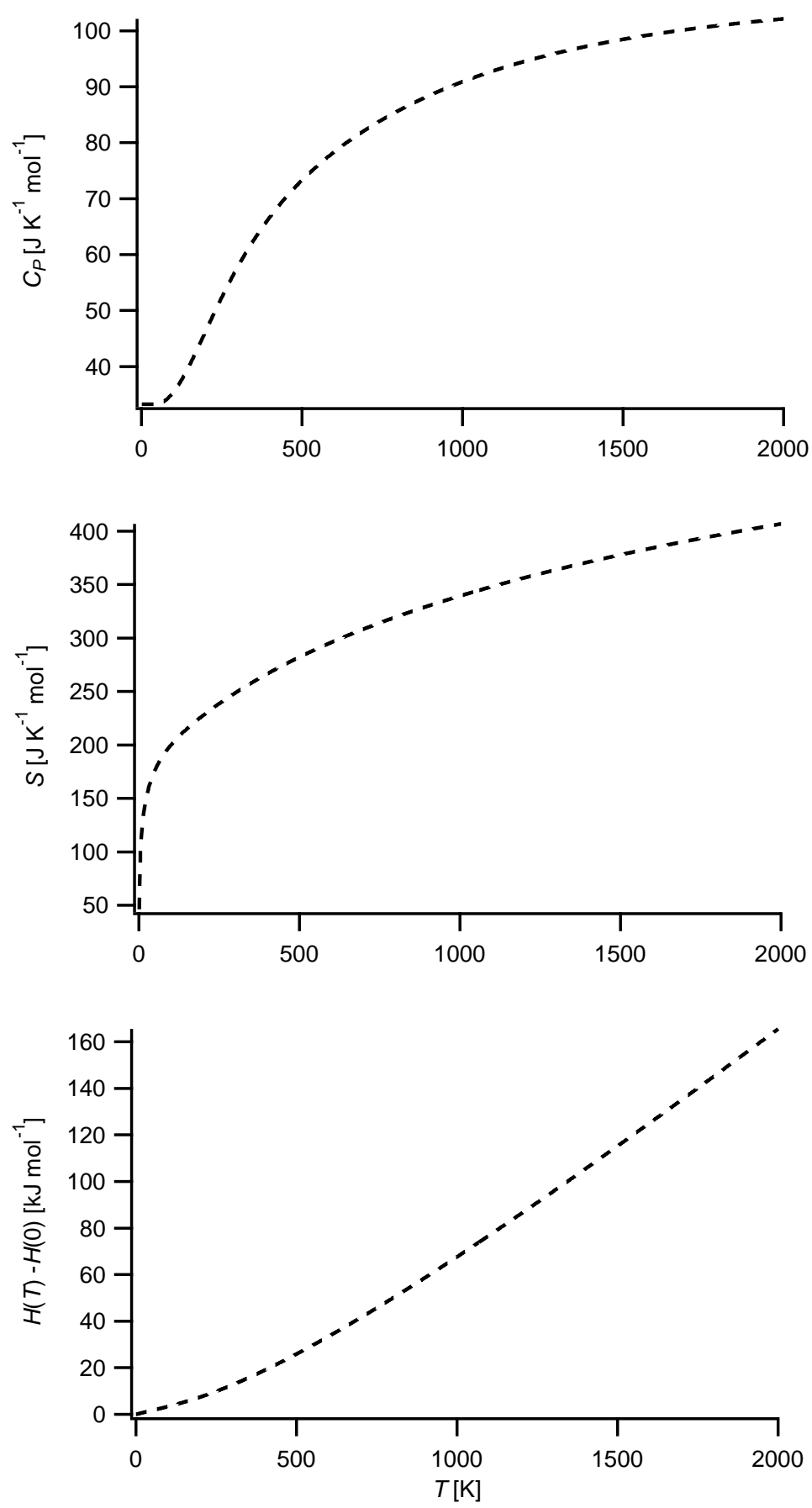

$T$ : Temperature. $C_{P}$ : Heat capacity at constant pressure. $S$ : Entropy. $H$ : Enthalpy. 
Table S.13.5. $\mathrm{B}_{3} \mathrm{H}_{2}$ : Fits (red solid lines) of the computed thermodynamic functions (blue dashed lines) with NASA type functions (Equations (1) - (3)) in the 200 - $900 \mathrm{~K}$ temperature range (anharmonic B3LYP-D2/cc-pVTZ results). In each case, the difference curve between the thermodynamic and NASA functions is plotted in the upper graph

Fit parameters

\begin{tabular}{llllll}
\hline $\mathrm{a}_{1}$ & $1.29198847 \mathrm{e}+00$ & $\mathrm{a}_{2}\left[\mathrm{~K}^{-1}\right]$ & $2.72533564 \mathrm{e}-02$ & $\mathrm{a}_{3}\left[\mathrm{~K}^{-2}\right]$ & $-3.39111664 \mathrm{e}-05$ \\
$\mathrm{a}_{4}\left[\mathrm{~K}^{-3}\right]$ & $2.16776350 \mathrm{e}-08$ & $\mathrm{a}_{5}\left[\mathrm{~K}^{-4}\right]$ & $-5.33384397 \mathrm{e}-12$ & $\mathrm{a}_{6}[\mathrm{~K}]$ & $1.70691846 \mathrm{e}+02$ \\
$\mathrm{a}_{7}$ & $1.56996468 \mathrm{e}+01$ & & & &
\end{tabular}
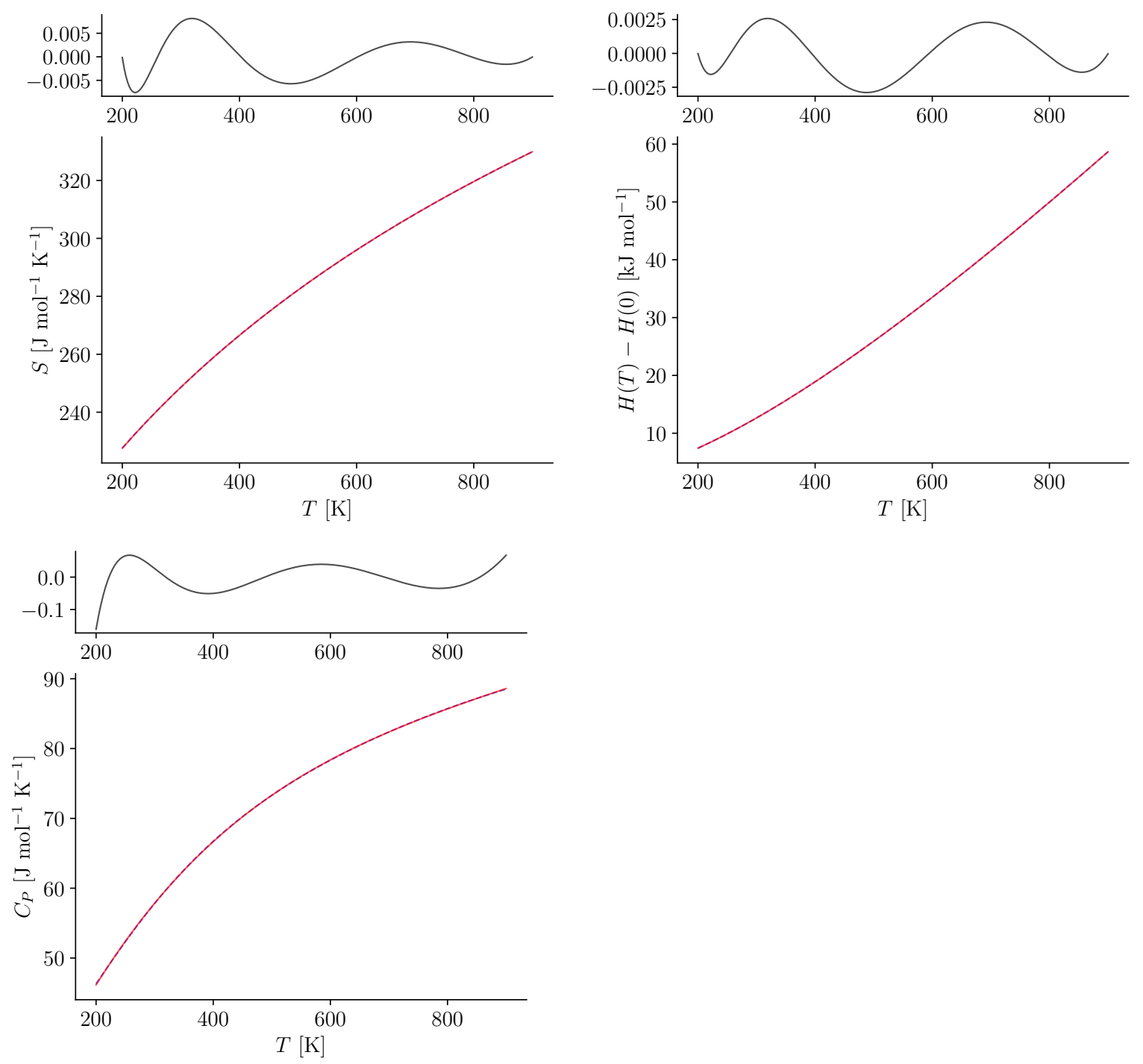


\section{$14 \mathrm{~B}_{3} \mathrm{H}_{3}$}

Figure S.14.1. Structure of $\mathrm{B}_{3} \mathrm{H}_{3}$

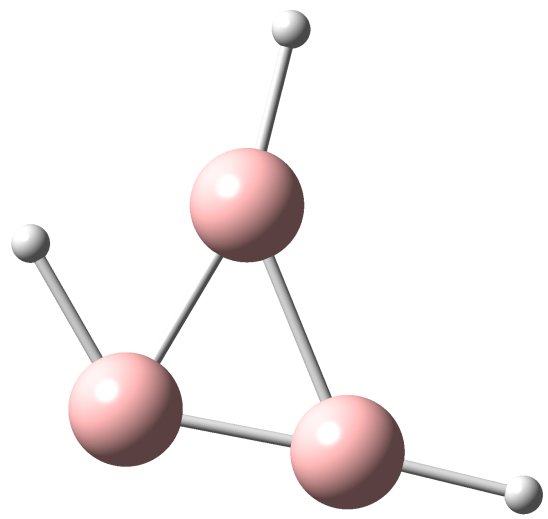

\begin{aligned} & \hline Charge 0 \\ & Spin multiplicity 1 \\ & Framework Group $\mathrm{C}_{\mathrm{s}} \\ &$ Rotor Type Asymmetric Top \\ & Symmetry number 1 \\ & \hline\end{aligned}

Table S.14.1. $\mathrm{B}_{3} \mathrm{H}_{3}$ : Selected anharmonic results obtained at the B3LYP-D2/cc-pVTZ level

\begin{tabular}{rll}
\hline $\mathrm{ZPE}_{\mathrm{v}}$ & 7718 & $\mathrm{~cm}^{-1}$ \\
$\mathrm{~A}$ & 1.12847 & $\mathrm{~cm}^{-1}$ \\
$\mathrm{~B}$ & 0.71698 & $\mathrm{~cm}^{-1}$ \\
$\mathrm{C}$ & 0.43843 & $\mathrm{~cm}^{-1}$ \\
\hline$C_{P}(300 \mathrm{~K})$ & 61.472 & $\mathrm{~J} \mathrm{~mol}^{-1} \mathrm{~K}-1$ \\
$S(300 \mathrm{~K})$ & 255.161 & $\mathrm{~J} \mathrm{~mol}^{-1} \mathrm{~K}-1$ \\
$H(300 \mathrm{~K})-H(0 \mathrm{~K})$ & 12.834 & $\mathrm{~kJ} \mathrm{~mol}^{-1}$ \\
\hline
\end{tabular}

$\mathrm{ZPE}_{\mathrm{v}}$ : vibrational contribution to the zero-point energy. A, B, C: Rotational constants. $C_{P}$ : Heat capacity at constant pressure. $S$ : Entropy. $H$ : Enthalpy. Ideal-gas calculations (1 bar). 
Table S.14.2. $\mathrm{B}_{3} \mathrm{H}_{3}$ : Cartesian coordinates $(\AA)$ of the B3LYP-D2/cc-pVTZ optimized structure

\begin{tabular}{cccc} 
atom & $\mathrm{x}$ & $\mathrm{y}$ & $\mathrm{z}$ \\
\hline B1 & -0.7846 & -0.4559 & 0.0000 \\
B2 & 0.6497 & -0.7317 & 0.0000 \\
B3 & 0.0000 & 0.8652 & -0.0000 \\
H4 & -1.3984 & 0.6175 & 0.0000 \\
H5 & 0.2853 & 2.0026 & -0.0000 \\
H6 & 1.7875 & -1.0080 & -0.0000 \\
\hline
\end{tabular}


Table S.14.3. $\mathrm{B}_{3} \mathrm{H}_{3}$ : Vibrational frequencies, infrared integrated intensities and Raman activities (B3LYP-D2/cc-pVTZ results)

\begin{tabular}{|c|c|c|c|c|c|c|c|}
\hline \multirow[b]{2}{*}{ mode } & \multirow[b]{2}{*}{ symm. } & \multicolumn{3}{|c|}{ Harmonic } & \multicolumn{3}{|c|}{ Anharmonic } \\
\hline & & $\begin{array}{c}\omega \\
{\left[\mathrm{cm}^{-1}\right]}\end{array}$ & $\begin{array}{c}\mathrm{IR} \\
{\left[\mathrm{km} \mathrm{mol}^{-1}\right]}\end{array}$ & $\begin{array}{c}\text { Raman } \\
{\left[\AA^{6}\right]}\end{array}$ & $\begin{array}{c}\omega \\
{\left[\mathrm{cm}^{-1}\right]}\end{array}$ & $\begin{array}{c}\mathrm{IR} \\
{\left[\mathrm{km} \mathrm{mol}^{-1}\right]}\end{array}$ & $\begin{array}{c}\text { Raman } \\
{\left[\AA^{6}\right]}\end{array}$ \\
\hline 1 & A" & 320 & 20.994 & 0.386 & 319 & 18.137 & 0.258 \\
\hline 2 & $A^{\prime}$ & 600 & 3.876 & 0.435 & 562 & 7.577 & 0.408 \\
\hline 3 & A" & 677 & 6.977 & 0.075 & 669 & 6.826 & 0.058 \\
\hline 4 & $A^{\prime}$ & 723 & 21.403 & 0.215 & 696 & 23.537 & 0.181 \\
\hline 5 & $A^{\prime}$ & 779 & 4.345 & 0.024 & 742 & 2.851 & 0.044 \\
\hline 6 & A" & 803 & 0.141 & 0.040 & 787 & 0.075 & 0.044 \\
\hline 7 & $A^{\prime}$ & 1090 & 13.439 & 0.305 & 979 & 19.339 & 0.115 \\
\hline 8 & $A^{\prime}$ & 1272 & 7.938 & 1.094 & 1179 & 6.268 & 0.663 \\
\hline 9 & $A^{\prime}$ & 1386 & 27.269 & 0.543 & 1276 & 40.635 & 1.580 \\
\hline 10 & $A^{\prime}$ & 2422 & 30.700 & 0.156 & 2339 & 21.420 & 0.184 \\
\hline 11 & $A^{\prime}$ & 2793 & 38.358 & 0.528 & 2686 & 42.639 & 0.609 \\
\hline 12 & $A^{\prime}$ & 2815 & 32.572 & 0.430 & 2712 & 31.280 & 0.463 \\
\hline
\end{tabular}


Figure S.14.2. Anharmonic IR spectrum of $\mathrm{B}_{3} \mathrm{H}_{3}$ obtained by convoluting the calculated intensities with Lorentzians having a FWHM of $4 \mathrm{~cm}^{-1}$ (B3LYP-D2/cc-pVTZ results): (top) full spectrum, (middle) contributions from fundamentals and combination bands, (bottom) contributions from overtones
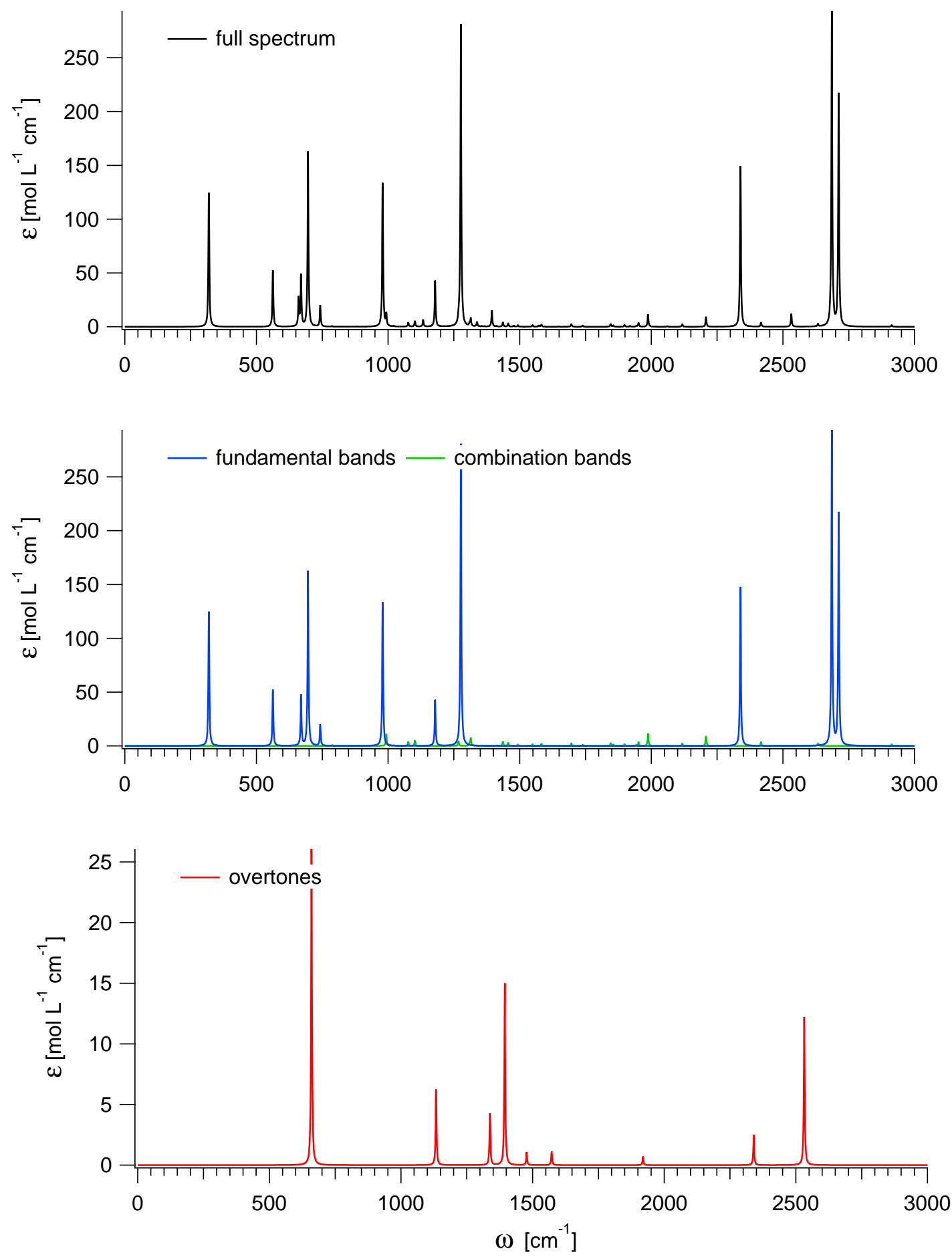
Figure S.14.3. Anharmonic Raman spectrum of $\mathrm{B}_{3} \mathrm{H}_{3}$ obtained by convoluting the calculated activities with Lorentzians having a FWHM of $4 \mathrm{~cm}^{-1}$ (B3LYP-D2/cc-pVTZ results): (top) full spectrum, (middle) contributions from fundamentals and combination bands, (bottom) contributions from overtones
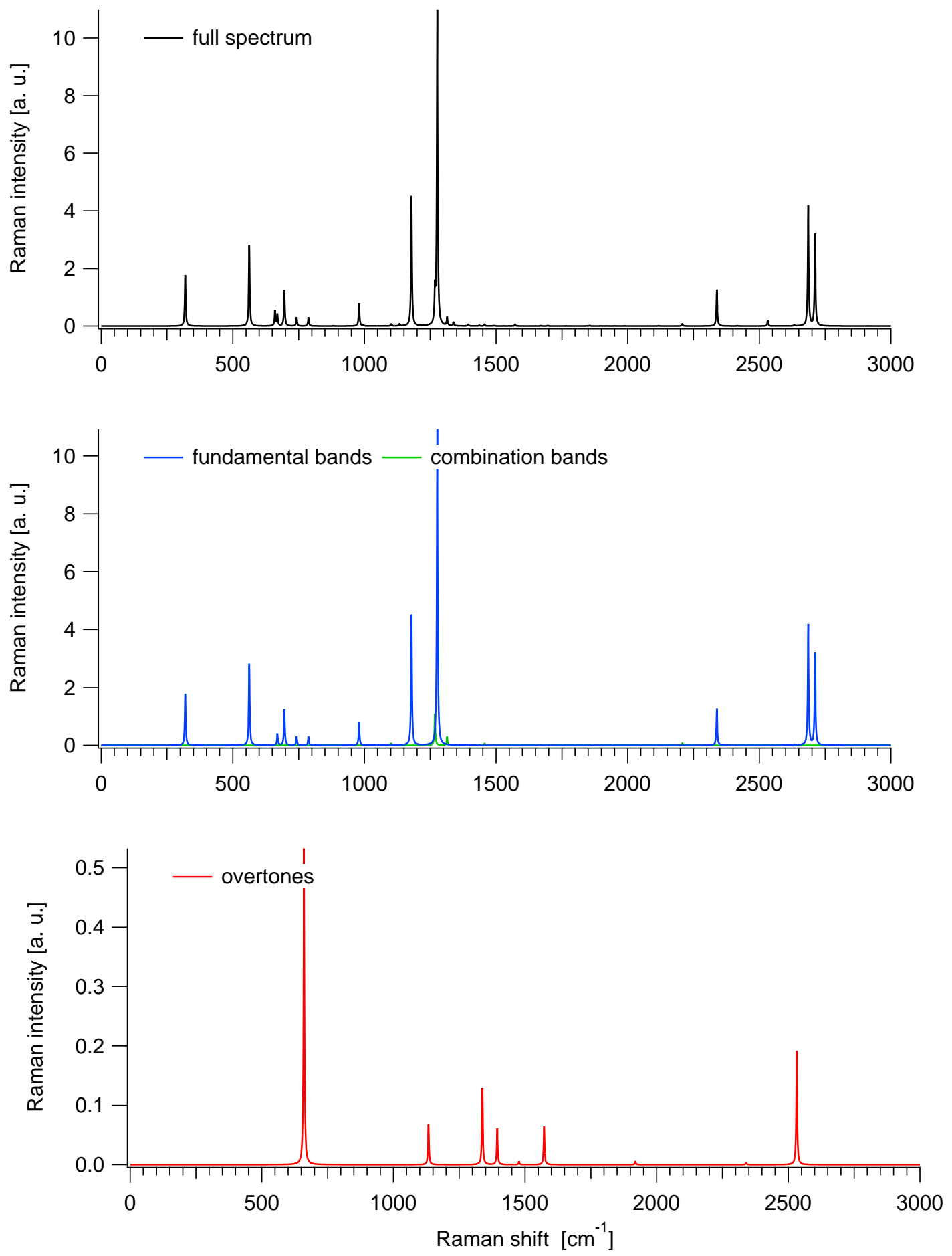

S.112 
Table S.14.4. $\mathrm{B}_{3} \mathrm{H}_{3}$ : Ideal-gas thermodynamic functions at 1 bar (anharmonic B3LYPD2/cc-pVTZ results)

\begin{tabular}{|c|c|c|c|}
\hline $\begin{array}{c}T \\
{[\mathrm{~K}]}\end{array}$ & $\begin{array}{c}C_{P} \\
{\left[\mathrm{~J} \mathrm{~mol}^{-1} \mathrm{~K}^{-1}\right]}\end{array}$ & $\begin{array}{c}S \\
{\left[\mathrm{~J} \mathrm{~mol}^{-1} \mathrm{~K}^{-1}\right]}\end{array}$ & $\begin{array}{c}H(T)-H(0 K) \\
{\left[\mathrm{kJ} \mathrm{mol}^{-1}\right]}\end{array}$ \\
\hline 100 & 35.362 & 205.807 & 3.368 \\
\hline 200 & 46.819 & 233.373 & 7.411 \\
\hline 300 & 61.472 & 255.161 & 12.834 \\
\hline 400 & 73.654 & 274.589 & 19.616 \\
\hline 500 & 82.959 & 292.070 & 27.468 \\
\hline 600 & 90.224 & 307.863 & 36.141 \\
\hline 700 & 96.106 & 322.227 & 45.467 \\
\hline 800 & 100.987 & 335.389 & 55.329 \\
\hline 900 & 105.085 & 347.527 & 65.638 \\
\hline 1000 & 108.546 & 358.783 & 76.324 \\
\hline 1100 & 111.479 & 369.270 & 87.330 \\
\hline 1200 & 113.971 & 379.079 & 98.605 \\
\hline 1300 & 116.096 & 388.288 & 110.112 \\
\hline 1400 & 117.915 & 396.960 & 121.814 \\
\hline 1500 & 119.477 & 405.150 & 133.686 \\
\hline 1600 & 120.825 & 412.905 & 145.703 \\
\hline 1700 & 121.993 & 420.266 & 157.845 \\
\hline 1800 & 123.011 & 427.268 & 170.096 \\
\hline 1900 & 123.900 & 433.943 & 182.443 \\
\hline 2000 & 124.681 & 440.319 & 194.873 \\
\hline
\end{tabular}

$T$ : Temperature. $C_{P}$ : Heat capacity at constant pressure. $S$ : Entropy. $H$ : Enthalpy. 
Figure S.14.4. $\mathrm{B}_{3} \mathrm{H}_{3}$ : Ideal-gas thermodynamic functions at 1 bar (anharmonic B3LYPD2/cc-pVTZ results)
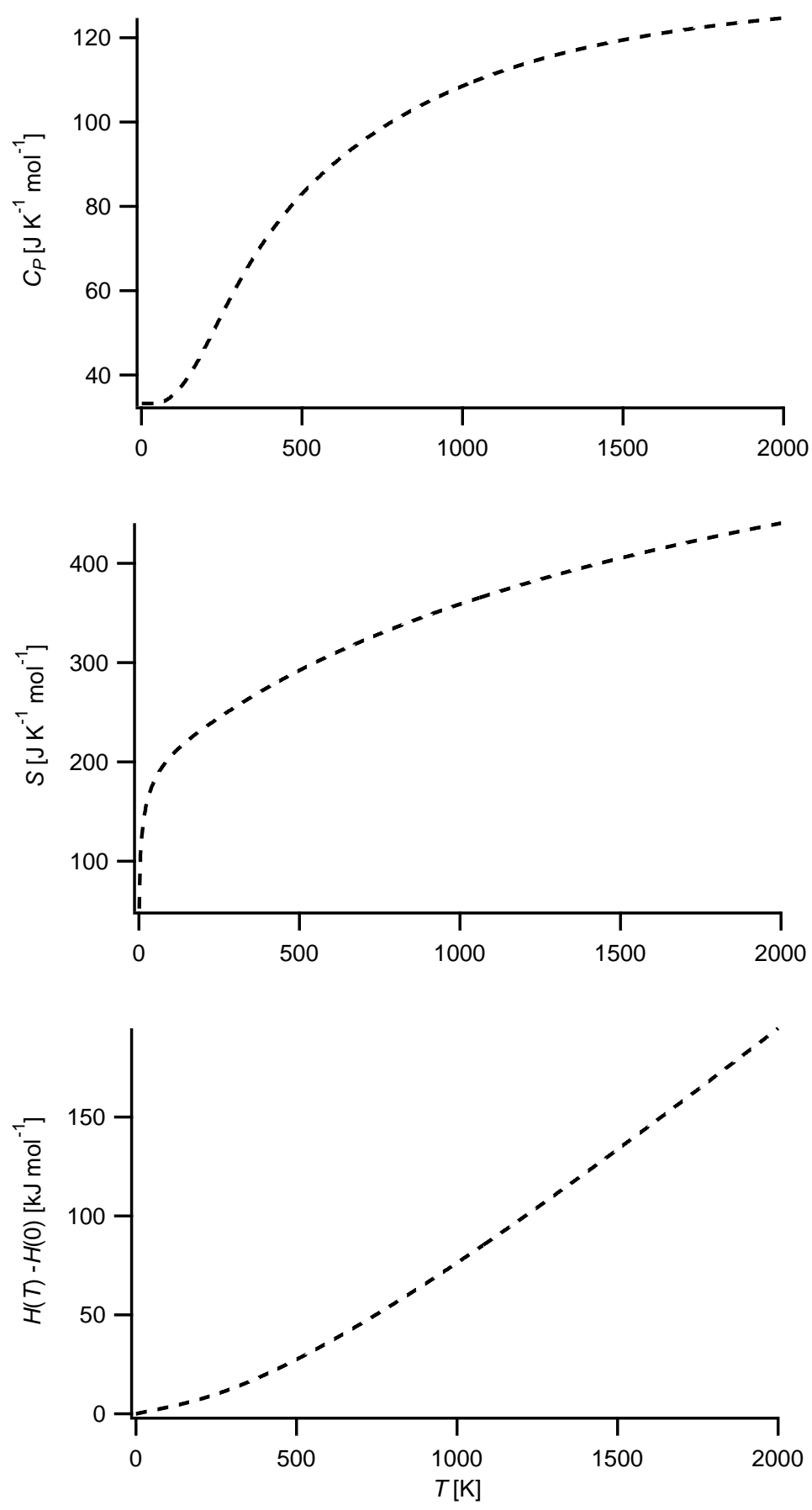

$T$ : Temperature. $C_{P}$ : Heat capacity at constant pressure. $S$ : Entropy. $H$ : Enthalpy. 
Table S.14.5. $\mathrm{B}_{3} \mathrm{H}_{3}$ : Fits (red solid lines) of the computed thermodynamic functions (blue dashed lines) with NASA type functions (Equations (1) - (3)) in the 200 - $900 \mathrm{~K}$ temperature range (anharmonic B3LYP-D2/cc-pVTZ results). In each case, the difference curve between the thermodynamic and NASA functions is plotted in the upper graph

Fit parameters

\begin{tabular}{llllll}
\hline $\mathrm{a}_{1}$ & $7.02006816 \mathrm{e}-01$ & $\mathrm{a}_{2}\left[\mathrm{~K}^{-1}\right]$ & $2.91185730 \mathrm{e}-02$ & $\mathrm{a}_{3}\left[\mathrm{~K}^{-2}\right]$ & $-2.45850153 \mathrm{e}-05$ \\
$\mathrm{a}_{4}\left[\mathrm{~K}^{-3}\right]$ & $5.84346534 \mathrm{e}-09$ & $\mathrm{a}_{5}\left[\mathrm{~K}^{-4}\right]$ & $2.14810147 \mathrm{e}-12$ & $\mathrm{a}_{6}[\mathrm{~K}]$ & $2.31595413 \mathrm{e}+02$ \\
$\mathrm{a}_{7}$ & $1.90004344 \mathrm{e}+01$ & & & &
\end{tabular}
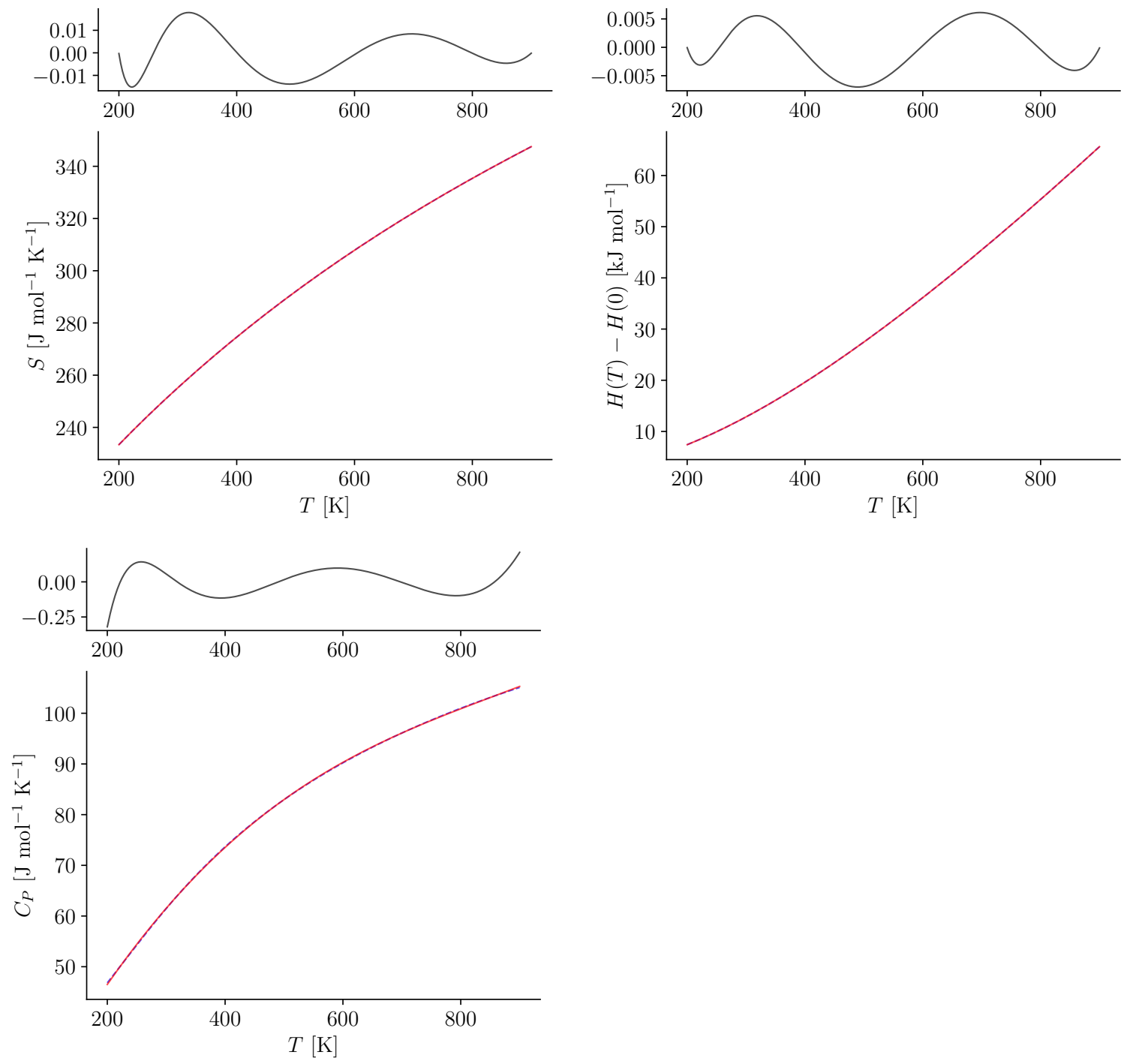


\section{$15 \mathrm{~B}_{3} \mathrm{H}_{5}$}

Figure S.15.1. Structure of $\mathrm{B}_{3} \mathrm{H}_{5}$

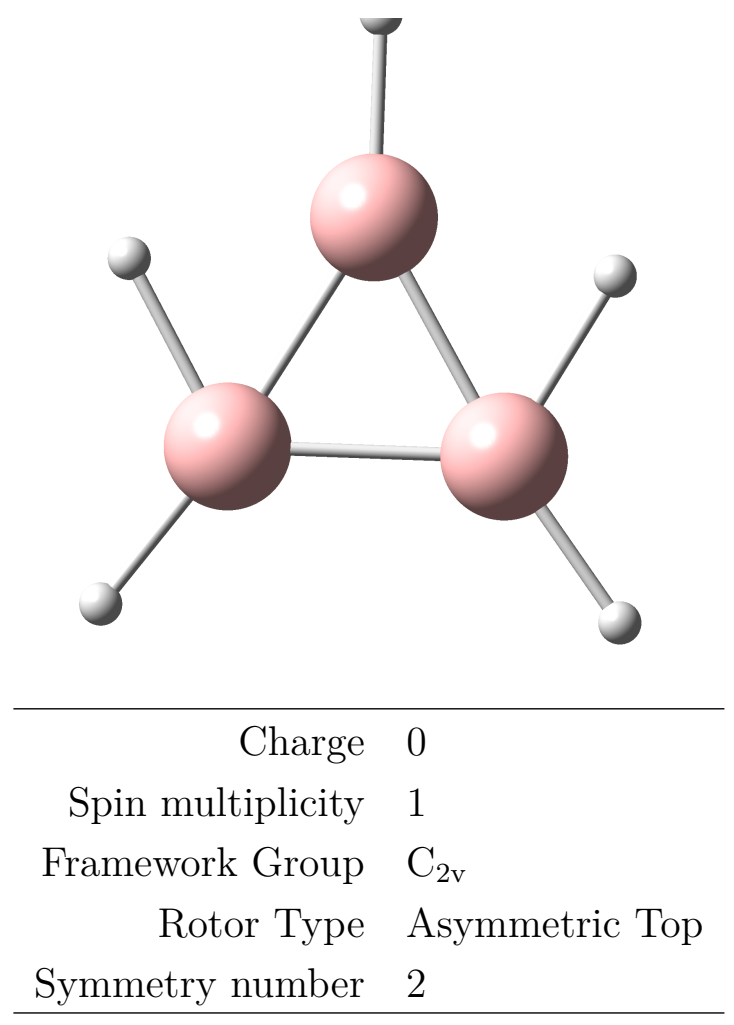

Table S.15.1. $\mathrm{B}_{3} \mathrm{H}_{5}$ : Selected anharmonic results obtained at the B3LYP-D2/cc-pVTZ level

\begin{tabular}{rll}
\hline $\mathrm{ZPE}_{\mathrm{v}}$ & 11990 & $\mathrm{~cm}^{-1}$ \\
$\mathrm{~A}$ & 0.74895 & $\mathrm{~cm}^{-1}$ \\
$\mathrm{~B}$ & 0.73944 & $\mathrm{~cm}^{-1}$ \\
$\mathrm{C}$ & 0.37208 & $\mathrm{~cm}^{-1}$ \\
\hline$C_{P}(300 \mathrm{~K})$ & 74.837 & $\mathrm{~J} \mathrm{~mol}^{-1} \mathrm{~K}-1$ \\
$S(300 \mathrm{~K})$ & 259.433 & $\mathrm{~J} \mathrm{~mol}^{-1} \mathrm{~K}-1$ \\
$H(300 \mathrm{~K})-H(0 \mathrm{~K})$ & 14.329 & $\mathrm{~kJ} \mathrm{~mol}^{-1}$ \\
\hline
\end{tabular}

$\mathrm{ZPE}_{\mathrm{v}}$ : vibrational contribution to the zero-point energy. A, B, C: Rotational constants. $C_{P}$ : Heat capacity at constant pressure. $S$ : Entropy. $H$ : Enthalpy. Ideal-gas calculations (1 bar). 
Table S.15.2. $\mathrm{B}_{3} \mathrm{H}_{5}$ : Cartesian coordinates $(\AA)$ of the B3LYP-D2/cc-pVTZ optimized structure

\begin{tabular}{cccc} 
atom & $\mathrm{x}$ & $\mathrm{y}$ & $\mathrm{z}$ \\
\hline B1 & -0.0000 & 0.8032 & -0.4785 \\
B2 & -0.0000 & -0.8032 & -0.4785 \\
B3 & 0.0000 & 0.0000 & 0.8772 \\
H4 & 0.0000 & -1.4112 & 0.5888 \\
H5 & -0.0000 & 1.5074 & -1.4166 \\
H6 & 0.0000 & 1.4112 & 0.5888 \\
H7 & -0.0000 & -1.5074 & -1.4166 \\
H8 & 0.0000 & 0.0000 & 2.0550 \\
\hline
\end{tabular}


Table S.15.3. $\mathrm{B}_{3} \mathrm{H}_{5}$ : Vibrational frequencies, infrared integrated intensities and Raman activities (B3LYP-D2/cc-pVTZ results)

\begin{tabular}{|c|c|c|c|c|c|c|c|}
\hline \multirow[b]{2}{*}{ mode } & \multirow[b]{2}{*}{ symm. } & \multicolumn{3}{|c|}{ Harmonic } & \multicolumn{3}{|c|}{ Anharmonic } \\
\hline & & $\begin{array}{c}\omega \\
{\left[\mathrm{cm}^{-1}\right]}\end{array}$ & $\begin{array}{c}\mathrm{IR} \\
{\left[\mathrm{km} \mathrm{mol}^{-1}\right]}\end{array}$ & $\begin{array}{c}\text { Raman } \\
{\left[\AA^{6}\right]}\end{array}$ & $\begin{array}{c}\omega \\
{\left[\mathrm{cm}^{-1}\right]}\end{array}$ & $\begin{array}{c}\mathrm{IR} \\
{\left[\mathrm{km} \mathrm{mol}^{-1}\right]}\end{array}$ & $\begin{array}{c}\text { Raman } \\
{\left[\AA^{6}\right]}\end{array}$ \\
\hline 1 & $\mathrm{~B}_{1}$ & 459 & 13.567 & 0.095 & 438 & 11.658 & 0.071 \\
\hline 2 & $\mathrm{~B}_{2}$ & 504 & 26.503 & 0.005 & 316 & 25.970 & 0.011 \\
\hline 3 & $\mathrm{~A}_{1}$ & 524 & 2.738 & 0.029 & 445 & 2.966 & 0.032 \\
\hline 4 & $\mathrm{~A}_{2}$ & 609 & 0.000 & 0.097 & 574 & 0.000 & 0.054 \\
\hline 5 & $\mathrm{~B}_{1}$ & 690 & 0.635 & 0.078 & 677 & 0.182 & 0.070 \\
\hline 6 & $\mathrm{~A}_{2}$ & 766 & 0.000 & 0.039 & 745 & 0.000 & 0.055 \\
\hline 7 & $\mathrm{~B}_{1}$ & 793 & 12.930 & 0.021 & 762 & 11.327 & 0.014 \\
\hline 8 & $\mathrm{~A}_{1}$ & 895 & 17.958 & 0.290 & 845 & 12.718 & 0.133 \\
\hline 9 & $\mathrm{~B}_{2}$ & 986 & 2.925 & 0.152 & 915 & 5.761 & 0.068 \\
\hline 10 & $\mathrm{~B}_{2}$ & 1015 & 7.453 & 0.027 & 976 & 0.602 & 0.121 \\
\hline 11 & $A_{1}$ & 1183 & 0.947 & 1.414 & 1152 & 1.152 & 1.082 \\
\hline 12 & $\mathrm{~B}_{2}$ & 1371 & 1.944 & 0.002 & 1226 & 0.003 & 0.000 \\
\hline 13 & $\mathrm{~A}_{1}$ & 1461 & 6.475 & 0.162 & 1340 & 0.884 & 0.068 \\
\hline 14 & $\mathrm{~B}_{2}$ & 2435 & 2.350 & 0.169 & 2326 & 0.466 & 0.143 \\
\hline 15 & $\mathrm{~A}_{1}$ & 2457 & 5.446 & 0.666 & 2347 & 2.738 & 0.658 \\
\hline 16 & $\mathrm{~A}_{1}$ & 2748 & 64.336 & 0.236 & 2641 & 60.669 & 0.393 \\
\hline 17 & $\mathrm{~B}_{2}$ & 2783 & 17.300 & 0.390 & 2674 & 18.037 & 0.415 \\
\hline 18 & $\mathrm{~A}_{1}$ & 2788 & 0.798 & 0.804 & 2682 & 4.828 & 0.826 \\
\hline
\end{tabular}


Figure S.15.2. Anharmonic IR spectrum of $\mathrm{B}_{3} \mathrm{H}_{5}$ obtained by convoluting the calculated intensities with Lorentzians having a FWHM of $4 \mathrm{~cm}^{-1}$ (B3LYP-D2/cc-pVTZ results): (top) full spectrum, (middle) contributions from fundamentals and combination bands, (bottom) contributions from overtones
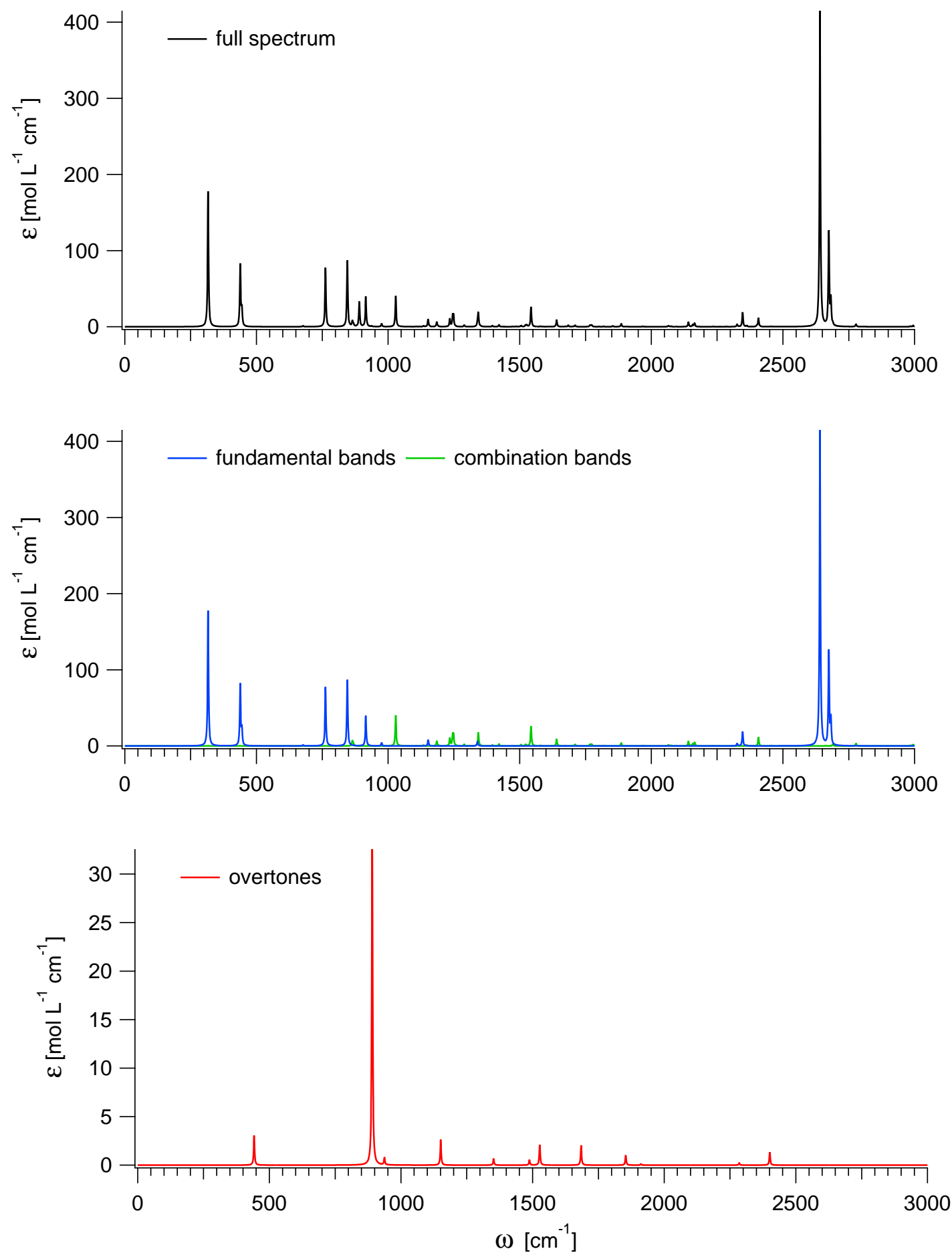
Figure S.15.3. Anharmonic Raman spectrum of $\mathrm{B}_{3} \mathrm{H}_{5}$ obtained by convoluting the calculated activities with Lorentzians having a FWHM of $4 \mathrm{~cm}^{-1}$ (B3LYP-D2/cc-pVTZ results): (top) full spectrum, (middle) contributions from fundamentals and combination bands, (bottom) contributions from overtones
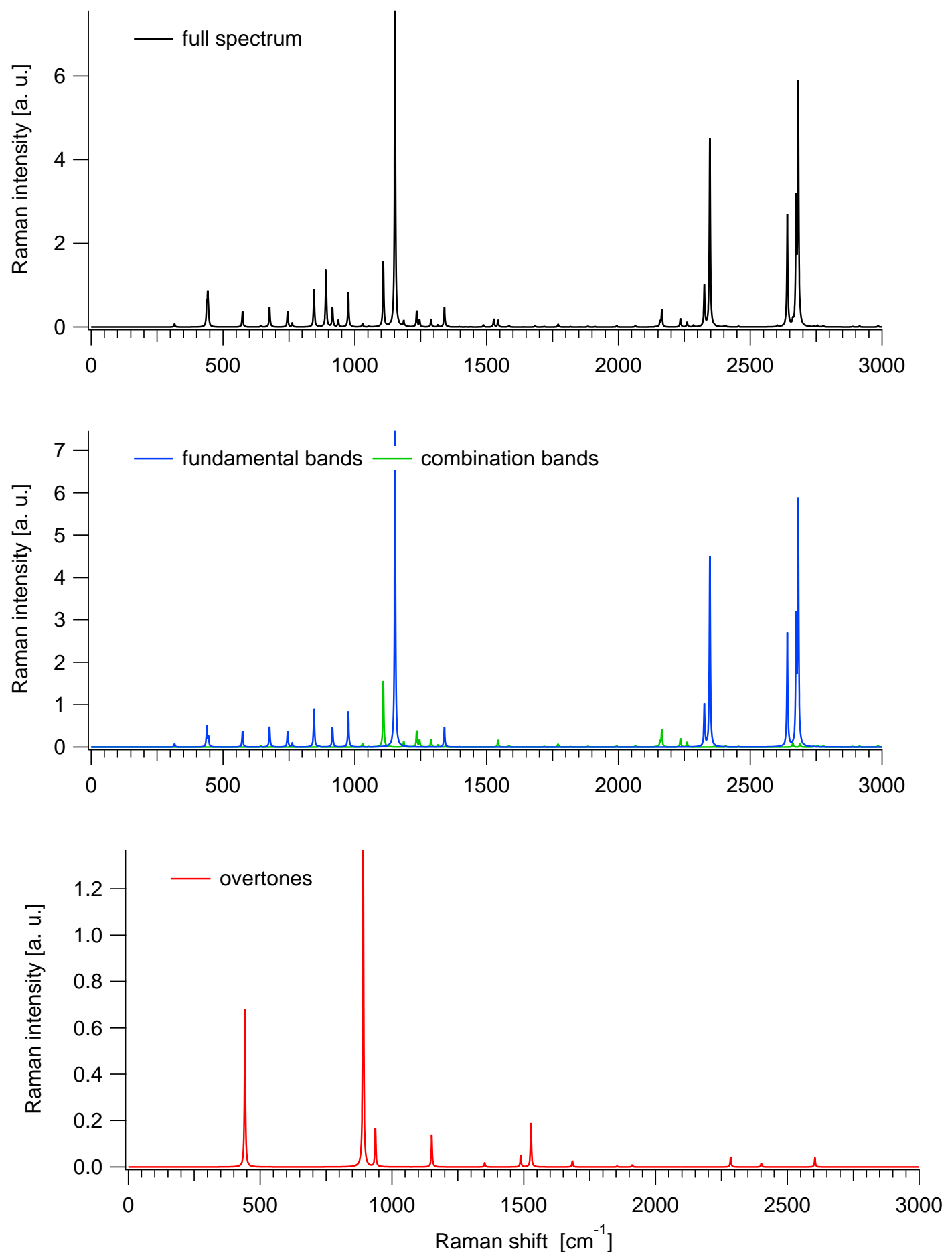

S. 120 
Table S.15.4. $\mathrm{B}_{3} \mathrm{H}_{5}$ : Ideal-gas thermodynamic functions at 1 bar (anharmonic B3LYPD2/cc-pVTZ results)

\begin{tabular}{cccc}
\hline$T$ & $C_{P}$ & $S$ & $H(T)-H(0 K)$ \\
{$[\mathrm{K}]$} & {$\left[\mathrm{J} \mathrm{mol}^{-1} \mathrm{~K}^{-1}\right]$} & {$\left[\mathrm{J} \mathrm{mol}^{-1} \mathrm{~K}^{-1}\right]$} & {$\left[\mathrm{kJ} \mathrm{mol}^{-1}\right]$} \\
\hline 100 & 36.531 & 203.203 & 3.387 \\
200 & 54.317 & 233.466 & 7.856 \\
300 & 74.837 & 259.433 & 14.329 \\
400 & 91.954 & 283.404 & 22.703 \\
500 & 105.359 & 305.423 & 32.595 \\
600 & 116.116 & 325.616 & 43.687 \\
700 & 125.026 & 344.204 & 55.757 \\
800 & 132.536 & 361.403 & 68.646 \\
900 & 138.908 & 377.392 & 82.227 \\
1000 & 144.323 & 392.315 & 96.396 \\
1100 & 148.929 & 406.293 & 111.064 \\
1200 & 152.852 & 419.424 & 126.159 \\
1300 & 156.202 & 431.794 & 141.616 \\
1400 & 159.071 & 443.478 & 157.383 \\
1500 & 161.536 & 454.539 & 173.416 \\
1600 & 163.664 & 465.034 & 189.679 \\
1700 & 165.509 & 475.012 & 206.140 \\
1800 & 167.115 & 484.519 & 222.773 \\
1900 & 168.519 & 493.593 & 239.556 \\
2000 & 169.752 & 502.269 & 256.471 \\
\hline & & & $S:$ \\
\hline
\end{tabular}

$T$ : Temperature. $C_{P}$ : Heat capacity at constant pressure. $S$ : Entropy. $H$ : Enthalpy. 
Figure S.15.4. $\mathrm{B}_{3} \mathrm{H}_{5}$ : Ideal-gas thermodynamic functions at 1 bar (anharmonic B3LYPD2/cc-pVTZ results)
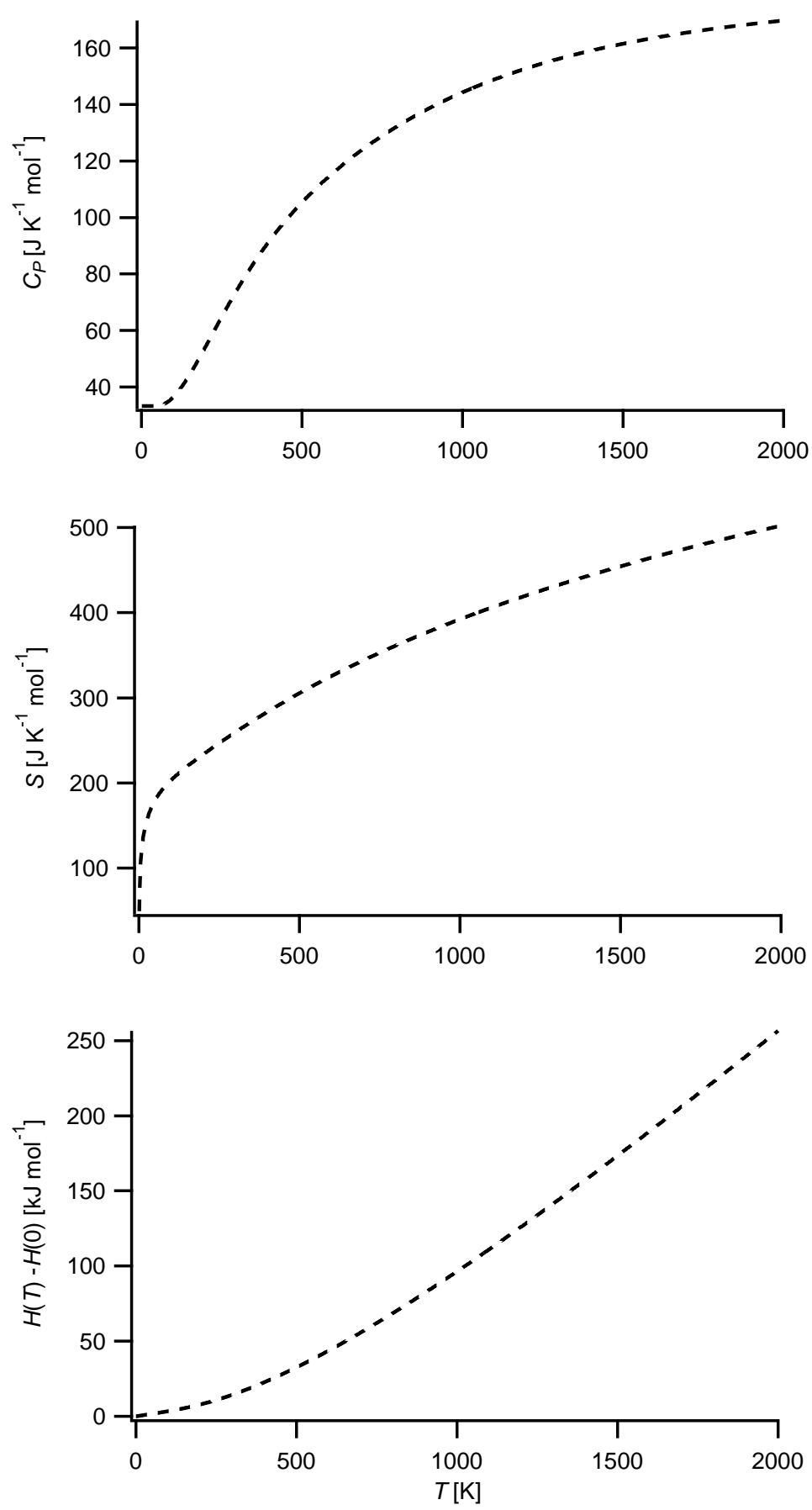

$T$ : Temperature. $C_{P}$ : Heat capacity at constant pressure. $S$ : Entropy. $H$ : Enthalpy. 
Table S.15.5. $\mathrm{B}_{3} \mathrm{H}_{5}$ : Fits (red solid lines) of the computed thermodynamic functions (blue dashed lines) with NASA type functions (Equations (1) - (3)) in the 200 - $900 \mathrm{~K}$ temperature range (anharmonic B3LYP-D2/cc-pVTZ results). In each case, the difference curve between the thermodynamic and NASA functions is plotted in the upper graph

Fit parameters

\begin{tabular}{llllll}
\hline $\mathrm{a}_{1}$ & $-2.61505190 \mathrm{e}-01$ & $\mathrm{a}_{2}\left[\mathrm{~K}^{-1}\right]$ & $4.02043832 \mathrm{e}-02$ & $\mathrm{a}_{3}\left[\mathrm{~K}^{-2}\right]$ & $-3.44100344 \mathrm{e}-05$ \\
$\mathrm{a}_{4}\left[\mathrm{~K}^{-3}\right]$ & $1.09717420 \mathrm{e}-08$ & $\mathrm{a}_{5}\left[\mathrm{~K}^{-4}\right]$ & $1.04860540 \mathrm{e}-12$ & $\mathrm{a}_{6}[\mathrm{~K}]$ & $2.80325765 \mathrm{e}+02$ \\
$\mathrm{a}_{7}$ & $2.20826645 \mathrm{e}+01$ & & & &
\end{tabular}
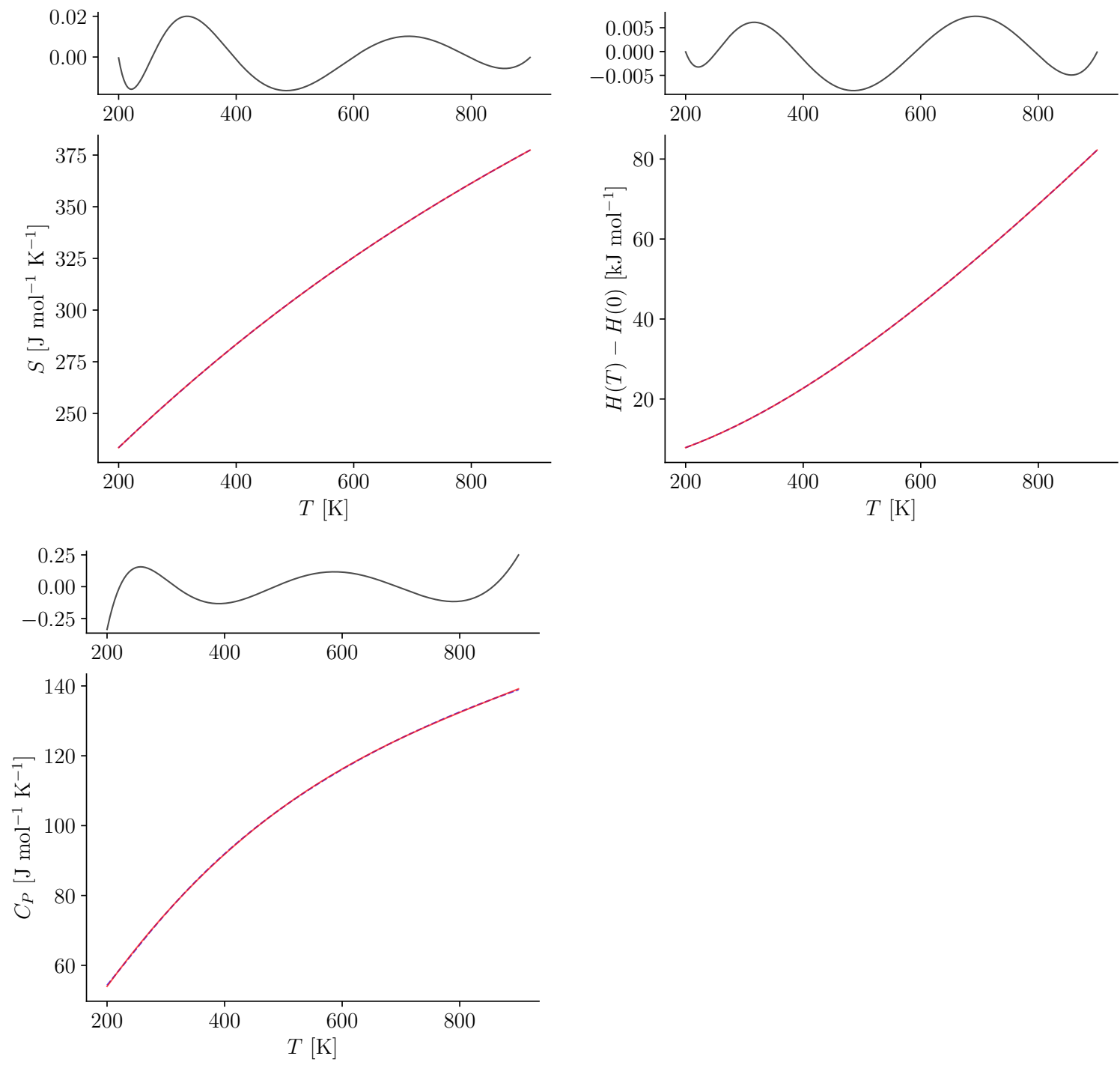


\section{$16 \mathrm{~B}_{3} \mathrm{H}_{6}$ - Isomer $\mathrm{A}$}

Figure S.16.1. Structure of $\mathrm{B}_{3} \mathrm{H}_{6}$ - Isomer A

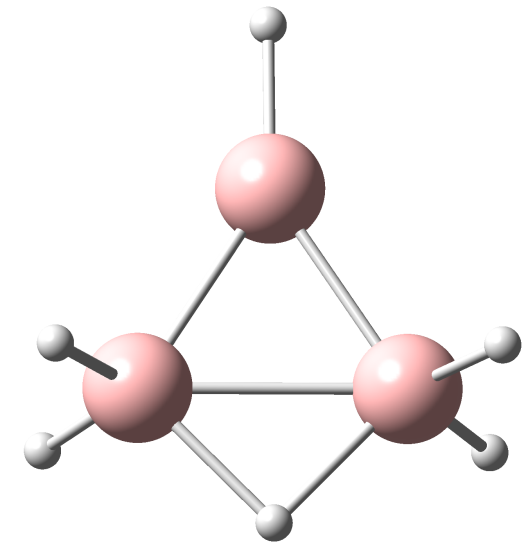

\begin{aligned} & \hline Charge 0 \\ & Spin multiplicity 2 \\ & Framework Group $\mathrm{C}_{2 \mathrm{v}} \\ &$ Rotor Type Asymmetric Top \\ & Symmetry number 2 \\ & \hline\end{aligned}

Table S.16.1. $\mathrm{B}_{3} \mathrm{H}_{6}$ - Isomer A: Selected anharmonic results obtained at the B3LYPD2/cc-pVTZ level

\begin{tabular}{rll}
\hline $\mathrm{ZPE}_{\mathrm{v}}$ & 13526 & $\mathrm{~cm}^{-1}$ \\
$\mathrm{~A}$ & 0.62015 & $\mathrm{~cm}^{-1}$ \\
$\mathrm{~B}$ & 0.53399 & $\mathrm{~cm}^{-1}$ \\
$\mathrm{C}$ & 0.33647 & $\mathrm{~cm}^{-1}$ \\
\hline$C_{P}(300 \mathrm{~K})$ & 83.875 & $\mathrm{~J} \mathrm{~mol}^{-1} \mathrm{~K}-1$ \\
$S(300 \mathrm{~K})$ & 267.598 & $\mathrm{~J} \mathrm{~mol}^{-1} \mathrm{~K}^{-1}$ \\
$H(300 \mathrm{~K})-H(0 \mathrm{~K})$ & 15.393 & $\mathrm{~kJ} \mathrm{~mol}^{-1}$ \\
\hline
\end{tabular}

$\mathrm{ZPE}_{\mathrm{v}}$ : vibrational contribution to the zero-point energy. A, B, C: Rotational constants. $C_{P}$ : Heat capacity at constant pressure. $S$ : Entropy. $H$ : Enthalpy. Ideal-gas calculations (1 bar). 
Table S.16.2. $\mathrm{B}_{3} \mathrm{H}_{6}$ - Isomer A: Cartesian coordinates $(\AA)$ of the B3LYP-D2/cc-pVTZ optimized structure

\begin{tabular}{cccc} 
atom & $\mathrm{x}$ & $\mathrm{y}$ & $\mathrm{z}$ \\
\hline B1 & 0.0000 & 0.9075 & -0.4064 \\
B2 & -0.0000 & -0.9075 & -0.4064 \\
B3 & 0.0000 & 0.0000 & 1.0265 \\
H4 & -0.0000 & 0.0000 & -1.3706 \\
H5 & 0.0000 & 0.0000 & 2.1972 \\
H6 & 1.0358 & 1.5032 & -0.4737 \\
H7 & -1.0358 & -1.5032 & -0.4737 \\
H8 & -1.0358 & 1.5032 & -0.4737 \\
H9 & 1.0358 & -1.5032 & -0.4737 \\
\hline
\end{tabular}


Table S.16.3. $\mathrm{B}_{3} \mathrm{H}_{6}$ - Isomer A: Vibrational frequencies, infrared integrated intensities and Raman activities (B3LYP-D2/cc-pVTZ results)

\begin{tabular}{|c|c|c|c|c|c|c|c|}
\hline \multirow[b]{2}{*}{ mode } & \multirow[b]{2}{*}{ symm. } & \multicolumn{3}{|c|}{ Harmonic } & \multicolumn{3}{|c|}{ Anharmonic } \\
\hline & & $\begin{array}{c}\omega \\
{\left[\mathrm{cm}^{-1}\right]}\end{array}$ & $\begin{array}{c}\mathrm{IR} \\
{\left[\mathrm{km} \mathrm{mol}^{-1}\right]}\end{array}$ & $\begin{array}{c}\text { Raman } \\
{\left[\AA^{6}\right]}\end{array}$ & $\begin{array}{c}\omega \\
{\left[\mathrm{cm}^{-1}\right]}\end{array}$ & $\begin{array}{c}\mathrm{IR} \\
{\left[\mathrm{km} \mathrm{mol}^{-1}\right]}\end{array}$ & $\begin{array}{c}\text { Raman } \\
{\left[\AA^{6}\right]}\end{array}$ \\
\hline 1 & $\mathrm{~B}_{1}$ & 288 & 6.473 & 0.136 & 267 & 6.773 & 0.147 \\
\hline 2 & $\mathrm{~B}_{2}$ & 451 & 4.368 & 0.001 & 444 & 8.028 & 0.000 \\
\hline 3 & $\mathrm{~B}_{1}$ & 453 & 6.806 & 0.031 & 450 & 6.010 & 0.035 \\
\hline 4 & $\mathrm{~B}_{2}$ & 553 & 8.161 & 0.046 & 531 & 9.532 & 0.046 \\
\hline 5 & $\mathrm{~A}_{2}$ & 555 & 0.000 & 0.018 & 507 & 0.000 & 0.017 \\
\hline 6 & $\mathrm{~A}_{1}$ & 724 & 2.105 & 0.249 & 681 & 0.281 & 0.289 \\
\hline 7 & $\mathrm{~A}_{1}$ & 756 & 16.933 & 0.244 & 744 & 9.937 & 0.105 \\
\hline 8 & $\mathrm{~B}_{2}$ & 766 & 0.536 & 0.008 & 696 & 1.727 & 0.025 \\
\hline 9 & $\mathrm{~B}_{1}$ & 784 & 30.584 & 0.020 & 758 & 23.872 & 0.018 \\
\hline 10 & $\mathrm{~A}_{2}$ & 950 & 0.000 & 0.030 & 907 & 0.000 & 0.033 \\
\hline 11 & $\mathrm{~A}_{1}$ & 1045 & 0.491 & 0.338 & 992 & 0.181 & 0.299 \\
\hline 12 & $\mathrm{~B}_{1}$ & 1065 & 1.505 & 0.055 & 965 & 0.943 & 0.056 \\
\hline 13 & $\mathrm{~B}_{2}$ & 1146 & 53.290 & 0.020 & 1094 & 50.571 & 0.017 \\
\hline 14 & $\mathrm{~A}_{1}$ & 1163 & 2.230 & 0.197 & 1115 & 2.317 & 0.195 \\
\hline 15 & $\mathrm{~B}_{2}$ & 1700 & 90.865 & 0.012 & 1503 & 30.625 & 0.006 \\
\hline 16 & $A_{1}$ & 2038 & 4.823 & 0.313 & 2001 & 1.827 & 0.221 \\
\hline 17 & $\mathrm{~B}_{2}$ & 2558 & 61.095 & 0.120 & 2431 & 58.251 & 0.120 \\
\hline 18 & $A_{1}$ & 2564 & 1.216 & 2.569 & 2439 & 2.251 & 2.821 \\
\hline 19 & $\mathrm{~A}_{2}$ & 2605 & 0.000 & 1.271 & 2487 & 0.000 & 1.051 \\
\hline 20 & $\mathrm{~B}_{1}$ & 2620 & 96.908 & 0.096 & 2499 & 95.290 & 0.087 \\
\hline 21 & $\mathrm{~A}_{1}$ & 2806 & 28.655 & 0.643 & 2710 & 30.886 & 0.706 \\
\hline
\end{tabular}


Figure S.16.2. Anharmonic IR spectrum of $\mathrm{B}_{3} \mathrm{H}_{6}$ - Isomer A obtained by convoluting the calculated intensities with Lorentzians having a FWHM of $4 \mathrm{~cm}^{-1}$ (B3LYP-D2/cc-pVTZ results): (top) full spectrum, (middle) contributions from fundamentals and combination bands, (bottom) contributions from overtones
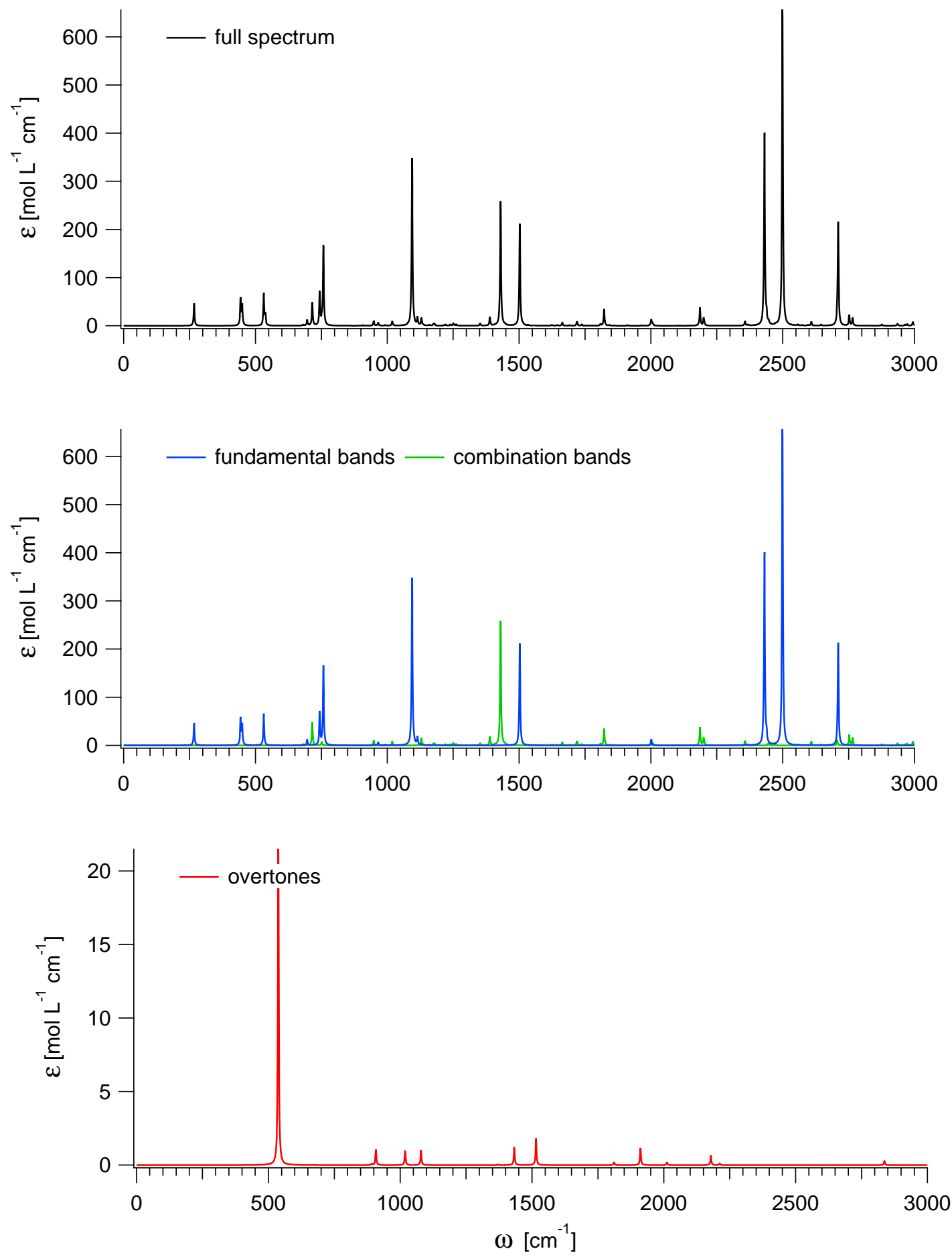
Figure S.16.3. Anharmonic Raman spectrum of $\mathrm{B}_{3} \mathrm{H}_{6}$ - Isomer A obtained by convoluting the calculated activities with Lorentzians having a FWHM of $4 \mathrm{~cm}^{-1}$ (B3LYP-D2/cc-pVTZ results): (top) full spectrum, (middle) contributions from fundamentals and combination bands, (bottom) contributions from overtones
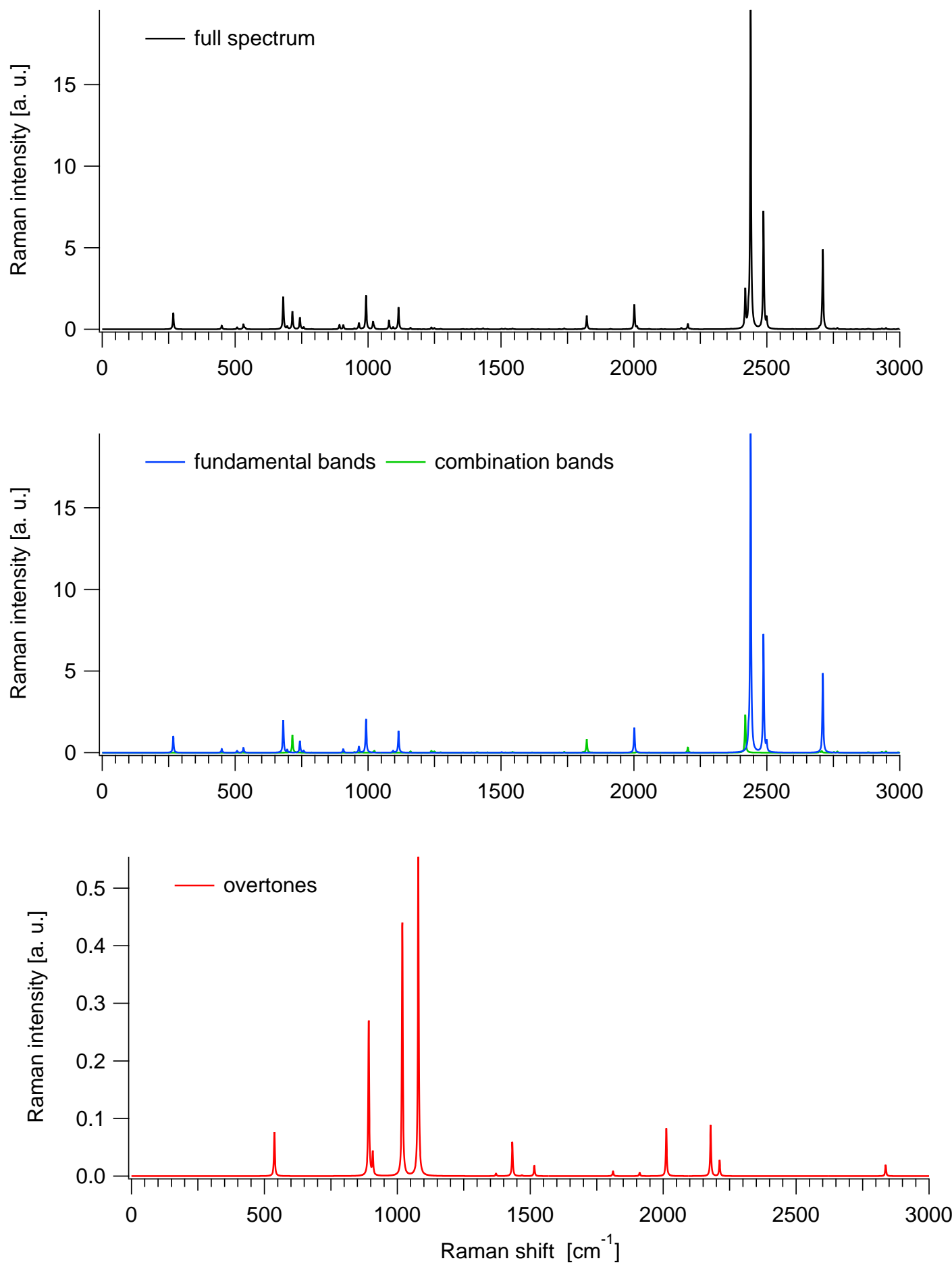

S.128 
Table S.16.4. $\mathrm{B}_{3} \mathrm{H}_{6}$ - Isomer A: Ideal-gas thermodynamic functions at 1 bar (anharmonic B3LYP-D2/cc-pVTZ results)

\begin{tabular}{|c|c|c|c|}
\hline $\begin{array}{c}T \\
{[\mathrm{~K}]}\end{array}$ & $\begin{array}{c}C_{P} \\
{\left[\mathrm{~J} \mathrm{~mol}^{-1} \mathrm{~K}^{-1}\right]}\end{array}$ & $\begin{array}{c}S \\
{\left[\mathrm{~J} \mathrm{~mol}^{-1} \mathrm{~K}^{-1}\right]}\end{array}$ & $\begin{array}{c}H(T)-H(0 K) \\
{\left[\mathrm{kJ} \mathrm{mol}^{-1}\right]}\end{array}$ \\
\hline 100 & 37.780 & 206.524 & 3.421 \\
\hline 200 & 59.508 & 238.755 & 8.197 \\
\hline 300 & 83.875 & 267.598 & 15.393 \\
\hline 400 & 103.656 & 294.557 & 24.811 \\
\hline 500 & 119.081 & 319.411 & 35.978 \\
\hline 600 & 131.528 & 342.259 & 48.529 \\
\hline 700 & 141.884 & 363.335 & 62.214 \\
\hline 800 & 150.617 & 382.867 & 76.852 \\
\hline 900 & 158.007 & 401.046 & 92.293 \\
\hline 1000 & 164.263 & 418.027 & 108.415 \\
\hline 1100 & 169.562 & 433.938 & 125.114 \\
\hline 1200 & 174.058 & 448.890 & 142.301 \\
\hline 1300 & 177.881 & 462.977 & 159.903 \\
\hline 1400 & 181.144 & 476.282 & 177.858 \\
\hline 1500 & 183.941 & 488.878 & 196.116 \\
\hline 1600 & 186.348 & 500.828 & 214.634 \\
\hline 1700 & 188.430 & 512.189 & 233.375 \\
\hline 1800 & 190.239 & 523.012 & 252.311 \\
\hline 1900 & 191.818 & 533.341 & 271.415 \\
\hline 2000 & 193.203 & 543.216 & 290.668 \\
\hline
\end{tabular}

$T$ : Temperature. $C_{P}$ : Heat capacity at constant pressure. $S$ : Entropy. $H$ : Enthalpy. 
Figure S.16.4. $\mathrm{B}_{3} \mathrm{H}_{6}$ - Isomer A: Ideal-gas thermodynamic functions at 1 bar (anharmonic B3LYP-D2/cc-pVTZ results)
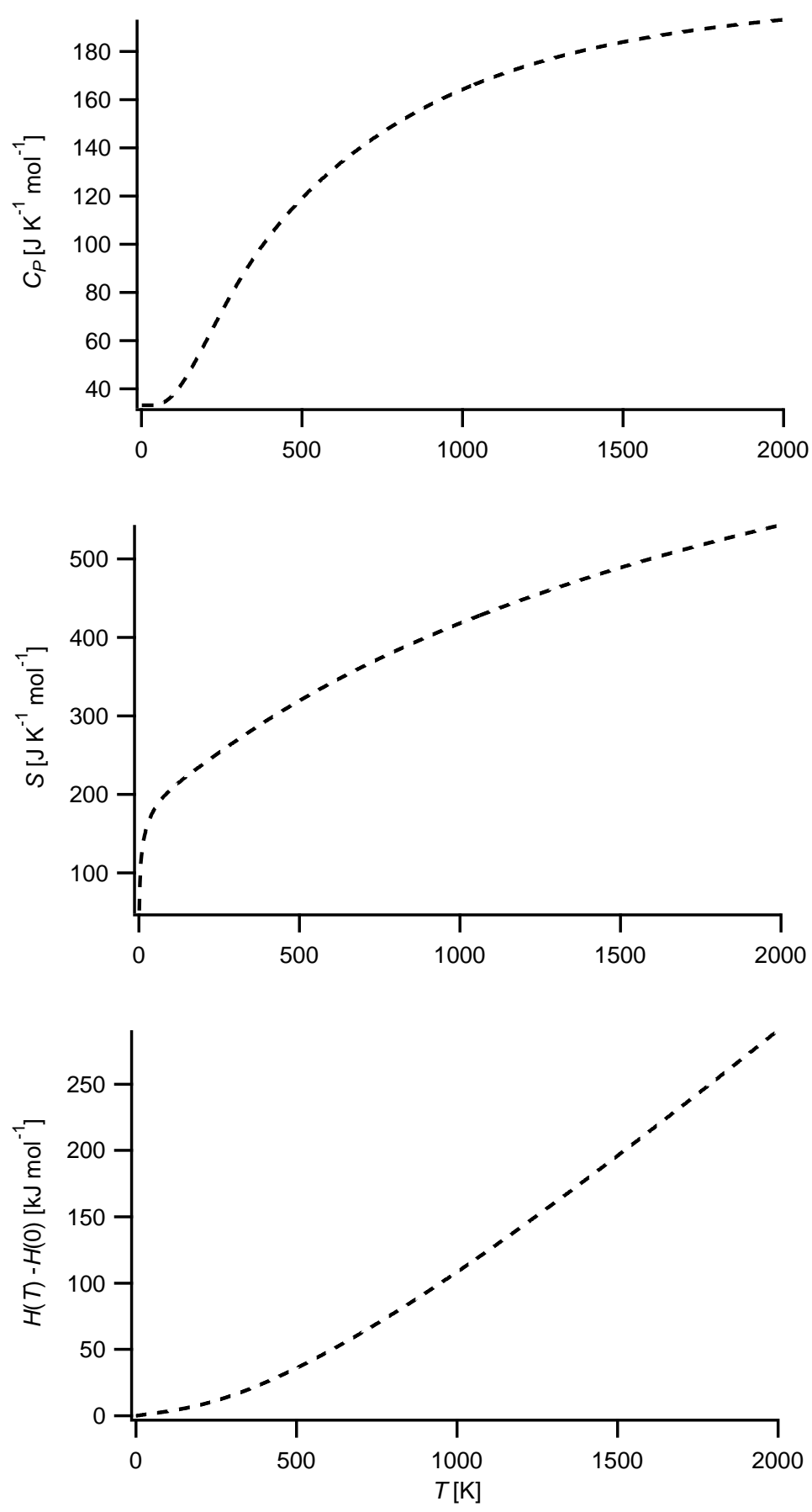

$T$ : Temperature. $C_{P}$ : Heat capacity at constant pressure. $S$ : Entropy. $H$ : Enthalpy. 
Table S.16.5. $\mathrm{B}_{3} \mathrm{H}_{6}$ - Isomer A: Fits (red solid lines) of the computed thermodynamic functions (blue dashed lines) with NASA type functions (Equations (1) - (3)) in the 200 - $900 \mathrm{~K}$ temperature range (anharmonic B3LYP-D2/cc-pVTZ results). In each case, the difference curve between the thermodynamic and NASA functions is plotted in the upper graph

Fit parameters

\begin{tabular}{llllll}
\hline $\mathrm{a}_{1}$ & $-1.28189391 \mathrm{e}+00$ & $\mathrm{a}_{2}\left[\mathrm{~K}^{-1}\right]$ & $5.17975644 \mathrm{e}-02$ & $\mathrm{a}_{3}\left[\mathrm{~K}^{-2}\right]$ & $-5.49280642 \mathrm{e}-05$ \\
$\mathrm{a}_{4}\left[\mathrm{~K}^{-3}\right]$ & $3.07857039 \mathrm{e}-08$ & $\mathrm{a}_{5}\left[\mathrm{~K}^{-4}\right]$ & $-6.49408825 \mathrm{e}-12$ & $\mathrm{a}_{6}[\mathrm{~K}]$ & $3.40815835 \mathrm{e}+02$ \\
$\mathrm{a}_{7}$ & $2.61670235 \mathrm{e}+01$ & & & &
\end{tabular}
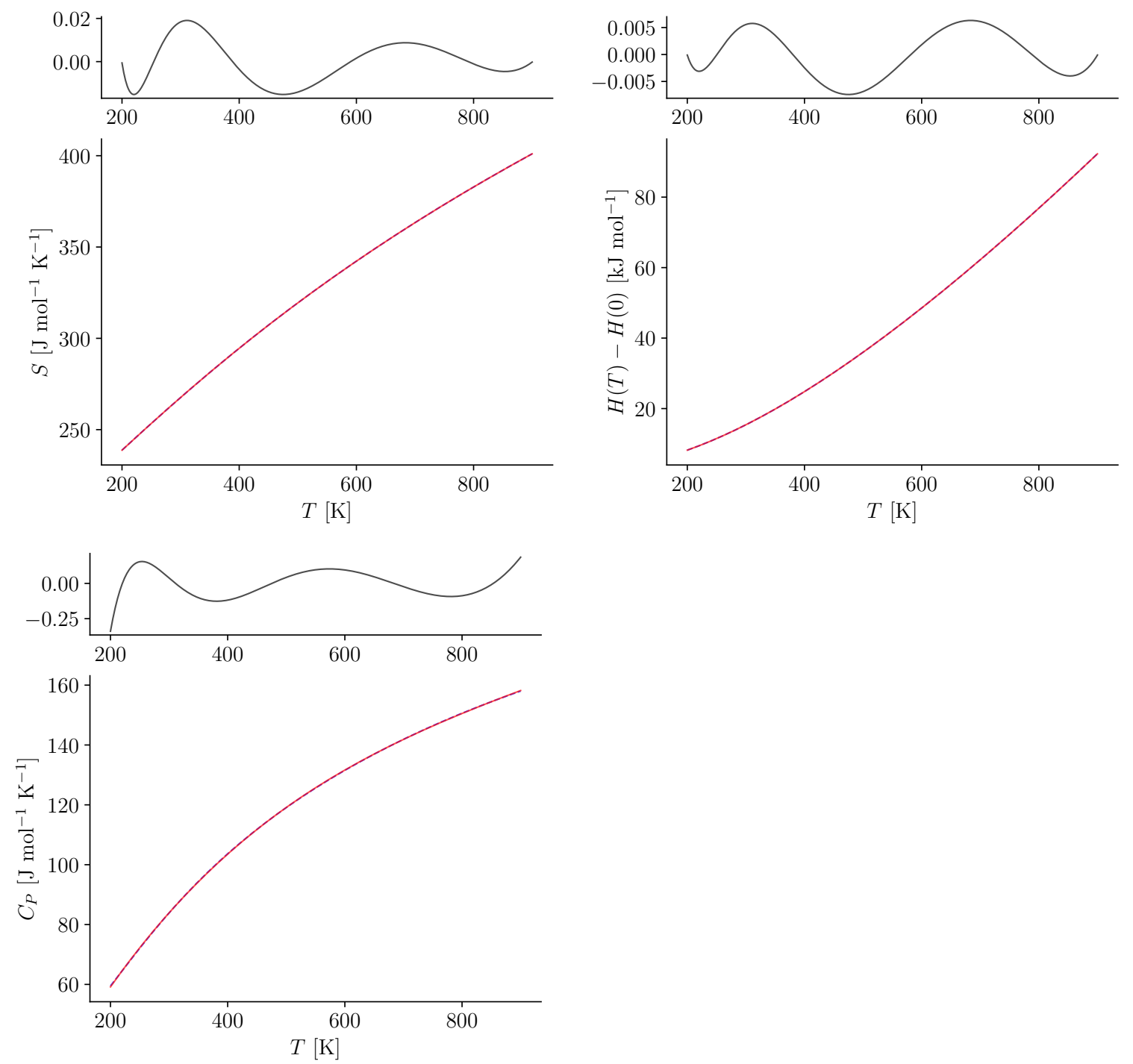


\section{$17 \mathrm{~B}_{3} \mathrm{H}_{6}$ - Isomer $\mathrm{B}$}

Figure S.17.1. Structure of $\mathrm{B}_{3} \mathrm{H}_{6}$ - Isomer B

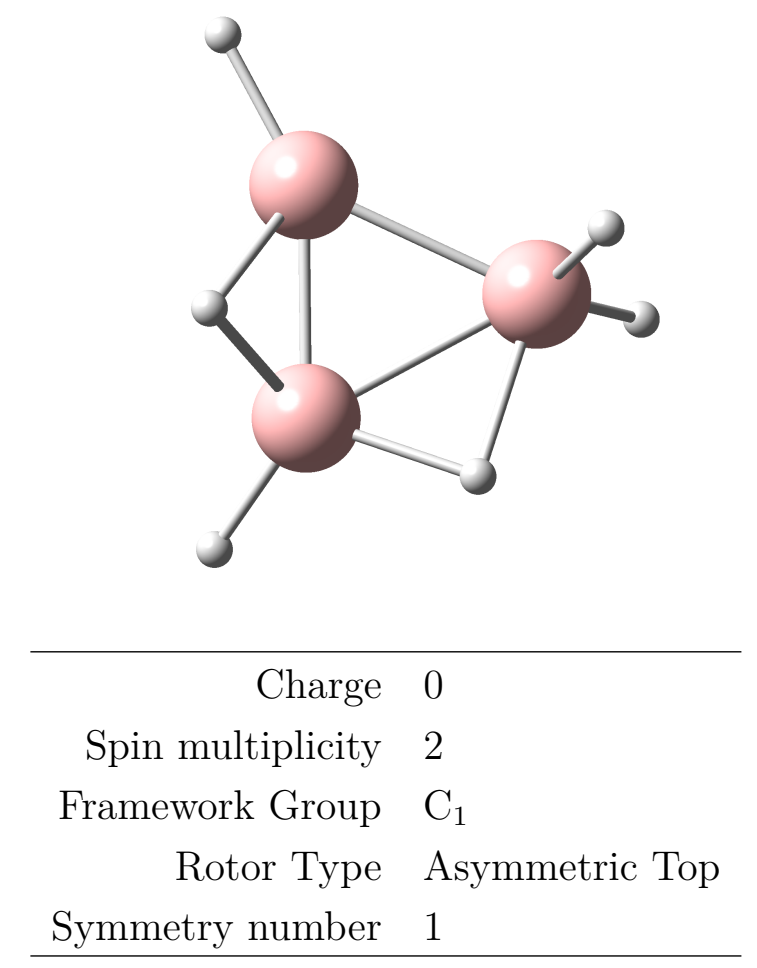

Table S.17.1. $\mathrm{B}_{3} \mathrm{H}_{6}$ - Isomer B: Selected anharmonic results obtained at the B3LYP-D2/ccpVTZ level

\begin{tabular}{rll}
\hline $\mathrm{ZPE}_{\mathrm{v}}$ & 13675 & $\mathrm{~cm}^{-1}$ \\
$\mathrm{~A}$ & 0.68013 & $\mathrm{~cm}^{-1}$ \\
$\mathrm{~B}$ & 0.54266 & $\mathrm{~cm}^{-1}$ \\
$\mathrm{C}$ & 0.34061 & $\mathrm{~cm}^{-1}$ \\
\hline$C_{P}(300 \mathrm{~K})$ & 79.889 & $\mathrm{~J} \mathrm{~mol}^{-1} \mathrm{~K}-1$ \\
$S(300 \mathrm{~K})$ & 267.629 & $\mathrm{~J} \mathrm{~mol}^{-1} \mathrm{~K}^{-1}$ \\
$H(300 \mathrm{~K})-H(0 \mathrm{~K})$ & 14.498 & $\mathrm{~kJ} \mathrm{~mol}^{-1}$ \\
\hline
\end{tabular}

$\mathrm{ZPE}_{\mathrm{v}}$ : vibrational contribution to the zero-point energy. A, B, C: Rotational constants. $C_{P}$ : Heat capacity at constant pressure. $S$ : Entropy. $H$ : Enthalpy. Ideal-gas calculations (1 bar). 
Table S.17.2. $\mathrm{B}_{3} \mathrm{H}_{6}$ - Isomer B: Cartesian coordinates $(\AA)$ of the B3LYP-D2/cc-pVTZ optimized structure

\begin{tabular}{cccc} 
atom & $\mathrm{x}$ & $\mathrm{y}$ & $\mathrm{z}$ \\
\hline B1 & -0.5579 & 0.8124 & -0.0912 \\
B2 & 1.0230 & 0.0731 & -0.0242 \\
B3 & -0.5416 & -0.7678 & 0.0008 \\
H4 & 1.7318 & -0.0975 & -0.9630 \\
H5 & 1.4934 & 0.5203 & 0.9876 \\
H6 & -1.1058 & 1.8319 & -0.2624 \\
H7 & 0.6244 & -1.1628 & 0.4178 \\
H8 & -1.1974 & -0.0220 & 0.8513 \\
H9 & -1.1635 & -1.6589 & -0.4582 \\
\hline
\end{tabular}


Table S.17.3. $\mathrm{B}_{3} \mathrm{H}_{6}$ - Isomer B: Vibrational frequencies, infrared integrated intensities and Raman activities (B3LYP-D2/cc-pVTZ results)

\begin{tabular}{|c|c|c|c|c|c|c|c|}
\hline \multirow[b]{2}{*}{ mode } & \multirow[b]{2}{*}{ symm. } & \multicolumn{3}{|c|}{ Harmonic } & \multicolumn{3}{|c|}{ Anharmonic } \\
\hline & & $\begin{array}{c}\omega \\
{\left[\mathrm{cm}^{-1}\right]}\end{array}$ & $\begin{array}{c}\mathrm{IR} \\
{\left[\mathrm{km} \mathrm{mol}^{-1}\right]}\end{array}$ & $\begin{array}{c}\text { Raman } \\
{\left[\AA^{6}\right]}\end{array}$ & $\begin{array}{c}\omega \\
{\left[\mathrm{cm}^{-1}\right]}\end{array}$ & $\begin{array}{c}\mathrm{IR} \\
{\left[\mathrm{km} \mathrm{mol}^{-1}\right]}\end{array}$ & $\begin{array}{c}\text { Raman } \\
{\left[\AA^{6}\right]}\end{array}$ \\
\hline 1 & A & 436 & 1.745 & 0.050 & 409 & 12.783 & 0.149 \\
\hline 2 & $\mathrm{~A}$ & 460 & 8.680 & 0.225 & 438 & 13.323 & 0.235 \\
\hline 3 & $\mathrm{~A}$ & 580 & 38.580 & 0.089 & 513 & 48.142 & 0.046 \\
\hline 4 & $\mathrm{~A}$ & 597 & 6.474 & 0.133 & 570 & 4.748 & 0.161 \\
\hline 5 & A & 656 & 7.703 & 0.077 & 619 & 5.333 & 0.093 \\
\hline 6 & $\mathrm{~A}$ & 715 & 2.213 & 0.163 & 690 & 5.404 & 0.096 \\
\hline 7 & $\mathrm{~A}$ & 735 & 9.438 & 0.010 & 674 & 2.721 & 0.083 \\
\hline 8 & $\mathrm{~A}$ & 821 & 2.064 & 0.101 & 772 & 1.451 & 0.124 \\
\hline 9 & $\mathrm{~A}$ & 903 & 1.804 & 0.325 & 853 & 2.297 & 0.146 \\
\hline 10 & $\mathrm{~A}$ & 952 & 10.469 & 0.050 & 899 & 9.345 & 0.092 \\
\hline 11 & $\mathrm{~A}$ & 1032 & 4.041 & 0.111 & 955 & 1.636 & 0.120 \\
\hline 12 & $\mathrm{~A}$ & 1110 & 2.296 & 0.687 & 1063 & 3.197 & 0.359 \\
\hline 13 & $\mathrm{~A}$ & 1173 & 20.962 & 0.149 & 1126 & 6.898 & 0.080 \\
\hline 14 & $\mathrm{~A}$ & 1336 & 93.966 & 0.137 & 1126 & 15.952 & 0.083 \\
\hline 15 & $\mathrm{~A}$ & 1579 & 61.205 & 0.040 & 1385 & 18.523 & 0.018 \\
\hline 16 & $\mathrm{~A}$ & 2039 & 11.327 & 0.580 & 2020 & 20.487 & 0.414 \\
\hline 17 & A & 2084 & 42.365 & 0.225 & 1985 & 4.285 & 0.226 \\
\hline 18 & $\mathrm{~A}$ & 2527 & 31.236 & 1.221 & 2409 & 28.650 & 1.323 \\
\hline 19 & $\mathrm{~A}$ & 2659 & 80.155 & 0.641 & 2533 & 76.082 & 0.695 \\
\hline 20 & $\mathrm{~A}$ & 2727 & 40.046 & 0.743 & 2602 & 33.406 & 0.694 \\
\hline 21 & $\mathrm{~A}$ & 2810 & 20.663 & 0.555 & 2704 & 20.682 & 0.551 \\
\hline
\end{tabular}


Figure S.17.2. Anharmonic IR spectrum of $\mathrm{B}_{3} \mathrm{H}_{6}$ - Isomer $\mathrm{B}$ obtained by convoluting the calculated intensities with Lorentzians having a FWHM of $4 \mathrm{~cm}^{-1}$ (B3LYP-D2/cc-pVTZ results): (top) full spectrum, (middle) contributions from fundamentals and combination bands, (bottom) contributions from overtones
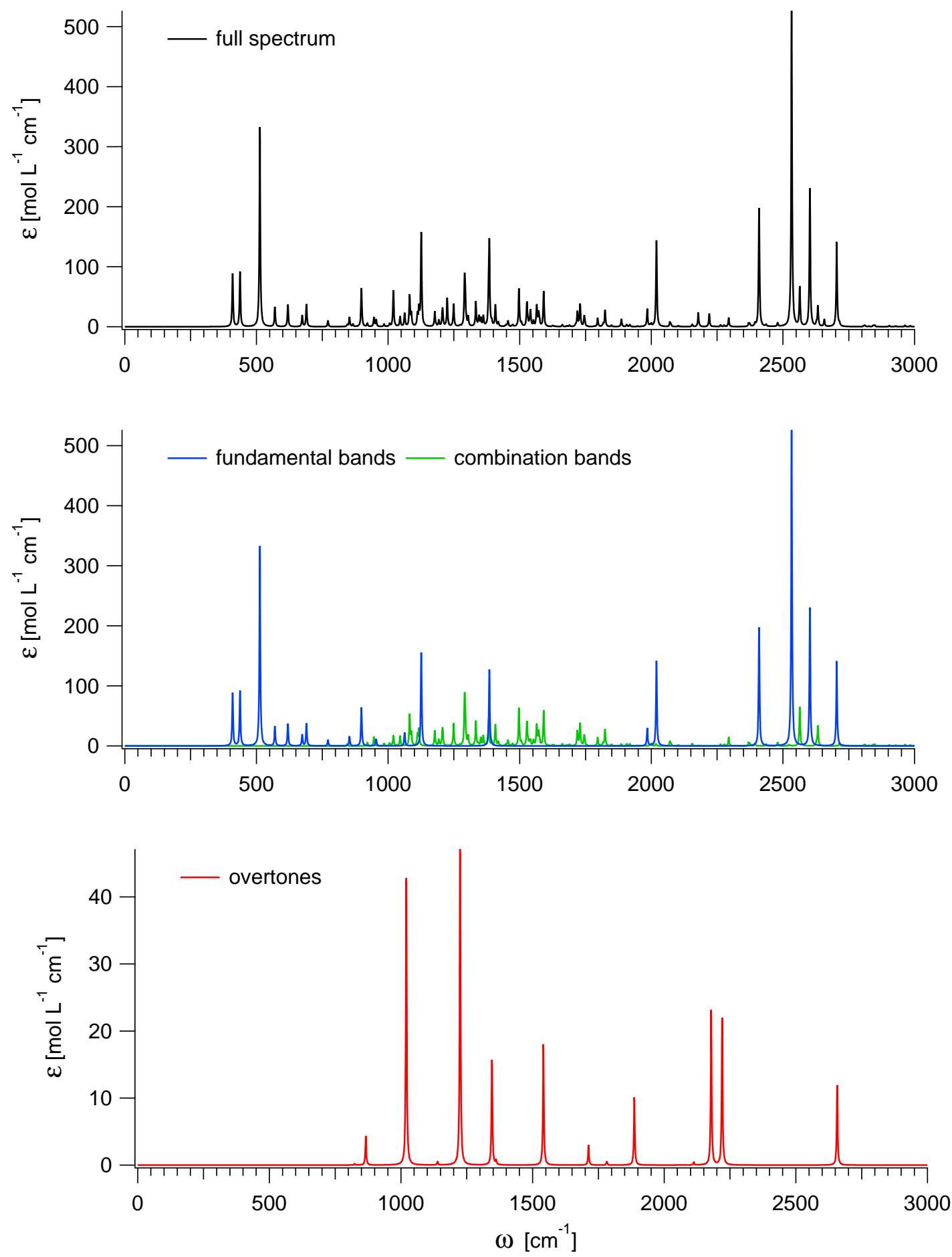
Figure S.17.3. Anharmonic Raman spectrum of $\mathrm{B}_{3} \mathrm{H}_{6}$ - Isomer B obtained by convoluting the calculated activities with Lorentzians having a FWHM of $4 \mathrm{~cm}^{-1}$ (B3LYP-D2/cc-pVTZ results): (top) full spectrum, (middle) contributions from fundamentals and combination bands, (bottom) contributions from overtones
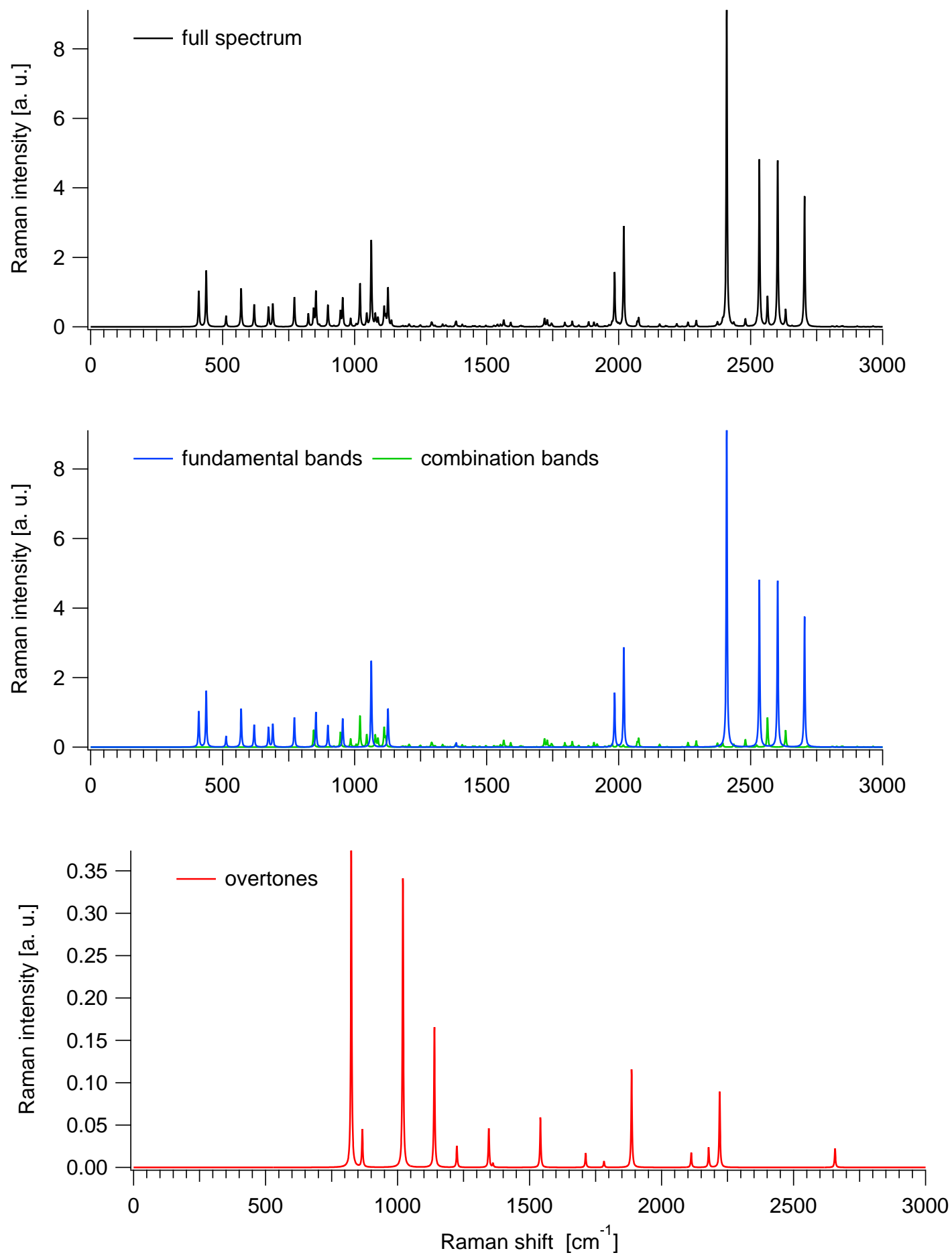

S.136 
Table S.17.4. $\mathrm{B}_{3} \mathrm{H}_{6}$ - Isomer B: Ideal-gas thermodynamic functions at 1 bar (anharmonic B3LYP-D2/cc-pVTZ results)

\begin{tabular}{cccc}
\hline$T$ & $C_{P}$ & $S$ & $H(T)-H(0 K)$ \\
{$[\mathrm{K}]$} & {$\left[\mathrm{J} \mathrm{mol}^{-1} \mathrm{~K}^{-1}\right]$} & {$\left[\mathrm{J} \mathrm{mol}^{-1} \mathrm{~K}^{-1}\right]$} & {$\left[\mathrm{kJ} \mathrm{mol}^{-1}\right]$} \\
\hline 100 & 35.309 & 210.970 & 3.357 \\
200 & 54.919 & 240.574 & 7.741 \\
300 & 79.889 & 267.629 & 14.498 \\
400 & 100.837 & 293.599 & 23.575 \\
500 & 117.331 & 317.943 & 34.516 \\
600 & 130.578 & 340.547 & 46.934 \\
700 & 141.471 & 361.520 & 60.553 \\
800 & 150.543 & 381.021 & 75.168 \\
900 & 158.137 & 399.204 & 90.613 \\
1000 & 164.509 & 416.205 & 106.754 \\
1100 & 169.870 & 432.143 & 123.481 \\
1200 & 174.393 & 447.123 & 140.700 \\
1300 & 178.224 & 461.238 & 158.336 \\
1400 & 181.482 & 474.568 & 176.326 \\
1500 & 184.267 & 487.186 & 194.617 \\
1600 & 186.659 & 499.157 & 213.166 \\
1700 & 188.725 & 510.537 & 231.938 \\
1800 & 190.516 & 521.376 & 250.902 \\
1900 & 192.078 & 531.719 & 270.034 \\
2000 & 193.447 & 541.607 & 289.312 \\
\hline
\end{tabular}

$T$ : Temperature. $C_{P}$ : Heat capacity at constant pressure. $S$ : Entropy. $H$ : Enthalpy. 
Figure S.17.4. $\mathrm{B}_{3} \mathrm{H}_{6}$ - Isomer B: Ideal-gas thermodynamic functions at 1 bar (anharmonic B3LYP-D2/cc-pVTZ results)
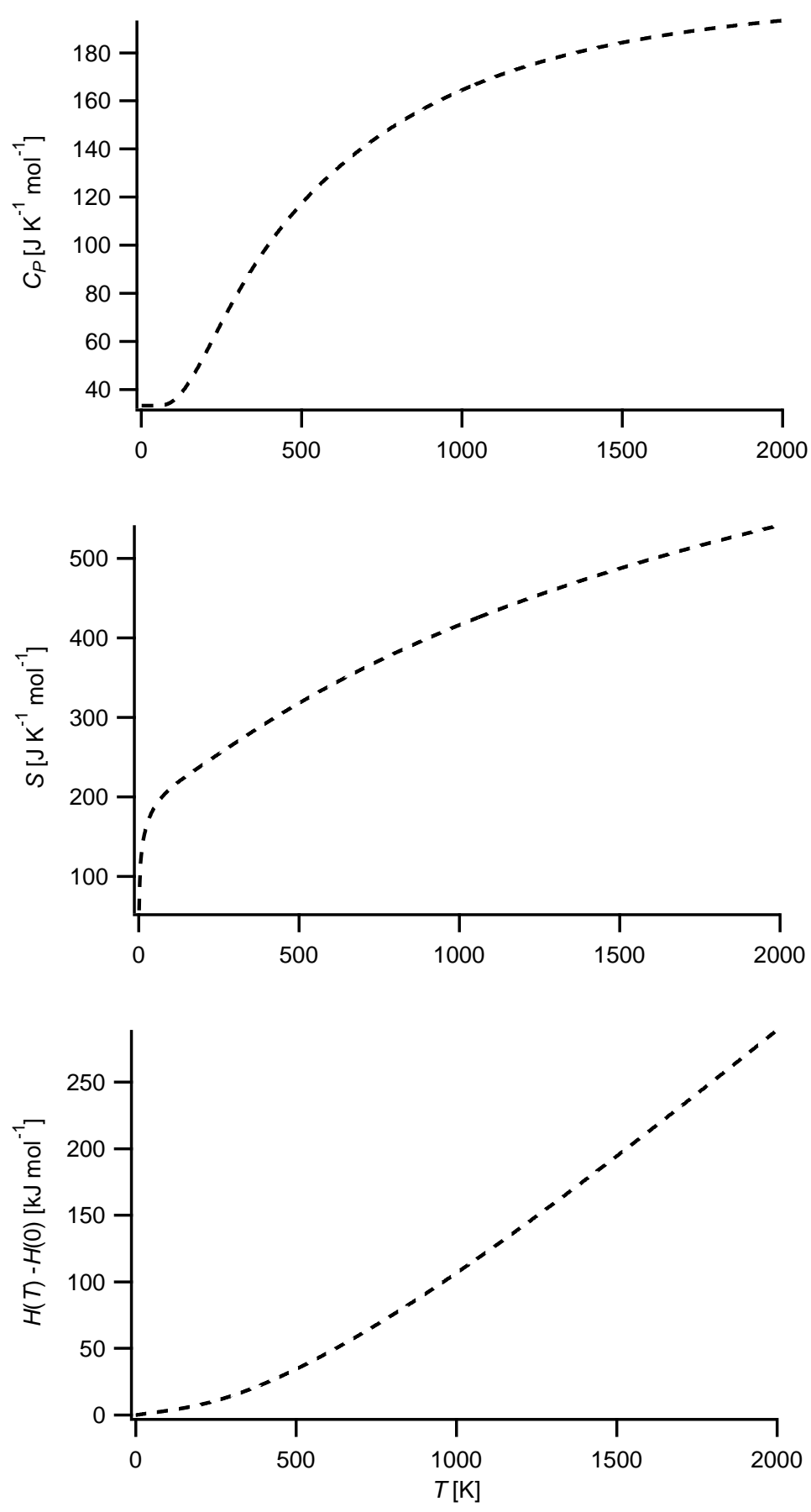

$T$ : Temperature. $C_{P}$ : Heat capacity at constant pressure. $S$ : Entropy. $H$ : Enthalpy. 
Table S.17.5. $\mathrm{B}_{3} \mathrm{H}_{6}$ - Isomer B: Fits (red solid lines) of the computed thermodynamic functions (blue dashed lines) with NASA type functions (Equations (1) - (3)) in the 200 - $900 \mathrm{~K}$ temperature range (anharmonic B3LYP-D2/cc-pVTZ results). In each case, the difference curve between the thermodynamic and NASA functions is plotted in the upper graph

Fit parameters

\begin{tabular}{llllll}
\hline $\mathrm{a}_{1}$ & $-1.51506009 \mathrm{e}+00$ & $\mathrm{a}_{2}\left[\mathrm{~K}^{-1}\right]$ & $4.74724730 \mathrm{e}-02$ & $\mathrm{a}_{3}\left[\mathrm{~K}^{-2}\right]$ & $-3.73635271 \mathrm{e}-05$ \\
$\mathrm{a}_{4}\left[\mathrm{~K}^{-3}\right]$ & $8.35670783 \mathrm{e}-09$ & $\mathrm{a}_{5}\left[\mathrm{~K}^{-4}\right]$ & $3.07712886 \mathrm{e}-12$ & $\mathrm{a}_{6}[\mathrm{~K}]$ & $3.80622538 \mathrm{e}+02$ \\
$\mathrm{a}_{7}$ & $2.81908687 \mathrm{e}+01$ & & & &
\end{tabular}
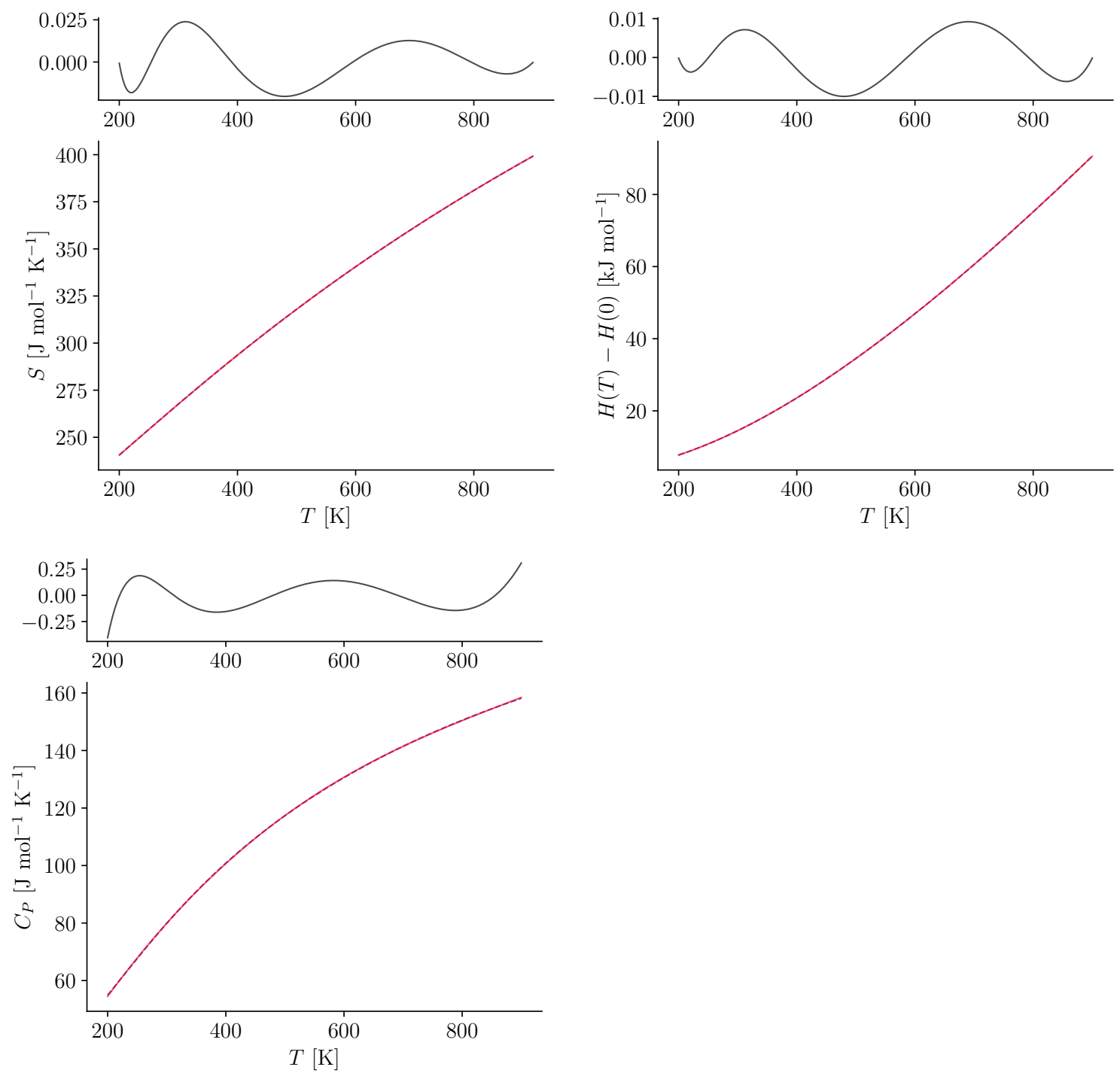


\section{$18 \mathrm{~B}_{3} \mathrm{H}_{7}$}

Figure S.18.1. Structure of $\mathrm{B}_{3} \mathrm{H}_{7}$

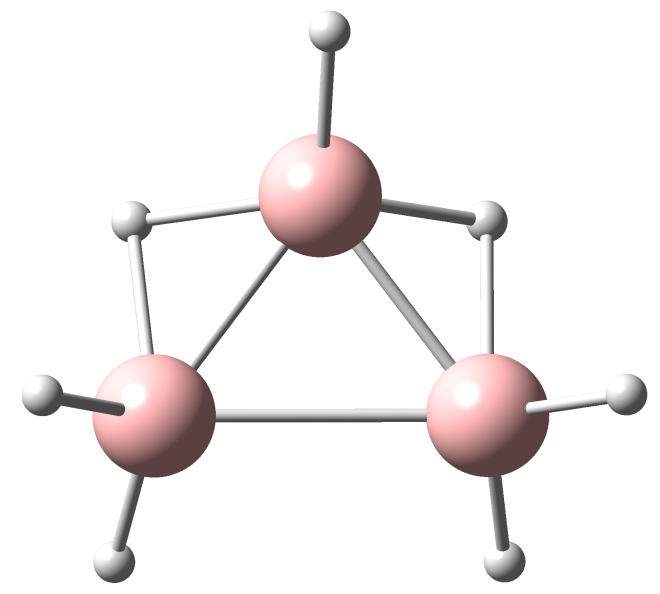

\begin{aligned} & \hline Charge 0 \\ & Spin multiplicity 1 \\ & Framework Group $\mathrm{C}_{\mathrm{s}} \\ &$ Rotor Type Asymmetric Top \\ & Symmetry number 1 \\ & \hline\end{aligned}

Table S.18.1. $\mathrm{B}_{3} \mathrm{H}_{7}$ : Selected anharmonic results obtained at the B3LYP-D2/cc-pVTZ level

\begin{tabular}{rll}
\hline $\mathrm{ZPE}_{\mathrm{v}}$ & 16407 & $\mathrm{~cm}^{-1}$ \\
$\mathrm{~A}$ & 0.66352 & $\mathrm{~cm}^{-1}$ \\
$\mathrm{~B}$ & 0.44643 & $\mathrm{~cm}^{-1}$ \\
$\mathrm{C}$ & 0.30849 & $\mathrm{~cm}^{-1}$ \\
\hline$C_{P}(300 \mathrm{~K})$ & 78.480 & $\mathrm{~J} \mathrm{~mol}^{-1} \mathrm{~K}-1$ \\
$S(300 \mathrm{~K})$ & 269.938 & $\mathrm{~J} \mathrm{~mol}^{-1} \mathrm{~K}-1$ \\
$H(300 \mathrm{~K})-H(0 \mathrm{~K})$ & 14.441 & $\mathrm{~kJ} \mathrm{~mol}^{-1}$ \\
\hline
\end{tabular}

$\mathrm{ZPE}_{\mathrm{v}}$ : vibrational contribution to the zero-point energy. A, B, C: Rotational constants. $C_{P}$ : Heat capacity at constant pressure. $S$ : Entropy. $H$ : Enthalpy. Ideal-gas calculations (1 bar). 
Table S.18.2. $\mathrm{B}_{3} \mathrm{H}_{7}$ : Cartesian coordinates $(\AA)$ of the B3LYP-D2/cc-pVTZ optimized structure

\begin{tabular}{cccc} 
atom & $\mathrm{x}$ & $\mathrm{y}$ & $\mathrm{z}$ \\
\hline B1 & -0.0242 & 0.8773 & -0.0000 \\
B2 & -0.0242 & -0.4422 & 1.0038 \\
B3 & -0.0242 & -0.4422 & -1.0038 \\
H4 & 0.6993 & 0.7324 & -1.0677 \\
H5 & -0.9379 & -0.3326 & -1.7580 \\
H6 & 0.7629 & -1.3077 & 1.1752 \\
H7 & 0.6993 & 0.7324 & 1.0677 \\
H8 & -0.6849 & 1.8520 & -0.0000 \\
H9 & -0.9379 & -0.3326 & 1.7580 \\
H10 & 0.7629 & -1.3077 & -1.1752 \\
\hline
\end{tabular}


Table S.18.3. $\mathrm{B}_{3} \mathrm{H}_{7}$ : Vibrational frequencies, infrared integrated intensities and Raman activities (B3LYP-D2/cc-pVTZ results)

\begin{tabular}{|c|c|c|c|c|c|c|c|}
\hline \multirow[b]{2}{*}{ mode } & \multirow[b]{2}{*}{ symm. } & \multicolumn{3}{|c|}{ Harmonic } & \multicolumn{3}{|c|}{ Anharmonic } \\
\hline & & $\begin{array}{c}\omega \\
{\left[\mathrm{cm}^{-1}\right]}\end{array}$ & $\begin{array}{c}\mathrm{IR} \\
{\left[\mathrm{km} \mathrm{mol}^{-1}\right]}\end{array}$ & $\begin{array}{c}\text { Raman } \\
{\left[\AA^{6}\right]}\end{array}$ & $\begin{array}{c}\omega \\
{\left[\mathrm{cm}^{-1}\right]}\end{array}$ & $\begin{array}{c}\mathrm{IR} \\
{\left[\mathrm{km} \mathrm{mol}^{-1}\right]}\end{array}$ & $\begin{array}{c}\text { Raman } \\
{\left[\AA^{6}\right]}\end{array}$ \\
\hline 1 & A" & 282 & 0.054 & 0.048 & 252 & 0.197 & 0.057 \\
\hline 2 & $A^{\prime}$ & 430 & 5.989 & 0.413 & 415 & 5.189 & 0.420 \\
\hline 3 & $\mathrm{~A}^{\prime}$ & 654 & 3.752 & 0.068 & 655 & 3.489 & 0.054 \\
\hline 4 & A" & 701 & 5.571 & 0.006 & 676 & 6.970 & 0.003 \\
\hline 5 & $A^{\prime}$ & 714 & 0.198 & 0.084 & 681 & 0.236 & 0.075 \\
\hline 6 & $\mathrm{~A}^{\prime}$ & 796 & 16.221 & 0.122 & 755 & 22.208 & 0.134 \\
\hline 7 & A" & 853 & 15.096 & 0.004 & 803 & 21.288 & 0.009 \\
\hline 8 & A" & 868 & 0.074 & 0.102 & 826 & 0.163 & 0.127 \\
\hline 9 & $\mathrm{~A}^{\prime}$ & 930 & 14.528 & 0.045 & 883 & 7.024 & 0.014 \\
\hline 10 & A" & 986 & 16.332 & 0.012 & 954 & 5.147 & 0.009 \\
\hline 11 & $\mathrm{~A}^{\prime}$ & 998 & 0.280 & 0.563 & 952 & 0.620 & 0.625 \\
\hline 12 & $\mathrm{~A}^{\prime}$ & 1097 & 4.563 & 0.159 & 1015 & 4.462 & 0.137 \\
\hline 13 & A" & 1121 & 31.716 & 0.034 & 1049 & 31.444 & 0.034 \\
\hline 14 & A" & 1197 & 21.706 & 0.135 & 1144 & 18.434 & 0.128 \\
\hline 15 & $A^{\prime}$ & 1226 & 20.063 & 0.025 & 1177 & 14.439 & 0.036 \\
\hline 16 & A" & 1487 & 56.044 & 0.051 & 1300 & 32.228 & 0.034 \\
\hline 17 & $A^{\prime}$ & 1550 & 158.088 & 0.088 & 1344 & 54.988 & 0.066 \\
\hline 18 & $A^{\prime}$ & 2086 & 3.971 & 0.660 & 1912 & 4.071 & 0.333 \\
\hline 19 & $A^{\prime \prime}$ & 2118 & 118.409 & 0.236 & 1913 & 52.723 & 0.105 \\
\hline 20 & A" & 2622 & 88.835 & 0.420 & 2504 & 84.842 & 0.467 \\
\hline 21 & $A^{\prime}$ & 2629 & 39.982 & 1.473 & 2508 & 36.300 & 1.376 \\
\hline 22 & $A^{\prime \prime}$ & 2705 & 0.041 & 0.344 & 2586 & 0.020 & 0.351 \\
\hline 23 & $\mathrm{~A}^{\prime}$ & 2721 & 79.624 & 0.660 & 2603 & 86.896 & 0.659 \\
\hline
\end{tabular}


Table S.18.3 - Continued

\begin{tabular}{cccccccc}
\hline & & \multicolumn{3}{c}{ Harmonic } & \multicolumn{3}{c}{ Anharmonic } \\
mode & symm. & $\omega$ & IR & Raman & $\omega$ & IR & Raman \\
& & {$\left[\mathrm{cm}^{-1}\right]$} & {$\left[\mathrm{km} \mathrm{mol}^{-1}\right]$} & {$\left[\AA^{6}\right]$} & {$\left[\mathrm{cm}^{-1}\right]$} & {$\left[\mathrm{km} \mathrm{mol}^{-1}\right]$} & {$\left[\AA^{6}\right]$} \\
\hline 24 & $\mathrm{~A}^{\prime}$ & 2756 & 56.898 & 0.619 & 2630 & 52.741 & 0.703 \\
\hline
\end{tabular}


Figure S.18.2. Anharmonic IR spectrum of $\mathrm{B}_{3} \mathrm{H}_{7}$ obtained by convoluting the calculated intensities with Lorentzians having a FWHM of $4 \mathrm{~cm}^{-1}$ (B3LYP-D2/cc-pVTZ results): (top) full spectrum, (middle) contributions from fundamentals and combination bands, (bottom) contributions from overtones
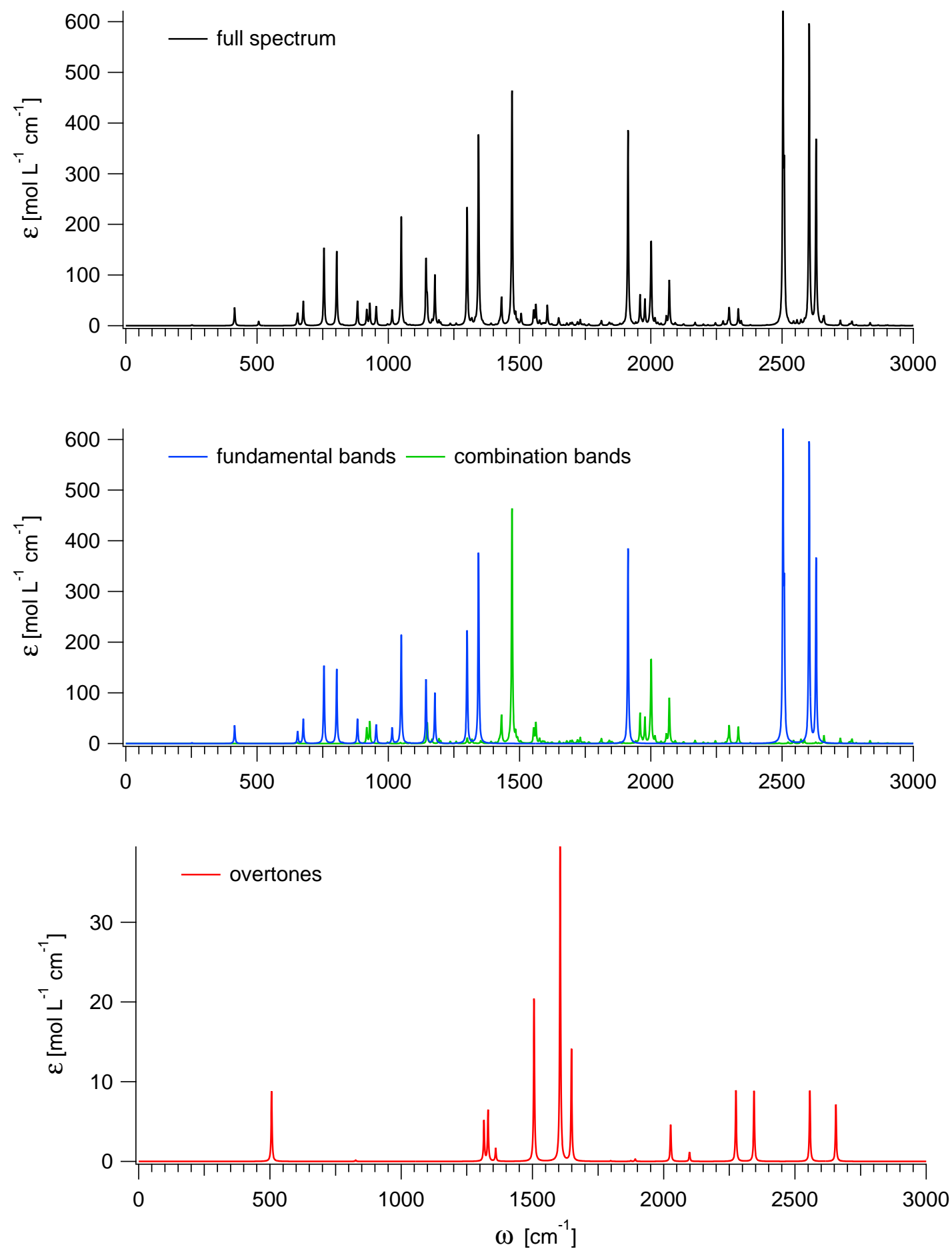
Figure S.18.3. Anharmonic Raman spectrum of $\mathrm{B}_{3} \mathrm{H}_{7}$ obtained by convoluting the calculated activities with Lorentzians having a FWHM of $4 \mathrm{~cm}^{-1}$ (B3LYP-D2/cc-pVTZ results): (top) full spectrum, (middle) contributions from fundamentals and combination bands, (bottom) contributions from overtones
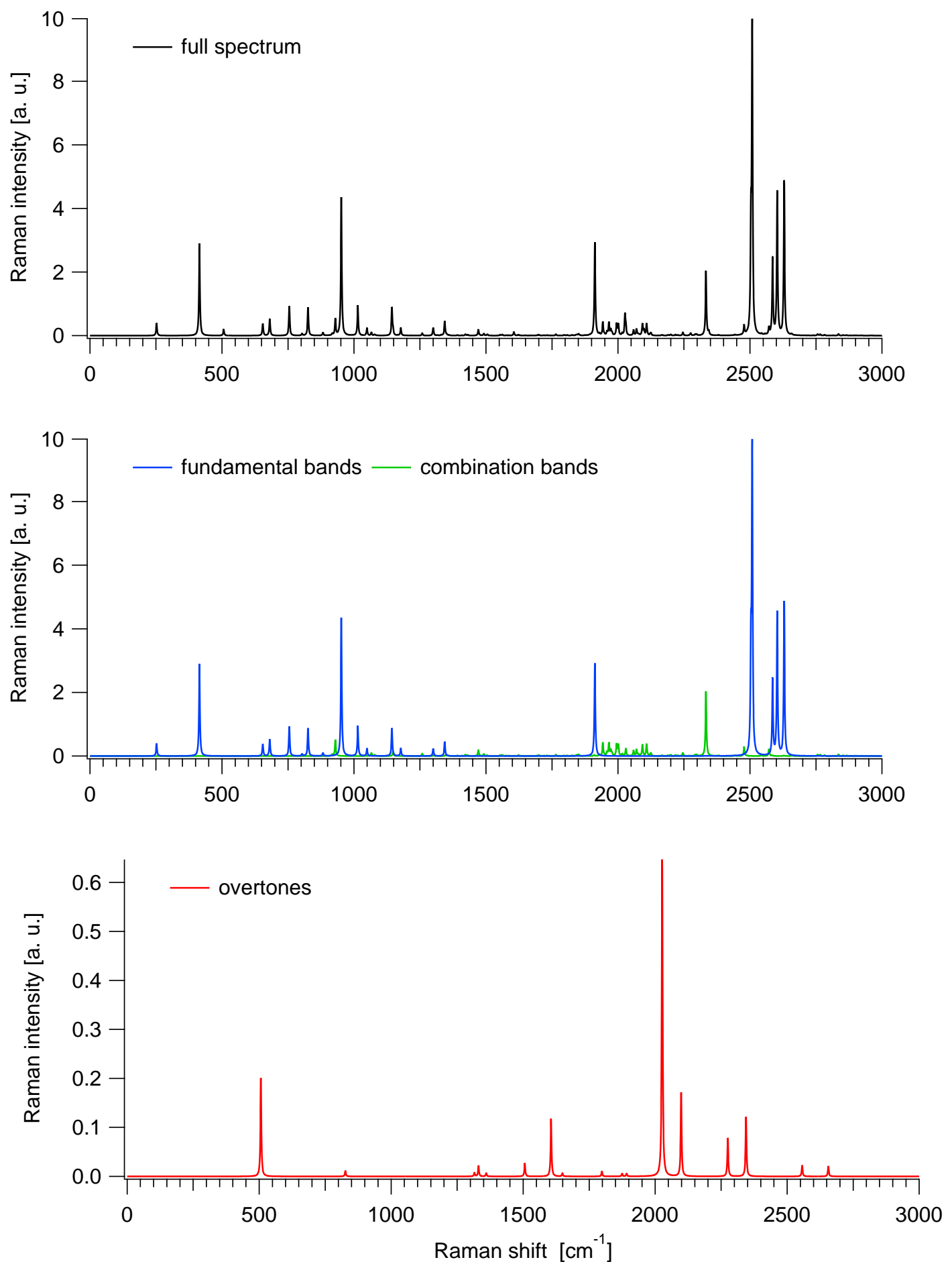

S.145 
Table S.18.4. $\mathrm{B}_{3} \mathrm{H}_{7}$ : Ideal-gas thermodynamic functions at 1 bar (anharmonic B3LYPD2/cc-pVTZ results)

\begin{tabular}{cccc}
\hline$T$ & $C_{P}$ & $S$ & $H(T)-H(0 K)$ \\
{$[\mathrm{K}]$} & {$\left[\mathrm{J} \mathrm{mol}^{-1} \mathrm{~K}^{-1}\right]$} & {$\left[\mathrm{J} \mathrm{mol}^{-1} \mathrm{~K}^{-1}\right]$} & {$\left[\mathrm{kJ} \mathrm{mol}^{-1}\right]$} \\
\hline 100 & 37.287 & 213.473 & 3.423 \\
200 & 53.603 & 243.599 & 7.861 \\
300 & 78.480 & 269.938 & 14.441 \\
400 & 102.343 & 295.870 & 23.513 \\
500 & 122.203 & 320.915 & 34.773 \\
600 & 138.455 & 344.680 & 47.833 \\
700 & 151.870 & 367.063 & 62.370 \\
800 & 163.027 & 388.093 & 78.132 \\
900 & 172.342 & 407.849 & 94.914 \\
1000 & 180.141 & 426.423 & 112.550 \\
1100 & 186.688 & 443.909 & 130.901 \\
1200 & 192.203 & 460.396 & 149.853 \\
1300 & 196.868 & 475.969 & 169.313 \\
1400 & 200.832 & 490.708 & 189.203 \\
1500 & 204.217 & 504.682 & 209.460 \\
1600 & 207.122 & 517.957 & 230.031 \\
1700 & 209.629 & 530.591 & 250.871 \\
1800 & 211.803 & 542.636 & 271.945 \\
1900 & 213.698 & 554.140 & 293.223 \\
2000 & 215.357 & 565.144 & 314.677 \\
\hline$T$ & & & $S$ \\
\hline & & &
\end{tabular}

$T$ : Temperature. $C_{P}$ : Heat capacity at constant pressure. $S$ : Entropy. $H$ : Enthalpy. 
Figure S.18.4. $\mathrm{B}_{3} \mathrm{H}_{7}$ : Ideal-gas thermodynamic functions at 1 bar (anharmonic B3LYPD2/cc-pVTZ results)
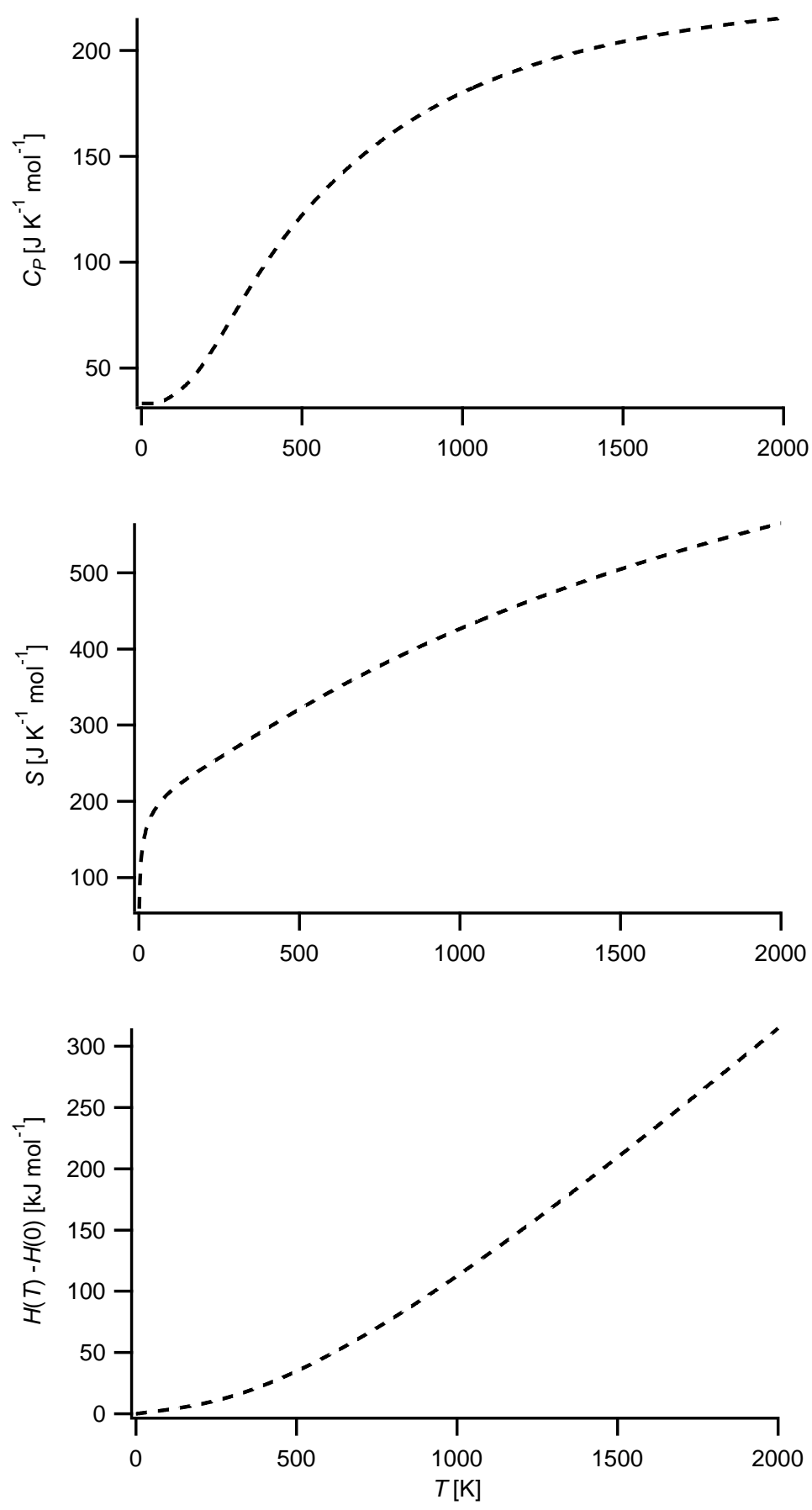

$T$ : Temperature. $C_{P}$ : Heat capacity at constant pressure. $S$ : Entropy. $H$ : Enthalpy. 
Table S.18.5. $\mathrm{B}_{3} \mathrm{H}_{7}$ : Fits (red solid lines) of the computed thermodynamic functions (blue dashed lines) with NASA type functions (Equations (1) - (3)) in the 200 - $900 \mathrm{~K}$ temperature range (anharmonic B3LYP-D2/cc-pVTZ results). In each case, the difference curve between the thermodynamic and NASA functions is plotted in the upper graph

Fit parameters

\begin{tabular}{llllll}
\hline $\mathrm{a}_{1}$ & $4.98142227 \mathrm{e}-01$ & $\mathrm{a}_{2}\left[\mathrm{~K}^{-1}\right]$ & $2.50164610 \mathrm{e}-02$ & $\mathrm{a}_{3}\left[\mathrm{~K}^{-2}\right]$ & $3.57745649 \mathrm{e}-05$ \\
$\mathrm{a}_{4}\left[\mathrm{~K}^{-3}\right]$ & $-7.69789757 \mathrm{e}-08$ & $\mathrm{a}_{5}\left[\mathrm{~K}^{-4}\right]$ & $3.80221878 \mathrm{e}-11$ & $\mathrm{a}_{6}[\mathrm{~K}]$ & $2.78437904 \mathrm{e}+02$
\end{tabular}

$\mathrm{a}_{7}$
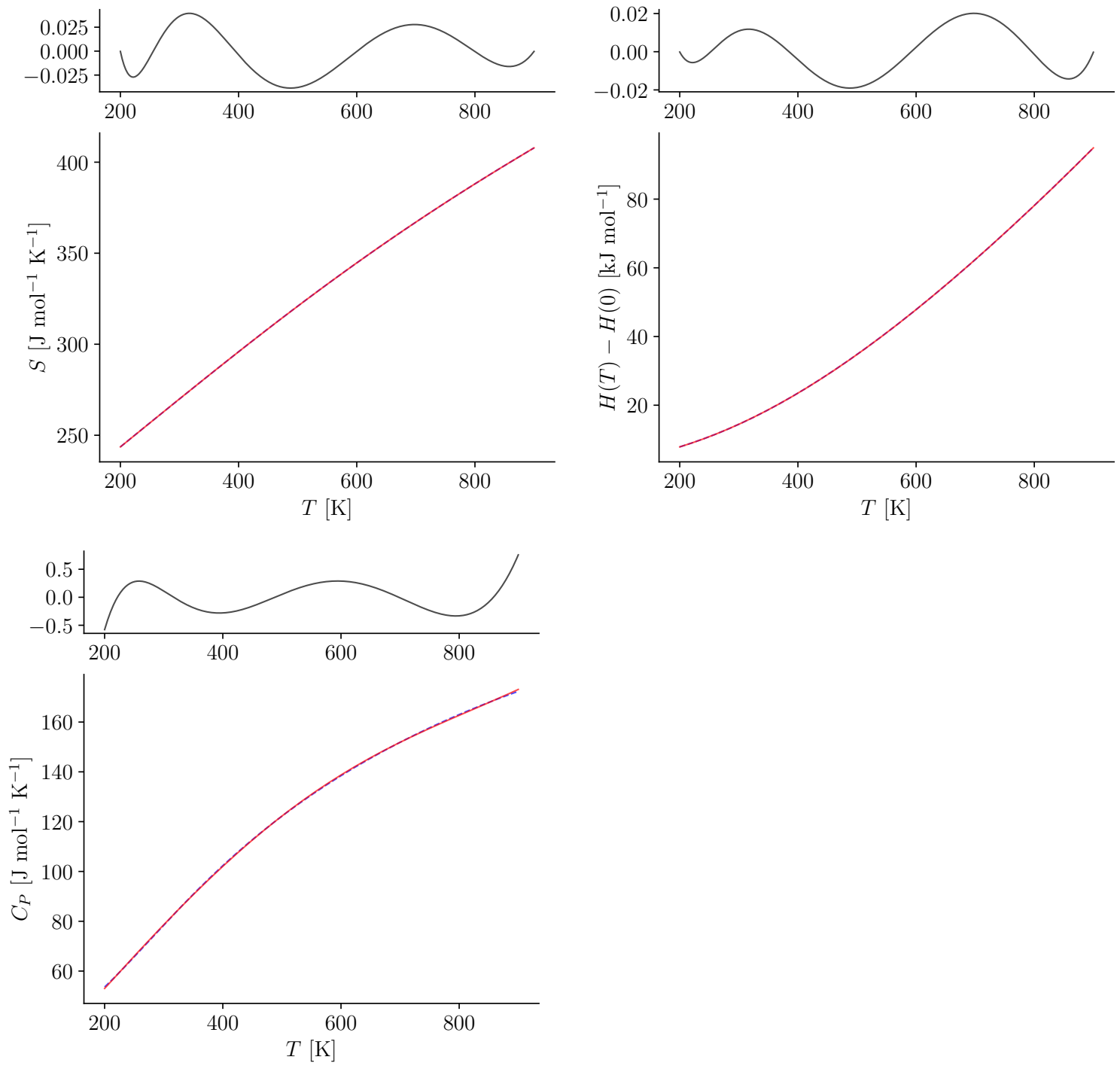


\section{$19 \mathrm{~B}_{3} \mathrm{H}_{8}$}

Figure S.19.1. Structure of $\mathrm{B}_{3} \mathrm{H}_{8}{ }^{-}$

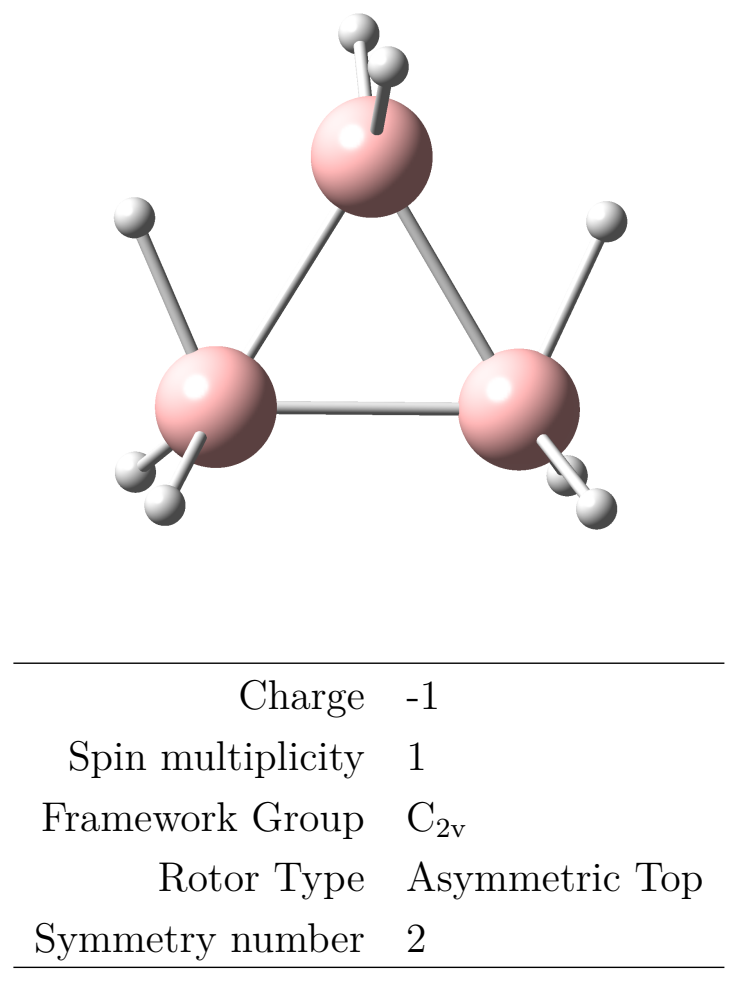

Table S.19.1. $\mathrm{B}_{3} \mathrm{H}_{8}{ }^{-}$: Selected anharmonic results obtained at the B3LYP-D2/cc-pVTZ level

\begin{tabular}{rll}
\hline $\mathrm{ZPE}_{\mathrm{v}}$ & 17868 & $\mathrm{~cm}^{-1}$ \\
$\mathrm{~A}$ & 0.49292 & $\mathrm{~cm}^{-1}$ \\
$\mathrm{~B}$ & 0.46503 & $\mathrm{~cm}^{-1}$ \\
$\mathrm{C}$ & 0.29165 & $\mathrm{~cm}^{-1}$ \\
\hline$C_{P}(300 \mathrm{~K})$ & 87.692 & $\mathrm{~J} \mathrm{~mol}^{-1} \mathrm{~K}-1$ \\
$S(300 \mathrm{~K})$ & 274.471 & $\mathrm{~J} \mathrm{~mol}^{-1} \mathrm{~K}-1$ \\
$H(300 \mathrm{~K})-H(0 \mathrm{~K})$ & 15.943 & $\mathrm{~kJ} \mathrm{~mol}^{-1}$ \\
\hline
\end{tabular}

$\mathrm{ZPE}_{\mathrm{v}}$ : vibrational contribution to the zero-point energy. A, B, C: Rotational constants. $C_{P}$ : Heat capacity at constant pressure. $S$ : Entropy. $H$ : Enthalpy. Ideal-gas calculations (1 bar). 
Table S.19.2. $\mathrm{B}_{3} \mathrm{H}_{8}{ }^{-}$: Cartesian coordinates $(\AA)$ of the B3LYP-D2/cc-pVTZ optimized structure

\begin{tabular}{cccc} 
atom & $\mathrm{x}$ & $\mathrm{y}$ & $\mathrm{z}$ \\
\hline B1 & 0.0000 & -0.0000 & 0.9962 \\
B2 & 0.0000 & 0.9224 & -0.5356 \\
B3 & -0.0000 & -0.9224 & -0.5356 \\
H4 & 1.0171 & -0.0000 & 1.6458 \\
H5 & -1.0171 & 0.0000 & 1.6458 \\
H6 & 1.0255 & 1.3162 & -1.0364 \\
H7 & -1.0255 & -1.3162 & -1.0364 \\
H8 & -1.0255 & 1.3162 & -1.0364 \\
H9 & 1.0255 & -1.3162 & -1.0364 \\
H10 & 0.0000 & 1.4377 & 0.6148 \\
H11 & -0.0000 & -1.4377 & 0.6148 \\
\hline
\end{tabular}


Table S.19.3. $\mathrm{B}_{3} \mathrm{H}_{8}{ }^{-}$: Vibrational frequencies, infrared integrated intensities and Raman activities (B3LYP-D2/cc-pVTZ results)

\begin{tabular}{|c|c|c|c|c|c|c|c|}
\hline \multirow[b]{2}{*}{ mode } & \multirow[b]{2}{*}{ symm. } & \multicolumn{3}{|c|}{ Harmonic } & \multicolumn{3}{|c|}{ Anharmonic } \\
\hline & & $\begin{array}{c}\omega \\
{\left[\mathrm{cm}^{-1}\right]}\end{array}$ & $\begin{array}{c}\mathrm{IR} \\
{\left[\mathrm{km} \mathrm{mol}^{-1}\right]}\end{array}$ & $\begin{array}{c}\text { Raman } \\
{\left[\AA^{6}\right]}\end{array}$ & $\begin{array}{c}\omega \\
{\left[\mathrm{cm}^{-1}\right]}\end{array}$ & $\begin{array}{c}\mathrm{IR} \\
{\left[\mathrm{km} \mathrm{mol}^{-1}\right]}\end{array}$ & $\begin{array}{c}\text { Raman } \\
{\left[\AA^{6}\right]}\end{array}$ \\
\hline 1 & $\mathrm{~A}_{2}$ & 206 & 0.000 & 0.070 & 178 & 0.000 & 0.078 \\
\hline 2 & $\mathrm{~B}_{1}$ & 433 & 12.741 & 0.001 & 408 & 11.081 & 0.003 \\
\hline 3 & $\mathrm{~B}_{2}$ & 487 & 157.337 & 0.000 & 382 & 150.555 & 0.000 \\
\hline 4 & $\mathrm{~A}_{1}$ & 521 & 2.363 & 0.200 & 484 & 1.658 & 0.231 \\
\hline 5 & $\mathrm{~A}_{1}$ & 720 & 1.549 & 0.198 & 655 & 2.184 & 0.098 \\
\hline 6 & $\mathrm{~B}_{1}$ & 735 & 4.737 & 0.006 & 705 & 3.992 & 0.007 \\
\hline 7 & $\mathrm{~B}_{2}$ & 803 & 16.368 & 0.189 & 746 & 9.739 & 0.187 \\
\hline 8 & $\mathrm{~A}_{1}$ & 831 & 8.745 & 0.683 & 820 & 6.025 & 0.548 \\
\hline 9 & $\mathrm{~A}_{2}$ & 837 & 0.000 & 0.001 & 793 & 0.000 & 0.001 \\
\hline 10 & $\mathrm{~B}_{1}$ & 917 & 1.276 & 0.001 & 870 & 1.325 & 0.000 \\
\hline 11 & $\mathrm{~A}_{2}$ & 1016 & 0.000 & 0.002 & 966 & 0.000 & 0.006 \\
\hline 12 & $\mathrm{~B}_{2}$ & 1067 & 93.406 & 0.000 & 1020 & 65.236 & 0.005 \\
\hline 13 & $\mathrm{~B}_{1}$ & 1093 & 0.269 & 0.064 & 1045 & 0.441 & 0.053 \\
\hline 14 & $\mathrm{~A}_{2}$ & 1185 & 0.000 & 0.306 & 1112 & 0.000 & 0.251 \\
\hline 15 & $\mathrm{~A}_{1}$ & 1195 & 69.157 & 0.033 & 1141 & 57.218 & 0.012 \\
\hline 16 & $\mathrm{~B}_{2}$ & 1201 & 41.124 & 0.039 & 1146 & 8.002 & 0.020 \\
\hline 17 & $\mathrm{~A}_{1}$ & 1217 & 16.963 & 0.103 & 1167 & 14.690 & 0.072 \\
\hline 18 & $\mathrm{~B}_{2}$ & 1297 & 44.853 & 0.041 & 1192 & 16.342 & 0.030 \\
\hline 19 & $\mathrm{~A}_{1}$ & 1411 & 2.198 & 0.011 & 1323 & 1.119 & 0.017 \\
\hline 20 & $\mathrm{~B}_{2}$ & 2185 & 31.773 & 0.327 & 2056 & 45.553 & 0.268 \\
\hline 21 & $\mathrm{~A}_{1}$ & 2228 & 177.476 & 0.901 & 2129 & 91.514 & 0.432 \\
\hline 22 & $\mathrm{~B}_{2}$ & 2468 & 192.737 & 0.323 & 2366 & 150.362 & 0.255 \\
\hline 23 & $\mathrm{~A}_{1}$ & 2480 & 391.006 & 0.394 & 2364 & 207.190 & 0.160 \\
\hline
\end{tabular}


Table S.19.3 - Continued

\begin{tabular}{cccccccc}
\hline & & \multicolumn{3}{c}{ Harmonic } & \multicolumn{3}{c}{ Anharmonic } \\
mode & symm. & $\omega$ & IR & Raman & $\omega$ & IR & Raman \\
& & {$\left[\mathrm{cm}^{-1}\right]$} & {$\left[\mathrm{km} \mathrm{mol}^{-1}\right]$} & {$\left[\AA^{6}\right]$} & {$\left[\mathrm{cm}^{-1}\right]$} & {$\left[\mathrm{km} \mathrm{mol}^{-1}\right]$} & {$\left[\AA^{6}\right]$} \\
\hline 24 & $\mathrm{~A}_{2}$ & 2483 & 0.000 & 0.608 & 2376 & 0.000 & 0.610 \\
25 & $\mathrm{~B}_{1}$ & 2493 & 87.102 & 1.284 & 2370 & 15.177 & 1.289 \\
26 & $\mathrm{~A}_{1}$ & 2505 & 24.108 & 2.538 & 2395 & 15.088 & 2.760 \\
27 & $\mathrm{~B}_{1}$ & 2519 & 622.475 & 0.047 & 2408 & 676.419 & 0.141 \\
\hline
\end{tabular}


Figure S.19.2. Anharmonic IR spectrum of $\mathrm{B}_{3} \mathrm{H}_{8}{ }^{-}$obtained by convoluting the calculated intensities with Lorentzians having a FWHM of $4 \mathrm{~cm}^{-1}$ (B3LYP-D2/cc-pVTZ results): (top) full spectrum, (middle) contributions from fundamentals and combination bands, (bottom) contributions from overtones
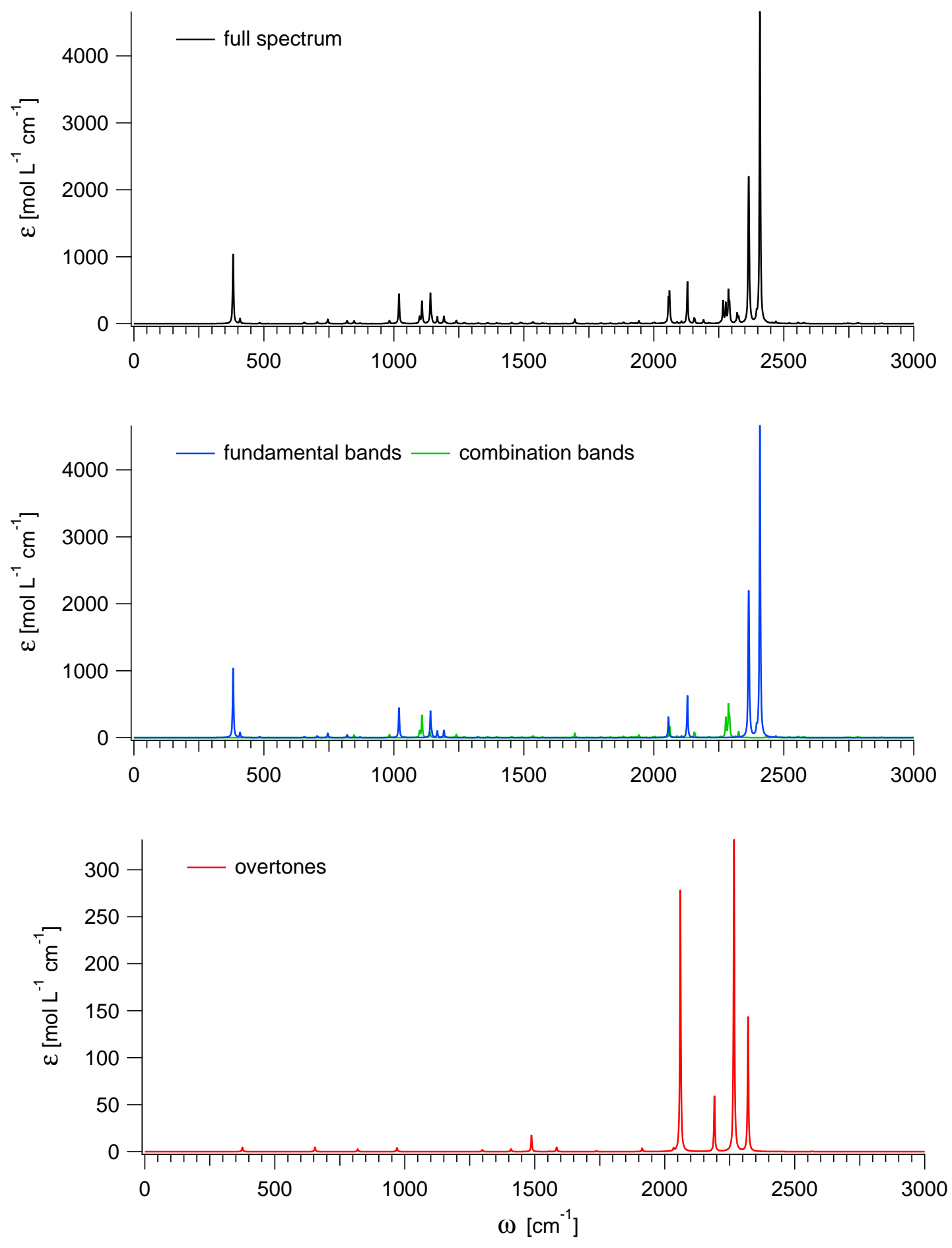

S.153 
Figure S.19.3. Anharmonic Raman spectrum of $\mathrm{B}_{3} \mathrm{H}_{8}{ }^{-}$obtained by convoluting the calculated activities with Lorentzians having a FWHM of $4 \mathrm{~cm}^{-1}$ (B3LYP-D2/cc-pVTZ results): (top) full spectrum, (middle) contributions from fundamentals and combination bands, (bottom) contributions from overtones
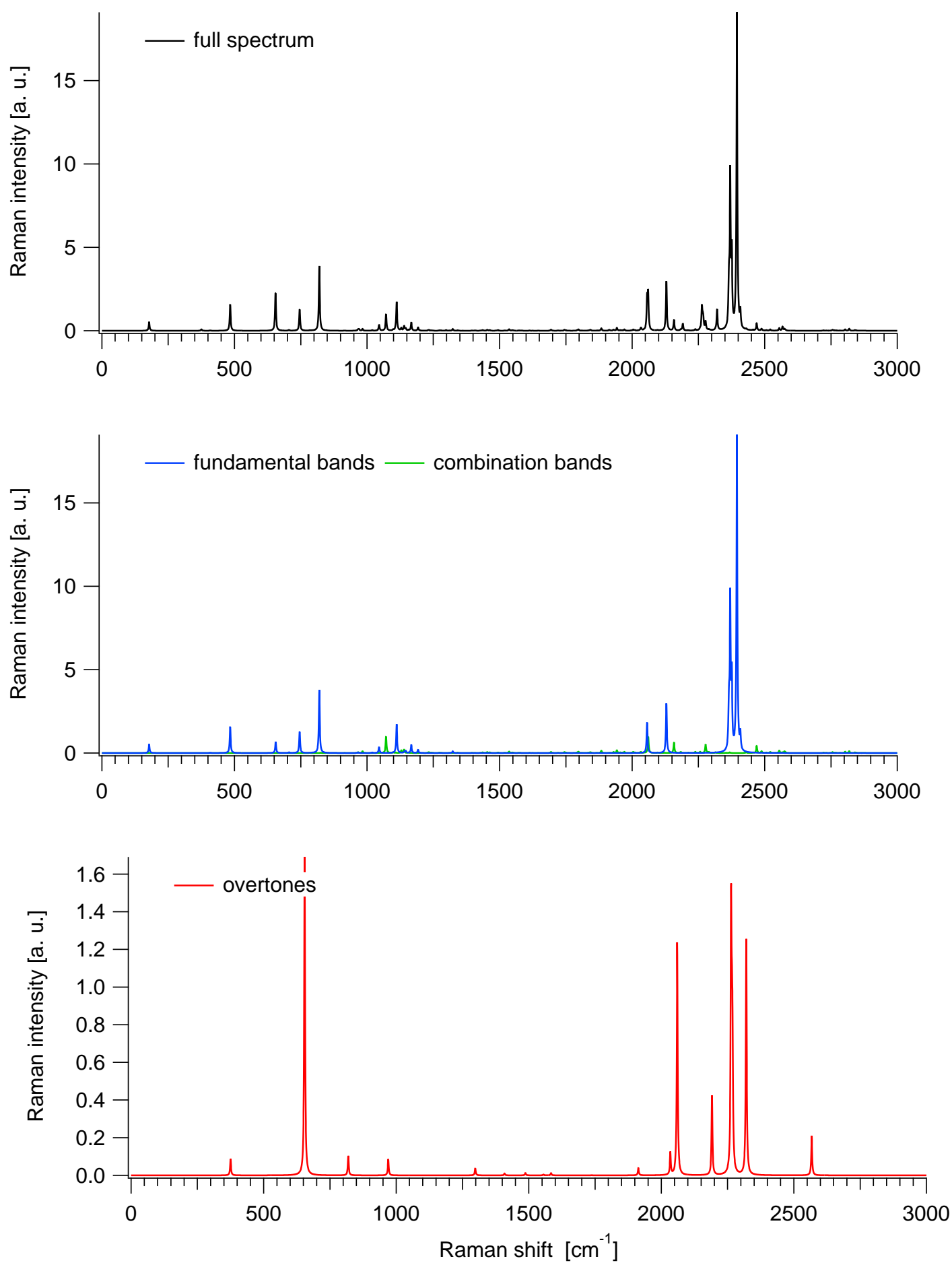

S.154 
Table S.19.4. $\mathrm{B}_{3} \mathrm{H}_{8}{ }^{-}$: Ideal-gas thermodynamic functions at 1 bar (anharmonic B3LYPD2/cc-pVTZ results)

\begin{tabular}{cccc}
\hline$T$ & $C_{P}$ & $S$ & $H(T)-H(0 K)$ \\
{$[\mathrm{K}]$} & {$\left[\mathrm{J} \mathrm{mol}^{-1} \mathrm{~K}^{-1}\right]$} & {$\left[\mathrm{J} \mathrm{mol}^{-1} \mathrm{~K}^{-1}\right]$} & {$\left[\mathrm{kJ} \mathrm{mol}^{-1}\right]$} \\
\hline 100 & 40.583 & 211.013 & 3.544 \\
200 & 60.885 & 244.784 & 8.531 \\
300 & 87.692 & 274.471 & 15.943 \\
400 & 113.195 & 303.276 & 26.018 \\
500 & 134.789 & 330.927 & 38.449 \\
600 & 152.801 & 357.144 & 52.856 \\
700 & 167.866 & 381.864 & 68.912 \\
800 & 180.470 & 405.127 & 86.347 \\
900 & 191.004 & 427.010 & 104.937 \\
1000 & 199.803 & 447.603 & 124.490 \\
1100 & 207.163 & 467.002 & 144.849 \\
1200 & 213.338 & 485.300 & 165.883 \\
1300 & 218.540 & 502.588 & 187.484 \\
1400 & 222.942 & 518.949 & 209.565 \\
1500 & 226.688 & 534.462 & 232.051 \\
1600 & 229.893 & 549.197 & 254.884 \\
1700 & 232.650 & 563.218 & 278.015 \\
1800 & 235.035 & 576.586 & 301.402 \\
1900 & 237.108 & 589.350 & 325.011 \\
2000 & 238.919 & 601.559 & 348.815 \\
\hline
\end{tabular}

$T$ : Temperature. $C_{P}$ : Heat capacity at constant pressure. $S$ : Entropy. $H$ : Enthalpy. 
Figure S.19.4. $\mathrm{B}_{3} \mathrm{H}_{8}{ }^{-}$: Ideal-gas thermodynamic functions at 1 bar (anharmonic B3LYPD2/cc-pVTZ results)
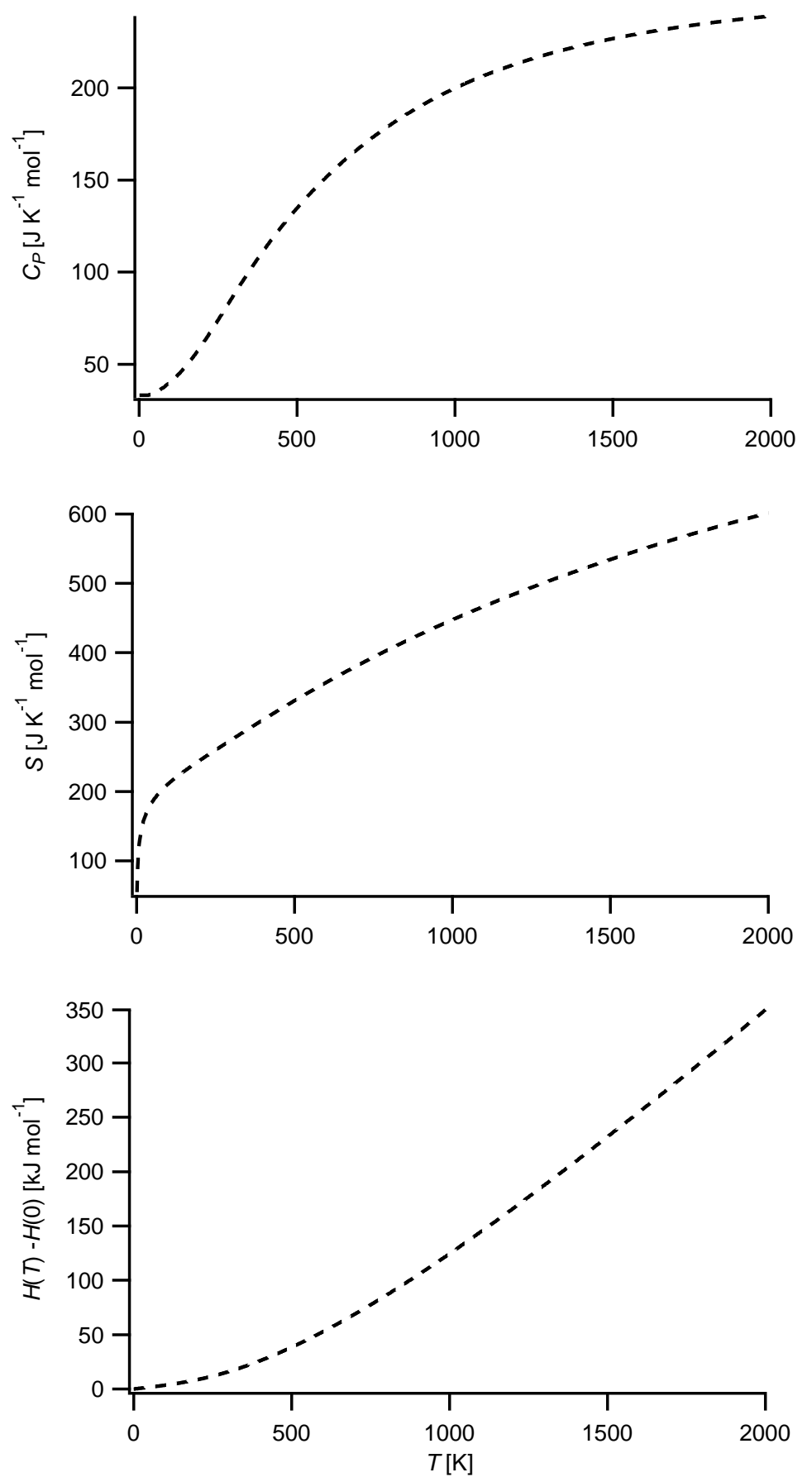

$T$ : Temperature. $C_{P}$ : Heat capacity at constant pressure. $S$ : Entropy. $H$ : Enthalpy. 
Table S.19.5. $\mathrm{B}_{3} \mathrm{H}_{8}{ }^{-}$: Fits (red solid lines) of the computed thermodynamic functions (blue dashed lines) with NASA type functions (Equations (1) - (3)) in the 200 - $900 \mathrm{~K}$ temperature range (anharmonic B3LYP-D2/cc-pVTZ results). In each case, the difference curve between the thermodynamic and NASA functions is plotted in the upper graph

Fit parameters

\begin{tabular}{llllll}
\hline $\mathrm{a}_{1}$ & $7.60427779 \mathrm{e}-01$ & $\mathrm{a}_{2}\left[\mathrm{~K}^{-1}\right]$ & $2.91917219 \mathrm{e}-02$ & $\mathrm{a}_{3}\left[\mathrm{~K}^{-2}\right]$ & $2.86907121 \mathrm{e}-05$ \\
$\mathrm{a}_{4}\left[\mathrm{~K}^{-3}\right]$ & $-6.69389972 \mathrm{e}-08$ & $\mathrm{a}_{5}\left[\mathrm{~K}^{-4}\right]$ & $3.28833637 \mathrm{e}-11$ & $\mathrm{a}_{6}[\mathrm{~K}]$ & $2.38279380 \mathrm{e}+02$ \\
$\mathrm{a}_{7}$ & $1.91649398 \mathrm{e}+01$ & & & &
\end{tabular}
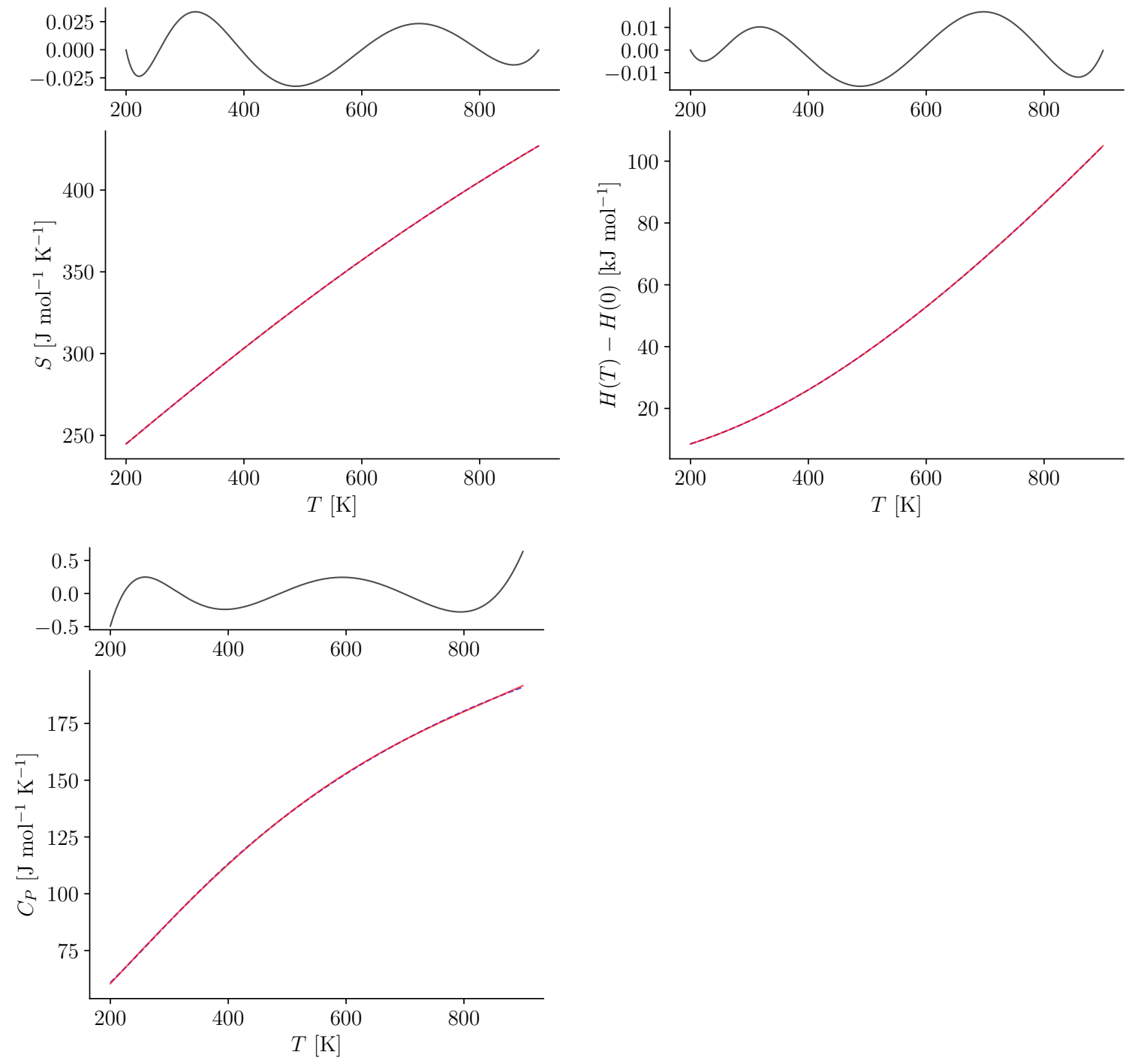


\section{$20 \mathbf{B}_{3} \mathbf{H}_{9}$}

Figure S.20.1. Structure of $\mathrm{B}_{3} \mathrm{H}_{9}$

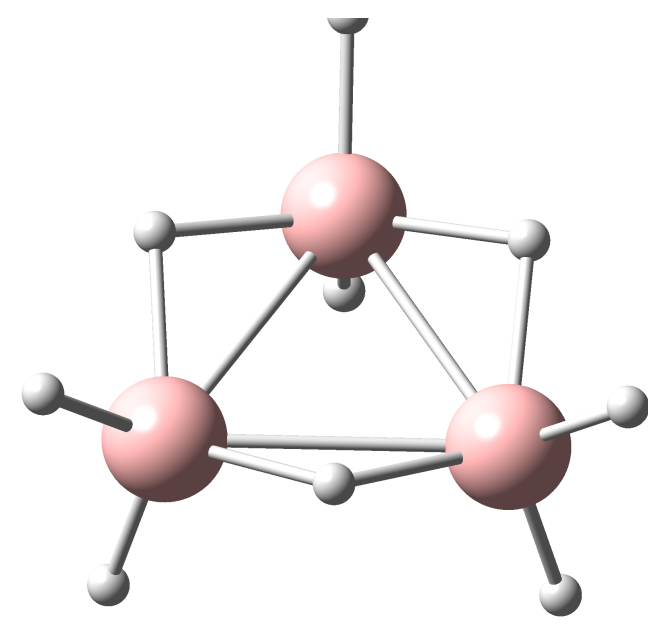

Charge 0

Spin multiplicity 1

Framework Group $\mathrm{C}_{\mathrm{s}}$

Rotor Type Asymmetric Top

Symmetry number 1

Table S.20.1. $\mathrm{B}_{3} \mathrm{H}_{9}$ : Selected anharmonic results obtained at the B3LYP-D2/cc-pVTZ level

\begin{tabular}{rll}
\hline $\mathrm{ZPE}_{\mathrm{v}}$ & 20861 & $\mathrm{~cm}^{-1}$ \\
$\mathrm{~A}$ & 0.41128 & $\mathrm{~cm}^{-1}$ \\
$\mathrm{~B}$ & 0.41128 & $\mathrm{~cm}^{-1}$ \\
$\mathrm{C}$ & 0.24561 & $\mathrm{~cm}^{-1}$ \\
\hline$C_{P}(300 \mathrm{~K})$ & 92.512 & $\mathrm{~J} \mathrm{~mol}^{-1} \mathrm{~K}-1$ \\
$S(300 \mathrm{~K})$ & 285.469 & $\mathrm{~J} \mathrm{~mol}^{-1} \mathrm{~K}-1$ \\
$H(300 \mathrm{~K})-H(0 \mathrm{~K})$ & 16.614 & $\mathrm{~kJ} \mathrm{~mol}^{-1}$ \\
\hline
\end{tabular}

$\mathrm{ZPE}_{\mathrm{v}}$ : vibrational contribution to the zero-point energy. A, B, C: Rotational constants. $C_{P}$ : Heat capacity at constant pressure. $S$ : Entropy. $H$ : Enthalpy. Ideal-gas calculations (1 bar). 
Table S.20.2. $\mathrm{B}_{3} \mathrm{H}_{9}$ : Cartesian coordinates $(\AA)$ of the B3LYP-D2/cc-pVTZ optimized structure

\begin{tabular}{cccc} 
atom & $\mathrm{x}$ & $\mathrm{y}$ & $\mathrm{z}$ \\
\hline B1 & 0.0243 & 1.1647 & -0.0000 \\
B2 & 0.0243 & -0.5824 & 1.0087 \\
B3 & 0.0243 & -0.5824 & -1.0087 \\
H4 & -0.4165 & 0.6340 & -1.0981 \\
H5 & 1.1436 & -0.7662 & 1.3267 \\
H6 & 1.1435 & 1.5322 & -0.0000 \\
H7 & -0.8484 & -0.9909 & -1.7164 \\
H8 & -0.4169 & -1.2678 & 0.0000 \\
H9 & -0.8484 & -0.9909 & 1.7164 \\
H10 & -0.8484 & 1.9818 & -0.0000 \\
H11 & 1.1436 & -0.7662 & -1.3267 \\
H12 & -0.4165 & 0.6340 & 1.0981 \\
\hline
\end{tabular}


Table S.20.3. $\mathrm{B}_{3} \mathrm{H}_{9}$ : Vibrational frequencies, infrared integrated intensities and Raman activities (B3LYP-D2/cc-pVTZ results)

\begin{tabular}{|c|c|c|c|c|c|c|c|}
\hline \multirow[b]{2}{*}{ mode } & \multirow[b]{2}{*}{ symm. } & \multicolumn{3}{|c|}{ Harmonic } & \multicolumn{3}{|c|}{ Anharmonic } \\
\hline & & $\begin{array}{c}\omega \\
{\left[\mathrm{cm}^{-1}\right]}\end{array}$ & $\begin{array}{c}\mathrm{IR} \\
{\left[\mathrm{km} \mathrm{mol}^{-1}\right]}\end{array}$ & $\begin{array}{c}\text { Raman } \\
{\left[\AA^{6}\right]}\end{array}$ & $\begin{array}{c}\omega \\
{\left[\mathrm{cm}^{-1}\right]}\end{array}$ & $\begin{array}{c}\mathrm{IR} \\
{\left[\mathrm{km} \mathrm{mol}^{-1}\right]}\end{array}$ & $\begin{array}{c}\text { Raman } \\
{\left[\AA^{6}\right]}\end{array}$ \\
\hline 1 & A" & 318 & 2.938 & 0.021 & 253 & 2.837 & 0.021 \\
\hline 2 & $A^{\prime}$ & 319 & 2.944 & 0.021 & 249 & 2.728 & 0.022 \\
\hline 3 & $\mathrm{~A}^{\prime}$ & 426 & 10.925 & 0.136 & 370 & 14.445 & 0.113 \\
\hline 4 & $A^{\prime}$ & 536 & 2.884 & 0.041 & 515 & 0.561 & 0.036 \\
\hline 5 & A" & 589 & 0.112 & 0.196 & 552 & 0.205 & 0.228 \\
\hline 6 & $\mathrm{~A}^{\prime}$ & 590 & 0.113 & 0.196 & 552 & 0.207 & 0.227 \\
\hline 7 & A" & 798 & 0.081 & 0.010 & 768 & 0.008 & 0.011 \\
\hline 8 & $A^{\prime}$ & 798 & 0.081 & 0.010 & 767 & 0.005 & 0.011 \\
\hline 9 & A" & 866 & 0.000 & 0.000 & 845 & 0.001 & 0.000 \\
\hline 10 & A" & 892 & 16.680 & 0.071 & 865 & 11.659 & 0.071 \\
\hline 11 & $\mathrm{~A}^{\prime}$ & 892 & 16.678 & 0.071 & 866 & 11.822 & 0.071 \\
\hline 12 & $A "$ & 984 & 0.000 & 0.000 & 932 & 0.006 & 0.000 \\
\hline 13 & $A^{\prime}$ & 1071 & 0.772 & 0.126 & 984 & 0.198 & 0.101 \\
\hline 14 & A" & 1121 & 42.968 & 0.050 & 1063 & 15.830 & 0.044 \\
\hline 15 & $A^{\prime}$ & 1121 & 43.202 & 0.050 & 1060 & 20.029 & 0.055 \\
\hline 16 & $A^{\prime \prime}$ & 1187 & 56.870 & 0.108 & 1129 & 42.850 & 0.096 \\
\hline 17 & $A^{\prime}$ & 1187 & 56.550 & 0.108 & 1128 & 46.885 & 0.120 \\
\hline 18 & $A^{\prime}$ & 1190 & 0.641 & 0.080 & 1149 & 15.081 & 0.085 \\
\hline 19 & $A^{\prime}$ & 1842 & 15.487 & 1.283 & 1716 & 9.515 & 1.136 \\
\hline 20 & $A^{\prime \prime}$ & 1930 & 2.620 & 0.100 & 1847 & 0.012 & 0.010 \\
\hline 21 & $A^{\prime}$ & 1931 & 2.581 & 0.099 & 1729 & 7.429 & 0.122 \\
\hline 22 & $A^{\prime \prime}$ & 1978 & 42.144 & 0.126 & 1732 & 7.459 & 0.112 \\
\hline 23 & $A^{\prime}$ & 1979 & 42.275 & 0.127 & 1817 & 0.635 & 0.008 \\
\hline
\end{tabular}


Table S.20.3 - Continued

\begin{tabular}{cccccccc}
\hline & & \multicolumn{3}{c}{ Harmonic } & \multicolumn{3}{c}{ Anharmonic } \\
mode & symm. & $\omega$ & IR & Raman & $\omega$ & IR & Raman \\
& & {$\left[\mathrm{cm}^{-1}\right]$} & {$\left[\mathrm{km} \mathrm{mol}^{-1}\right]$} & {$\left[\AA^{6}\right]$} & {$\left[\mathrm{cm}^{-1}\right]$} & {$\left[\mathrm{km} \mathrm{mol}^{-1}\right]$} & {$\left[\AA^{6}\right]$} \\
\hline 24 & A $^{\prime \prime}$ & 2149 & 0.001 & 0.000 & 1934 & 0.047 & 0.000 \\
25 & A $^{\prime \prime}$ & 2573 & 57.689 & 0.581 & 2460 & 54.752 & 0.596 \\
26 & A' $^{\prime}$ & 2573 & 57.684 & 0.580 & 2441 & 46.903 & 1.349 \\
27 & A' $^{\prime}$ & 2579 & 37.690 & 2.256 & 2481 & 44.714 & 1.596 \\
29 & A' $^{\prime}$ & 2738 & 21.051 & 0.301 & 2590 & 46.486 & 0.427 \\
30 & A $^{\prime \prime}$ & 2738 & 21.037 & 0.301 & 2604 & 15.504 & 0.331 \\
\hline
\end{tabular}


Figure S.20.2. Anharmonic IR spectrum of $\mathrm{B}_{3} \mathrm{H}_{9}$ obtained by convoluting the calculated intensities with Lorentzians having a FWHM of $4 \mathrm{~cm}^{-1}$ (B3LYP-D2/cc-pVTZ results): (top) full spectrum, (middle) contributions from fundamentals and combination bands, (bottom) contributions from overtones
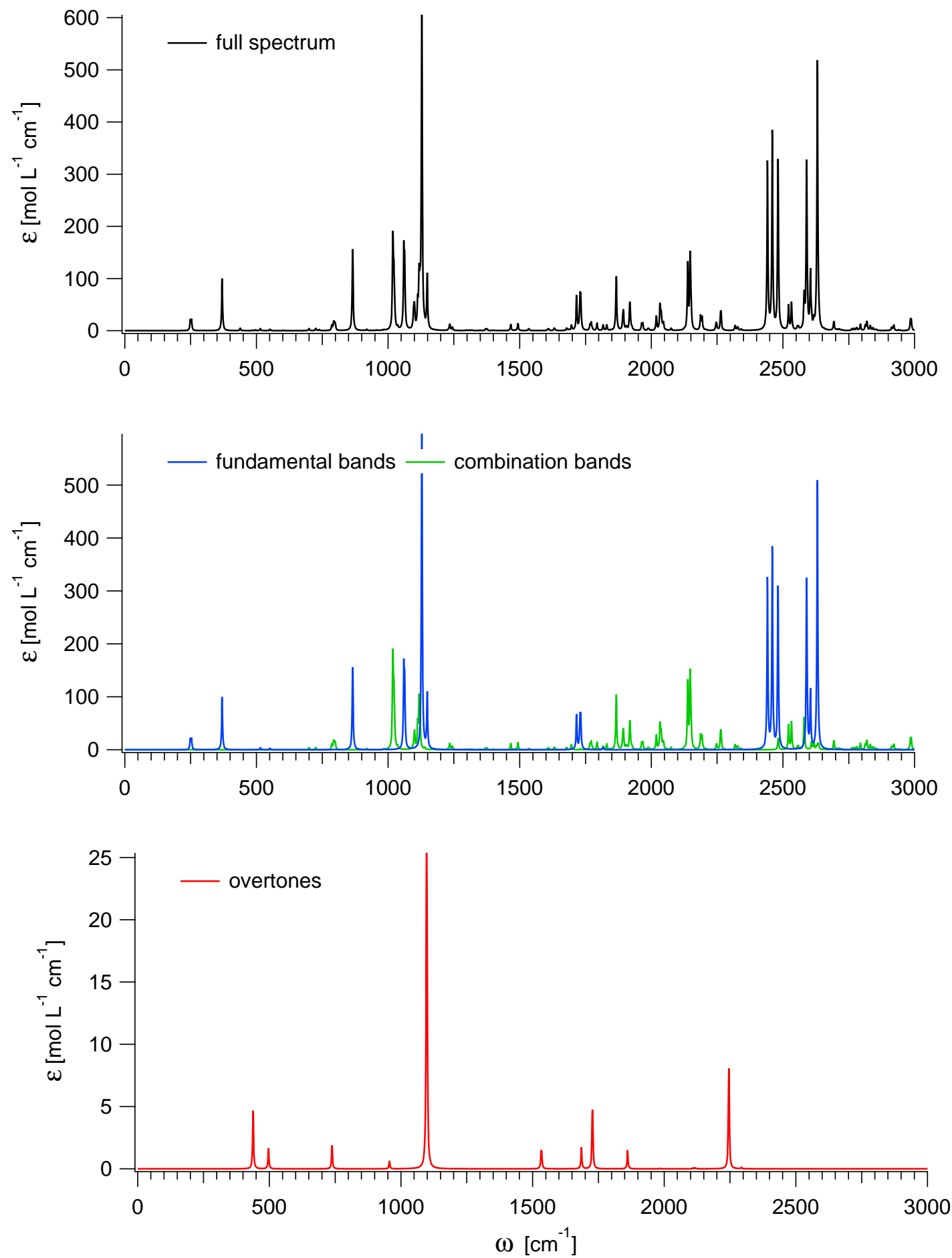
Figure S.20.3. Anharmonic Raman spectrum of $\mathrm{B}_{3} \mathrm{H}_{9}$ obtained by convoluting the calculated activities with Lorentzians having a FWHM of $4 \mathrm{~cm}^{-1}$ (B3LYP-D2/cc-pVTZ results): (top) full spectrum, (middle) contributions from fundamentals and combination bands, (bottom) contributions from overtones
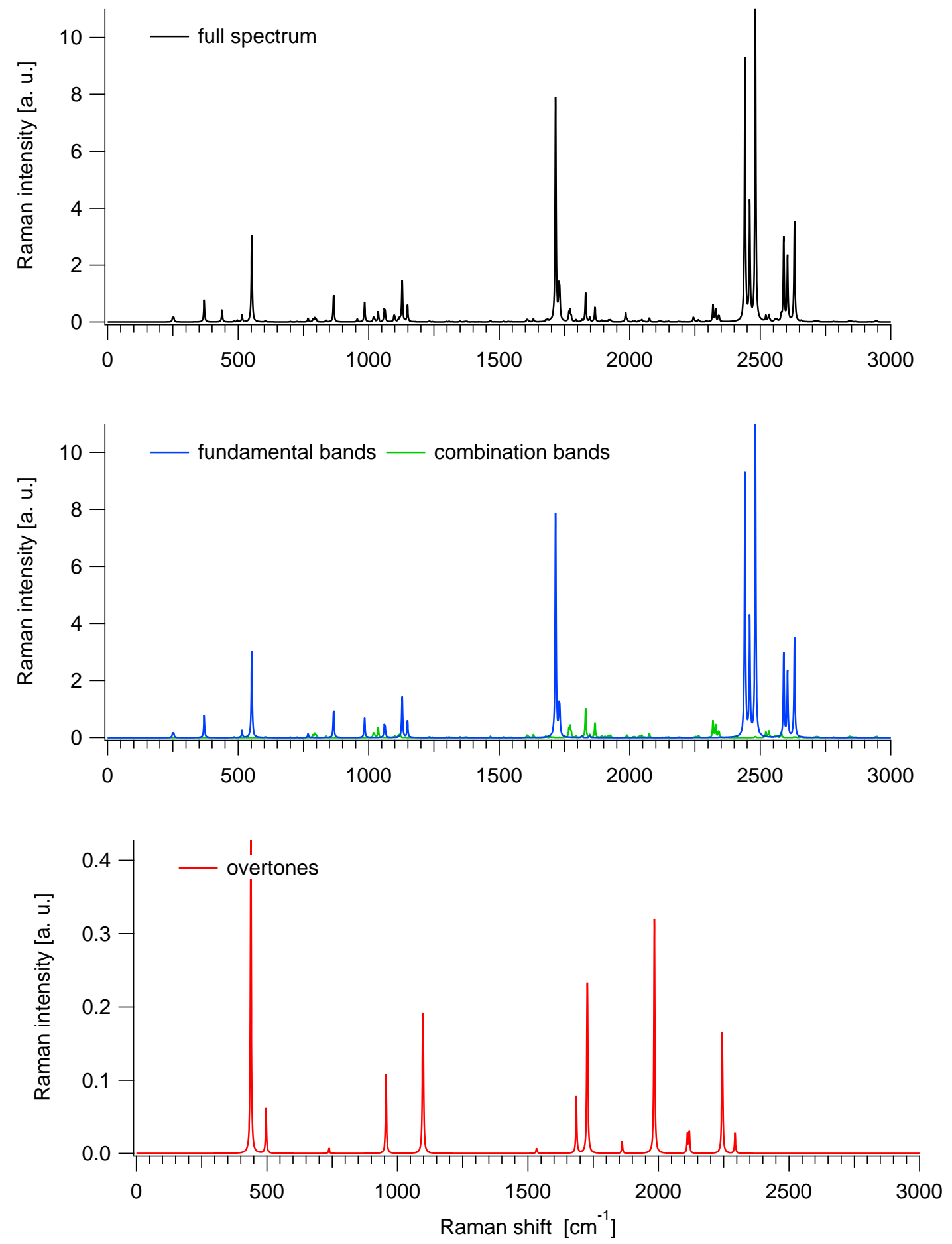

S.163 
Table S.20.4. $\mathrm{B}_{3} \mathrm{H}_{9}$ : Ideal-gas thermodynamic functions at 1 bar (anharmonic B3LYPD2/cc-pVTZ results)

\begin{tabular}{cccc}
\hline$T$ & $C_{P}$ & $S$ & $H(T)-H(0 K)$ \\
{$[\mathrm{K}]$} & {$\left[\mathrm{J} \mathrm{mol}^{-1} \mathrm{~K}^{-1}\right]$} & {$\left[\mathrm{J} \mathrm{mol}^{-1} \mathrm{~K}^{-1}\right]$} & {$\left[\mathrm{kJ} \mathrm{mol}^{-1}\right]$} \\
\hline 100 & 41.319 & 218.628 & 3.523 \\
200 & 64.678 & 254.012 & 8.761 \\
300 & 92.512 & 285.469 & 16.614 \\
400 & 118.754 & 315.753 & 27.204 \\
500 & 141.589 & 344.769 & 40.249 \\
600 & 161.200 & 372.363 & 55.414 \\
700 & 177.954 & 398.505 & 72.394 \\
800 & 192.176 & 423.222 & 90.920 \\
900 & 204.187 & 446.571 & 110.756 \\
1000 & 214.302 & 468.623 & 131.695 \\
1100 & 222.817 & 489.459 & 153.563 \\
1200 & 229.999 & 509.163 & 176.214 \\
1300 & 236.075 & 527.820 & 199.526 \\
1400 & 241.237 & 545.509 & 223.398 \\
1500 & 245.643 & 562.307 & 247.748 \\
1600 & 249.423 & 578.284 & 272.506 \\
1700 & 252.683 & 593.505 & 297.615 \\
1800 & 255.507 & 608.030 & 323.028 \\
1900 & 257.968 & 621.912 & 348.704 \\
2000 & 260.121 & 635.200 & 374.611 \\
\hline & & & $S:$ \\
\hline
\end{tabular}

$T$ : Temperature. $C_{P}$ : Heat capacity at constant pressure. $S$ : Entropy. $H$ : Enthalpy. 
Figure S.20.4. $\mathrm{B}_{3} \mathrm{H}_{9}$ : Ideal-gas thermodynamic functions at 1 bar (anharmonic B3LYPD2/cc-pVTZ results)
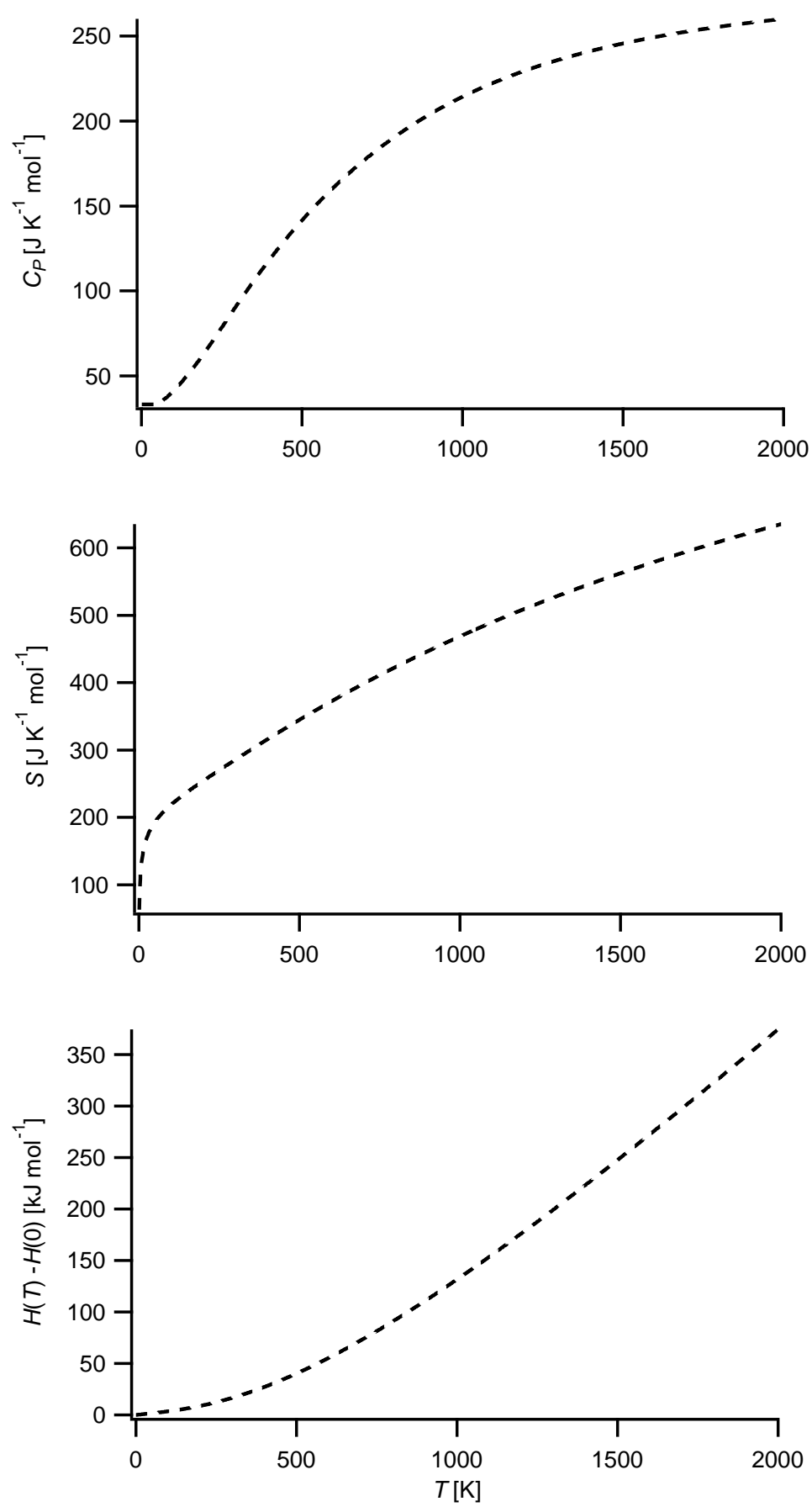

$T$ : Temperature. $C_{P}$ : Heat capacity at constant pressure. $S$ : Entropy. $H$ : Enthalpy. 
Table S.20.5. $\mathrm{B}_{3} \mathrm{H}_{9}$ : Fits (red solid lines) of the computed thermodynamic functions (blue dashed lines) with NASA type functions (Equations (1) - (3)) in the 200 - $900 \mathrm{~K}$ temperature range (anharmonic B3LYP-D2/cc-pVTZ results). In each case, the difference curve between the thermodynamic and NASA functions is plotted in the upper graph

Fit parameters

\begin{tabular}{llllll}
\hline $\mathrm{a}_{1}$ & $7.83801266 \mathrm{e}-01$ & $\mathrm{a}_{2}\left[\mathrm{~K}^{-1}\right]$ & $3.31150340 \mathrm{e}-02$ & $\mathrm{a}_{3}\left[\mathrm{~K}^{-2}\right]$ & $1.68570110 \mathrm{e}-05$ \\
$\mathrm{a}_{4}\left[\mathrm{~K}^{-3}\right]$ & $-4.77272724 \mathrm{e}-08$ & $\mathrm{a}_{5}\left[\mathrm{~K}^{-4}\right]$ & $2.31053521 \mathrm{e}-11$ & $\mathrm{a}_{6}[\mathrm{~K}]$ & $2.07340315 \mathrm{e}+02$
\end{tabular}

$\mathrm{a}_{7}$
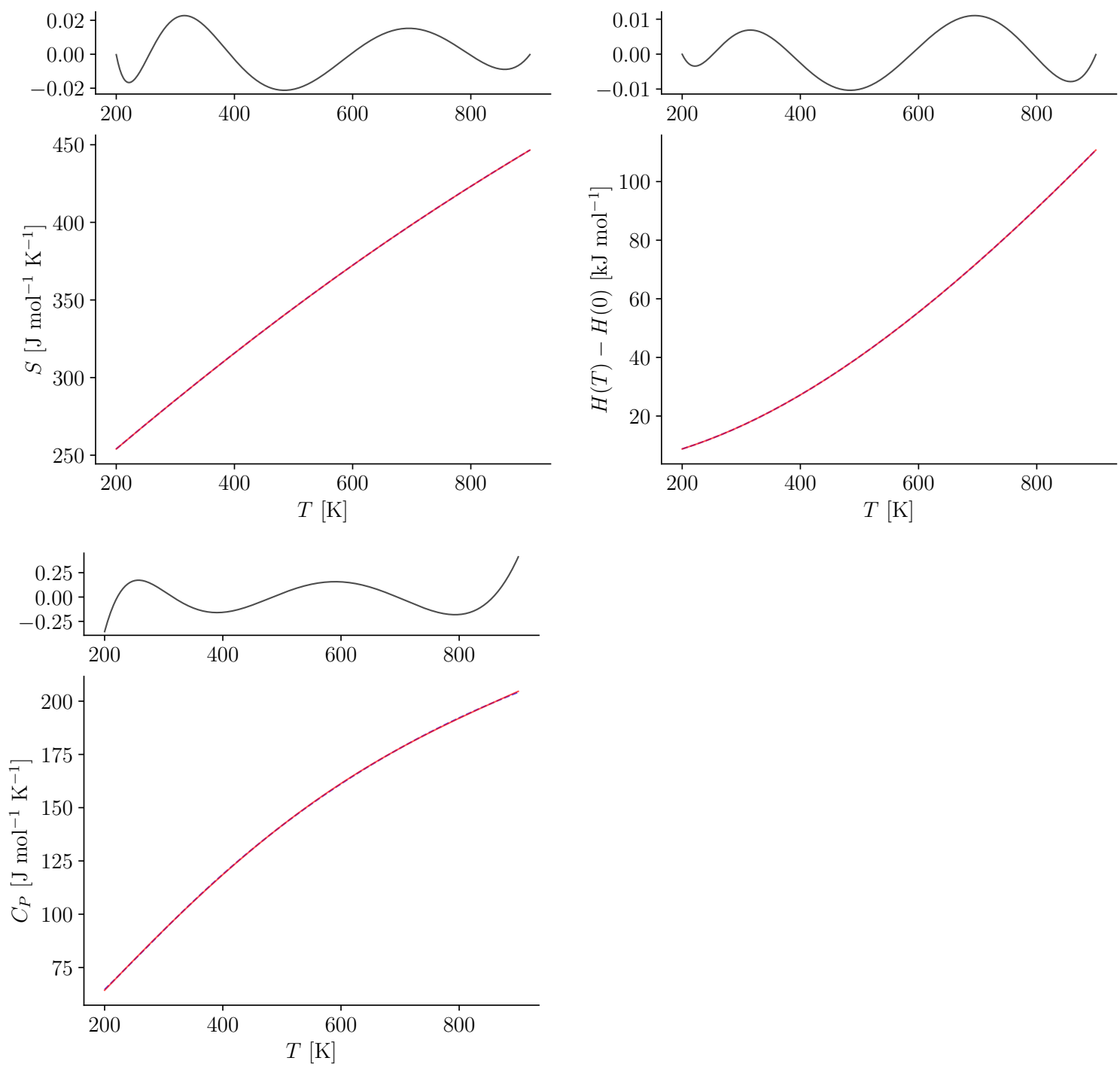


\section{$21 \quad \mathbf{B}_{3} \mathbf{H}_{10}$}

Figure S.21.1. Structure of $\mathrm{B}_{3} \mathrm{H}_{10}$

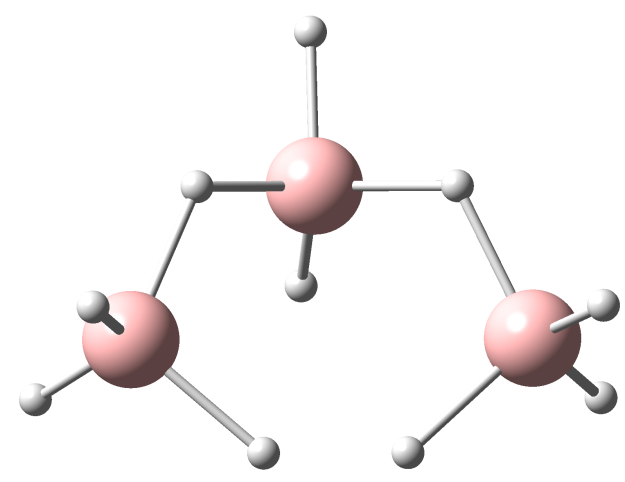

\begin{aligned} & \hline Charge 0 \\ & Spin multiplicity 2 \\ & Framework Group $\mathrm{C}_{\mathrm{s}} \\ &$ Rotor Type Asymmetric Top \\ & Symmetry number 1 \\ & \hline\end{aligned}

Table S.21.1. $\mathrm{B}_{3} \mathrm{H}_{10}$ : Selected anharmonic results obtained at the B3LYP-D2/cc-pVTZ level

\begin{tabular}{rll}
\hline $\mathrm{ZPE}_{\mathrm{v}}$ & 20919 & $\mathrm{~cm}^{-1}$ \\
$\mathrm{~A}$ & 0.45007 & $\mathrm{~cm}^{-1}$ \\
$\mathrm{~B}$ & 0.21487 & $\mathrm{~cm}^{-1}$ \\
$\mathrm{C}$ & 0.16427 & $\mathrm{~cm}^{-1}$ \\
\hline$C_{P}(300 \mathrm{~K})$ & 116.813 & $\mathrm{~J} \mathrm{~mol}^{-1} \mathrm{~K}-1$ \\
$S(300 \mathrm{~K})$ & 314.564 & $\mathrm{~J} \mathrm{~mol}^{-1} \mathrm{~K}-1$ \\
$H(300 \mathrm{~K})-H(0 \mathrm{~K})$ & 20.770 & $\mathrm{~kJ} \mathrm{~mol}^{-1}$ \\
\hline
\end{tabular}

$\mathrm{ZPE}_{\mathrm{v}}$ : vibrational contribution to the zero-point energy. A, B, C: Rotational constants. $C_{P}$ : Heat capacity at constant pressure. $S$ : Entropy. $H$ : Enthalpy. Ideal-gas calculations (1 bar). 
Table S.21.2. $\mathrm{B}_{3} \mathrm{H}_{10}$ : Cartesian coordinates $(\AA)$ of the B3LYP-D2/cc-pVTZ optimized structure

\begin{tabular}{cccc} 
atom & $\mathrm{x}$ & $\mathrm{y}$ & $\mathrm{z}$ \\
\hline B1 & -0.0067 & 1.1099 & 0.0000 \\
B2 & -0.0067 & -0.4847 & 1.5376 \\
B3 & -0.0067 & -0.4847 & -1.5376 \\
H4 & -0.9718 & -1.0489 & -1.9534 \\
H5 & 0.9585 & -0.2037 & 2.1647 \\
H6 & 0.4126 & -1.2802 & -0.5527 \\
H7 & 0.4126 & -1.2802 & 0.5527 \\
H8 & 1.1680 & 1.1687 & 0.0000 \\
H9 & -0.6409 & 2.1194 & 0.0000 \\
H10 & -0.6127 & 0.5380 & -0.9966 \\
H11 & -0.6127 & 0.5380 & 0.9966 \\
H12 & -0.9718 & -1.0489 & 1.9534 \\
H13 & 0.9585 & -0.2037 & -2.1647 \\
\hline
\end{tabular}


Table S.21.3. $\mathrm{B}_{3} \mathrm{H}_{10}$ : Vibrational frequencies, infrared integrated intensities and Raman activities (B3LYP-D2/cc-pVTZ results)

\begin{tabular}{|c|c|c|c|c|c|c|c|}
\hline \multirow[b]{2}{*}{ mode } & \multirow[b]{2}{*}{ symm. } & \multicolumn{3}{|c|}{ Harmonic } & \multicolumn{3}{|c|}{ Anharmonic } \\
\hline & & $\begin{array}{c}\omega \\
{\left[\mathrm{cm}^{-1}\right]}\end{array}$ & $\begin{array}{c}\mathrm{IR} \\
{\left[\mathrm{km} \mathrm{mol}^{-1}\right]}\end{array}$ & $\begin{array}{c}\text { Raman } \\
{\left[\AA^{6}\right]}\end{array}$ & $\begin{array}{c}\omega \\
{\left[\mathrm{cm}^{-1}\right]}\end{array}$ & $\begin{array}{c}\mathrm{IR} \\
{\left[\mathrm{km} \mathrm{mol}^{-1}\right]}\end{array}$ & $\begin{array}{c}\text { Raman } \\
{\left[\AA^{6}\right]}\end{array}$ \\
\hline 1 & $\mathrm{~A}^{\prime}$ & 184 & 4.381 & 0.057 & 144 & 4.009 & 0.072 \\
\hline 2 & A" & 204 & 0.434 & 0.102 & 204 & 0.068 & 0.037 \\
\hline 3 & A" & 302 & 0.001 & 0.437 & 281 & 0.056 & 0.521 \\
\hline 4 & $A^{\prime}$ & 325 & 2.700 & 0.155 & 251 & 2.404 & 0.123 \\
\hline 5 & A" & 345 & 0.042 & 0.376 & 326 & 0.000 & 0.550 \\
\hline 6 & $\mathrm{~A}^{\prime}$ & 406 & 0.108 & 0.363 & 380 & 0.298 & 0.371 \\
\hline 7 & A" & 595 & 2.956 & 0.030 & 588 & 0.136 & 0.022 \\
\hline 8 & $A^{\prime}$ & 610 & 0.205 & 0.093 & 594 & 0.001 & 0.107 \\
\hline 9 & A" & 745 & 0.383 & 1.033 & 659 & 0.007 & 0.679 \\
\hline 10 & $\mathrm{~A}^{\prime}$ & 753 & 3.985 & 0.080 & 704 & 0.253 & 0.363 \\
\hline 11 & A" & 774 & 0.548 & 0.029 & 714 & 3.005 & 0.050 \\
\hline 12 & $\mathrm{~A}^{\prime}$ & 804 & 6.864 & 0.121 & 720 & 9.288 & 0.112 \\
\hline 13 & A" & 855 & 17.690 & 0.049 & 805 & 46.873 & 0.038 \\
\hline 14 & $A^{\prime}$ & 868 & 35.596 & 0.452 & 803 & 20.897 & 1.100 \\
\hline 15 & A" & 946 & 44.040 & 0.102 & 905 & 9.613 & 0.061 \\
\hline 16 & $\mathrm{~A}^{\prime}$ & 1042 & 21.532 & 0.470 & 986 & 8.447 & 0.268 \\
\hline 17 & $A "$ & 1081 & 306.145 & 0.024 & 1039 & 106.980 & 0.006 \\
\hline 18 & $A^{\prime \prime}$ & 1116 & 27.697 & 0.066 & 1080 & 100.786 & 0.078 \\
\hline 19 & $A^{\prime}$ & 1126 & 29.075 & 0.028 & 1087 & 19.454 & 0.097 \\
\hline 20 & $A^{\prime}$ & 1179 & 11.769 & 0.126 & 1122 & 2.500 & 0.655 \\
\hline 21 & $\mathrm{~A}^{\prime}$ & 1186 & 47.637 & 2.823 & 969 & 40.273 & 0.993 \\
\hline 22 & $\mathrm{~A}^{\prime}$ & 1672 & 0.357 & 1.287 & 1490 & 1.121 & 1.089 \\
\hline 23 & A" & 1686 & 12.837 & 0.077 & 1540 & 1.275 & 0.032 \\
\hline
\end{tabular}


Table S.21.3 - Continued

\begin{tabular}{|c|c|c|c|c|c|c|c|}
\hline \multirow[b]{2}{*}{ mode } & \multirow[b]{2}{*}{ symm. } & \multicolumn{3}{|c|}{ Harmonic } & \multicolumn{3}{|c|}{ Anharmonic } \\
\hline & & $\begin{array}{c}\omega \\
{\left[\mathrm{cm}^{-1}\right]}\end{array}$ & $\begin{array}{c}\mathrm{IR} \\
{\left[\mathrm{km} \mathrm{mol}^{-1}\right]}\end{array}$ & $\begin{array}{c}\text { Raman } \\
{\left[\AA^{6}\right]}\end{array}$ & $\begin{array}{c}\omega \\
{\left[\mathrm{cm}^{-1}\right]}\end{array}$ & $\begin{array}{c}\mathrm{IR} \\
{\left[\mathrm{km} \mathrm{mol}^{-1}\right]}\end{array}$ & $\begin{array}{c}\text { Raman } \\
{\left[\AA^{6}\right]} \\
\end{array}$ \\
\hline 24 & $A^{\prime \prime}$ & 1779 & 135.934 & 0.047 & 1728 & 33.068 & 0.019 \\
\hline 25 & $\mathrm{~A}^{\prime}$ & 1833 & 1.287 & 3.493 & 1523 & 0.524 & 0.792 \\
\hline 26 & $A^{\prime \prime}$ & 2179 & 271.594 & 0.035 & 1971 & 156.049 & 0.036 \\
\hline 27 & $A^{\prime}$ & 2237 & 249.489 & 0.120 & 1994 & 107.909 & 0.141 \\
\hline 28 & $A^{\prime \prime}$ & 2585 & 35.130 & 0.192 & 2474 & 14.244 & 0.071 \\
\hline 29 & $A^{\prime}$ & 2588 & 38.451 & 2.492 & 2478 & 17.136 & 3.618 \\
\hline 30 & $A^{\prime}$ & 2605 & 39.851 & 1.434 & 2504 & 41.586 & 1.208 \\
\hline 31 & $A^{\prime}$ & 2704 & 62.516 & 0.302 & 2574 & 74.186 & 0.277 \\
\hline 32 & $A^{\prime \prime}$ & 2704 & 10.501 & 1.279 & 2574 & 0.935 & 1.176 \\
\hline 33 & $A^{\prime}$ & 2757 & 46.489 & 0.326 & 2629 & 39.818 & 0.276 \\
\hline
\end{tabular}


Figure S.21.2. Anharmonic IR spectrum of $\mathrm{B}_{3} \mathrm{H}_{10}$ obtained by convoluting the calculated intensities with Lorentzians having a FWHM of $4 \mathrm{~cm}^{-1}$ (B3LYP-D2/cc-pVTZ results): (top) full spectrum, (middle) contributions from fundamentals and combination bands, (bottom) contributions from overtones
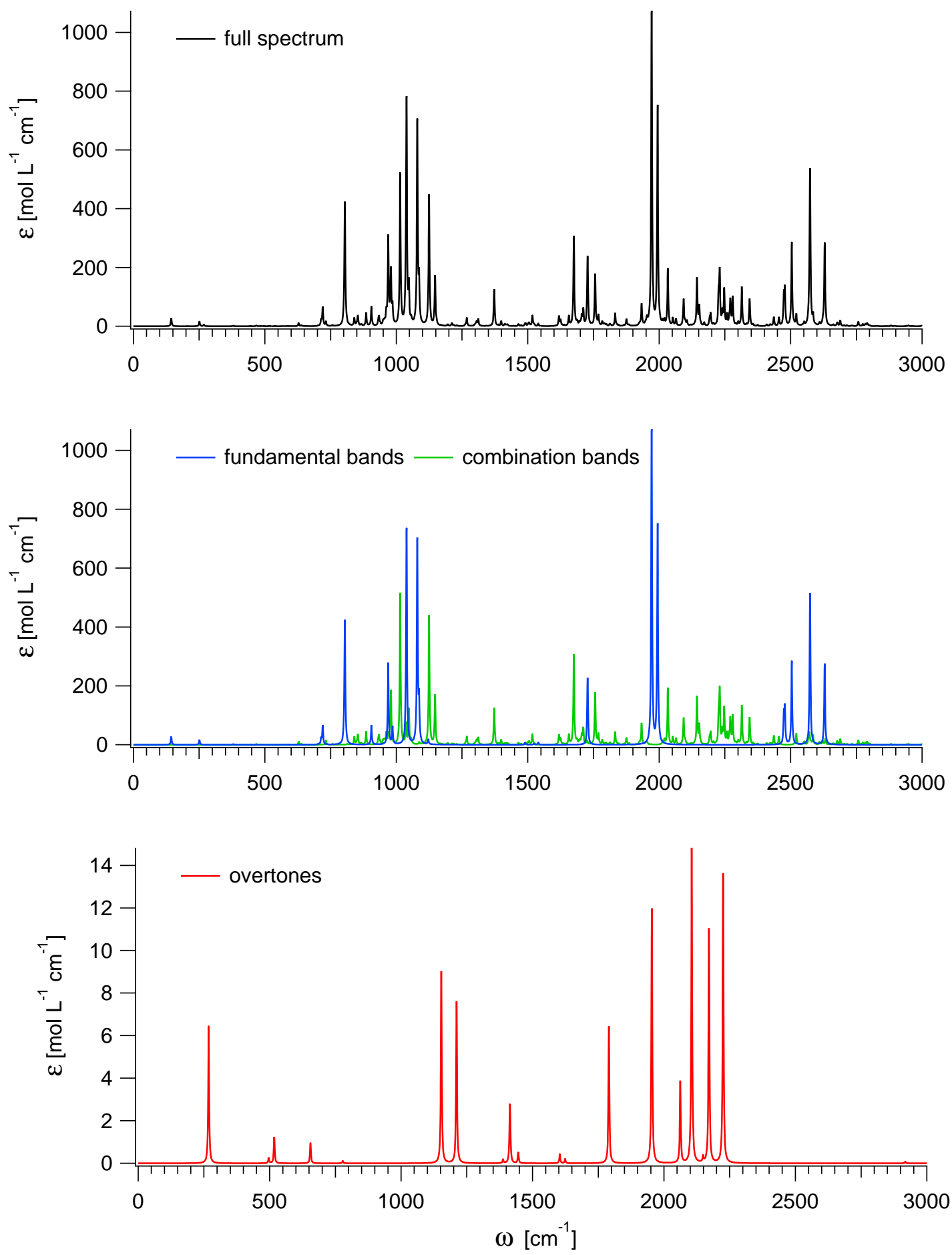
Figure S.21.3. Anharmonic Raman spectrum of $\mathrm{B}_{3} \mathrm{H}_{10}$ obtained by convoluting the calculated activities with Lorentzians having a FWHM of $4 \mathrm{~cm}^{-1}$ (B3LYP-D2/cc-pVTZ results): (top) full spectrum, (middle) contributions from fundamentals and combination bands, (bottom) contributions from overtones
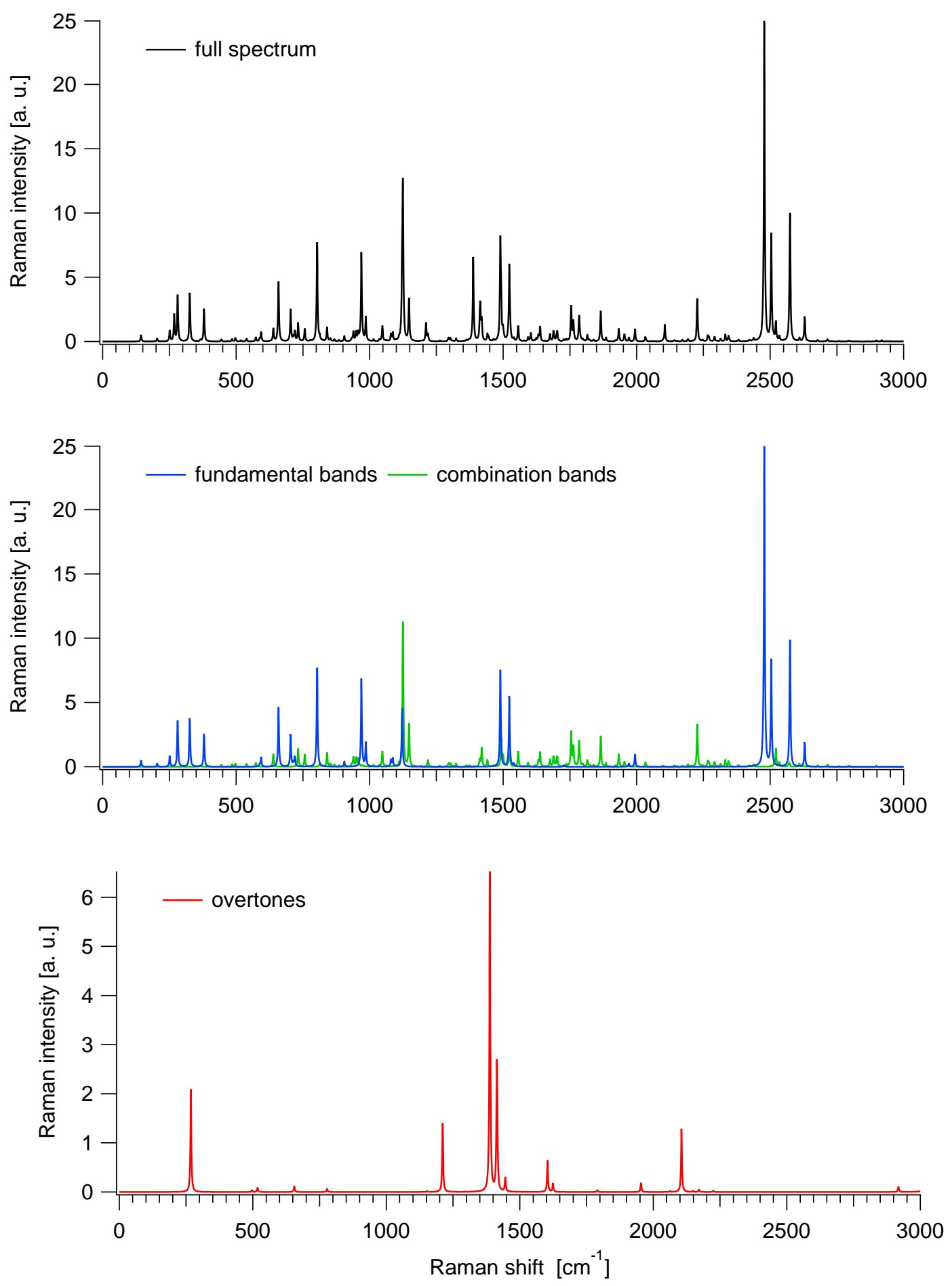
Table S.21.4. $\mathrm{B}_{3} \mathrm{H}_{10}$ : Ideal-gas thermodynamic functions at 1 bar (anharmonic B3LYP$\mathrm{D} 2 / \mathrm{cc}-\mathrm{pVTZ}$ results)

\begin{tabular}{|c|c|c|c|}
\hline $\begin{array}{c}T \\
{[\mathrm{~K}]}\end{array}$ & $\begin{array}{c}C_{P} \\
{\left[\mathrm{~J} \mathrm{~mol}^{-1} \mathrm{~K}^{-1}\right]}\end{array}$ & $\begin{array}{c}S \\
{\left[\mathrm{~J} \mathrm{~mol}^{-1} \mathrm{~K}^{-1}\right]}\end{array}$ & $\begin{array}{c}H(T)-H(0 K) \\
{\left[\mathrm{kJ} \mathrm{mol}^{-1}\right]}\end{array}$ \\
\hline 100 & 52.135 & 228.382 & 3.914 \\
\hline 200 & 84.074 & 274.217 & 10.709 \\
\hline 300 & 116.813 & 314.564 & 20.770 \\
\hline 400 & 145.530 & 352.224 & 33.930 \\
\hline 500 & 169.373 & 387.343 & 49.712 \\
\hline 600 & 189.222 & 420.031 & 67.672 \\
\hline 700 & 205.858 & 450.487 & 87.450 \\
\hline 800 & 219.825 & 478.914 & 108.754 \\
\hline 900 & 231.546 & 505.503 & 131.340 \\
\hline 1000 & 241.382 & 530.423 & 155.001 \\
\hline 1100 & 249.646 & 553.828 & 179.564 \\
\hline 1200 & 256.607 & 575.857 & 204.887 \\
\hline 1300 & 262.493 & 596.635 & 230.850 \\
\hline 1400 & 267.492 & 616.276 & 257.356 \\
\hline 1500 & 271.758 & 634.880 & 284.324 \\
\hline 1600 & 275.417 & 652.539 & 311.687 \\
\hline 1700 & 278.573 & 669.333 & 339.390 \\
\hline 1800 & 281.307 & 685.335 & 367.388 \\
\hline 1900 & 283.689 & 700.610 & 395.640 \\
\hline 2000 & 285.774 & 715.215 & 424.116 \\
\hline
\end{tabular}

$T$ : Temperature. $C_{P}$ : Heat capacity at constant pressure. $S$ : Entropy. $H$ : Enthalpy. 
Figure S.21.4. $\mathrm{B}_{3} \mathrm{H}_{10}$ : Ideal-gas thermodynamic functions at 1 bar (anharmonic B3LYPD2/cc-pVTZ results)
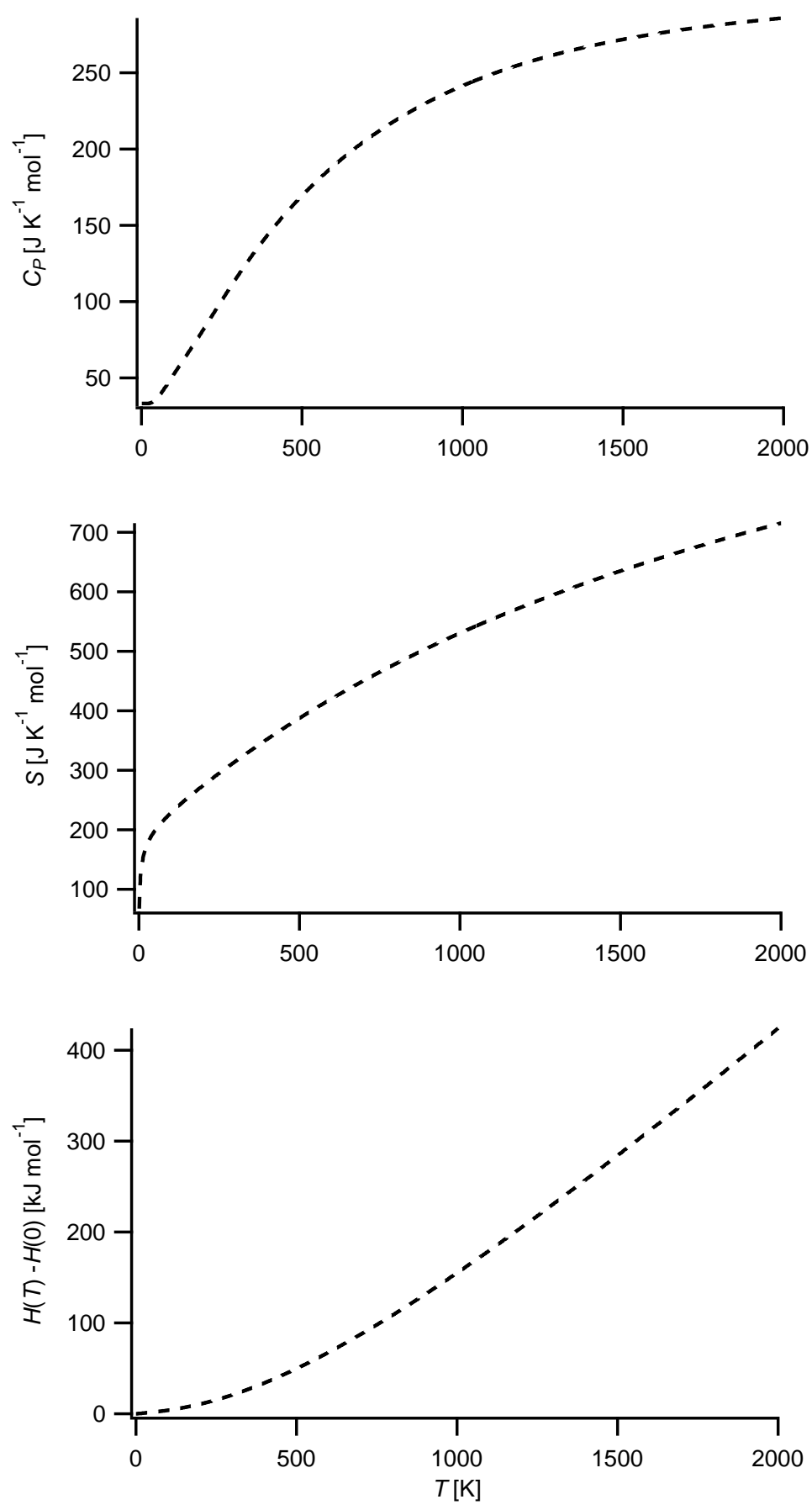

$T$ : Temperature. $C_{P}$ : Heat capacity at constant pressure. $S$ : Entropy. $H$ : Enthalpy. 
Table S.21.5. $\mathrm{B}_{3} \mathrm{H}_{10}$ : Fits (red solid lines) of the computed thermodynamic functions (blue dashed lines) with NASA type functions (Equations (1) - (3)) in the $200-900 \mathrm{~K}$ temperature range (anharmonic B3LYP-D2/cc-pVTZ results). In each case, the difference curve between the thermodynamic and NASA functions is plotted in the upper graph

Fit parameters

\begin{tabular}{llllll}
\hline $\mathrm{a}_{1}$ & $4.31719851 \mathrm{e}-01$ & $\mathrm{a}_{2}\left[\mathrm{~K}^{-1}\right]$ & $5.33706886 \mathrm{e}-02$ & $\mathrm{a}_{3}\left[\mathrm{~K}^{-2}\right]$ & $-2.42758148 \mathrm{e}-05$ \\
$\mathrm{a}_{4}\left[\mathrm{~K}^{-3}\right]$ & $-1.05496823 \mathrm{e}-08$ & $\mathrm{a}_{5}\left[\mathrm{~K}^{-4}\right]$ & $1.03291324 \mathrm{e}-11$ & $\mathrm{a}_{6}[\mathrm{~K}]$ & $2.02493664 \mathrm{e}+02$ \\
$\mathrm{a}_{7}$ & $2.05286862 \mathrm{e}+01$ & & & &
\end{tabular}
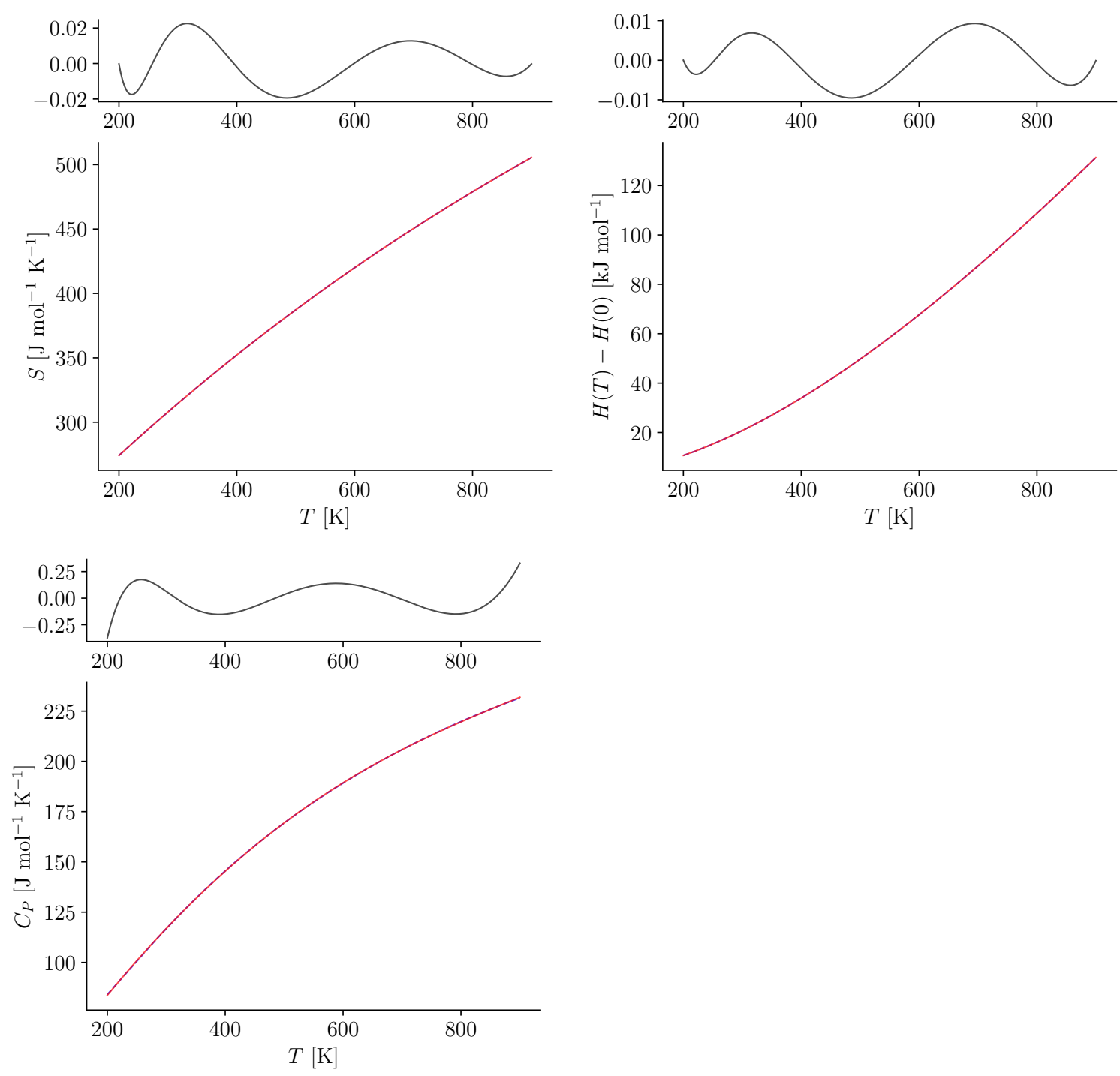


\section{$22 \quad \mathrm{~B}_{4} \mathrm{H}$}

Figure S.22.1. Structure of $\mathrm{B}_{4} \mathrm{H}$

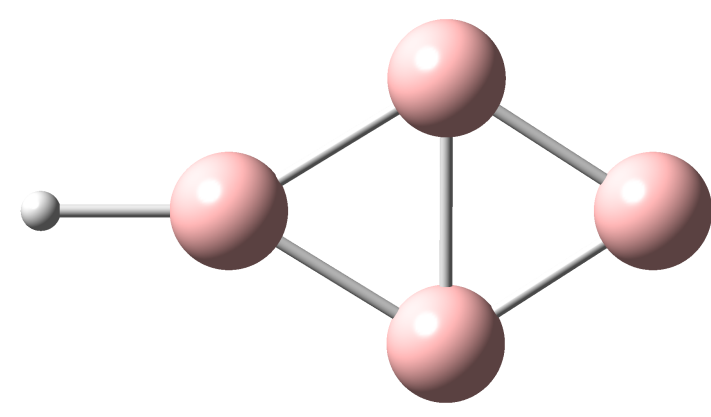

Charge 0

Spin multiplicity 2

Framework Group $\mathrm{C}_{2 \mathrm{v}}$

Rotor Type Asymmetric Top

Symmetry number 2

Table S.22.1. $\mathrm{B}_{4} \mathrm{H}$ : Selected anharmonic results obtained at the B3LYP-D2/cc-pVTZ level

\begin{tabular}{rll}
\hline $\mathrm{ZPE}_{\mathrm{v}}$ & 4524 & $\mathrm{~cm}^{-1}$ \\
$\mathrm{~A}$ & 1.07387 & $\mathrm{~cm}^{-1}$ \\
$\mathrm{~B}$ & 0.37506 & $\mathrm{~cm}^{-1}$ \\
$\mathrm{C}$ & 0.27798 & $\mathrm{~cm}^{-1}$ \\
\hline$C_{P}(300 \mathrm{~K})$ & 56.245 & $\mathrm{~J} \mathrm{~mol}^{-1} \mathrm{~K}-1$ \\
$S(300 \mathrm{~K})$ & 255.050 & $\mathrm{~J} \mathrm{~mol}^{-1} \mathrm{~K}^{-1}$ \\
$H(300 \mathrm{~K})-H(0 \mathrm{~K})$ & 12.344 & $\mathrm{~kJ} \mathrm{~mol}^{-1}$ \\
\hline
\end{tabular}

$\mathrm{ZPE}_{\mathrm{v}}$ : vibrational contribution to the zero-point energy. A, B, C: Rotational constants. $C_{P}$ : Heat capacity at constant pressure. $S$ : Entropy. $H$ : Enthalpy. Ideal-gas calculations (1 bar). 
Table S.22.2. $\mathrm{B}_{4} \mathrm{H}$ : Cartesian coordinates $(\AA)$ of the B3LYP-D2/cc-pVTZ optimized structure

\begin{tabular}{cccc} 
atom & $\mathrm{x}$ & $\mathrm{y}$ & $\mathrm{z}$ \\
\hline B1 & -0.0000 & 0.0000 & 1.4293 \\
B2 & 0.0000 & 0.8444 & 0.1361 \\
B3 & 0.0000 & -0.0000 & -1.2219 \\
B4 & -0.0000 & -0.8444 & 0.1361 \\
H5 & 0.0000 & -0.0000 & -2.3975 \\
\hline
\end{tabular}


Table S.22.3. $\mathrm{B}_{4} \mathrm{H}$ : Vibrational frequencies, infrared integrated intensities and Raman activities (B3LYP-D2/cc-pVTZ results)

\begin{tabular}{|c|c|c|c|c|c|c|c|}
\hline \multirow[b]{2}{*}{ mode } & \multirow[b]{2}{*}{ symm. } & \multicolumn{3}{|c|}{ Harmonic } & \multicolumn{3}{|c|}{ Anharmonic } \\
\hline & & $\begin{array}{c}\omega \\
{\left[\mathrm{cm}^{-1}\right]}\end{array}$ & $\begin{array}{c}\mathrm{IR} \\
{\left[\mathrm{km} \mathrm{mol}^{-1}\right]}\end{array}$ & $\begin{array}{c}\text { Raman } \\
{\left[\AA^{6}\right]}\end{array}$ & $\begin{array}{c}\omega \\
{\left[\mathrm{cm}^{-1}\right]}\end{array}$ & $\begin{array}{c}\mathrm{IR} \\
{\left[\mathrm{km} \mathrm{mol}^{-1}\right]}\end{array}$ & $\begin{array}{c}\text { Raman } \\
{\left[\AA^{6}\right]}\end{array}$ \\
\hline 1 & $\mathrm{~B}_{2}$ & 214 & 2.593 & 0.001 & 870 & 12.756 & 0.303 \\
\hline 2 & $\mathrm{~B}_{1}$ & 296 & 0.542 & 0.079 & 281 & 0.417 & 0.063 \\
\hline 3 & $\mathrm{~A}_{1}$ & 728 & 25.231 & 0.027 & 794 & 7.986 & 0.638 \\
\hline 4 & $\mathrm{~B}_{1}$ & 735 & 0.605 & 0.019 & 734 & 1.595 & 0.000 \\
\hline 5 & $\mathrm{~B}_{2}$ & 743 & 0.054 & 0.002 & 698 & 0.117 & 0.005 \\
\hline 6 & $\mathrm{~B}_{2}$ & 1008 & 9.306 & 0.039 & 971 & 7.850 & 0.027 \\
\hline 7 & $\mathrm{~A}_{1}$ & 1083 & 6.184 & 1.315 & 1047 & 4.712 & 1.383 \\
\hline 8 & $\mathrm{~A}_{1}$ & 1227 & 0.463 & 0.463 & 1223 & 1.241 & 0.050 \\
\hline 9 & $\mathrm{~A}_{1}$ & 2756 & 40.534 & 1.139 & 2664 & 43.682 & 0.804 \\
\hline
\end{tabular}


Figure S.22.2. Anharmonic IR spectrum of $\mathrm{B}_{4} \mathrm{H}$ obtained by convoluting the calculated intensities with Lorentzians having a FWHM of $4 \mathrm{~cm}^{-1}$ (B3LYP-D2/cc-pVTZ results): (top) full spectrum, (middle) contributions from fundamentals and combination bands, (bottom) contributions from overtones
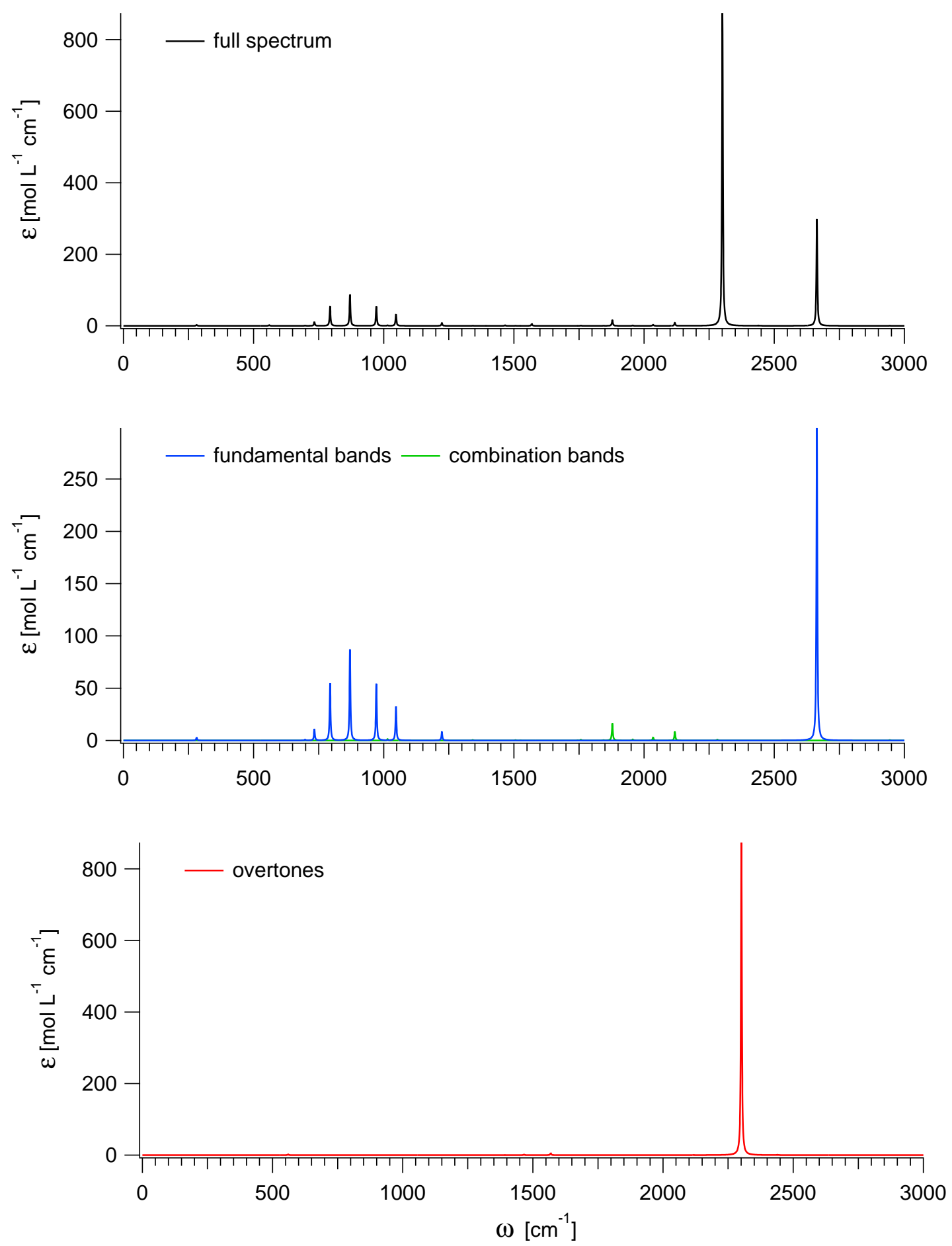
Figure S.22.3. Anharmonic Raman spectrum of $\mathrm{B}_{4} \mathrm{H}$ obtained by convoluting the calculated activities with Lorentzians having a FWHM of $4 \mathrm{~cm}^{-1}$ (B3LYP-D2/cc-pVTZ results): (top) full spectrum, (middle) contributions from fundamentals and combination bands, (bottom) contributions from overtones
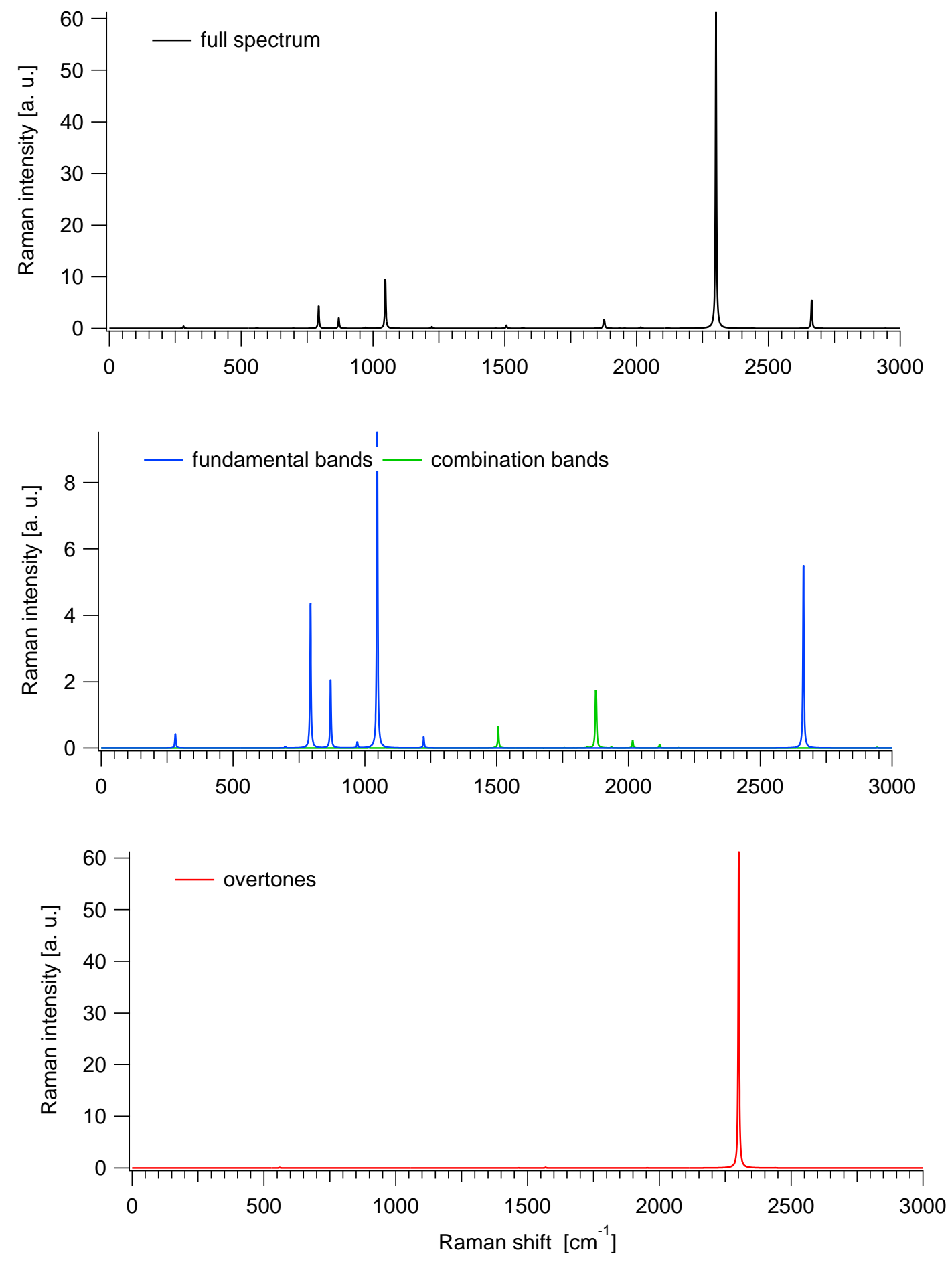

S.180 
Table S.22.4. $\mathrm{B}_{4} \mathrm{H}$ : Ideal-gas thermodynamic functions at 1 bar (anharmonic B3LYP$\mathrm{D} 2 / \mathrm{cc}-\mathrm{pVTZ}$ results)

\begin{tabular}{|c|c|c|c|}
\hline $\begin{array}{c}T \\
{[\mathrm{~K}]}\end{array}$ & $\begin{array}{c}C_{P} \\
{\left[\mathrm{~J} \mathrm{~mol}^{-1} \mathrm{~K}^{-1}\right]}\end{array}$ & $\begin{array}{c}S \\
{\left[\mathrm{~J} \mathrm{~mol}^{-1} \mathrm{~K}^{-1}\right]}\end{array}$ & $\begin{array}{c}H(T)-H(0 K) \\
{\left[\mathrm{kJ} \mathrm{mol}^{-1}\right]}\end{array}$ \\
\hline 100 & 35.804 & 207.857 & 3.386 \\
\hline 200 & 44.085 & 234.912 & 7.336 \\
\hline 300 & 56.245 & 255.050 & 12.344 \\
\hline 400 & 67.243 & 272.799 & 18.540 \\
\hline 500 & 75.553 & 288.744 & 25.701 \\
\hline 600 & 81.658 & 303.087 & 33.577 \\
\hline 700 & 86.222 & 316.033 & 41.982 \\
\hline 800 & 89.727 & 327.785 & 50.786 \\
\hline 900 & 92.481 & 338.519 & 59.902 \\
\hline 1000 & 94.684 & 348.381 & 69.264 \\
\hline 1100 & 96.474 & 357.492 & 78.825 \\
\hline 1200 & 97.945 & 365.952 & 88.548 \\
\hline 1300 & 99.166 & 373.841 & 98.406 \\
\hline 1400 & 100.189 & 381.228 & 108.375 \\
\hline 1500 & 101.052 & 388.171 & 118.438 \\
\hline 1600 & 101.788 & 394.717 & 128.581 \\
\hline 1700 & 102.417 & 400.907 & 138.792 \\
\hline 1800 & 102.960 & 406.777 & 149.062 \\
\hline 1900 & 103.431 & 412.357 & 159.382 \\
\hline 2000 & 103.842 & 417.673 & 169.746 \\
\hline
\end{tabular}

$T$ : Temperature. $C_{P}$ : Heat capacity at constant pressure. $S$ : Entropy. $H$ : Enthalpy. 
Figure S.22.4. $\mathrm{B}_{4} \mathrm{H}$ : Ideal-gas thermodynamic functions at 1 bar (anharmonic B3LYPD2/cc-pVTZ results)
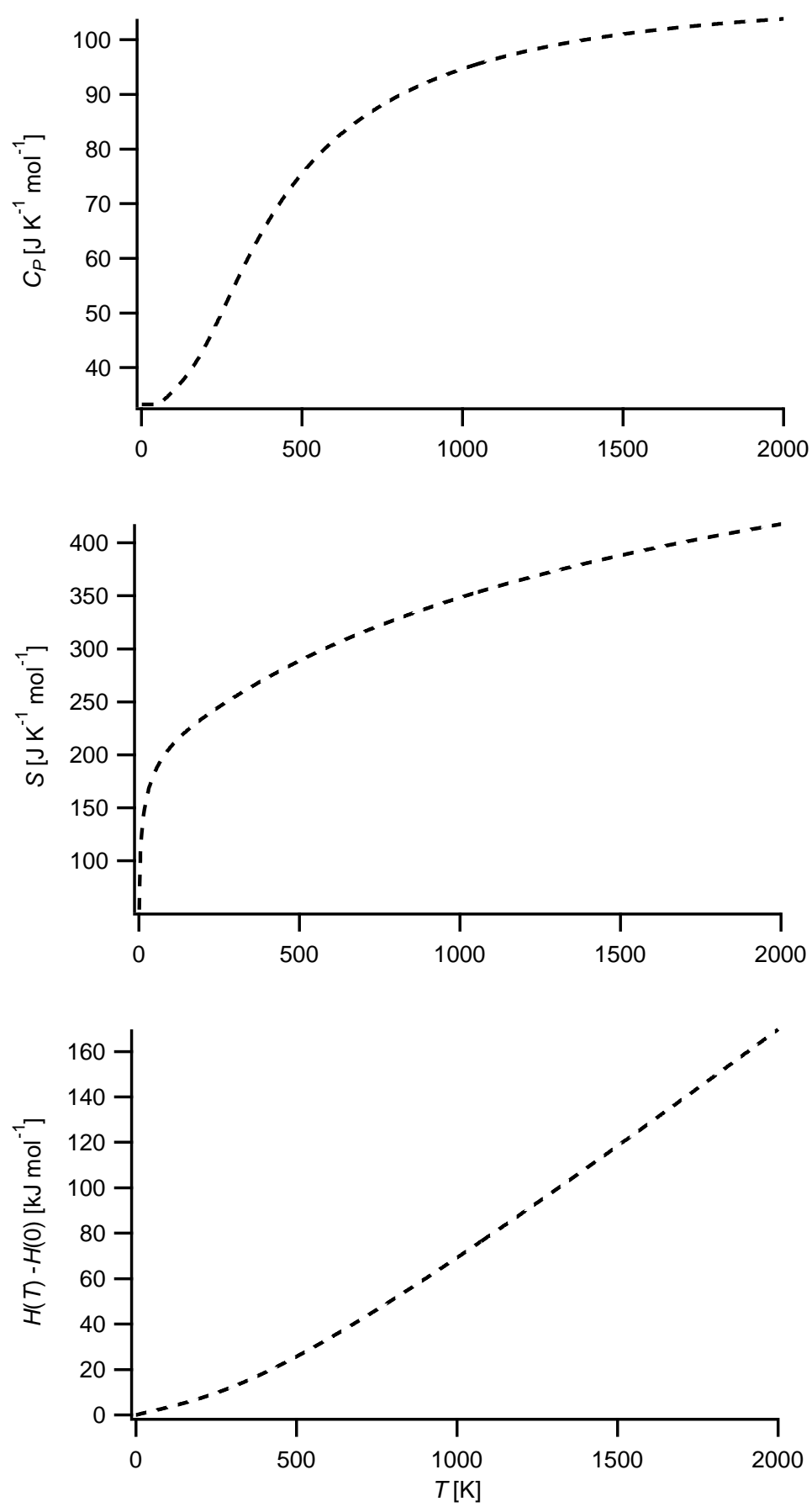

$T$ : Temperature. $C_{P}$ : Heat capacity at constant pressure. $S$ : Entropy. $H$ : Enthalpy. 
Table S.22.5. $\mathrm{B}_{4} \mathrm{H}$ : Fits (red solid lines) of the computed thermodynamic functions (blue dashed lines) with NASA type functions (Equations (1) - (3)) in the 200 - $900 \mathrm{~K}$ temperature range (anharmonic B3LYP-D2/cc-pVTZ results). In each case, the difference curve between the thermodynamic and NASA functions is plotted in the upper graph

Fit parameters

\begin{tabular}{llllll}
\hline $\mathrm{a}_{1}$ & $1.85253850 \mathrm{e}+00$ & $\mathrm{a}_{2}\left[\mathrm{~K}^{-1}\right]$ & $1.68691508 \mathrm{e}-02$ & $\mathrm{a}_{3}\left[\mathrm{~K}^{-2}\right]$ & $5.99769614 \mathrm{e}-06$ \\
$\mathrm{a}_{4}\left[\mathrm{~K}^{-3}\right]$ & $-3.02053831 \mathrm{e}-08$ & $\mathrm{a}_{5}\left[\mathrm{~K}^{-4}\right]$ & $1.72131660 \mathrm{e}-11$ & $\mathrm{a}_{6}[\mathrm{~K}]$ & $1.69413313 \mathrm{e}+02$
\end{tabular}

$\mathrm{a}_{7}$
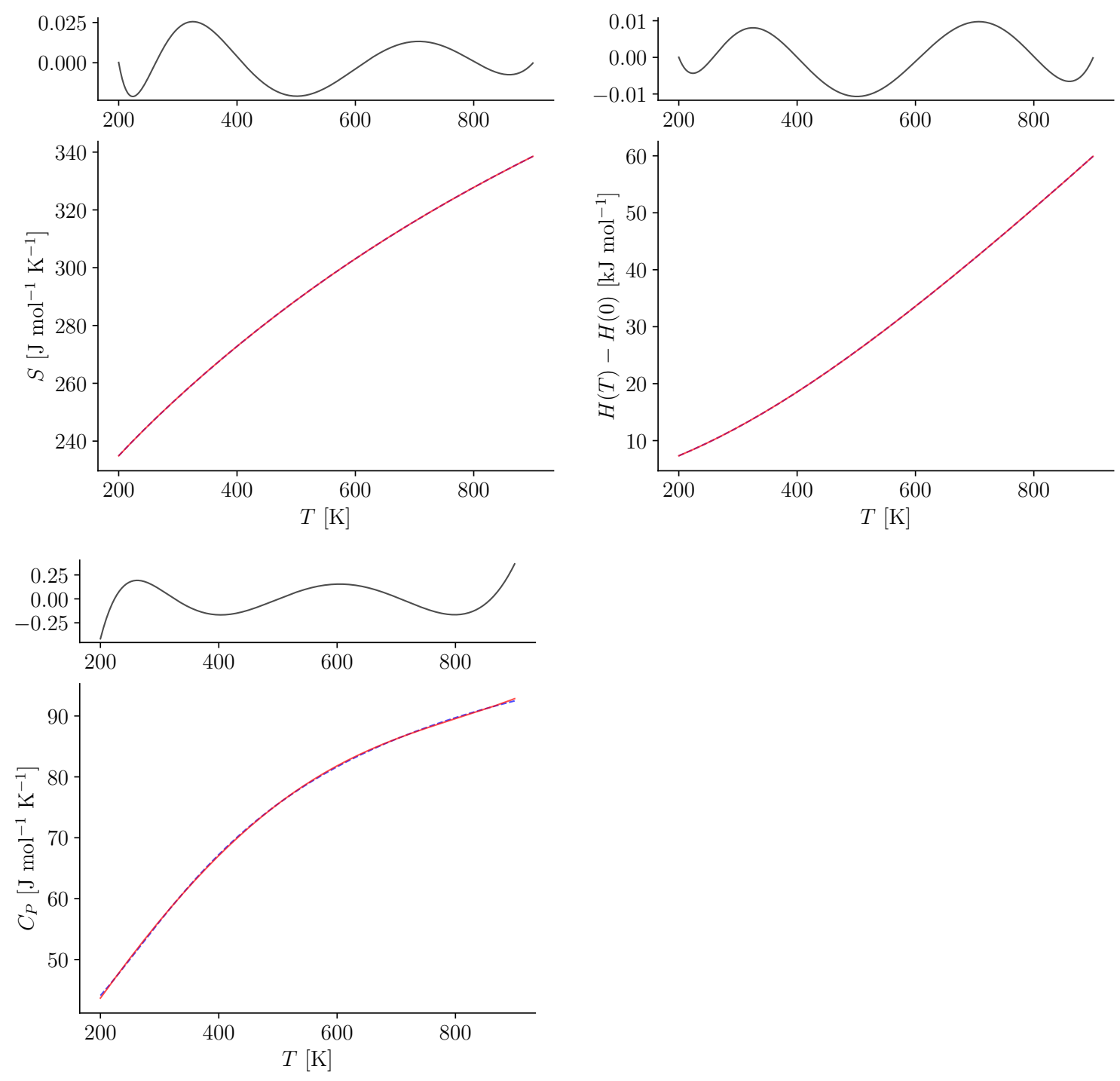


\section{$23 \quad \mathbf{B}_{4} \mathbf{H}_{2}$}

Figure S.23.1. Structure of $\mathrm{B}_{4} \mathrm{H}_{2}$

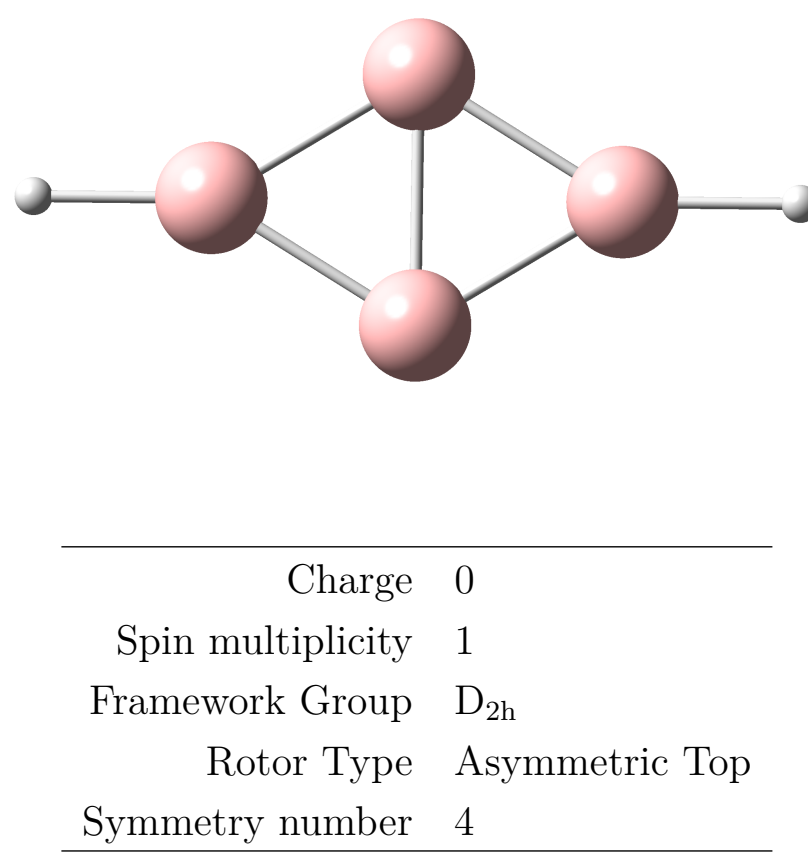

Table S.23.1. $\mathrm{B}_{4} \mathrm{H}_{2}$ : Selected anharmonic results obtained at the B3LYP-D2/cc-pVTZ level

\begin{tabular}{rll}
\hline $\mathrm{ZPE}_{\mathrm{v}}$ & 6612 & $\mathrm{~cm}^{-1}$ \\
$\mathrm{~A}$ & 1.11981 & $\mathrm{~cm}^{-1}$ \\
$\mathrm{~B}$ & 0.31485 & $\mathrm{~cm}^{-1}$ \\
$\mathrm{C}$ & 0.24575 & $\mathrm{~cm}^{-1}$ \\
\hline$C_{P}(300 \mathrm{~K})$ & 64.320 & $\mathrm{~J} \mathrm{~mol}^{-1} \mathrm{~K}-1$ \\
$S(300 \mathrm{~K})$ & 254.177 & $\mathrm{~J} \mathrm{~mol}^{-1} \mathrm{~K}-1$ \\
$H(300 \mathrm{~K})-H(0 \mathrm{~K})$ & 13.122 & $\mathrm{~kJ} \mathrm{~mol}^{-1}$ \\
\hline
\end{tabular}

$\mathrm{ZPE}_{\mathrm{v}}$ : vibrational contribution to the zero-point energy. A, B, C: Rotational constants. $C_{P}$ : Heat capacity at constant pressure. $S$ : Entropy. $H$ : Enthalpy. Ideal-gas calculations (1 bar). 
Table S.23.2. $\mathrm{B}_{4} \mathrm{H}_{2}$ : Cartesian coordinates $(\AA)$ of the B3LYP-D2/cc-pVTZ optimized structure

\begin{tabular}{cccc} 
atom & $\mathrm{x}$ & $\mathrm{y}$ & $\mathrm{z}$ \\
\hline B1 & -0.0000 & 0.8269 & 0.0000 \\
B2 & 0.0000 & -0.0000 & 1.3581 \\
B3 & 0.0000 & -0.0000 & -1.3581 \\
B4 & -0.0000 & -0.8269 & 0.0000 \\
H5 & 0.0000 & -0.0000 & 2.5327 \\
H6 & 0.0000 & -0.0000 & -2.5327 \\
\hline
\end{tabular}


Table S.23.3. $\mathrm{B}_{4} \mathrm{H}_{2}$ : Vibrational frequencies, infrared integrated intensities and Raman activities (B3LYP-D2/cc-pVTZ results)

\begin{tabular}{cccccccc}
\hline & & \multicolumn{3}{c}{ Harmonic } & \multicolumn{3}{c}{ Anharmonic } \\
mode & symm. & $\omega$ & IR & Raman & $\omega$ & IR & Raman \\
& & {$\left[\mathrm{cm}^{-1}\right]$} & {$\left[\mathrm{km} \mathrm{mol}^{-1}\right]$} & {$\left[\AA^{6}\right]$} & {$\left[\mathrm{cm}^{-1}\right]$} & {$\left[\mathrm{km} \mathrm{mol}^{-1}\right]$} & {$\left[\AA^{6}\right]$} \\
\hline 1 & $\mathrm{~B}_{3 \mathrm{G}}$ & 124 & 0.000 & 0.561 & 654 & 0.000 & 1.627 \\
2 & $\mathrm{~B}_{3 \mathrm{U}}$ & 284 & 0.223 & 0.000 & 268 & 0.167 & 0.000 \\
3 & $\mathrm{~B}_{2 \mathrm{U}}$ & 657 & 2.521 & 0.000 & 616 & 1.099 & 0.000 \\
4 & $\mathrm{~B}_{3 \mathrm{U}}$ & 728 & 0.763 & 0.000 & 723 & 1.608 & 0.000 \\
5 & $\mathrm{~B}_{2 \mathrm{G}}$ & 804 & 0.000 & 0.008 & 796 & 0.000 & 0.005 \\
6 & $\mathrm{~B}_{2 \mathrm{U}}$ & 867 & 52.959 & 0.000 & 814 & 49.409 & 0.000 \\
7 & $\mathrm{~A}_{\mathrm{G}}$ & 873 & 0.000 & 0.558 & 833 & 0.000 & 0.405 \\
8 & $\mathrm{~B}_{3 \mathrm{G}}$ & 902 & 0.000 & 0.025 & 869 & 0.000 & 0.061 \\
9 & $\mathrm{~A}_{\mathrm{G}}$ & 1092 & 0.000 & 1.494 & 1081 & 0.000 & 1.384 \\
10 & $\mathrm{~B}_{1 \mathrm{U}}$ & 1204 & 29.093 & 0.000 & 1219 & 35.409 & 0.000 \\
11 & $\mathrm{~B}_{1 \mathrm{U}}$ & 2766 & 71.864 & 0.000 & 2672 & 76.314 & 0.000 \\
12 & $\mathrm{~A}_{\mathrm{G}}$ & 2769 & 0.000 & 1.656 & 2675 & 0.000 & 1.732 \\
\hline
\end{tabular}


Figure S.23.2. Anharmonic IR spectrum of $\mathrm{B}_{4} \mathrm{H}_{2}$ obtained by convoluting the calculated intensities with Lorentzians having a FWHM of $4 \mathrm{~cm}^{-1}$ (B3LYP-D2/cc-pVTZ results): (top) full spectrum, (bottom) contributions from fundamentals and combination bands
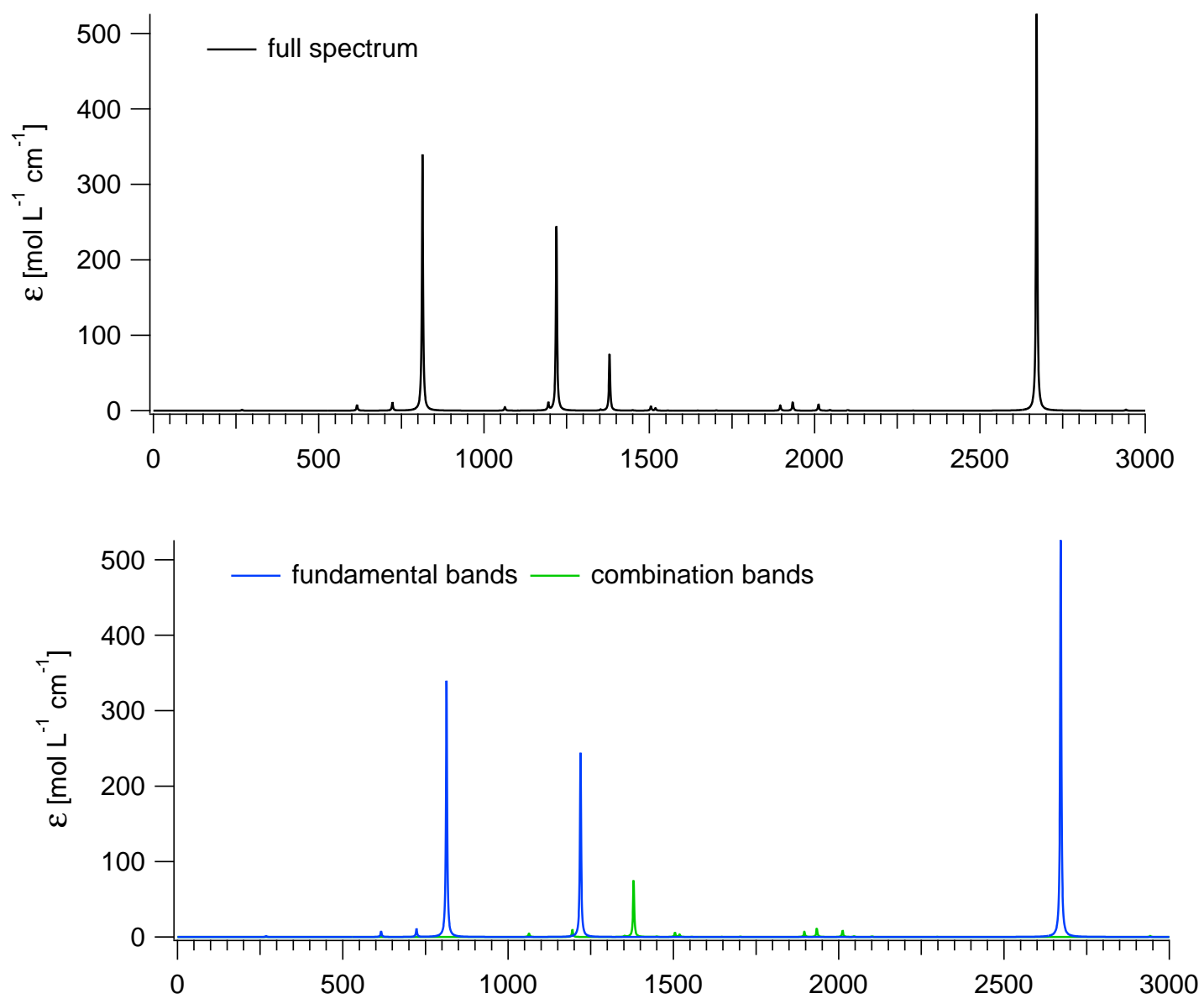
Figure S.23.3. Anharmonic Raman spectrum of $\mathrm{B}_{4} \mathrm{H}_{2}$ obtained by convoluting the calculated activities with Lorentzians having a FWHM of $4 \mathrm{~cm}^{-1}$ (B3LYP-D2/cc-pVTZ results): (top) full spectrum, (middle) contributions from fundamentals and combination bands, (bottom) contributions from overtones
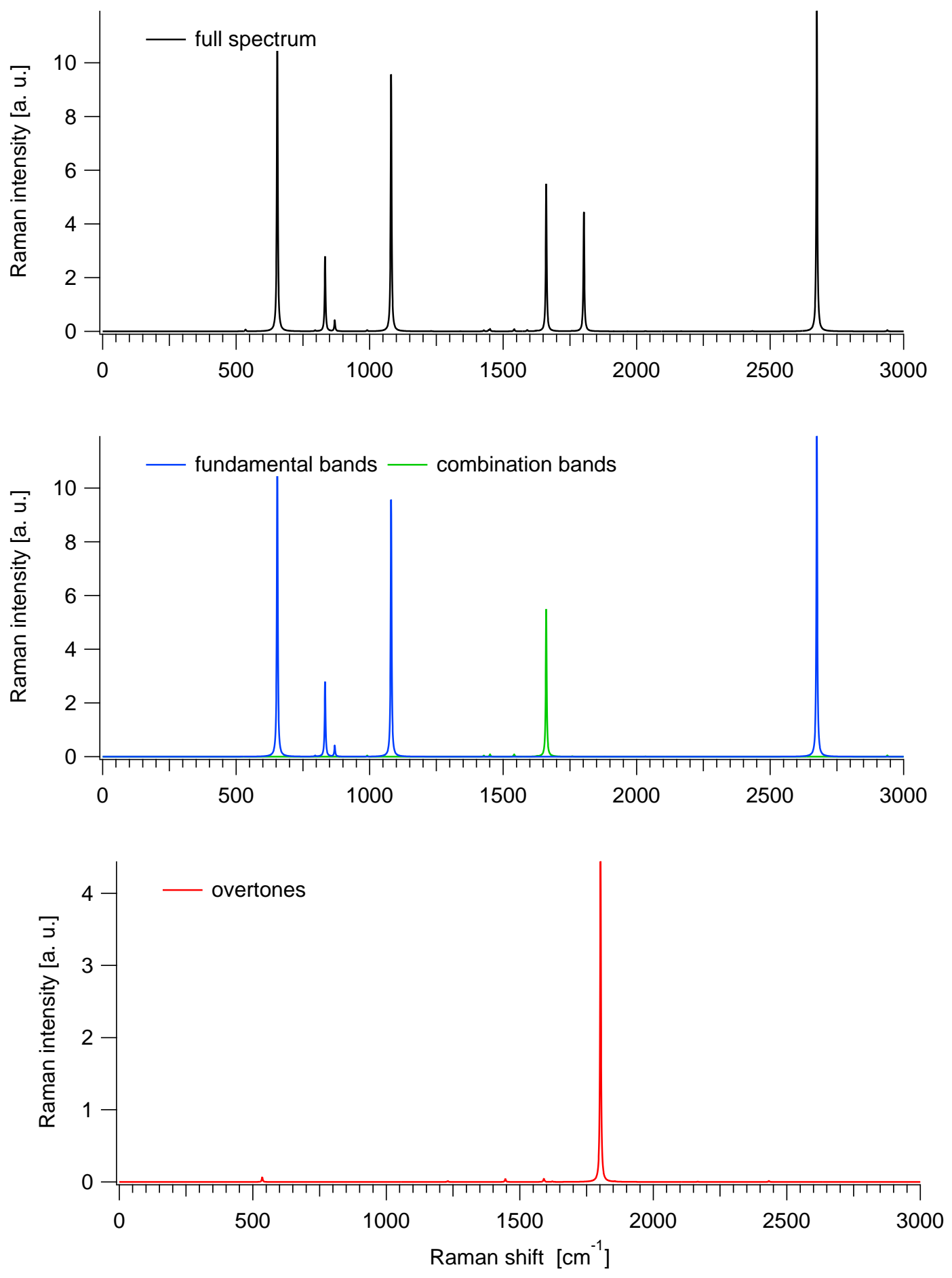

S.188 
Table S.23.4. $\mathrm{B}_{4} \mathrm{H}_{2}$ : Ideal-gas thermodynamic functions at 1 bar (anharmonic B3LYPD2/cc-pVTZ results)

\begin{tabular}{cccc}
\hline$T$ & $C_{P}$ & $S$ & $H(T)-H(0 K)$ \\
{$[\mathrm{K}]$} & {$\left[\mathrm{J} \mathrm{mol}^{-1} \mathrm{~K}^{-1}\right]$} & {$\left[\mathrm{J} \mathrm{mol}^{-1} \mathrm{~K}^{-1}\right]$} & {$\left[\mathrm{kJ} \mathrm{mol}^{-1}\right]$} \\
\hline 100 & 36.198 & 203.575 & 3.397 \\
200 & 47.755 & 231.679 & 7.517 \\
300 & 64.320 & 254.177 & 13.122 \\
400 & 78.282 & 274.687 & 20.285 \\
500 & 88.512 & 293.316 & 28.652 \\
600 & 96.028 & 310.151 & 37.897 \\
700 & 101.760 & 325.402 & 47.798 \\
800 & 106.287 & 339.297 & 58.209 \\
900 & 109.951 & 352.035 & 69.027 \\
1000 & 112.963 & 363.780 & 80.178 \\
1100 & 115.466 & 374.668 & 91.603 \\
1200 & 117.564 & 384.807 & 103.257 \\
1300 & 119.333 & 394.289 & 115.105 \\
1400 & 120.835 & 403.189 & 127.115 \\
1500 & 122.118 & 411.571 & 139.265 \\
1600 & 123.220 & 419.488 & 151.533 \\
1700 & 124.171 & 426.988 & 163.904 \\
1800 & 124.996 & 434.109 & 176.363 \\
1900 & 125.716 & 440.887 & 188.899 \\
2000 & 126.348 & 447.352 & 201.503 \\
\hline & & & $S:$ \\
\hline
\end{tabular}

$T$ : Temperature. $C_{P}$ : Heat capacity at constant pressure. $S$ : Entropy. $H$ : Enthalpy. 
Figure S.23.4. $\mathrm{B}_{4} \mathrm{H}_{2}$ : Ideal-gas thermodynamic functions at 1 bar (anharmonic B3LYPD2/cc-pVTZ results)
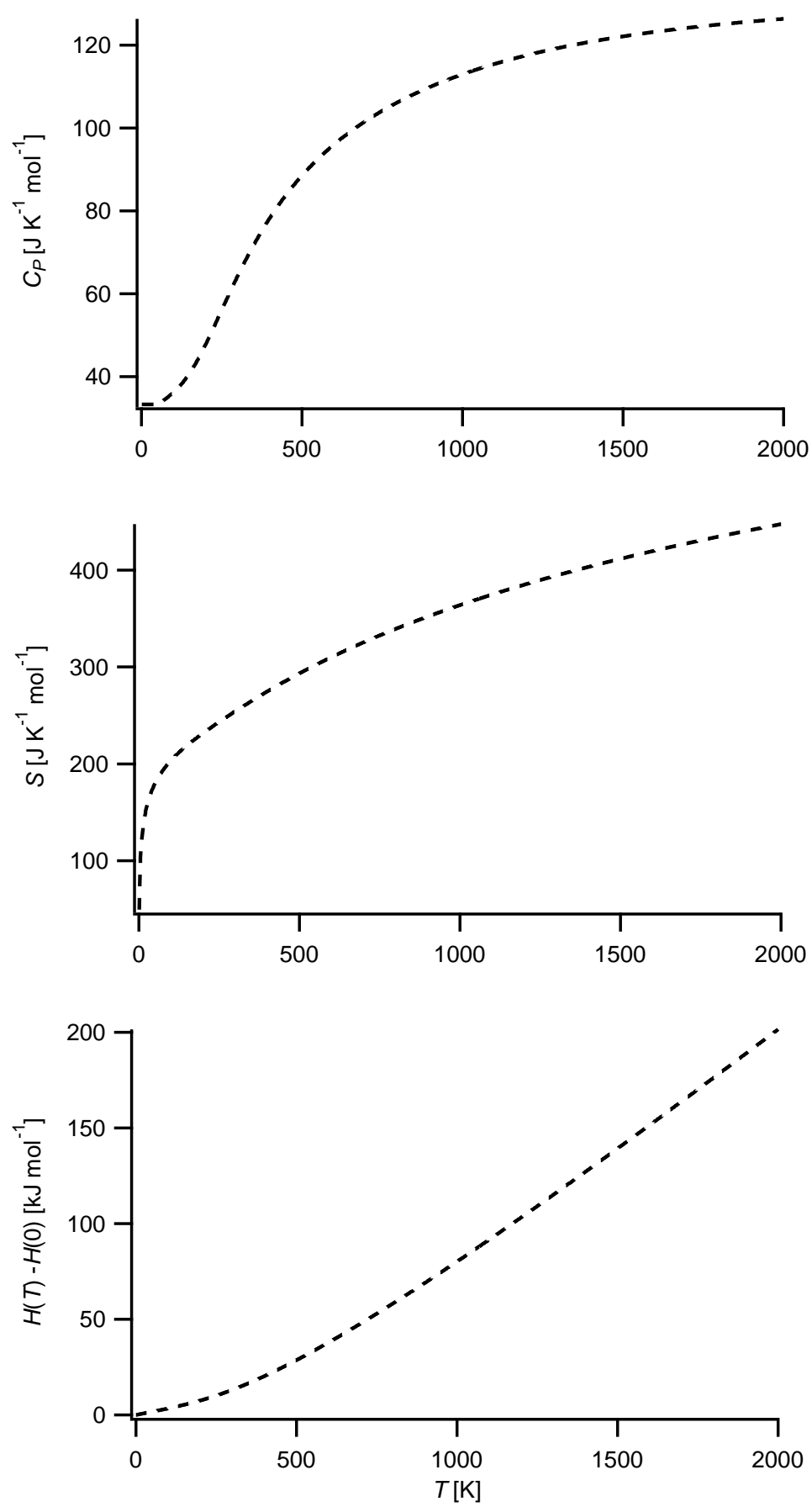

$T$ : Temperature. $C_{P}$ : Heat capacity at constant pressure. $S$ : Entropy. $H$ : Enthalpy. 
Table S.23.5. $\mathrm{B}_{4} \mathrm{H}_{2}$ : Fits (red solid lines) of the computed thermodynamic functions (blue dashed lines) with NASA type functions (Equations (1) - (3)) in the 200 - $900 \mathrm{~K}$ temperature range (anharmonic B3LYP-D2/cc-pVTZ results). In each case, the difference curve between the thermodynamic and NASA functions is plotted in the upper graph

Fit parameters

\begin{tabular}{llllll}
\hline $\mathrm{a}_{1}$ & $3.08953875 \mathrm{e}-01$ & $\mathrm{a}_{2}\left[\mathrm{~K}^{-1}\right]$ & $3.07579788 \mathrm{e}-02$ & $\mathrm{a}_{3}\left[\mathrm{~K}^{-2}\right]$ & $-1.78590382 \mathrm{e}-05$ \\
$\mathrm{a}_{4}\left[\mathrm{~K}^{-3}\right]$ & $-9.91836591 \mathrm{e}-09$ & $\mathrm{a}_{5}\left[\mathrm{~K}^{-4}\right]$ & $1.06318954 \mathrm{e}-11$ & $\mathrm{a}_{6}[\mathrm{~K}]$ & $2.78010933 \mathrm{e}+02$ \\
$\mathrm{a}_{7}$ & $2.04554152 \mathrm{e}+01$ & & & &
\end{tabular}
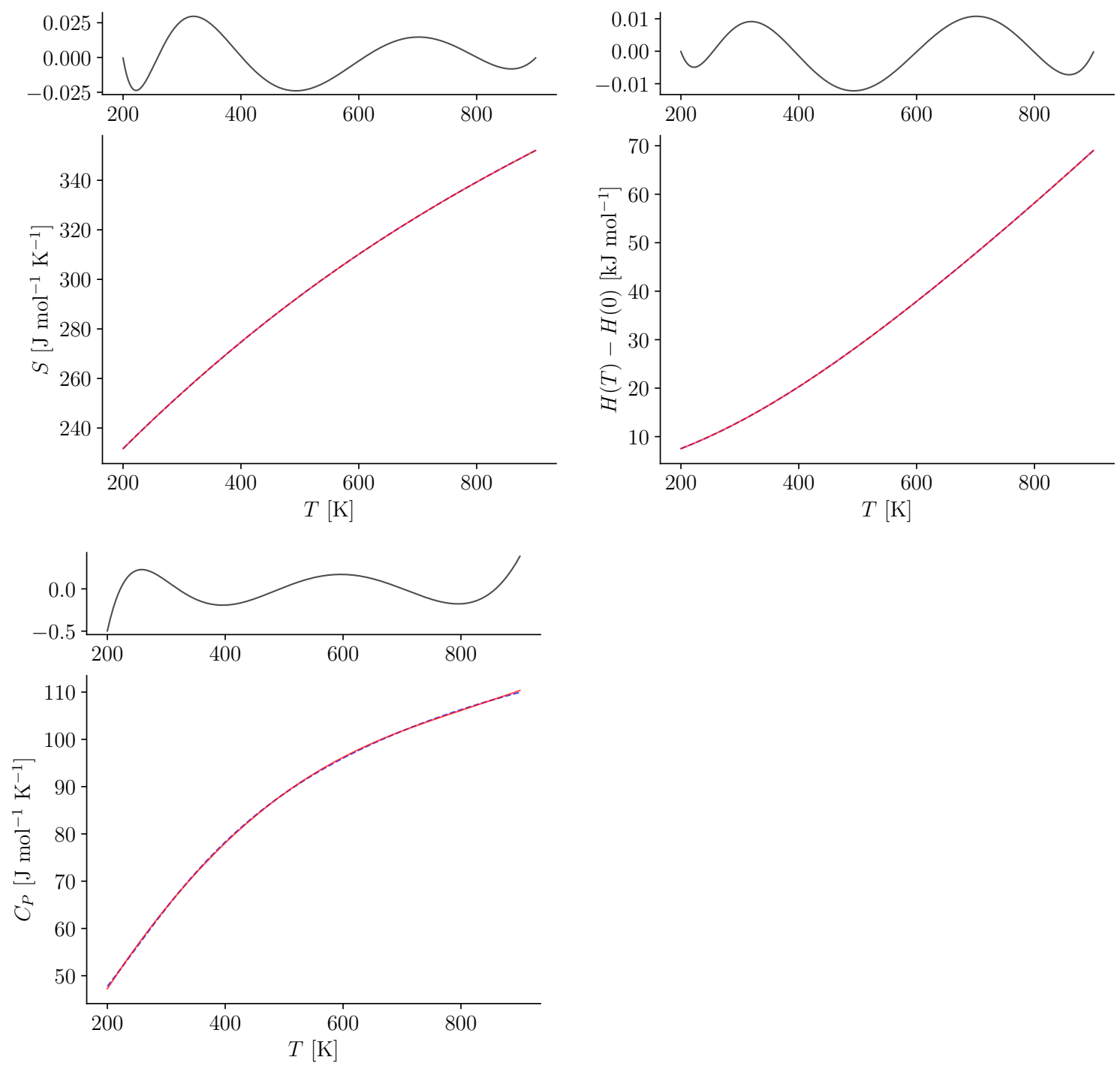


\section{$24 \quad \mathbf{B}_{4} \mathrm{H}_{3}$}

Figure S.24.1. Structure of $\mathrm{B}_{4} \mathrm{H}_{3}$

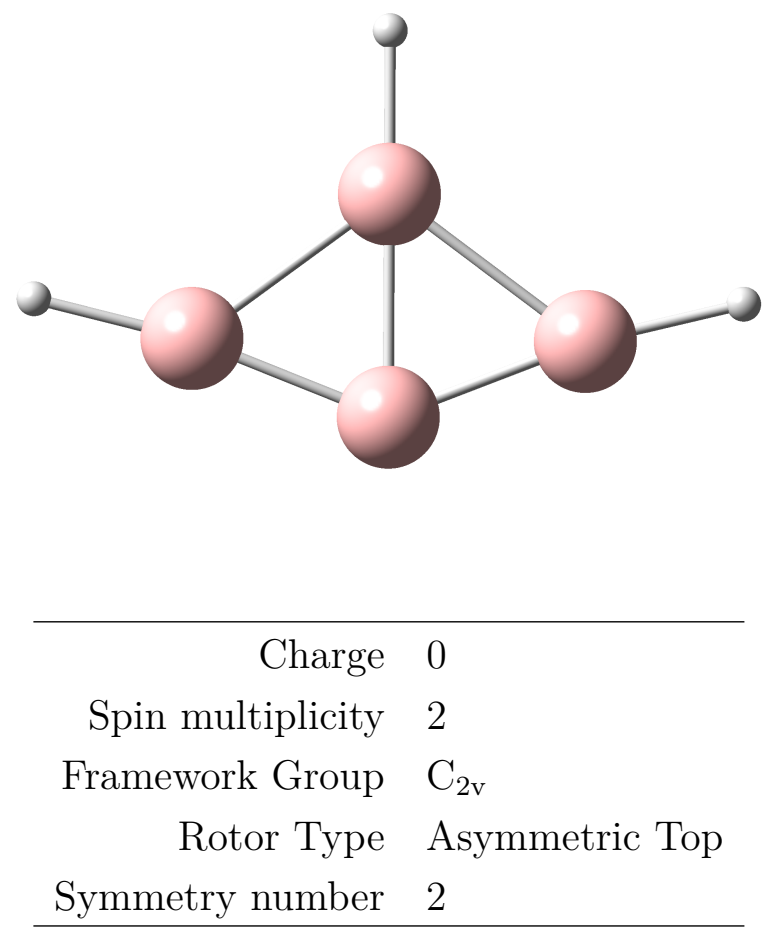

Table S.24.1. $\mathrm{B}_{4} \mathrm{H}_{3}$ : Selected anharmonic results obtained at the B3LYP-D2/cc-pVTZ level

\begin{tabular}{rll}
\hline $\mathrm{ZPE}_{\mathrm{v}}$ & 8484 & $\mathrm{~cm}^{-1}$ \\
$\mathrm{~A}$ & 0.87733 & $\mathrm{~cm}^{-1}$ \\
$\mathrm{~B}$ & 0.29485 & $\mathrm{~cm}^{-1}$ \\
$\mathrm{C}$ & 0.22069 & $\mathrm{~cm}^{-1}$ \\
\hline$C_{P}(300 \mathrm{~K})$ & 76.776 & $\mathrm{~J} \mathrm{~mol}^{-1} \mathrm{~K}-1$ \\
$S(300 \mathrm{~K})$ & 270.692 & $\mathrm{~J} \mathrm{~mol}^{-1} \mathrm{~K}-1$ \\
$H(300 \mathrm{~K})-H(0 \mathrm{~K})$ & 14.750 & $\mathrm{~kJ} \mathrm{~mol}^{-1}$ \\
\hline
\end{tabular}

$\mathrm{ZPE}_{\mathrm{v}}$ : vibrational contribution to the zero-point energy. A, B, C: Rotational constants. $C_{P}$ : Heat capacity at constant pressure. $S$ : Entropy. $H$ : Enthalpy. Ideal-gas calculations (1 bar). 
Table S.24.2. $\mathrm{B}_{4} \mathrm{H}_{3}$ : Cartesian coordinates $(\AA)$ of the B3LYP-D2/cc-pVTZ optimized structure

\begin{tabular}{cccc} 
atom & $\mathrm{x}$ & $\mathrm{y}$ & $\mathrm{z}$ \\
\hline B1 & -0.0000 & 0.0000 & -0.7837 \\
B2 & 0.0000 & -0.0000 & 0.8207 \\
B3 & -0.0000 & 1.4145 & -0.2280 \\
B4 & -0.0000 & -1.4145 & -0.2280 \\
H5 & 0.0000 & -0.0000 & 1.9976 \\
H6 & 0.0000 & 2.5513 & 0.0487 \\
H7 & -0.0000 & -2.5513 & 0.0487 \\
\hline
\end{tabular}


Table S.24.3. $\mathrm{B}_{4} \mathrm{H}_{3}$ : Vibrational frequencies, infrared integrated intensities and Raman activities (B3LYP-D2/cc-pVTZ results)

\begin{tabular}{cccccccc}
\hline & & \multicolumn{3}{c}{ Harmonic } & \multicolumn{3}{c}{ Anharmonic } \\
mode & symm. & $\omega$ & IR & Raman & $\omega$ & IR & Raman \\
& & {$\left[\mathrm{cm}^{-1}\right]$} & {$\left[\mathrm{km} \mathrm{mol}^{-1}\right]$} & {$\left[\AA^{6}\right]$} & {$\left[\mathrm{cm}^{-1}\right]$} & {$\left[\mathrm{km} \mathrm{mol}^{-1}\right]$} & {$\left[\AA^{6}\right]$} \\
\hline 1 & $\mathrm{~B}_{1}$ & 188 & 0.422 & 0.050 & 186 & 0.350 & 0.046 \\
2 & $\mathrm{~A}_{1}$ & 516 & 0.351 & 0.042 & 502 & 0.345 & 0.063 \\
3 & $\mathrm{~B}_{2}$ & 574 & 20.914 & 0.012 & 564 & 20.498 & 0.002 \\
4 & $\mathrm{~A}_{1}$ & 655 & 1.372 & 0.050 & 640 & 1.461 & 0.052 \\
5 & $\mathrm{~B}_{1}$ & 667 & 2.721 & 0.028 & 659 & 2.223 & 0.018 \\
6 & $\mathrm{~B}_{2}$ & 683 & 5.344 & 0.003 & 671 & 3.220 & 0.003 \\
7 & $\mathrm{~B}_{2}$ & 718 & 90.486 & 0.196 & 691 & 86.069 & 0.210 \\
8 & $\mathrm{~A}_{2}$ & 780 & 0.000 & 0.051 & 771 & 0.000 & 0.034 \\
9 & $\mathrm{~B}_{1}$ & 782 & 10.763 & 0.004 & 770 & 9.935 & 0.002 \\
10 & $\mathrm{~A}_{1}$ & 850 & 0.752 & 0.587 & 829 & 0.680 & 0.538 \\
11 & $\mathrm{~A}_{1}$ & 1049 & 0.583 & 1.959 & 1028 & 0.551 & 1.872 \\
12 & $\mathrm{~B}_{2}$ & 1315 & 229.735 & 0.060 & 1280 & 170.323 & 0.047 \\
13 & $\mathrm{~A}_{1}$ & 2744 & 40.512 & 0.775 & 2633 & 43.361 & 0.872 \\
14 & $\mathrm{~B}_{2}$ & 2813 & 122.801 & 0.053 & 2713 & 124.657 & 0.045 \\
15 & $\mathrm{~A}_{1}$ & 2816 & 0.748 & 1.106 & 2716 & 0.633 & 1.260 \\
\hline
\end{tabular}


Figure S.24.2. Anharmonic IR spectrum of $\mathrm{B}_{4} \mathrm{H}_{3}$ obtained by convoluting the calculated intensities with Lorentzians having a FWHM of $4 \mathrm{~cm}^{-1}$ (B3LYP-D2/cc-pVTZ results): (top) full spectrum, (middle) contributions from fundamentals and combination bands, (bottom) contributions from overtones
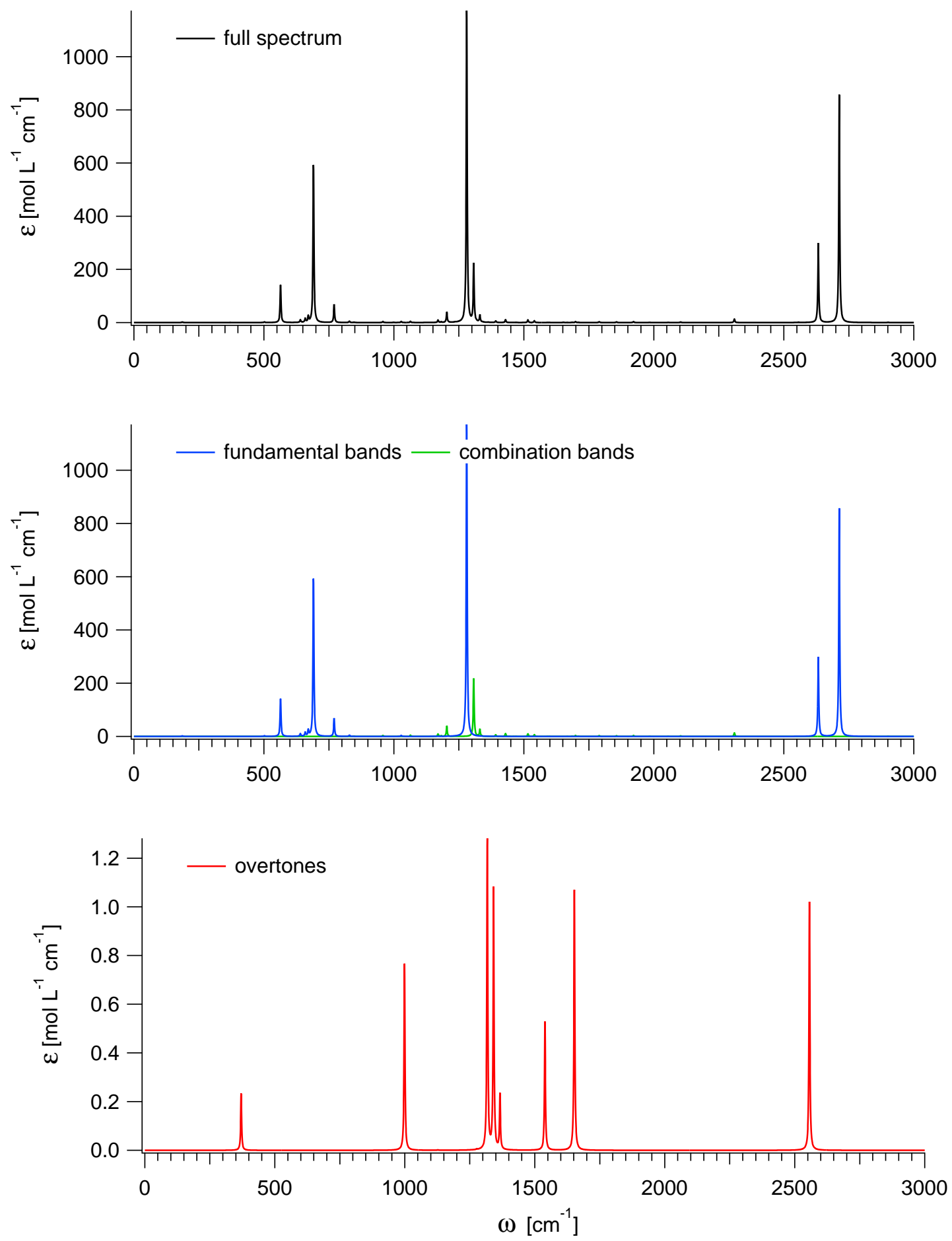
Figure S.24.3. Anharmonic Raman spectrum of $\mathrm{B}_{4} \mathrm{H}_{3}$ obtained by convoluting the calculated activities with Lorentzians having a FWHM of $4 \mathrm{~cm}^{-1}$ (B3LYP-D2/cc-pVTZ results): (top) full spectrum, (middle) contributions from fundamentals and combination bands, (bottom) contributions from overtones
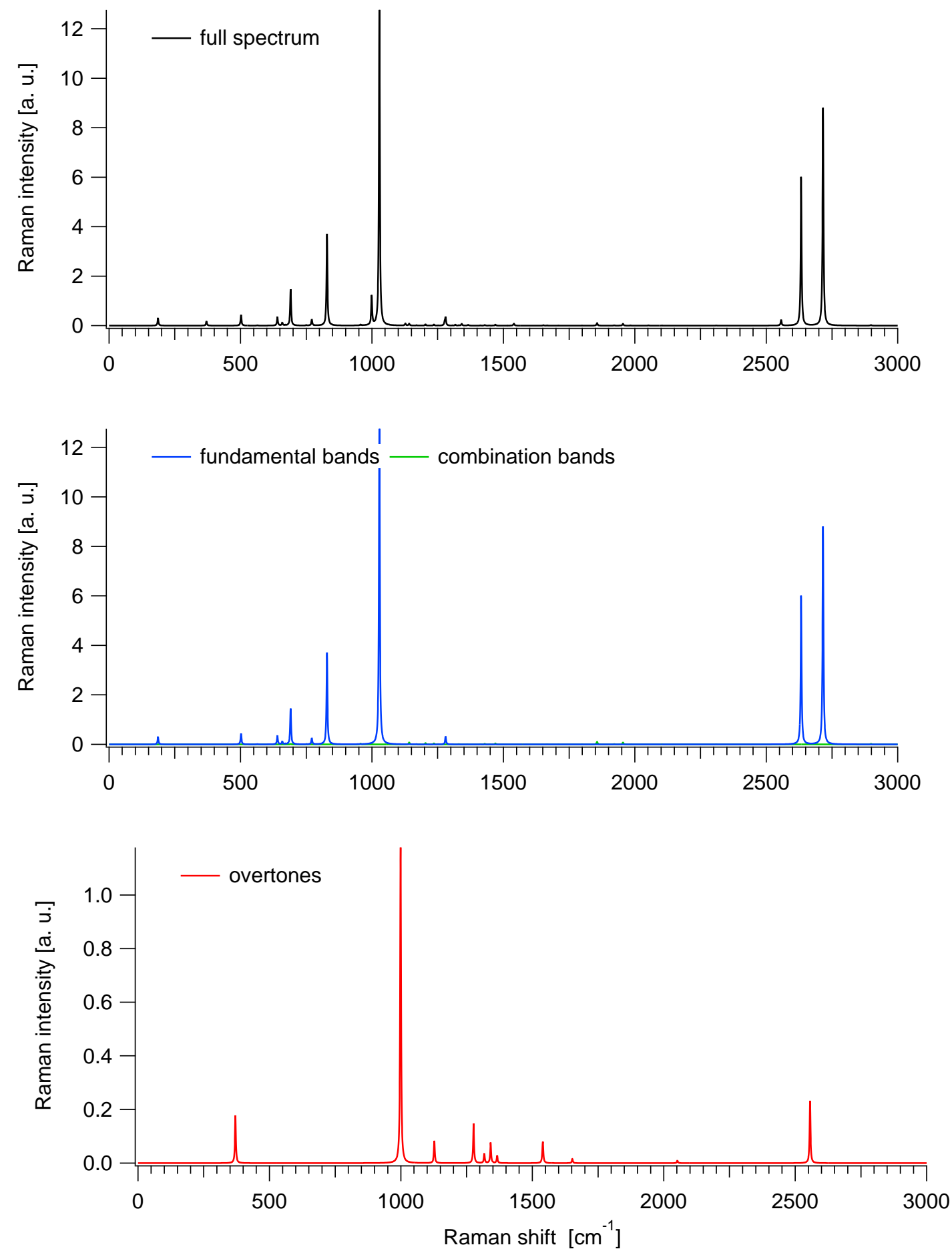

S.196 
Table S.24.4. $\mathrm{B}_{4} \mathrm{H}_{3}$ : Ideal-gas thermodynamic functions at 1 bar (anharmonic B3LYPD2/cc-pVTZ results)

\begin{tabular}{|c|c|c|c|}
\hline $\begin{array}{c}T \\
{[\mathrm{~K}]}\end{array}$ & $\begin{array}{c}C_{P} \\
{\left[\mathrm{~J} \mathrm{~mol}^{-1} \mathrm{~K}^{-1}\right]}\end{array}$ & $\begin{array}{c}S \\
{\left[\mathrm{~J} \mathrm{~mol}^{-1} \mathrm{~K}^{-1}\right]}\end{array}$ & $\begin{array}{c}H(T)-H(0 K) \\
{\left[\mathrm{kJ} \mathrm{mol}^{-1}\right]}\end{array}$ \\
\hline 100 & 38.717 & 212.783 & 3.499 \\
\hline 200 & 55.592 & 244.038 & 8.104 \\
\hline 300 & 76.776 & 270.692 & 14.750 \\
\hline 400 & 92.853 & 295.116 & 23.279 \\
\hline 500 & 104.149 & 317.121 & 33.161 \\
\hline 600 & 112.423 & 336.876 & 44.009 \\
\hline 700 & 118.850 & 354.707 & 55.584 \\
\hline 800 & 124.059 & 370.929 & 67.738 \\
\hline 900 & 128.384 & 385.798 & 80.367 \\
\hline 1000 & 132.019 & 399.518 & 93.392 \\
\hline 1100 & 135.096 & 412.250 & 106.752 \\
\hline 1200 & 137.714 & 424.120 & 120.396 \\
\hline 1300 & 139.948 & 435.233 & 134.282 \\
\hline 1400 & 141.864 & 445.676 & 148.375 \\
\hline 1500 & 143.513 & 455.522 & 162.646 \\
\hline 1600 & 144.938 & 464.830 & 177.070 \\
\hline 1700 & 146.176 & 473.655 & 191.627 \\
\hline 1800 & 147.255 & 482.041 & 206.300 \\
\hline 1900 & 148.200 & 490.029 & 221.074 \\
\hline 2000 & 149.030 & 497.652 & 235.936 \\
\hline
\end{tabular}

$T$ : Temperature. $C_{P}$ : Heat capacity at constant pressure. $S$ : Entropy. $H$ : Enthalpy. 
Figure S.24.4. $\mathrm{B}_{4} \mathrm{H}_{3}$ : Ideal-gas thermodynamic functions at 1 bar (anharmonic B3LYPD2/cc-pVTZ results)
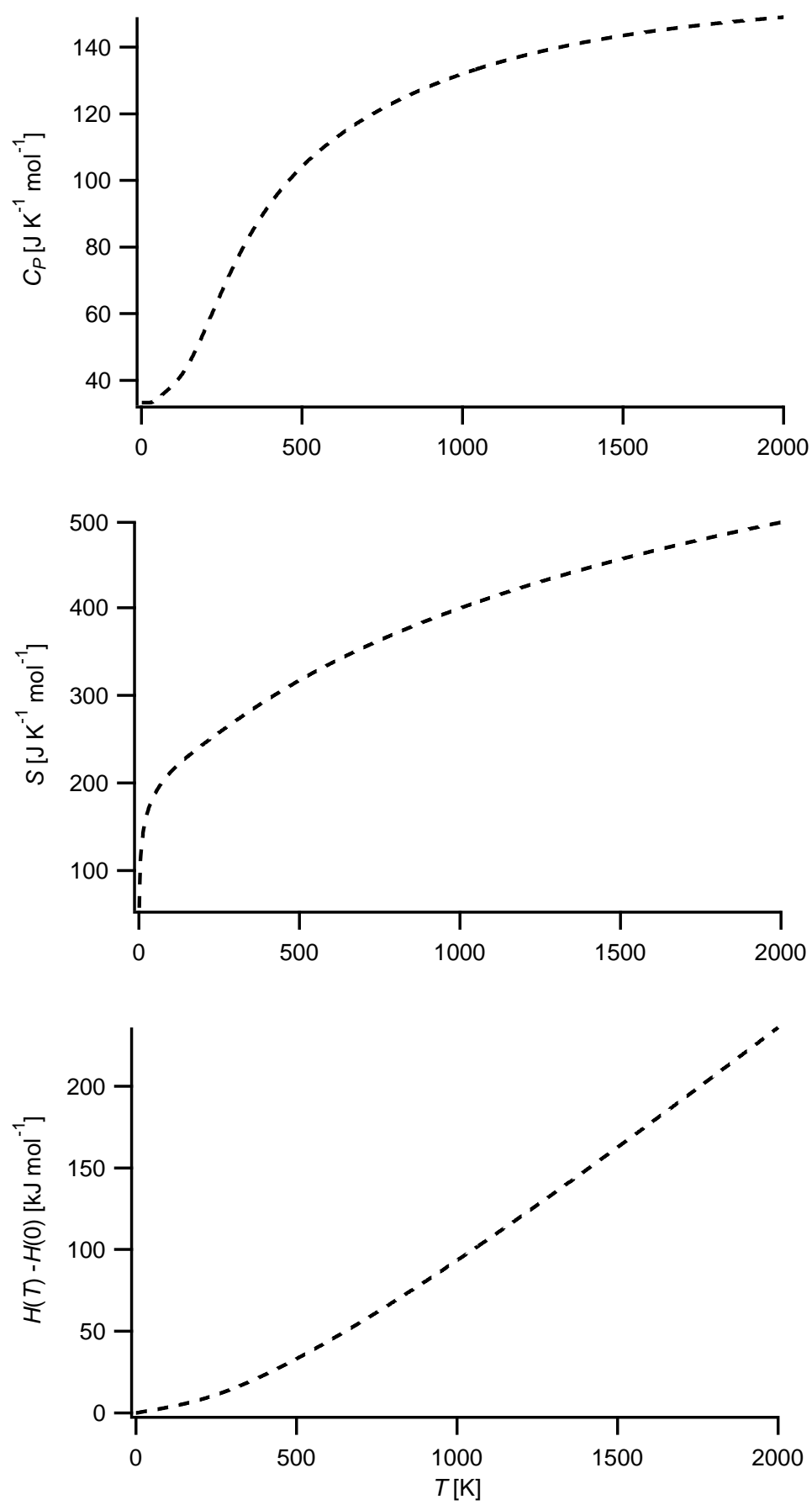

$T$ : Temperature. $C_{P}$ : Heat capacity at constant pressure. $S$ : Entropy. $H$ : Enthalpy. 
Table S.24.5. $\mathrm{B}_{4} \mathrm{H}_{3}$ : Fits (red solid lines) of the computed thermodynamic functions (blue dashed lines) with NASA type functions (Equations (1) - (3)) in the 200 - $900 \mathrm{~K}$ temperature range (anharmonic B3LYP-D2/cc-pVTZ results). In each case, the difference curve between the thermodynamic and NASA functions is plotted in the upper graph

Fit parameters

\begin{tabular}{llllll}
\hline $\mathrm{a}_{1}$ & $-1.49006898 \mathrm{e}+00$ & $\mathrm{a}_{2}\left[\mathrm{~K}^{-1}\right]$ & $5.25014897 \mathrm{e}-02$ & $\mathrm{a}_{3}\left[\mathrm{~K}^{-2}\right]$ & $-6.74328199 \mathrm{e}-05$ \\
$\mathrm{a}_{4}\left[\mathrm{~K}^{-3}\right]$ & $4.16002550 \mathrm{e}-08$ & $\mathrm{a}_{5}\left[\mathrm{~K}^{-4}\right]$ & $-9.14410110 \mathrm{e}-12$ & $\mathrm{a}_{6}[\mathrm{~K}]$ & $3.86449967 \mathrm{e}+02$ \\
$\mathrm{a}_{7}$ & $2.79868350 \mathrm{e}+01$ & & & &
\end{tabular}
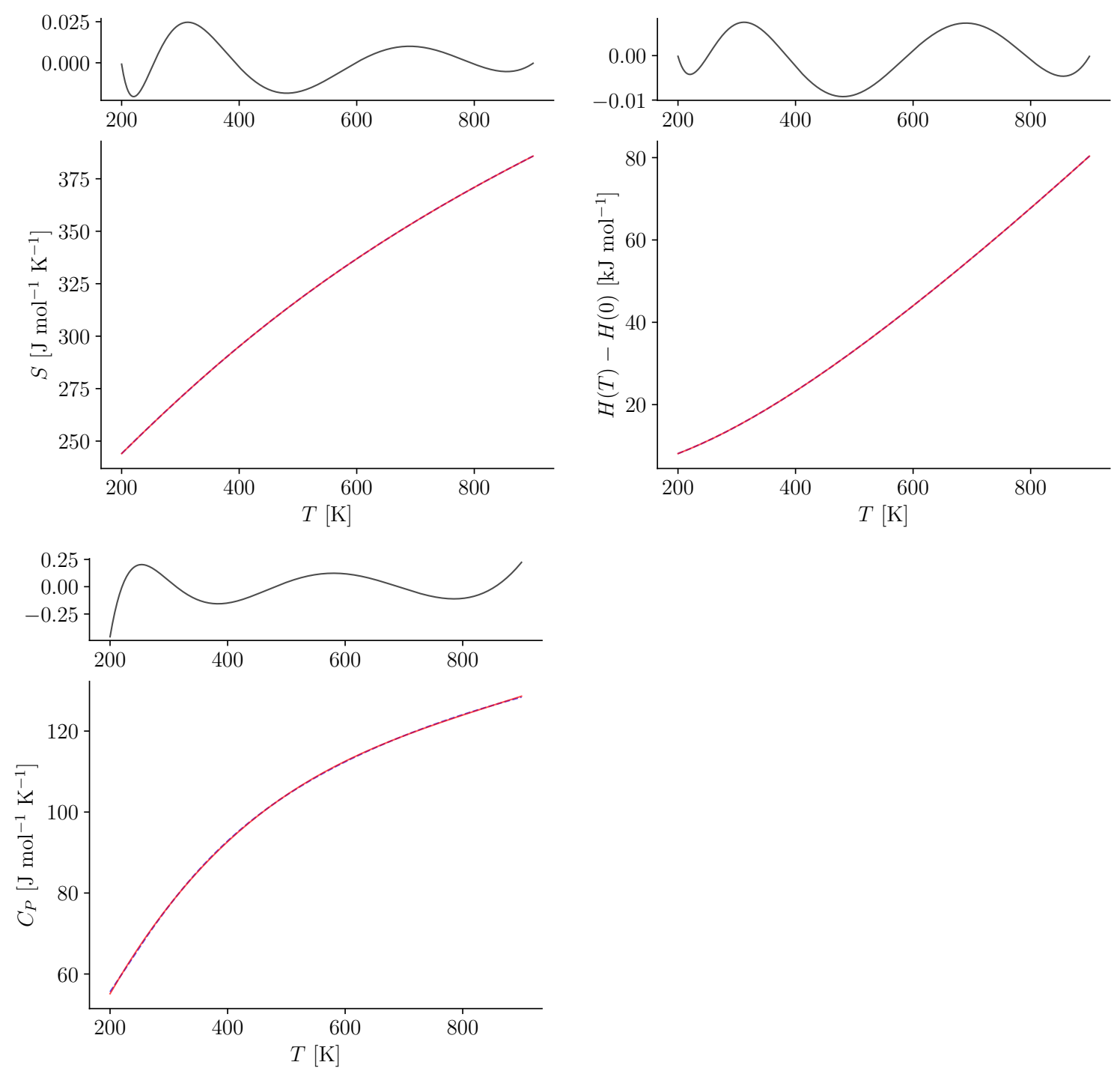


\section{$25 \mathrm{~B}_{4} \mathrm{H}_{4}$}

Figure S.25.1. Structure of $\mathrm{B}_{4} \mathrm{H}_{4}$

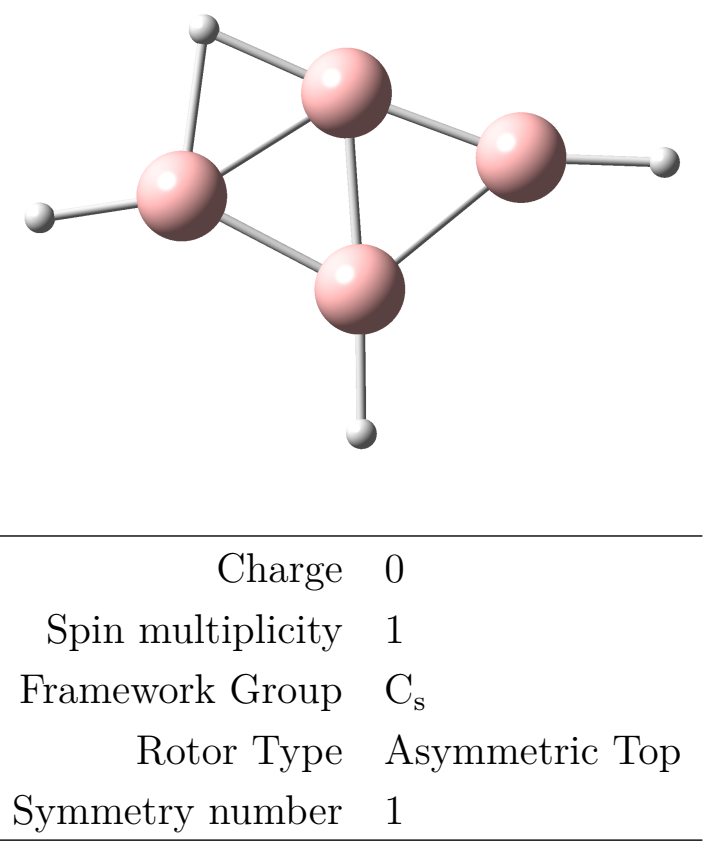

Table S.25.1. $\mathrm{B}_{4} \mathrm{H}_{4}$ : Selected anharmonic results obtained at the B3LYP-D2/cc-pVTZ level

\begin{tabular}{rll}
\hline $\mathrm{ZPE}_{\mathrm{v}}$ & 10759 & $\mathrm{~cm}^{-1}$ \\
$\mathrm{~A}$ & 0.82628 & $\mathrm{~cm}^{-1}$ \\
$\mathrm{~B}$ & 0.29547 & $\mathrm{~cm}^{-1}$ \\
$\mathrm{C}$ & 0.21764 & $\mathrm{~cm}^{-1}$ \\
\hline$C_{P}(300 \mathrm{~K})$ & 79.581 & $\mathrm{~J} \mathrm{~mol}^{-1} \mathrm{~K}-1$ \\
$S(300 \mathrm{~K})$ & 276.191 & $\mathrm{~J} \mathrm{~mol}^{-1} \mathrm{~K}-1$ \\
$H(300 \mathrm{~K})-H(0 \mathrm{~K})$ & 14.789 & $\mathrm{~kJ} \mathrm{~mol}^{-1}$ \\
\hline
\end{tabular}

$\mathrm{ZPE}_{\mathrm{v}}$ : vibrational contribution to the zero-point energy. A, B, C: Rotational constants. $C_{P}$ : Heat capacity at constant pressure. $S$ : Entropy. $H$ : Enthalpy. Ideal-gas calculations (1 bar). 
Table S.25.2. $\mathrm{B}_{4} \mathrm{H}_{4}$ : Cartesian coordinates $(\AA)$ of the B3LYP-D2/cc-pVTZ optimized structure

\begin{tabular}{cccc} 
atom & $\mathrm{x}$ & $\mathrm{y}$ & $\mathrm{z}$ \\
\hline B1 & 0.1089 & -0.8229 & -0.0000 \\
B2 & -1.3436 & -0.0616 & 0.0000 \\
B3 & 1.4239 & 0.2521 & -0.0000 \\
B4 & 0.0000 & 0.7790 & 0.0000 \\
H5 & 2.5944 & 0.2123 & -0.0000 \\
H6 & -1.1617 & 1.2959 & 0.0000 \\
H7 & 0.1223 & -2.0012 & -0.0000 \\
H8 & -2.5011 & -0.2401 & 0.0000 \\
\hline
\end{tabular}


Table S.25.3. $\mathrm{B}_{4} \mathrm{H}_{4}$ : Vibrational frequencies, infrared integrated intensities and Raman activities (B3LYP-D2/cc-pVTZ results)

\begin{tabular}{|c|c|c|c|c|c|c|c|}
\hline \multirow[b]{2}{*}{ mode } & \multirow[b]{2}{*}{ symm. } & \multicolumn{3}{|c|}{ Harmonic } & \multicolumn{3}{|c|}{ Anharmonic } \\
\hline & & $\begin{array}{c}\omega \\
{\left[\mathrm{cm}^{-1}\right]}\end{array}$ & $\begin{array}{c}\mathrm{IR} \\
{\left[\mathrm{km} \mathrm{mol}^{-1}\right]}\end{array}$ & $\begin{array}{c}\text { Raman } \\
{\left[\AA^{6}\right]}\end{array}$ & $\begin{array}{c}\omega \\
{\left[\mathrm{cm}^{-1}\right]}\end{array}$ & $\begin{array}{c}\mathrm{IR} \\
{\left[\mathrm{km} \mathrm{mol}^{-1}\right]}\end{array}$ & $\begin{array}{c}\text { Raman } \\
{\left[\AA^{6}\right]}\end{array}$ \\
\hline 1 & A" & 246 & 0.509 & 0.041 & 247 & 0.376 & 0.033 \\
\hline 2 & $\mathrm{~A}^{\prime}$ & 470 & 34.576 & 0.193 & 454 & 33.297 & 0.181 \\
\hline 3 & A" & 558 & 12.655 & 0.050 & 535 & 10.967 & 0.033 \\
\hline 4 & $\mathrm{~A}^{\prime}$ & 595 & 4.333 & 0.160 & 579 & 5.264 & 0.157 \\
\hline 5 & $A^{\prime \prime}$ & 703 & 0.001 & 0.005 & 686 & 0.000 & 0.002 \\
\hline 6 & $A^{\prime}$ & 743 & 2.008 & 0.093 & 711 & 1.797 & 0.068 \\
\hline 7 & $A^{\prime \prime}$ & 759 & 4.503 & 0.054 & 748 & 5.560 & 0.041 \\
\hline 8 & $A^{\prime \prime}$ & 796 & 13.783 & 0.012 & 787 & 11.982 & 0.008 \\
\hline 9 & $A^{\prime}$ & 809 & 0.242 & 0.178 & 774 & 0.916 & 0.262 \\
\hline 10 & $\mathrm{~A}^{\prime}$ & 858 & 26.034 & 0.232 & 833 & 21.053 & 0.233 \\
\hline 11 & $A^{\prime}$ & 918 & 20.299 & 0.599 & 891 & 19.305 & 0.467 \\
\hline 12 & $A^{\prime}$ & 1079 & 1.408 & 1.522 & 1049 & 0.758 & 1.303 \\
\hline 13 & $A^{\prime}$ & 1288 & 80.609 & 0.076 & 1202 & 33.864 & 0.059 \\
\hline 14 & $A^{\prime}$ & 1413 & 4.705 & 0.180 & 1231 & 44.069 & 0.296 \\
\hline 15 & $A^{\prime}$ & 2254 & 3.298 & 0.286 & 2227 & 2.276 & 0.279 \\
\hline 16 & $A^{\prime}$ & 2733 & 10.247 & 0.781 & 2626 & 10.901 & 0.864 \\
\hline 17 & $\mathrm{~A}^{\prime}$ & 2803 & 74.539 & 0.490 & 2704 & 88.618 & 0.438 \\
\hline 18 & $\mathrm{~A}^{\prime}$ & 2811 & 26.389 & 0.639 & 2701 & 20.995 & 0.838 \\
\hline
\end{tabular}


Figure S.25.2. Anharmonic IR spectrum of $\mathrm{B}_{4} \mathrm{H}_{4}$ obtained by convoluting the calculated intensities with Lorentzians having a FWHM of $4 \mathrm{~cm}^{-1}$ (B3LYP-D2/cc-pVTZ results): (top) full spectrum, (middle) contributions from fundamentals and combination bands, (bottom) contributions from overtones
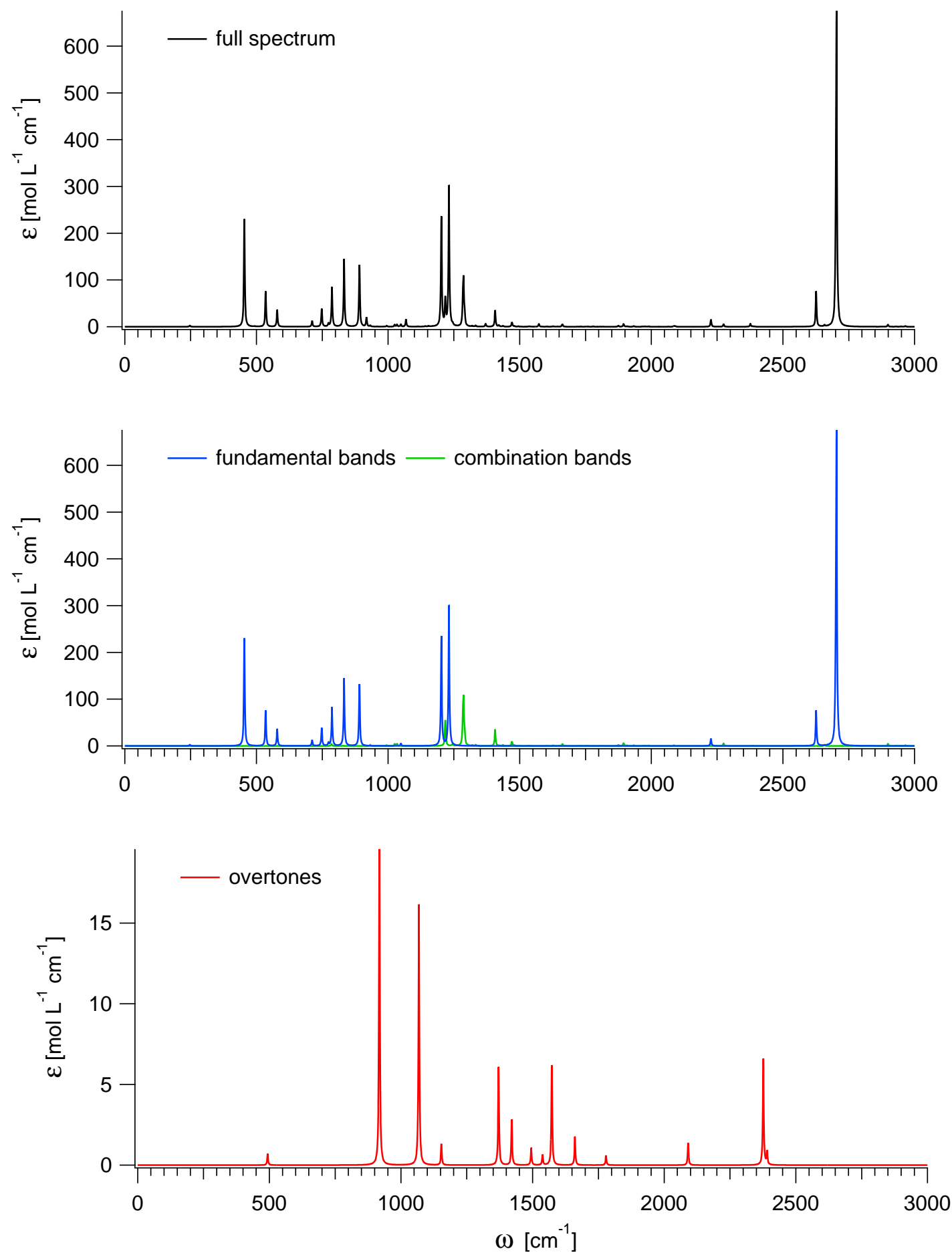
Figure S.25.3. Anharmonic Raman spectrum of $\mathrm{B}_{4} \mathrm{H}_{4}$ obtained by convoluting the calculated activities with Lorentzians having a FWHM of $4 \mathrm{~cm}^{-1}$ (B3LYP-D2/cc-pVTZ results): (top) full spectrum, (middle) contributions from fundamentals and combination bands, (bottom) contributions from overtones
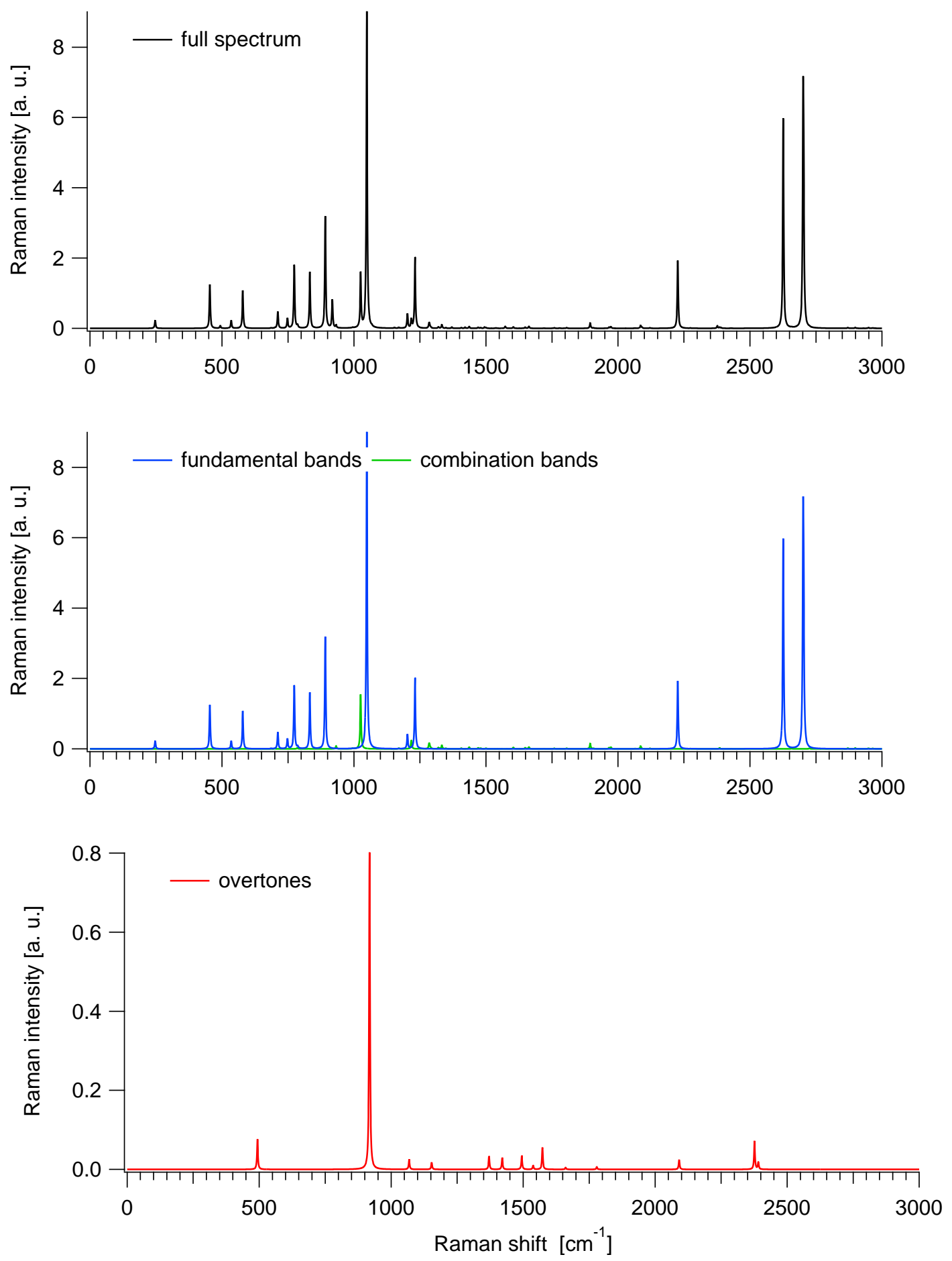
Table S.25.4. $\mathrm{B}_{4} \mathrm{H}_{4}$ : Ideal-gas thermodynamic functions at 1 bar (anharmonic B3LYPD2/cc-pVTZ results)

\begin{tabular}{|c|c|c|c|}
\hline $\begin{array}{c}T \\
{[\mathrm{~K}]}\end{array}$ & $\begin{array}{c}C_{P} \\
{\left[\mathrm{~J} \mathrm{~mol}^{-1} \mathrm{~K}^{-1}\right]}\end{array}$ & $\begin{array}{c}S \\
{\left[\mathrm{~J} \mathrm{~mol}^{-1} \mathrm{~K}^{-1}\right]}\end{array}$ & $\begin{array}{c}H(T)-H(0 K) \\
{\left[\mathrm{kJ} \mathrm{mol}^{-1}\right]}\end{array}$ \\
\hline 100 & 37.460 & 218.045 & 3.427 \\
\hline 200 & 56.011 & 248.955 & 7.992 \\
\hline 300 & 79.581 & 276.191 & 14.789 \\
\hline 400 & 98.684 & 301.834 & 23.749 \\
\hline 500 & 112.843 & 325.453 & 34.360 \\
\hline 600 & 123.592 & 347.018 & 46.204 \\
\hline 700 & 132.100 & 366.732 & 59.004 \\
\hline 800 & 139.037 & 384.840 & 72.572 \\
\hline 900 & 144.790 & 401.558 & 86.772 \\
\hline 1000 & 149.603 & 417.070 & 101.499 \\
\hline 1100 & 153.655 & 431.524 & 116.667 \\
\hline 1200 & 157.081 & 445.045 & 132.209 \\
\hline 1300 & 159.991 & 457.736 & 148.066 \\
\hline 1400 & 162.474 & 469.686 & 164.193 \\
\hline 1500 & 164.602 & 480.970 & 180.549 \\
\hline 1600 & 166.435 & 491.653 & 197.103 \\
\hline 1700 & 168.021 & 501.792 & 213.828 \\
\hline 1800 & 169.400 & 511.435 & 230.701 \\
\hline 1900 & 170.605 & 520.628 & 247.702 \\
\hline 2000 & 171.663 & 529.406 & 264.817 \\
\hline
\end{tabular}

$T$ : Temperature. $C_{P}$ : Heat capacity at constant pressure. $S$ : Entropy. $H$ : Enthalpy. 
Figure S.25.4. $\mathrm{B}_{4} \mathrm{H}_{4}$ : Ideal-gas thermodynamic functions at 1 bar (anharmonic B3LYPD2/cc-pVTZ results)
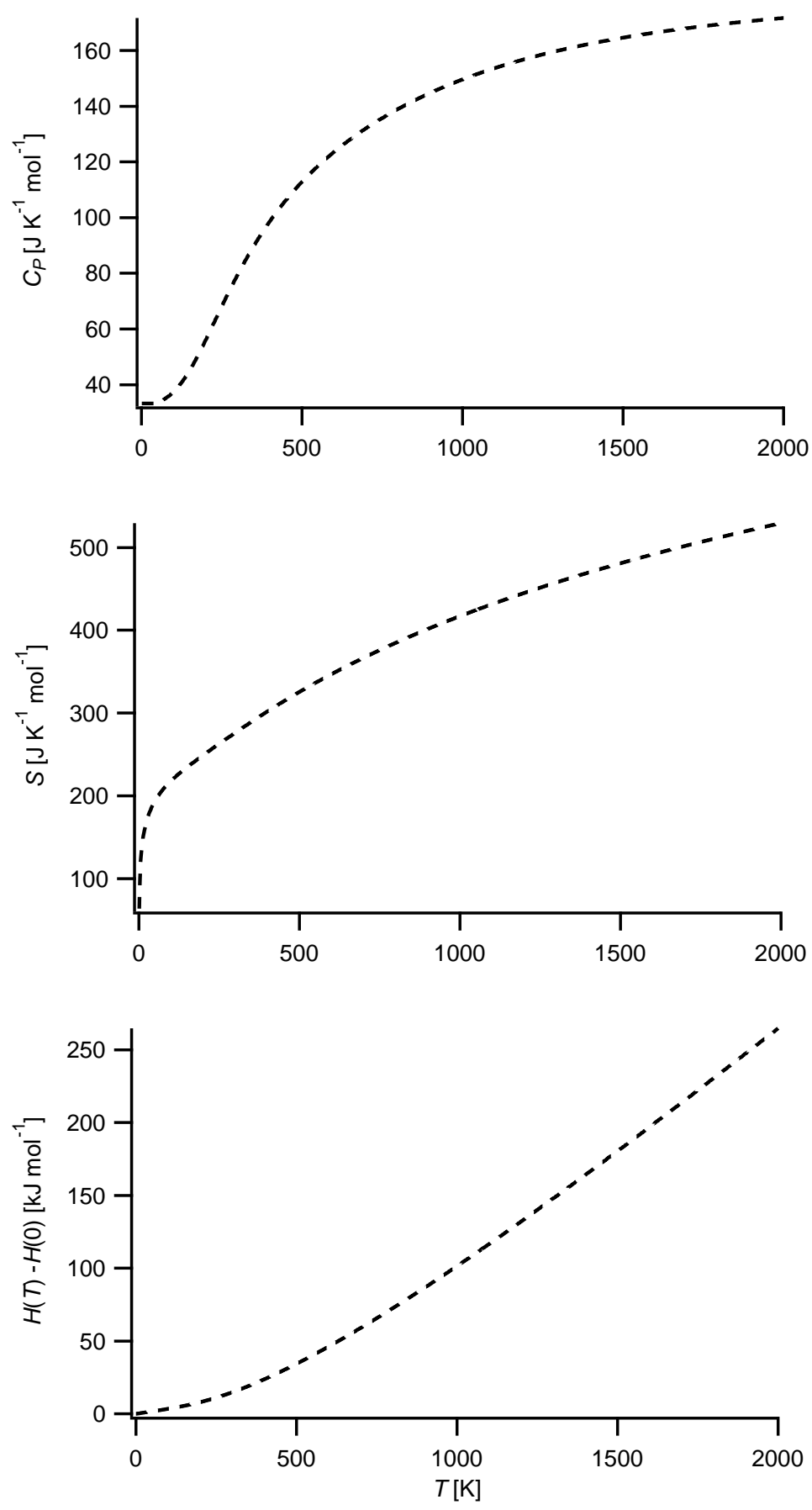

$T$ : Temperature. $C_{P}$ : Heat capacity at constant pressure. $S$ : Entropy. $H$ : Enthalpy. 
Table S.25.5. $\mathrm{B}_{4} \mathrm{H}_{4}$ : Fits (red solid lines) of the computed thermodynamic functions (blue dashed lines) with NASA type functions (Equations (1) - (3)) in the 200 - $900 \mathrm{~K}$ temperature range (anharmonic B3LYP-D2/cc-pVTZ results). In each case, the difference curve between the thermodynamic and NASA functions is plotted in the upper graph

Fit parameters

\begin{tabular}{llllll}
\hline $\mathrm{a}_{1}$ & $-1.45008503 \mathrm{e}+00$ & $\mathrm{a}_{2}\left[\mathrm{~K}^{-1}\right]$ & $4.92877986 \mathrm{e}-02$ & $\mathrm{a}_{3}\left[\mathrm{~K}^{-2}\right]$ & $-4.63009233 \mathrm{e}-05$ \\
$\mathrm{a}_{4}\left[\mathrm{~K}^{-3}\right]$ & $1.45821323 \mathrm{e}-08$ & $\mathrm{a}_{5}\left[\mathrm{~K}^{-4}\right]$ & $2.16874272 \mathrm{e}-12$ & $\mathrm{a}_{6}[\mathrm{~K}]$ & $3.82947082 \mathrm{e}+02$ \\
$\mathrm{a}_{7}$ & $2.86540506 \mathrm{e}+01$ & & & &
\end{tabular}
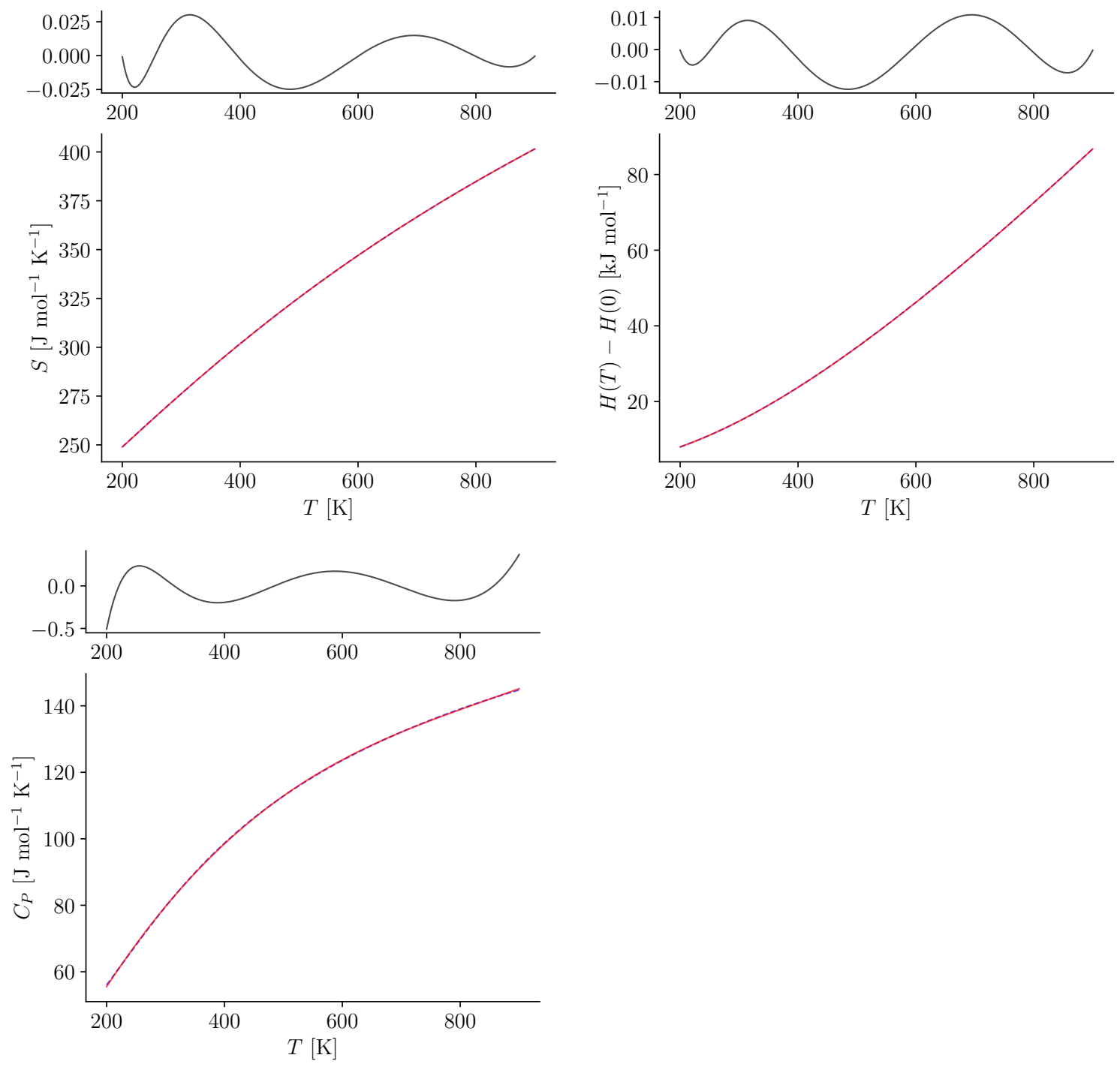


\section{$26 \quad \mathrm{~B}_{4} \mathrm{H}_{5}$ - Isomer $\mathrm{A}$}

Figure S.26.1. Structure of $\mathrm{B}_{4} \mathrm{H}_{5}$ - Isomer A

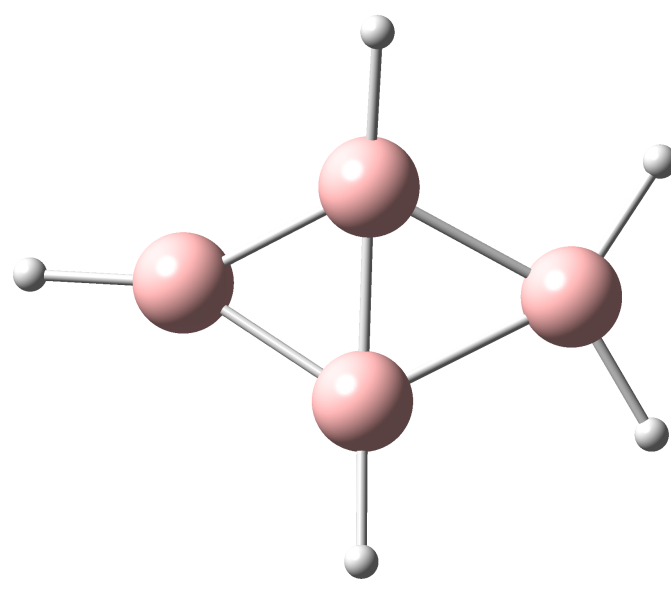

\begin{aligned} & \hline Charge 0 \\ & Spin multiplicity 2 \\ & Framework Group $\mathrm{C}_{\mathrm{s}} \\ &$ Rotor Type Asymmetric Top \\ & Symmetry number 1 \\ & \hline\end{aligned}

Table S.26.1. $\mathrm{B}_{4} \mathrm{H}_{5}$ - Isomer A: Selected anharmonic results obtained at the B3LYPD2/cc-pVTZ level

\begin{tabular}{rll}
\hline $\mathrm{ZPE}_{\mathrm{v}}$ & 12240 & $\mathrm{~cm}^{-1}$ \\
$\mathrm{~A}$ & 0.64488 & $\mathrm{~cm}^{-1}$ \\
$\mathrm{~B}$ & 0.26993 & $\mathrm{~cm}^{-1}$ \\
$\mathrm{C}$ & 0.20368 & $\mathrm{~cm}^{-1}$ \\
\hline$C_{P}(300 \mathrm{~K})$ & 95.255 & $\mathrm{~J} \mathrm{~mol}^{-1} \mathrm{~K}-1$ \\
$S(300 \mathrm{~K})$ & 297.493 & $\mathrm{~J} \mathrm{~mol}^{-1} \mathrm{~K}^{-1}$ \\
$H(300 \mathrm{~K})-H(0 \mathrm{~K})$ & 17.645 & $\mathrm{~kJ} \mathrm{~mol}^{-1}$ \\
\hline
\end{tabular}

$\mathrm{ZPE}_{\mathrm{v}}$ : vibrational contribution to the zero-point energy. A, B, C: Rotational constants. $C_{P}$ : Heat capacity at constant pressure. $S$ : Entropy. $H$ : Enthalpy. Ideal-gas calculations (1 bar). 
Table S.26.2. $\mathrm{B}_{4} \mathrm{H}_{5}$ - Isomer A: Cartesian coordinates $(\AA)$ of the B3LYP-D2/cc-pVTZ optimized structure

\begin{tabular}{cccc} 
atom & $\mathrm{x}$ & $\mathrm{y}$ & $\mathrm{z}$ \\
\hline B1 & -0.2424 & -0.0205 & 0.7861 \\
B2 & -0.2424 & -0.0205 & -0.7861 \\
B3 & 0.5986 & 1.2645 & -0.0000 \\
B4 & -0.2424 & -1.4533 & 0.0000 \\
H5 & -0.4609 & 0.0945 & 1.9416 \\
H6 & 0.9480 & 1.7903 & -1.0012 \\
H7 & -0.3308 & -2.6206 & 0.0000 \\
H8 & 0.9480 & 1.7903 & 1.0012 \\
H9 & -0.4609 & 0.0945 & -1.9416 \\
\hline
\end{tabular}


Table S.26.3. $\mathrm{B}_{4} \mathrm{H}_{5}$ - Isomer A: Vibrational frequencies, infrared integrated intensities and Raman activities (B3LYP-D2/cc-pVTZ results)

\begin{tabular}{|c|c|c|c|c|c|c|c|}
\hline \multirow[b]{2}{*}{ mode } & \multirow[b]{2}{*}{ symm. } & \multicolumn{3}{|c|}{ Harmonic } & \multicolumn{3}{|c|}{ Anharmonic } \\
\hline & & $\begin{array}{c}\omega \\
{\left[\mathrm{cm}^{-1}\right]}\end{array}$ & $\begin{array}{c}\mathrm{IR} \\
{\left[\mathrm{km} \mathrm{mol}^{-1}\right]}\end{array}$ & $\begin{array}{c}\text { Raman } \\
{\left[\AA^{6}\right]}\end{array}$ & $\begin{array}{c}\omega \\
{\left[\mathrm{cm}^{-1}\right]}\end{array}$ & $\begin{array}{c}\mathrm{IR} \\
{\left[\mathrm{km} \mathrm{mol}^{-1}\right]}\end{array}$ & $\begin{array}{c}\text { Raman } \\
{\left[\AA^{6}\right]}\end{array}$ \\
\hline 1 & $A^{\prime}$ & 113 & 0.743 & 1.368 & 89 & 0.667 & 1.534 \\
\hline 2 & A" & 313 & 11.614 & 0.012 & 260 & 7.310 & 0.010 \\
\hline 3 & A" & 430 & 1.164 & 0.096 & 413 & 1.792 & 0.083 \\
\hline 4 & A" & 498 & 0.022 & 0.023 & 472 & 0.089 & 0.023 \\
\hline 5 & A" & 575 & 10.193 & 0.090 & 556 & 8.435 & 0.087 \\
\hline 6 & $A^{\prime}$ & 635 & 3.134 & 0.178 & 624 & 1.026 & 0.312 \\
\hline 7 & $A^{\prime}$ & 646 & 0.975 & 0.256 & 603 & 3.392 & 0.102 \\
\hline 8 & A" & 675 & 0.979 & 0.021 & 650 & 0.834 & 0.015 \\
\hline 9 & $A^{\prime}$ & 764 & 12.375 & 0.195 & 750 & 6.882 & 0.155 \\
\hline 10 & A" & 783 & 2.141 & 0.219 & 755 & 1.864 & 0.193 \\
\hline 11 & $A^{\prime}$ & 861 & 22.686 & 0.024 & 831 & 13.624 & 0.003 \\
\hline 12 & A" & 862 & 4.987 & 0.108 & 834 & 3.018 & 0.133 \\
\hline 13 & $A^{\prime}$ & 881 & 9.321 & 0.046 & 855 & 5.358 & 0.030 \\
\hline 14 & $A^{\prime}$ & 1008 & 6.361 & 0.133 & 966 & 2.407 & 0.075 \\
\hline 15 & $A^{\prime}$ & 1101 & 5.407 & 1.549 & 1078 & 3.426 & 0.948 \\
\hline 16 & $A^{\prime}$ & 1173 & 4.429 & 0.181 & 1144 & 2.063 & 0.154 \\
\hline 17 & $A^{\prime}$ & 2648 & 31.254 & 1.035 & 2539 & 32.842 & 1.241 \\
\hline 18 & $A^{\prime \prime}$ & 2675 & 37.580 & 0.203 & 2562 & 16.486 & 0.326 \\
\hline 19 & A" & 2700 & 19.352 & 0.566 & 2582 & 42.007 & 0.518 \\
\hline 20 & $A^{\prime}$ & 2702 & 4.516 & 1.442 & 2590 & 4.211 & 1.657 \\
\hline 21 & $\mathrm{~A}^{\prime}$ & 2803 & 44.610 & 0.704 & 2707 & 47.029 & 0.772 \\
\hline
\end{tabular}


Figure S.26.2. Anharmonic IR spectrum of $\mathrm{B}_{4} \mathrm{H}_{5}$ - Isomer A obtained by convoluting the calculated intensities with Lorentzians having a FWHM of $4 \mathrm{~cm}^{-1}$ (B3LYP-D2/cc-pVTZ results): (top) full spectrum, (middle) contributions from fundamentals and combination bands, (bottom) contributions from overtones
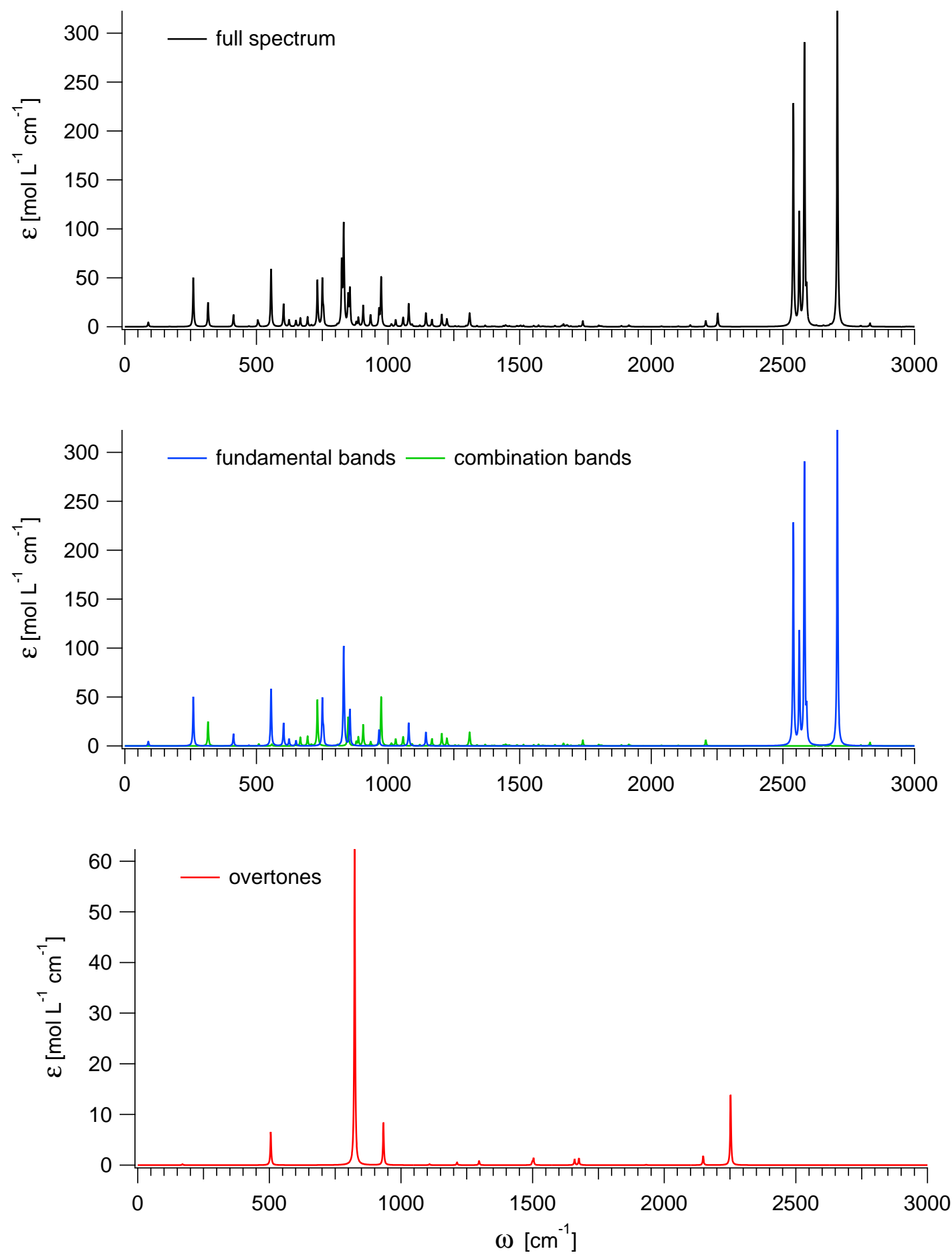
Figure S.26.3. Anharmonic Raman spectrum of $\mathrm{B}_{4} \mathrm{H}_{5}$ - Isomer A obtained by convoluting the calculated activities with Lorentzians having a FWHM of $4 \mathrm{~cm}^{-1}$ (B3LYP-D2/cc-pVTZ results): (top) full spectrum, (middle) contributions from fundamentals and combination bands, (bottom) contributions from overtones
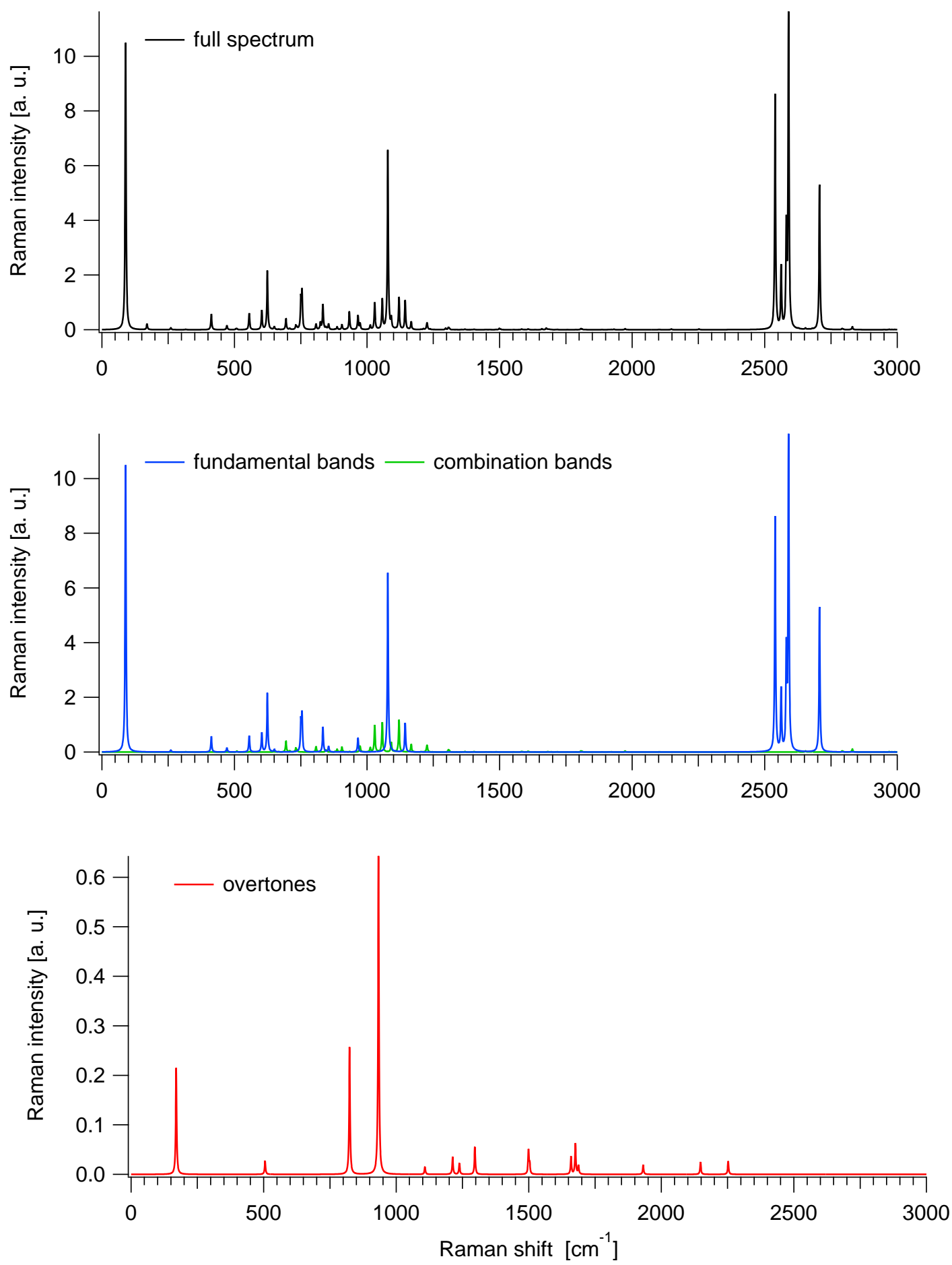
Table S.26.4. $\mathrm{B}_{4} \mathrm{H}_{5}$ - Isomer A: Ideal-gas thermodynamic functions at 1 bar (anharmonic B3LYP-D2/cc-pVTZ results)

\begin{tabular}{cccc}
\hline$T$ & $C_{P}$ & $S$ & $H(T)-H(0 K)$ \\
{$[\mathrm{K}]$} & {$\left[\mathrm{J} \mathrm{mol}^{-1} \mathrm{~K}^{-1}\right]$} & {$\left[\mathrm{J} \mathrm{mol}^{-1} \mathrm{~K}^{-1}\right]$} & {$\left[\mathrm{kJ} \mathrm{mol}^{-1}\right]$} \\
\hline 100 & 45.119 & 226.739 & 3.835 \\
200 & 68.579 & 264.501 & 9.417 \\
300 & 95.255 & 297.493 & 17.645 \\
400 & 115.713 & 327.861 & 28.250 \\
500 & 130.430 & 355.347 & 40.595 \\
600 & 141.546 & 380.152 & 54.217 \\
700 & 150.452 & 402.663 & 68.832 \\
800 & 157.850 & 423.250 & 84.257 \\
900 & 164.098 & 442.213 & 100.363 \\
1000 & 169.408 & 459.785 & 117.046 \\
1100 & 173.935 & 476.149 & 134.219 \\
1200 & 177.801 & 491.454 & 151.811 \\
1300 & 181.111 & 505.820 & 169.761 \\
1400 & 183.953 & 519.348 & 188.018 \\
1500 & 186.402 & 532.125 & 206.538 \\
1600 & 188.519 & 544.224 & 225.287 \\
1700 & 190.358 & 555.710 & 244.233 \\
1800 & 191.961 & 566.637 & 263.351 \\
1900 & 193.365 & 577.054 & 282.618 \\
2000 & 194.600 & 587.004 & 302.018 \\
\hline$T$ & & & $S: E n t o p y$ \\
\hline
\end{tabular}

$T$ : Temperature. $C_{P}$ : Heat capacity at constant pressure. $S$ : Entropy. $H$ : Enthalpy. 
Figure S.26.4. $\mathrm{B}_{4} \mathrm{H}_{5}$ - Isomer A: Ideal-gas thermodynamic functions at 1 bar (anharmonic B3LYP-D2/cc-pVTZ results)
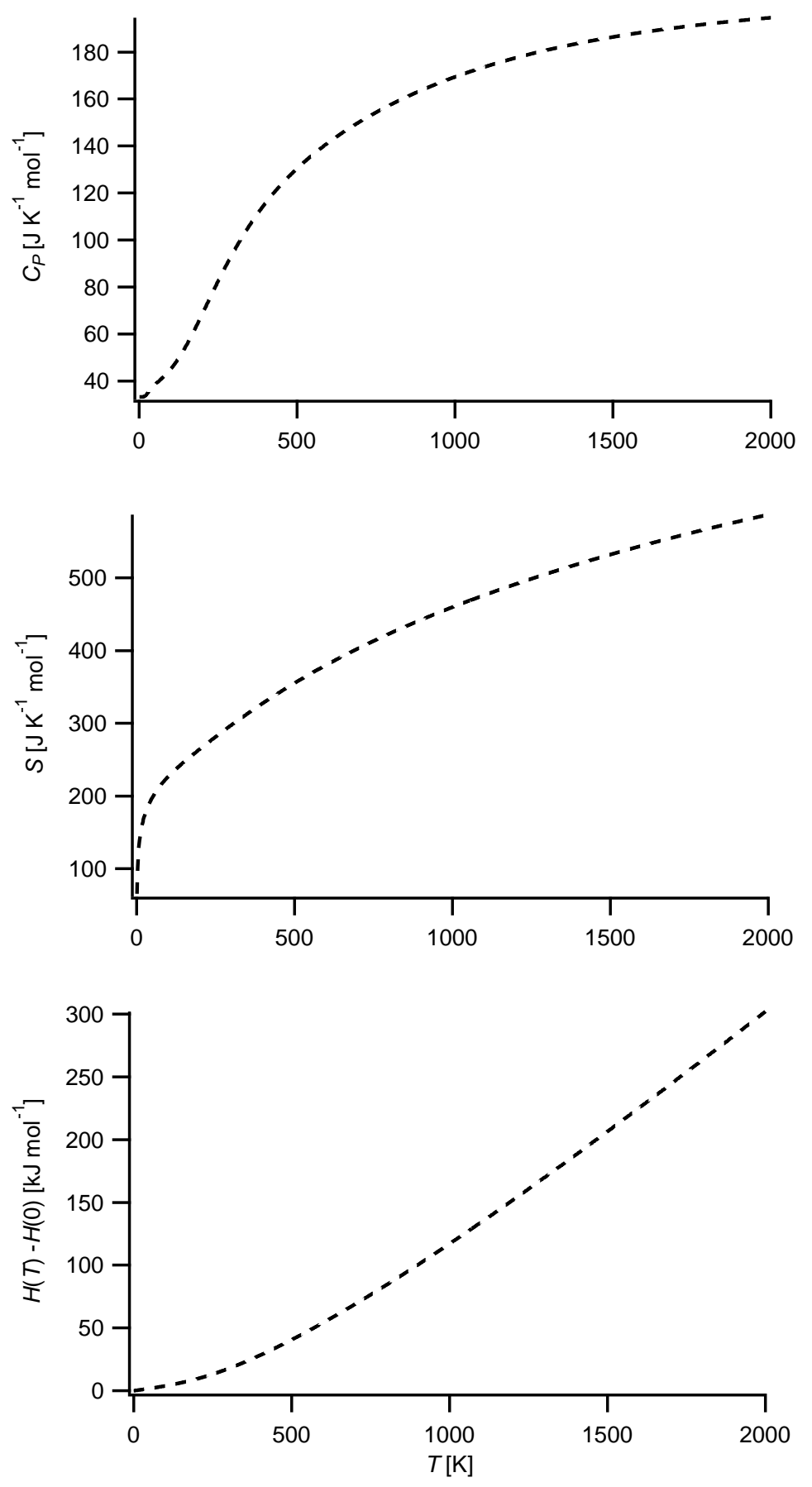

$T$ : Temperature. $C_{P}$ : Heat capacity at constant pressure. $S$ : Entropy. $H$ : Enthalpy. 
Table S.26.5. $\mathrm{B}_{4} \mathrm{H}_{5}$ - Isomer A: Fits (red solid lines) of the computed thermodynamic functions (blue dashed lines) with NASA type functions (Equations (1) - (3)) in the 200 - $900 \mathrm{~K}$ temperature range (anharmonic B3LYP-D2/cc-pVTZ results). In each case, the difference curve between the thermodynamic and NASA functions is plotted in the upper graph

Fit parameters

\begin{tabular}{llllll}
\hline $\mathrm{a}_{1}$ & $-1.91597228 \mathrm{e}+00$ & $\mathrm{a}_{2}\left[\mathrm{~K}^{-1}\right]$ & $6.51429263 \mathrm{e}-02$ & $\mathrm{a}_{3}\left[\mathrm{~K}^{-2}\right]$ & $-8.32870010 \mathrm{e}-05$ \\
$\mathrm{a}_{4}\left[\mathrm{~K}^{-3}\right]$ & $5.31442140 \mathrm{e}-08$ & $\mathrm{a}_{5}\left[\mathrm{~K}^{-4}\right]$ & $-1.25356799 \mathrm{e}-11$ & $\mathrm{a}_{6}[\mathrm{~K}]$ & $4.14617946 \mathrm{e}+02$ \\
$\mathrm{a}_{7}$ & $3.04639079 \mathrm{e}+01$ & & & &
\end{tabular}
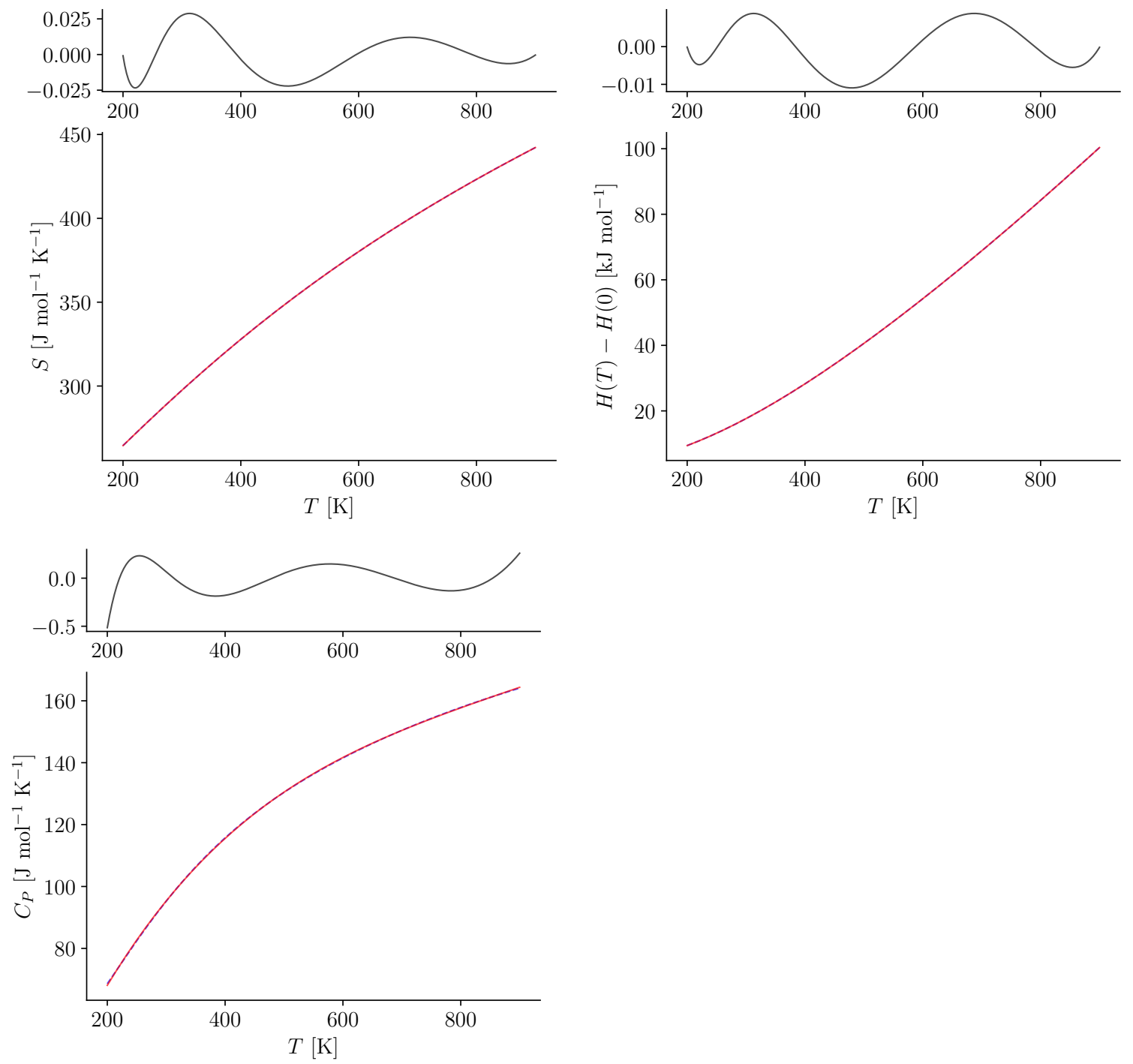


\section{$27 \quad \mathrm{~B}_{4} \mathrm{H}_{5}$ - Isomer $\mathrm{B}$}

Figure S.27.1. Structure of $\mathrm{B}_{4} \mathrm{H}_{5}$ - Isomer B

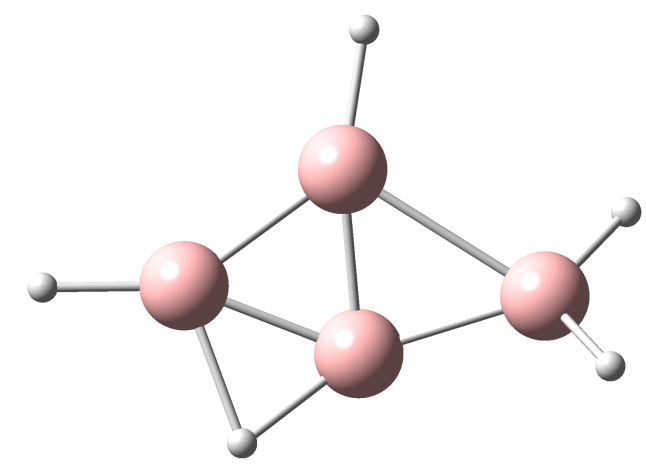

\begin{aligned} & \hline Charge 0 \\ & Spin multiplicity 2 \\ & Framework Group $\mathrm{C}_{1} \\ &$ Rotor Type Asymmetric Top \\ & Symmetry number 1 \\ & \hline\end{aligned}

Table S.27.1. $\mathrm{B}_{4} \mathrm{H}_{5}$ - Isomer B: Selected anharmonic results obtained at the B3LYP-D2/ccpVTZ level

\begin{tabular}{rll}
\hline $\mathrm{ZPE}_{\mathrm{v}}$ & 12356 & $\mathrm{~cm}^{-1}$ \\
$\mathrm{~A}$ & 0.75839 & $\mathrm{~cm}^{-1}$ \\
$\mathrm{~B}$ & 0.24706 & $\mathrm{~cm}^{-1}$ \\
$\mathrm{C}$ & 0.19385 & $\mathrm{~cm}^{-1}$ \\
\hline$C_{P}(300 \mathrm{~K})$ & 93.423 & $\mathrm{~J} \mathrm{~mol}^{-1} \mathrm{~K}-1$ \\
$S(300 \mathrm{~K})$ & 290.540 & $\mathrm{~J} \mathrm{~mol}^{-1} \mathrm{~K}-1$ \\
$H(300 \mathrm{~K})-H(0 \mathrm{~K})$ & 17.035 & $\mathrm{~kJ} \mathrm{~mol}^{-1}$ \\
\hline
\end{tabular}

$\mathrm{ZPE}_{\mathrm{v}}$ : vibrational contribution to the zero-point energy. A, B, C: Rotational constants. $C_{P}$ : Heat capacity at constant pressure. $S$ : Entropy. $H$ : Enthalpy. Ideal-gas calculations (1 bar). 
Table S.27.2. $\mathrm{B}_{4} \mathrm{H}_{5}$ - Isomer B: Cartesian coordinates $(\AA)$ of the B3LYP-D2/cc-pVTZ optimized structure

\begin{tabular}{cccc} 
atom & $\mathrm{x}$ & $\mathrm{y}$ & $\mathrm{z}$ \\
\hline B1 & -1.4681 & -0.0926 & 0.0156 \\
B2 & -0.0222 & -0.6545 & -0.1988 \\
B3 & 1.5170 & -0.1784 & 0.0060 \\
B4 & -0.1560 & 0.8698 & 0.0876 \\
H5 & 2.0588 & -0.7594 & 0.9017 \\
H6 & 2.1905 & 0.5168 & -0.6847 \\
H7 & -0.9875 & -1.3970 & 0.0960 \\
H8 & 0.0228 & 2.0257 & 0.0788 \\
H9 & -2.6383 & -0.1078 & 0.0561 \\
\hline
\end{tabular}


Table S.27.3. $\mathrm{B}_{4} \mathrm{H}_{5}$ - Isomer B: Vibrational frequencies, infrared integrated intensities and Raman activities (B3LYP-D2/cc-pVTZ results)

\begin{tabular}{|c|c|c|c|c|c|c|c|}
\hline \multirow[b]{2}{*}{ mode } & \multirow[b]{2}{*}{ symm. } & \multicolumn{3}{|c|}{ Harmonic } & \multicolumn{3}{|c|}{ Anharmonic } \\
\hline & & $\begin{array}{c}\omega \\
{\left[\mathrm{cm}^{-1}\right]}\end{array}$ & $\begin{array}{c}\mathrm{IR} \\
{\left[\mathrm{km} \mathrm{mol}^{-1}\right]}\end{array}$ & $\begin{array}{c}\text { Raman } \\
{\left[\AA^{6}\right]}\end{array}$ & $\begin{array}{c}\omega \\
{\left[\mathrm{cm}^{-1}\right]}\end{array}$ & $\begin{array}{c}\mathrm{IR} \\
{\left[\mathrm{km} \mathrm{mol}^{-1}\right]}\end{array}$ & $\begin{array}{c}\text { Raman } \\
{\left[\AA^{6}\right]}\end{array}$ \\
\hline 1 & $\mathrm{~A}$ & 207 & 0.689 & 0.141 & 204 & 0.486 & 0.130 \\
\hline 2 & $\mathrm{~A}$ & 271 & 3.168 & 0.193 & 255 & 2.560 & 0.185 \\
\hline 3 & $\mathrm{~A}$ & 452 & 3.516 & 0.355 & 422 & 6.822 & 0.124 \\
\hline 4 & $\mathrm{~A}$ & 464 & 19.468 & 0.215 & 441 & 12.060 & 0.474 \\
\hline 5 & $\mathrm{~A}$ & 581 & 15.795 & 0.076 & 561 & 16.149 & 0.053 \\
\hline 6 & $\mathrm{~A}$ & 626 & 6.335 & 0.026 & 591 & 5.006 & 0.015 \\
\hline 7 & $\mathrm{~A}$ & 653 & 3.250 & 0.123 & 629 & 3.172 & 0.100 \\
\hline 8 & $\mathrm{~A}$ & 722 & 3.730 & 0.186 & 693 & 3.049 & 0.155 \\
\hline 9 & $\mathrm{~A}$ & 773 & 13.587 & 0.219 & 749 & 4.774 & 0.243 \\
\hline 10 & $\mathrm{~A}$ & 781 & 4.411 & 0.038 & 743 & 15.818 & 0.078 \\
\hline 11 & $\mathrm{~A}$ & 824 & 14.612 & 0.307 & 789 & 6.124 & 0.353 \\
\hline 12 & $\mathrm{~A}$ & 855 & 4.531 & 0.118 & 830 & 5.137 & 0.023 \\
\hline 13 & $\mathrm{~A}$ & 1108 & 1.957 & 0.688 & 1059 & 1.069 & 0.307 \\
\hline 14 & A & 1185 & 1.154 & 0.586 & 1151 & 13.037 & 0.855 \\
\hline 15 & $\mathrm{~A}$ & 1188 & 39.151 & 0.601 & 1113 & 21.900 & 0.269 \\
\hline 16 & A & 1303 & 8.921 & 0.064 & 1064 & 6.499 & 0.132 \\
\hline 17 & $\mathrm{~A}$ & 2333 & 6.497 & 0.303 & 2265 & 3.959 & 0.302 \\
\hline 18 & $\mathrm{~A}$ & 2558 & 57.905 & 1.564 & 2441 & 51.010 & 1.748 \\
\hline 19 & $\mathrm{~A}$ & 2648 & 52.534 & 0.776 & 2531 & 49.688 & 0.926 \\
\hline 20 & $\mathrm{~A}$ & 2808 & 23.773 & 0.551 & 2698 & 23.552 & 0.583 \\
\hline 21 & $\mathrm{~A}$ & 2816 & 16.762 & 0.592 & 2704 & 19.571 & 0.746 \\
\hline
\end{tabular}


Figure S.27.2. Anharmonic IR spectrum of $\mathrm{B}_{4} \mathrm{H}_{5}$ - Isomer B obtained by convoluting the calculated intensities with Lorentzians having a FWHM of $4 \mathrm{~cm}^{-1}$ (B3LYP-D2/cc-pVTZ results): (top) full spectrum, (middle) contributions from fundamentals and combination bands, (bottom) contributions from overtones
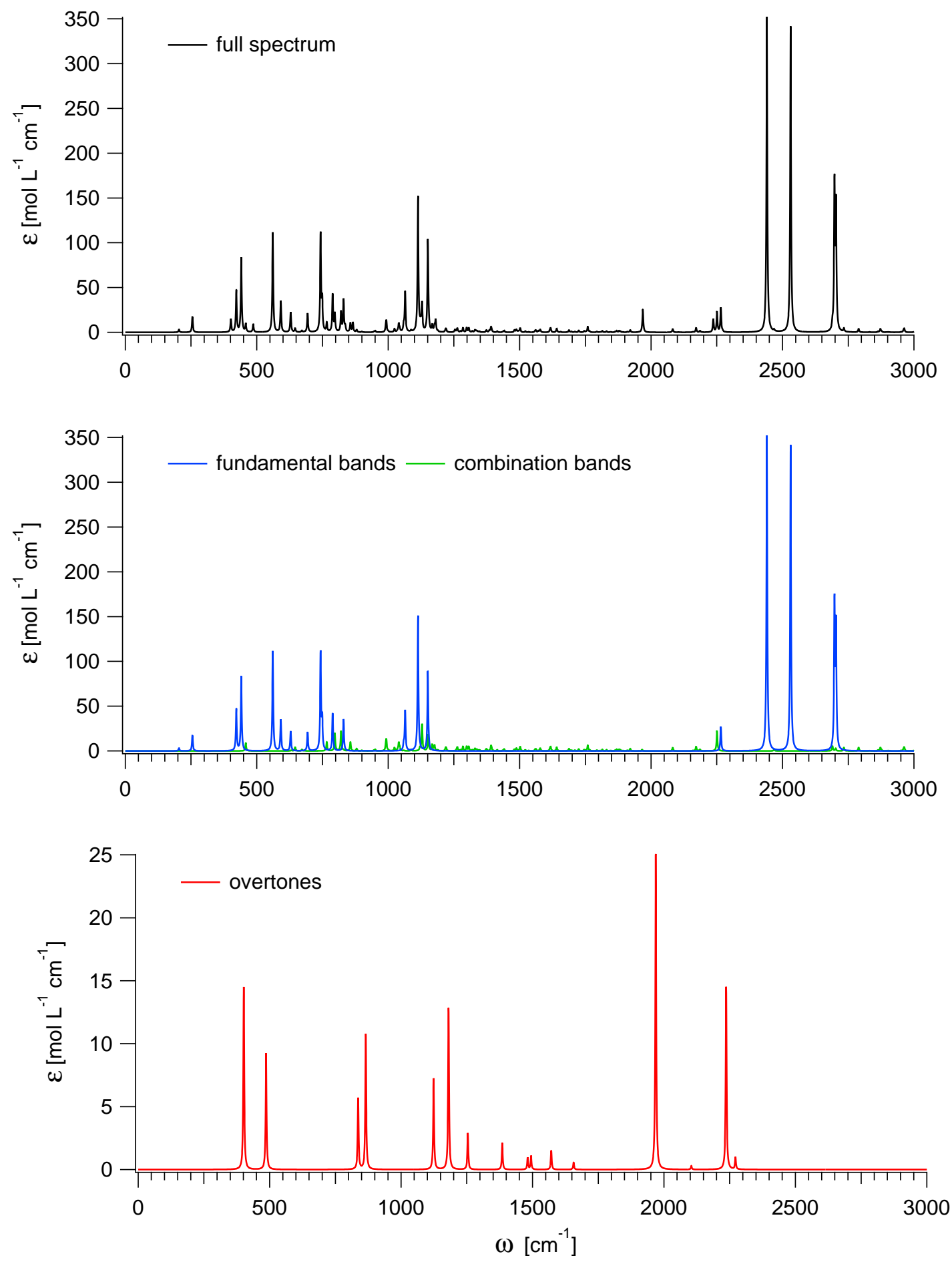
Figure S.27.3. Anharmonic Raman spectrum of $\mathrm{B}_{4} \mathrm{H}_{5}$ - Isomer B obtained by convoluting the calculated activities with Lorentzians having a FWHM of $4 \mathrm{~cm}^{-1}$ (B3LYP-D2/cc-pVTZ results): (top) full spectrum, (middle) contributions from fundamentals and combination bands, (bottom) contributions from overtones
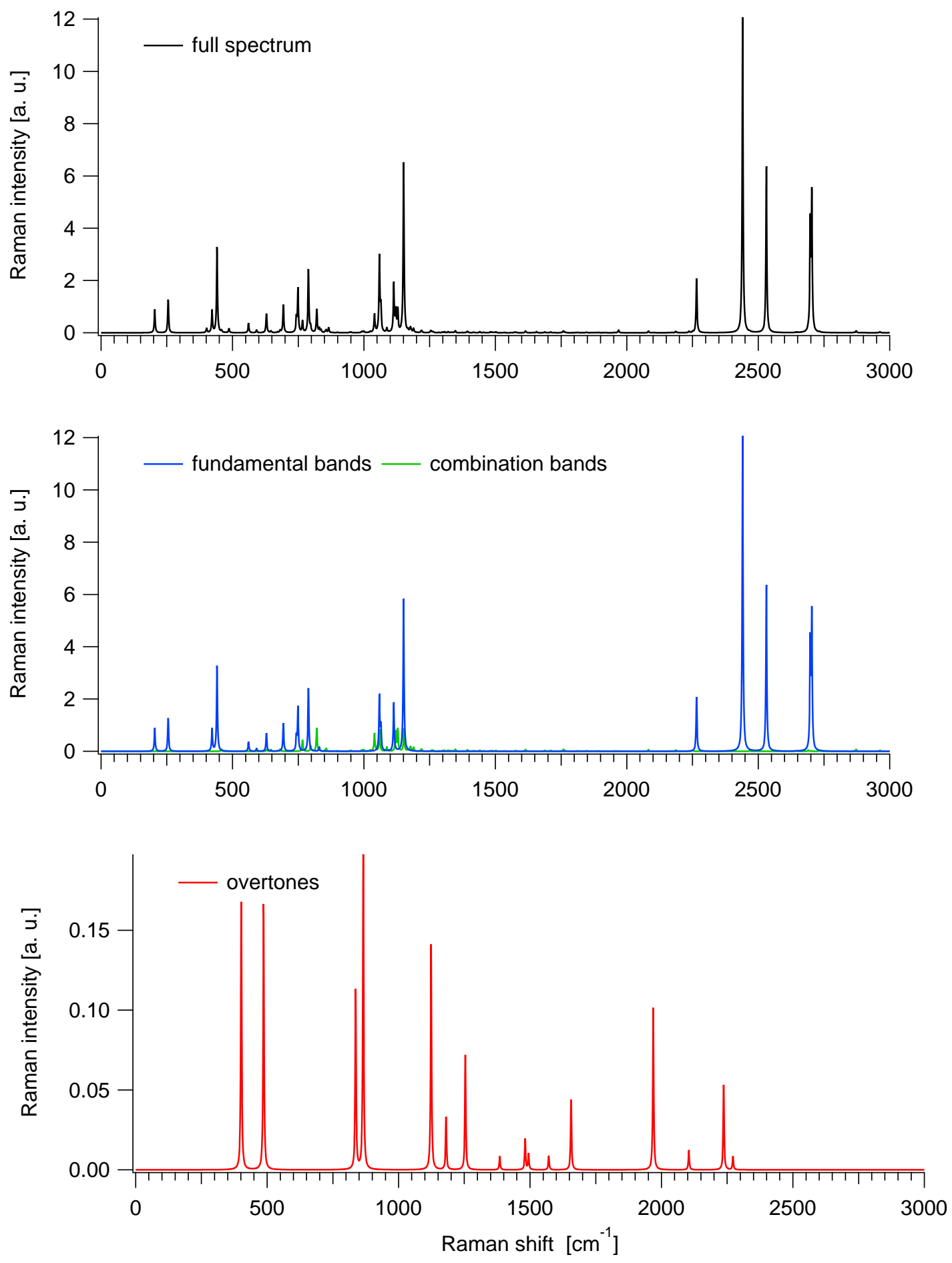
Table S.27.4. $\mathrm{B}_{4} \mathrm{H}_{5}$ - Isomer B: Ideal-gas thermodynamic functions at 1 bar (anharmonic B3LYP-D2/cc-pVTZ results)

\begin{tabular}{|c|c|c|c|}
\hline $\begin{array}{c}T \\
{[\mathrm{~K}]}\end{array}$ & $\begin{array}{c}C_{P} \\
{\left[\mathrm{~J} \mathrm{~mol}^{-1} \mathrm{~K}^{-1}\right]}\end{array}$ & $\begin{array}{c}S \\
{\left[\mathrm{~J} \mathrm{~mol}^{-1} \mathrm{~K}^{-1}\right]}\end{array}$ & $\begin{array}{c}H(T)-H(0 K) \\
{\left[\mathrm{kJ} \mathrm{mol}^{-1}\right]}\end{array}$ \\
\hline 100 & 42.269 & 221.748 & 3.568 \\
\hline 200 & 67.157 & 258.199 & 8.970 \\
\hline 300 & 93.423 & 290.540 & 17.035 \\
\hline 400 & 113.843 & 320.363 & 27.451 \\
\hline 500 & 128.910 & 347.466 & 39.625 \\
\hline 600 & 140.482 & 372.033 & 53.117 \\
\hline 700 & 149.797 & 394.412 & 67.647 \\
\hline 800 & 157.509 & 414.933 & 83.024 \\
\hline 900 & 163.980 & 433.870 & 99.107 \\
\hline 1000 & 169.441 & 451.438 & 115.786 \\
\hline 1100 & 174.065 & 467.810 & 132.968 \\
\hline 1200 & 177.992 & 483.129 & 150.576 \\
\hline 1300 & 181.336 & 497.511 & 168.547 \\
\hline 1400 & 184.196 & 511.057 & 186.827 \\
\hline 1500 & 186.651 & 523.851 & 205.372 \\
\hline 1600 & 188.768 & 535.967 & 224.146 \\
\hline 1700 & 190.602 & 547.467 & 243.117 \\
\hline 1800 & 192.197 & 558.408 & 262.258 \\
\hline 1900 & 193.592 & 568.837 & 281.549 \\
\hline 2000 & 194.816 & 578.799 & 300.971 \\
\hline
\end{tabular}

$T$ : Temperature. $C_{P}$ : Heat capacity at constant pressure. $S$ : Entropy. $H$ : Enthalpy. 
Figure S.27.4. $\mathrm{B}_{4} \mathrm{H}_{5}$ - Isomer B: Ideal-gas thermodynamic functions at 1 bar (anharmonic B3LYP-D2/cc-pVTZ results)
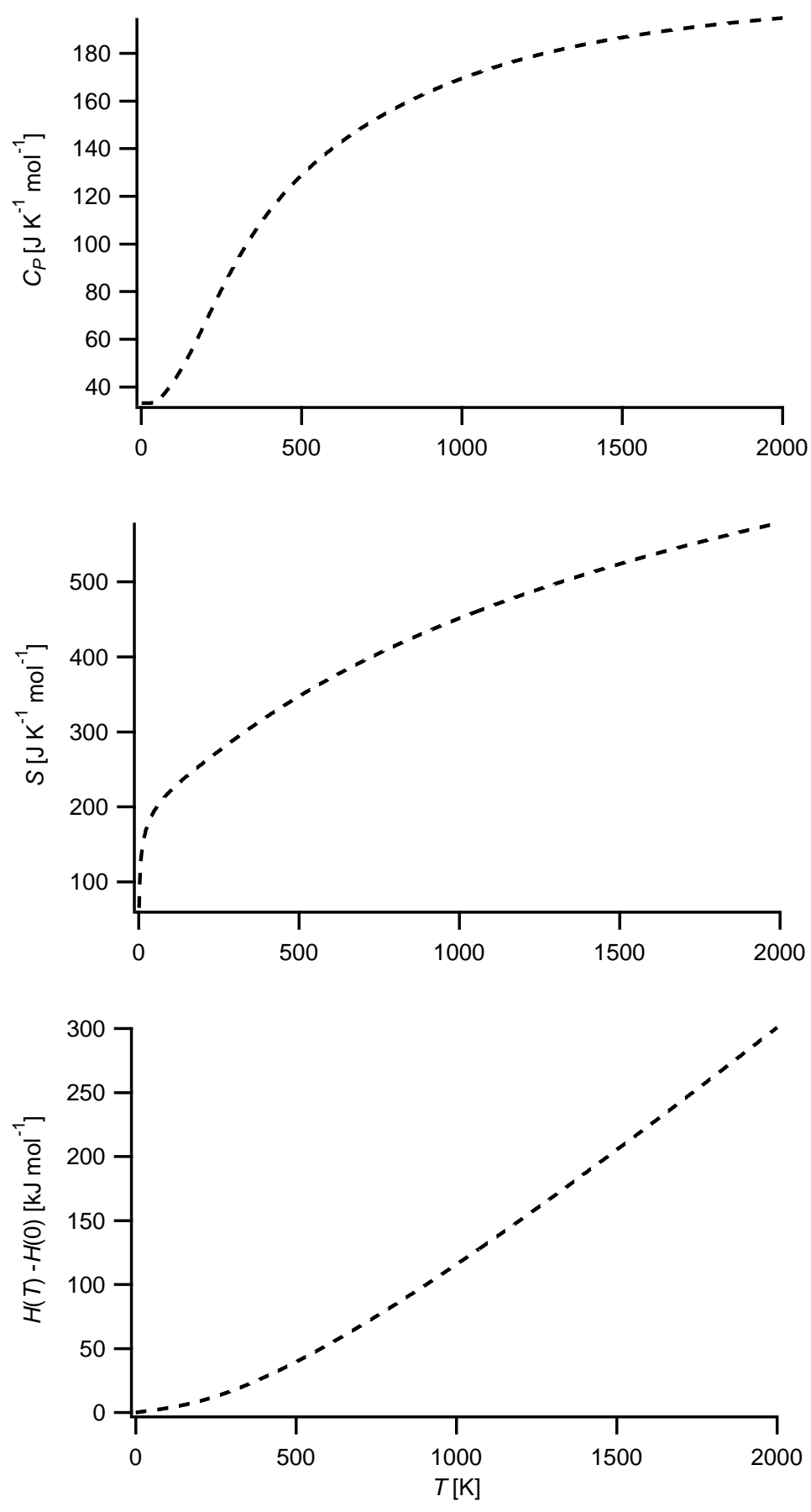

$T$ : Temperature. $C_{P}$ : Heat capacity at constant pressure. $S$ : Entropy. $H$ : Enthalpy. 
Table S.27.5. $\mathrm{B}_{4} \mathrm{H}_{5}$ - Isomer B: Fits (red solid lines) of the computed thermodynamic functions (blue dashed lines) with NASA type functions (Equations (1) - (3)) in the 200 - $900 \mathrm{~K}$ temperature range (anharmonic B3LYP-D2/cc-pVTZ results). In each case, the difference curve between the thermodynamic and NASA functions is plotted in the upper graph

Fit parameters

\begin{tabular}{llllll}
\hline $\mathrm{a}_{1}$ & $-1.63470483 \mathrm{e}+00$ & $\mathrm{a}_{2}\left[\mathrm{~K}^{-1}\right]$ & $6.15278359 \mathrm{e}-02$ & $\mathrm{a}_{3}\left[\mathrm{~K}^{-2}\right]$ & $-7.48926430 \mathrm{e}-05$ \\
$\mathrm{a}_{4}\left[\mathrm{~K}^{-3}\right]$ & $4.61273685 \mathrm{e}-08$ & $\mathrm{a}_{5}\left[\mathrm{~K}^{-4}\right]$ & $-1.06011046 \mathrm{e}-11$ & $\mathrm{a}_{6}[\mathrm{~K}]$ & $3.57155748 \mathrm{e}+02$ \\
$\mathrm{a}_{7}$ & $2.87888157 \mathrm{e}+01$ & & & &
\end{tabular}
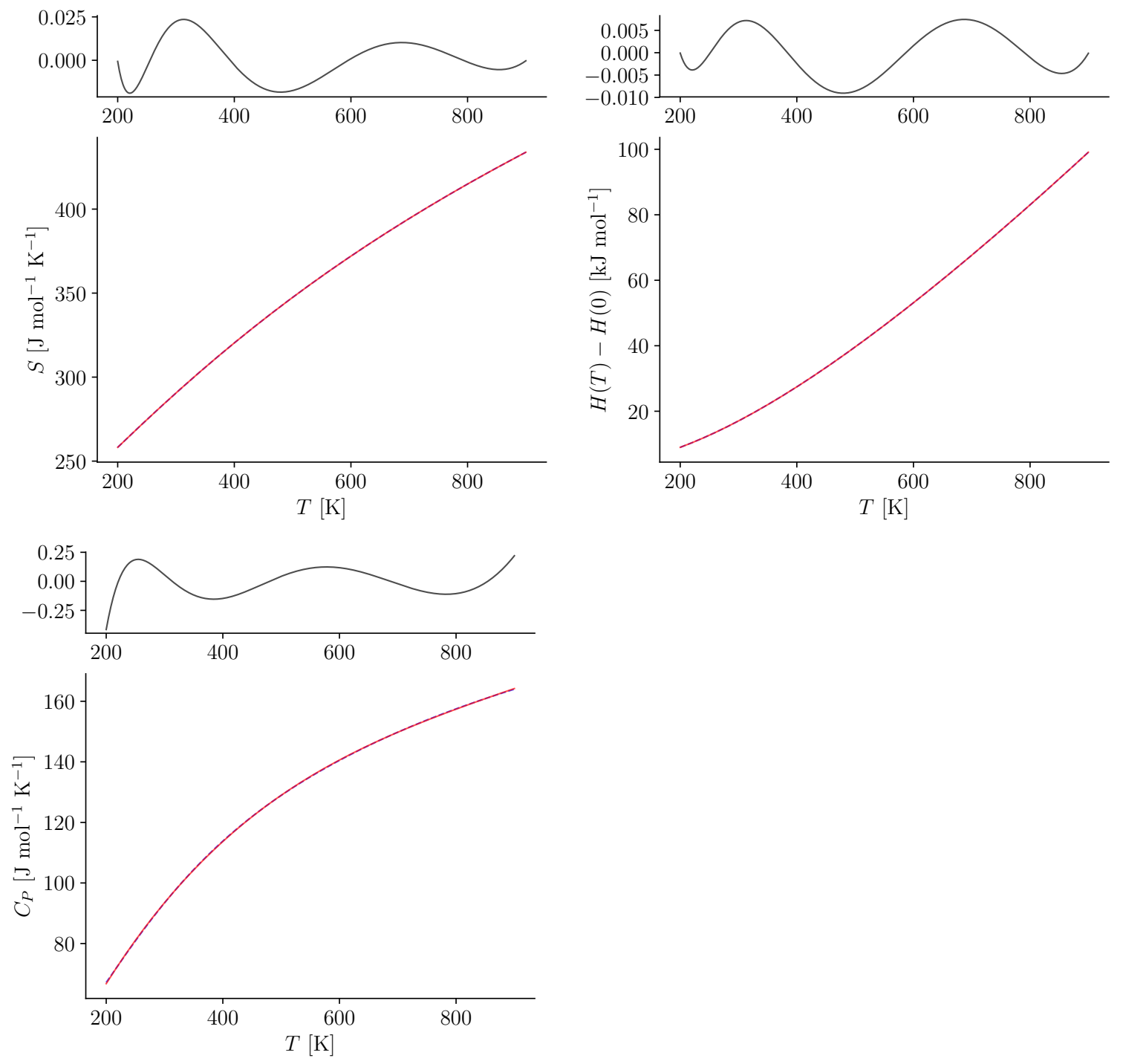


\section{$28 \quad \mathbf{B}_{4} \mathbf{H}_{5}$ - Isomer $\mathbf{C}$}

Figure S.28.1. Structure of $\mathrm{B}_{4} \mathrm{H}_{5}$ - Isomer C

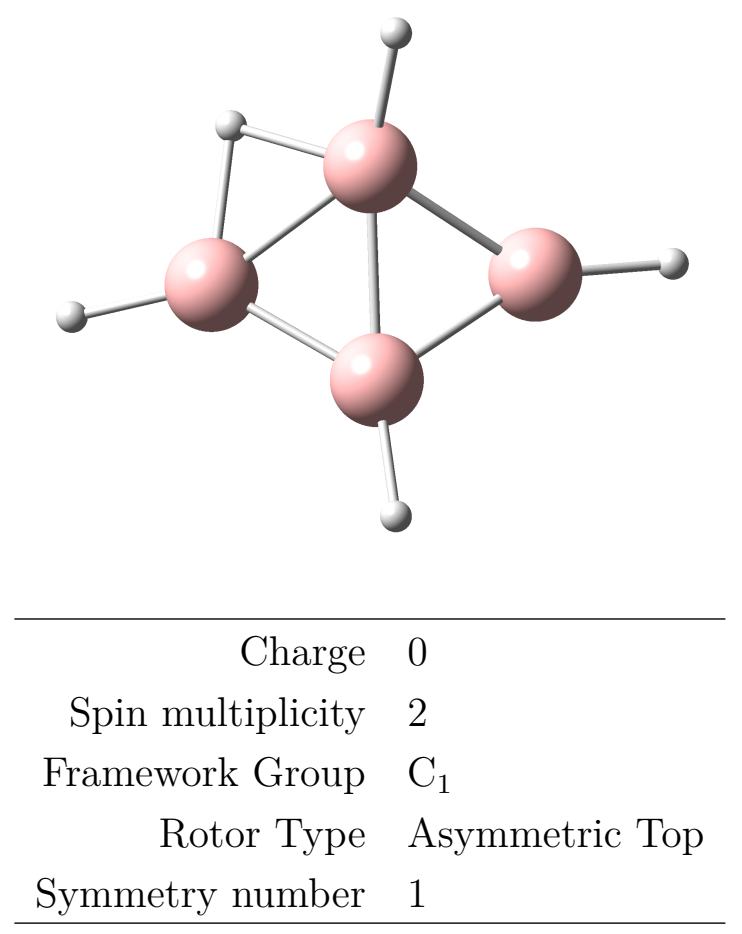

Table S.28.1. $\mathrm{B}_{4} \mathrm{H}_{5}$ - Isomer C: Selected anharmonic results obtained at the B3LYP-D2/ccpVTZ level

\begin{tabular}{rll}
\hline $\mathrm{ZPE}_{\mathrm{v}}$ & 12443 & $\mathrm{~cm}^{-1}$ \\
$\mathrm{~A}$ & 0.54137 & $\mathrm{~cm}^{-1}$ \\
$\mathrm{~B}$ & 0.30614 & $\mathrm{~cm}^{-1}$ \\
$\mathrm{C}$ & 0.23154 & $\mathrm{~cm}^{-1}$ \\
\hline$C_{P}(300 \mathrm{~K})$ & 90.924 & $\mathrm{~J} \mathrm{~mol}^{-1} \mathrm{~K}-1$ \\
$S(300 \mathrm{~K})$ & 288.146 & $\mathrm{~J} \mathrm{~mol}^{-1} \mathrm{~K}-1$ \\
$H(300 \mathrm{~K})-H(0 \mathrm{~K})$ & 16.508 & $\mathrm{~kJ} \mathrm{~mol}^{-1}$ \\
\hline
\end{tabular}

$\mathrm{ZPE}_{\mathrm{v}}$ : vibrational contribution to the zero-point energy. A, B, C: Rotational constants. $C_{P}$ : Heat capacity at constant pressure. $S$ : Entropy. $H$ : Enthalpy. Ideal-gas calculations (1 bar). 
Table S.28.2. $\mathrm{B}_{4} \mathrm{H}_{5}$ - Isomer C: Cartesian coordinates $(\AA)$ of the B3LYP-D2/cc-pVTZ optimized structure

\begin{tabular}{cccc} 
atom & $\mathrm{x}$ & $\mathrm{y}$ & $\mathrm{z}$ \\
\hline B1 & 1.3050 & -0.0253 & -0.3303 \\
B2 & 0.0577 & -0.8551 & 0.3039 \\
B3 & 0.0059 & 0.8275 & 0.3267 \\
B4 & -1.2437 & -0.1059 & -0.2680 \\
H5 & -1.0889 & 1.1445 & -0.4274 \\
H6 & 0.2087 & -1.9274 & 0.7902 \\
H7 & 2.3875 & 0.0607 & -0.7718 \\
H8 & -2.3414 & -0.3582 & -0.6085 \\
H9 & 0.2097 & 1.8749 & 0.8561 \\
\hline
\end{tabular}


Table S.28.3. $\mathrm{B}_{4} \mathrm{H}_{5}$ - Isomer C: Vibrational frequencies, infrared integrated intensities and Raman activities (B3LYP-D2/cc-pVTZ results)

\begin{tabular}{|c|c|c|c|c|c|c|c|}
\hline \multirow[b]{2}{*}{ mode } & \multirow[b]{2}{*}{ symm. } & \multicolumn{3}{|c|}{ Harmonic } & \multicolumn{3}{|c|}{ Anharmonic } \\
\hline & & $\begin{array}{c}\omega \\
{\left[\mathrm{cm}^{-1}\right]}\end{array}$ & $\begin{array}{c}\mathrm{IR} \\
{\left[\mathrm{km} \mathrm{mol}^{-1}\right]}\end{array}$ & $\begin{array}{c}\text { Raman } \\
{\left[\AA^{6}\right]}\end{array}$ & $\begin{array}{c}\omega \\
{\left[\mathrm{cm}^{-1}\right]}\end{array}$ & $\begin{array}{c}\mathrm{IR} \\
{\left[\mathrm{km} \mathrm{mol}^{-1}\right]}\end{array}$ & $\begin{array}{c}\text { Raman } \\
{\left[\AA^{6}\right]}\end{array}$ \\
\hline 1 & A & 150 & 0.944 & 1.514 & 146 & 1.127 & 1.541 \\
\hline 2 & $\mathrm{~A}$ & 418 & 7.631 & 0.037 & 384 & 20.275 & 0.026 \\
\hline 3 & $\mathrm{~A}$ & 449 & 27.743 & 0.035 & 416 & 18.803 & 0.061 \\
\hline 4 & $\mathrm{~A}$ & 596 & 10.707 & 0.184 & 576 & 10.404 & 0.147 \\
\hline 5 & $\mathrm{~A}$ & 635 & 10.931 & 0.023 & 608 & 5.446 & 0.040 \\
\hline 6 & $\mathrm{~A}$ & 654 & 5.507 & 0.231 & 632 & 6.074 & 0.228 \\
\hline 7 & $\mathrm{~A}$ & 704 & 10.248 & 0.030 & 664 & 13.355 & 0.046 \\
\hline 8 & $\mathrm{~A}$ & 758 & 3.515 & 0.160 & 739 & 6.129 & 0.055 \\
\hline 9 & $\mathrm{~A}$ & 775 & 13.190 & 0.048 & 718 & 6.530 & 0.064 \\
\hline 10 & $\mathrm{~A}$ & 782 & 2.445 & 0.078 & 767 & 0.148 & 0.078 \\
\hline 11 & $\mathrm{~A}$ & 821 & 9.724 & 0.053 & 794 & 15.810 & 0.064 \\
\hline 12 & $\mathrm{~A}$ & 847 & 8.276 & 0.276 & 814 & 3.944 & 0.224 \\
\hline 13 & $\mathrm{~A}$ & 904 & 4.220 & 0.314 & 872 & 2.028 & 0.291 \\
\hline 14 & $\mathrm{~A}$ & 1051 & 0.785 & 1.279 & 1014 & 0.342 & 0.315 \\
\hline 15 & $\mathrm{~A}$ & 1103 & 19.875 & 0.121 & 1067 & 10.443 & 0.103 \\
\hline 16 & $\mathrm{~A}$ & 1608 & 12.406 & 0.087 & 1427 & 3.926 & 0.050 \\
\hline 17 & $\mathrm{~A}$ & 2225 & 5.742 & 0.377 & 2183 & 0.737 & 0.294 \\
\hline 18 & $\mathrm{~A}$ & 2608 & 68.889 & 0.879 & 2498 & 63.378 & 0.921 \\
\hline 19 & $\mathrm{~A}$ & 2650 & 15.967 & 1.180 & 2536 & 15.009 & 1.239 \\
\hline 20 & $\mathrm{~A}$ & 2753 & 36.243 & 0.795 & 2656 & 35.390 & 0.820 \\
\hline 21 & A & 2788 & 44.034 & 0.772 & 2691 & 45.978 & 0.853 \\
\hline
\end{tabular}


Figure S.28.2. Anharmonic IR spectrum of $\mathrm{B}_{4} \mathrm{H}_{5}$ - Isomer $\mathrm{C}$ obtained by convoluting the calculated intensities with Lorentzians having a FWHM of $4 \mathrm{~cm}^{-1}$ (B3LYP-D2/cc-pVTZ results): (top) full spectrum, (middle) contributions from fundamentals and combination bands, (bottom) contributions from overtones
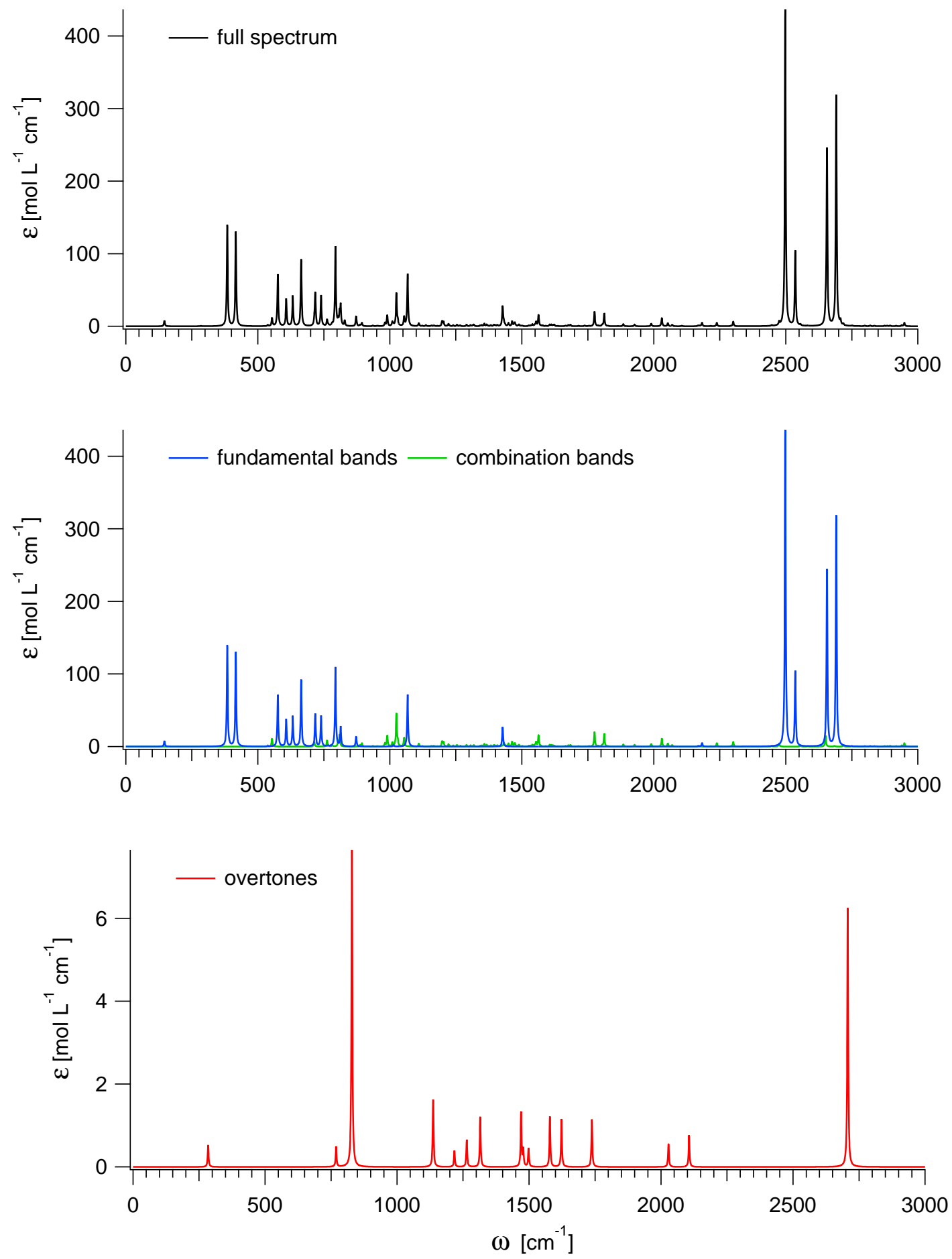
Figure S.28.3. Anharmonic Raman spectrum of $\mathrm{B}_{4} \mathrm{H}_{5}$ - Isomer $\mathrm{C}$ obtained by convoluting the calculated activities with Lorentzians having a FWHM of $4 \mathrm{~cm}^{-1}$ (B3LYP-D2/cc-pVTZ results): (top) full spectrum, (middle) contributions from fundamentals and combination bands, (bottom) contributions from overtones
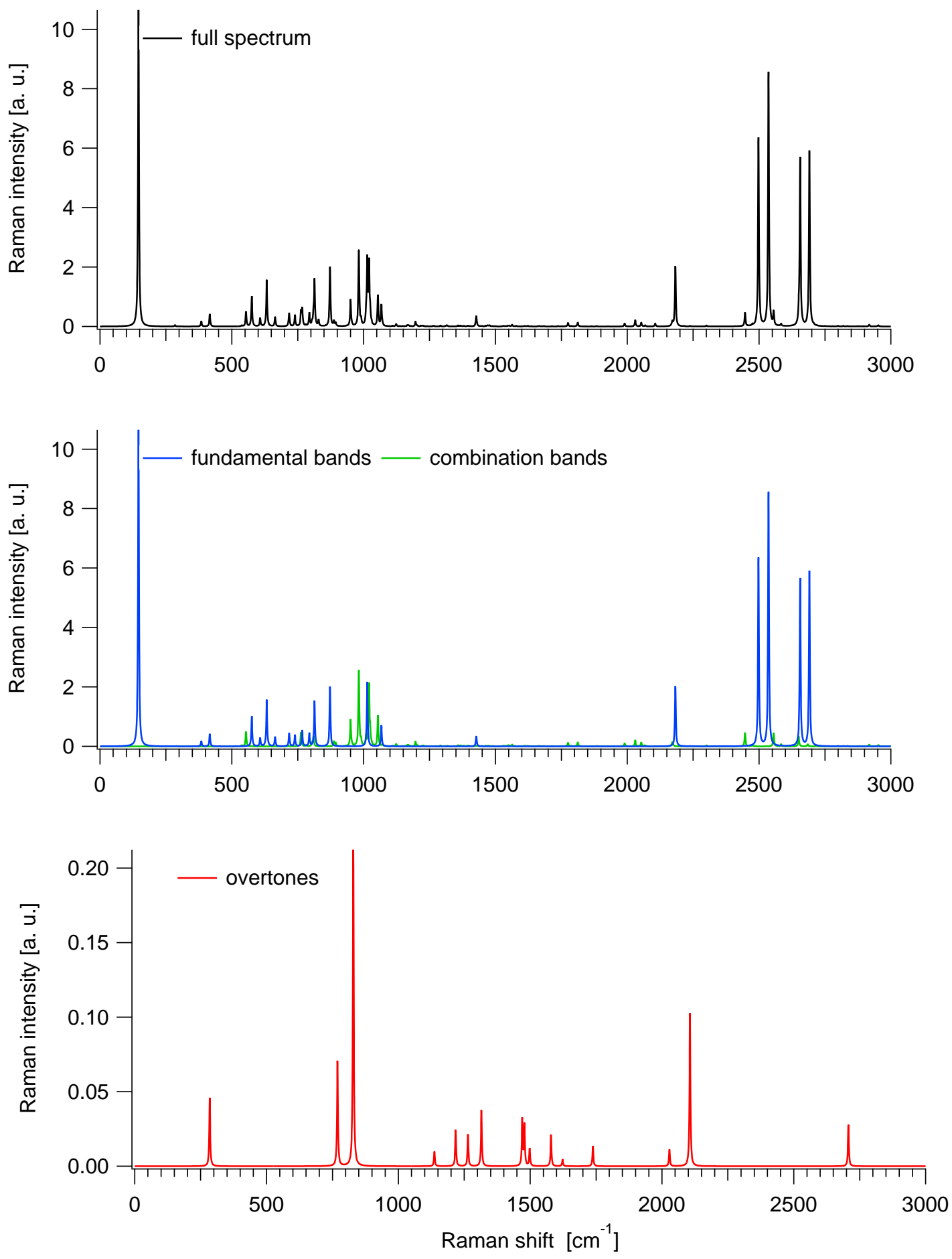

S.228 
Table S.28.4. $\mathrm{B}_{4} \mathrm{H}_{5}$ - Isomer C: Ideal-gas thermodynamic functions at 1 bar (anharmonic B3LYP-D2/cc-pVTZ results)

\begin{tabular}{|c|c|c|c|}
\hline $\begin{array}{c}T \\
{[\mathrm{~K}]}\end{array}$ & $\begin{array}{c}C_{P} \\
{\left[\mathrm{~J} \mathrm{~mol}^{-1} \mathrm{~K}^{-1}\right]}\end{array}$ & $\begin{array}{c}S \\
{\left[\mathrm{~J} \mathrm{~mol}^{-1} \mathrm{~K}^{-1}\right]}\end{array}$ & $\begin{array}{c}H(T)-H(0 K) \\
{\left[\mathrm{kJ} \mathrm{mol}^{-1}\right]}\end{array}$ \\
\hline 100 & 41.327 & 222.327 & 3.605 \\
\hline 200 & 63.803 & 257.022 & 8.739 \\
\hline 300 & 90.924 & 288.146 & 16.508 \\
\hline 400 & 112.068 & 317.358 & 26.713 \\
\hline 500 & 127.571 & 344.115 & 38.732 \\
\hline 600 & 139.446 & 368.467 & 52.107 \\
\hline 700 & 148.987 & 390.704 & 66.545 \\
\hline 800 & 156.872 & 411.129 & 81.849 \\
\hline 900 & 163.474 & 429.998 & 97.876 \\
\hline 1000 & 169.035 & 447.518 & 114.510 \\
\hline 1100 & 173.735 & 463.856 & 131.655 \\
\hline 1200 & 177.720 & 479.148 & 149.233 \\
\hline 1300 & 181.111 & 493.511 & 167.179 \\
\hline 1400 & 184.006 & 507.041 & 185.439 \\
\hline 1500 & 186.490 & 519.823 & 203.966 \\
\hline 1600 & 188.630 & 531.929 & 222.725 \\
\hline 1700 & 190.482 & 543.421 & 241.683 \\
\hline 1800 & 192.092 & 554.356 & 260.813 \\
\hline 1900 & 193.499 & 564.780 & 280.094 \\
\hline 2000 & 194.734 & 574.737 & 299.507 \\
\hline
\end{tabular}

$T$ : Temperature. $C_{P}$ : Heat capacity at constant pressure. $S$ : Entropy. $H$ : Enthalpy. 
Figure S.28.4. $\mathrm{B}_{4} \mathrm{H}_{5}$ - Isomer C: Ideal-gas thermodynamic functions at 1 bar (anharmonic B3LYP-D2/cc-pVTZ results)
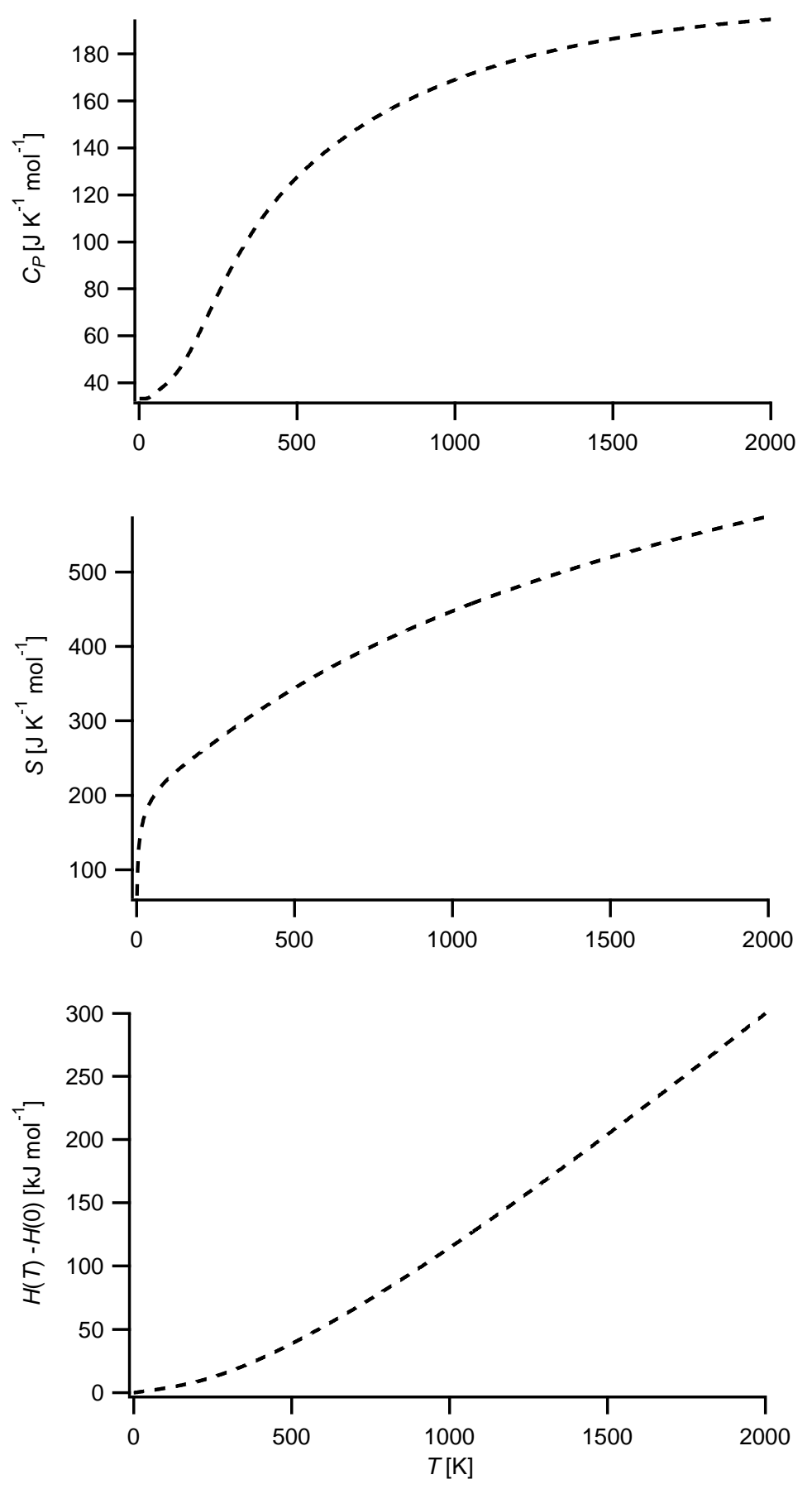

$T$ : Temperature. $C_{P}$ : Heat capacity at constant pressure. $S$ : Entropy. $H$ : Enthalpy. 
Table S.28.5. $\mathrm{B}_{4} \mathrm{H}_{5}$ - Isomer C: Fits (red solid lines) of the computed thermodynamic functions (blue dashed lines) with NASA type functions (Equations (1) - (3)) in the 200 - $900 \mathrm{~K}$ temperature range (anharmonic B3LYP-D2/cc-pVTZ results). In each case, the difference curve between the thermodynamic and NASA functions is plotted in the upper graph

Fit parameters

\begin{tabular}{llllll}
\hline $\mathrm{a}_{1}$ & $-2.38211263 \mathrm{e}+00$ & $\mathrm{a}_{2}\left[\mathrm{~K}^{-1}\right]$ & $6.35388385 \mathrm{e}-02$ & $\mathrm{a}_{3}\left[\mathrm{~K}^{-2}\right]$ & $-7.66537515 \mathrm{e}-05$ \\
$\mathrm{a}_{4}\left[\mathrm{~K}^{-3}\right]$ & $4.60138736 \mathrm{e}-08$ & $\mathrm{a}_{5}\left[\mathrm{~K}^{-4}\right]$ & $-1.00040903 \mathrm{e}-11$ & $\mathrm{a}_{6}[\mathrm{~K}]$ & $4.43368622 \mathrm{e}+02$ \\
$\mathrm{a}_{7}$ & $3.22403242 \mathrm{e}+01$ & & & &
\end{tabular}
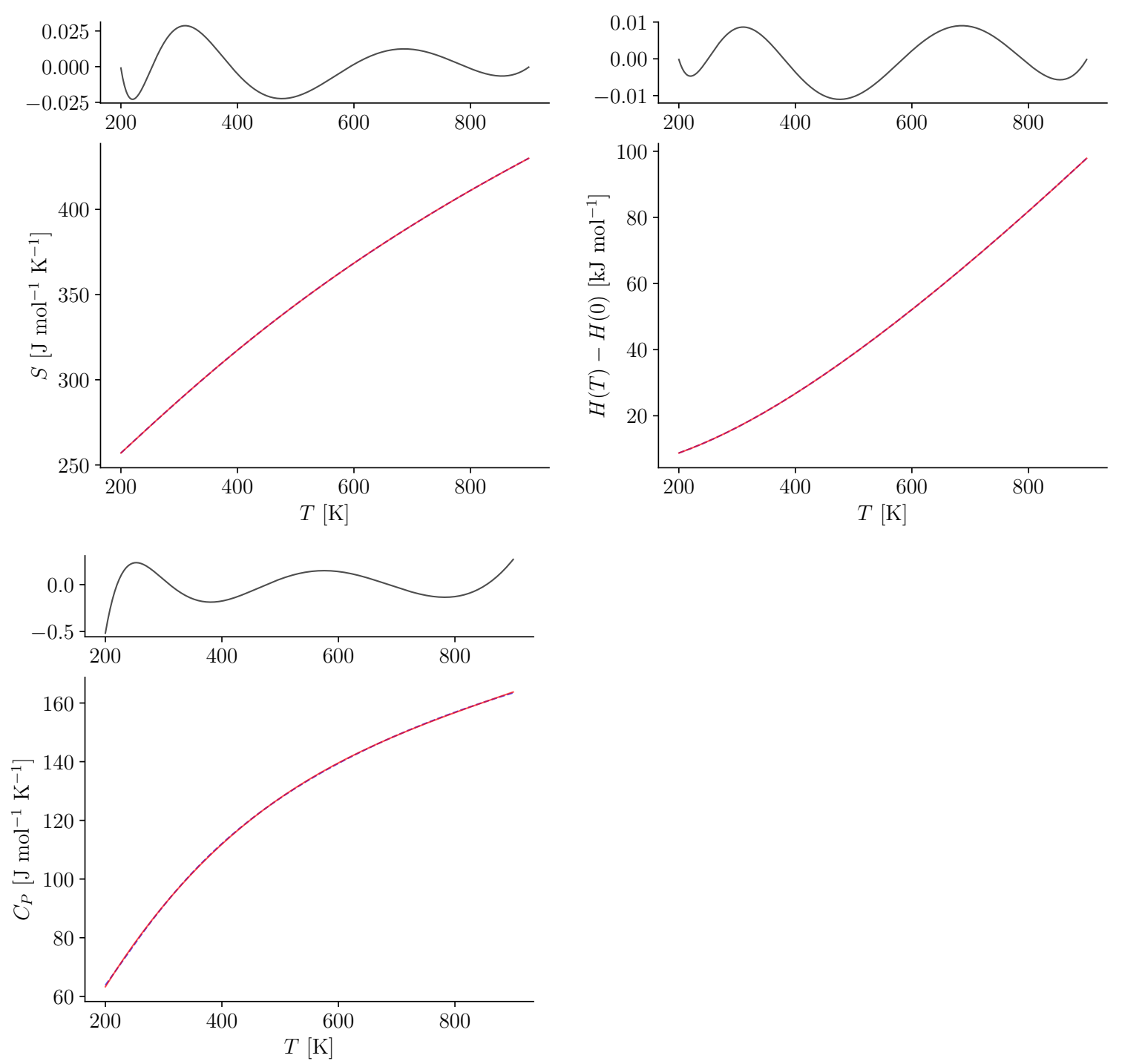


\section{$29 \quad \mathrm{~B}_{4} \mathrm{H}_{7}$ - Isomer $\mathrm{A}$}

Figure S.29.1. Structure of $\mathrm{B}_{4} \mathrm{H}_{7}$ - Isomer A

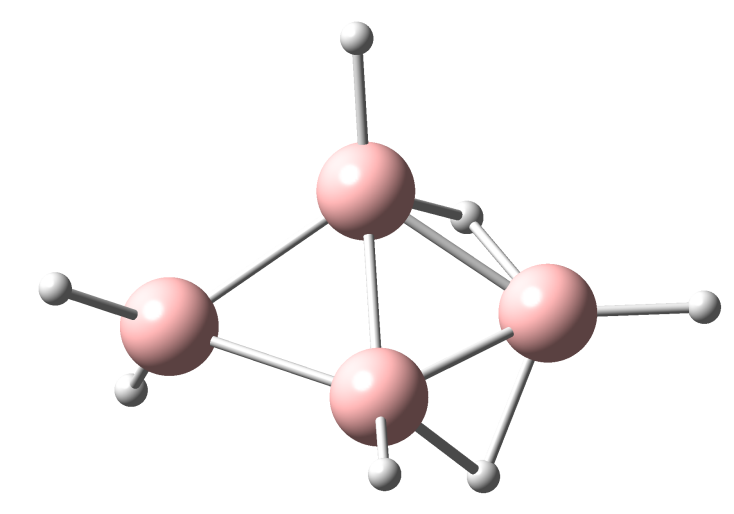

\begin{aligned} & \hline Charge 0 \\ & Spin multiplicity 2 \\ & Framework Group $\mathrm{C}_{\mathrm{s}} \\ &$ Rotor Type Asymmetric Top \\ & Symmetry number 1 \\ & \hline\end{aligned}

Table S.29.1. $\mathrm{B}_{4} \mathrm{H}_{7}$ - Isomer A: Selected anharmonic results obtained at the B3LYPD2/cc-pVTZ level

\begin{tabular}{rll}
\hline $\mathrm{ZPE}_{\mathrm{v}}$ & 16786 & $\mathrm{~cm}^{-1}$ \\
$\mathrm{~A}$ & 0.52172 & $\mathrm{~cm}^{-1}$ \\
$\mathrm{~B}$ & 0.24380 & $\mathrm{~cm}^{-1}$ \\
$\mathrm{C}$ & 0.19716 & $\mathrm{~cm}^{-1}$ \\
\hline$C_{P}(300 \mathrm{~K})$ & 98.918 & $\mathrm{~J} \mathrm{~mol}^{-1} \mathrm{~K}-1$ \\
$S(300 \mathrm{~K})$ & 292.177 & $\mathrm{~J} \mathrm{~mol}^{-1} \mathrm{~K}-1$ \\
$H(300 \mathrm{~K})-H(0 \mathrm{~K})$ & 17.106 & $\mathrm{~kJ} \mathrm{~mol}^{-1}$ \\
\hline
\end{tabular}

$\mathrm{ZPE}_{\mathrm{v}}$ : vibrational contribution to the zero-point energy. A, B, C: Rotational constants. $C_{P}$ : Heat capacity at constant pressure. $S$ : Entropy. $H$ : Enthalpy. Ideal-gas calculations (1 bar). 
Table S.29.2. $\mathrm{B}_{4} \mathrm{H}_{7}$ - Isomer A: Cartesian coordinates $(\AA)$ of the B3LYP-D2/cc-pVTZ optimized structure

\begin{tabular}{cccc} 
atom & $\mathrm{x}$ & $\mathrm{y}$ & $\mathrm{z}$ \\
\hline B1 & 0.6847 & -1.3180 & 0.0000 \\
B2 & -0.2637 & 1.3629 & -0.0000 \\
B3 & -0.2637 & -0.0584 & 0.8430 \\
B4 & -0.2637 & -0.0584 & -0.8430 \\
H5 & -0.9184 & -0.3386 & 1.7847 \\
H6 & 0.4617 & 1.0687 & -1.0601 \\
H7 & -0.6634 & 2.4681 & -0.0000 \\
H8 & -0.9184 & -0.3386 & -1.7847 \\
H9 & 0.4617 & 1.0687 & 1.0601 \\
H10 & 0.2444 & -2.4214 & 0.0000 \\
H11 & 1.8650 & -1.1474 & 0.0000 \\
\hline
\end{tabular}


Table S.29.3. $\mathrm{B}_{4} \mathrm{H}_{7}$ - Isomer A: Vibrational frequencies, infrared integrated intensities and Raman activities (B3LYP-D2/cc-pVTZ results)

\begin{tabular}{|c|c|c|c|c|c|c|c|}
\hline \multirow[b]{2}{*}{ mode } & \multirow[b]{2}{*}{ symm. } & \multicolumn{3}{|c|}{ Harmonic } & \multicolumn{3}{|c|}{ Anharmonic } \\
\hline & & $\begin{array}{c}\omega \\
{\left[\mathrm{cm}^{-1}\right]}\end{array}$ & $\begin{array}{c}\mathrm{IR} \\
{\left[\mathrm{km} \mathrm{mol}^{-1}\right]}\end{array}$ & $\begin{array}{c}\text { Raman } \\
{\left[\AA^{6}\right]}\end{array}$ & $\begin{array}{c}\omega \\
{\left[\mathrm{cm}^{-1}\right]}\end{array}$ & $\begin{array}{c}\mathrm{IR} \\
{\left[\mathrm{km} \mathrm{mol}^{-1}\right]}\end{array}$ & $\begin{array}{c}\text { Raman } \\
{\left[\AA^{6}\right]}\end{array}$ \\
\hline 1 & A" & 217 & 3.931 & 0.154 & 194 & 4.824 & 0.222 \\
\hline 2 & $\mathrm{~A}^{\prime}$ & 309 & 0.711 & 0.182 & 301 & 0.593 & 0.175 \\
\hline 3 & A" & 448 & 4.056 & 0.108 & 422 & 3.326 & 0.133 \\
\hline 4 & A" & 575 & 0.678 & 0.001 & 553 & 0.728 & 0.000 \\
\hline 5 & $\mathrm{~A}^{\prime}$ & 643 & 4.645 & 0.179 & 620 & 2.823 & 0.033 \\
\hline 6 & A" & 648 & 9.737 & 0.028 & 616 & 10.544 & 0.034 \\
\hline 7 & $\mathrm{~A}^{\prime}$ & 680 & 29.282 & 0.225 & 658 & 26.350 & 0.188 \\
\hline 8 & $A^{\prime}$ & 728 & 6.892 & 0.447 & 697 & 7.986 & 0.565 \\
\hline 9 & A" & 746 & 7.911 & 0.001 & 693 & 4.291 & 0.002 \\
\hline 10 & $A^{\prime}$ & 791 & 2.330 & 0.208 & 716 & 1.708 & 0.077 \\
\hline 11 & $\mathrm{~A}^{\prime}$ & 810 & 0.101 & 0.103 & 757 & 1.282 & 0.037 \\
\hline 12 & A" & 836 & 2.604 & 0.001 & 800 & 2.381 & 0.003 \\
\hline 13 & A" & 921 & 0.622 & 0.018 & 883 & 0.820 & 0.027 \\
\hline 14 & $A^{\prime}$ & 988 & 0.734 & 0.398 & 956 & 0.644 & 0.366 \\
\hline 15 & A" & 1002 & 0.036 & 0.043 & 944 & 0.036 & 0.041 \\
\hline 16 & $\mathrm{~A}^{\prime}$ & 1038 & 2.612 & 0.191 & 969 & 2.110 & 0.090 \\
\hline 17 & $A^{\prime}$ & 1069 & 6.162 & 0.146 & 1022 & 7.019 & 0.193 \\
\hline 18 & $A^{\prime}$ & 1196 & 29.392 & 0.082 & 1143 & 21.126 & 0.082 \\
\hline 19 & $A^{\prime \prime}$ & 1491 & 37.672 & 0.033 & 1292 & 9.060 & 0.016 \\
\hline 20 & $A^{\prime}$ & 1536 & 137.616 & 0.065 & 1326 & 57.486 & 0.043 \\
\hline 21 & $\mathrm{~A}^{\prime}$ & 2050 & 0.630 & 0.521 & 1813 & 1.138 & 0.128 \\
\hline 22 & $A "$ & 2084 & 20.331 & 0.177 & 2121 & 2.360 & 0.094 \\
\hline 23 & $\mathrm{~A}^{\prime}$ & 2603 & 56.513 & 1.065 & 2472 & 47.866 & 1.049 \\
\hline
\end{tabular}


Table S.29.3 - Continued

\begin{tabular}{cccccccc}
\hline & & \multicolumn{3}{c}{ Harmonic } & \multicolumn{3}{c}{ Anharmonic } \\
mode & symm. & $\omega$ & IR & Raman & $\omega$ & IR & Raman \\
& & {$\left[\mathrm{cm}^{-1}\right]$} & {$\left[\mathrm{km} \mathrm{mol}^{-1}\right]$} & {$\left[\AA^{6}\right]$} & {$\left[\mathrm{cm}^{-1}\right]$} & {$\left[\mathrm{km} \mathrm{mol}^{-1}\right]$} & {$\left[\AA^{6}\right]$} \\
\hline 24 & A' $^{\prime}$ & 2670 & 83.563 & 0.557 & 2565 & 82.073 & 0.800 \\
25 & A $^{\prime \prime}$ & 2711 & 53.620 & 0.522 & 2588 & 52.589 & 0.582 \\
26 & A' $^{\prime}$ & 2722 & 26.892 & 1.104 & 2598 & 25.236 & 0.930 \\
27 & A' $^{\prime}$ & 2773 & 37.375 & 0.913 & 2648 & 31.924 & 1.039 \\
\hline
\end{tabular}


Figure S.29.2. Anharmonic IR spectrum of $\mathrm{B}_{4} \mathrm{H}_{7}$ - Isomer A obtained by convoluting the calculated intensities with Lorentzians having a FWHM of $4 \mathrm{~cm}^{-1}$ (B3LYP-D2/cc-pVTZ results): (top) full spectrum, (middle) contributions from fundamentals and combination bands, (bottom) contributions from overtones
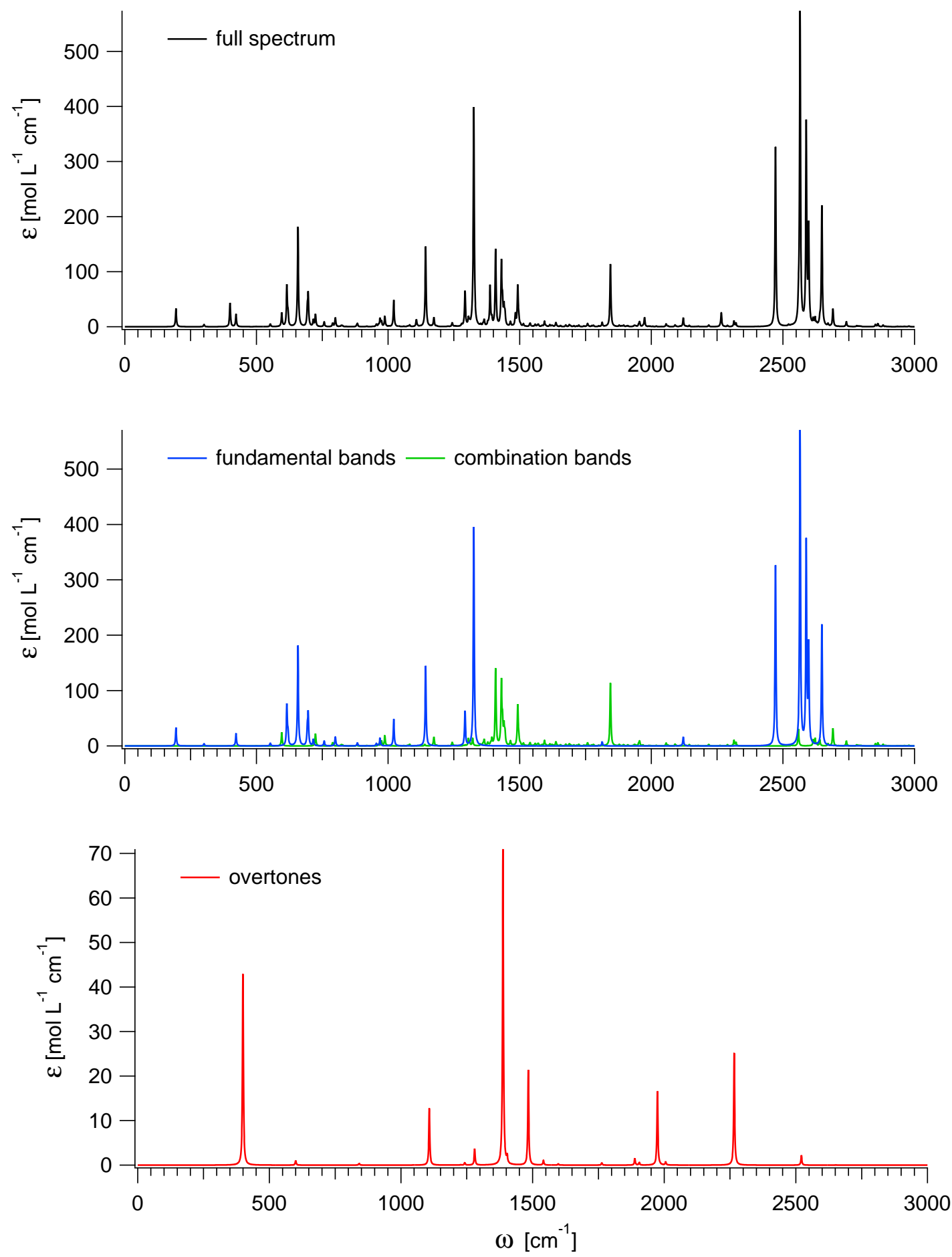
Figure S.29.3. Anharmonic Raman spectrum of $\mathrm{B}_{4} \mathrm{H}_{7}$ - Isomer A obtained by convoluting the calculated activities with Lorentzians having a FWHM of $4 \mathrm{~cm}^{-1}$ (B3LYP-D2/cc-pVTZ results): (top) full spectrum, (middle) contributions from fundamentals and combination bands, (bottom) contributions from overtones
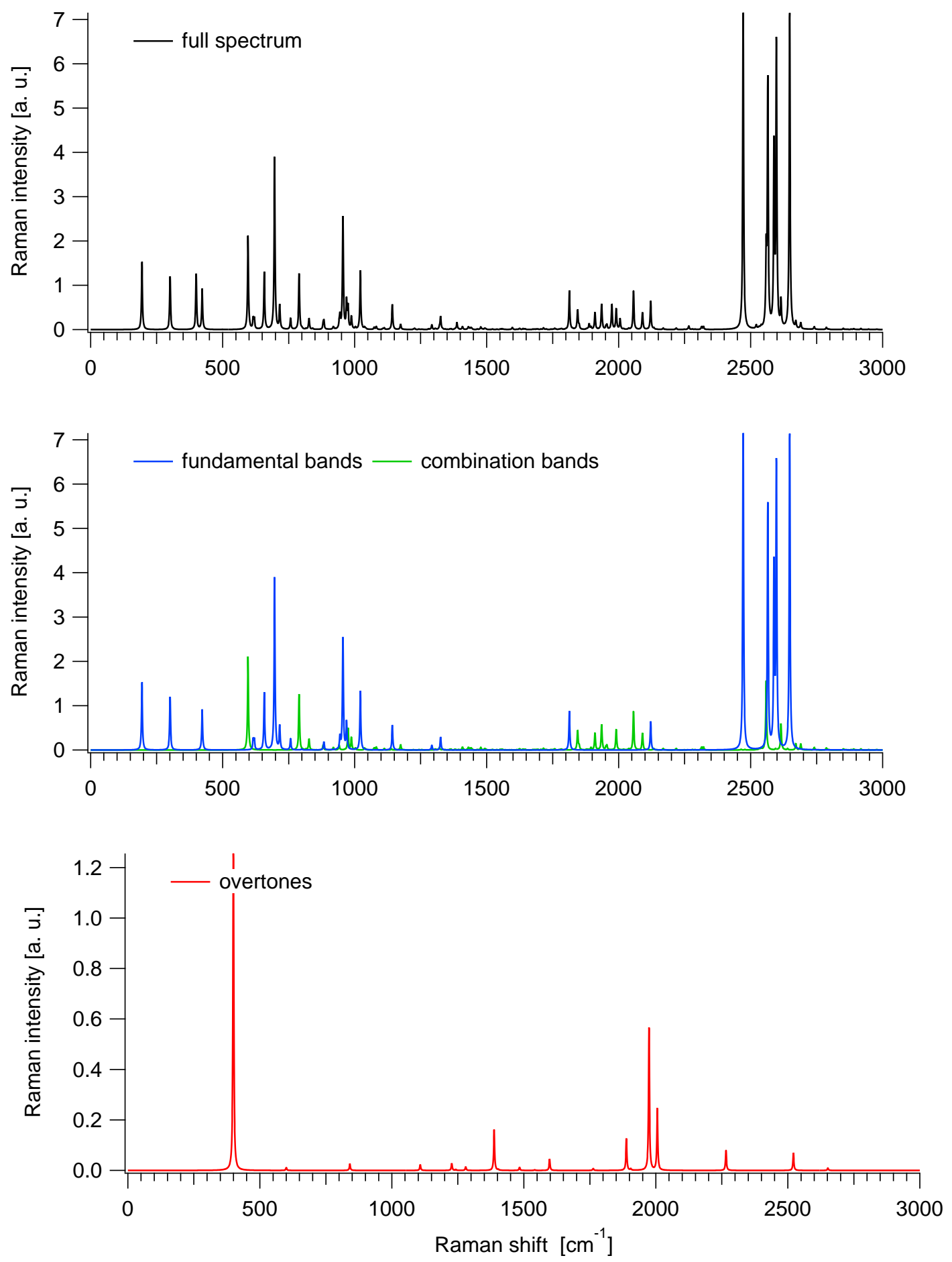
Table S.29.4. $\mathrm{B}_{4} \mathrm{H}_{7}$ - Isomer A: Ideal-gas thermodynamic functions at 1 bar (anharmonic B3LYP-D2/cc-pVTZ results)

\begin{tabular}{|c|c|c|c|}
\hline $\begin{array}{c}T \\
{[\mathrm{~K}]}\end{array}$ & $\begin{array}{c}C_{P} \\
{\left[\mathrm{~J} \mathrm{~mol}^{-1} \mathrm{~K}^{-1}\right]}\end{array}$ & $\begin{array}{c}S \\
{\left[\mathrm{~J} \mathrm{~mol}^{-1} \mathrm{~K}^{-1}\right]}\end{array}$ & $\begin{array}{c}H(T)-H(0 K) \\
{\left[\mathrm{kJ} \mathrm{mol}^{-1}\right]}\end{array}$ \\
\hline 100 & 41.144 & 223.489 & 3.543 \\
\hline 200 & 66.737 & 258.972 & 8.808 \\
\hline 300 & 98.918 & 292.177 & 17.106 \\
\hline 400 & 126.043 & 324.508 & 28.410 \\
\hline 500 & 147.092 & 354.993 & 42.111 \\
\hline 600 & 163.701 & 383.335 & 57.681 \\
\hline 700 & 177.171 & 409.615 & 74.747 \\
\hline 800 & 188.287 & 434.022 & 93.037 \\
\hline 900 & 197.541 & 456.750 & 112.342 \\
\hline 1000 & 205.281 & 477.975 & 132.495 \\
\hline 1100 & 211.779 & 497.854 & 153.357 \\
\hline 1200 & 217.257 & 516.523 & 174.817 \\
\hline 1300 & 221.893 & 534.101 & 196.780 \\
\hline 1400 & 225.835 & 550.693 & 219.172 \\
\hline 1500 & 229.204 & 566.392 & 241.928 \\
\hline 1600 & 232.097 & 581.279 & 264.997 \\
\hline 1700 & 234.595 & 595.427 & 288.334 \\
\hline 1800 & 236.762 & 608.899 & 311.905 \\
\hline 1900 & 238.651 & 621.751 & 335.678 \\
\hline 2000 & 240.306 & 634.036 & 359.627 \\
\hline
\end{tabular}

$T$ : Temperature. $C_{P}$ : Heat capacity at constant pressure. $S$ : Entropy. $H$ : Enthalpy. 
Figure S.29.4. $\mathrm{B}_{4} \mathrm{H}_{7}$ - Isomer A: Ideal-gas thermodynamic functions at 1 bar (anharmonic B3LYP-D2/cc-pVTZ results)
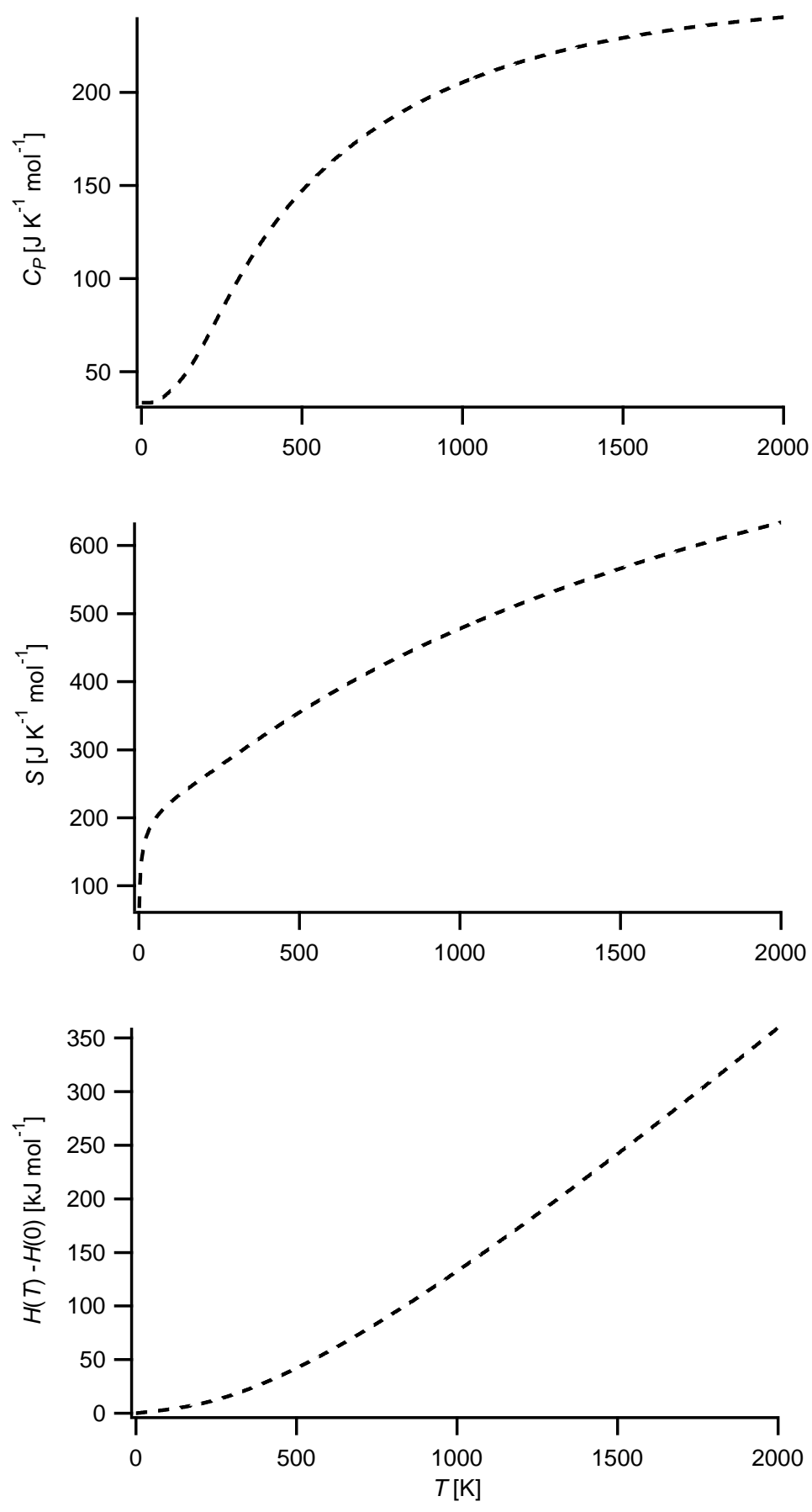

$T$ : Temperature. $C_{P}$ : Heat capacity at constant pressure. $S$ : Entropy. $H$ : Enthalpy. 
Table S.29.5. $\mathrm{B}_{4} \mathrm{H}_{7}$ - Isomer A: Fits (red solid lines) of the computed thermodynamic functions (blue dashed lines) with NASA type functions (Equations (1) - (3)) in the 200 - $900 \mathrm{~K}$ temperature range (anharmonic B3LYP-D2/cc-pVTZ results). In each case, the difference curve between the thermodynamic and NASA functions is plotted in the upper graph

Fit parameters

\begin{tabular}{llllll}
\hline $\mathrm{a}_{1}$ & $-2.33562715 \mathrm{e}+00$ & $\mathrm{a}_{2}\left[\mathrm{~K}^{-1}\right]$ & $5.96272229 \mathrm{e}-02$ & $\mathrm{a}_{3}\left[\mathrm{~K}^{-2}\right]$ & $-4.11013127 \mathrm{e}-05$ \\
$\mathrm{a}_{4}\left[\mathrm{~K}^{-3}\right]$ & $-9.85335732 \mathrm{e}-10$ & $\mathrm{a}_{5}\left[\mathrm{~K}^{-4}\right]$ & $9.91260739 \mathrm{e}-12$ & $\mathrm{a}_{6}[\mathrm{~K}]$ & $4.43262610 \mathrm{e}+02$ \\
$\mathrm{a}_{7}$ & $3.24172413 \mathrm{e}+01$ & & & &
\end{tabular}
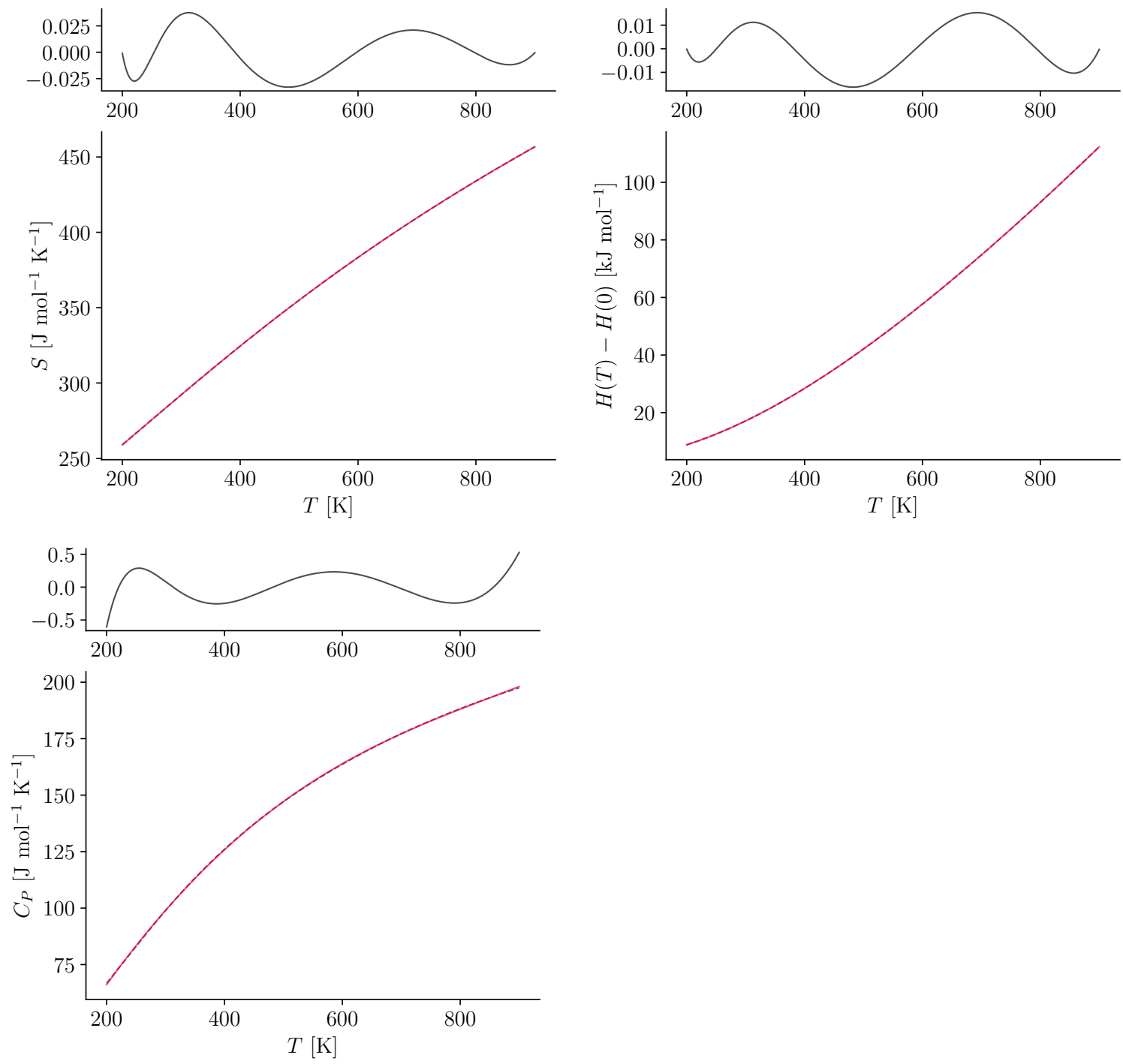


\section{$30 \quad \mathbf{B}_{4} \mathbf{H}_{7}$ - Isomer B}

Figure S.30.1. Structure of $\mathrm{B}_{4} \mathrm{H}_{7}$ - Isomer B

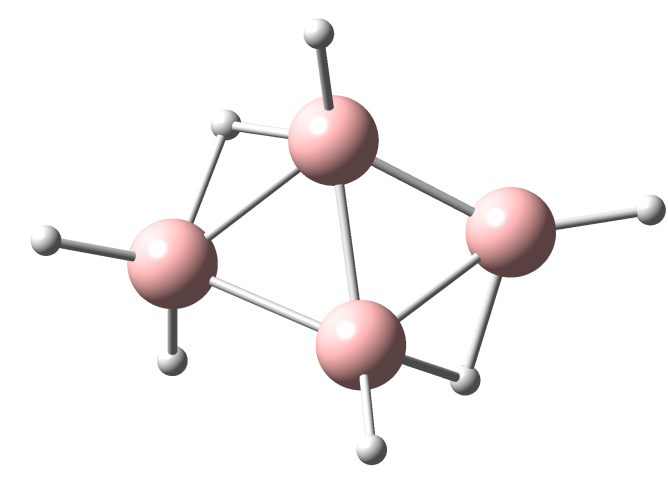

\begin{aligned} & \hline Charge 0 \\ & Spin multiplicity 2 \\ & Framework Group $\mathrm{C}_{1} \\ &$ Rotor Type Asymmetric Top \\ & Symmetry number 1 \\ & \hline\end{aligned}

Table S.30.1. $\mathrm{B}_{4} \mathrm{H}_{7}$ - Isomer B: Selected anharmonic results obtained at the B3LYP-D2/ccpVTZ level

\begin{tabular}{rll}
\hline $\mathrm{ZPE}_{\mathrm{v}}$ & 16755 & $\mathrm{~cm}^{-1}$ \\
$\mathrm{~A}$ & 0.50887 & $\mathrm{~cm}^{-1}$ \\
$\mathrm{~B}$ & 0.25154 & $\mathrm{~cm}^{-1}$ \\
$\mathrm{C}$ & 0.20195 & $\mathrm{~cm}^{-1}$ \\
\hline$C_{P}(300 \mathrm{~K})$ & 98.456 & $\mathrm{~J} \mathrm{~mol}^{-1} \mathrm{~K}-1$ \\
$S(300 \mathrm{~K})$ & 289.419 & $\mathrm{~J} \mathrm{~mol}^{-1} \mathrm{~K}^{-1}$ \\
$H(300 \mathrm{~K})-H(0 \mathrm{~K})$ & 16.793 & $\mathrm{~kJ} \mathrm{~mol}^{-1}$ \\
\hline
\end{tabular}

$\mathrm{ZPE}_{\mathrm{v}}$ : vibrational contribution to the zero-point energy. A, B, C: Rotational constants. $C_{P}$ : Heat capacity at constant pressure. $S$ : Entropy. $H$ : Enthalpy. Ideal-gas calculations (1 bar). 
Table S.30.2. $\mathrm{B}_{4} \mathrm{H}_{7}$ - Isomer B: Cartesian coordinates $(\AA)$ of the B3LYP-D2/cc-pVTZ optimized structure

\begin{tabular}{cccc} 
atom & $\mathrm{x}$ & $\mathrm{y}$ & $\mathrm{z}$ \\
\hline B1 & -0.0415 & 0.8826 & 0.2059 \\
B2 & 0.1833 & -0.7900 & 0.3776 \\
B3 & 1.4085 & 0.1325 & -0.2468 \\
B4 & -1.3562 & -0.1255 & -0.2325 \\
H5 & -0.1610 & 1.7549 & 0.9932 \\
H6 & -0.9182 & 1.0098 & -0.7932 \\
H7 & 0.2715 & -1.6456 & 1.1870 \\
H8 & 2.5500 & 0.3142 & -0.4517 \\
H9 & -2.3902 & 0.0642 & 0.3202 \\
H10 & 1.0353 & -1.0771 & -0.6383 \\
H11 & -1.3574 & -0.9186 & -1.1386 \\
\hline
\end{tabular}


Table S.30.3. $\mathrm{B}_{4} \mathrm{H}_{7}$ - Isomer B: Vibrational frequencies, infrared integrated intensities and Raman activities (B3LYP-D2/cc-pVTZ results)

\begin{tabular}{|c|c|c|c|c|c|c|c|}
\hline \multirow[b]{2}{*}{ mode } & \multirow[b]{2}{*}{ symm. } & \multicolumn{3}{|c|}{ Harmonic } & \multicolumn{3}{|c|}{ Anharmonic } \\
\hline & & $\begin{array}{c}\omega \\
{\left[\mathrm{cm}^{-1}\right]}\end{array}$ & $\begin{array}{c}\mathrm{IR} \\
{\left[\mathrm{km} \mathrm{mol}^{-1}\right]}\end{array}$ & $\begin{array}{c}\text { Raman } \\
{\left[\AA^{6}\right]}\end{array}$ & $\begin{array}{c}\omega \\
{\left[\mathrm{cm}^{-1}\right]}\end{array}$ & $\begin{array}{c}\mathrm{IR} \\
{\left[\mathrm{km} \mathrm{mol}^{-1}\right]}\end{array}$ & $\begin{array}{c}\text { Raman } \\
{\left[\AA^{6}\right]}\end{array}$ \\
\hline 1 & $\mathrm{~A}$ & 313 & 21.578 & 0.202 & 252 & 59.187 & 0.214 \\
\hline 2 & $\mathrm{~A}$ & 354 & 38.484 & 0.049 & 335 & 1.776 & 0.053 \\
\hline 3 & $\mathrm{~A}$ & 472 & 2.187 & 0.060 & 452 & 3.748 & 0.042 \\
\hline 4 & $\mathrm{~A}$ & 561 & 7.565 & 0.075 & 538 & 9.659 & 0.090 \\
\hline 5 & $\mathrm{~A}$ & 595 & 12.470 & 0.133 & 574 & 8.001 & 0.128 \\
\hline 6 & $\mathrm{~A}$ & 633 & 3.353 & 0.165 & 609 & 7.461 & 0.112 \\
\hline 7 & $\mathrm{~A}$ & 673 & 2.009 & 0.043 & 642 & 11.753 & 0.036 \\
\hline 8 & $\mathrm{~A}$ & 689 & 11.148 & 0.044 & 658 & 6.871 & 0.140 \\
\hline 9 & $\mathrm{~A}$ & 754 & 2.309 & 0.097 & 722 & 2.708 & 0.097 \\
\hline 10 & $\mathrm{~A}$ & 778 & 2.595 & 0.540 & 746 & 1.900 & 0.224 \\
\hline 11 & $\mathrm{~A}$ & 804 & 16.964 & 0.068 & 766 & 20.835 & 0.192 \\
\hline 12 & $\mathrm{~A}$ & 869 & 1.080 & 0.153 & 831 & 0.446 & 0.127 \\
\hline 13 & $\mathrm{~A}$ & 923 & 5.907 & 0.285 & 882 & 5.463 & 0.192 \\
\hline 14 & $\mathrm{~A}$ & 962 & 1.522 & 0.187 & 906 & 1.669 & 0.127 \\
\hline 15 & $\mathrm{~A}$ & 1031 & 2.650 & 0.237 & 965 & 0.774 & 0.473 \\
\hline 16 & $\mathrm{~A}$ & 1053 & 7.407 & 0.405 & 977 & 4.071 & 0.094 \\
\hline 17 & $\mathrm{~A}$ & 1099 & 15.715 & 0.155 & 1046 & 16.459 & 0.213 \\
\hline 18 & $\mathrm{~A}$ & 1165 & 7.646 & 0.196 & 1106 & 3.312 & 0.079 \\
\hline 19 & $\mathrm{~A}$ & 1521 & 20.646 & 0.079 & 1296 & 14.218 & 0.017 \\
\hline 20 & $\mathrm{~A}$ & 1542 & 177.186 & 0.074 & 1333 & 35.048 & 0.032 \\
\hline 21 & $\mathrm{~A}$ & 2015 & 9.153 & 0.337 & 1843 & 1.707 & 0.111 \\
\hline 22 & $\mathrm{~A}$ & 2046 & 19.593 & 0.371 & 2073 & 2.489 & 0.128 \\
\hline 23 & $\mathrm{~A}$ & 2528 & 18.029 & 1.173 & 2388 & 18.211 & 1.203 \\
\hline
\end{tabular}


Table S.30.3 - Continued

\begin{tabular}{cccccccc}
\hline & & \multicolumn{3}{c}{ Harmonic } & \multicolumn{3}{c}{ Anharmonic } \\
mode & symm. & $\omega$ & IR & Raman & $\omega$ & IR & Raman \\
& & {$\left[\mathrm{cm}^{-1}\right]$} & {$\left[\mathrm{km} \mathrm{mol}^{-1}\right]$} & {$\left[\AA^{6}\right]$} & {$\left[\mathrm{cm}^{-1}\right]$} & {$\left[\mathrm{km} \mathrm{mol}^{-1}\right]$} & {$\left[\AA^{6}\right]$} \\
\hline 24 & $\mathrm{~A}$ & 2658 & 62.607 & 1.077 & 2537 & 53.563 & 1.147 \\
25 & $\mathrm{~A}$ & 2710 & 68.475 & 0.521 & 2582 & 36.003 & 0.691 \\
26 & $\mathrm{~A}$ & 2718 & 41.369 & 0.978 & 2605 & 46.609 & 0.668 \\
27 & $\mathrm{~A}$ & 2776 & 34.626 & 0.829 & 2665 & 34.222 & 0.959 \\
\hline
\end{tabular}


Figure S.30.2. Anharmonic IR spectrum of $\mathrm{B}_{4} \mathrm{H}_{7}$ - Isomer B obtained by convoluting the calculated intensities with Lorentzians having a FWHM of $4 \mathrm{~cm}^{-1}$ (B3LYP-D2/cc-pVTZ results): (top) full spectrum, (middle) contributions from fundamentals and combination bands, (bottom) contributions from overtones
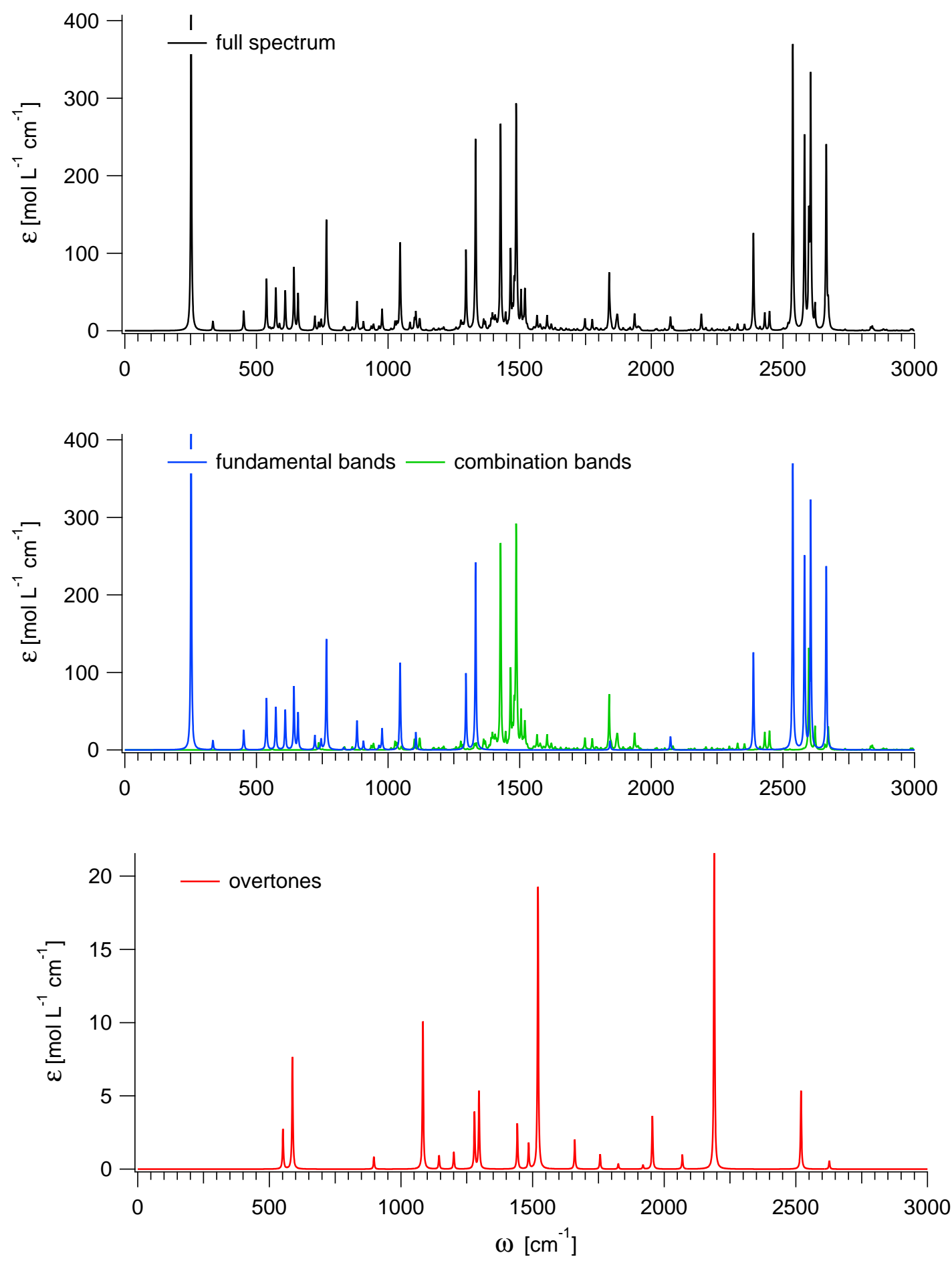
Figure S.30.3. Anharmonic Raman spectrum of $\mathrm{B}_{4} \mathrm{H}_{7}$ - Isomer B obtained by convoluting the calculated activities with Lorentzians having a FWHM of $4 \mathrm{~cm}^{-1}$ (B3LYP-D2/cc-pVTZ results): (top) full spectrum, (middle) contributions from fundamentals and combination bands, (bottom) contributions from overtones
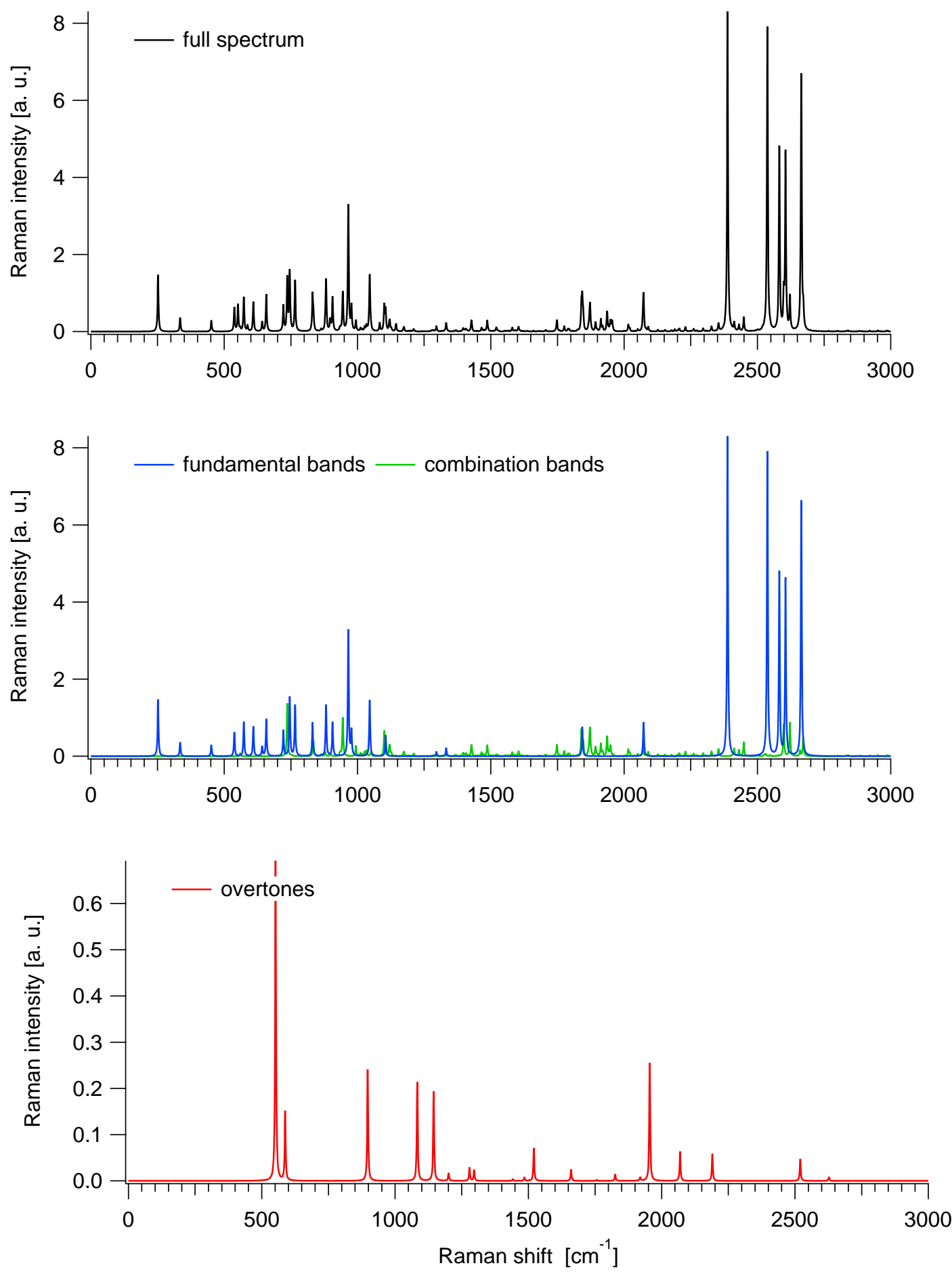

S. 246 
Table S.30.4. $\mathrm{B}_{4} \mathrm{H}_{7}$ - Isomer B: Ideal-gas thermodynamic functions at 1 bar (anharmonic B3LYP-D2/cc-pVTZ results)

\begin{tabular}{|c|c|c|c|}
\hline $\begin{array}{c}T \\
{[\mathrm{~K}]}\end{array}$ & $\begin{array}{c}C_{P} \\
{\left[\mathrm{~J} \mathrm{~mol}^{-1} \mathrm{~K}^{-1}\right]}\end{array}$ & $\begin{array}{c}S \\
{\left[\mathrm{~J} \mathrm{~mol}^{-1} \mathrm{~K}^{-1}\right]}\end{array}$ & $\begin{array}{c}H(T)-H(0 K) \\
{\left[\mathrm{kJ} \mathrm{mol}^{-1}\right]}\end{array}$ \\
\hline 100 & 39.122 & 222.157 & 3.457 \\
\hline 200 & 65.713 & 256.503 & 8.564 \\
\hline 300 & 98.456 & 289.419 & 16.793 \\
\hline 400 & 125.811 & 321.651 & 28.063 \\
\hline 500 & 147.010 & 352.102 & 41.748 \\
\hline 600 & 163.735 & 380.439 & 57.317 \\
\hline 700 & 177.291 & 406.732 & 74.390 \\
\hline 800 & 188.463 & 431.158 & 92.696 \\
\hline 900 & 197.748 & 453.909 & 112.020 \\
\hline 1000 & 205.504 & 475.157 & 132.194 \\
\hline 1100 & 212.006 & 495.058 & 153.079 \\
\hline 1200 & 217.479 & 513.746 & 174.561 \\
\hline 1300 & 222.107 & 531.341 & 196.547 \\
\hline 1400 & 226.039 & 547.949 & 218.959 \\
\hline 1500 & 229.396 & 563.662 & 241.736 \\
\hline 1600 & 232.277 & 578.561 & 264.823 \\
\hline 1700 & 234.763 & 592.719 & 288.178 \\
\hline 1800 & 236.919 & 606.200 & 311.764 \\
\hline 1900 & 238.798 & 619.061 & 335.552 \\
\hline 2000 & 240.443 & 631.352 & 359.516 \\
\hline
\end{tabular}

$T$ : Temperature. $C_{P}$ : Heat capacity at constant pressure. $S$ : Entropy. $H$ : Enthalpy. 
Figure S.30.4. $\mathrm{B}_{4} \mathrm{H}_{7}$ - Isomer B: Ideal-gas thermodynamic functions at 1 bar (anharmonic B3LYP-D2/cc-pVTZ results)
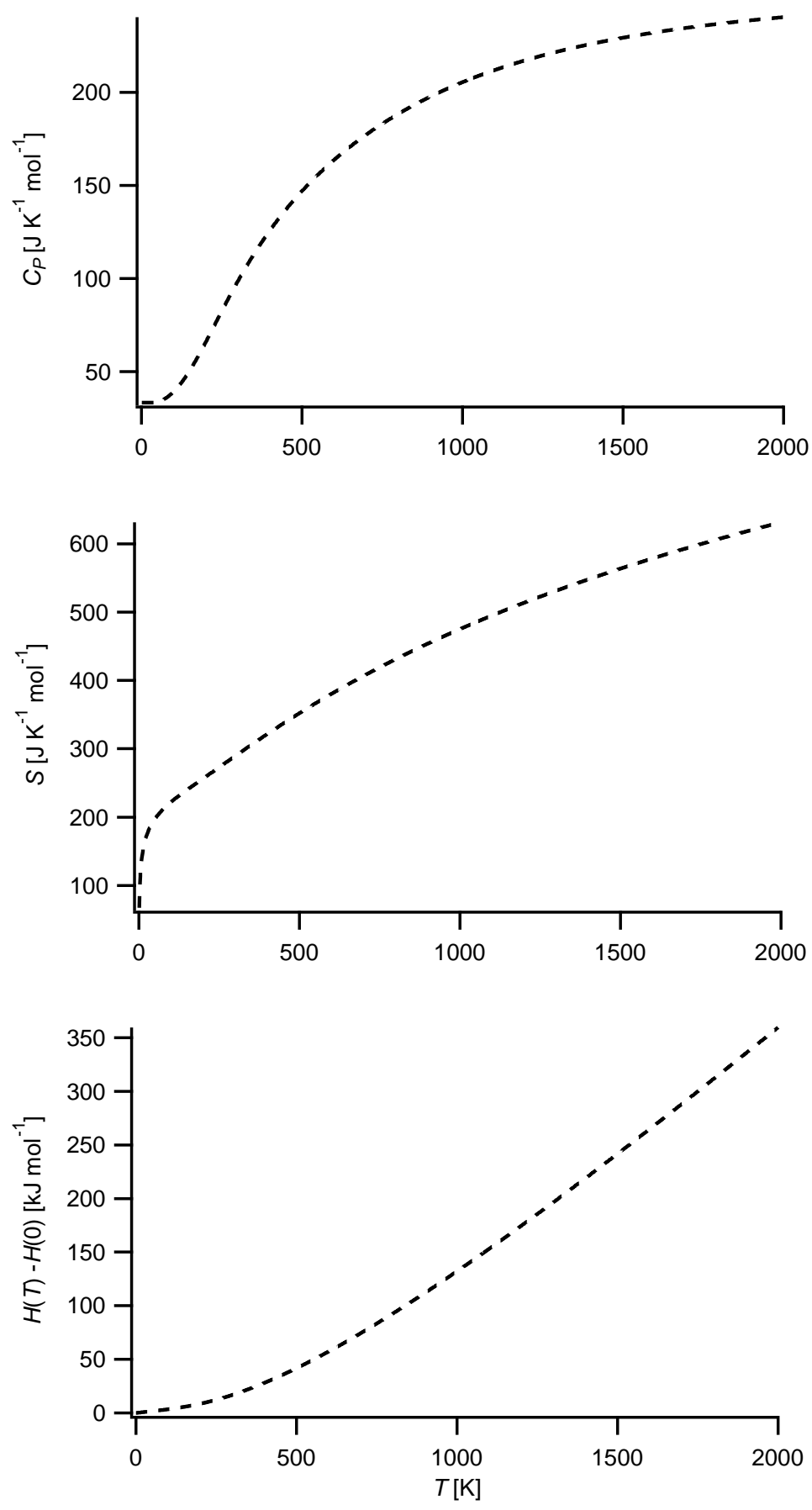

$T$ : Temperature. $C_{P}$ : Heat capacity at constant pressure. $S$ : Entropy. $H$ : Enthalpy. 
Table S.30.5. $\mathrm{B}_{4} \mathrm{H}_{7}$ - Isomer B: Fits (red solid lines) of the computed thermodynamic functions (blue dashed lines) with NASA type functions (Equations (1) - (3)) in the 200 - $900 \mathrm{~K}$ temperature range (anharmonic B3LYP-D2/cc-pVTZ results). In each case, the difference curve between the thermodynamic and NASA functions is plotted in the upper graph

Fit parameters

\begin{tabular}{llllll}
\hline $\mathrm{a}_{1}$ & $-2.77357836 \mathrm{e}+00$ & $\mathrm{a}_{2}\left[\mathrm{~K}^{-1}\right]$ & $6.21887652 \mathrm{e}-02$ & $\mathrm{a}_{3}\left[\mathrm{~K}^{-2}\right]$ & $-4.71496877 \mathrm{e}-05$ \\
$\mathrm{a}_{4}\left[\mathrm{~K}^{-3}\right]$ & $5.65068724 \mathrm{e}-09$ & $\mathrm{a}_{5}\left[\mathrm{~K}^{-4}\right]$ & $7.19100268 \mathrm{e}-12$ & $\mathrm{a}_{6}[\mathrm{~K}]$ & $4.63953772 \mathrm{e}+02$ \\
$\mathrm{a}_{7}$ & $3.40327228 \mathrm{e}+01$ & & & &
\end{tabular}
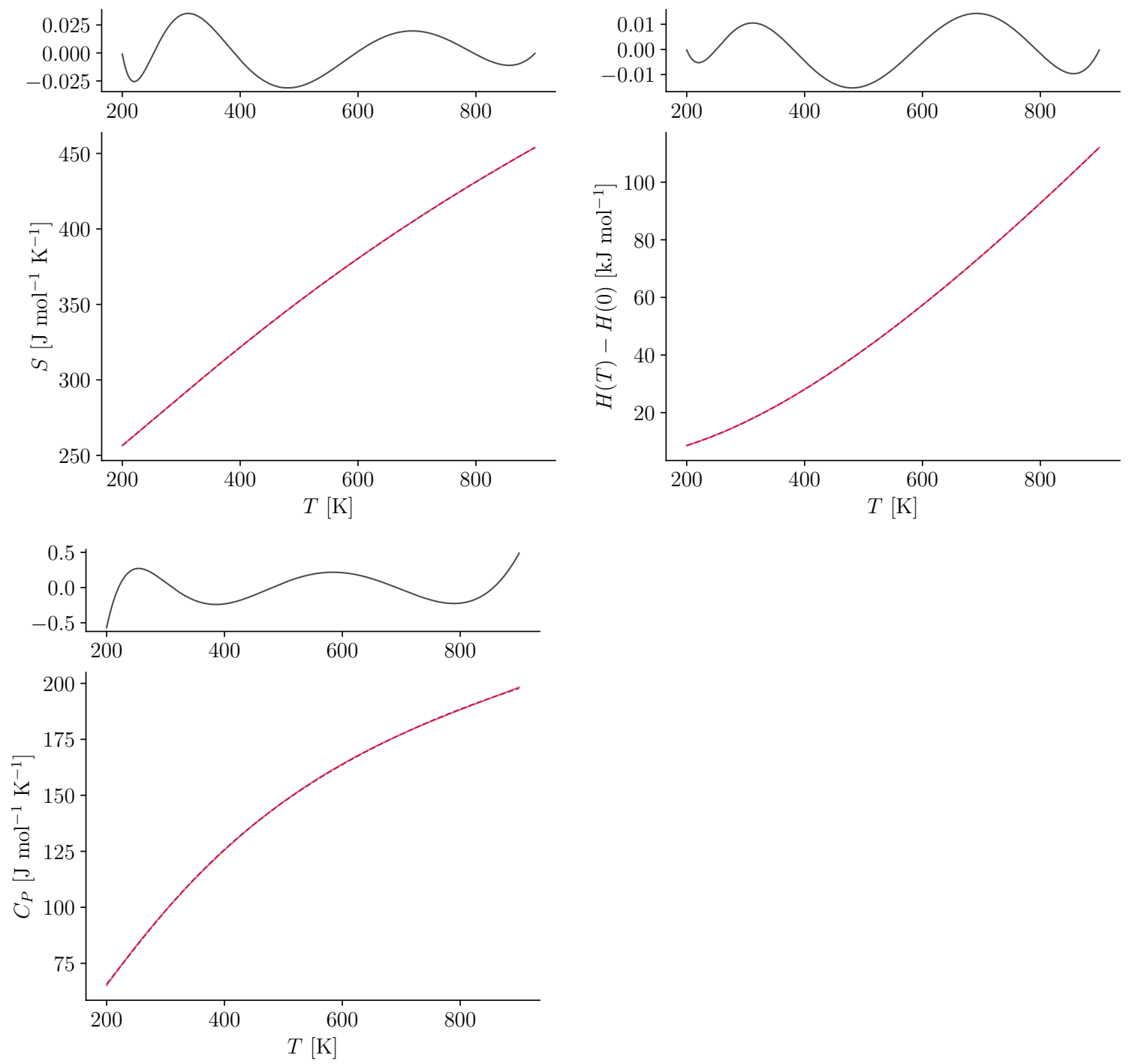


\section{$31 \quad \mathbf{B}_{4} \mathbf{H}_{7}$ - Isomer $\mathbf{C}$}

Figure S.31.1. Structure of $\mathrm{B}_{4} \mathrm{H}_{7}$ - Isomer C

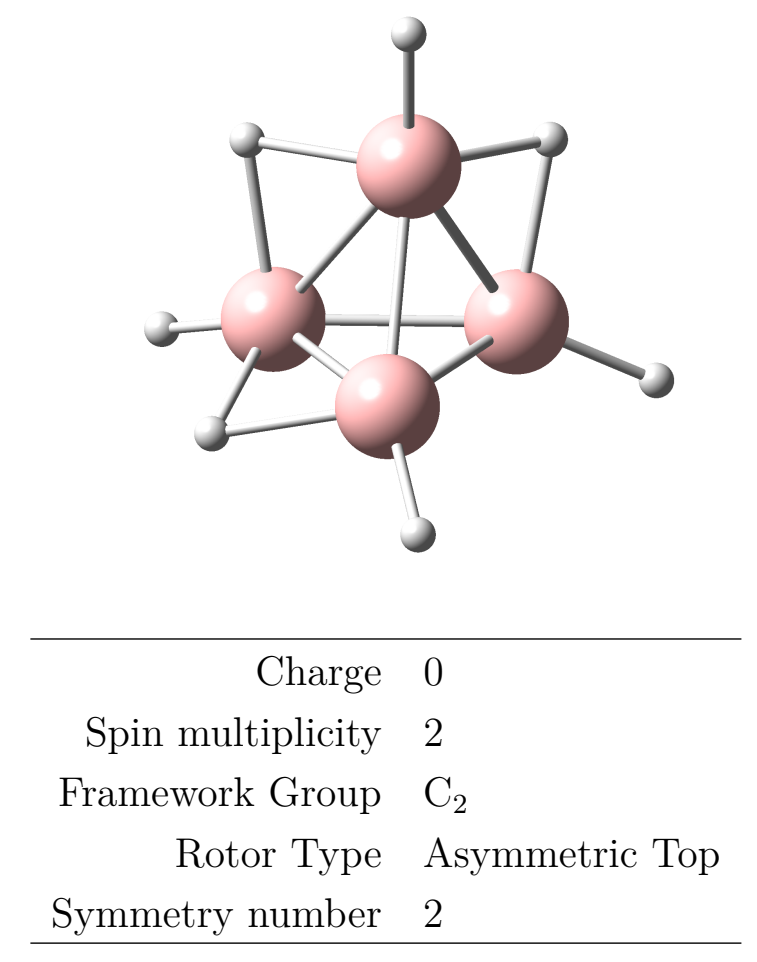

Table S.31.1. $\mathrm{B}_{4} \mathrm{H}_{7}$ - Isomer C: Selected anharmonic results obtained at the B3LYP-D2/ccpVTZ level

\begin{tabular}{rll}
\hline $\mathrm{ZPE}_{\mathrm{v}}$ & 16985 & $\mathrm{~cm}^{-1}$ \\
$\mathrm{~A}$ & 0.34444 & $\mathrm{~cm}^{-1}$ \\
$\mathrm{~B}$ & 0.31710 & $\mathrm{~cm}^{-1}$ \\
$\mathrm{C}$ & 0.31424 & $\mathrm{~cm}^{-1}$ \\
\hline$C_{P}(300 \mathrm{~K})$ & 95.811 & $\mathrm{~J} \mathrm{~mol}^{-1} \mathrm{~K}-1$ \\
$S(300 \mathrm{~K})$ & 277.567 & $\mathrm{~J} \mathrm{~mol}^{-1} \mathrm{~K}-1$ \\
$H(300 \mathrm{~K})-H(0 \mathrm{~K})$ & 16.037 & $\mathrm{~kJ} \mathrm{~mol}^{-1}$ \\
\hline
\end{tabular}

$\mathrm{ZPE}_{\mathrm{v}}$ : vibrational contribution to the zero-point energy. A, B, C: Rotational constants. $C_{P}$ : Heat capacity at constant pressure. $S$ : Entropy. $H$ : Enthalpy. Ideal-gas calculations (1 bar). 
Table S.31.2. $\mathrm{B}_{4} \mathrm{H}_{7}$ - Isomer C: Cartesian coordinates $(\AA)$ of the B3LYP-D2/cc-pVTZ optimized structure

\begin{tabular}{cccc} 
atom & $\mathrm{x}$ & $\mathrm{y}$ & $\mathrm{z}$ \\
\hline B1 & -0.6334 & -0.6766 & 0.5366 \\
B2 & 0.6334 & -0.5785 & -0.6311 \\
B3 & -0.6334 & 0.5785 & -0.6311 \\
B4 & 0.6334 & 0.6766 & 0.5366 \\
H5 & -0.1967 & 1.5942 & 0.1153 \\
H6 & 1.2132 & -1.0659 & -1.5292 \\
H7 & 0.1967 & -1.5942 & 0.1153 \\
H8 & 1.4847 & 1.2493 & 1.1236 \\
H9 & -1.2132 & 1.0659 & -1.5292 \\
H10 & 0.0000 & 0.0000 & 1.5248 \\
H11 & -1.4847 & -1.2493 & 1.1236 \\
\hline
\end{tabular}


Table S.31.3. $\mathrm{B}_{4} \mathrm{H}_{7}$ - Isomer C: Vibrational frequencies, infrared integrated intensities and Raman activities (B3LYP-D2/cc-pVTZ results)

\begin{tabular}{|c|c|c|c|c|c|c|c|}
\hline \multirow[b]{2}{*}{ mode } & \multirow[b]{2}{*}{ symm. } & \multicolumn{3}{|c|}{ Harmonic } & \multicolumn{3}{|c|}{ Anharmonic } \\
\hline & & $\begin{array}{c}\omega \\
{\left[\mathrm{cm}^{-1}\right]}\end{array}$ & $\begin{array}{c}\mathrm{IR} \\
{\left[\mathrm{km} \mathrm{mol}^{-1}\right]}\end{array}$ & $\begin{array}{c}\text { Raman } \\
{\left[\AA^{6}\right]}\end{array}$ & $\begin{array}{c}\omega \\
{\left[\mathrm{cm}^{-1}\right]}\end{array}$ & $\begin{array}{c}\mathrm{IR} \\
{\left[\mathrm{km} \mathrm{mol}^{-1}\right]}\end{array}$ & $\begin{array}{c}\text { Raman } \\
{\left[\AA^{6}\right]}\end{array}$ \\
\hline 1 & A & 422 & 0.188 & 0.253 & 409 & 0.117 & 0.234 \\
\hline 2 & $\mathrm{~B}$ & 456 & 16.466 & 0.033 & 389 & 22.048 & 0.068 \\
\hline 3 & $\mathrm{~A}$ & 515 & 1.185 & 0.061 & 488 & 0.412 & 0.057 \\
\hline 4 & $\mathrm{~A}$ & 591 & 0.536 & 0.140 & 547 & 1.393 & 0.160 \\
\hline 5 & $\mathrm{~B}$ & 622 & 12.092 & 0.032 & 583 & 12.989 & 0.040 \\
\hline 6 & $\mathrm{~B}$ & 691 & 1.021 & 0.067 & 651 & 6.607 & 0.057 \\
\hline 7 & $\mathrm{~B}$ & 705 & 14.384 & 0.013 & 637 & 9.733 & 0.035 \\
\hline 8 & $\mathrm{~A}$ & 713 & 5.683 & 0.149 & 675 & 5.942 & 0.182 \\
\hline 9 & $\mathrm{~A}$ & 758 & 0.174 & 0.057 & 714 & 2.422 & 0.025 \\
\hline 10 & $\mathrm{~B}$ & 764 & 3.076 & 0.190 & 733 & 3.123 & 0.163 \\
\hline 11 & $\mathrm{~A}$ & 794 & 5.028 & 0.117 & 758 & 4.163 & 0.133 \\
\hline 12 & $\mathrm{~B}$ & 822 & 6.679 & 0.022 & 790 & 2.237 & 0.088 \\
\hline 13 & $\mathrm{~B}$ & 897 & 2.079 & 0.044 & 854 & 0.833 & 0.046 \\
\hline 14 & $\mathrm{~A}$ & 911 & 5.523 & 0.166 & 863 & 5.987 & 0.158 \\
\hline 15 & $\mathrm{~A}$ & 929 & 4.168 & 0.017 & 896 & 3.115 & 0.012 \\
\hline 16 & A & 1035 & 0.008 & 1.044 & 1009 & 0.108 & 0.761 \\
\hline 17 & B & 1072 & 3.494 & 0.081 & 1017 & 2.936 & 0.059 \\
\hline 18 & B & 1575 & 36.298 & 0.101 & 1367 & 13.196 & 0.075 \\
\hline 19 & $\mathrm{~A}$ & 1676 & 25.507 & 0.059 & 1490 & 9.601 & 0.040 \\
\hline 20 & B & 1680 & 16.178 & 0.039 & 1499 & 7.642 & 0.018 \\
\hline 21 & $\mathrm{~A}$ & 1902 & 8.273 & 0.583 & 1917 & 3.281 & 0.381 \\
\hline 22 & B & 2099 & 6.779 & 0.014 & 2018 & 5.888 & 0.009 \\
\hline 23 & $\mathrm{~A}$ & 2127 & 1.326 & 0.646 & 2078 & 0.014 & 0.257 \\
\hline
\end{tabular}


Table S.31.3 - Continued

\begin{tabular}{cccccccc}
\hline & & \multicolumn{3}{c}{ Harmonic } & \multicolumn{3}{c}{ Anharmonic } \\
mode & symm. & $\omega$ & IR & Raman & $\omega$ & IR & Raman \\
& & {$\left[\mathrm{cm}^{-1}\right]$} & {$\left[\mathrm{km} \mathrm{mol}^{-1}\right]$} & {$\left[\AA^{6}\right]$} & {$\left[\mathrm{cm}^{-1}\right]$} & {$\left[\mathrm{km} \mathrm{mol}^{-1}\right]$} & {$\left[\AA^{6}\right]$} \\
\hline 24 & B & 2692 & 64.951 & 0.482 & 2592 & 68.205 & 0.512 \\
25 & A & 2698 & 29.157 & 1.271 & 2595 & 28.997 & 1.318 \\
26 & B & 2754 & 33.772 & 0.539 & 2646 & 32.614 & 0.521 \\
27 & A & 2760 & 24.559 & 1.042 & 2652 & 26.137 & 1.181 \\
\hline
\end{tabular}


Figure S.31.2. Anharmonic IR spectrum of $\mathrm{B}_{4} \mathrm{H}_{7}$ - Isomer $\mathrm{C}$ obtained by convoluting the calculated intensities with Lorentzians having a FWHM of $4 \mathrm{~cm}^{-1}$ (B3LYP-D2/cc-pVTZ results): (top) full spectrum, (middle) contributions from fundamentals and combination bands, (bottom) contributions from overtones
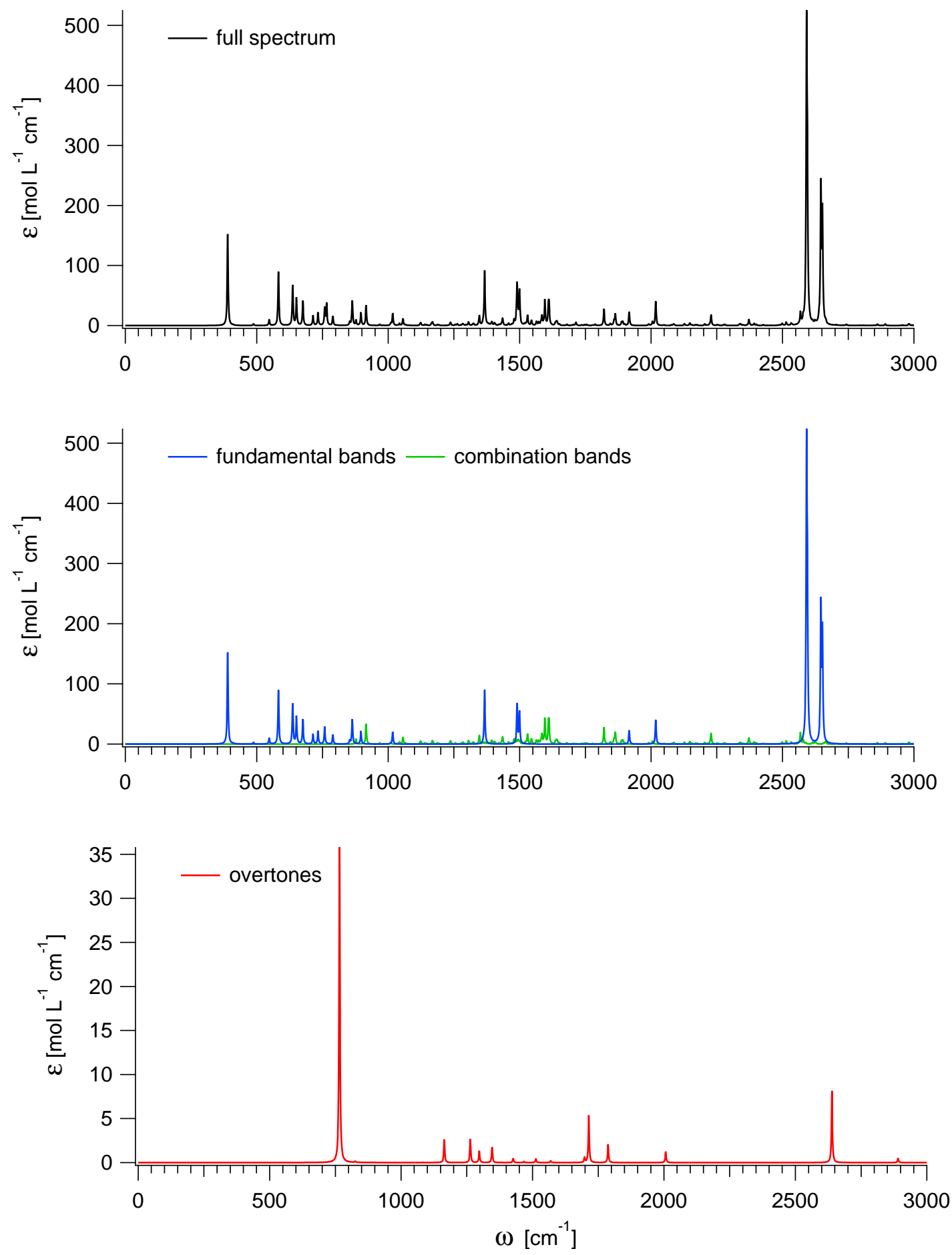
Figure S.31.3. Anharmonic Raman spectrum of $\mathrm{B}_{4} \mathrm{H}_{7}$ - Isomer $\mathrm{C}$ obtained by convoluting the calculated activities with Lorentzians having a FWHM of $4 \mathrm{~cm}^{-1}$ (B3LYP-D2/cc-pVTZ results): (top) full spectrum, (middle) contributions from fundamentals and combination bands, (bottom) contributions from overtones
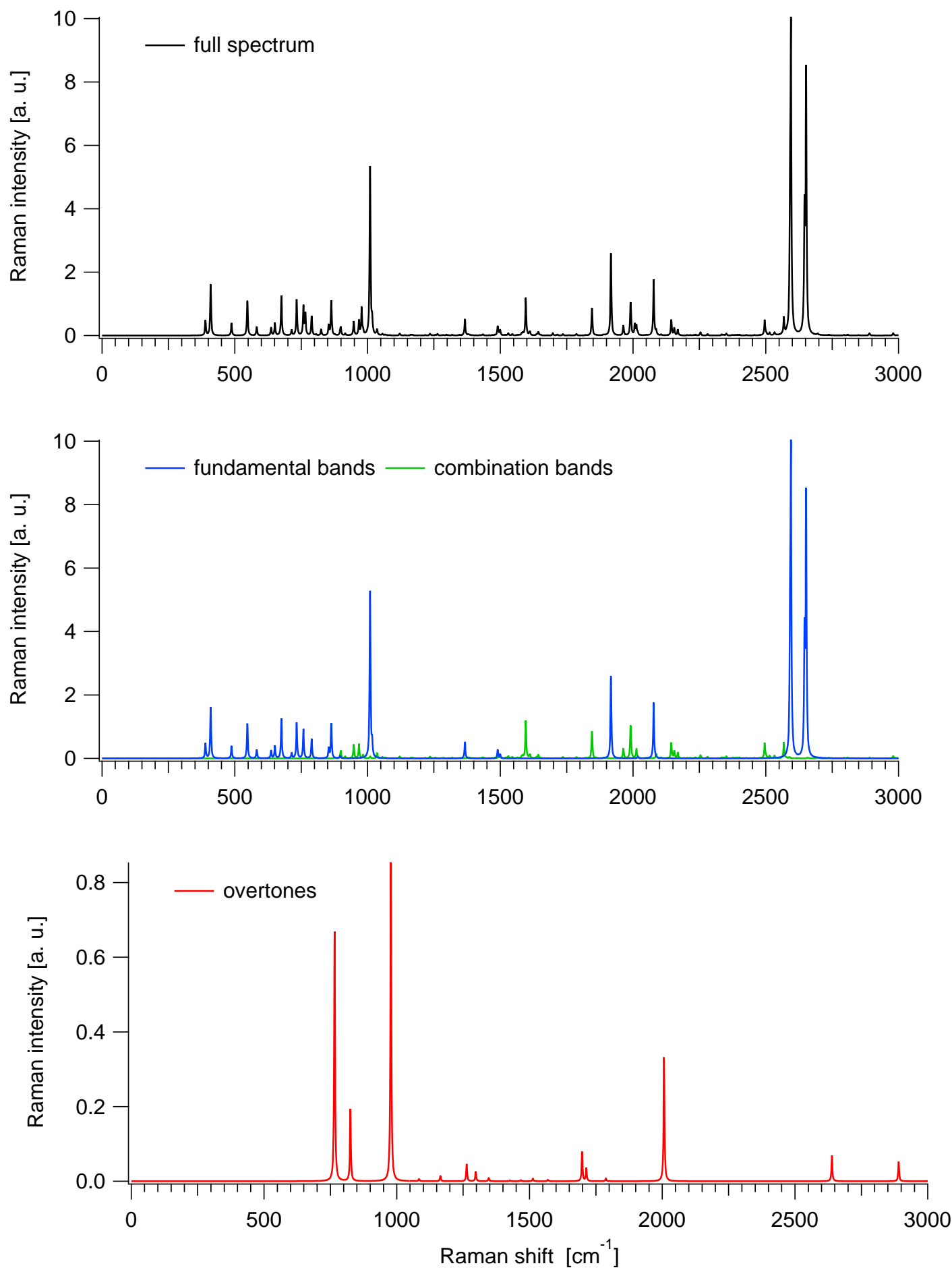

$\mathrm{S} .255$ 
Table S.31.4. $\mathrm{B}_{4} \mathrm{H}_{7}$ - Isomer C: Ideal-gas thermodynamic functions at 1 bar (anharmonic B3LYP-D2/cc-pVTZ results)

\begin{tabular}{cccc}
\hline$T$ & $C_{P}$ & $S$ & $H(T)-H(0 K)$ \\
{$[\mathrm{K}]$} & {$\left[\mathrm{J} \mathrm{mol}^{-1} \mathrm{~K}^{-1}\right]$} & {$\left[\mathrm{J} \mathrm{mol}^{-1} \mathrm{~K}^{-1}\right]$} & {$\left[\mathrm{kJ} \mathrm{mol}^{-1}\right]$} \\
\hline 100 & 36.015 & 214.090 & 3.369 \\
200 & 62.275 & 245.854 & 8.102 \\
300 & 95.811 & 277.567 & 16.037 \\
400 & 123.208 & 309.049 & 27.047 \\
500 & 144.517 & 338.926 & 40.475 \\
600 & 161.523 & 366.833 & 55.807 \\
700 & 175.415 & 392.809 & 72.677 \\
800 & 186.903 & 417.007 & 90.810 \\
900 & 196.457 & 439.589 & 109.993 \\
1000 & 204.429 & 460.713 & 130.049 \\
1100 & 211.105 & 480.520 & 150.836 \\
1200 & 216.717 & 499.136 & 172.235 \\
1300 & 221.456 & 516.675 & 194.150 \\
1400 & 225.477 & 533.238 & 216.502 \\
1500 & 228.907 & 548.914 & 239.226 \\
1600 & 231.849 & 563.784 & 262.267 \\
1700 & 234.385 & 577.918 & 285.582 \\
1800 & 236.583 & 591.378 & 309.133 \\
1900 & 238.497 & 604.222 & 332.889 \\
2000 & 240.172 & 616.499 & 356.824 \\
\hline$T$ & & & $S$ \\
\hline
\end{tabular}

$T$ : Temperature. $C_{P}$ : Heat capacity at constant pressure. $S$ : Entropy. $H$ : Enthalpy. 
Figure S.31.4. $\mathrm{B}_{4} \mathrm{H}_{7}$ - Isomer C: Ideal-gas thermodynamic functions at 1 bar (anharmonic B3LYP-D2/cc-pVTZ results)
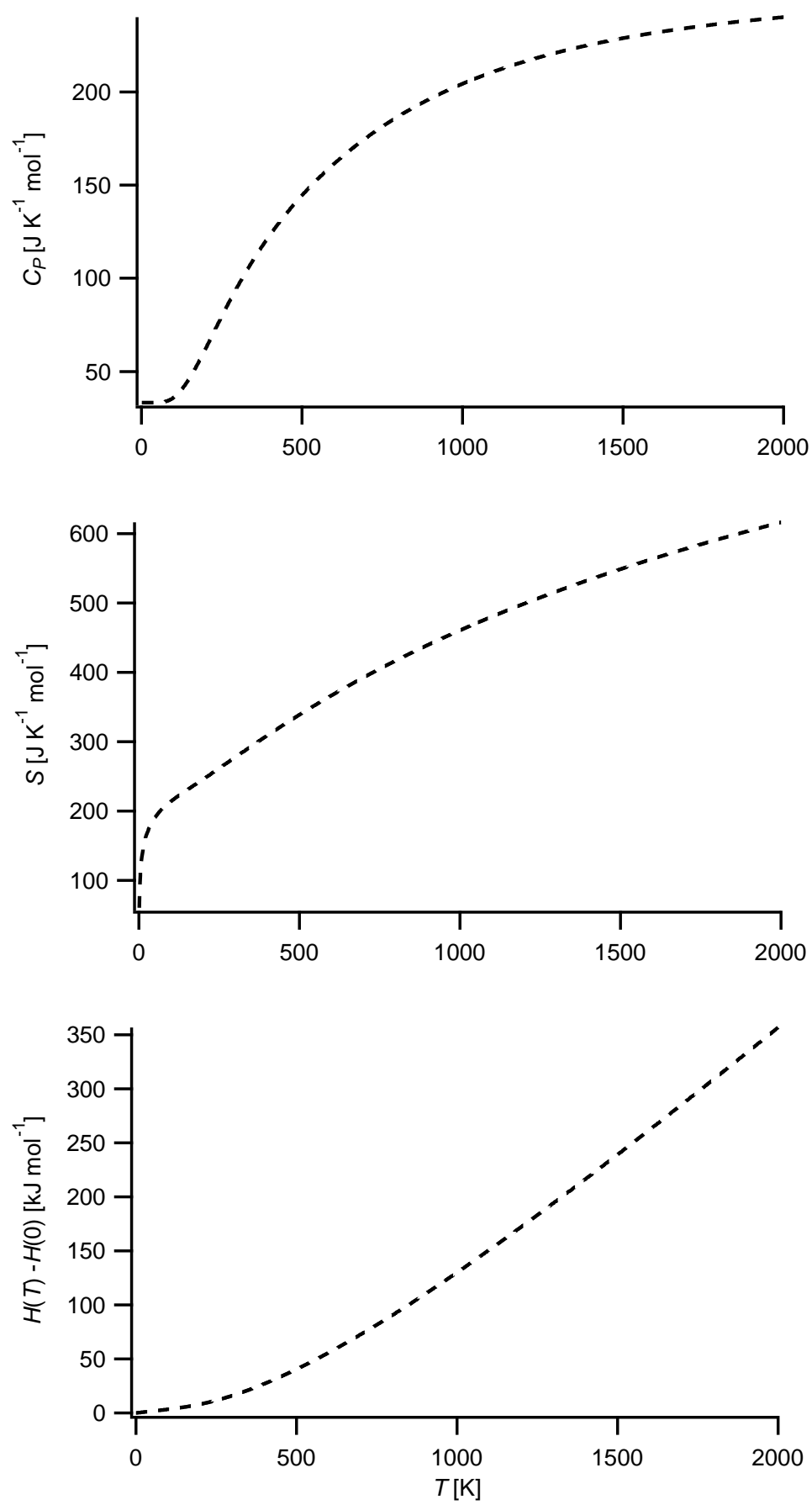

$T$ : Temperature. $C_{P}$ : Heat capacity at constant pressure. $S$ : Entropy. $H$ : Enthalpy. 
Table S.31.5. $\mathrm{B}_{4} \mathrm{H}_{7}$ - Isomer C: Fits (red solid lines) of the computed thermodynamic functions (blue dashed lines) with NASA type functions (Equations (1) - (3)) in the 200 - $900 \mathrm{~K}$ temperature range (anharmonic B3LYP-D2/cc-pVTZ results). In each case, the difference curve between the thermodynamic and NASA functions is plotted in the upper graph

Fit parameters

\begin{tabular}{llllll}
\hline $\mathrm{a}_{1}$ & $-3.86802260 \mathrm{e}+00$ & $\mathrm{a}_{2}\left[\mathrm{~K}^{-1}\right]$ & $6.84829445 \mathrm{e}-02$ & $\mathrm{a}_{3}\left[\mathrm{~K}^{-2}\right]$ & $-6.56151834 \mathrm{e}-05$ \\
$\mathrm{a}_{4}\left[\mathrm{~K}^{-3}\right]$ & $2.89097660 \mathrm{e}-08$ & $\mathrm{a}_{5}\left[\mathrm{~K}^{-4}\right]$ & $-3.08829068 \mathrm{e}-12$ & $\mathrm{a}_{6}[\mathrm{~K}]$ & $5.42010038 \mathrm{e}+02$ \\
$\mathrm{a}_{7}$ & $3.76032108 \mathrm{e}+01$ & & & &
\end{tabular}
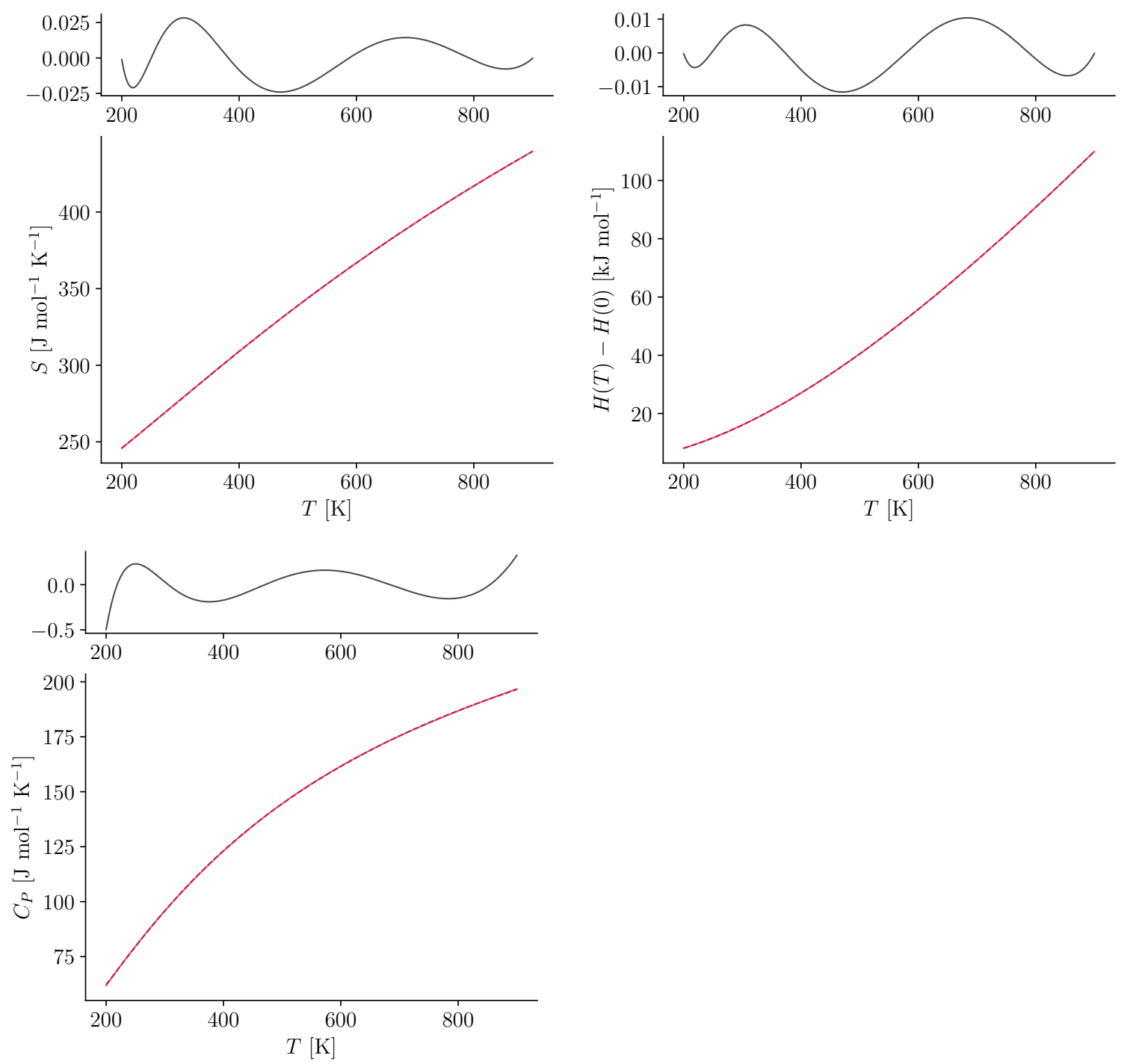


\section{$32 \quad \mathrm{~B}_{4} \mathrm{H}_{8}$}

Figure S.32.1. Structure of $\mathrm{B}_{4} \mathrm{H}_{8}$

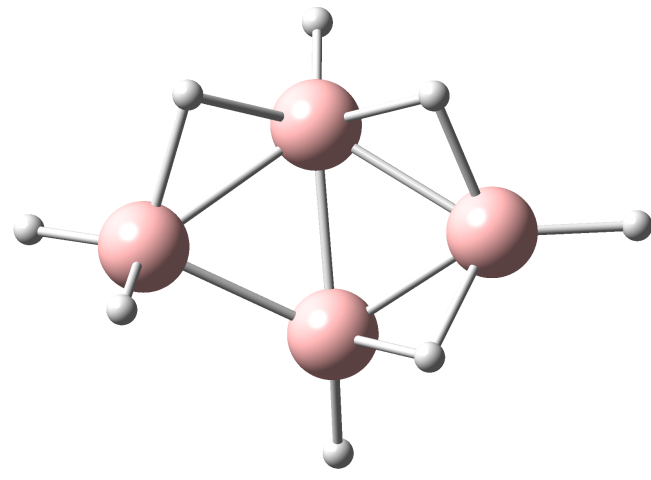

\begin{aligned} & \hline Charge 0 \\ & Spin multiplicity 1 \\ & Framework Group $\mathrm{C}_{1} \\ &$ Rotor Type Asymmetric Top \\ & Symmetry number 1 \\ & \hline\end{aligned}

Table S.32.1. $\mathrm{B}_{4} \mathrm{H}_{8}$ : Selected anharmonic results obtained at the B3LYP-D2/cc-pVTZ level

\begin{tabular}{rll}
\hline $\mathrm{ZPE}_{\mathrm{v}}$ & 19378 & $\mathrm{~cm}^{-1}$ \\
$\mathrm{~A}$ & 0.49100 & $\mathrm{~cm}^{-1}$ \\
$\mathrm{~B}$ & 0.24189 & $\mathrm{~cm}^{-1}$ \\
$\mathrm{C}$ & 0.19340 & $\mathrm{~cm}^{-1}$ \\
\hline$C_{P}(300 \mathrm{~K})$ & 96.503 & $\mathrm{~J} \mathrm{~mol}^{-1} \mathrm{~K}-1$ \\
$S(300 \mathrm{~K})$ & 285.405 & $\mathrm{~J} \mathrm{~mol}^{-1} \mathrm{~K}-1$ \\
$H(300 \mathrm{~K})-H(0 \mathrm{~K})$ & 16.038 & $\mathrm{~kJ} \mathrm{~mol}^{-1}$ \\
\hline
\end{tabular}

$\mathrm{ZPE}_{\mathrm{v}}$ : vibrational contribution to the zero-point energy. A, B, C: Rotational constants. $C_{P}$ : Heat capacity at constant pressure. $S$ : Entropy. $H$ : Enthalpy. Ideal-gas calculations (1 bar). 
Table S.32.2. $\mathrm{B}_{4} \mathrm{H}_{8}$ : Cartesian coordinates $(\AA)$ of the B3LYP-D2/cc-pVTZ optimized structure

\begin{tabular}{cccc} 
atom & $\mathrm{x}$ & $\mathrm{y}$ & $\mathrm{z}$ \\
\hline B1 & -0.0197 & 0.8607 & -0.2419 \\
B2 & 1.4009 & -0.0073 & 0.1961 \\
B3 & -1.4120 & -0.1243 & 0.2002 \\
B4 & 0.1158 & -0.8309 & -0.3344 \\
H5 & -1.0547 & 1.1021 & 0.5836 \\
H6 & -2.3368 & 0.0147 & -0.5342 \\
H7 & -0.0090 & 1.6886 & -1.0841 \\
H8 & 0.8972 & -1.0155 & 0.8294 \\
H9 & 0.1589 & -1.7908 & -1.0148 \\
H10 & -1.5877 & -0.6322 & 1.2675 \\
H11 & 0.9339 & 1.0951 & 0.7298 \\
H12 & 2.5732 & 0.0469 & 0.1223 \\
\hline
\end{tabular}


Table S.32.3. $\mathrm{B}_{4} \mathrm{H}_{8}$ : Vibrational frequencies, infrared integrated intensities and Raman activities (B3LYP-D2/cc-pVTZ results)

\begin{tabular}{|c|c|c|c|c|c|c|c|}
\hline \multirow[b]{2}{*}{ mode } & \multirow[b]{2}{*}{ symm. } & \multicolumn{3}{|c|}{ Harmonic } & \multicolumn{3}{|c|}{ Anharmonic } \\
\hline & & $\begin{array}{c}\omega \\
{\left[\mathrm{cm}^{-1}\right]}\end{array}$ & $\begin{array}{c}\mathrm{IR} \\
{\left[\mathrm{km} \mathrm{mol}^{-1}\right]}\end{array}$ & $\begin{array}{c}\text { Raman } \\
{\left[\AA^{6}\right]}\end{array}$ & $\begin{array}{c}\omega \\
{\left[\mathrm{cm}^{-1}\right]}\end{array}$ & $\begin{array}{c}\mathrm{IR} \\
{\left[\mathrm{km} \mathrm{mol}^{-1}\right]}\end{array}$ & $\begin{array}{c}\text { Raman } \\
{\left[\AA^{6}\right]}\end{array}$ \\
\hline 1 & $\mathrm{~A}$ & 348 & 1.800 & 0.086 & 338 & 3.883 & 0.102 \\
\hline 2 & $\mathrm{~A}$ & 439 & 22.285 & 0.104 & 416 & 26.630 & 0.088 \\
\hline 3 & $\mathrm{~A}$ & 548 & 24.157 & 0.079 & 510 & 42.571 & 0.055 \\
\hline 4 & $\mathrm{~A}$ & 569 & 0.786 & 0.031 & 540 & 2.761 & 0.030 \\
\hline 5 & $\mathrm{~A}$ & 599 & 12.013 & 0.013 & 555 & 6.025 & 0.042 \\
\hline 6 & $\mathrm{~A}$ & 662 & 15.984 & 0.224 & 617 & 12.465 & 0.293 \\
\hline 7 & $\mathrm{~A}$ & 727 & 6.656 & 0.024 & 673 & 5.398 & 0.070 \\
\hline 8 & $\mathrm{~A}$ & 741 & 1.819 & 0.175 & 715 & 5.033 & 0.182 \\
\hline 9 & $\mathrm{~A}$ & 773 & 45.572 & 0.153 & 729 & 68.087 & 0.123 \\
\hline 10 & $\mathrm{~A}$ & 810 & 4.388 & 0.160 & 772 & 4.515 & 0.218 \\
\hline 11 & $\mathrm{~A}$ & 864 & 27.603 & 0.119 & 817 & 25.382 & 0.080 \\
\hline 12 & $\mathrm{~A}$ & 913 & 1.110 & 0.081 & 873 & 0.705 & 0.044 \\
\hline 13 & $\mathrm{~A}$ & 955 & 4.439 & 0.077 & 913 & 3.352 & 0.119 \\
\hline 14 & $\mathrm{~A}$ & 991 & 13.521 & 0.168 & 948 & 1.535 & 0.030 \\
\hline 15 & $\mathrm{~A}$ & 1025 & 3.219 & 0.204 & 980 & 3.530 & 0.077 \\
\hline 16 & $\mathrm{~A}$ & 1070 & 13.419 & 0.401 & 1008 & 9.589 & 0.325 \\
\hline 17 & $\mathrm{~A}$ & 1108 & 1.529 & 0.082 & 1043 & 1.640 & 0.137 \\
\hline 18 & $\mathrm{~A}$ & 1155 & 26.463 & 0.135 & 1092 & 8.943 & 0.103 \\
\hline 19 & $\mathrm{~A}$ & 1180 & 18.815 & 0.112 & 1134 & 9.585 & 0.055 \\
\hline 20 & $\mathrm{~A}$ & 1370 & 52.823 & 0.115 & 1162 & 10.999 & 0.030 \\
\hline 21 & $\mathrm{~A}$ & 1482 & 211.851 & 0.071 & 1265 & 55.214 & 0.028 \\
\hline 22 & $\mathrm{~A}$ & 1619 & 20.517 & 0.022 & 1427 & 2.465 & 0.014 \\
\hline 23 & $\mathrm{~A}$ & 1985 & 8.221 & 0.156 & 1898 & 1.997 & 0.026 \\
\hline
\end{tabular}


Table S.32.3 - Continued

\begin{tabular}{cccccccc}
\hline & & \multicolumn{3}{c}{ Harmonic } & \multicolumn{3}{c}{ Anharmonic } \\
mode & symm. & $\omega$ & IR & Raman & $\omega$ & IR & Raman \\
& & {$\left[\mathrm{cm}^{-1}\right]$} & {$\left[\mathrm{km} \mathrm{mol}^{-1}\right]$} & {$\left[\AA^{6}\right]$} & {$\left[\mathrm{cm}^{-1}\right]$} & {$\left[\mathrm{km} \mathrm{mol}^{-1}\right]$} & {$\left[\AA^{6}\right]$} \\
\hline 24 & A & 2057 & 18.715 & 0.676 & 1975 & 1.492 & 0.086 \\
25 & A & 2131 & 47.674 & 0.226 & 2070 & 4.551 & 0.101 \\
26 & A & 2586 & 30.460 & 1.088 & 2476 & 26.091 & 1.219 \\
27 & A & 2660 & 73.739 & 0.658 & 2544 & 32.631 & 0.354 \\
28 & A & 2720 & 70.202 & 0.635 & 2594 & 53.644 & 0.597 \\
29 & A & 2744 & 25.694 & 0.709 & 2629 & 26.966 & 0.703 \\
30 & A & 2765 & 37.741 & 0.965 & 2642 & 36.720 & 1.023 \\
\hline
\end{tabular}


Figure S.32.2. Anharmonic IR spectrum of $\mathrm{B}_{4} \mathrm{H}_{8}$ obtained by convoluting the calculated intensities with Lorentzians having a FWHM of $4 \mathrm{~cm}^{-1}$ (B3LYP-D2/cc-pVTZ results): (top) full spectrum, (middle) contributions from fundamentals and combination bands, (bottom) contributions from overtones
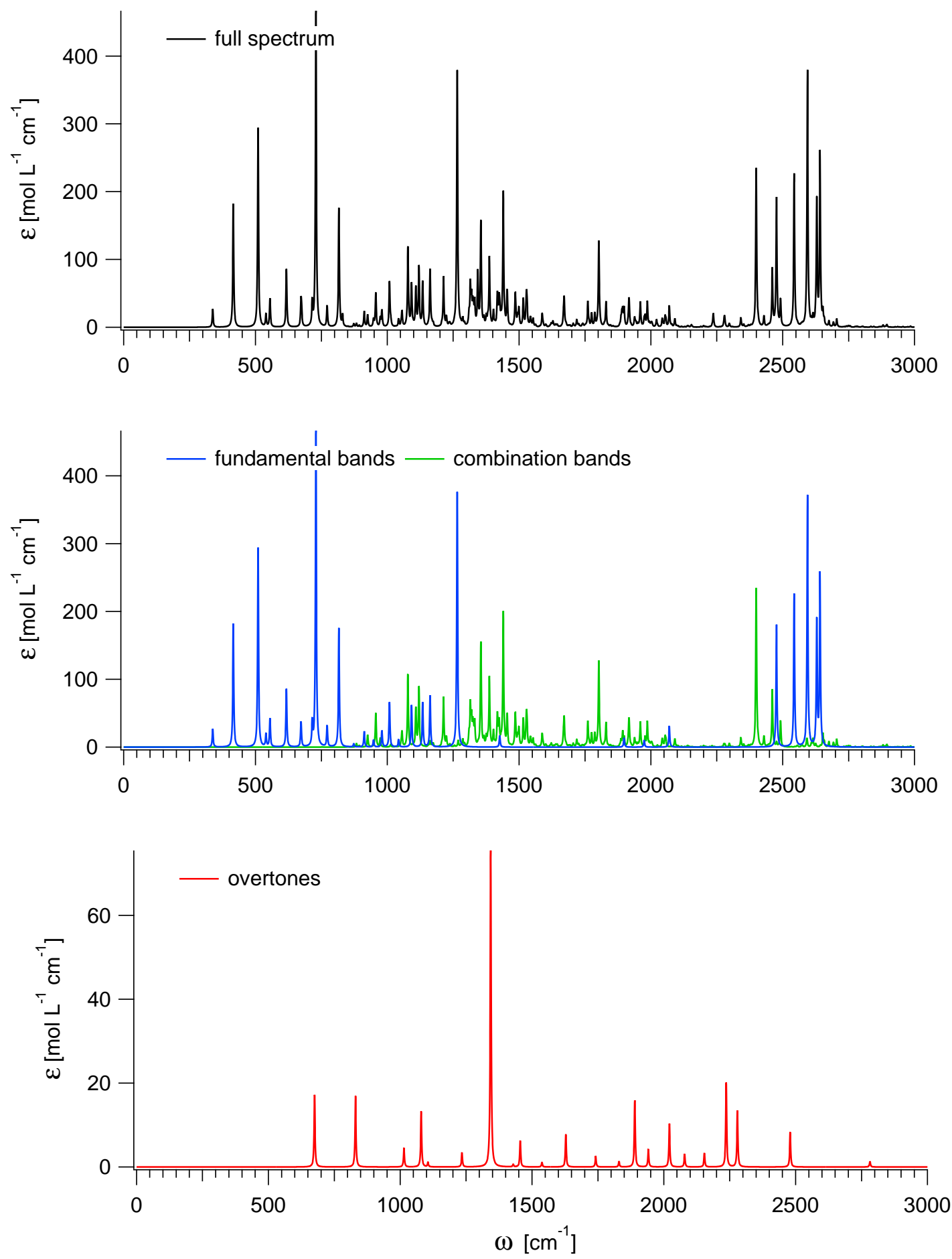
Figure S.32.3. Anharmonic Raman spectrum of $\mathrm{B}_{4} \mathrm{H}_{8}$ obtained by convoluting the calculated activities with Lorentzians having a FWHM of $4 \mathrm{~cm}^{-1}$ (B3LYP-D2/cc-pVTZ results): (top) full spectrum, (middle) contributions from fundamentals and combination bands, (bottom) contributions from overtones
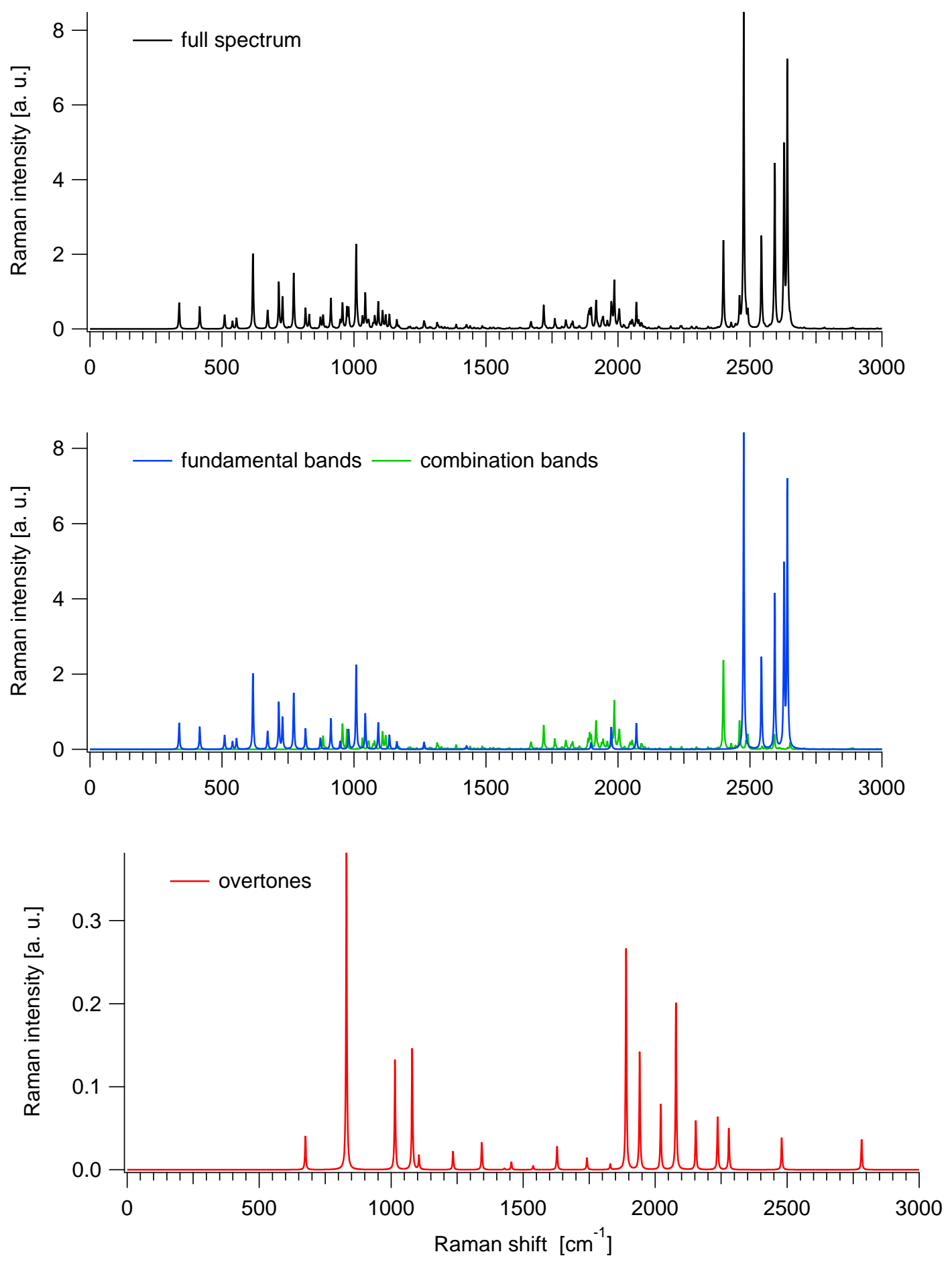

S.264 
Table S.32.4. $\mathrm{B}_{4} \mathrm{H}_{8}$ : Ideal-gas thermodynamic functions at 1 bar (anharmonic B3LYPD2/cc-pVTZ results)

\begin{tabular}{cccc}
\hline$T$ & $C_{P}$ & $S$ & $H(T)-H(0 K)$ \\
{$[\mathrm{K}]$} & {$\left[\mathrm{J} \mathrm{mol}^{-1} \mathrm{~K}^{-1}\right]$} & {$\left[\mathrm{J} \mathrm{mol}^{-1} \mathrm{~K}^{-1}\right]$} & {$\left[\mathrm{kJ} \mathrm{mol}^{-1}\right]$} \\
\hline 100 & 36.474 & 221.920 & 3.381 \\
200 & 61.781 & 253.795 & 8.124 \\
300 & 96.503 & 285.405 & 16.038 \\
400 & 127.367 & 317.542 & 27.283 \\
500 & 152.169 & 348.732 & 41.307 \\
600 & 172.064 & 378.298 & 57.554 \\
700 & 188.262 & 406.079 & 75.597 \\
800 & 201.593 & 432.117 & 95.111 \\
900 & 212.635 & 456.519 & 115.839 \\
1000 & 221.820 & 479.412 & 137.576 \\
1100 & 229.491 & 500.925 & 160.153 \\
1200 & 235.928 & 521.177 & 183.433 \\
1300 & 241.354 & 540.282 & 207.304 \\
1400 & 245.953 & 558.341 & 231.676 \\
1500 & 249.872 & 575.447 & 256.472 \\
1600 & 253.230 & 591.683 & 281.632 \\
1700 & 256.123 & 607.124 & 307.103 \\
1800 & 258.628 & 621.836 & 332.843 \\
1900 & 260.809 & 635.879 & 358.818 \\
2000 & 262.717 & 649.307 & 384.996 \\
\hline & & & $S:$ \\
\hline
\end{tabular}

$T$ : Temperature. $C_{P}$ : Heat capacity at constant pressure. $S$ : Entropy. $H$ : Enthalpy. 
Figure S.32.4. $\mathrm{B}_{4} \mathrm{H}_{8}$ : Ideal-gas thermodynamic functions at 1 bar (anharmonic B3LYPD2/cc-pVTZ results)
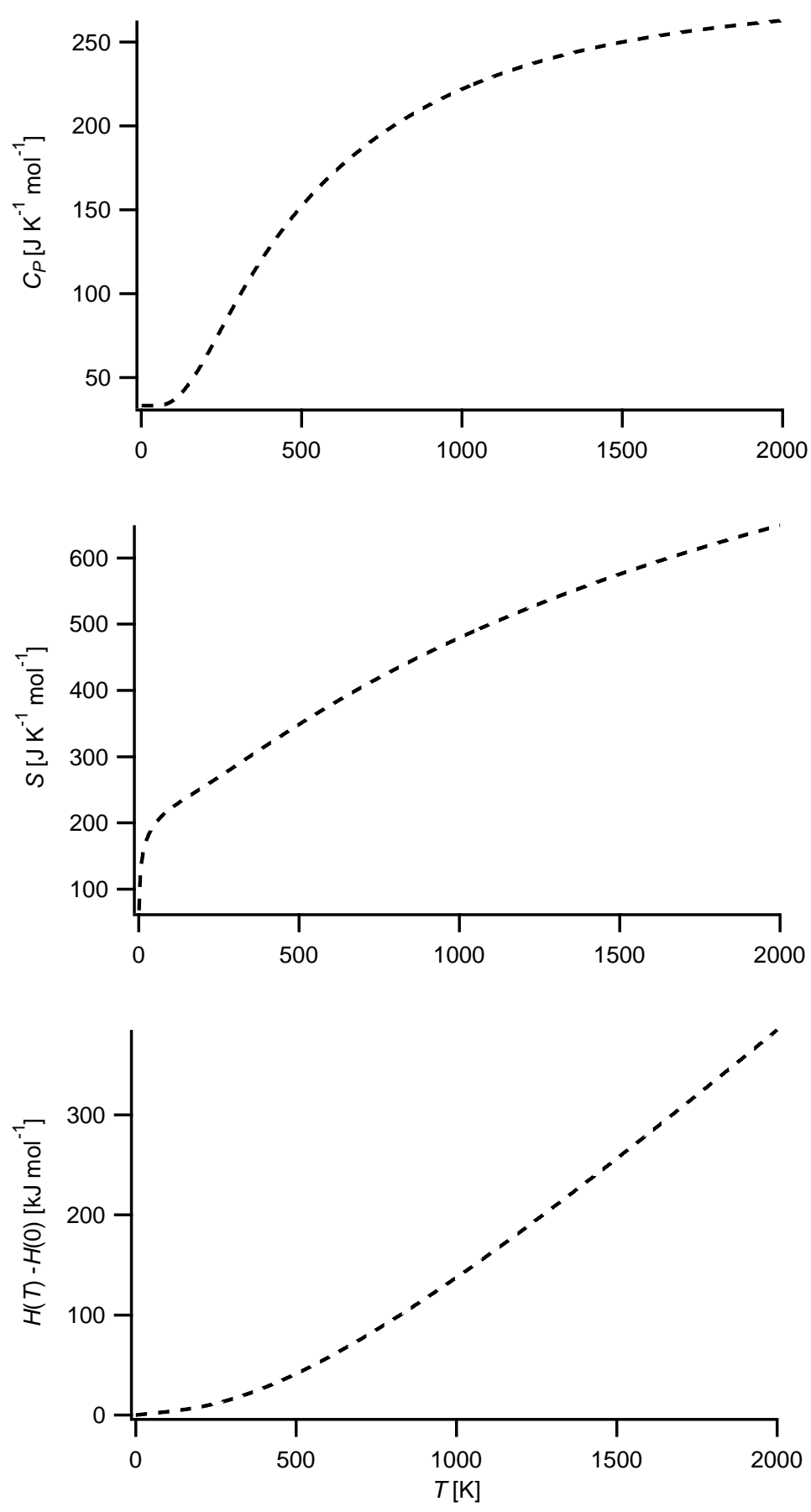

$T$ : Temperature. $C_{P}$ : Heat capacity at constant pressure. $S$ : Entropy. $H$ : Enthalpy. 
Table S.32.5. $\mathrm{B}_{4} \mathrm{H}_{8}$ : Fits (red solid lines) of the computed thermodynamic functions (blue dashed lines) with NASA type functions (Equations (1) - (3)) in the 200 - $900 \mathrm{~K}$ temperature range (anharmonic B3LYP-D2/cc-pVTZ results). In each case, the difference curve between the thermodynamic and NASA functions is plotted in the upper graph

Fit parameters

\begin{tabular}{llllll}
\hline $\mathrm{a}_{1}$ & $-2.45465439 \mathrm{e}+00$ & $\mathrm{a}_{2}\left[\mathrm{~K}^{-1}\right]$ & $5.13752984 \mathrm{e}-02$ & $\mathrm{a}_{3}\left[\mathrm{~K}^{-2}\right]$ & $-3.36992512 \mathrm{e}-06$ \\
$\mathrm{a}_{4}\left[\mathrm{~K}^{-3}\right]$ & $-4.70452758 \mathrm{e}-08$ & $\mathrm{a}_{5}\left[\mathrm{~K}^{-4}\right]$ & $2.88185813 \mathrm{e}-11$ & $\mathrm{a}_{6}[\mathrm{~K}]$ & $4.66478218 \mathrm{e}+02$
\end{tabular}

$\mathrm{a}_{7}$
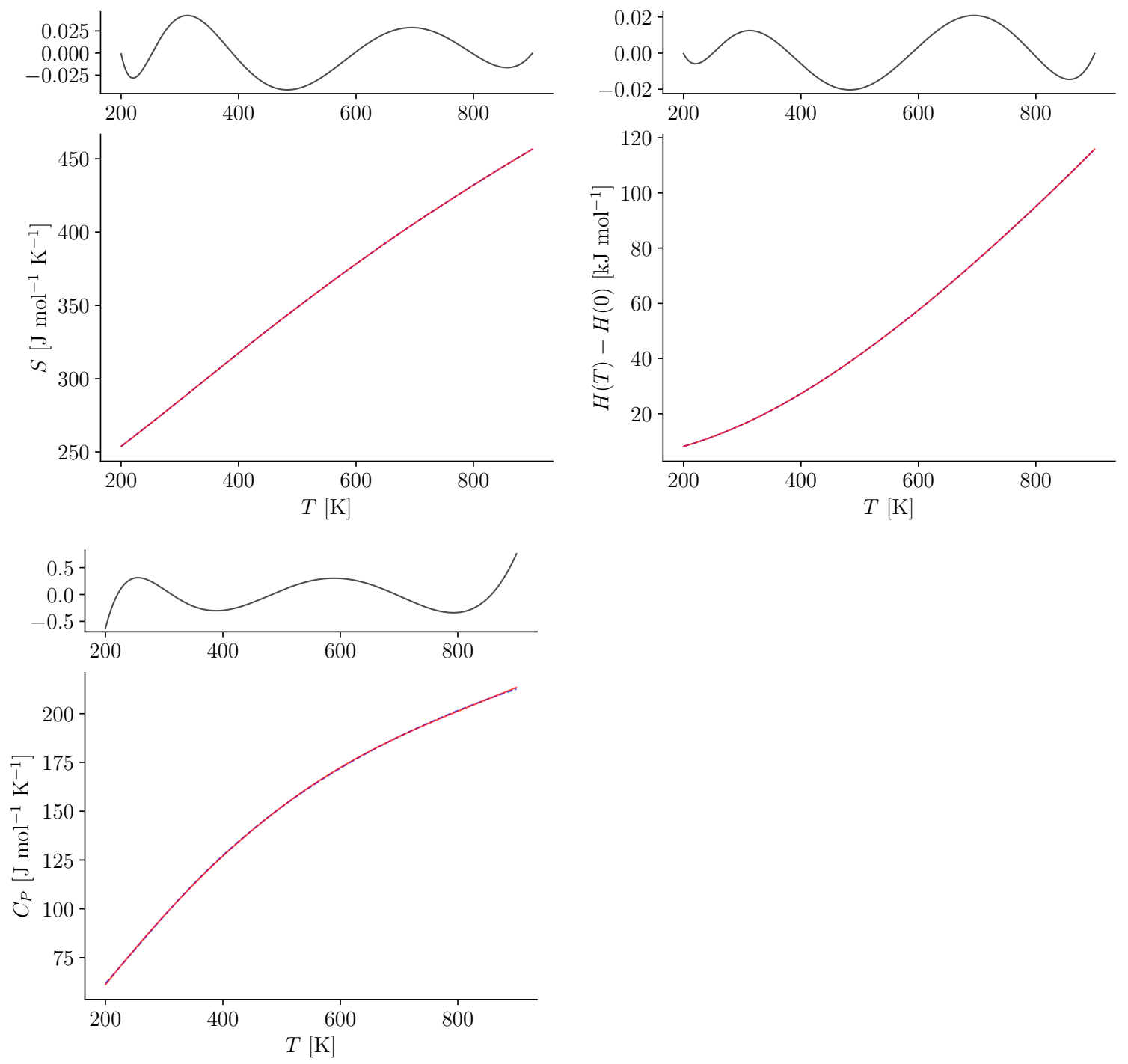


\section{$33 \quad \mathbf{B}_{4} \mathbf{H}_{9}$}

Figure S.33.1. Structure of $\mathrm{B}_{4} \mathrm{H}_{9}$

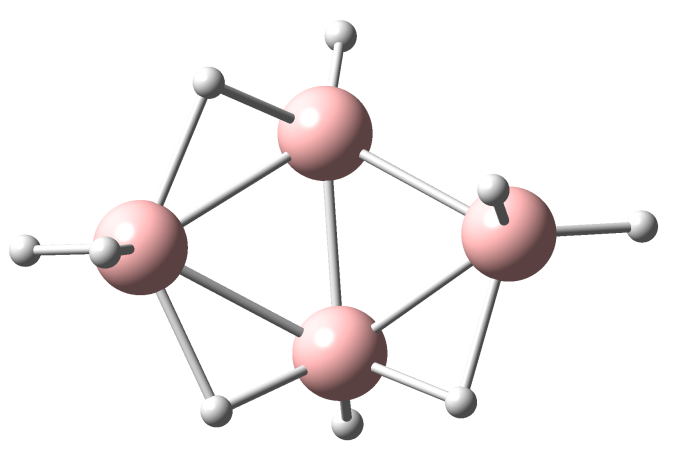

\begin{aligned} & \hline Charge 0 \\ & Spin multiplicity 2 \\ & Framework Group $\mathrm{C}_{1} \\ &$ Rotor Type Asymmetric Top \\ & Symmetry number 1 \\ & \hline\end{aligned}

Table S.33.1. $\mathrm{B}_{4} \mathrm{H}_{9}$ : Selected anharmonic results obtained at the B3LYP-D2/cc-pVTZ level

\begin{tabular}{rll}
\hline $\mathrm{ZPE}_{\mathrm{v}}$ & 21294 & $\mathrm{~cm}^{-1}$ \\
$\mathrm{~A}$ & 0.41080 & $\mathrm{~cm}^{-1}$ \\
$\mathrm{~B}$ & 0.21192 & $\mathrm{~cm}^{-1}$ \\
$\mathrm{C}$ & 0.18714 & $\mathrm{~cm}^{-1}$ \\
\hline$C_{P}(300 \mathrm{~K})$ & 107.437 & $\mathrm{~J} \mathrm{~mol}^{-1} \mathrm{~K}-1$ \\
$S(300 \mathrm{~K})$ & 297.092 & $\mathrm{~J} \mathrm{~mol}^{-1} \mathrm{~K}^{-1}$ \\
$H(300 \mathrm{~K})-H(0 \mathrm{~K})$ & 17.794 & $\mathrm{~kJ} \mathrm{~mol}^{-1}$ \\
\hline
\end{tabular}

$\mathrm{ZPE}_{\mathrm{v}}$ : vibrational contribution to the zero-point energy. A, B, C: Rotational constants. $C_{P}$ : Heat capacity at constant pressure. $S$ : Entropy. $H$ : Enthalpy. Ideal-gas calculations (1 bar). 
Table S.33.2. $\mathrm{B}_{4} \mathrm{H}_{9}$ : Cartesian coordinates $(\AA)$ of the B3LYP-D2/cc-pVTZ optimized structure

\begin{tabular}{cccc} 
atom & $\mathrm{x}$ & $\mathrm{y}$ & $\mathrm{z}$ \\
\hline B1 & -1.4318 & -0.0168 & 0.2973 \\
B2 & 0.0042 & 0.8712 & -0.4184 \\
B3 & 1.4331 & 0.0890 & 0.3880 \\
B4 & 0.1193 & -0.8385 & -0.3952 \\
H5 & -0.8997 & 1.2647 & 0.3743 \\
H6 & 0.1262 & 1.6262 & -1.3146 \\
H7 & -0.8391 & -1.2889 & 0.3079 \\
H8 & 1.0609 & -1.2194 & 0.3999 \\
H9 & 2.4672 & 0.1473 & -0.1868 \\
H10 & 1.3132 & 0.4302 & 1.5256 \\
H11 & 0.1782 & -1.3892 & -1.4382 \\
H12 & -1.7048 & -0.0545 & 1.4596 \\
H13 & -2.3256 & -0.0407 & -0.4863 \\
\hline
\end{tabular}


Table S.33.3. $\mathrm{B}_{4} \mathrm{H}_{9}$ : Vibrational frequencies, infrared integrated intensities and Raman activities (B3LYP-D2/cc-pVTZ results)

\begin{tabular}{|c|c|c|c|c|c|c|c|}
\hline \multirow[b]{2}{*}{ mode } & \multirow[b]{2}{*}{ symm. } & \multicolumn{3}{|c|}{ Harmonic } & \multicolumn{3}{|c|}{ Anharmonic } \\
\hline & & $\begin{array}{c}\omega \\
{\left[\mathrm{cm}^{-1}\right]}\end{array}$ & $\begin{array}{c}\mathrm{IR} \\
{\left[\mathrm{km} \mathrm{mol}^{-1}\right]}\end{array}$ & $\begin{array}{c}\text { Raman } \\
{\left[\AA^{6}\right]}\end{array}$ & $\begin{array}{c}\omega \\
{\left[\mathrm{cm}^{-1}\right]}\end{array}$ & $\begin{array}{c}\mathrm{IR} \\
{\left[\mathrm{km} \mathrm{mol}^{-1}\right]}\end{array}$ & $\begin{array}{c}\text { Raman } \\
{\left[\AA^{6}\right]}\end{array}$ \\
\hline 1 & A & 209 & 8.317 & 0.403 & 198 & 7.964 & 0.471 \\
\hline 2 & $\mathrm{~A}$ & 400 & 0.300 & 0.029 & 381 & 0.392 & 0.037 \\
\hline 3 & $\mathrm{~A}$ & 470 & 1.693 & 0.024 & 444 & 1.835 & 0.026 \\
\hline 4 & $\mathrm{~A}$ & 506 & 0.899 & 0.017 & 482 & 0.605 & 0.019 \\
\hline 5 & $\mathrm{~A}$ & 524 & 0.420 & 0.101 & 504 & 1.699 & 0.099 \\
\hline 6 & $\mathrm{~A}$ & 554 & 8.427 & 0.036 & 527 & 8.639 & 0.062 \\
\hline 7 & $\mathrm{~A}$ & 630 & 5.257 & 0.098 & 612 & 6.799 & 0.139 \\
\hline 8 & $\mathrm{~A}$ & 685 & 8.384 & 0.145 & 644 & 7.364 & 0.111 \\
\hline 9 & $\mathrm{~A}$ & 718 & 0.374 & 0.105 & 682 & 0.341 & 0.085 \\
\hline 10 & $\mathrm{~A}$ & 769 & 3.339 & 0.094 & 739 & 3.056 & 0.092 \\
\hline 11 & $\mathrm{~A}$ & 803 & 4.220 & 0.035 & 768 & 2.466 & 0.048 \\
\hline 12 & $\mathrm{~A}$ & 831 & 4.417 & 0.116 & 795 & 3.343 & 0.093 \\
\hline 13 & $\mathrm{~A}$ & 859 & 3.368 & 0.266 & 825 & 1.471 & 0.150 \\
\hline 14 & $\mathrm{~A}$ & 895 & 9.341 & 0.033 & 832 & 4.917 & 0.058 \\
\hline 15 & $\mathrm{~A}$ & 965 & 21.334 & 0.162 & 945 & 30.501 & 0.070 \\
\hline 16 & A & 981 & 12.874 & 0.059 & 914 & 1.935 & 0.118 \\
\hline 17 & $\mathrm{~A}$ & 1035 & 21.235 & 0.058 & 972 & 1.939 & 0.014 \\
\hline 18 & $\mathrm{~A}$ & 1073 & 14.451 & 0.030 & 999 & 1.126 & 0.041 \\
\hline 19 & $\mathrm{~A}$ & 1110 & 5.152 & 0.127 & 1035 & 3.195 & 0.098 \\
\hline 20 & $\mathrm{~A}$ & 1162 & 20.130 & 0.330 & 1099 & 8.026 & 0.325 \\
\hline 21 & $\mathrm{~A}$ & 1185 & 37.280 & 0.064 & 1134 & 9.549 & 0.019 \\
\hline 22 & $\mathrm{~A}$ & 1450 & 3.136 & 0.067 & 1290 & 1.514 & 0.008 \\
\hline 23 & $\mathrm{~A}$ & 1525 & 24.524 & 0.043 & 1340 & 2.340 & 0.004 \\
\hline
\end{tabular}


Table S.33.3 - Continued

\begin{tabular}{cccccccc}
\hline & & \multicolumn{3}{c}{ Harmonic } & \multicolumn{3}{c}{ Anharmonic } \\
mode & symm. & $\omega$ & IR & Raman & $\omega$ & IR & Raman \\
& & {$\left[\mathrm{cm}^{-1}\right]$} & {$\left[\mathrm{km} \mathrm{mol}^{-1}\right]$} & {$\left[\AA^{6}\right]$} & {$\left[\mathrm{cm}^{-1}\right]$} & {$\left[\mathrm{km} \mathrm{mol}^{-1}\right]$} & {$\left[\AA^{6}\right]$} \\
\hline 24 & A & 1668 & 69.700 & 0.030 & 1513 & 7.679 & 0.004 \\
25 & A & 2084 & 23.901 & 0.373 & 1914 & 14.094 & 0.026 \\
26 & A & 2149 & 47.868 & 0.638 & 2020 & 6.844 & 0.361 \\
27 & A & 2203 & 14.939 & 0.276 & 2095 & 3.370 & 0.084 \\
28 & A & 2582 & 42.395 & 1.001 & 2478 & 40.402 & 1.042 \\
29 & A & 2597 & 36.443 & 1.222 & 2477 & 26.176 & 1.329 \\
30 & A & 2663 & 40.599 & 0.860 & 2537 & 30.091 & 0.749 \\
31 & A & 2702 & 60.494 & 0.534 & 2580 & 53.580 & 0.579 \\
32 & A & 2716 & 54.284 & 0.659 & 2591 & 41.688 & 0.556 \\
33 & A & 2730 & 43.694 & 0.931 & 2615 & 56.017 & 1.116 \\
\hline
\end{tabular}


Figure S.33.2. Anharmonic IR spectrum of $\mathrm{B}_{4} \mathrm{H}_{9}$ obtained by convoluting the calculated intensities with Lorentzians having a FWHM of $4 \mathrm{~cm}^{-1}$ (B3LYP-D2/cc-pVTZ results): (top) full spectrum, (middle) contributions from fundamentals and combination bands, (bottom) contributions from overtones
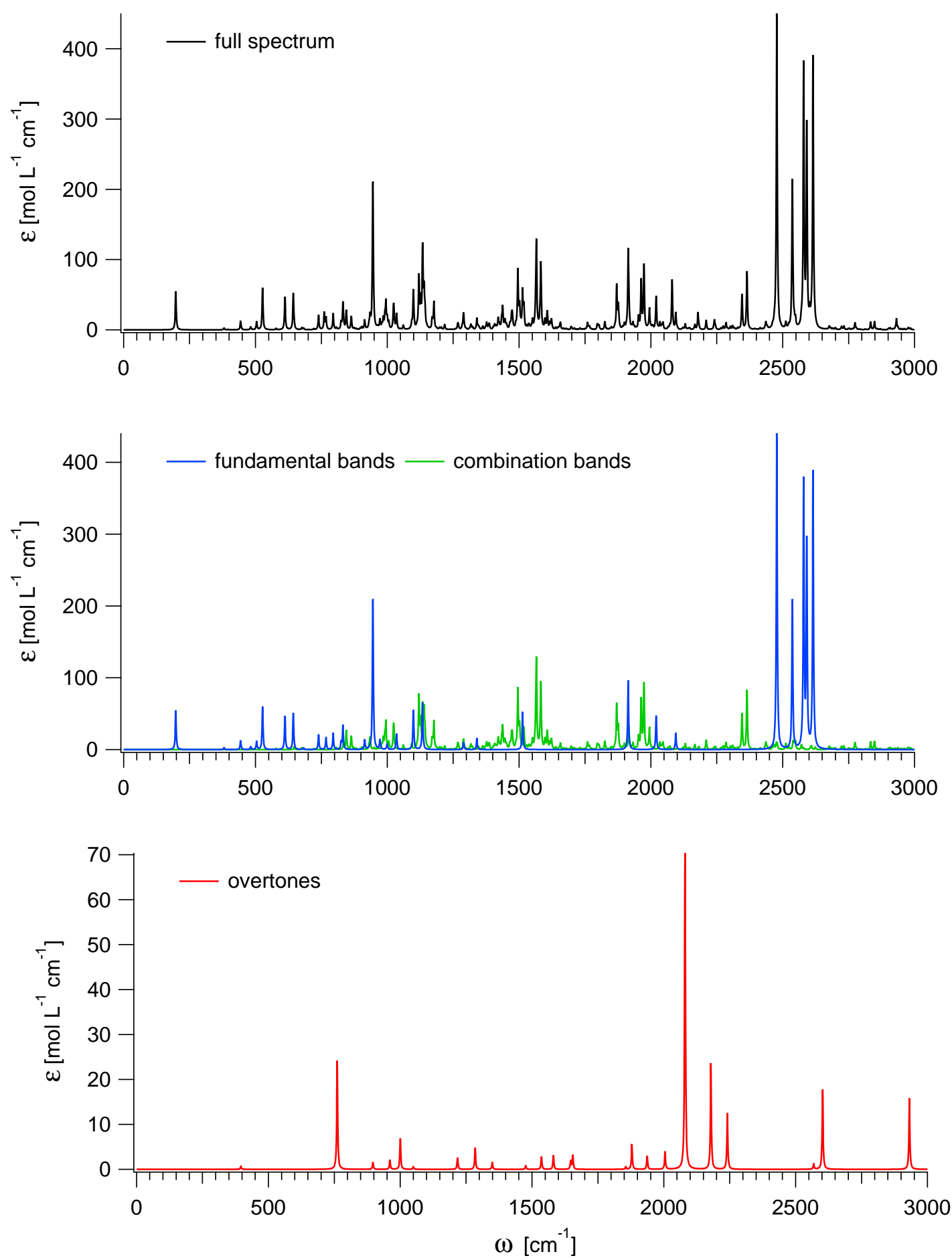
Figure S.33.3. Anharmonic Raman spectrum of $\mathrm{B}_{4} \mathrm{H}_{9}$ obtained by convoluting the calculated activities with Lorentzians having a FWHM of $4 \mathrm{~cm}^{-1}$ (B3LYP-D2/cc-pVTZ results): (top) full spectrum, (middle) contributions from fundamentals and combination bands, (bottom) contributions from overtones
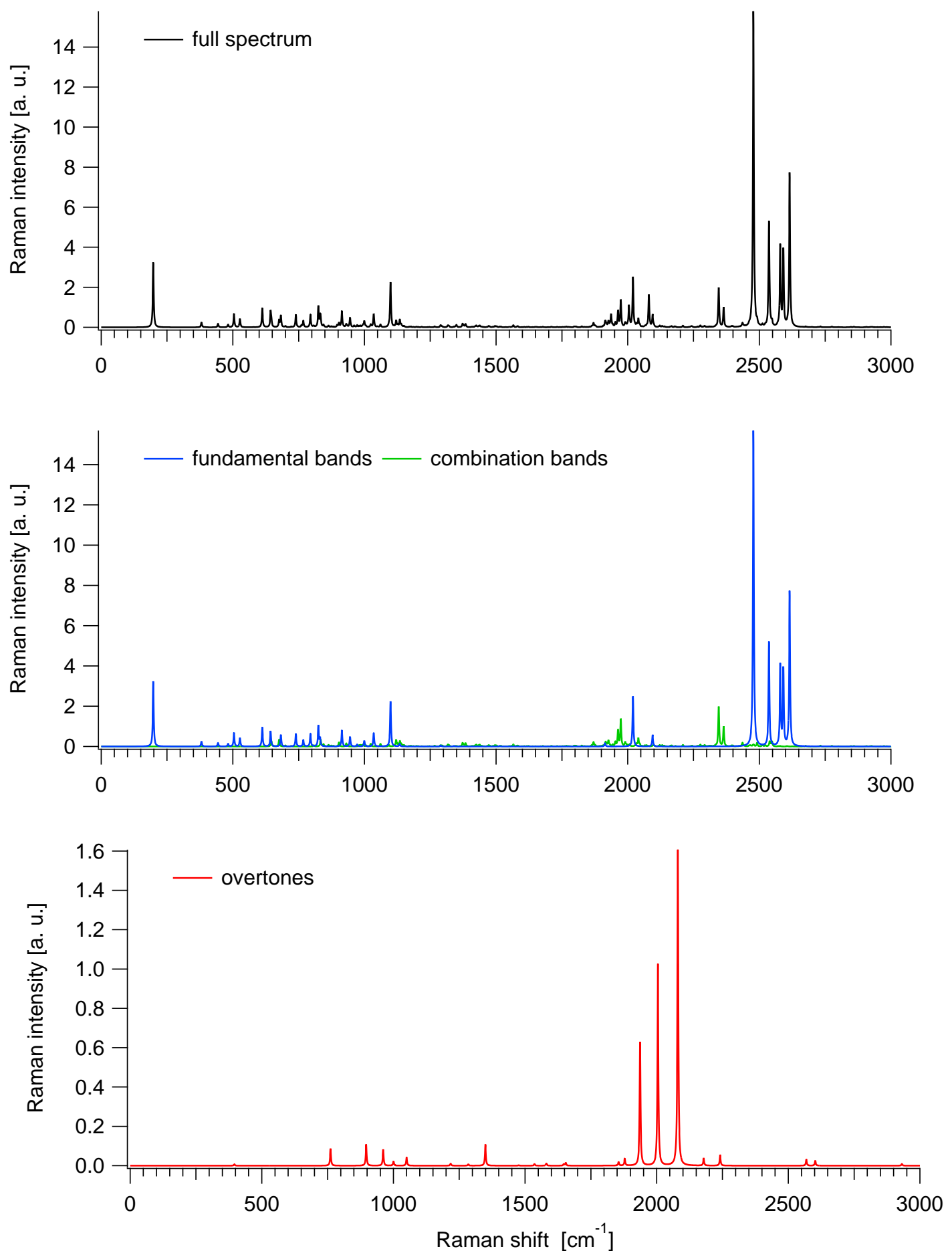
Table S.33.4. $\mathrm{B}_{4} \mathrm{H}_{9}$ : Ideal-gas thermodynamic functions at 1 bar (anharmonic B3LYPD2/cc-pVTZ results)

\begin{tabular}{|c|c|c|c|}
\hline $\begin{array}{c}T \\
{[\mathrm{~K}]}\end{array}$ & $\begin{array}{c}C_{P} \\
{\left[\mathrm{~J} \mathrm{~mol}^{-1} \mathrm{~K}^{-1}\right]}\end{array}$ & $\begin{array}{c}S \\
{\left[\mathrm{~J} \mathrm{~mol}^{-1} \mathrm{~K}^{-1}\right]}\end{array}$ & $\begin{array}{c}H(T)-H(0 K) \\
{\left[\mathrm{kJ} \mathrm{mol}^{-1}\right]}\end{array}$ \\
\hline 100 & 40.520 & 225.404 & 3.516 \\
\hline 200 & 70.212 & 261.532 & 8.898 \\
\hline 300 & 107.437 & 297.092 & 17.794 \\
\hline 400 & 139.665 & 332.575 & 30.206 \\
\hline 500 & 165.536 & 366.625 & 45.513 \\
\hline 600 & 186.496 & 398.723 & 63.150 \\
\hline 700 & 203.768 & 428.810 & 82.690 \\
\hline 800 & 218.135 & 456.986 & 103.807 \\
\hline 900 & 230.130 & 483.392 & 126.238 \\
\hline 1000 & 240.169 & 508.174 & 149.768 \\
\hline 1100 & 248.592 & 531.471 & 174.218 \\
\hline 1200 & 255.682 & 553.414 & 199.442 \\
\hline 1300 & 261.674 & 574.123 & 225.318 \\
\hline 1400 & 266.762 & 593.706 & 251.747 \\
\hline 1500 & 271.104 & 612.263 & 278.646 \\
\hline 1600 & 274.828 & 629.881 & 305.947 \\
\hline 1700 & 278.040 & 646.642 & 333.594 \\
\hline 1800 & 280.823 & 662.615 & 361.541 \\
\hline 1900 & 283.248 & 677.864 & 389.747 \\
\hline 2000 & 285.370 & 692.448 & 418.180 \\
\hline
\end{tabular}

$T$ : Temperature. $C_{P}$ : Heat capacity at constant pressure. $S$ : Entropy. $H$ : Enthalpy. 
Figure S.33.4. $\mathrm{B}_{4} \mathrm{H}_{9}$ : Ideal-gas thermodynamic functions at 1 bar (anharmonic B3LYPD2/cc-pVTZ results)
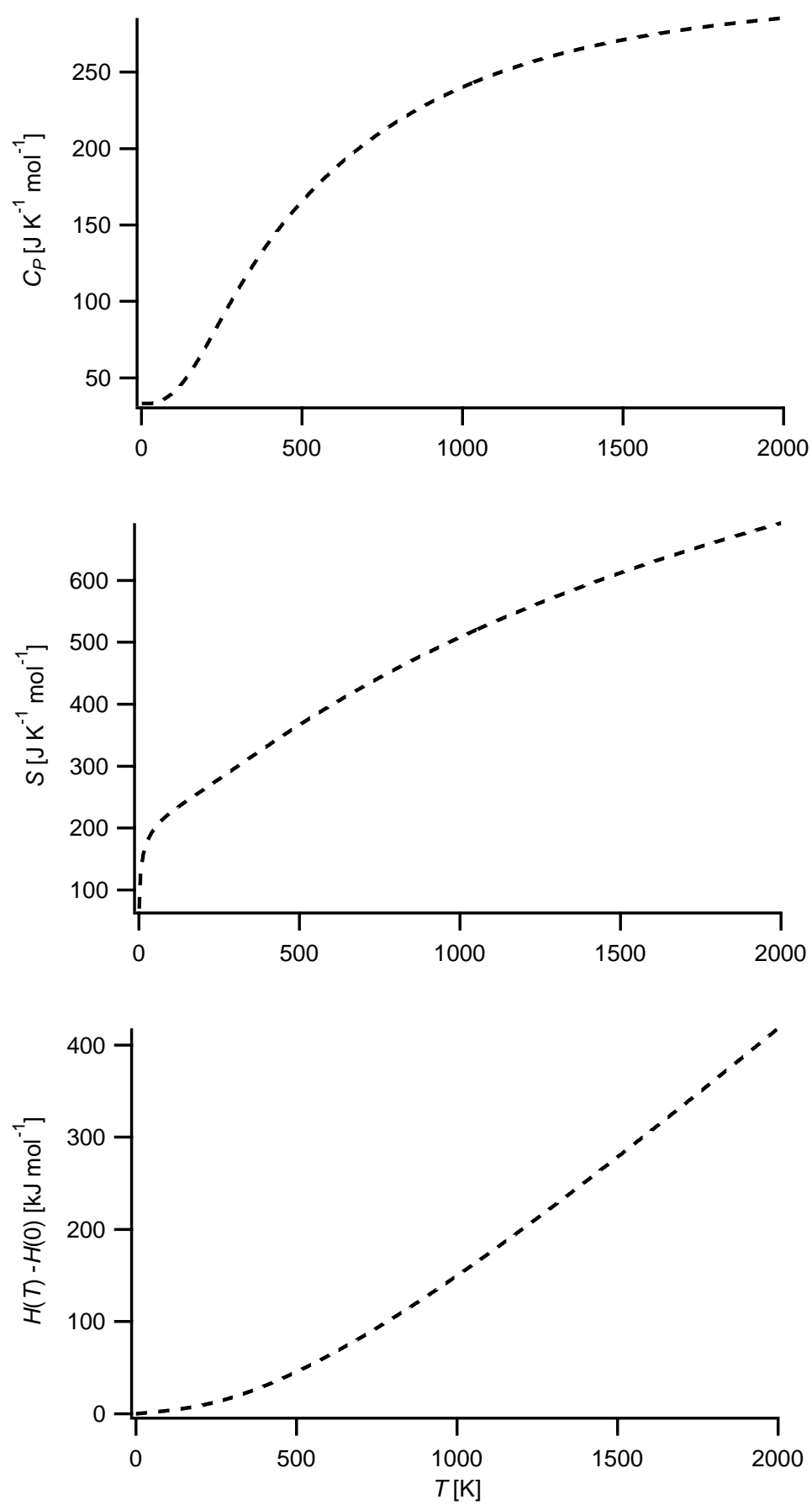

$T$ : Temperature. $C_{P}$ : Heat capacity at constant pressure. $S$ : Entropy. $H$ : Enthalpy. 
Table S.33.5. $\mathrm{B}_{4} \mathrm{H}_{9}$ : Fits (red solid lines) of the computed thermodynamic functions (blue dashed lines) with NASA type functions (Equations (1) - (3)) in the 200 - $900 \mathrm{~K}$ temperature range (anharmonic B3LYP-D2/cc-pVTZ results). In each case, the difference curve between the thermodynamic and NASA functions is plotted in the upper graph

Fit parameters

\begin{tabular}{llllll}
\hline $\mathrm{a}_{1}$ & $-2.85164894 \mathrm{e}+00$ & $\mathrm{a}_{2}\left[\mathrm{~K}^{-1}\right]$ & $6.26512970 \mathrm{e}-02$ & $\mathrm{a}_{3}\left[\mathrm{~K}^{-2}\right]$ & $-2.99315509 \mathrm{e}-05$ \\
$\mathrm{a}_{4}\left[\mathrm{~K}^{-3}\right]$ & $-1.66649429 \mathrm{e}-08$ & $\mathrm{a}_{5}\left[\mathrm{~K}^{-4}\right]$ & $1.61692636 \mathrm{e}-11$ & $\mathrm{a}_{6}[\mathrm{~K}]$ & $4.72926004 \mathrm{e}+02$
\end{tabular}

$\mathrm{a}_{7}$

$3.46702493 \mathrm{e}+01$
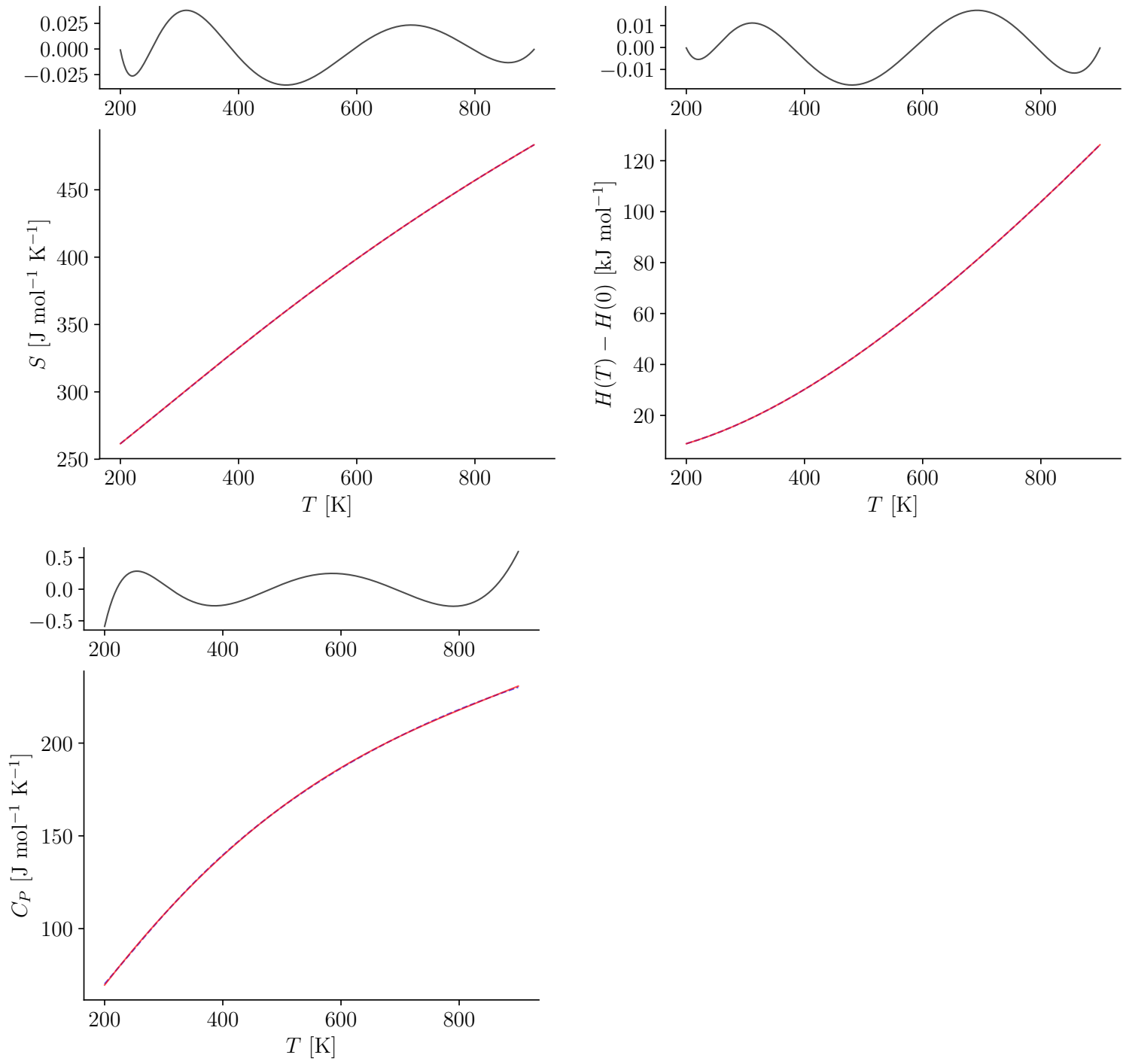


\section{$34 \quad \mathbf{B}_{4} \mathbf{H}_{10}$}

Figure S.34.1. Structure of $\mathrm{B}_{4} \mathrm{H}_{10}$

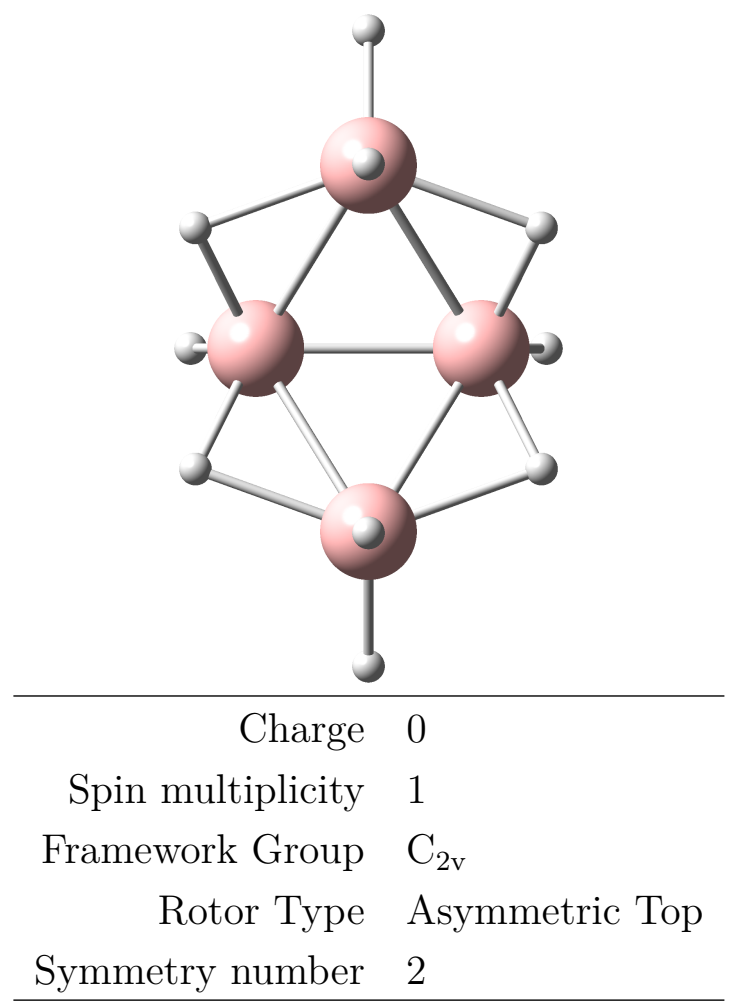

Table S.34.1. $\mathrm{B}_{4} \mathrm{H}_{10}$ : Selected anharmonic results obtained at the B3LYP-D2/cc-pVTZ level

\begin{tabular}{rll}
\hline $\mathrm{ZPE}_{\mathrm{v}}$ & 24018 & $\mathrm{~cm}^{-1}$ \\
$\mathrm{~A}$ & 0.37088 & $\mathrm{~cm}^{-1}$ \\
$\mathrm{~B}$ & 0.20863 & $\mathrm{~cm}^{-1}$ \\
$\mathrm{C}$ & 0.18809 & $\mathrm{~cm}^{-1}$ \\
\hline$C_{P}(300 \mathrm{~K})$ & 107.219 & $\mathrm{~J} \mathrm{~mol}^{-1} \mathrm{~K}-1$ \\
$S(300 \mathrm{~K})$ & 292.586 & $\mathrm{~J} \mathrm{~mol}^{-1} \mathrm{~K}-1$ \\
$H(300 \mathrm{~K})-H(0 \mathrm{~K})$ & 17.809 & $\mathrm{~kJ} \mathrm{~mol}^{-1}$ \\
\hline
\end{tabular}

$\mathrm{ZPE}_{\mathrm{v}}$ : vibrational contribution to the zero-point energy. A, B, C: Rotational constants. $C_{P}$ : Heat capacity at constant pressure. $S$ : Entropy. $H$ : Enthalpy. Ideal-gas calculations (1 bar). 
Table S.34.2. $\mathrm{B}_{4} \mathrm{H}_{10}$ : Cartesian coordinates $(\AA)$ of the B3LYP-D2/cc-pVTZ optimized structure

\begin{tabular}{cccc} 
atom & $\mathrm{x}$ & $\mathrm{y}$ & $\mathrm{z}$ \\
\hline B1 & 0.0000 & 1.3999 & 0.3938 \\
B2 & -0.0000 & -1.3999 & 0.3938 \\
B3 & -0.8619 & 0.0000 & -0.4652 \\
B4 & 0.8619 & -0.0000 & -0.4652 \\
H5 & -1.3228 & 0.9218 & 0.2506 \\
H6 & 1.3228 & -0.9218 & 0.2506 \\
H7 & 0.0000 & -1.4106 & 1.5853 \\
H8 & 0.0000 & 1.4106 & 1.5853 \\
H9 & 0.0000 & -2.4300 & -0.1941 \\
H10 & 0.0000 & 2.4300 & -0.1941 \\
H11 & -1.3228 & -0.9218 & 0.2506 \\
H12 & 1.3228 & 0.9218 & 0.2506 \\
H13 & -1.3598 & 0.0000 & -1.5357 \\
H14 & 1.3598 & -0.0000 & -1.5357 \\
\hline
\end{tabular}


Table S.34.3. $\mathrm{B}_{4} \mathrm{H}_{10}$ : Vibrational frequencies, infrared integrated intensities and Raman activities (B3LYP-D2/cc-pVTZ results)

\begin{tabular}{|c|c|c|c|c|c|c|c|}
\hline \multirow[b]{2}{*}{ mode } & \multirow[b]{2}{*}{ symm. } & \multicolumn{3}{|c|}{ Harmonic } & \multicolumn{3}{|c|}{ Anharmonic } \\
\hline & & $\begin{array}{c}\omega \\
{\left[\mathrm{cm}^{-1}\right]}\end{array}$ & $\begin{array}{c}\mathrm{IR} \\
{\left[\mathrm{km} \mathrm{mol}^{-1}\right]}\end{array}$ & $\begin{array}{c}\text { Raman } \\
{\left[\AA^{6}\right]}\end{array}$ & $\begin{array}{c}\omega \\
{\left[\mathrm{cm}^{-1}\right]}\end{array}$ & $\begin{array}{c}\mathrm{IR} \\
{\left[\mathrm{km} \mathrm{mol}^{-1}\right]}\end{array}$ & $\begin{array}{c}\text { Raman } \\
{\left[\AA^{6}\right]}\end{array}$ \\
\hline 1 & $\mathrm{~A}_{1}$ & 225 & 7.458 & 0.086 & 186 & 7.212 & 0.129 \\
\hline 2 & $\mathrm{~B}_{2}$ & 379 & 2.554 & 0.020 & 361 & 1.385 & 0.026 \\
\hline 3 & $\mathrm{~A}_{2}$ & 422 & 0.000 & 0.016 & 405 & 0.000 & 0.019 \\
\hline 4 & $\mathrm{~B}_{2}$ & 482 & 11.240 & 0.011 & 415 & 9.217 & 0.010 \\
\hline 5 & $\mathrm{~A}_{1}$ & 572 & 0.057 & 0.198 & 541 & 0.097 & 0.248 \\
\hline 6 & $\mathrm{~B}_{1}$ & 585 & 20.012 & 0.023 & 544 & 22.619 & 0.020 \\
\hline 7 & $\mathrm{~A}_{2}$ & 684 & 0.000 & 0.045 & 645 & 0.000 & 0.048 \\
\hline 8 & $\mathrm{~A}_{1}$ & 693 & 0.047 & 0.022 & 672 & 0.009 & 0.028 \\
\hline 9 & $\mathrm{~B}_{1}$ & 776 & 1.484 & 0.006 & 744 & 0.036 & 0.004 \\
\hline 10 & $\mathrm{~A}_{1}$ & 816 & 1.008 & 0.362 & 785 & 0.724 & 0.280 \\
\hline 11 & $\mathrm{~A}_{1}$ & 874 & 0.190 & 0.177 & 840 & 0.011 & 0.239 \\
\hline 12 & $\mathrm{~B}_{2}$ & 895 & 30.604 & 0.044 & 844 & 41.242 & 0.065 \\
\hline 13 & $\mathrm{~A}_{2}$ & 928 & 0.000 & 0.045 & 858 & 0.000 & 0.050 \\
\hline 14 & $\mathrm{~B}_{1}$ & 931 & 18.543 & 0.001 & 887 & 20.434 & 0.001 \\
\hline 15 & $\mathrm{~B}_{2}$ & 972 & 20.000 & 0.030 & 892 & 3.486 & 0.005 \\
\hline 16 & $\mathrm{~A}_{1}$ & 1022 & 5.589 & 0.048 & 963 & 5.998 & 0.076 \\
\hline 17 & $\mathrm{~B}_{1}$ & 1027 & 51.444 & 0.067 & 992 & 38.773 & 0.065 \\
\hline 18 & $\mathrm{~A}_{2}$ & 1054 & 0.000 & 0.003 & 1015 & 0.000 & 0.025 \\
\hline 19 & $\mathrm{~A}_{2}$ & 1123 & 0.000 & 0.066 & 1042 & 0.000 & 0.014 \\
\hline 20 & $\mathrm{~B}_{1}$ & 1139 & 14.627 & 0.104 & 1063 & 10.090 & 0.111 \\
\hline 21 & $\mathrm{~B}_{2}$ & 1189 & 31.684 & 0.116 & 1118 & 13.134 & 0.098 \\
\hline 22 & $\mathrm{~A}_{1}$ & 1207 & 9.574 & 0.081 & 1148 & 9.041 & 0.089 \\
\hline 23 & $\mathrm{~B}_{2}$ & 1349 & 7.149 & 0.000 & 1239 & 3.758 & 0.000 \\
\hline
\end{tabular}


Table S.34.3 - Continued

\begin{tabular}{cccccccc}
\hline & & \multicolumn{3}{c}{ Harmonic } & \multicolumn{3}{c}{ Anharmonic } \\
mode & symm. & $\omega$ & IR & Raman & $\omega$ & IR & Raman \\
& & {$\left[\mathrm{cm}^{-1}\right]$} & {$\left[\mathrm{km} \mathrm{mol}^{-1}\right]$} & {$\left[\AA^{6}\right]$} & {$\left[\mathrm{cm}^{-1}\right]$} & {$\left[\mathrm{km} \mathrm{mol}^{-1}\right]$} & {$\left[\AA^{6}\right]$} \\
\hline 24 & $\mathrm{~A}_{2}$ & 1481 & 0.000 & 0.094 & 1317 & 0.000 & 0.034 \\
25 & $\mathrm{~B}_{1}$ & 1540 & 33.093 & 0.001 & 1362 & 12.389 & 0.002 \\
26 & $\mathrm{~A}_{1}$ & 1578 & 7.261 & 0.093 & 1437 & 1.849 & 0.038 \\
27 & $\mathrm{~A}_{1}$ & 2240 & 14.058 & 1.322 & 2138 & 6.454 & 0.723 \\
28 & $\mathrm{~B}_{1}$ & 2249 & 12.957 & 0.045 & 2138 & 2.123 & 0.041 \\
29 & $\mathrm{~B}_{2}$ & 2259 & 113.186 & 0.094 & 2132 & 62.357 & 0.069 \\
30 & $\mathrm{~A}_{2}$ & 2270 & 0.000 & 0.150 & 2146 & 0.000 & 0.078 \\
31 & $\mathrm{~B}_{2}$ & 2615 & 52.613 & 0.043 & 2511 & 64.166 & 0.022 \\
32 & $\mathrm{~A}_{1}$ & 2620 & 63.067 & 1.618 & 2516 & 50.860 & 1.764 \\
33 & $\mathrm{~A}_{1}$ & 2692 & 17.996 & 0.775 & 2572 & 6.634 & 0.548 \\
34 & $\mathrm{~B}_{2}$ & 2694 & 101.460 & 0.260 & 2574 & 74.516 & 0.317 \\
35 & $\mathrm{~B}_{1}$ & 2707 & 30.715 & 0.406 & 2595 & 24.010 & 0.370 \\
36 & $\mathrm{~A}_{1}$ & 2714 & 92.008 & 1.110 & 2605 & 92.642 & 1.117 \\
\hline
\end{tabular}


Figure S.34.2. Anharmonic IR spectrum of $\mathrm{B}_{4} \mathrm{H}_{10}$ obtained by convoluting the calculated intensities with Lorentzians having a FWHM of $4 \mathrm{~cm}^{-1}$ (B3LYP-D2/cc-pVTZ results): (top) full spectrum, (middle) contributions from fundamentals and combination bands, (bottom) contributions from overtones
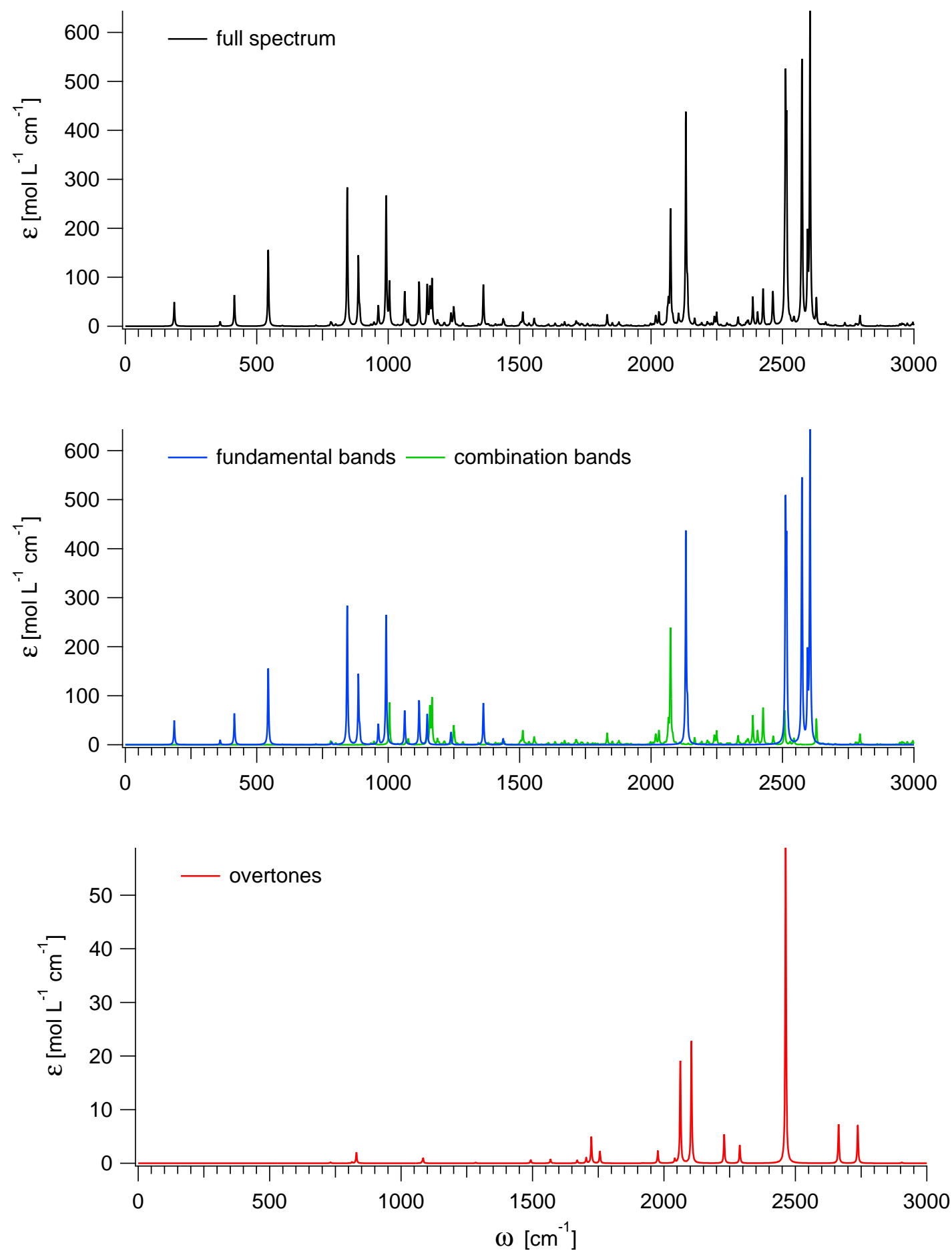
Figure S.34.3. Anharmonic Raman spectrum of $\mathrm{B}_{4} \mathrm{H}_{10}$ obtained by convoluting the calculated activities with Lorentzians having a FWHM of $4 \mathrm{~cm}^{-1}$ (B3LYP-D2/cc-pVTZ results): (top) full spectrum, (middle) contributions from fundamentals and combination bands, (bottom) contributions from overtones
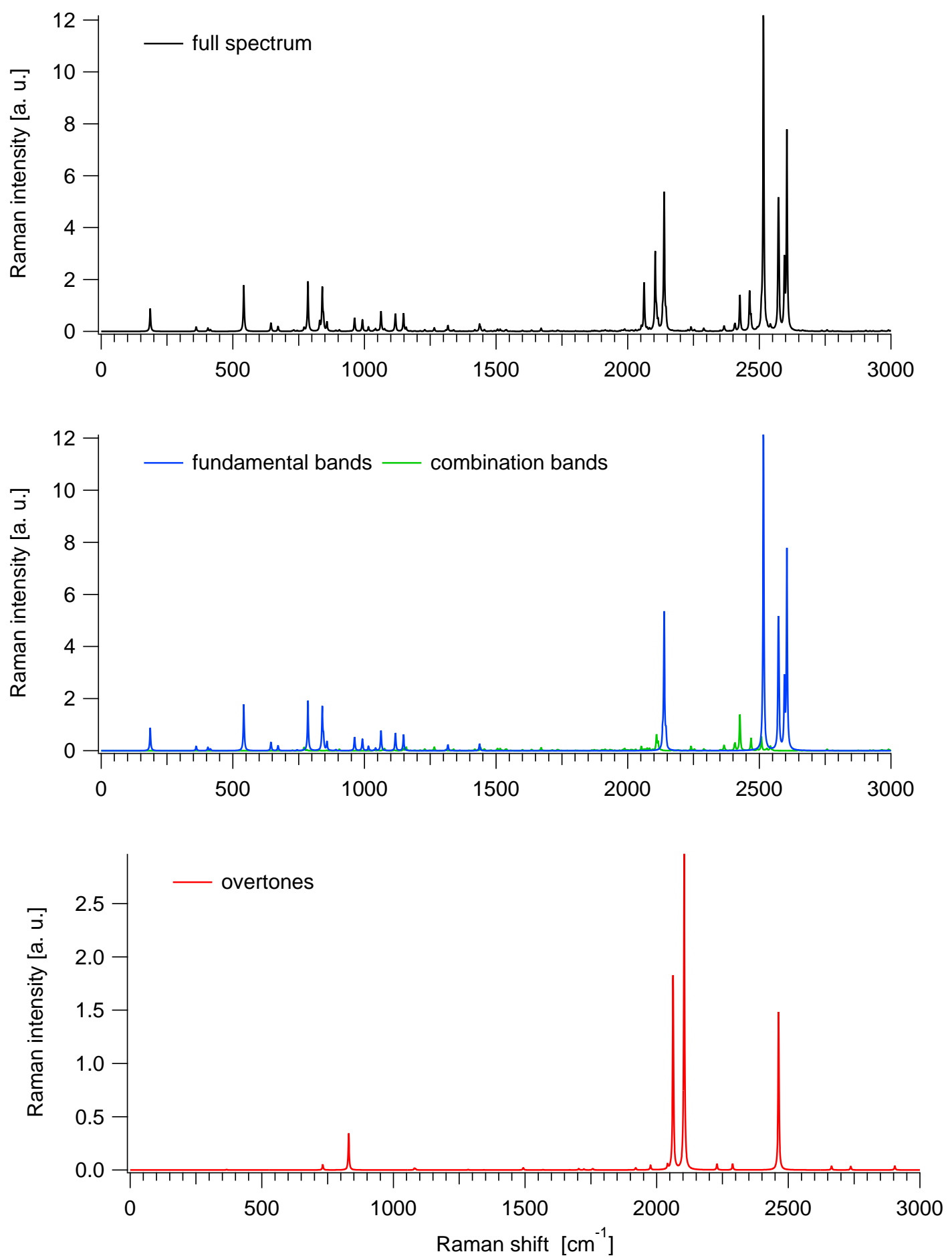
Table S.34.4. $\mathrm{B}_{4} \mathrm{H}_{10}$ : Ideal-gas thermodynamic functions at 1 bar (anharmonic B3LYP$\mathrm{D} 2 / \mathrm{cc}-\mathrm{pVTZ}$ results)

\begin{tabular}{cccc}
\hline$T$ & $C_{P}$ & $S$ & $H(T)-H(0 K)$ \\
{$[\mathrm{K}]$} & {$\left[\mathrm{J} \mathrm{mol}^{-1} \mathrm{~K}^{-1}\right]$} & {$\left[\mathrm{J} \mathrm{mol}^{-1} \mathrm{~K}^{-1}\right]$} & {$\left[\mathrm{kJ} \mathrm{mol}^{-1}\right]$} \\
\hline 100 & 41.428 & 220.788 & 3.548 \\
200 & 69.781 & 257.272 & 8.973 \\
300 & 107.219 & 292.586 & 17.809 \\
400 & 141.899 & 328.315 & 30.312 \\
500 & 170.664 & 363.176 & 45.988 \\
600 & 194.309 & 396.450 & 64.275 \\
700 & 213.927 & 427.922 & 84.716 \\
800 & 230.298 & 457.589 & 106.952 \\
900 & 243.991 & 485.529 & 130.686 \\
1000 & 255.460 & 511.847 & 155.676 \\
1100 & 265.087 & 536.660 & 181.717 \\
1200 & 273.193 & 560.083 & 208.643 \\
1300 & 280.044 & 582.228 & 236.314 \\
1400 & 285.862 & 603.201 & 264.617 \\
1500 & 290.826 & 623.097 & 293.458 \\
1600 & 295.084 & 642.006 & 322.759 \\
1700 & 298.756 & 660.008 & 352.455 \\
1800 & 301.938 & 677.177 & 382.494 \\
1900 & 304.710 & 693.577 & 412.829 \\
2000 & 307.136 & 709.270 & 443.424 \\
\hline$T$ & & & $S$ \\
\hline
\end{tabular}

$T$ : Temperature. $C_{P}$ : Heat capacity at constant pressure. $S$ : Entropy. $H$ : Enthalpy. 
Figure S.34.4. $\mathrm{B}_{4} \mathrm{H}_{10}$ : Ideal-gas thermodynamic functions at 1 bar (anharmonic B3LYPD2/cc-pVTZ results)
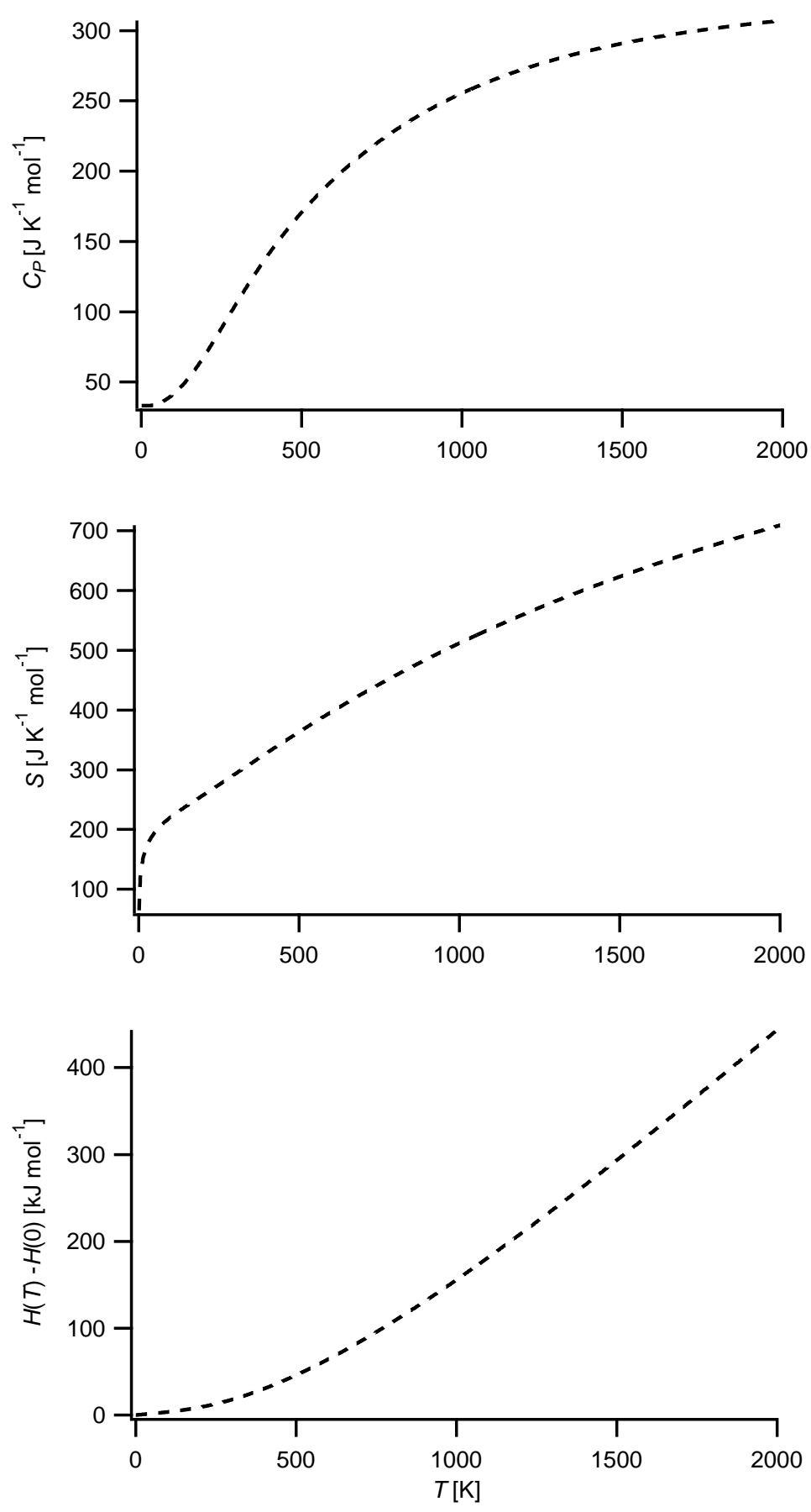

$T$ : Temperature. $C_{P}$ : Heat capacity at constant pressure. $S$ : Entropy. $H$ : Enthalpy. 
Table S.34.5. $\mathrm{B}_{4} \mathrm{H}_{10}$ : Fits (red solid lines) of the computed thermodynamic functions (blue dashed lines) with NASA type functions (Equations (1) - (3)) in the $200-900 \mathrm{~K}$ temperature range (anharmonic B3LYP-D2/cc-pVTZ results). In each case, the difference curve between the thermodynamic and NASA functions is plotted in the upper graph

Fit parameters

\begin{tabular}{llllll}
\hline $\mathrm{a}_{1}$ & $-1.31964869 \mathrm{e}+00$ & $\mathrm{a}_{2}\left[\mathrm{~K}^{-1}\right]$ & $4.61278735 \mathrm{e}-02$ & $\mathrm{a}_{3}\left[\mathrm{~K}^{-2}\right]$ & $2.43129463 \mathrm{e}-05$ \\
$\mathrm{a}_{4}\left[\mathrm{~K}^{-3}\right]$ & $-7.89747607 \mathrm{e}-08$ & $\mathrm{a}_{5}\left[\mathrm{~K}^{-4}\right]$ & $4.13635659 \mathrm{e}-11$ & $\mathrm{a}_{6}[\mathrm{~K}]$ & $3.84676066 \mathrm{e}+02$ \\
$\mathrm{a}_{7}$ & $2.84168277 \mathrm{e}+01$ & & & &
\end{tabular}
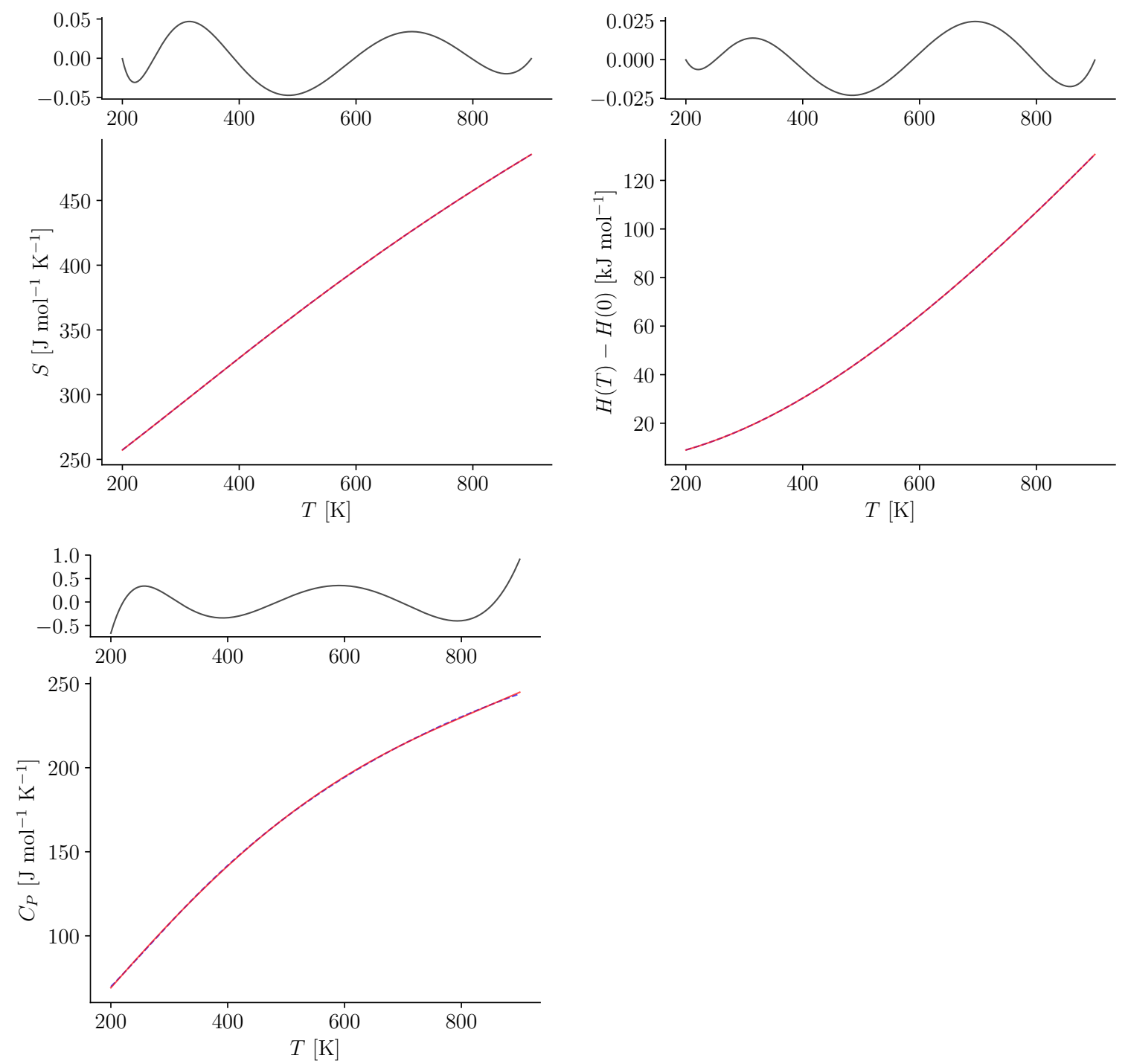


\section{$35 \quad \mathbf{B}_{5} \mathbf{H}_{9}$}

Figure S.35.1. Structure of $\mathrm{B}_{5} \mathrm{H}_{9}$

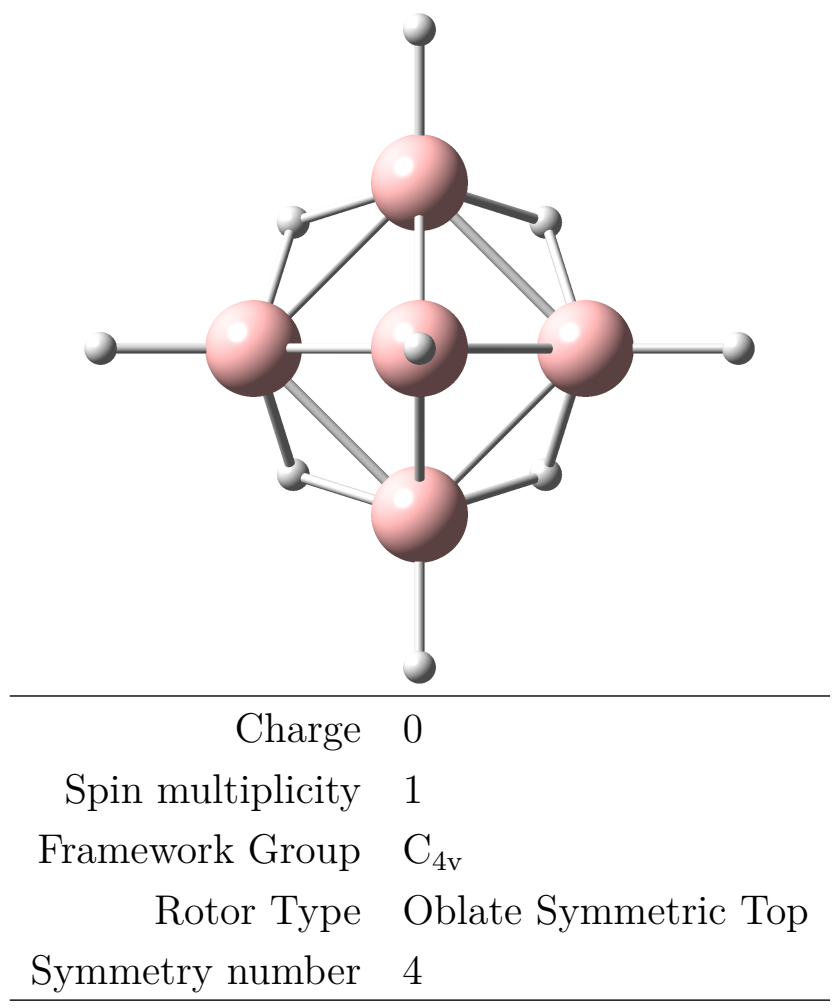

Table S.35.1. $\mathrm{B}_{5} \mathrm{H}_{9}$ : Selected anharmonic results obtained at the B3LYP-D2/cc-pVTZ level

\begin{tabular}{rll}
\hline $\mathrm{ZPE}_{\mathrm{v}}$ & 23139 & $\mathrm{~cm}^{-1}$ \\
$\mathrm{~A}$ & 0.23629 & $\mathrm{~cm}^{-1}$ \\
$\mathrm{~B}$ & 0.23629 & $\mathrm{~cm}^{-1}$ \\
$\mathrm{C}$ & 0.16499 & $\mathrm{~cm}^{-1}$ \\
\hline$C_{P}(300 \mathrm{~K})$ & 102.415 & $\mathrm{~J} \mathrm{~mol}^{-1} \mathrm{~K}-1$ \\
$S(300 \mathrm{~K})$ & 278.207 & $\mathrm{~J} \mathrm{~mol}^{-1} \mathrm{~K}-1$ \\
$H(300 \mathrm{~K})-H(0 \mathrm{~K})$ & 15.852 & $\mathrm{~kJ} \mathrm{~mol}^{-1}$ \\
\hline
\end{tabular}

$\mathrm{ZPE}_{\mathrm{v}}$ : vibrational contribution to the zero-point energy. A, B, C: Rotational constants. $C_{P}$ : Heat capacity at constant pressure. $S$ : Entropy. $H$ : Enthalpy. Ideal-gas calculations (1 bar). 
Table S.35.2. $\mathrm{B}_{5} \mathrm{H}_{9}$ : Cartesian coordinates $(\AA)$ of the B3LYP-D2/cc-pVTZ optimized structure

\begin{tabular}{cccc} 
atom & $\mathrm{x}$ & $\mathrm{y}$ & $\mathrm{z}$ \\
\hline B1 & 0.0000 & 0.0000 & 0.9735 \\
B2 & 0.0000 & 1.2676 & -0.1453 \\
B3 & 1.2676 & -0.0000 & -0.1453 \\
B4 & -0.0000 & -1.2676 & -0.1453 \\
B5 & -1.2676 & 0.0000 & -0.1453 \\
H6 & 0.0000 & 0.0000 & 2.1504 \\
H7 & 0.0000 & 2.4359 & 0.0069 \\
H8 & 2.4359 & -0.0000 & 0.0069 \\
H9 & -0.0000 & -2.4359 & 0.0069 \\
H10 & -2.4359 & 0.0000 & 0.0069 \\
H11 & -0.9641 & 0.9641 & -1.0348 \\
H12 & -0.9641 & -0.9641 & -1.0348 \\
H13 & 0.9641 & -0.9641 & -1.0348 \\
H14 & 0.9641 & 0.9641 & -1.0348 \\
\hline
\end{tabular}


Table S.35.3. $\mathrm{B}_{5} \mathrm{H}_{9}$ : Vibrational frequencies, infrared integrated intensities and Raman activities (B3LYP-D2/cc-pVTZ results)

\begin{tabular}{|c|c|c|c|c|c|c|c|}
\hline \multirow[b]{2}{*}{ mode } & \multirow[b]{2}{*}{ symm. } & \multicolumn{3}{|c|}{ Harmonic } & \multicolumn{3}{|c|}{ Anharmonic } \\
\hline & & $\begin{array}{c}\omega \\
{\left[\mathrm{cm}^{-1}\right]}\end{array}$ & $\begin{array}{c}\mathrm{IR} \\
{\left[\mathrm{km} \mathrm{mol}{ }^{-1}\right]}\end{array}$ & $\begin{array}{c}\text { Raman } \\
{\left[\AA^{6}\right]}\end{array}$ & $\begin{array}{c}\omega \\
{\left[\mathrm{cm}^{-1}\right]}\end{array}$ & $\begin{array}{c}\mathrm{IR} \\
{\left[\mathrm{km} \mathrm{mol}^{-1}\right]}\end{array}$ & $\begin{array}{c}\text { Raman } \\
{\left[\AA^{6}\right]}\end{array}$ \\
\hline 1 & $\mathrm{~B}_{2}$ & 481 & 0.000 & 0.001 & 460 & 0.002 & 0.001 \\
\hline 2 & $\mathrm{E}$ & 587 & 2.135 & 0.010 & 570 & 2.620 & 0.005 \\
\hline 3 & $\mathrm{E}$ & 587 & 2.135 & 0.010 & 570 & 2.620 & 0.005 \\
\hline 4 & $\mathrm{~B}_{1}$ & 624 & 0.000 & 0.100 & 608 & 0.000 & 0.115 \\
\hline 5 & $\mathrm{E}$ & 641 & 20.588 & 0.015 & 617 & 19.149 & 0.023 \\
\hline 6 & $\mathrm{E}$ & 641 & 20.588 & 0.015 & 617 & 19.149 & 0.023 \\
\hline 7 & $\mathrm{~B}_{2}$ & 712 & 0.000 & 0.121 & 686 & 0.002 & 0.133 \\
\hline 8 & $\mathrm{~A}_{1}$ & 736 & 0.274 & 0.050 & 711 & 0.507 & 0.085 \\
\hline 9 & $\mathrm{~B}_{1}$ & 772 & 0.000 & 0.136 & 733 & 0.000 & 0.150 \\
\hline 10 & $\mathrm{E}$ & 806 & 0.004 & 0.077 & 784 & 0.013 & 0.086 \\
\hline 11 & $\mathrm{E}$ & 806 & 0.004 & 0.077 & 784 & 0.013 & 0.086 \\
\hline 12 & $\mathrm{~B}_{2}$ & 818 & 0.000 & 0.025 & 791 & 5.118 & 1.098 \\
\hline 13 & $A_{1}$ & 822 & 2.044 & 0.449 & 800 & 2.006 & 0.543 \\
\hline 14 & $\mathrm{~A}_{2}$ & 852 & 0.000 & 0.000 & 808 & 0.001 & 0.000 \\
\hline 15 & $\mathrm{E}$ & 924 & 22.024 & 0.024 & 896 & 18.905 & 0.022 \\
\hline 16 & $\mathrm{E}$ & 924 & 22.024 & 0.024 & 896 & 18.905 & 0.022 \\
\hline 17 & $\mathrm{E}$ & 953 & 15.176 & 0.005 & 935 & 13.700 & 0.007 \\
\hline 18 & $\mathrm{E}$ & 953 & 15.176 & 0.005 & 935 & 13.700 & 0.007 \\
\hline 19 & $\mathrm{~A}_{1}$ & 1035 & 0.133 & 0.907 & 1009 & 0.041 & 0.855 \\
\hline 20 & $\mathrm{~B}_{1}$ & 1063 & 0.000 & 0.093 & 1001 & 0.000 & 0.103 \\
\hline 21 & $\mathrm{E}$ & 1119 & 3.234 & 0.009 & 1047 & 2.875 & 0.010 \\
\hline 22 & $\mathrm{E}$ & 1119 & 3.234 & 0.009 & 1047 & 2.875 & 0.010 \\
\hline 23 & $\mathrm{~A}_{1}$ & 1199 & 3.340 & 0.115 & 1159 & 2.472 & 0.111 \\
\hline
\end{tabular}


Table S.35.3 - Continued

\begin{tabular}{cccccccc}
\hline & & \multicolumn{3}{c}{ Harmonic } & \multicolumn{3}{c}{ Anharmonic } \\
mode & symm. & $\omega$ & IR & Raman & $\omega$ & IR & Raman \\
& & {$\left[\mathrm{cm}^{-1}\right]$} & {$\left[\mathrm{km} \mathrm{mol}^{-1}\right]$} & {$\left[\AA^{6}\right]$} & {$\left[\mathrm{cm}^{-1}\right]$} & {$\left[\mathrm{km} \mathrm{mol}^{-1}\right]$} & {$\left[\AA^{6}\right]$} \\
\hline 24 & $\mathrm{~A}_{2}$ & 1451 & 0.000 & 0.000 & 1238 & 0.000 & 0.000 \\
25 & $\mathrm{E}$ & 1568 & 78.830 & 0.001 & 1437 & 57.386 & 0.002 \\
26 & $\mathrm{E}$ & 1568 & 78.830 & 0.001 & 1437 & 57.386 & 0.002 \\
27 & $\mathrm{~B}_{2}$ & 1704 & 0.000 & 0.008 & 1524 & 0.000 & 0.006 \\
28 & $\mathrm{~B}_{1}$ & 1950 & 0.000 & 0.076 & 1892 & 0.001 & 0.061 \\
29 & $\mathrm{E}$ & 1961 & 22.794 & 0.256 & 1944 & 14.795 & 0.171 \\
30 & $\mathrm{E}$ & 1961 & 22.794 & 0.256 & 1944 & 14.795 & 0.171 \\
31 & $\mathrm{~A}_{1}$ & 2009 & 17.202 & 0.814 & 1884 & 14.723 & 0.626 \\
32 & $\mathrm{~B}_{2}$ & 2734 & 0.000 & 1.080 & 2628 & 0.000 & 1.034 \\
33 & $\mathrm{E}$ & 2740 & 106.489 & 0.013 & 2653 & 103.824 & 0.015 \\
34 & $\mathrm{E}$ & 2740 & 106.489 & 0.013 & 2653 & 103.824 & 0.015 \\
35 & $\mathrm{~A}_{1}$ & 2740 & 29.383 & 0.786 & 2612 & 24.834 & 0.700 \\
36 & $\mathrm{~A}_{1}$ & 2750 & 19.040 & 2.124 & 2680 & 14.699 & 1.646 \\
\hline
\end{tabular}


Figure S.35.2. Anharmonic IR spectrum of $\mathrm{B}_{5} \mathrm{H}_{9}$ obtained by convoluting the calculated intensities with Lorentzians having a FWHM of $4 \mathrm{~cm}^{-1}$ (B3LYP-D2/cc-pVTZ results): (top) full spectrum, (middle) contributions from fundamentals and combination bands, (bottom) contributions from overtones
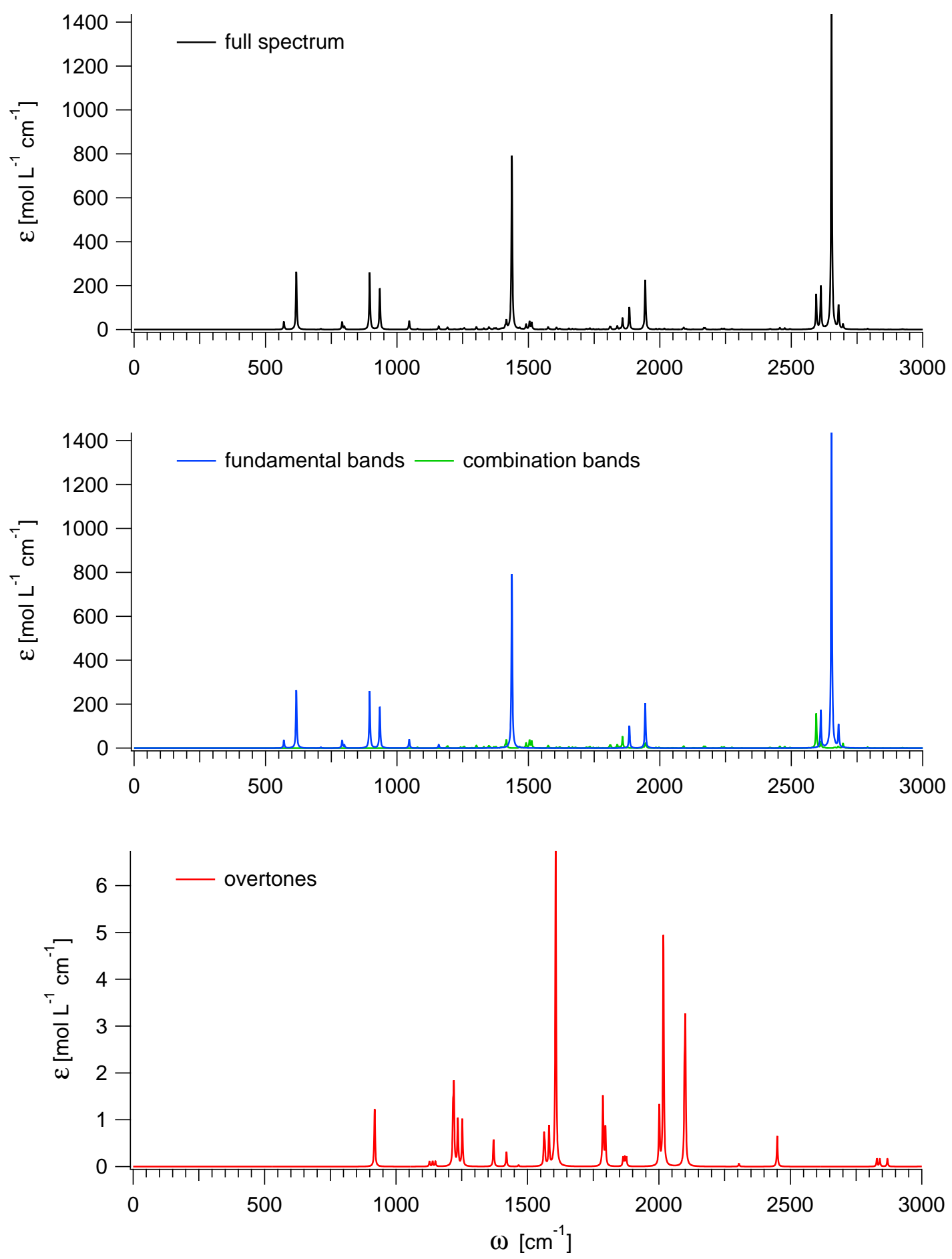
Figure S.35.3. Anharmonic Raman spectrum of $\mathrm{B}_{5} \mathrm{H}_{9}$ obtained by convoluting the calculated activities with Lorentzians having a FWHM of $4 \mathrm{~cm}^{-1}$ (B3LYP-D2/cc-pVTZ results): (top) full spectrum, (middle) contributions from fundamentals and combination bands, (bottom) contributions from overtones
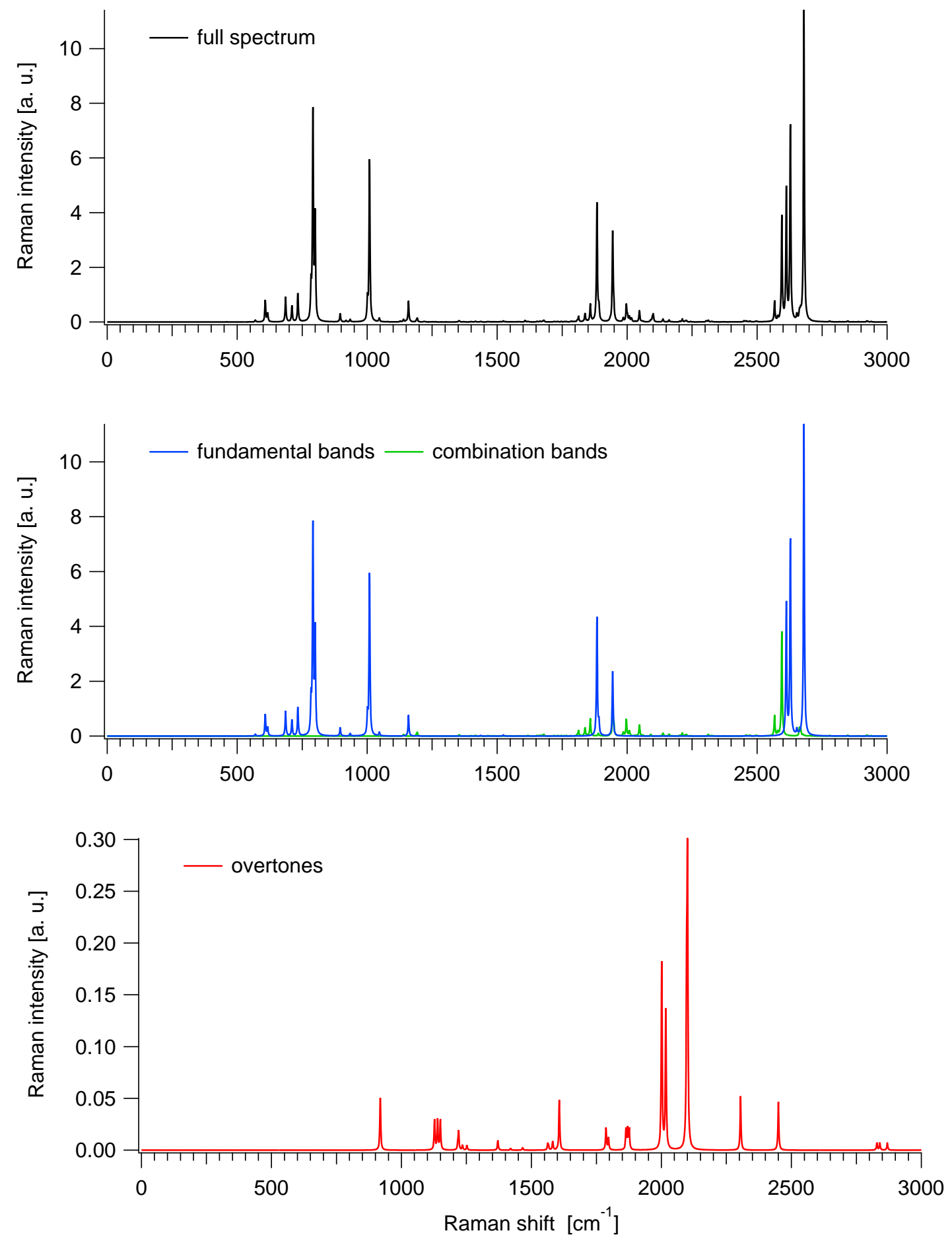

S.291 
Table S.35.4. $\mathrm{B}_{5} \mathrm{H}_{9}$ : Ideal-gas thermodynamic functions at 1 bar (anharmonic B3LYPD2/cc-pVTZ results)

\begin{tabular}{cccc}
\hline$T$ & $C_{P}$ & $S$ & $H(T)-H(0 K)$ \\
{$[\mathrm{K}]$} & {$\left[\mathrm{J} \mathrm{mol}^{-1} \mathrm{~K}^{-1}\right]$} & {$\left[\mathrm{J} \mathrm{mol}^{-1} \mathrm{~K}^{-1}\right]$} & {$\left[\mathrm{kJ} \mathrm{mol}^{-1}\right]$} \\
\hline 100 & 34.510 & 216.307 & 3.342 \\
200 & 59.633 & 246.038 & 7.773 \\
300 & 102.415 & 278.207 & 15.852 \\
400 & 141.603 & 313.214 & 28.114 \\
500 & 173.226 & 348.343 & 43.916 \\
600 & 198.454 & 382.241 & 62.547 \\
700 & 218.781 & 414.414 & 83.444 \\
800 & 235.321 & 444.745 & 106.177 \\
900 & 248.882 & 473.271 & 130.409 \\
1000 & 260.069 & 500.091 & 155.874 \\
1100 & 269.353 & 525.327 & 182.360 \\
1200 & 277.102 & 549.106 & 209.694 \\
1300 & 283.609 & 571.550 & 237.739 \\
1400 & 289.107 & 592.775 & 266.382 \\
1500 & 293.780 & 612.885 & 295.533 \\
1600 & 297.776 & 631.976 & 325.116 \\
1700 & 301.213 & 650.134 & 355.069 \\
1800 & 304.185 & 667.437 & 385.343 \\
1900 & 306.770 & 683.954 & 415.893 \\
2000 & 309.029 & 699.748 & 446.686 \\
\hline$T$ & & & $S$ \\
\hline
\end{tabular}

$T$ : Temperature. $C_{P}$ : Heat capacity at constant pressure. $S$ : Entropy. $H$ : Enthalpy. 
Figure S.35.4. $\mathrm{B}_{5} \mathrm{H}_{9}$ : Ideal-gas thermodynamic functions at 1 bar (anharmonic B3LYPD2/cc-pVTZ results)
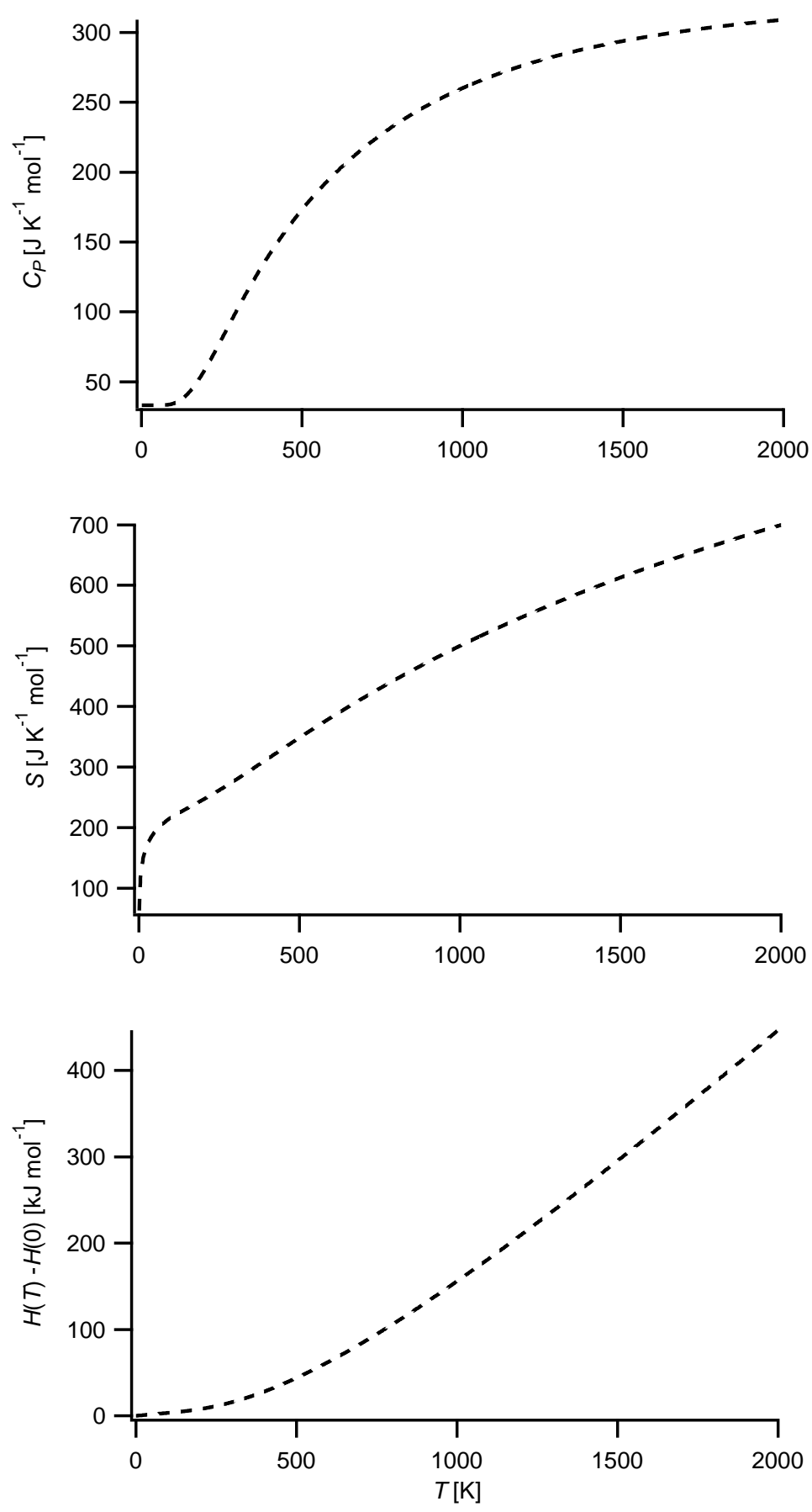

$T$ : Temperature. $C_{P}$ : Heat capacity at constant pressure. $S$ : Entropy. $H$ : Enthalpy. 
Table S.35.5. $\mathrm{B}_{5} \mathrm{H}_{9}$ : Fits (red solid lines) of the computed thermodynamic functions (blue dashed lines) with NASA type functions (Equations (1) - (3)) in the 200 - $900 \mathrm{~K}$ temperature range (anharmonic B3LYP-D2/cc-pVTZ results). In each case, the difference curve between the thermodynamic and NASA functions is plotted in the upper graph

Fit parameters

\begin{tabular}{llllll}
\hline $\mathrm{a}_{1}$ & $-3.97374108 \mathrm{e}+00$ & $\mathrm{a}_{2}\left[\mathrm{~K}^{-1}\right]$ & $5.22817757 \mathrm{e}-02$ & $\mathrm{a}_{3}\left[\mathrm{~K}^{-2}\right]$ & $3.34596103 \mathrm{e}-05$ \\
$\mathrm{a}_{4}\left[\mathrm{~K}^{-3}\right]$ & $-1.05480072 \mathrm{e}-07$ & $\mathrm{a}_{5}\left[\mathrm{~K}^{-4}\right]$ & $5.60911507 \mathrm{e}-11$ & $\mathrm{a}_{6}[\mathrm{~K}]$ & $6.33242189 \mathrm{e}+02$
\end{tabular}

$\mathrm{a}_{7}$
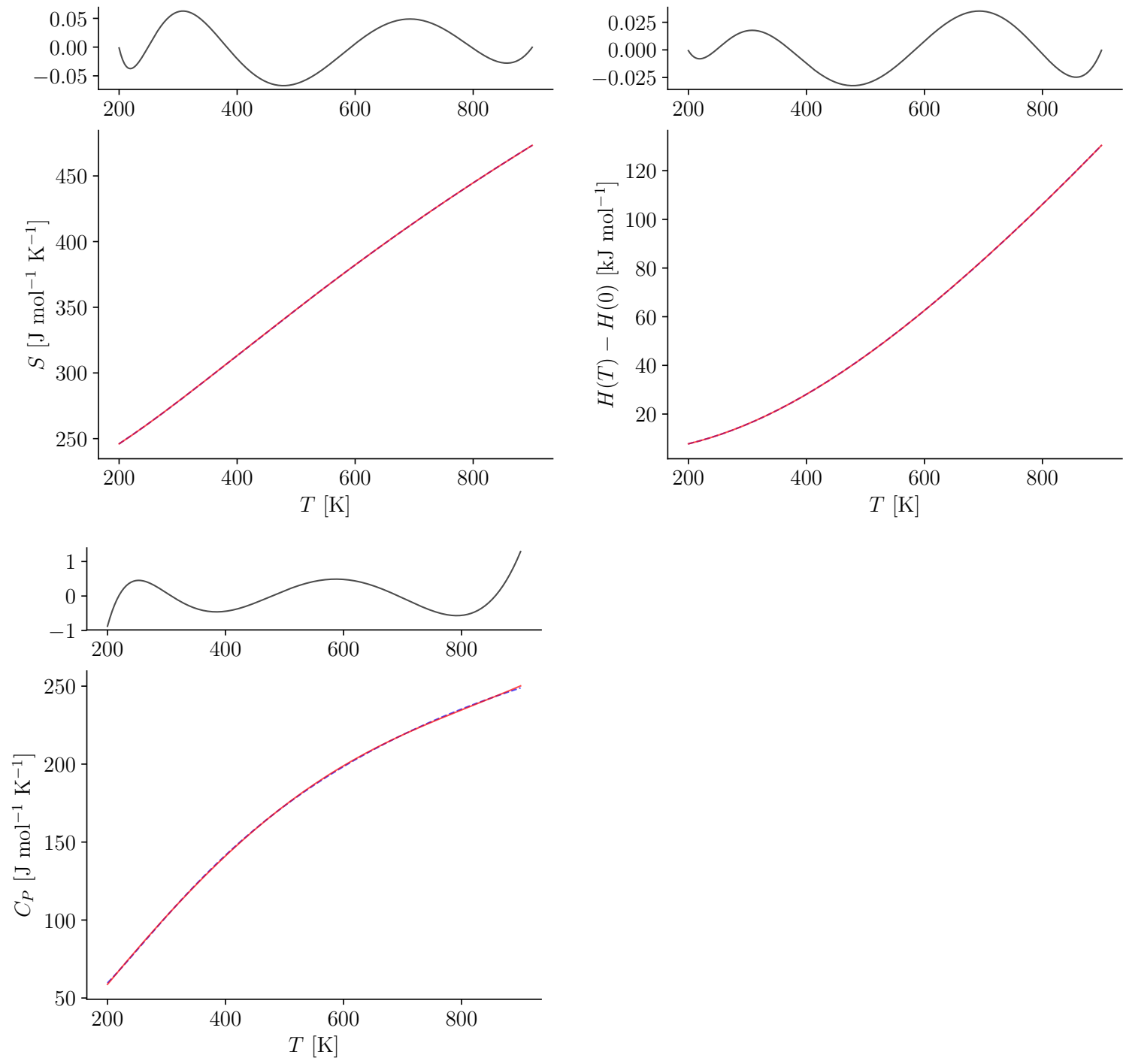


\section{$36 \quad \mathbf{B}_{5} \mathbf{H}_{11}$}

Figure S.36.1. Structure of $\mathrm{B}_{5} \mathrm{H}_{11}$

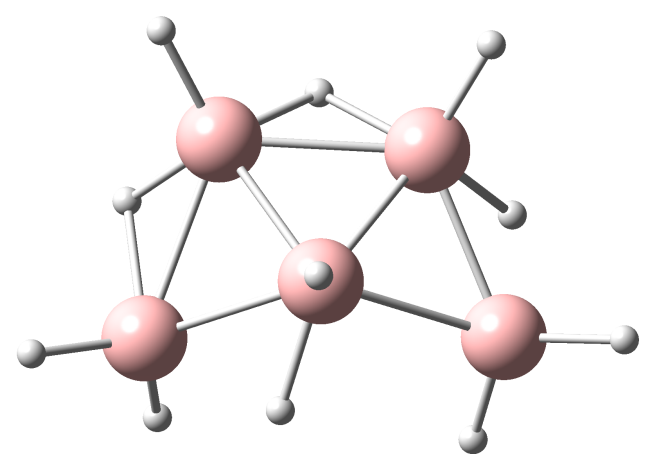

\begin{aligned} & \hline Charge 0 \\ & Spin multiplicity 1 \\ & Framework Group $\mathrm{C}_{1} \\ &$ Rotor Type Asymmetric Top \\ & Symmetry number 1 \\ & \hline\end{aligned}

Table S.36.1. $\mathrm{B}_{5} \mathrm{H}_{11}$ : Selected anharmonic results obtained at the B3LYP-D2/cc-pVTZ level

\begin{tabular}{rll}
\hline $\mathrm{ZPE}_{\mathrm{v}}$ & 27013 & $\mathrm{~cm}^{-1}$ \\
$\mathrm{~A}$ & 0.25447 & $\mathrm{~cm}^{-1}$ \\
$\mathrm{~B}$ & 0.14702 & $\mathrm{~cm}^{-1}$ \\
$\mathrm{C}$ & 0.11606 & $\mathrm{~cm}^{-1}$ \\
\hline$C_{P}(300 \mathrm{~K})$ & 125.653 & $\mathrm{~J} \mathrm{~mol}^{-1} \mathrm{~K}^{-1}$ \\
$S(300 \mathrm{~K})$ & 315.567 & $\mathrm{~J} \mathrm{~mol}^{-1} \mathrm{~K}-1$ \\
$H(300 \mathrm{~K})-H(0 \mathrm{~K})$ & 19.798 & $\mathrm{~kJ} \mathrm{~mol}^{-1}$ \\
\hline
\end{tabular}

$\mathrm{ZPE}_{\mathrm{v}}$ : vibrational contribution to the zero-point energy. A, B, C: Rotational constants. $C_{P}$ : Heat capacity at constant pressure. $S$ : Entropy. $H$ : Enthalpy. Ideal-gas calculations (1 bar). 
Table S.36.2. $\mathrm{B}_{5} \mathrm{H}_{11}$ : Cartesian coordinates $(\AA)$ of the B3LYP-D2/cc-pVTZ optimized structure

\begin{tabular}{cccc} 
atom & $\mathrm{x}$ & $\mathrm{y}$ & $\mathrm{z}$ \\
\hline B1 & -1.5228 & -0.7472 & -0.1891 \\
B2 & -0.8810 & 0.9634 & -0.0969 \\
B3 & -0.0056 & -0.2567 & 0.7945 \\
B4 & 0.9097 & 0.8715 & -0.1473 \\
B5 & 1.5686 & -0.7360 & -0.1538 \\
H6 & -2.4947 & -0.8803 & 0.4752 \\
H7 & -1.4110 & -1.4283 & -1.1642 \\
H8 & -1.3785 & 1.8986 & 0.4206 \\
H9 & -0.0244 & -0.2082 & 1.9737 \\
H10 & 1.4608 & 1.7777 & 0.3713 \\
H11 & 1.3036 & -1.6184 & -0.9027 \\
H12 & 2.6050 & -0.7600 & 0.4241 \\
H13 & -1.6728 & 0.4326 & -0.9245 \\
H14 & -0.0195 & 1.3653 & -1.0090 \\
H15 & 1.6411 & 0.3142 & -1.0437 \\
H16 & -0.3539 & -1.3681 & 0.3429 \\
\hline
\end{tabular}


Table S.36.3. $\mathrm{B}_{5} \mathrm{H}_{11}$ : Vibrational frequencies, infrared integrated intensities and Raman activities (B3LYP-D2/cc-pVTZ results)

\begin{tabular}{|c|c|c|c|c|c|c|c|}
\hline \multirow[b]{2}{*}{ mode } & \multirow[b]{2}{*}{ symm. } & \multicolumn{3}{|c|}{ Harmonic } & \multicolumn{3}{|c|}{ Anharmonic } \\
\hline & & $\begin{array}{c}\omega \\
{\left[\mathrm{cm}^{-1}\right]}\end{array}$ & $\begin{array}{c}\mathrm{IR} \\
{\left[\mathrm{km} \mathrm{mol}^{-1}\right]}\end{array}$ & $\begin{array}{c}\text { Raman } \\
{\left[\AA^{6}\right]}\end{array}$ & $\begin{array}{c}\omega \\
{\left[\mathrm{cm}^{-1}\right]}\end{array}$ & $\begin{array}{c}\mathrm{IR} \\
{\left[\mathrm{km} \mathrm{mol}^{-1}\right]}\end{array}$ & $\begin{array}{c}\text { Raman } \\
{\left[\AA^{6}\right]}\end{array}$ \\
\hline 1 & $\mathrm{~A}$ & 207 & 1.731 & 0.099 & 195 & 2.155 & 0.125 \\
\hline 2 & $\mathrm{~A}$ & 247 & 3.259 & 0.318 & 249 & 3.068 & 0.345 \\
\hline 3 & $\mathrm{~A}$ & 405 & 2.065 & 0.052 & 367 & 1.214 & 0.035 \\
\hline 4 & $\mathrm{~A}$ & 468 & 7.691 & 0.127 & 445 & 2.267 & 0.088 \\
\hline 5 & $\mathrm{~A}$ & 539 & 12.387 & 0.046 & 500 & 17.445 & 0.068 \\
\hline 6 & $\mathrm{~A}$ & 567 & 3.418 & 0.090 & 523 & 8.984 & 0.064 \\
\hline 7 & $\mathrm{~A}$ & 611 & 10.066 & 0.173 & 581 & 6.128 & 0.215 \\
\hline 8 & $\mathrm{~A}$ & 630 & 1.230 & 0.200 & 590 & 1.337 & 0.235 \\
\hline 9 & $\mathrm{~A}$ & 682 & 7.452 & 0.112 & 638 & 6.347 & 0.104 \\
\hline 10 & $\mathrm{~A}$ & 719 & 0.649 & 0.066 & 688 & 1.632 & 0.045 \\
\hline 11 & $\mathrm{~A}$ & 741 & 10.962 & 0.019 & 680 & 4.426 & 0.044 \\
\hline 12 & $\mathrm{~A}$ & 765 & 3.716 & 0.082 & 731 & 4.035 & 0.057 \\
\hline 13 & $\mathrm{~A}$ & 810 & 2.005 & 0.226 & 770 & 2.961 & 0.225 \\
\hline 14 & $\mathrm{~A}$ & 866 & 1.222 & 0.193 & 833 & 0.552 & 0.105 \\
\hline 15 & $\mathrm{~A}$ & 892 & 0.085 & 0.040 & 859 & 0.599 & 0.019 \\
\hline 16 & $\mathrm{~A}$ & 904 & 3.332 & 0.081 & 873 & 1.750 & 0.115 \\
\hline 17 & $\mathrm{~A}$ & 926 & 0.184 & 0.031 & 873 & 0.869 & 0.031 \\
\hline 18 & $\mathrm{~A}$ & 952 & 7.137 & 0.021 & 919 & 17.628 & 0.021 \\
\hline 19 & $\mathrm{~A}$ & 960 & 29.128 & 0.048 & 892 & 7.784 & 0.005 \\
\hline 20 & $\mathrm{~A}$ & 987 & 24.590 & 0.057 & 942 & 8.987 & 0.050 \\
\hline 21 & $\mathrm{~A}$ & 1003 & 23.039 & 0.065 & 983 & 17.124 & 0.066 \\
\hline 22 & $\mathrm{~A}$ & 1060 & 8.531 & 0.052 & 962 & 7.889 & 0.121 \\
\hline 23 & $\mathrm{~A}$ & 1104 & 1.659 & 0.145 & 1024 & 6.041 & 0.050 \\
\hline
\end{tabular}


Table S.36.3 - Continued

\begin{tabular}{|c|c|c|c|c|c|c|c|}
\hline \multirow[b]{2}{*}{ mode } & \multirow[b]{2}{*}{ symm. } & \multicolumn{3}{|c|}{ Harmonic } & \multicolumn{3}{|c|}{ Anharmonic } \\
\hline & & $\begin{array}{c}\omega \\
{\left[\mathrm{cm}^{-1}\right]} \\
\end{array}$ & $\begin{array}{c}\text { IR } \\
{\left[\mathrm{km} \mathrm{mol}^{-1}\right]} \\
\end{array}$ & $\begin{array}{c}\text { Raman } \\
{\left[\AA^{6}\right]}\end{array}$ & $\begin{array}{c}\omega \\
{\left[\mathrm{cm}^{-1}\right]} \\
\end{array}$ & $\begin{array}{c}\mathrm{IR} \\
{\left[\mathrm{km} \mathrm{mol}^{-1}\right]} \\
\end{array}$ & $\begin{array}{c}\text { Raman } \\
{\left[\AA^{6}\right]} \\
\end{array}$ \\
\hline 24 & A & 1119 & 42.926 & 0.050 & 1041 & 55.632 & 0.077 \\
\hline 25 & A & 1161 & 18.654 & 0.058 & 1083 & 13.704 & 0.060 \\
\hline 26 & $\mathrm{~A}$ & 1201 & 15.406 & 0.137 & 1137 & 2.248 & 0.088 \\
\hline 27 & $\mathrm{~A}$ & 1236 & 28.392 & 0.058 & 1171 & 5.385 & 0.020 \\
\hline 28 & $\mathrm{~A}$ & 1320 & 21.542 & 0.063 & 1069 & 35.528 & 0.062 \\
\hline 29 & $\mathrm{~A}$ & 1485 & 23.717 & 0.085 & 1295 & 1.047 & 0.007 \\
\hline 30 & $\mathrm{~A}$ & 1558 & 145.198 & 0.023 & 1383 & 1.571 & 0.009 \\
\hline 31 & $\mathrm{~A}$ & 1652 & 21.335 & 0.016 & 1508 & 4.784 & 0.003 \\
\hline 32 & $\mathrm{~A}$ & 2005 & 17.545 & 0.284 & 1840 & 5.440 & 0.037 \\
\hline 33 & $\mathrm{~A}$ & 2114 & 57.573 & 0.460 & 2007 & 2.352 & 0.050 \\
\hline 34 & $\mathrm{~A}$ & 2195 & 32.759 & 0.659 & 2076 & 4.528 & 0.255 \\
\hline 35 & $\mathrm{~A}$ & 2288 & 9.681 & 0.219 & 2181 & 2.502 & 0.028 \\
\hline 36 & $\mathrm{~A}$ & 2587 & 58.424 & 0.686 & 2494 & 57.577 & 0.742 \\
\hline 37 & $\mathrm{~A}$ & 2634 & 69.760 & 0.950 & 2519 & 50.417 & 0.903 \\
\hline 38 & $\mathrm{~A}$ & 2689 & 66.909 & 0.406 & 2580 & 25.034 & 0.584 \\
\hline 39 & $\mathrm{~A}$ & 2693 & 45.917 & 0.663 & 2569 & 57.452 & 0.383 \\
\hline 40 & $\mathrm{~A}$ & 2712 & 63.845 & 0.584 & 2589 & 65.573 & 0.654 \\
\hline 41 & $\mathrm{~A}$ & 2725 & 47.026 & 0.490 & 2603 & 42.237 & 0.441 \\
\hline 42 & $\mathrm{~A}$ & 2736 & 70.015 & 1.123 & 2615 & 34.260 & 0.564 \\
\hline
\end{tabular}


Figure S.36.2. Anharmonic IR spectrum of $\mathrm{B}_{5} \mathrm{H}_{11}$ obtained by convoluting the calculated intensities with Lorentzians having a FWHM of $4 \mathrm{~cm}^{-1}$ (B3LYP-D2/cc-pVTZ results): (top) full spectrum, (middle) contributions from fundamentals and combination bands, (bottom) contributions from overtones
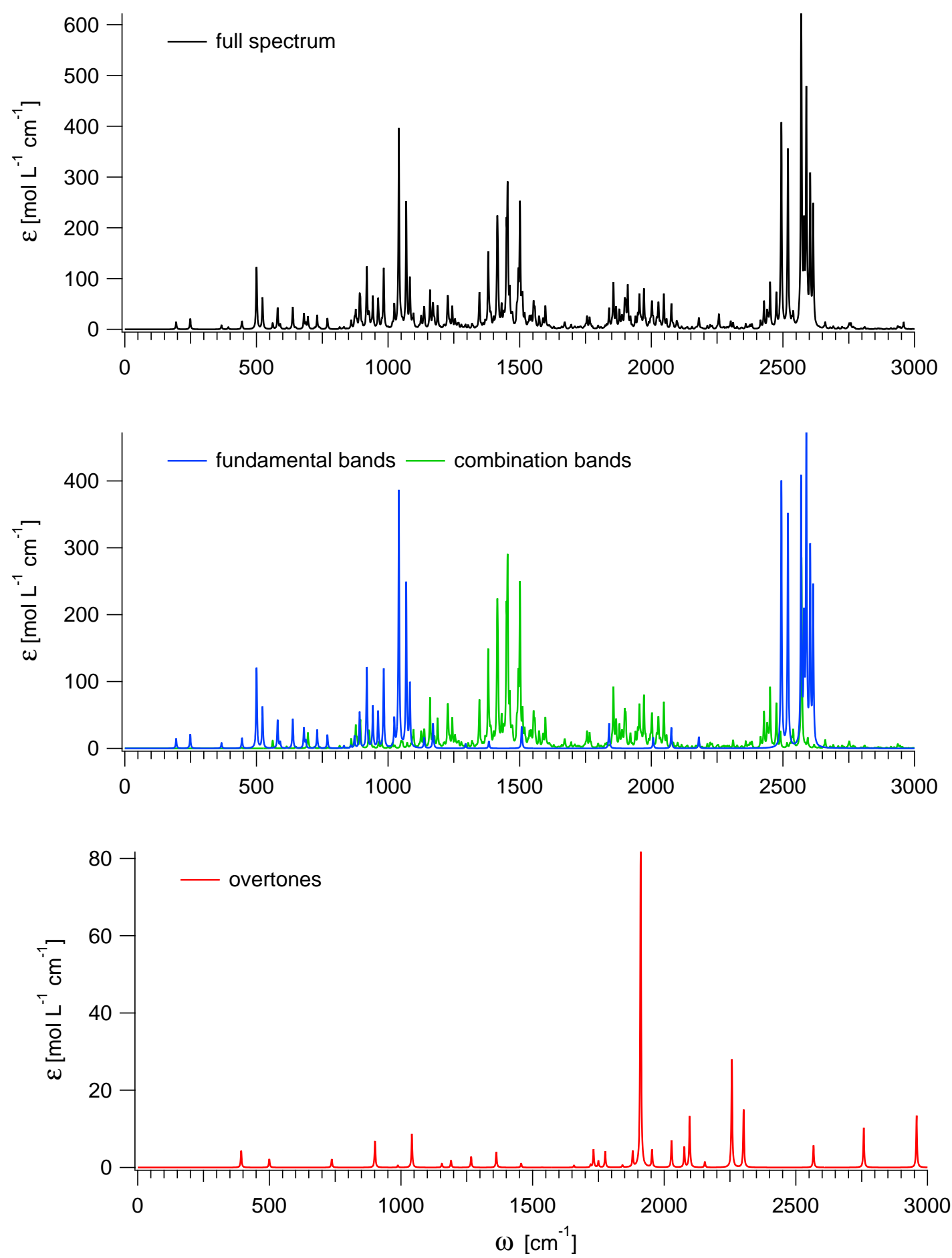
Figure S.36.3. Anharmonic Raman spectrum of $\mathrm{B}_{5} \mathrm{H}_{11}$ obtained by convoluting the calculated activities with Lorentzians having a FWHM of $4 \mathrm{~cm}^{-1}$ (B3LYP-D2/cc-pVTZ results): (top) full spectrum, (middle) contributions from fundamentals and combination bands, (bottom) contributions from overtones
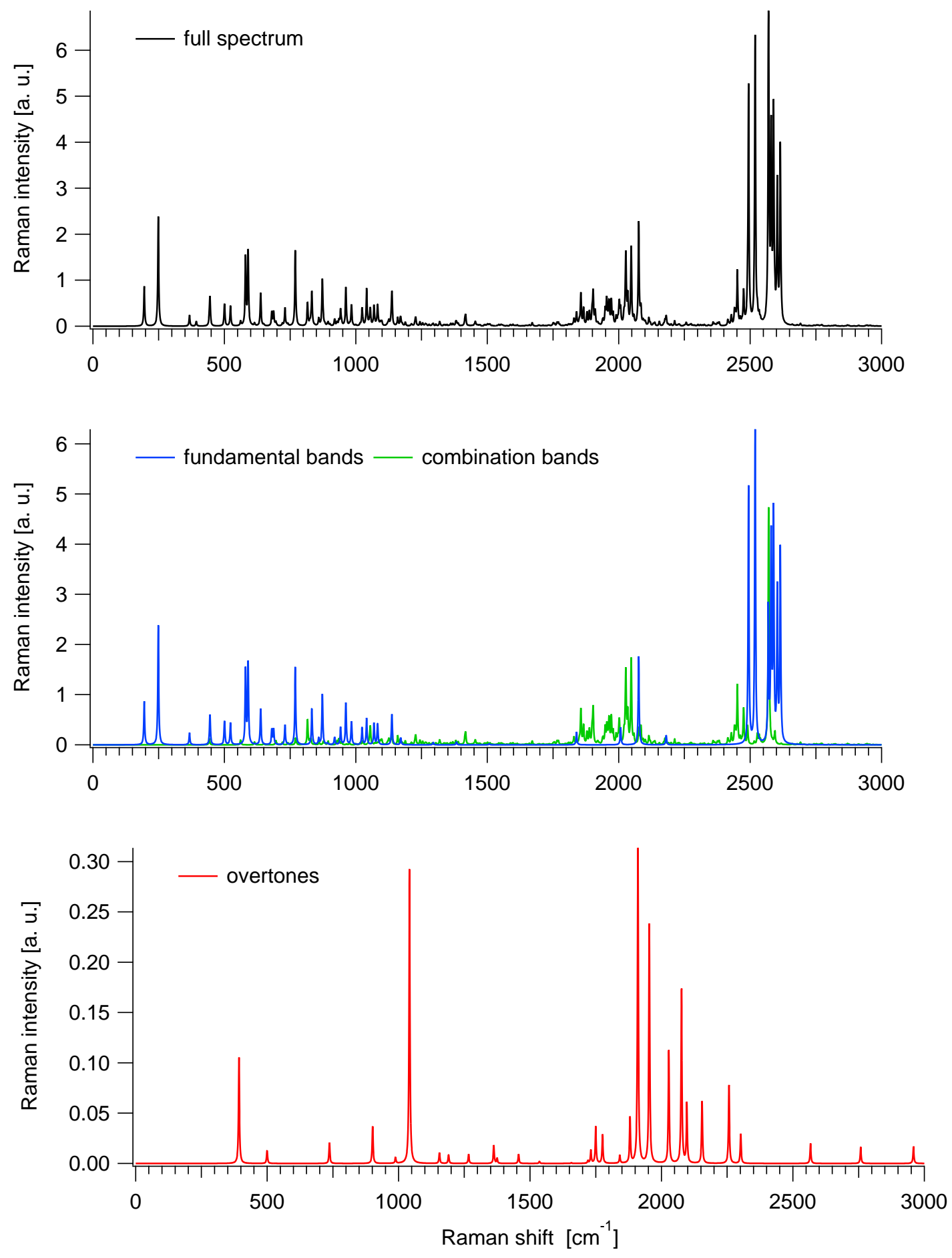

S.300 
Table S.36.4. $\mathrm{B}_{5} \mathrm{H}_{11}$ : Ideal-gas thermodynamic functions at 1 bar (anharmonic B3LYPD2/cc-pVTZ results)

\begin{tabular}{|c|c|c|c|}
\hline $\begin{array}{c}T \\
{[\mathrm{~K}]}\end{array}$ & $\begin{array}{c}C_{P} \\
{\left[\mathrm{~J} \mathrm{~mol}^{-1} \mathrm{~K}^{-1}\right]}\end{array}$ & $\begin{array}{c}S \\
{\left[\mathrm{~J} \mathrm{~mol}^{-1} \mathrm{~K}^{-1}\right]}\end{array}$ & $\begin{array}{c}H(T)-H(0 K) \\
{\left[\mathrm{kJ} \mathrm{mol}^{-1}\right]}\end{array}$ \\
\hline 100 & 43.714 & 234.793 & 3.606 \\
\hline 200 & 79.081 & 274.726 & 9.568 \\
\hline 300 & 125.653 & 315.567 & 19.798 \\
\hline 400 & 167.543 & 357.643 & 34.525 \\
\hline 500 & 201.428 & 398.809 & 53.036 \\
\hline 600 & 228.739 & 438.034 & 74.592 \\
\hline 700 & 251.067 & 475.027 & 98.619 \\
\hline 800 & 269.504 & 509.795 & 124.676 \\
\hline 900 & 284.812 & 542.449 & 152.415 \\
\hline 1000 & 297.570 & 573.138 & 181.554 \\
\hline 1100 & 308.240 & 602.014 & 211.860 \\
\hline 1200 & 317.201 & 629.230 & 243.145 \\
\hline 1300 & 324.762 & 654.927 & 275.253 \\
\hline 1400 & 331.173 & 679.235 & 308.059 \\
\hline 1500 & 336.638 & 702.275 & 341.457 \\
\hline 1600 & 341.323 & 724.155 & 375.361 \\
\hline 1700 & 345.359 & 744.971 & 409.700 \\
\hline 1800 & 348.856 & 764.813 & 444.414 \\
\hline 1900 & 351.901 & 783.758 & 479.456 \\
\hline 2000 & 354.564 & 801.877 & 514.782 \\
\hline
\end{tabular}

$T$ : Temperature. $C_{P}$ : Heat capacity at constant pressure. $S$ : Entropy. $H$ : Enthalpy. 
Figure S.36.4. $\mathrm{B}_{5} \mathrm{H}_{11}$ : Ideal-gas thermodynamic functions at 1 bar (anharmonic B3LYPD2/cc-pVTZ results)
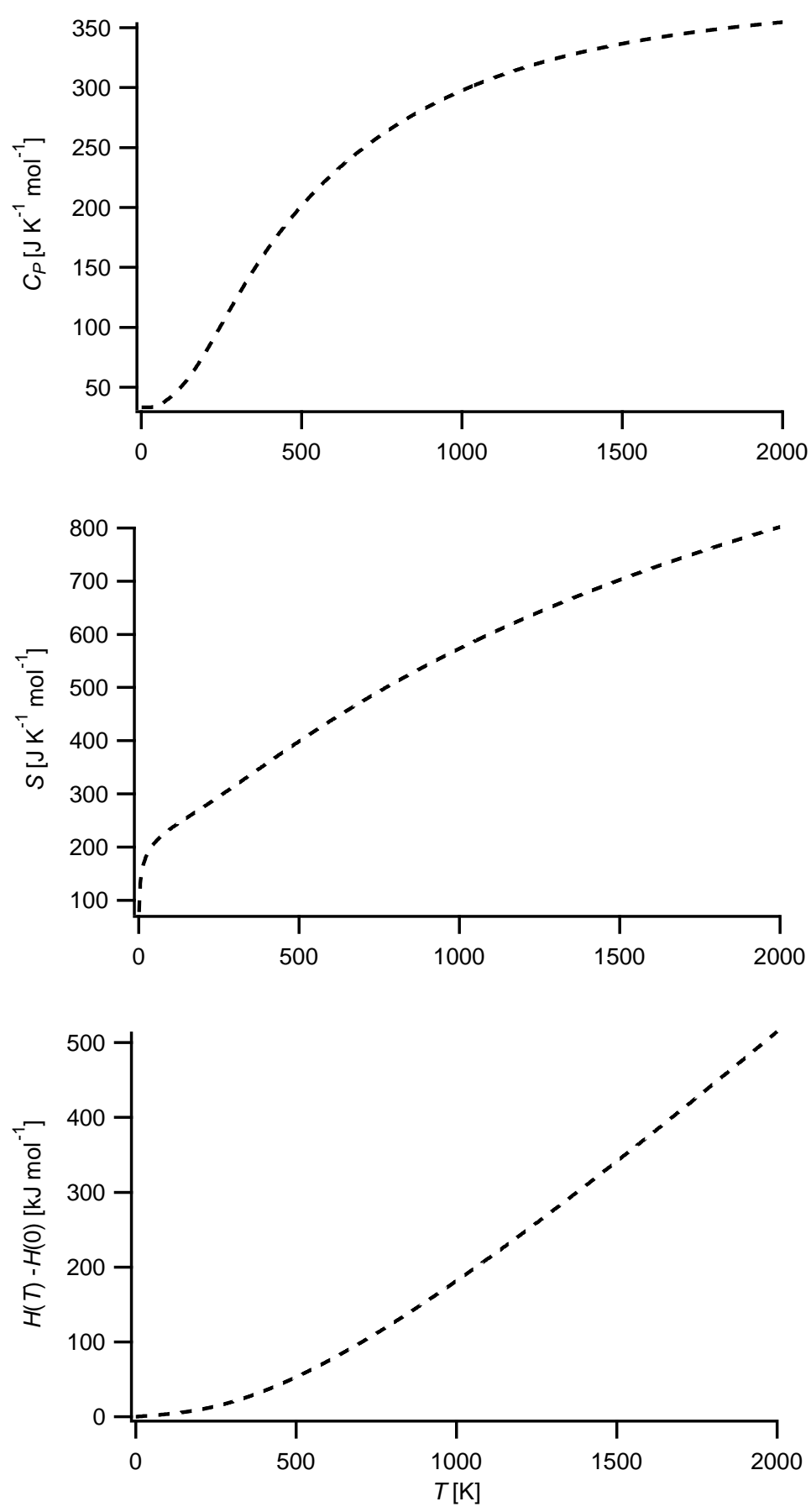

$T$ : Temperature. $C_{P}$ : Heat capacity at constant pressure. $S$ : Entropy. $H$ : Enthalpy. 
Table S.36.5. $\mathrm{B}_{5} \mathrm{H}_{11}$ : Fits (red solid lines) of the computed thermodynamic functions (blue dashed lines) with NASA type functions (Equations (1) - (3)) in the $200-900 \mathrm{~K}$ temperature range (anharmonic B3LYP-D2/cc-pVTZ results). In each case, the difference curve between the thermodynamic and NASA functions is plotted in the upper graph

Fit parameters

\begin{tabular}{llllll}
\hline $\mathrm{a}_{1}$ & $-3.37543950 \mathrm{e}+00$ & $\mathrm{a}_{2}\left[\mathrm{~K}^{-1}\right]$ & $6.51623062 \mathrm{e}-02$ & $\mathrm{a}_{3}\left[\mathrm{~K}^{-2}\right]$ & $7.36631132 \mathrm{e}-06$ \\
$\mathrm{a}_{4}\left[\mathrm{~K}^{-3}\right]$ & $-7.64940724 \mathrm{e}-08$ & $\mathrm{a}_{5}\left[\mathrm{~K}^{-4}\right]$ & $4.40709360 \mathrm{e}-11$ & $\mathrm{a}_{6}[\mathrm{~K}]$ & $5.30707109 \mathrm{e}+02$
\end{tabular}

$a_{7}$
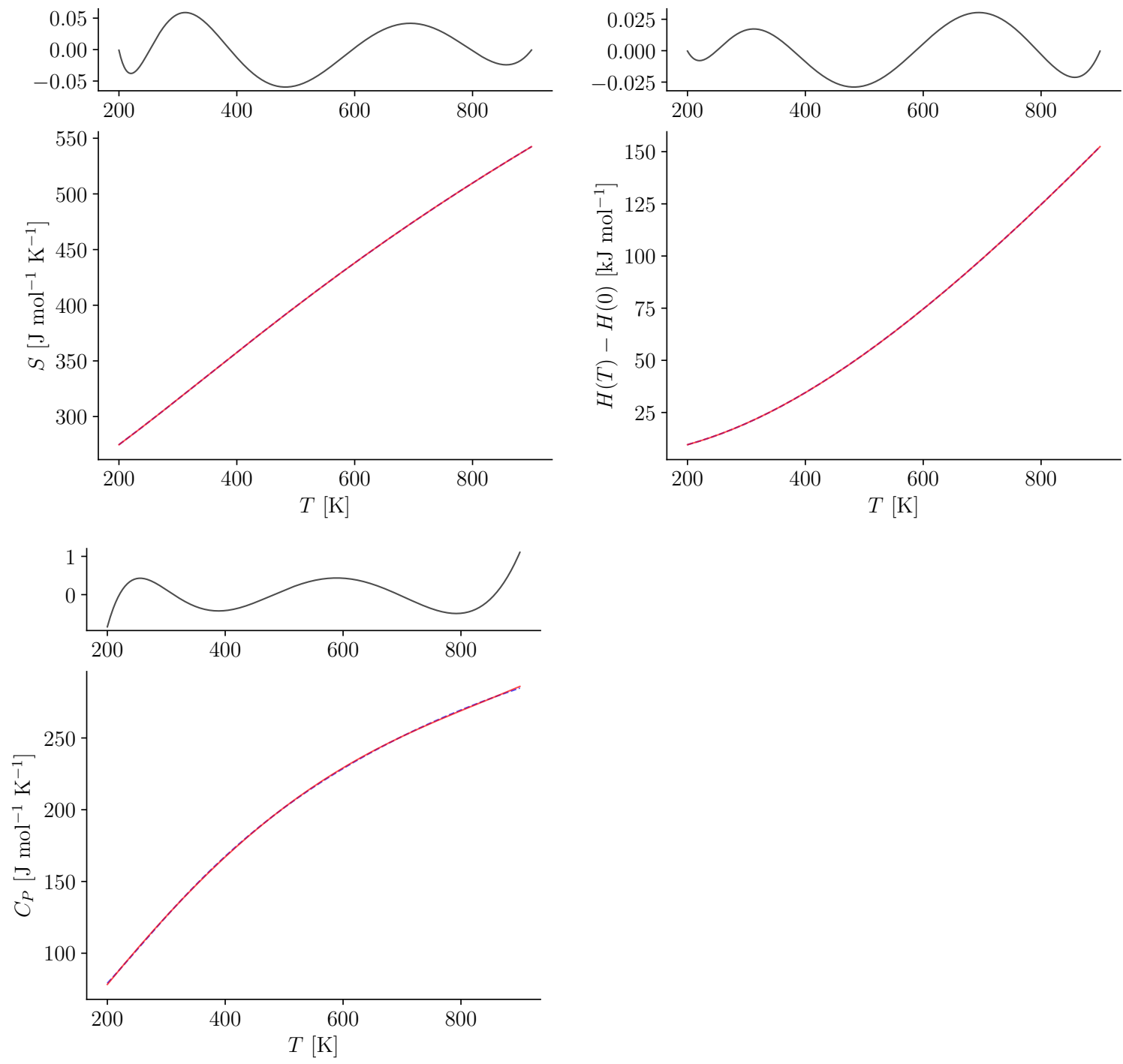


\section{$37 \quad \mathbf{B}_{6} \mathbf{H}_{10}$}

Figure S.37.1. Structure of $\mathrm{B}_{6} \mathrm{H}_{10}$

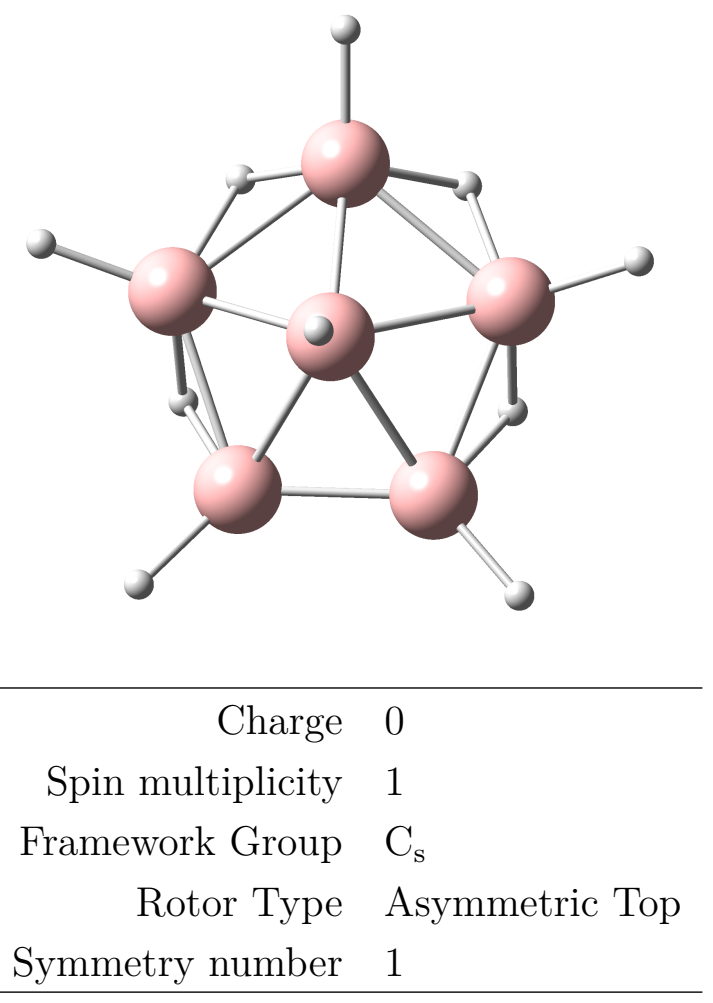

Table S.37.1. $\mathrm{B}_{6} \mathrm{H}_{10}$ : Selected anharmonic results obtained at the B3LYP-D2/cc-pVTZ level

\begin{tabular}{rll}
\hline $\mathrm{ZPE}_{\mathrm{v}}$ & 26202 & $\mathrm{~cm}^{-1}$ \\
$\mathrm{~A}$ & 0.16882 & $\mathrm{~cm}^{-1}$ \\
$\mathrm{~B}$ & 0.16753 & $\mathrm{~cm}^{-1}$ \\
$\mathrm{C}$ & 0.10195 & $\mathrm{~cm}^{-1}$ \\
\hline$C_{P}(300 \mathrm{~K})$ & 122.946 & $\mathrm{~J} \mathrm{~mol}^{-1} \mathrm{~K}-1$ \\
$S(300 \mathrm{~K})$ & 307.953 & $\mathrm{~J} \mathrm{~mol}^{-1} \mathrm{~K}-1$ \\
$H(300 \mathrm{~K})-H(0 \mathrm{~K})$ & 18.187 & $\mathrm{~kJ} \mathrm{~mol}^{-1}$ \\
\hline
\end{tabular}

$\mathrm{ZPE}_{\mathrm{v}}$ : vibrational contribution to the zero-point energy. A, B, C: Rotational constants. $C_{P}$ : Heat capacity at constant pressure. $S$ : Entropy. $H$ : Enthalpy. Ideal-gas calculations (1 bar). 
Table S.37.2. $\mathrm{B}_{6} \mathrm{H}_{10}$ : Cartesian coordinates $(\AA)$ of the B3LYP-D2/cc-pVTZ optimized structure

\begin{tabular}{cccc} 
atom & $\mathrm{x}$ & $\mathrm{y}$ & $\mathrm{z}$ \\
\hline B1 & 0.8492 & 0.0205 & -0.0000 \\
B2 & -0.1199 & 1.4757 & -0.0000 \\
B3 & -0.1266 & 0.3674 & 1.4155 \\
B4 & -0.1266 & -1.2676 & 0.8189 \\
B5 & -0.1266 & -1.2676 & -0.8189 \\
B6 & -0.1266 & 0.3674 & -1.4155 \\
H7 & 2.0283 & 0.0579 & -0.0000 \\
H8 & 0.2626 & 2.5905 & -0.0000 \\
H9 & 0.1497 & 0.7327 & 2.5013 \\
H10 & 0.1497 & 0.7327 & -2.5013 \\
H11 & 0.2329 & -2.0854 & -1.5936 \\
H12 & 0.2329 & -2.0854 & 1.5936 \\
H13 & -0.9916 & 1.3290 & 0.9496 \\
H14 & -0.9916 & 1.3290 & -0.9496 \\
H15 & -1.0935 & -0.5398 & 1.3806 \\
H16 & -1.0935 & -0.5398 & -1.3806 \\
\hline
\end{tabular}


Table S.37.3. $\mathrm{B}_{6} \mathrm{H}_{10}$ : Vibrational frequencies, infrared integrated intensities and Raman activities (B3LYP-D2/cc-pVTZ results)

\begin{tabular}{|c|c|c|c|c|c|c|c|}
\hline \multirow[b]{2}{*}{ mode } & \multirow[b]{2}{*}{ symm. } & \multicolumn{3}{|c|}{ Harmonic } & \multicolumn{3}{|c|}{ Anharmonic } \\
\hline & & $\begin{array}{c}\omega \\
{\left[\mathrm{cm}^{-1}\right]}\end{array}$ & $\begin{array}{c}\mathrm{IR} \\
{\left[\mathrm{km} \mathrm{mol}^{-1}\right]}\end{array}$ & $\begin{array}{c}\text { Raman } \\
{\left[\AA^{6}\right]}\end{array}$ & $\begin{array}{c}\omega \\
{\left[\mathrm{cm}^{-1}\right]}\end{array}$ & $\begin{array}{c}\mathrm{IR} \\
{\left[\mathrm{km} \mathrm{mol}^{-1}\right]}\end{array}$ & $\begin{array}{c}\text { Raman } \\
{\left[\AA^{6}\right]}\end{array}$ \\
\hline 1 & A" & 358 & 0.585 & 0.019 & 351 & 0.474 & 0.018 \\
\hline 2 & $\mathrm{~A}^{\prime}$ & 387 & 0.454 & 0.028 & 373 & 0.494 & 0.028 \\
\hline 3 & $A^{\prime}$ & 558 & 0.688 & 0.132 & 539 & 0.413 & 0.147 \\
\hline 4 & A" & 592 & 0.412 & 0.119 & 572 & 1.363 & 0.077 \\
\hline 5 & $A^{\prime}$ & 605 & 3.128 & 0.083 & 594 & 4.047 & 0.066 \\
\hline 6 & $A^{\prime \prime}$ & 608 & 1.829 & 0.017 & 586 & 1.268 & 0.031 \\
\hline 7 & $A^{\prime}$ & 643 & 9.616 & 0.074 & 618 & 10.876 & 0.094 \\
\hline 8 & $A "$ & 667 & 2.502 & 0.066 & 643 & 3.849 & 0.100 \\
\hline 9 & $A^{\prime}$ & 695 & 1.553 & 0.079 & 665 & 1.881 & 0.072 \\
\hline 10 & $A^{\prime}$ & 717 & 12.297 & 0.012 & 700 & 2.743 & 0.011 \\
\hline 11 & A" & 737 & 3.412 & 0.084 & 712 & 0.787 & 0.104 \\
\hline 12 & $A^{\prime}$ & 769 & 7.022 & 0.835 & 748 & 4.942 & 0.787 \\
\hline 13 & $A^{\prime \prime}$ & 771 & 0.008 & 0.014 & 741 & 6.624 & 0.054 \\
\hline 14 & $A^{\prime \prime}$ & 775 & 15.651 & 0.040 & 741 & 14.105 & 0.025 \\
\hline 15 & $A^{\prime}$ & 800 & 2.471 & 0.261 & 781 & 4.601 & 0.440 \\
\hline 16 & $\mathrm{~A}^{\prime}$ & 838 & 1.386 & 0.023 & 810 & 1.377 & 0.018 \\
\hline 17 & A" & 872 & 1.757 & 0.017 & 841 & 0.358 & 0.014 \\
\hline 18 & $\mathrm{~A}^{\prime}$ & 899 & 5.999 & 0.422 & 878 & 0.117 & 0.446 \\
\hline 19 & $\mathrm{~A}^{\prime}$ & 912 & 24.035 & 0.069 & 874 & 31.178 & 0.064 \\
\hline 20 & $A^{\prime \prime}$ & 927 & 15.890 & 0.028 & 879 & 18.561 & 0.042 \\
\hline 21 & $\mathrm{~A}^{\prime}$ & 951 & 2.965 & 0.027 & 925 & 1.297 & 0.047 \\
\hline 22 & $A^{\prime \prime}$ & 965 & 0.031 & 0.009 & 932 & 0.485 & 0.016 \\
\hline 23 & A" & 993 & 5.894 & 0.023 & 952 & 3.285 & 0.053 \\
\hline
\end{tabular}


Table S.37.3 - Continued

\begin{tabular}{|c|c|c|c|c|c|c|c|}
\hline \multirow[b]{2}{*}{ mode } & \multirow[b]{2}{*}{ symm. } & \multicolumn{3}{|c|}{ Harmonic } & \multicolumn{3}{|c|}{ Anharmonic } \\
\hline & & $\begin{array}{c}\omega \\
{\left[\mathrm{cm}^{-1}\right]} \\
\end{array}$ & $\begin{array}{c}\mathrm{IR} \\
{\left[\mathrm{km} \mathrm{mol}^{-1}\right]} \\
\end{array}$ & $\begin{array}{c}\text { Raman } \\
{\left[\AA^{6}\right]}\end{array}$ & $\begin{array}{c}\omega \\
{\left[\mathrm{cm}^{-1}\right]} \\
\end{array}$ & $\begin{array}{c}\mathrm{IR} \\
{\left[\mathrm{km} \mathrm{mol}^{-1}\right]} \\
\end{array}$ & $\begin{array}{c}\text { Raman } \\
{\left[\AA^{6}\right]} \\
\end{array}$ \\
\hline 24 & $\mathrm{~A}^{\prime \prime}$ & 1042 & 1.454 & 0.062 & 982 & 3.897 & 0.017 \\
\hline 25 & $\mathrm{~A}^{\prime}$ & 1075 & 13.031 & 0.076 & 1015 & 5.275 & 0.057 \\
\hline 26 & $\mathrm{~A}^{\prime}$ & 1096 & 1.173 & 0.173 & 1048 & 7.149 & 0.157 \\
\hline 27 & A" & 1097 & 0.510 & 0.082 & 1032 & 0.062 & 0.084 \\
\hline 28 & $\mathrm{~A}^{\prime}$ & 1153 & 4.685 & 0.096 & 1105 & 3.070 & 0.101 \\
\hline 29 & $A^{\prime \prime}$ & 1506 & 12.622 & 0.048 & 1333 & 1.438 & 0.012 \\
\hline 30 & $A "$ & 1602 & 0.071 & 0.003 & 1431 & 0.643 & 0.002 \\
\hline 31 & $\mathrm{~A}^{\prime}$ & 1625 & 185.489 & 0.090 & 1427 & 15.188 & 0.023 \\
\hline 32 & $\mathrm{~A}^{\prime}$ & 1671 & 33.510 & 0.034 & 1501 & 5.106 & 0.016 \\
\hline 33 & $A^{\prime}$ & 1983 & 0.855 & 0.133 & 1868 & 0.077 & 0.019 \\
\hline 34 & A" & 1989 & 5.595 & 0.062 & 1885 & 0.008 & 0.005 \\
\hline 35 & A" & 2059 & 46.096 & 0.182 & 1933 & 27.933 & 0.092 \\
\hline 36 & $A^{\prime}$ & 2065 & 2.125 & 0.794 & 1939 & 3.709 & 0.335 \\
\hline 37 & A" & 2699 & 46.959 & 0.604 & 2588 & 40.778 & 0.625 \\
\hline 38 & $A^{\prime}$ & 2705 & 87.966 & 0.771 & 2590 & 51.221 & 0.637 \\
\hline 39 & $\mathrm{~A}^{\prime}$ & 2719 & 56.257 & 0.690 & 2606 & 85.446 & 0.665 \\
\hline 40 & $A^{\prime}$ & 2736 & 16.553 & 0.543 & 2598 & 41.293 & 0.954 \\
\hline 41 & $A "$ & 2741 & 115.826 & 0.179 & 2618 & 105.043 & 0.176 \\
\hline 42 & $\mathrm{~A}^{\prime}$ & 2746 & 41.149 & 1.993 & 2638 & 11.645 & 1.872 \\
\hline
\end{tabular}


Figure S.37.2. Anharmonic IR spectrum of $\mathrm{B}_{6} \mathrm{H}_{10}$ obtained by convoluting the calculated intensities with Lorentzians having a FWHM of $4 \mathrm{~cm}^{-1}$ (B3LYP-D2/cc-pVTZ results): (top) full spectrum, (middle) contributions from fundamentals and combination bands, (bottom) contributions from overtones
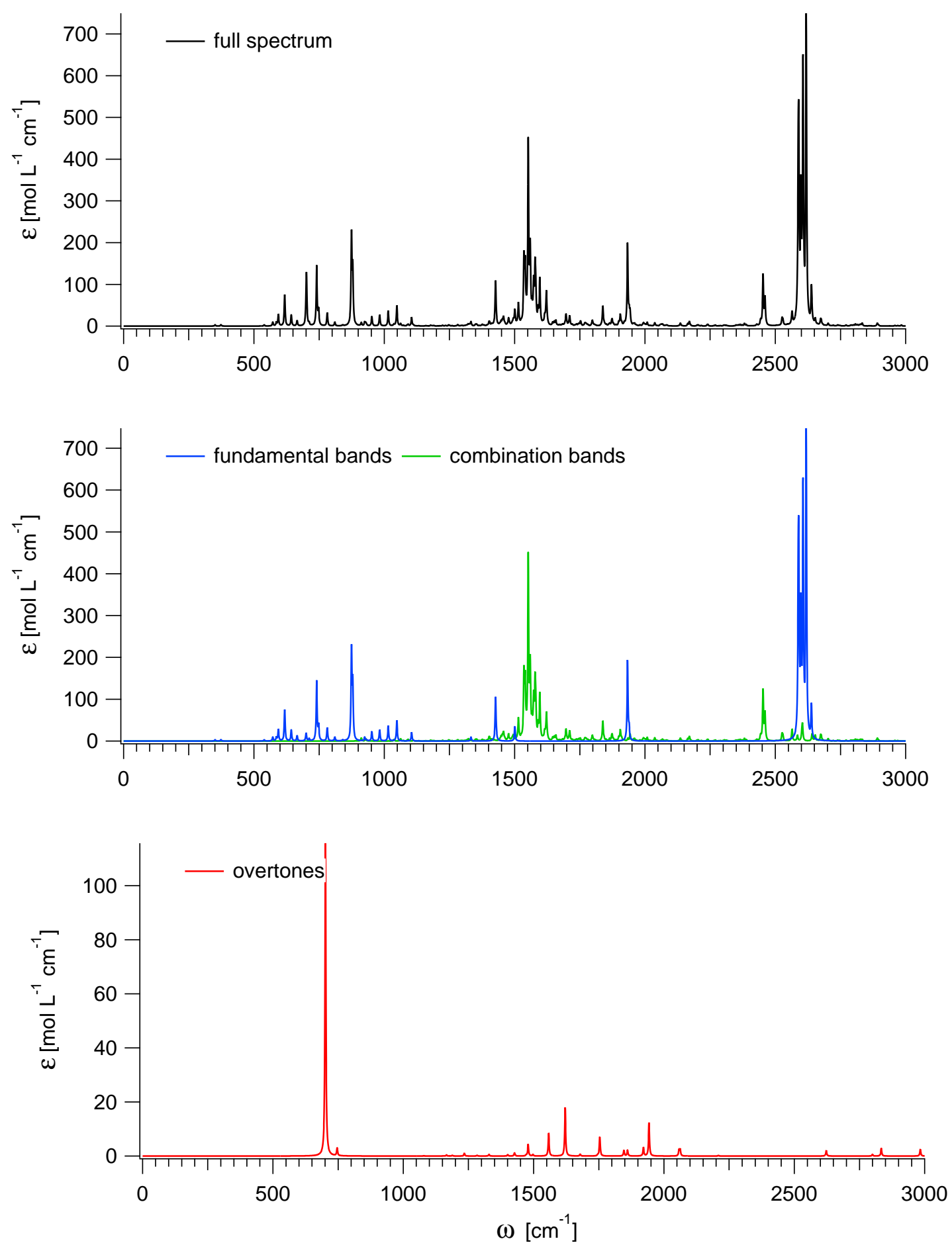
Figure S.37.3. Anharmonic Raman spectrum of $\mathrm{B}_{6} \mathrm{H}_{10}$ obtained by convoluting the calculated activities with Lorentzians having a FWHM of $4 \mathrm{~cm}^{-1}$ (B3LYP-D2/cc-pVTZ results): (top) full spectrum, (middle) contributions from fundamentals and combination bands, (bottom) contributions from overtones
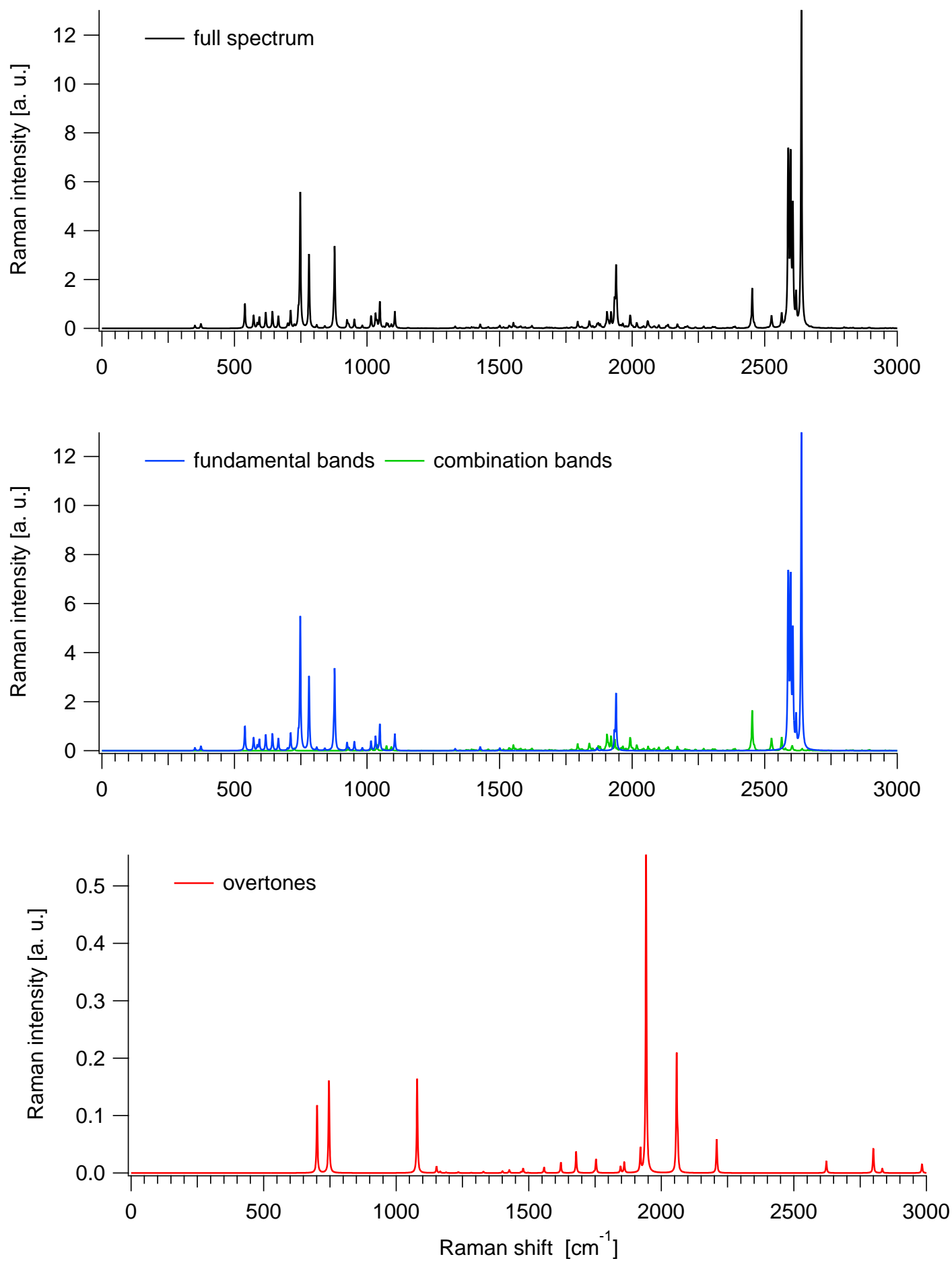
Table S.37.4. $\mathrm{B}_{6} \mathrm{H}_{10}$ : Ideal-gas thermodynamic functions at 1 bar (anharmonic B3LYPD2/cc-pVTZ results)

\begin{tabular}{|c|c|c|c|}
\hline $\begin{array}{c}T \\
{[\mathrm{~K}]}\end{array}$ & $\begin{array}{c}C_{P} \\
{\left[\mathrm{~J} \mathrm{~mol}^{-1} \mathrm{~K}^{-1}\right]}\end{array}$ & $\begin{array}{c}S \\
{\left[\mathrm{~J} \mathrm{~mol}^{-1} \mathrm{~K}^{-1}\right]}\end{array}$ & $\begin{array}{c}H(T)-H(0 K) \\
{\left[\mathrm{kJ} \mathrm{mol}^{-1}\right]}\end{array}$ \\
\hline 100 & 36.768 & 235.324 & 3.385 \\
\hline 200 & 71.165 & 269.334 & 8.487 \\
\hline 300 & 122.946 & 307.953 & 18.187 \\
\hline 400 & 168.610 & 349.812 & 32.848 \\
\hline 500 & 204.678 & 391.475 & 51.585 \\
\hline 600 & 233.108 & 431.404 & 73.529 \\
\hline 700 & 255.870 & 469.110 & 98.019 \\
\hline 800 & 274.334 & 504.524 & 124.560 \\
\hline 900 & 289.448 & 537.738 & 152.774 \\
\hline 1000 & 301.906 & 568.900 & 182.362 \\
\hline 1100 & 312.237 & 598.174 & 213.085 \\
\hline 1200 & 320.857 & 625.723 & 244.752 \\
\hline 1300 & 328.093 & 651.699 & 277.210 \\
\hline 1400 & 334.204 & 676.243 & 310.333 \\
\hline 1500 & 339.398 & 699.483 & 344.020 \\
\hline 1600 & 343.838 & 721.533 & 378.188 \\
\hline 1700 & 347.656 & 742.495 & 412.767 \\
\hline 1800 & 350.958 & 762.462 & 447.702 \\
\hline 1900 & 353.828 & 781.516 & 482.944 \\
\hline 2000 & 356.337 & 799.731 & 518.455 \\
\hline
\end{tabular}

$T$ : Temperature. $C_{P}$ : Heat capacity at constant pressure. $S$ : Entropy. $H$ : Enthalpy. 
Figure S.37.4. $\mathrm{B}_{6} \mathrm{H}_{10}$ : Ideal-gas thermodynamic functions at 1 bar (anharmonic B3LYPD2/cc-pVTZ results)
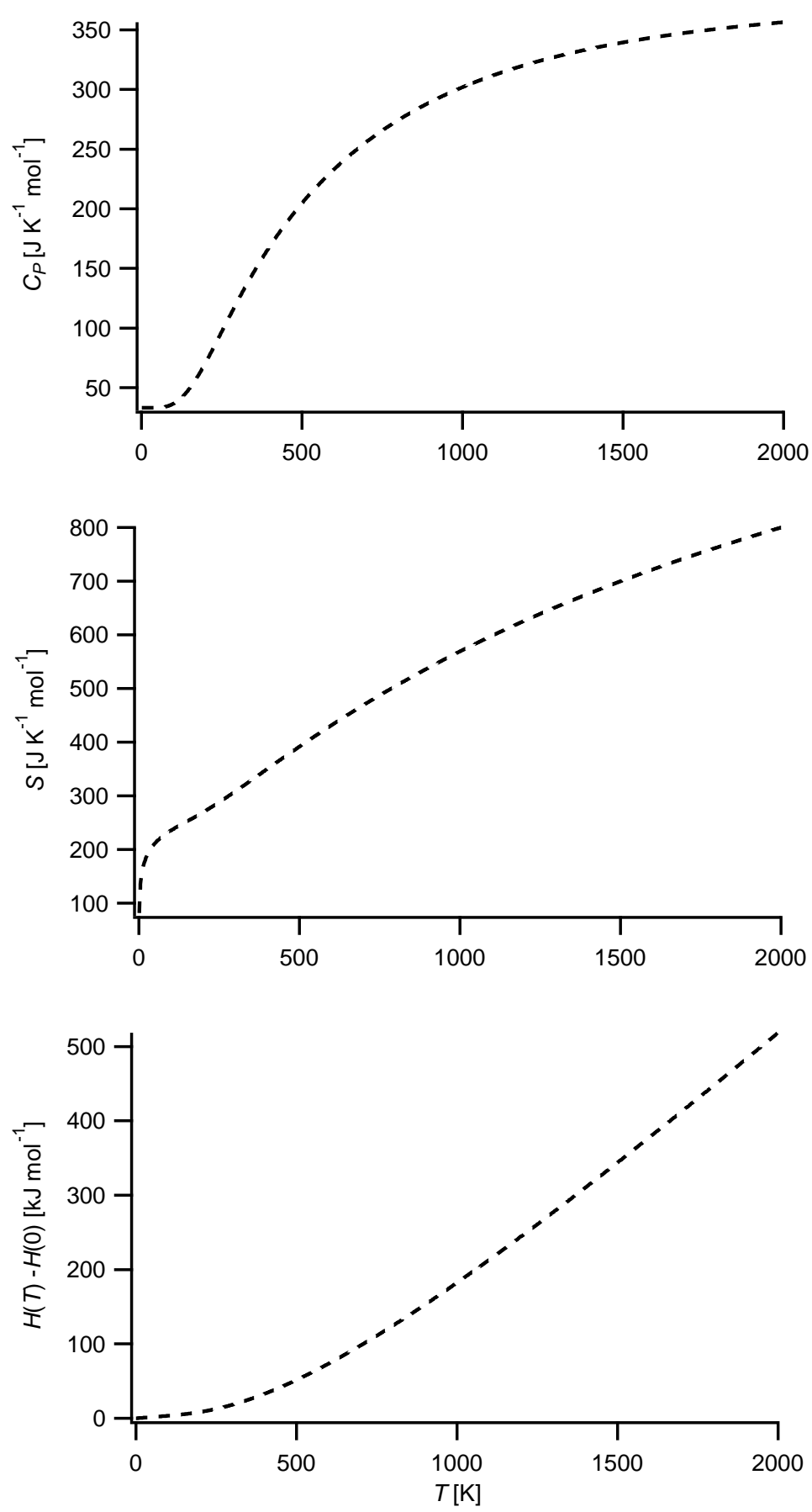

$T$ : Temperature. $C_{P}$ : Heat capacity at constant pressure. $S$ : Entropy. $H$ : Enthalpy. 
Table S.37.5. $\mathrm{B}_{6} \mathrm{H}_{10}$ : Fits (red solid lines) of the computed thermodynamic functions (blue dashed lines) with NASA type functions (Equations (1) - (3)) in the $200-900 \mathrm{~K}$ temperature range (anharmonic B3LYP-D2/cc-pVTZ results). In each case, the difference curve between the thermodynamic and NASA functions is plotted in the upper graph

Fit parameters

\begin{tabular}{llllll}
\hline $\mathrm{a}_{1}$ & $-6.21937684 \mathrm{e}+00$ & $\mathrm{a}_{2}\left[\mathrm{~K}^{-1}\right]$ & $7.64435521 \mathrm{e}-02$ & $\mathrm{a}_{3}\left[\mathrm{~K}^{-2}\right]$ & $-1.69083198 \mathrm{e}-06$ \\
$\mathrm{a}_{4}\left[\mathrm{~K}^{-3}\right]$ & $-7.99613718 \mathrm{e}-08$ & $\mathrm{a}_{5}\left[\mathrm{~K}^{-4}\right]$ & $4.88558744 \mathrm{e}-11$ & $\mathrm{a}_{6}[\mathrm{~K}]$ & $7.69030741 \mathrm{e}+02$ \\
$\mathrm{a}_{7}$ & $5.02842137 \mathrm{e}+01$ & & & &
\end{tabular}
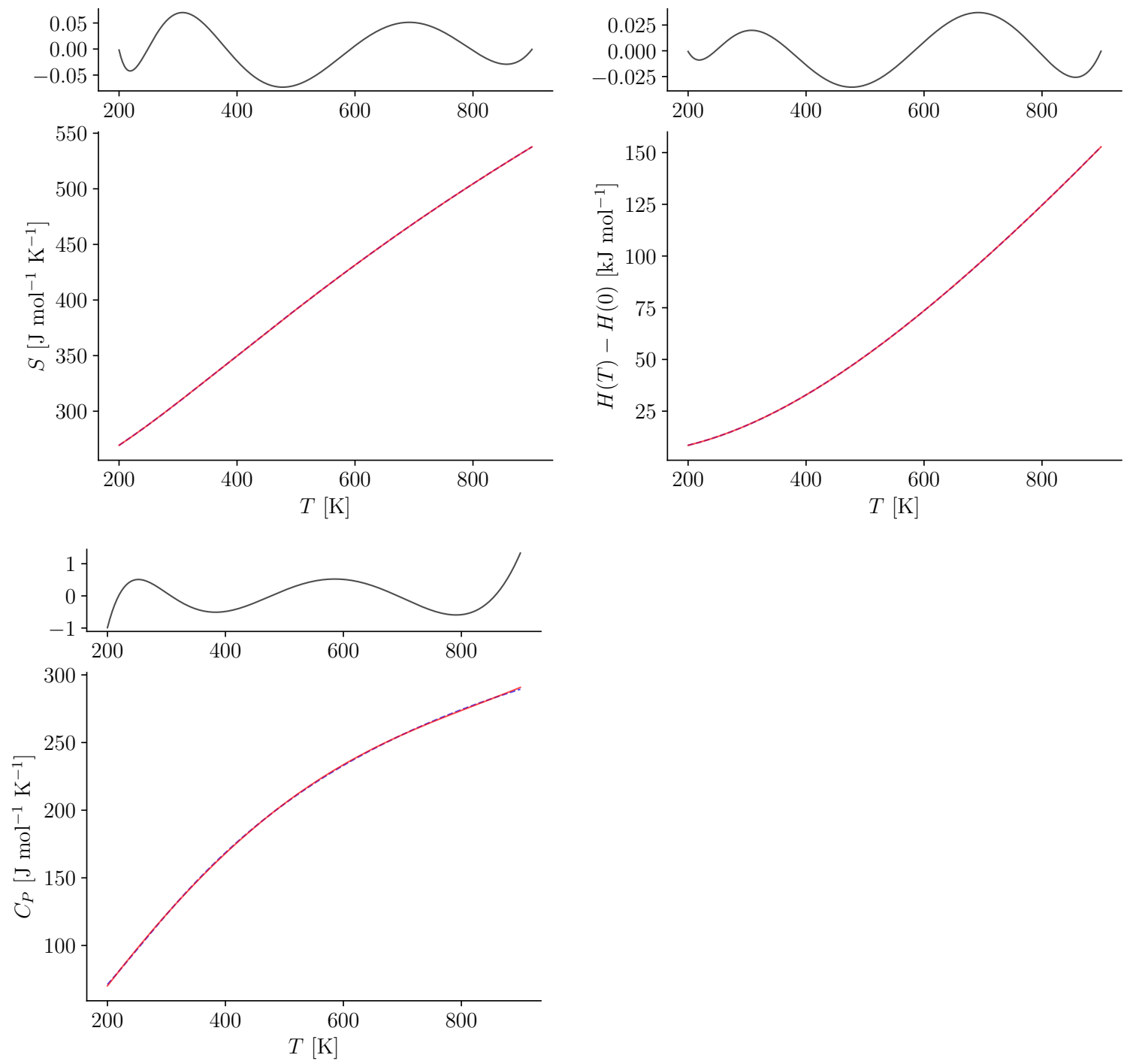


\section{$38 \quad \mathbf{B}_{6} \mathbf{H}_{12}$}

Figure S.38.1. Structure of $\mathrm{B}_{6} \mathrm{H}_{12}$

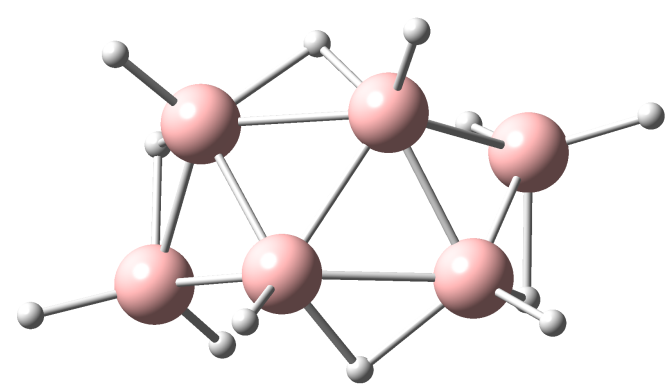

Charge 0

Spin multiplicity 1

Framework Group $\mathrm{C}_{1}$

Rotor Type Asymmetric Top

Symmetry number 1

Table S.38.1. $\mathrm{B}_{6} \mathrm{H}_{12}$ : Selected anharmonic results obtained at the B3LYP-D2/cc-pVTZ level

\begin{tabular}{rll}
\hline $\mathrm{ZPE}_{\mathrm{v}}$ & 30322 & $\mathrm{~cm}^{-1}$ \\
$\mathrm{~A}$ & 0.19309 & $\mathrm{~cm}^{-1}$ \\
$\mathrm{~B}$ & 0.09280 & $\mathrm{~cm}^{-1}$ \\
$\mathrm{C}$ & 0.08976 & $\mathrm{~cm}^{-1}$ \\
\hline$C_{P}(300 \mathrm{~K})$ & 140.814 & $\mathrm{~J} \mathrm{~mol}^{-1} \mathrm{~K}^{-1}$ \\
$S(300 \mathrm{~K})$ & 331.805 & $\mathrm{~J} \mathrm{~mol}^{-1} \mathrm{~K}^{-1}$ \\
$H(300 \mathrm{~K})-H(0 \mathrm{~K})$ & 21.616 & $\mathrm{~kJ} \mathrm{~mol}^{-1}$ \\
\hline
\end{tabular}

$\mathrm{ZPE}_{\mathrm{v}}$ : vibrational contribution to the zero-point energy. A, B, C: Rotational constants. $C_{P}$ : Heat capacity at constant pressure. $S$ : Entropy. $H$ : Enthalpy. Ideal-gas calculations (1 bar). 
Table S.38.2. $\mathrm{B}_{6} \mathrm{H}_{12}$ : Cartesian coordinates $(\AA)$ of the B3LYP-D2/cc-pVTZ optimized structure

\begin{tabular}{cccc} 
atom & $\mathrm{x}$ & $\mathrm{y}$ & $\mathrm{z}$ \\
\hline B1 & 1.7823 & -0.3875 & 0.7672 \\
B2 & 1.1547 & 0.8756 & -0.2337 \\
B3 & 0.5787 & -0.6825 & -0.6880 \\
B4 & -0.5787 & 0.6825 & -0.6880 \\
B5 & -1.1547 & -0.8756 & -0.2337 \\
H6 & 2.9453 & -0.5525 & 0.6058 \\
H7 & 1.2508 & -0.8705 & 1.7137 \\
H8 & 1.8413 & 1.5027 & -0.9605 \\
H9 & 0.9494 & -1.2412 & -1.6586 \\
H10 & -0.9494 & 1.2412 & -1.6586 \\
H11 & -1.5928 & -0.9342 & 0.9855 \\
H12 & -1.8413 & -1.5027 & -0.9605 \\
H13 & -0.0083 & -1.5323 & 0.0868 \\
B14 & -1.7823 & 0.3875 & 0.7672 \\
H15 & -1.2508 & 0.8705 & 1.7137 \\
H16 & -2.9453 & 0.5525 & 0.6058 \\
H17 & 0.0083 & 1.5323 & 0.0868 \\
H18 & 1.5928 & 0.9342 & 0.9855 \\
\hline
\end{tabular}


Table S.38.3. $\mathrm{B}_{6} \mathrm{H}_{12}$ : Vibrational frequencies, infrared integrated intensities and Raman activities (B3LYP-D2/cc-pVTZ results)

\begin{tabular}{|c|c|c|c|c|c|c|c|}
\hline \multirow[b]{2}{*}{ mode } & \multirow[b]{2}{*}{ symm. } & \multicolumn{3}{|c|}{ Harmonic } & \multicolumn{3}{|c|}{ Anharmonic } \\
\hline & & $\begin{array}{c}\omega \\
{\left[\mathrm{cm}^{-1}\right]}\end{array}$ & $\begin{array}{c}\mathrm{IR} \\
{\left[\mathrm{km} \mathrm{mol}^{-1}\right]}\end{array}$ & $\begin{array}{c}\text { Raman } \\
{\left[\AA^{6}\right]}\end{array}$ & $\begin{array}{c}\omega \\
{\left[\mathrm{cm}^{-1}\right]}\end{array}$ & $\begin{array}{c}\mathrm{IR} \\
{\left[\mathrm{km} \mathrm{mol}^{-1}\right]}\end{array}$ & $\begin{array}{c}\text { Raman } \\
{\left[\AA^{6}\right]}\end{array}$ \\
\hline 1 & $\mathrm{~A}$ & 134 & 1.513 & 0.747 & 154 & 1.404 & 0.710 \\
\hline 2 & $\mathrm{~A}$ & 251 & 8.518 & 0.088 & 236 & 6.514 & 0.102 \\
\hline 3 & $\mathrm{~A}$ & 280 & 0.343 & 0.167 & 275 & 0.246 & 0.141 \\
\hline 4 & $\mathrm{~A}$ & 456 & 0.465 & 0.438 & 439 & 0.604 & 0.489 \\
\hline 5 & $\mathrm{~A}$ & 467 & 20.603 & 0.103 & 449 & 18.540 & 0.116 \\
\hline 6 & $\mathrm{~A}$ & 545 & 12.747 & 0.035 & 525 & 14.665 & 0.037 \\
\hline 7 & $\mathrm{~A}$ & 577 & 1.175 & 0.253 & 559 & 0.629 & 0.231 \\
\hline 8 & $\mathrm{~A}$ & 599 & 0.927 & 0.021 & 560 & 0.012 & 0.021 \\
\hline 9 & $\mathrm{~A}$ & 605 & 0.096 & 0.147 & 586 & 0.153 & 0.119 \\
\hline 10 & $\mathrm{~A}$ & 680 & 0.000 & 0.074 & 655 & 1.034 & 0.066 \\
\hline 11 & $\mathrm{~A}$ & 712 & 8.927 & 0.423 & 685 & 5.009 & 0.327 \\
\hline 12 & $\mathrm{~A}$ & 741 & 0.098 & 0.033 & 724 & 0.051 & 0.019 \\
\hline 13 & $\mathrm{~A}$ & 747 & 25.927 & 0.058 & 718 & 22.769 & 0.017 \\
\hline 14 & $\mathrm{~A}$ & 762 & 8.240 & 0.144 & 730 & 3.942 & 0.181 \\
\hline 15 & $\mathrm{~A}$ & 786 & 11.494 & 0.024 & 758 & 6.712 & 0.044 \\
\hline 16 & $\mathrm{~A}$ & 804 & 0.743 & 0.073 & 774 & 0.387 & 0.068 \\
\hline 17 & $\mathrm{~A}$ & 876 & 0.753 & 0.053 & 829 & 0.615 & 0.050 \\
\hline 18 & $\mathrm{~A}$ & 876 & 4.823 & 0.022 & 847 & 5.715 & 0.017 \\
\hline 19 & $\mathrm{~A}$ & 887 & 4.048 & 0.500 & 840 & 4.773 & 0.382 \\
\hline 20 & $\mathrm{~A}$ & 909 & 7.370 & 0.047 & 866 & 18.663 & 0.049 \\
\hline 21 & $\mathrm{~A}$ & 911 & 1.002 & 0.060 & 874 & 1.129 & 0.035 \\
\hline 22 & $\mathrm{~A}$ & 942 & 25.809 & 0.007 & 914 & 23.589 & 0.010 \\
\hline 23 & $\mathrm{~A}$ & 982 & 0.264 & 0.059 & 926 & 0.036 & 0.134 \\
\hline
\end{tabular}


Table S.38.3 - Continued

\begin{tabular}{|c|c|c|c|c|c|c|c|}
\hline \multirow[b]{2}{*}{ mode } & \multirow[b]{2}{*}{ symm. } & \multicolumn{3}{|c|}{ Harmonic } & \multicolumn{3}{|c|}{ Anharmonic } \\
\hline & & $\begin{array}{c}\omega \\
{\left[\mathrm{cm}^{-1}\right]} \\
\end{array}$ & $\begin{array}{c}\mathrm{IR} \\
{\left[\mathrm{km} \mathrm{mol}^{-1}\right]} \\
\end{array}$ & $\begin{array}{c}\text { Raman } \\
{\left[\AA^{6}\right]}\end{array}$ & $\begin{array}{c}\omega \\
{\left[\mathrm{cm}^{-1}\right]} \\
\end{array}$ & $\begin{array}{c}\mathrm{IR} \\
{\left[\mathrm{km} \mathrm{mol}^{-1}\right]} \\
\end{array}$ & $\begin{array}{c}\text { Raman } \\
{\left[\AA^{6}\right]}\end{array}$ \\
\hline 24 & $\mathrm{~A}$ & 990 & 25.563 & 0.016 & 937 & 14.713 & 0.011 \\
\hline 25 & $\mathrm{~A}$ & 1008 & 1.178 & 0.115 & 962 & 0.399 & 0.038 \\
\hline 26 & $\mathrm{~A}$ & 1023 & 15.243 & 0.006 & 966 & 6.443 & 0.012 \\
\hline 27 & $\mathrm{~A}$ & 1102 & 54.840 & 0.086 & 1028 & 33.568 & 0.082 \\
\hline 28 & $\mathrm{~A}$ & 1119 & 0.494 & 0.066 & 1046 & 0.646 & 0.086 \\
\hline 29 & $\mathrm{~A}$ & 1127 & 9.661 & 0.143 & 1056 & 1.127 & 0.033 \\
\hline 30 & $\mathrm{~A}$ & 1128 & 39.108 & 0.022 & 1062 & 56.484 & 0.005 \\
\hline 31 & $\mathrm{~A}$ & 1225 & 37.774 & 0.047 & 1175 & 29.082 & 0.054 \\
\hline 32 & $\mathrm{~A}$ & 1231 & 9.317 & 0.085 & 1184 & 7.051 & 0.047 \\
\hline 33 & $\mathrm{~A}$ & 1555 & 93.701 & 0.024 & 1372 & 48.356 & 0.018 \\
\hline 34 & $\mathrm{~A}$ & 1570 & 40.854 & 0.196 & 1384 & 5.817 & 0.043 \\
\hline 35 & $\mathrm{~A}$ & 1650 & 178.765 & 0.009 & 1483 & 5.339 & 0.001 \\
\hline 36 & $\mathrm{~A}$ & 1678 & 18.147 & 0.036 & 1507 & 0.293 & 0.006 \\
\hline 37 & $\mathrm{~A}$ & 2055 & 20.103 & 0.079 & 1902 & 13.624 & 0.019 \\
\hline 38 & $\mathrm{~A}$ & 2059 & 16.277 & 0.098 & 1906 & 1.493 & 0.020 \\
\hline 39 & $\mathrm{~A}$ & 2096 & 97.313 & 0.123 & 1971 & 0.056 & 0.004 \\
\hline 40 & $\mathrm{~A}$ & 2104 & 6.644 & 0.827 & 2008 & 0.241 & 0.113 \\
\hline 41 & $\mathrm{~A}$ & 2633 & 55.870 & 0.152 & 2523 & 7.692 & 0.030 \\
\hline 42 & $\mathrm{~A}$ & 2634 & 54.992 & 1.489 & 2521 & 39.462 & 1.337 \\
\hline 43 & $\mathrm{~A}$ & 2692 & 111.774 & 0.084 & 2577 & 71.780 & 0.106 \\
\hline 44 & $\mathrm{~A}$ & 2694 & 6.791 & 0.933 & 2578 & 1.352 & 0.136 \\
\hline 45 & $\mathrm{~A}$ & 2719 & 14.842 & 0.411 & 2599 & 7.913 & 0.423 \\
\hline 46 & $\mathrm{~A}$ & 2719 & 53.881 & 0.461 & 2601 & 54.136 & 0.505 \\
\hline 47 & $\mathrm{~A}$ & 2728 & 108.713 & 0.378 & 2605 & 96.407 & 0.393 \\
\hline
\end{tabular}

To be continued 
Table S.38.3 - Continued

\begin{tabular}{cccccccc}
\hline & & \multicolumn{3}{c}{ Harmonic } & \multicolumn{3}{c}{ Anharmonic } \\
mode & symm. & $\omega$ & IR & Raman & $\omega$ & IR & Raman \\
& & {$\left[\mathrm{cm}^{-1}\right]$} & {$\left[\mathrm{km} \mathrm{mol}^{-1}\right]$} & {$\left[\AA^{6}\right]$} & {$\left[\mathrm{cm}^{-1}\right]$} & {$\left[\mathrm{km} \mathrm{mol}^{-1}\right]$} & {$\left[\AA^{6}\right]$} \\
\hline 48 & $\mathrm{~A}$ & 2734 & 78.504 & 1.861 & 2612 & 84.987 & 2.074 \\
\hline
\end{tabular}


Figure S.38.2. Anharmonic IR spectrum of $\mathrm{B}_{6} \mathrm{H}_{12}$ obtained by convoluting the calculated intensities with Lorentzians having a FWHM of $4 \mathrm{~cm}^{-1}$ (B3LYP-D2/cc-pVTZ results): (top) full spectrum, (middle) contributions from fundamentals and combination bands, (bottom) contributions from overtones
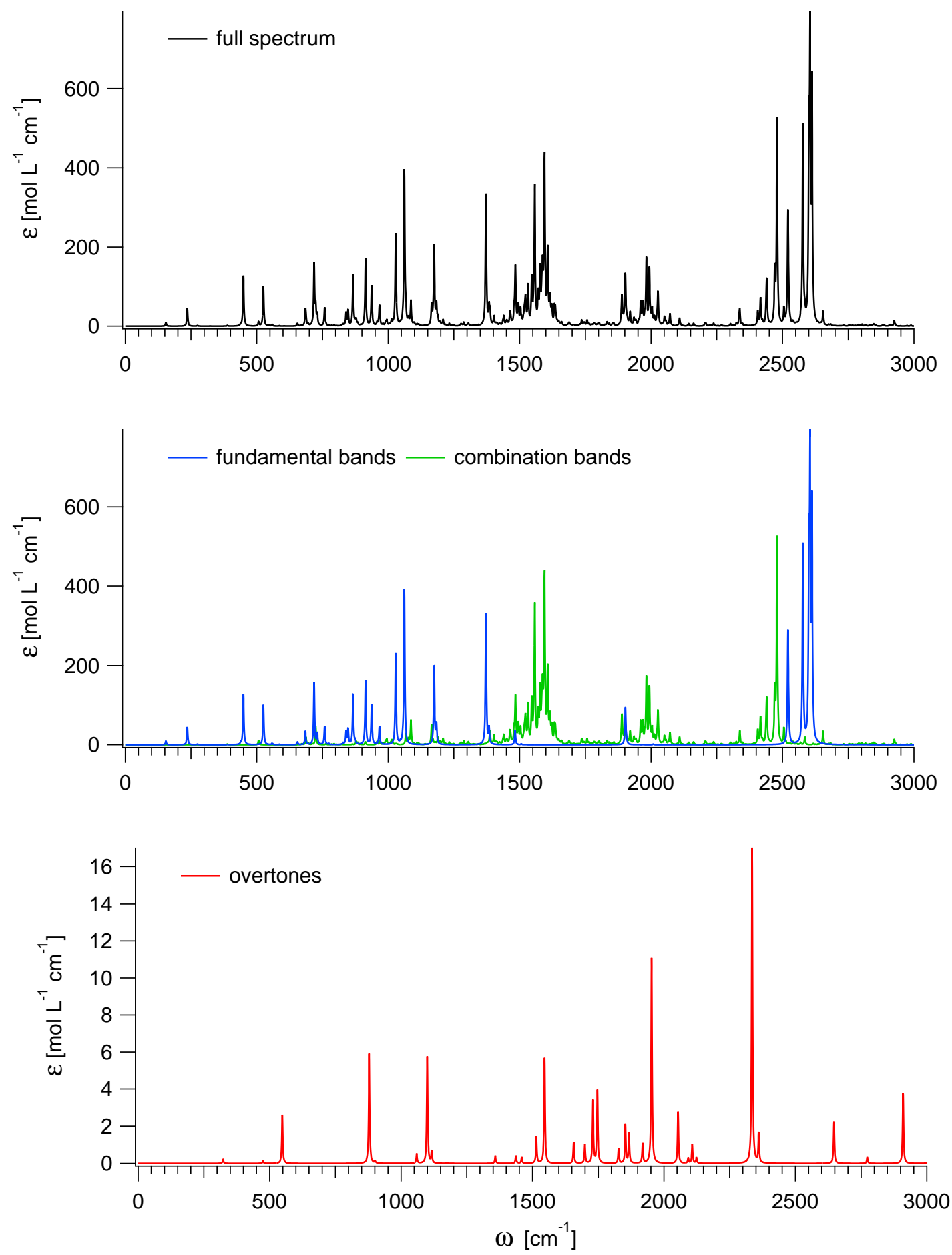
Figure S.38.3. Anharmonic Raman spectrum of $\mathrm{B}_{6} \mathrm{H}_{12}$ obtained by convoluting the calculated activities with Lorentzians having a FWHM of $4 \mathrm{~cm}^{-1}$ (B3LYP-D2/cc-pVTZ results): (top) full spectrum, (middle) contributions from fundamentals and combination bands, (bottom) contributions from overtones
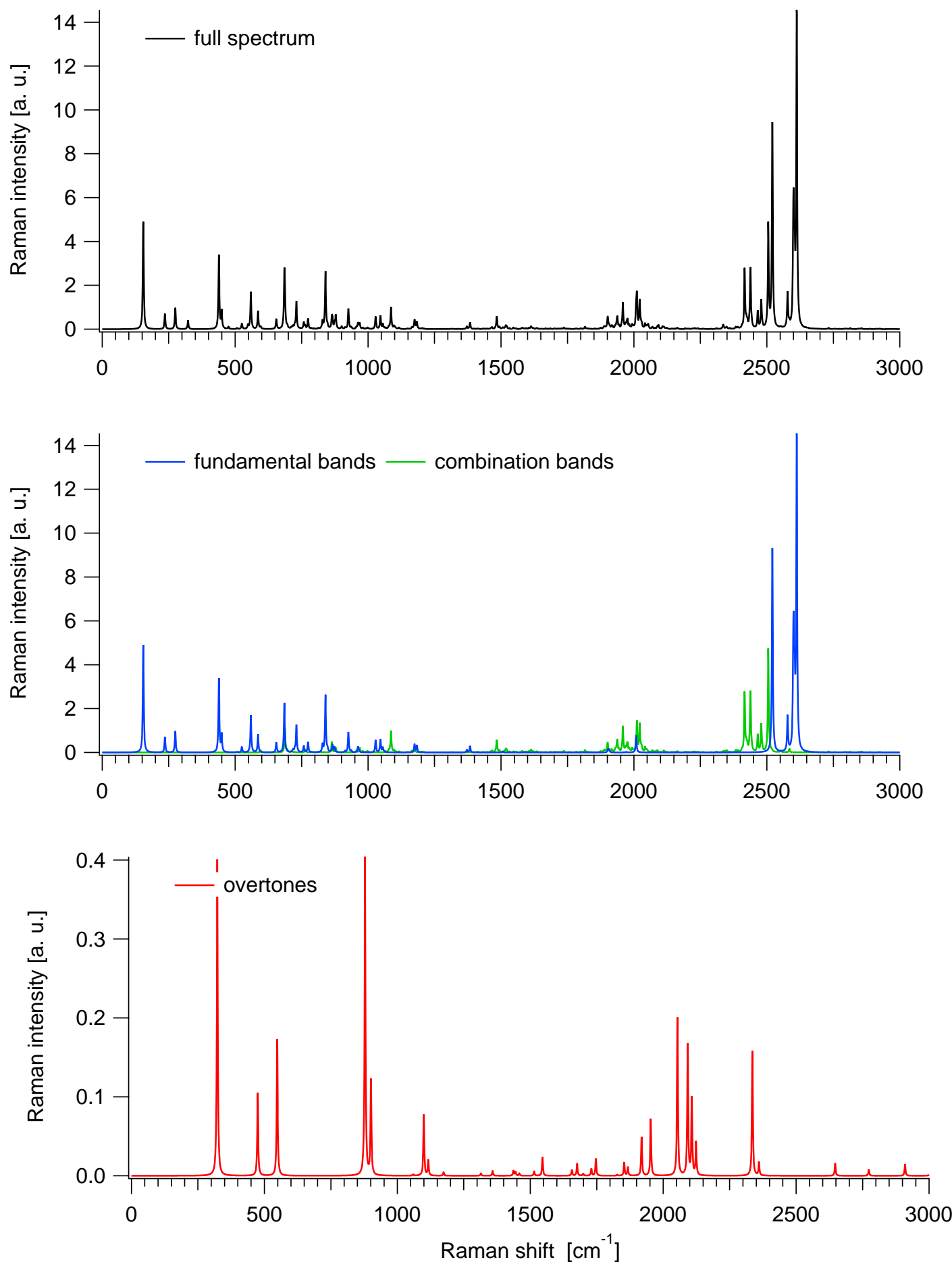
Table S.38.4. $\mathrm{B}_{6} \mathrm{H}_{12}$ : Ideal-gas thermodynamic functions at 1 bar (anharmonic B3LYPD2/cc-pVTZ results)

\begin{tabular}{cccc}
\hline$T$ & $C_{P}$ & $S$ & $H(T)-H(0 K)$ \\
{$[\mathrm{K}]$} & {$\left[\mathrm{J} \mathrm{mol}^{-1} \mathrm{~K}^{-1}\right]$} & {$\left[\mathrm{J} \mathrm{mol}^{-1} \mathrm{~K}^{-1}\right]$} & {$\left[\mathrm{kJ} \mathrm{mol}^{-1}\right]$} \\
\hline 100 & 47.016 & 242.982 & 3.742 \\
200 & 87.044 & 286.379 & 10.230 \\
300 & 140.814 & 331.805 & 21.616 \\
400 & 189.038 & 379.143 & 38.187 \\
500 & 227.935 & 425.666 & 59.108 \\
600 & 259.178 & 470.086 & 83.519 \\
700 & 284.612 & 512.014 & 110.751 \\
800 & 305.529 & 551.428 & 140.291 \\
900 & 322.837 & 588.445 & 171.736 \\
1000 & 337.224 & 623.227 & 204.761 \\
1100 & 349.232 & 655.948 & 239.102 \\
1200 & 359.302 & 686.780 & 274.543 \\
1300 & 367.788 & 715.884 & 310.909 \\
1400 & 374.978 & 743.410 & 348.058 \\
1500 & 381.103 & 769.496 & 385.870 \\
1600 & 386.350 & 794.263 & 424.249 \\
1700 & 390.870 & 817.825 & 463.115 \\
1800 & 394.784 & 840.279 & 502.403 \\
1900 & 398.190 & 861.718 & 542.055 \\
2000 & 401.170 & 882.220 & 582.027 \\
\hline & & & $S:$ \\
\hline
\end{tabular}

$T$ : Temperature. $C_{P}$ : Heat capacity at constant pressure. $S$ : Entropy. $H$ : Enthalpy. 
Figure S.38.4. $\mathrm{B}_{6} \mathrm{H}_{12}$ : Ideal-gas thermodynamic functions at 1 bar (anharmonic B3LYPD2/cc-pVTZ results)
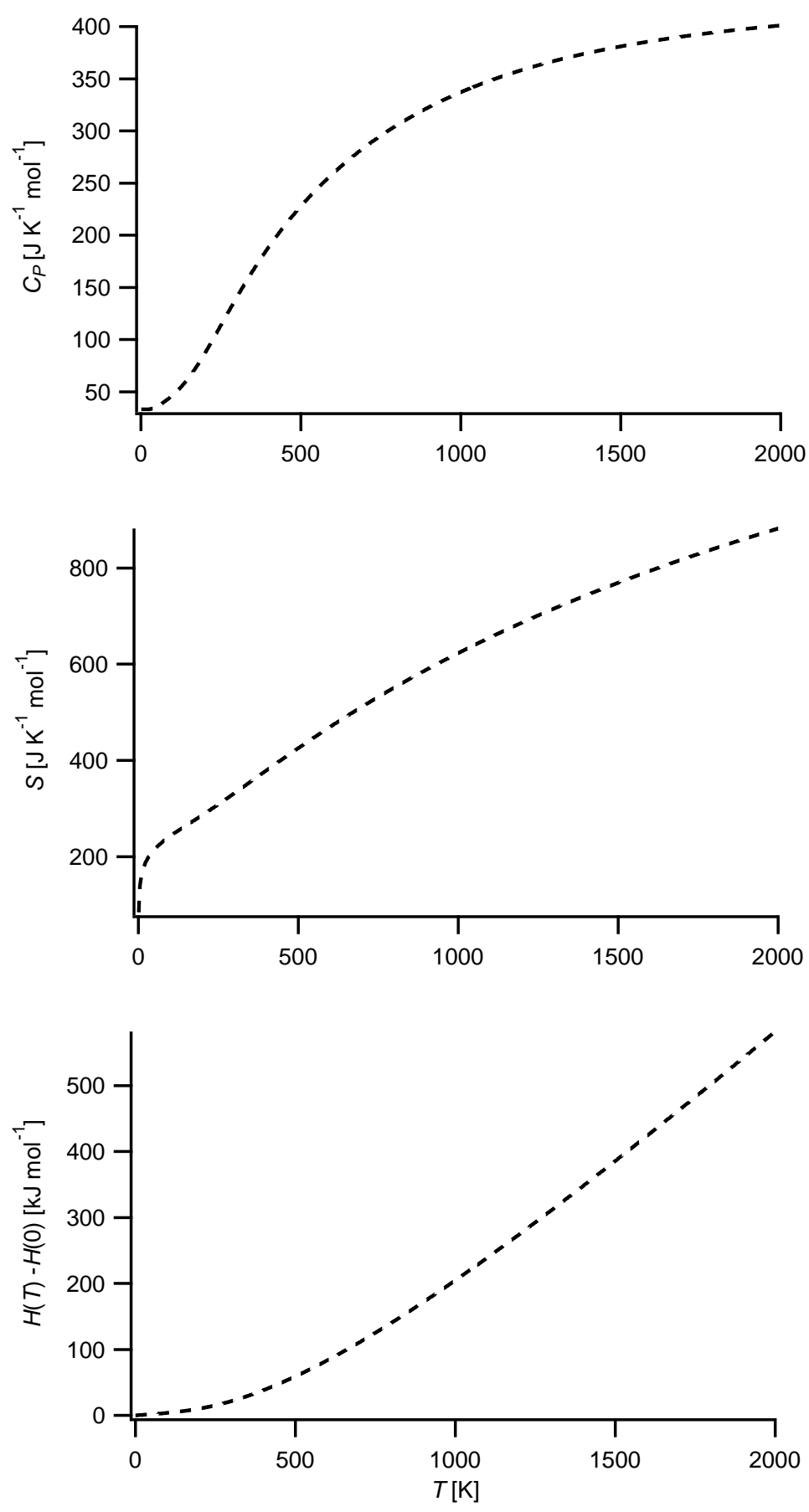

$T$ : Temperature. $C_{P}$ : Heat capacity at constant pressure. $S$ : Entropy. $H$ : Enthalpy. 
Table S.38.5. $\mathrm{B}_{6} \mathrm{H}_{12}$ : Fits (red solid lines) of the computed thermodynamic functions (blue dashed lines) with NASA type functions (Equations (1) - (3)) in the $200-900 \mathrm{~K}$ temperature range (anharmonic B3LYP-D2/cc-pVTZ results). In each case, the difference curve between the thermodynamic and NASA functions is plotted in the upper graph

Fit parameters

\begin{tabular}{llllll}
\hline $\mathrm{a}_{1}$ & $-4.45043454 \mathrm{e}+00$ & $\mathrm{a}_{2}\left[\mathrm{~K}^{-1}\right]$ & $7.55806736 \mathrm{e}-02$ & $\mathrm{a}_{3}\left[\mathrm{~K}^{-2}\right]$ & $7.79783604 \mathrm{e}-06$ \\
$\mathrm{a}_{4}\left[\mathrm{~K}^{-3}\right]$ & $-8.84548969 \mathrm{e}-08$ & $\mathrm{a}_{5}\left[\mathrm{~K}^{-4}\right]$ & $5.11787646 \mathrm{e}-11$ & $\mathrm{a}_{6}[\mathrm{~K}]$ & $6.20139227 \mathrm{e}+02$ \\
$\mathrm{a}_{7}$ & $4.29664666 \mathrm{e}+01$ & & & &
\end{tabular}
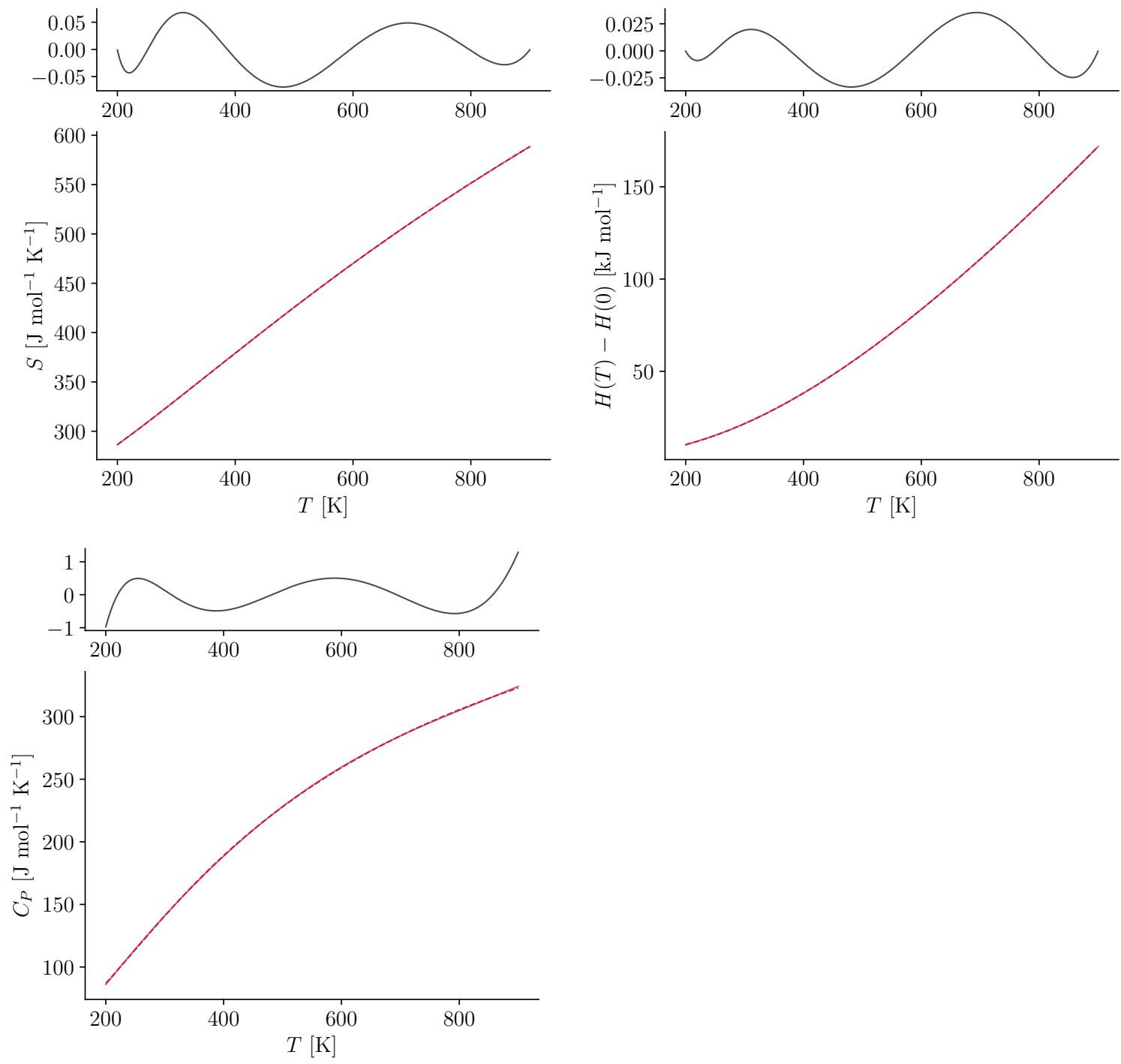


\section{$39 \mathrm{~B}_{8} \mathrm{H}_{8}{ }^{2-}$}

Figure S.39.1. Structure of $\mathrm{B}_{8} \mathrm{H}_{8}{ }^{2-}$

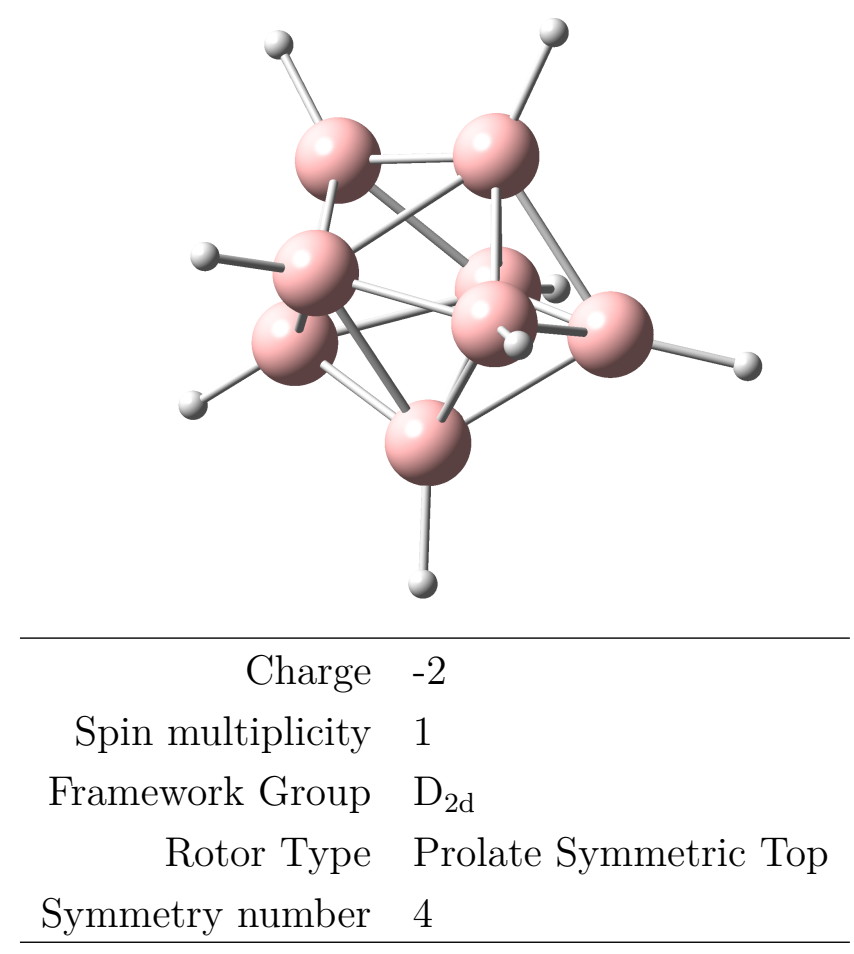

Table S.39.1. $\mathrm{B}_{8} \mathrm{H}_{8}{ }^{2-}$ : Selected anharmonic results obtained at the B3LYP-D2/cc-pVTZ level

\begin{tabular}{rll}
\hline $\mathrm{ZPE}_{\mathrm{v}}$ & 22391 & $\mathrm{~cm}^{-1}$ \\
$\mathrm{~A}$ & 0.12628 & $\mathrm{~cm}^{-1}$ \\
$\mathrm{~B}$ & 0.09822 & $\mathrm{~cm}^{-1}$ \\
$\mathrm{C}$ & 0.09822 & $\mathrm{~cm}^{-1}$ \\
\hline$C_{P}(300 \mathrm{~K})$ & 148.660 & $\mathrm{~J} \mathrm{~mol}^{-1} \mathrm{~K}^{-1}$ \\
$S(300 \mathrm{~K})$ & 319.791 & $\mathrm{~J} \mathrm{~mol}^{-1} \mathrm{~K}^{-1}$ \\
$H(300 \mathrm{~K})-H(0 \mathrm{~K})$ & 21.531 & $\mathrm{~kJ} \mathrm{~mol}^{-1}$ \\
\hline
\end{tabular}

$\mathrm{ZPE}_{\mathrm{v}}$ : vibrational contribution to the zero-point energy. A, B, C: Rotational constants. $C_{P}$ : Heat capacity at constant pressure. $S$ : Entropy. $H$ : Enthalpy. Ideal-gas calculations (1 bar). 
Table S.39.2. $\mathrm{B}_{8} \mathrm{H}_{8}{ }^{2-}$ : Cartesian coordinates $(\AA)$ of the B3LYP-D2/cc-pVTZ optimized structure

\begin{tabular}{cccc} 
atom & $\mathrm{x}$ & $\mathrm{y}$ & $\mathrm{z}$ \\
\hline B1 & 0.0000 & 1.2672 & 0.3128 \\
B2 & 0.0000 & 0.8056 & -1.3365 \\
H3 & 0.0000 & 2.4383 & 0.6126 \\
H4 & 0.0000 & 1.5934 & -2.2495 \\
B5 & -1.2672 & 0.0000 & -0.3128 \\
B6 & -0.8056 & 0.0000 & 1.3365 \\
H7 & -2.4383 & 0.0000 & -0.6126 \\
H8 & -1.5934 & 0.0000 & 2.2495 \\
B9 & 1.2672 & -0.0000 & -0.3128 \\
B10 & 0.8056 & -0.0000 & 1.3365 \\
H11 & 2.4383 & -0.0000 & -0.6126 \\
H12 & 1.5934 & -0.0000 & 2.2495 \\
B13 & -0.0000 & -1.2672 & 0.3128 \\
B14 & -0.0000 & -0.8056 & -1.3365 \\
H15 & -0.0000 & -2.4383 & 0.6126 \\
H16 & -0.0000 & -1.5934 & -2.2495 \\
\hline
\end{tabular}


Table S.39.3. $\mathrm{B}_{8} \mathrm{H}_{8}{ }^{2-}$ : Vibrational frequencies, infrared integrated intensities and Raman activities (B3LYP-D2/cc-pVTZ results)

\begin{tabular}{|c|c|c|c|c|c|c|c|}
\hline \multirow[b]{2}{*}{ mode } & \multirow[b]{2}{*}{ symm. } & \multicolumn{3}{|c|}{ Harmonic } & \multicolumn{3}{|c|}{ Anharmonic } \\
\hline & & $\begin{array}{c}\omega \\
{\left[\mathrm{cm}^{-1}\right]}\end{array}$ & $\begin{array}{c}\mathrm{IR} \\
{\left[\mathrm{km} \mathrm{mol}^{-1}\right]}\end{array}$ & $\begin{array}{c}\text { Raman } \\
{\left[\AA^{6}\right]}\end{array}$ & $\begin{array}{c}\omega \\
{\left[\mathrm{cm}^{-1}\right]}\end{array}$ & $\begin{array}{c}\mathrm{IR} \\
{\left[\mathrm{km} \mathrm{mol}^{-1}\right]}\end{array}$ & $\begin{array}{c}\text { Raman } \\
{\left[\AA^{6}\right]}\end{array}$ \\
\hline 1 & $\mathrm{E}$ & 324 & 1.804 & 0.051 & 297 & 1.663 & 0.059 \\
\hline 2 & $\mathrm{E}$ & 324 & 1.804 & 0.051 & 297 & 1.663 & 0.059 \\
\hline 3 & $\mathrm{~B}_{1}$ & 368 & 0.000 & 0.291 & 341 & 0.000 & 0.355 \\
\hline 4 & $\mathrm{~A}_{1}$ & 432 & 0.000 & 0.307 & 419 & 0.000 & 0.241 \\
\hline 5 & $\mathrm{~B}_{1}$ & 462 & 0.000 & 0.144 & 448 & 0.000 & 0.152 \\
\hline 6 & $\mathrm{~A}_{2}$ & 577 & 0.000 & 0.000 & 555 & 0.000 & 0.000 \\
\hline 7 & $\mathrm{~B}_{2}$ & 591 & 7.549 & 0.044 & 579 & 5.981 & 0.043 \\
\hline 8 & $\mathrm{E}$ & 613 & 2.315 & 0.165 & 597 & 2.864 & 0.130 \\
\hline 9 & $\mathrm{E}$ & 613 & 2.315 & 0.165 & 597 & 2.863 & 0.129 \\
\hline 10 & $\mathrm{~A}_{1}$ & 627 & 0.000 & 0.066 & 605 & 0.000 & 0.071 \\
\hline 11 & $\mathrm{E}$ & 652 & 1.928 & 0.104 & 650 & 0.974 & 0.085 \\
\hline 12 & $\mathrm{E}$ & 652 & 1.928 & 0.104 & 650 & 0.973 & 0.085 \\
\hline 13 & $\mathrm{E}$ & 755 & 0.056 & 0.011 & 737 & 0.211 & 0.016 \\
\hline 14 & $\mathrm{E}$ & 755 & 0.056 & 0.011 & 737 & 0.211 & 0.016 \\
\hline 15 & $\mathrm{~B}_{2}$ & 771 & 0.163 & 0.001 & 747 & 0.042 & 0.000 \\
\hline 16 & $\mathrm{~A}_{1}$ & 772 & 0.000 & 0.112 & 752 & 0.000 & 0.181 \\
\hline 17 & $\mathrm{~B}_{1}$ & 782 & 0.000 & 0.072 & 766 & 0.000 & 0.112 \\
\hline 18 & $\mathrm{~B}_{2}$ & 817 & 0.512 & 0.082 & 799 & 1.614 & 0.107 \\
\hline 19 & $\mathrm{~A}_{1}$ & 838 & 0.000 & 2.702 & 820 & 0.000 & 1.936 \\
\hline 20 & $\mathrm{~A}_{2}$ & 848 & 0.000 & 0.000 & 828 & 0.000 & 0.000 \\
\hline 21 & $\mathrm{E}$ & 868 & 9.035 & 0.000 & 848 & 9.599 & 0.000 \\
\hline 22 & $\mathrm{E}$ & 868 & 9.035 & 0.000 & 848 & 9.601 & 0.000 \\
\hline 23 & $\mathrm{~A}_{2}$ & 875 & 0.000 & 0.000 & 858 & 0.000 & 0.000 \\
\hline
\end{tabular}

To be continued 
Table S.39.3 - Continued

\begin{tabular}{|c|c|c|c|c|c|c|c|}
\hline \multirow[b]{2}{*}{ mode } & \multirow[b]{2}{*}{ symm. } & \multicolumn{3}{|c|}{ Harmonic } & \multicolumn{3}{|c|}{ Anharmonic } \\
\hline & & $\begin{array}{c}\omega \\
{\left[\mathrm{cm}^{-1}\right]}\end{array}$ & $\begin{array}{c}\text { IR } \\
{\left[\mathrm{km} \mathrm{mol}^{-1}\right]}\end{array}$ & $\begin{array}{c}\text { Raman } \\
{\left[\AA^{6}\right]}\end{array}$ & $\begin{array}{c}\omega \\
{\left[\mathrm{cm}^{-1}\right]} \\
\end{array}$ & $\begin{array}{c}\mathrm{IR} \\
{\left[\mathrm{km} \mathrm{mol}^{-1}\right]}\end{array}$ & $\begin{array}{c}\text { Raman } \\
{\left[\AA^{6}\right]}\end{array}$ \\
\hline 24 & $\mathrm{~B}_{1}$ & 876 & 0.000 & 0.004 & 862 & 0.000 & 0.005 \\
\hline 25 & $\mathrm{E}$ & 884 & 0.009 & 0.006 & 856 & 0.441 & 0.011 \\
\hline 26 & $\mathrm{E}$ & 884 & 0.009 & 0.006 & 856 & 0.433 & 0.011 \\
\hline 27 & $\mathrm{~B}_{2}$ & 890 & 4.047 & 0.000 & 861 & 3.710 & 0.001 \\
\hline 28 & $\mathrm{E}$ & 943 & 3.228 & 0.003 & 917 & 3.192 & 0.005 \\
\hline 29 & $\mathrm{E}$ & 943 & 3.228 & 0.003 & 917 & 3.191 & 0.005 \\
\hline 30 & $\mathrm{~A}_{1}$ & 947 & 0.000 & 0.066 & 921 & 0.000 & 0.065 \\
\hline 31 & $\mathrm{E}$ & 996 & 3.782 & 0.000 & 975 & 3.282 & 0.000 \\
\hline 32 & $\mathrm{E}$ & 996 & 3.782 & 0.000 & 975 & 3.288 & 0.000 \\
\hline 33 & $\mathrm{~A}_{1}$ & 1054 & 0.000 & 0.320 & 1013 & 0.000 & 0.193 \\
\hline 34 & $\mathrm{~B}_{2}$ & 1138 & 28.192 & 0.068 & 1103 & 25.493 & 0.067 \\
\hline 35 & $\mathrm{~B}_{2}$ & 2431 & 56.971 & 2.609 & 2303 & 68.223 & 2.763 \\
\hline 36 & $\mathrm{E}$ & 2441 & 484.632 & 0.793 & 2313 & 633.068 & 0.663 \\
\hline 37 & $\mathrm{E}$ & 2441 & 484.632 & 0.793 & 2313 & 629.315 & 0.668 \\
\hline 38 & $\mathrm{~A}_{1}$ & 2446 & 0.000 & 1.415 & 2319 & 0.000 & 1.697 \\
\hline 39 & $\mathrm{E}$ & 2469 & 657.945 & 0.874 & 2348 & 541.056 & 1.123 \\
\hline 40 & $\mathrm{E}$ & 2469 & 657.945 & 0.874 & 2348 & 537.866 & 1.129 \\
\hline 41 & $\mathrm{~B}_{2}$ & 2483 & 972.575 & 0.238 & 2364 & 21.910 & 0.270 \\
\hline 42 & $\mathrm{~A}_{1}$ & 2507 & 0.000 & 3.131 & 2387 & 0.000 & 3.412 \\
\hline
\end{tabular}


Figure S.39.2. Anharmonic IR spectrum of $\mathrm{B}_{8} \mathrm{H}_{8}{ }^{2-}$ obtained by convoluting the calculated intensities with Lorentzians having a FWHM of $4 \mathrm{~cm}^{-1}$ (B3LYP-D2/cc-pVTZ results): (top) full spectrum, (middle) contributions from fundamentals and combination bands, (bottom) contributions from overtones
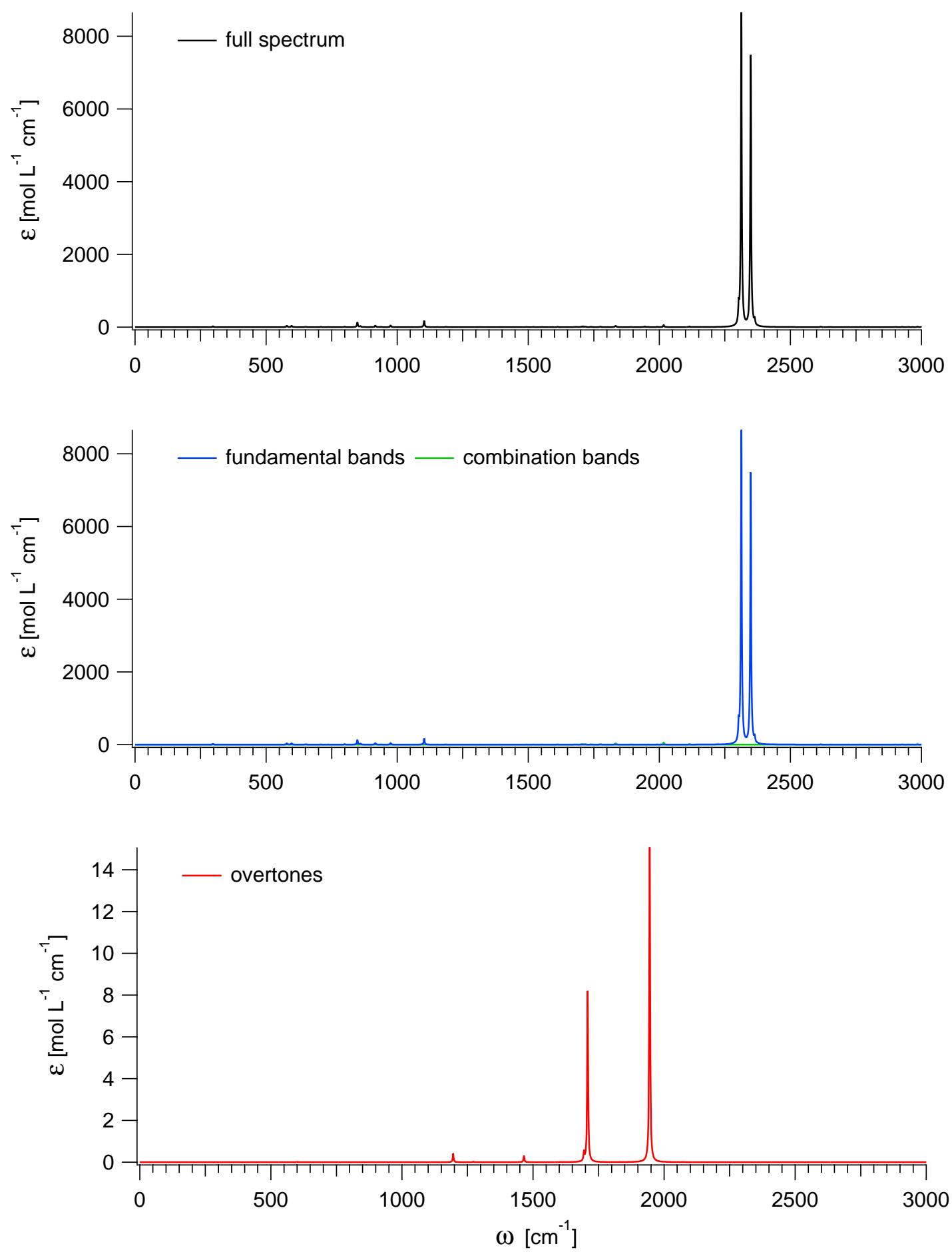
Figure S.39.3. Anharmonic Raman spectrum of $\mathrm{B}_{8} \mathrm{H}_{8}{ }^{2-}$ obtained by convoluting the calculated activities with Lorentzians having a FWHM of $4 \mathrm{~cm}^{-1}$ (B3LYP-D2/cc-pVTZ results): (top) full spectrum, (middle) contributions from fundamentals and combination bands, (bottom) contributions from overtones
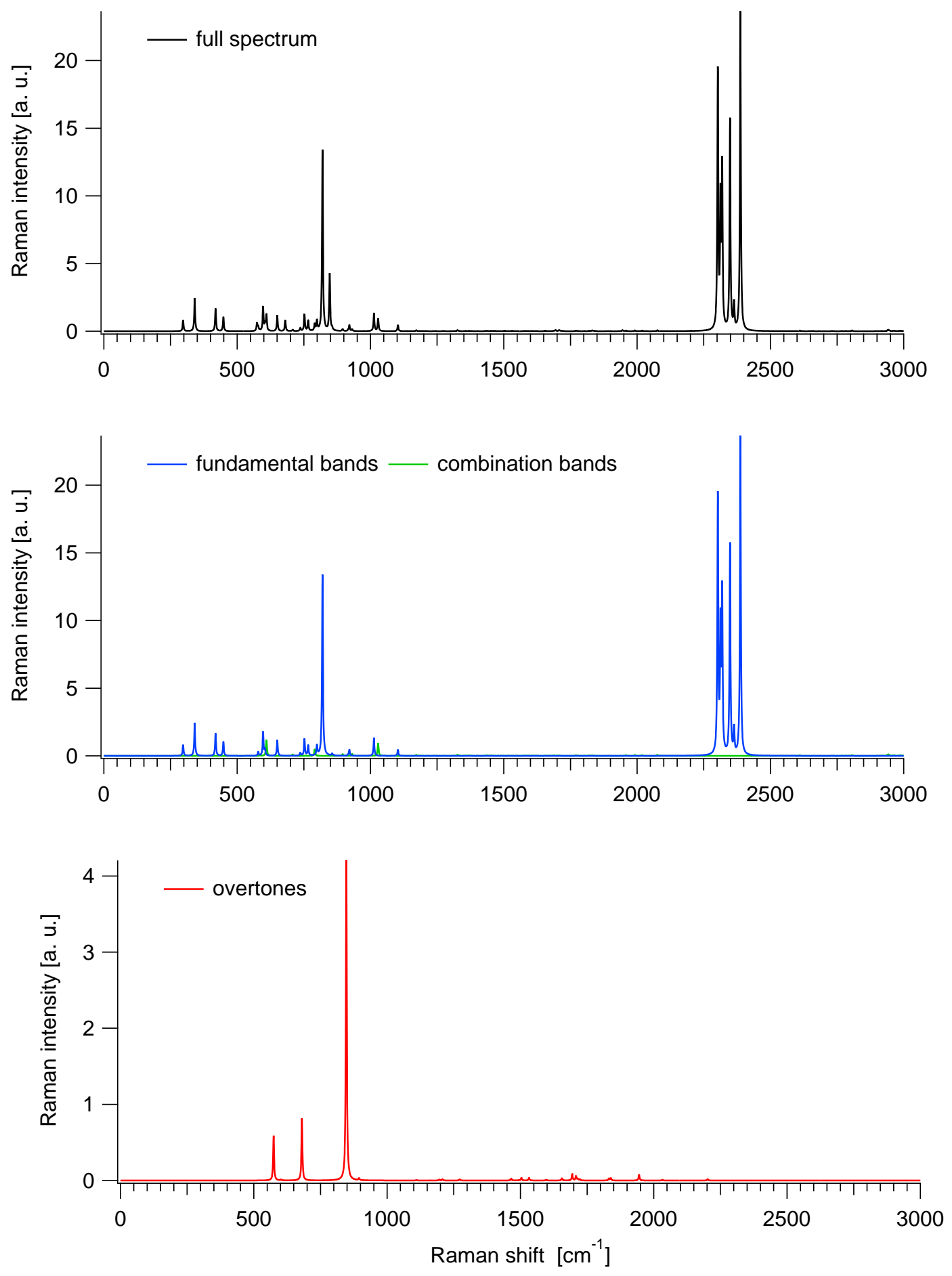
Table S.39.4. $\mathrm{B}_{8} \mathrm{H}_{8}{ }^{2-}$ : Ideal-gas thermodynamic functions at 1 bar (anharmonic B3LYPD2/cc-pVTZ results)

\begin{tabular}{cccc}
\hline$T$ & $C_{P}$ & $S$ & $H(T)-H(0 K)$ \\
{$[\mathrm{K}]$} & {$\left[\mathrm{J} \mathrm{mol}^{-1} \mathrm{~K}^{-1}\right]$} & {$\left[\mathrm{J} \mathrm{mol}^{-1} \mathrm{~K}^{-1}\right]$} & {$\left[\mathrm{kJ} \mathrm{mol}^{-1}\right]$} \\
\hline 100 & 41.351 & 231.549 & 3.488 \\
200 & 87.891 & 272.499 & 9.661 \\
300 & 148.660 & 319.791 & 21.531 \\
400 & 197.303 & 369.586 & 38.957 \\
500 & 232.443 & 417.597 & 60.538 \\
600 & 258.436 & 462.384 & 85.142 \\
700 & 278.443 & 503.788 & 112.027 \\
800 & 294.286 & 542.042 & 140.693 \\
900 & 307.051 & 577.467 & 170.782 \\
1000 & 317.455 & 610.375 & 202.025 \\
1100 & 326.008 & 641.046 & 234.212 \\
1200 & 333.096 & 669.726 & 267.178 \\
1300 & 339.012 & 696.628 & 300.792 \\
1400 & 343.984 & 721.939 & 334.949 \\
1500 & 348.192 & 745.819 & 369.563 \\
1600 & 351.778 & 768.408 & 404.566 \\
1700 & 354.851 & 789.829 & 439.902 \\
1800 & 357.502 & 810.189 & 475.522 \\
1900 & 359.801 & 829.581 & 511.390 \\
2000 & 361.807 & 848.088 & 547.473 \\
\hline
\end{tabular}

$T$ : Temperature. $C_{P}$ : Heat capacity at constant pressure. $S$ : Entropy. $H$ : Enthalpy. 
Figure S.39.4. $\mathrm{B}_{8} \mathrm{H}_{8}{ }^{2-}$ : Ideal-gas thermodynamic functions at 1 bar (anharmonic B3LYPD2/cc-pVTZ results)
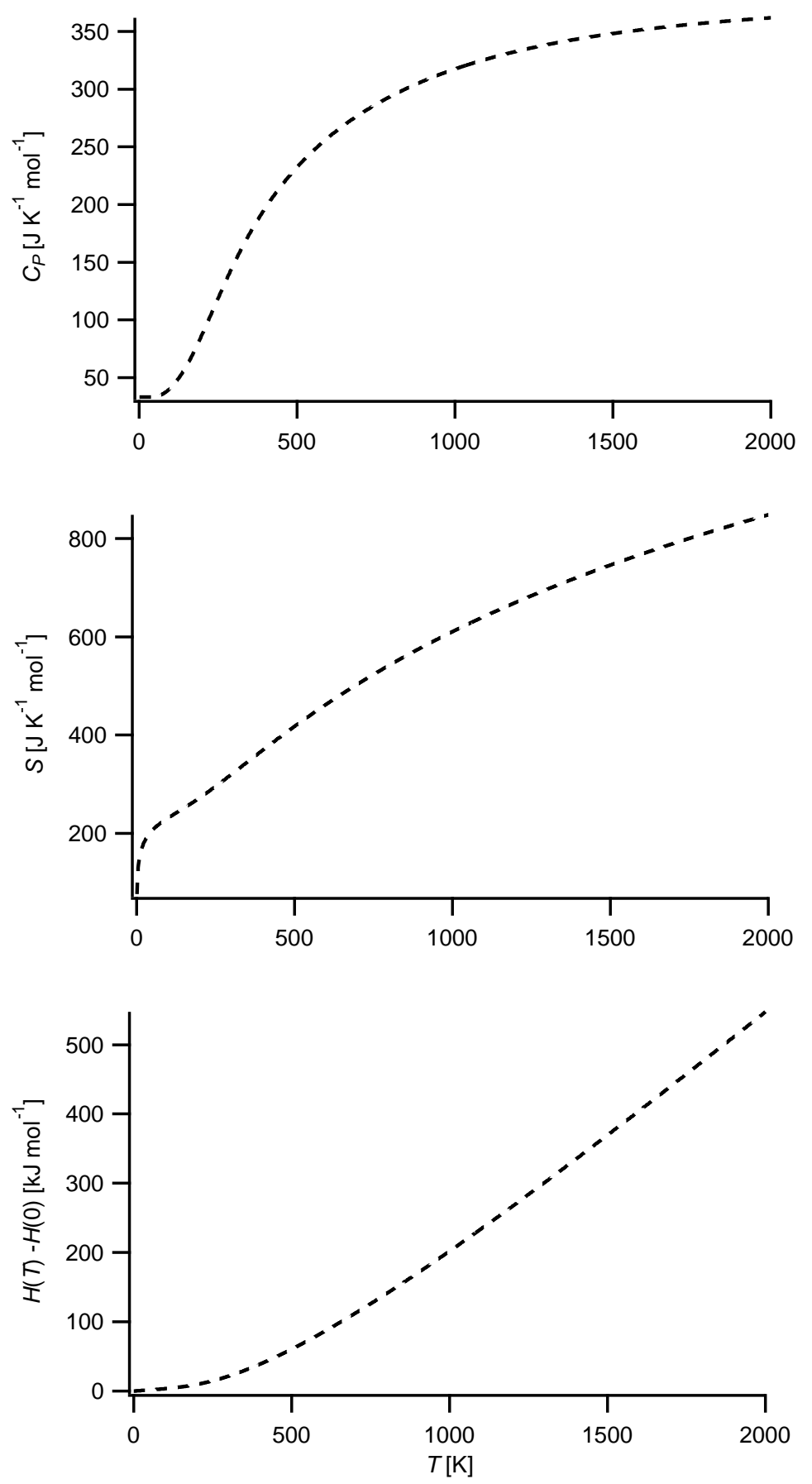

$T$ : Temperature. $C_{P}$ : Heat capacity at constant pressure. $S$ : Entropy. $H$ : Enthalpy. 
Table S.39.5. $\mathrm{B}_{8} \mathrm{H}_{8}{ }^{2-}$ : Fits (red solid lines) of the computed thermodynamic functions (blue dashed lines) with NASA type functions (Equations (1) - (3)) in the $200-900 \mathrm{~K}$ temperature range (anharmonic B3LYP-D2/cc-pVTZ results). In each case, the difference curve between the thermodynamic and NASA functions is plotted in the upper graph

Fit parameters

\begin{tabular}{llllll}
\hline $\mathrm{a}_{1}$ & $-1.05717585 \mathrm{e}+01$ & $\mathrm{a}_{2}\left[\mathrm{~K}^{-1}\right]$ & $1.27005298 \mathrm{e}-01$ & $\mathrm{a}_{3}\left[\mathrm{~K}^{-2}\right]$ & $-1.16140371 \mathrm{e}-04$ \\
$\mathrm{a}_{4}\left[\mathrm{~K}^{-3}\right]$ & $2.65367043 \mathrm{e}-08$ & $\mathrm{a}_{5}\left[\mathrm{~K}^{-4}\right]$ & $1.23014344 \mathrm{e}-11$ & $\mathrm{a}_{6}[\mathrm{~K}]$ & $1.03439458 \mathrm{e}+03$ \\
$\mathrm{a}_{7}$ & $6.56323785 \mathrm{e}+01$ & & & &
\end{tabular}
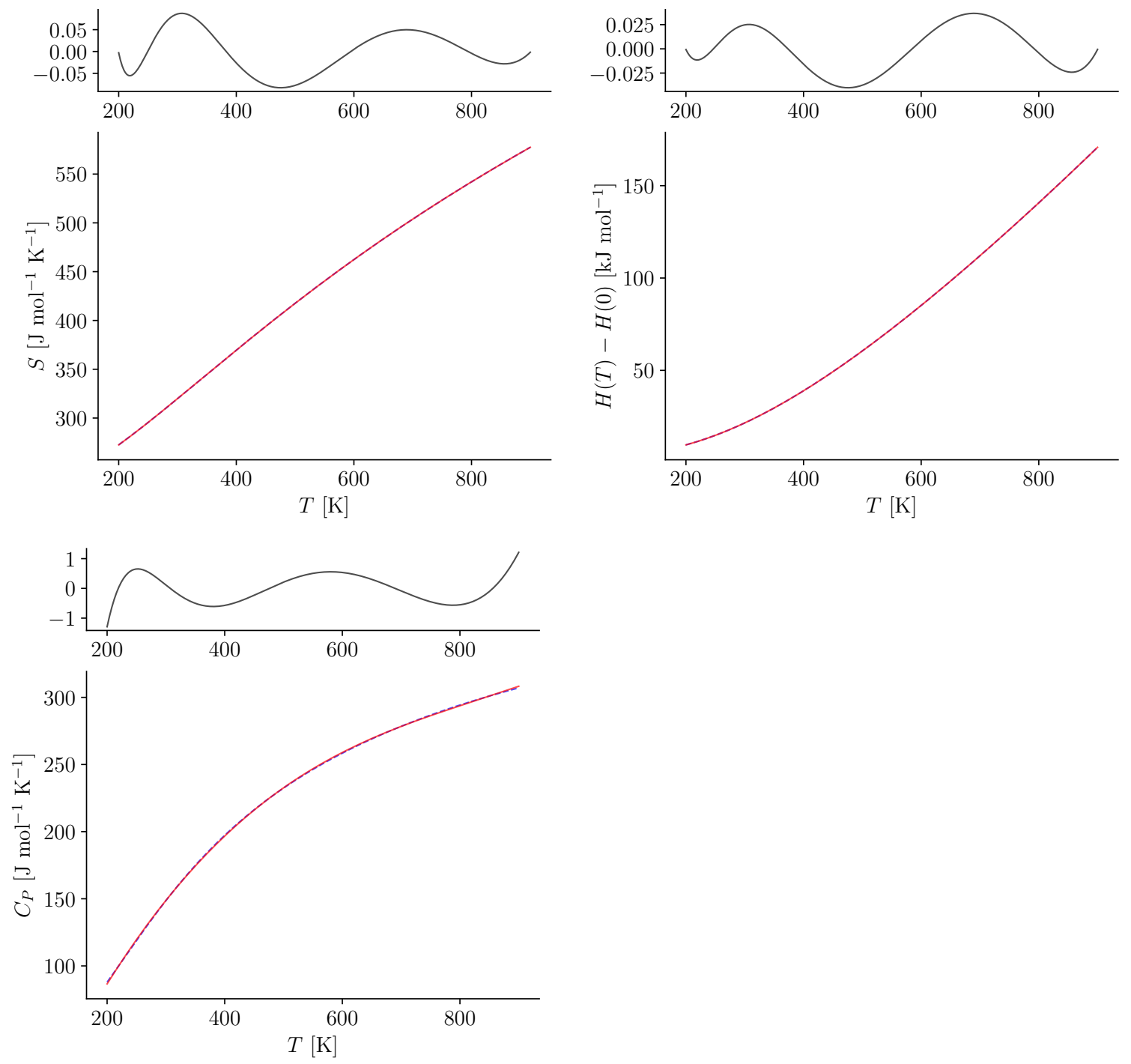


\section{$40 \quad \mathbf{B}_{8} \mathbf{H}_{14}$}

Figure S.40.1. Structure of $\mathrm{B}_{8} \mathrm{H}_{14}$

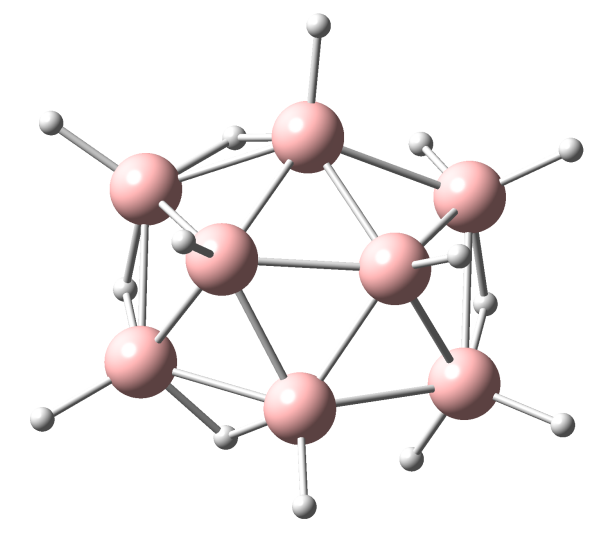

\begin{aligned} & \hline Charge 0 \\ & Spin multiplicity 1 \\ & Framework Group $\mathrm{C}_{1} \\ &$ Rotor Type Asymmetric Top \\ & Symmetry number 1 \\ & \hline\end{aligned}

Table S.40.1. $\mathrm{B}_{8} \mathrm{H}_{14}$ : Selected anharmonic results obtained at the B3LYP-D2/cc-pVTZ level

\begin{tabular}{rll}
\hline $\mathrm{ZPE}_{\mathrm{v}}$ & 36443 & $\mathrm{~cm}^{-1}$ \\
$\mathrm{~A}$ & 0.10803 & $\mathrm{~cm}^{-1}$ \\
$\mathrm{~B}$ & 0.07455 & $\mathrm{~cm}^{-1}$ \\
$\mathrm{C}$ & 0.05716 & $\mathrm{~cm}^{-1}$ \\
\hline$C_{P}(300 \mathrm{~K})$ & 178.830 & $\mathrm{~J} \mathrm{~mol}^{-1} \mathrm{~K}-1$ \\
$S(300 \mathrm{~K})$ & 359.922 & $\mathrm{~J} \mathrm{~mol}^{-1} \mathrm{~K}-1$ \\
$H(300 \mathrm{~K})-H(0 \mathrm{~K})$ & 25.656 & $\mathrm{~kJ} \mathrm{~mol}^{-1}$ \\
\hline
\end{tabular}

$\mathrm{ZPE}_{\mathrm{v}}$ : vibrational contribution to the zero-point energy. A, B, C: Rotational constants. $C_{P}$ : Heat capacity at constant pressure. $S$ : Entropy. $H$ : Enthalpy. Ideal-gas calculations (1 bar). 
Table S.40.2. $\mathrm{B}_{8} \mathrm{H}_{14}$ : Cartesian coordinates $(\AA)$ of the B3LYP-D2/cc-pVTZ optimized structure

\begin{tabular}{cccc} 
atom & $\mathrm{x}$ & $\mathrm{y}$ & $\mathrm{z}$ \\
\hline B1 & 1.0020 & 0.0000 & 0.8917 \\
B2 & -0.7866 & 0.0000 & 0.8272 \\
B3 & 0.0744 & -1.4004 & 0.2568 \\
B4 & 1.6313 & -0.8917 & -0.4582 \\
B5 & 1.6313 & 0.8917 & -0.4582 \\
B6 & 0.0744 & 1.4004 & 0.2568 \\
B7 & -1.6879 & 0.9630 & -0.3898 \\
B8 & -1.6879 & -0.9630 & -0.3898 \\
H9 & 1.5336 & 0.0000 & 1.9442 \\
H10 & -1.3104 & 0.0000 & 1.8864 \\
H11 & 0.0580 & -2.4818 & 0.7251 \\
H12 & 2.6230 & -1.5285 & -0.4765 \\
H13 & 2.6230 & 1.5285 & -0.4765 \\
H14 & 0.0580 & 2.4818 & 0.7251 \\
H15 & -2.6480 & 1.4191 & 0.1302 \\
H16 & -2.6480 & -1.4191 & 0.1302 \\
H17 & -1.2890 & -1.6254 & -1.3098 \\
H18 & -1.2890 & 1.6254 & -1.3098 \\
H19 & 1.6860 & 0.0000 & -1.4485 \\
H20 & -1.9902 & -0.0000 & -1.2389 \\
H21 & 0.6691 & -1.5455 & -0.9815 \\
H22 & 0.6691 & 1.5455 & -0.9815 \\
\hline & & & \\
\hline
\end{tabular}


Table S.40.3. $\mathrm{B}_{8} \mathrm{H}_{14}$ : Vibrational frequencies, infrared integrated intensities and Raman activities (B3LYP-D2/cc-pVTZ results)

\begin{tabular}{|c|c|c|c|c|c|c|c|}
\hline \multirow[b]{2}{*}{ mode } & \multirow[b]{2}{*}{ symm. } & \multicolumn{3}{|c|}{ Harmonic } & \multicolumn{3}{|c|}{ Anharmonic } \\
\hline & & $\begin{array}{c}\omega \\
{\left[\mathrm{cm}^{-1}\right]}\end{array}$ & $\begin{array}{c}\mathrm{IR} \\
{\left[\mathrm{km} \mathrm{mol}^{-1}\right]}\end{array}$ & $\begin{array}{c}\text { Raman } \\
{\left[\AA^{6}\right]}\end{array}$ & $\begin{array}{c}\omega \\
{\left[\mathrm{cm}^{-1}\right]}\end{array}$ & $\begin{array}{c}\mathrm{IR} \\
{\left[\mathrm{km} \mathrm{mol}^{-1}\right]}\end{array}$ & $\begin{array}{c}\text { Raman } \\
{\left[\AA^{6}\right]}\end{array}$ \\
\hline 1 & $\mathrm{~A}$ & 163 & 0.580 & 0.266 & 127 & 0.669 & 0.338 \\
\hline 2 & $\mathrm{~A}$ & 275 & 1.079 & 0.185 & 250 & 1.593 & 0.192 \\
\hline 3 & $\mathrm{~A}$ & 367 & 12.769 & 0.065 & 290 & 16.137 & 0.143 \\
\hline 4 & $\mathrm{~A}$ & 394 & 35.577 & 0.158 & 322 & 40.409 & 0.104 \\
\hline 5 & $\mathrm{~A}$ & 447 & 5.242 & 0.017 & 427 & 3.139 & 0.012 \\
\hline 6 & $\mathrm{~A}$ & 458 & 1.104 & 0.226 & 427 & 4.352 & 0.070 \\
\hline 7 & $\mathrm{~A}$ & 500 & 0.022 & 0.243 & 473 & 0.056 & 0.248 \\
\hline 8 & $\mathrm{~A}$ & 508 & 0.055 & 0.181 & 471 & 2.949 & 0.455 \\
\hline 9 & $\mathrm{~A}$ & 556 & 1.177 & 0.129 & 525 & 1.722 & 0.089 \\
\hline 10 & $\mathrm{~A}$ & 587 & 2.559 & 0.094 & 564 & 3.243 & 0.154 \\
\hline 11 & $\mathrm{~A}$ & 609 & 0.315 & 0.015 & 563 & 0.057 & 0.016 \\
\hline 12 & $\mathrm{~A}$ & 649 & 3.401 & 0.020 & 624 & 3.891 & 0.021 \\
\hline 13 & $\mathrm{~A}$ & 659 & 15.570 & 0.051 & 628 & 24.619 & 0.082 \\
\hline 14 & $\mathrm{~A}$ & 680 & 12.505 & 0.235 & 652 & 5.232 & 0.194 \\
\hline 15 & $\mathrm{~A}$ & 702 & 2.967 & 0.038 & 672 & 0.328 & 0.033 \\
\hline 16 & $\mathrm{~A}$ & 718 & 3.188 & 0.434 & 688 & 2.674 & 0.215 \\
\hline 17 & $\mathrm{~A}$ & 735 & 0.528 & 0.027 & 703 & 5.521 & 0.247 \\
\hline 18 & $\mathrm{~A}$ & 737 & 7.153 & 0.018 & 709 & 4.585 & 0.011 \\
\hline 19 & $\mathrm{~A}$ & 762 & 0.880 & 0.016 & 731 & 1.813 & 0.024 \\
\hline 20 & $\mathrm{~A}$ & 767 & 3.224 & 0.372 & 738 & 3.609 & 0.495 \\
\hline 21 & $\mathrm{~A}$ & 780 & 4.475 & 0.081 & 761 & 26.740 & 0.417 \\
\hline 22 & $\mathrm{~A}$ & 788 & 7.367 & 0.066 & 780 & 32.464 & 0.220 \\
\hline 23 & $\mathrm{~A}$ & 829 & 6.131 & 0.063 & 796 & 4.593 & 0.056 \\
\hline
\end{tabular}


Table S.40.3 - Continued

\begin{tabular}{|c|c|c|c|c|c|c|c|}
\hline \multirow[b]{2}{*}{ mode } & \multirow[b]{2}{*}{ symm. } & \multicolumn{3}{|c|}{ Harmonic } & \multicolumn{3}{|c|}{ Anharmonic } \\
\hline & & $\begin{array}{c}\omega \\
{\left[\mathrm{cm}^{-1}\right]} \\
\end{array}$ & $\begin{array}{c}\mathrm{IR} \\
{\left[\mathrm{km} \mathrm{mol}^{-1}\right]} \\
\end{array}$ & $\begin{array}{c}\text { Raman } \\
{\left[\AA^{6}\right]}\end{array}$ & $\begin{array}{c}\omega \\
{\left[\mathrm{cm}^{-1}\right]} \\
\end{array}$ & $\begin{array}{c}\mathrm{IR} \\
{\left[\mathrm{km} \mathrm{mol}^{-1}\right]} \\
\end{array}$ & $\begin{array}{c}\text { Raman } \\
{\left[\AA^{6}\right]}\end{array}$ \\
\hline 24 & $\mathrm{~A}$ & 854 & 0.942 & 0.023 & 826 & 1.982 & 0.024 \\
\hline 25 & $\mathrm{~A}$ & 862 & 0.682 & 0.003 & 821 & 0.463 & 0.002 \\
\hline 26 & $\mathrm{~A}$ & 885 & 6.453 & 0.002 & 832 & 3.740 & 0.009 \\
\hline 27 & $\mathrm{~A}$ & 891 & 9.805 & 0.107 & 853 & 6.571 & 0.062 \\
\hline 28 & $\mathrm{~A}$ & 897 & 0.150 & 0.020 & 867 & 0.294 & 0.012 \\
\hline 29 & $\mathrm{~A}$ & 920 & 14.035 & 0.261 & 892 & 11.683 & 0.270 \\
\hline 30 & $\mathrm{~A}$ & 946 & 15.666 & 0.011 & 895 & 19.575 & 0.023 \\
\hline 31 & $\mathrm{~A}$ & 977 & 3.166 & 0.022 & 940 & 1.775 & 0.037 \\
\hline 32 & $\mathrm{~A}$ & 980 & 0.477 & 0.017 & 947 & 1.978 & 0.013 \\
\hline 33 & $\mathrm{~A}$ & 989 & 5.290 & 0.009 & 949 & 3.746 & 0.008 \\
\hline 34 & $\mathrm{~A}$ & 1004 & 24.482 & 0.050 & 964 & 24.616 & 0.043 \\
\hline 35 & $\mathrm{~A}$ & 1020 & 5.981 & 0.012 & 976 & 1.060 & 0.007 \\
\hline 36 & $\mathrm{~A}$ & 1062 & 45.809 & 0.016 & 1023 & 27.398 & 0.010 \\
\hline 37 & $\mathrm{~A}$ & 1103 & 46.242 & 0.072 & 1038 & 19.356 & 0.090 \\
\hline 38 & $\mathrm{~A}$ & 1114 & 3.549 & 0.094 & 1032 & 2.579 & 0.088 \\
\hline 39 & $\mathrm{~A}$ & 1124 & 2.061 & 0.114 & 1032 & 3.635 & 0.070 \\
\hline 40 & $\mathrm{~A}$ & 1140 & 1.586 & 0.104 & 1069 & 0.900 & 0.050 \\
\hline 41 & $\mathrm{~A}$ & 1203 & 9.921 & 0.127 & 1148 & 6.192 & 0.070 \\
\hline 42 & $\mathrm{~A}$ & 1204 & 29.895 & 0.147 & 1147 & 8.609 & 0.077 \\
\hline 43 & $\mathrm{~A}$ & 1509 & 12.846 & 0.028 & 1281 & 0.062 & 0.007 \\
\hline 44 & $\mathrm{~A}$ & 1570 & 111.490 & 0.046 & 1361 & 1.697 & 0.005 \\
\hline 45 & $\mathrm{~A}$ & 1677 & 24.572 & 0.027 & 1493 & 2.246 & 0.004 \\
\hline 46 & $\mathrm{~A}$ & 1772 & 4.684 & 0.055 & 1537 & 0.908 & 0.031 \\
\hline 47 & $\mathrm{~A}$ & 1908 & 3.763 & 0.466 & 1801 & 1.515 & 0.009 \\
\hline
\end{tabular}

To be continued 
Table S.40.3 - Continued

\begin{tabular}{cccccccc}
\hline & & \multicolumn{3}{c}{ Harmonic } & \multicolumn{3}{c}{ Anharmonic } \\
mode & symm. & $\omega$ & IR & Raman & $\omega$ & IR & Raman \\
& & {$\left[\mathrm{cm}^{-1}\right]$} & {$\left[\mathrm{km} \mathrm{mol}^{-1}\right]$} & {$\left[\AA^{6}\right]$} & {$\left[\mathrm{cm}^{-1}\right]$} & {$\left[\mathrm{km} \mathrm{mol}^{-1}\right]$} & {$\left[\AA^{6}\right]$} \\
\hline 48 & A & 1991 & 19.643 & 0.329 & 1858 & 2.623 & 0.044 \\
49 & A & 2171 & 12.667 & 0.040 & 2062 & 4.193 & 0.018 \\
50 & A & 2181 & 18.661 & 0.210 & 2063 & 0.812 & 0.146 \\
51 & A & 2524 & 18.904 & 0.512 & 2414 & 6.971 & 0.355 \\
52 & A & 2530 & 25.481 & 1.199 & 2425 & 33.517 & 1.080 \\
53 & A & 2681 & 34.055 & 0.421 & 2569 & 8.634 & 0.150 \\
54 & A & 2686 & 52.849 & 0.598 & 2573 & 18.119 & 0.400 \\
55 & A & 2699 & 101.345 & 1.009 & 2595 & 121.142 & 1.137 \\
56 & A & 2722 & 43.064 & 0.646 & 2606 & 65.458 & 1.097 \\
57 & A & 2735 & 11.098 & 0.396 & 2613 & 5.472 & 0.438 \\
58 & A & 2737 & 44.141 & 0.610 & 2615 & 72.648 & 0.489 \\
59 & A & 2740 & 110.102 & 0.332 & 2625 & 87.055 & 0.195 \\
60 & A & 2746 & 55.931 & 2.513 & 2632 & 17.618 & 2.424 \\
\hline
\end{tabular}


Figure S.40.2. Anharmonic IR spectrum of $\mathrm{B}_{8} \mathrm{H}_{14}$ obtained by convoluting the calculated intensities with Lorentzians having a FWHM of $4 \mathrm{~cm}^{-1}$ (B3LYP-D2/cc-pVTZ results): (top) full spectrum, (middle) contributions from fundamentals and combination bands, (bottom) contributions from overtones
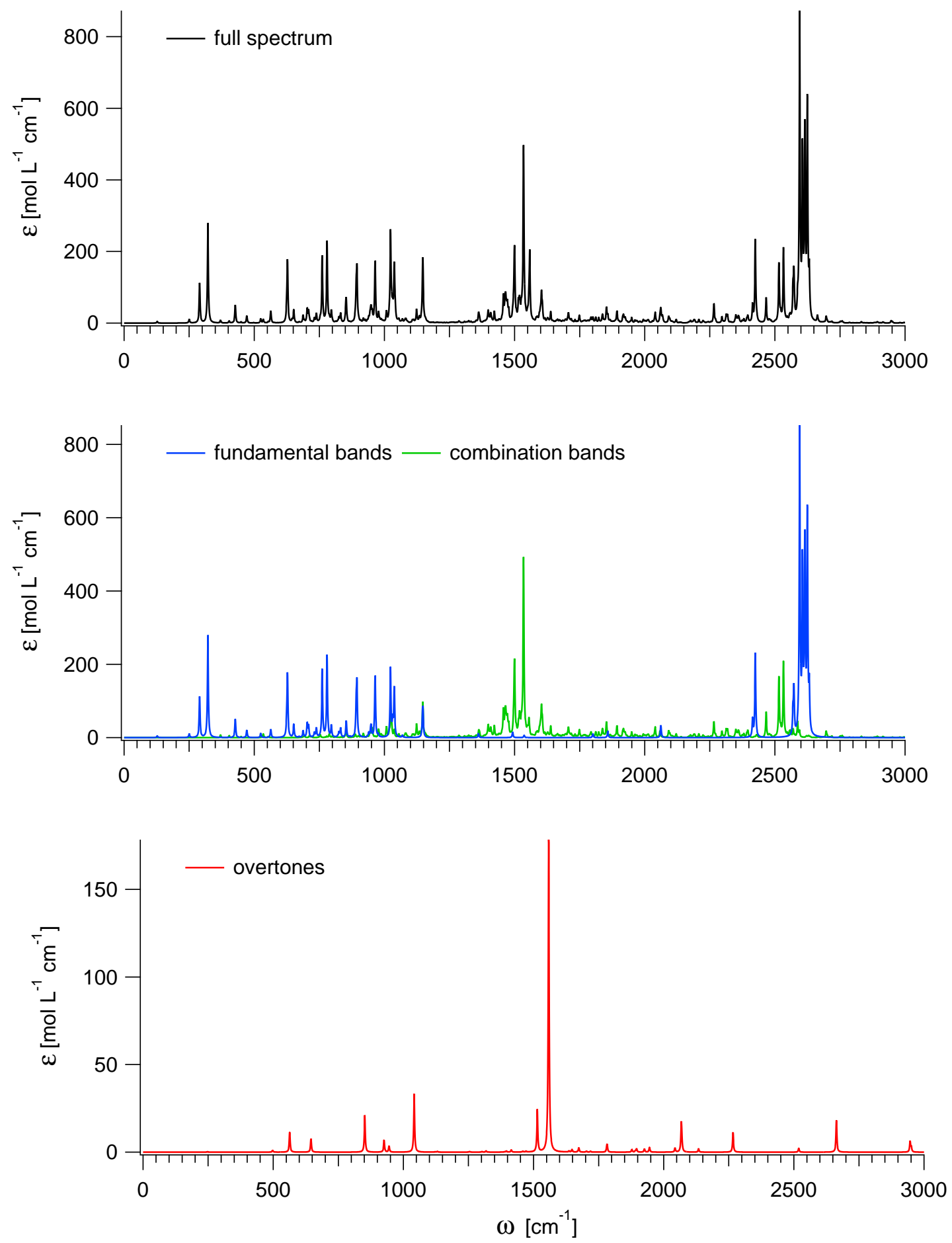
Figure S.40.3. Anharmonic Raman spectrum of $\mathrm{B}_{8} \mathrm{H}_{14}$ obtained by convoluting the calculated activities with Lorentzians having a FWHM of $4 \mathrm{~cm}^{-1}$ (B3LYP-D2/cc-pVTZ results): (top) full spectrum, (middle) contributions from fundamentals and combination bands, (bottom) contributions from overtones
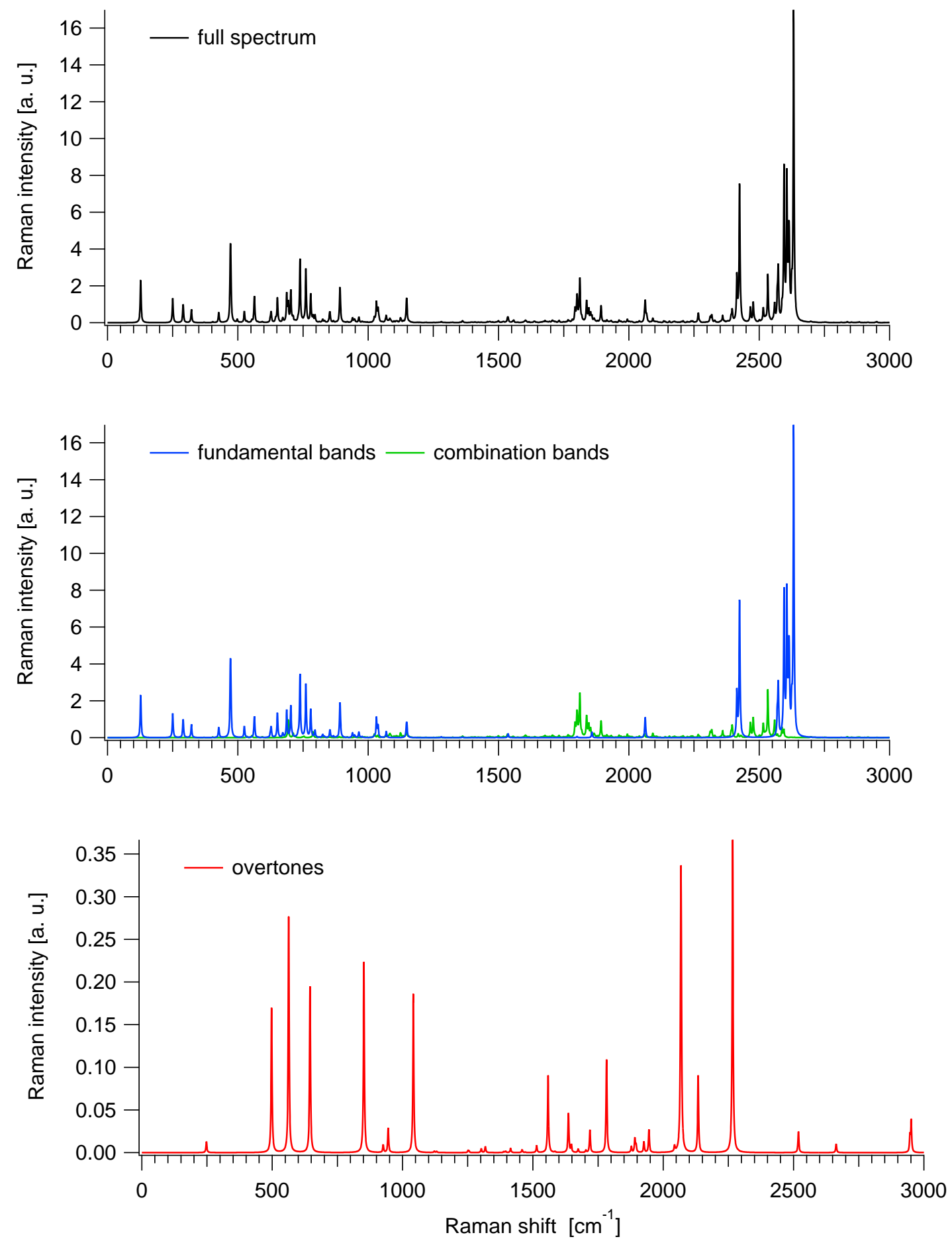
Table S.40.4. $\mathrm{B}_{8} \mathrm{H}_{14}$ : Ideal-gas thermodynamic functions at 1 bar (anharmonic B3LYP$\mathrm{D} 2 / \mathrm{cc}-\mathrm{pVTZ}$ results)

\begin{tabular}{cccc}
\hline$T$ & $C_{P}$ & $S$ & $H(T)-H(0 K)$ \\
{$[\mathrm{K}]$} & {$\left[\mathrm{J} \mathrm{mol}^{-1} \mathrm{~K}^{-1}\right]$} & {$\left[\mathrm{J} \mathrm{mol}^{-1} \mathrm{~K}^{-1}\right]$} & {$\left[\mathrm{kJ} \mathrm{mol}^{-1}\right]$} \\
\hline 100 & 50.099 & 253.024 & 3.840 \\
200 & 106.454 & 302.994 & 11.370 \\
300 & 178.830 & 359.922 & 25.656 \\
400 & 240.874 & 420.213 & 46.762 \\
500 & 289.223 & 479.384 & 73.369 \\
600 & 327.158 & 535.601 & 104.261 \\
700 & 357.607 & 588.402 & 138.552 \\
800 & 382.452 & 637.830 & 175.596 \\
900 & 402.927 & 684.097 & 214.898 \\
1000 & 419.909 & 727.456 & 256.065 \\
1100 & 434.070 & 768.161 & 298.785 \\
1200 & 445.940 & 806.454 & 342.803 \\
1300 & 455.942 & 842.555 & 387.911 \\
1400 & 464.416 & 876.662 & 433.941 \\
1500 & 471.636 & 908.956 & 480.753 \\
1600 & 477.822 & 939.597 & 528.233 \\
1700 & 483.152 & 968.729 & 576.289 \\
1800 & 487.767 & 996.479 & 624.840 \\
1900 & 491.786 & 1022.961 & 673.822 \\
2000 & 495.302 & 1048.278 & 723.180 \\
\hline & & & $S:$ \\
\hline
\end{tabular}

$T$ : Temperature. $C_{P}$ : Heat capacity at constant pressure. $S$ : Entropy. $H$ : Enthalpy. 
Figure S.40.4. $\mathrm{B}_{8} \mathrm{H}_{14}$ : Ideal-gas thermodynamic functions at 1 bar (anharmonic B3LYPD2/cc-pVTZ results)
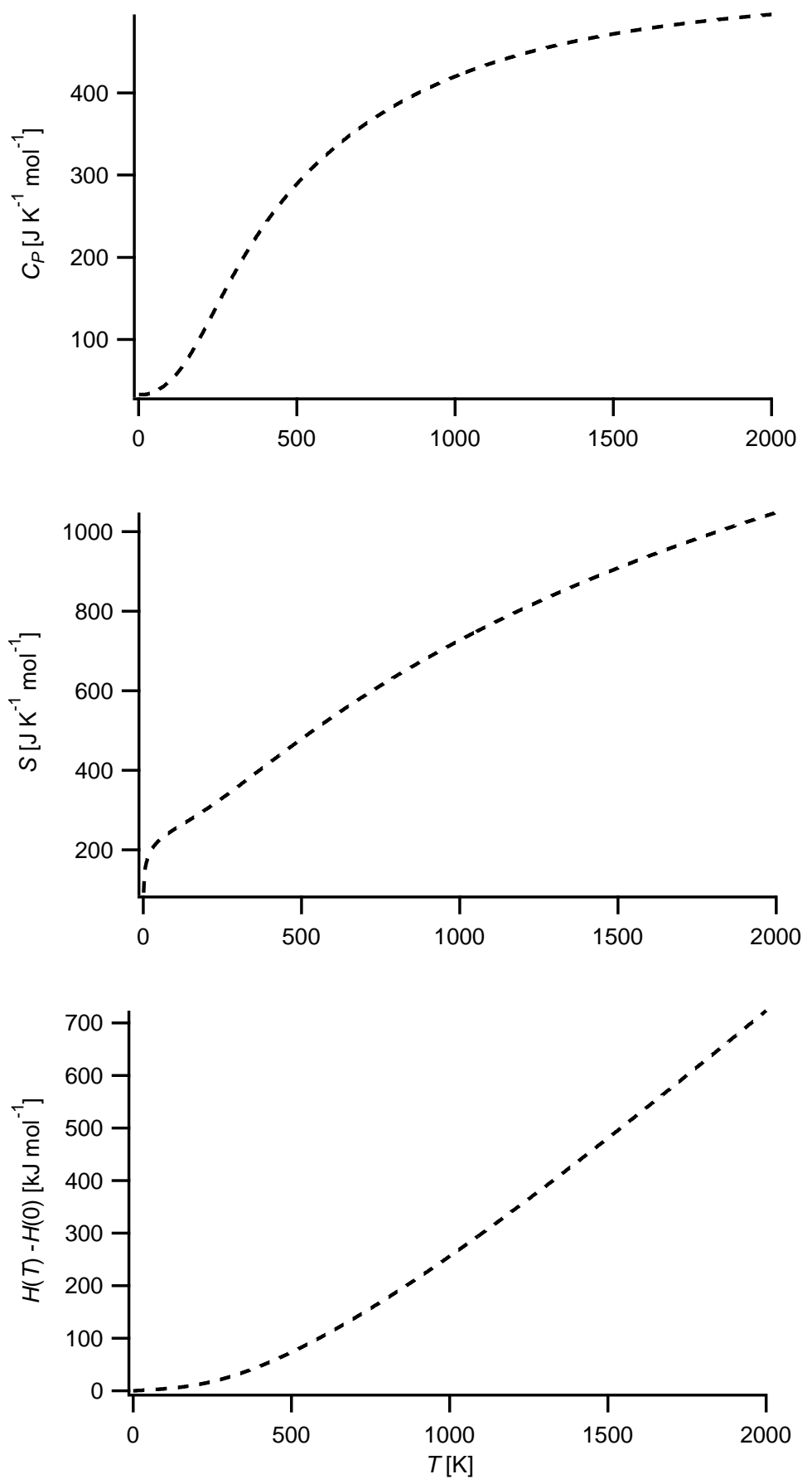

$T$ : Temperature. $C_{P}$ : Heat capacity at constant pressure. $S$ : Entropy. $H$ : Enthalpy. 
Table S.40.5. $\mathrm{B}_{8} \mathrm{H}_{14}$ : Fits (red solid lines) of the computed thermodynamic functions (blue dashed lines) with NASA type functions (Equations (1) - (3)) in the $200-900 \mathrm{~K}$ temperature range (anharmonic B3LYP-D2/cc-pVTZ results). In each case, the difference curve between the thermodynamic and NASA functions is plotted in the upper graph

Fit parameters

\begin{tabular}{llllll}
\hline $\mathrm{a}_{1}$ & $-9.25712045 \mathrm{e}+00$ & $\mathrm{a}_{2}\left[\mathrm{~K}^{-1}\right]$ & $1.21424164 \mathrm{e}-01$ & $\mathrm{a}_{3}\left[\mathrm{~K}^{-2}\right]$ & $-5.01284569 \mathrm{e}-05$ \\
$\mathrm{a}_{4}\left[\mathrm{~K}^{-3}\right]$ & $-5.55556321 \mathrm{e}-08$ & $\mathrm{a}_{5}\left[\mathrm{~K}^{-4}\right]$ & $4.53110512 \mathrm{e}-11$ & $\mathrm{a}_{6}[\mathrm{~K}]$ & $9.43397270 \mathrm{e}+02$
\end{tabular}

$\mathrm{a}_{7}$
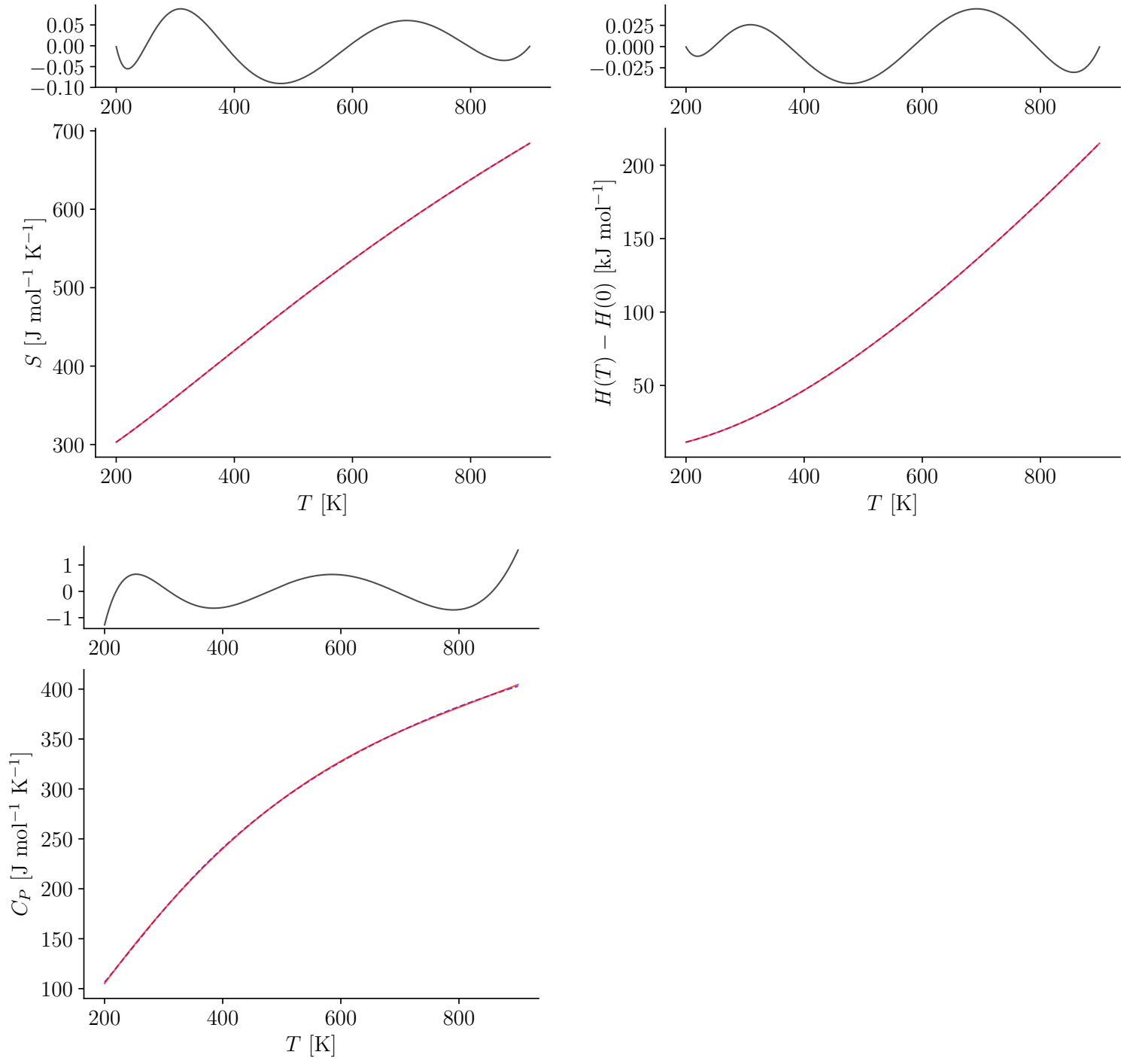


\section{$41 \mathbf{B}_{9} \mathbf{H}_{9}{ }^{2-}$}

Figure S.41.1. Structure of $\mathrm{B}_{9} \mathrm{H}_{9}{ }^{2-}$

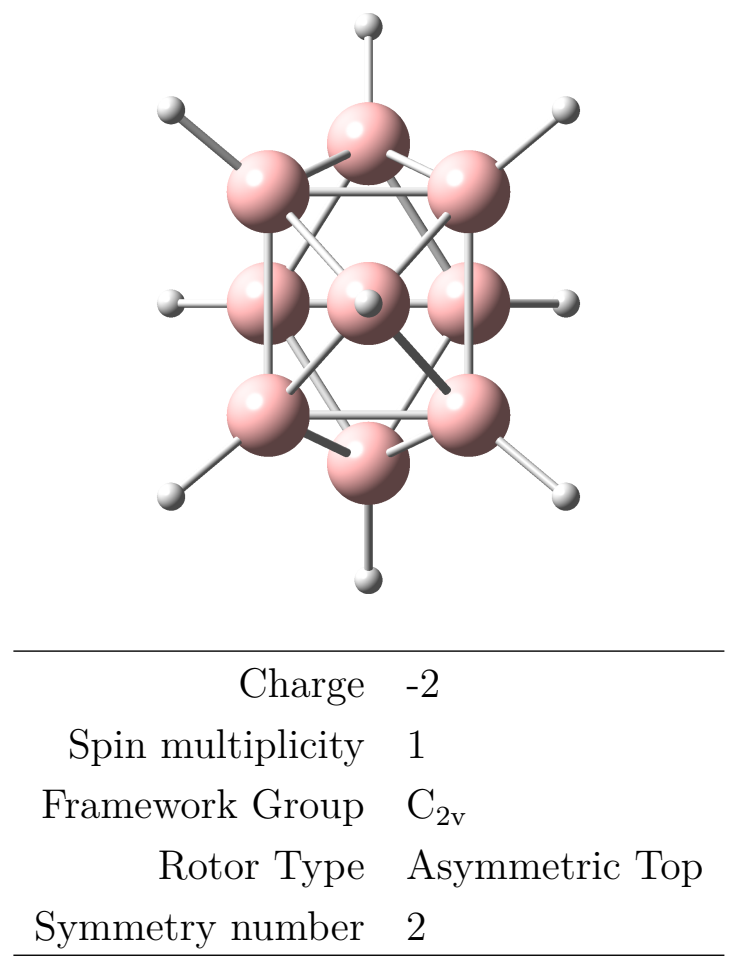

Table S.41.1. $\mathrm{B}_{9} \mathrm{H}_{9}{ }^{2-}$ : Selected anharmonic results obtained at the B3LYP-D2/cc-pVTZ level

\begin{tabular}{rll}
\hline $\mathrm{ZPE}_{\mathrm{v}}$ & 25657 & $\mathrm{~cm}^{-1}$ \\
$\mathrm{~A}$ & 0.09181 & $\mathrm{~cm}^{-1}$ \\
$\mathrm{~B}$ & 0.09180 & $\mathrm{~cm}^{-1}$ \\
$\mathrm{C}$ & 0.07502 & $\mathrm{~cm}^{-1}$ \\
\hline$C_{P}(300 \mathrm{~K})$ & 165.007 & $\mathrm{~J} \mathrm{~mol}^{-1} \mathrm{~K}-1$ \\
$S(300 \mathrm{~K})$ & 339.504 & $\mathrm{~J} \mathrm{~mol}^{-1} \mathrm{~K}-1$ \\
$H(300 \mathrm{~K})-H(0 \mathrm{~K})$ & 23.342 & $\mathrm{~kJ} \mathrm{~mol}^{-1}$ \\
\hline
\end{tabular}

$\mathrm{ZPE}_{\mathrm{v}}$ : vibrational contribution to the zero-point energy. A, B, C: Rotational constants. $C_{P}$ : Heat capacity at constant pressure. $S$ : Entropy. $H$ : Enthalpy. Ideal-gas calculations (1 bar). 
Table S.41.2. $\mathrm{B}_{9} \mathrm{H}_{9}{ }^{2-}$ : Cartesian coordinates $(\AA)$ of the B3LYP-D2/cc-pVTZ optimized structure

\begin{tabular}{cccc} 
atom & $\mathrm{x}$ & $\mathrm{y}$ & $\mathrm{z}$ \\
\hline H1 & 0.0000 & 2.4630 & -1.4222 \\
H2 & -0.0000 & -2.4630 & -1.4222 \\
H3 & 0.0000 & 0.0000 & 2.8441 \\
B4 & -0.8922 & 0.9954 & 0.5746 \\
B5 & 0.0000 & 0.0000 & 1.6428 \\
B6 & 0.0000 & 1.4228 & -0.8214 \\
B7 & 0.8922 & 0.9954 & 0.5746 \\
B8 & -0.8922 & -0.9954 & 0.5746 \\
B9 & -0.8922 & 0.0000 & -1.1493 \\
B10 & -0.0000 & -1.4228 & -0.8214 \\
B11 & 0.8922 & -0.0000 & -1.1493 \\
B12 & 0.8922 & -0.9954 & 0.5746 \\
H13 & -1.7574 & 1.7204 & 0.9933 \\
H14 & 1.7574 & 1.7204 & 0.9933 \\
H15 & -1.7574 & -1.7204 & 0.9933 \\
H16 & 1.7574 & -1.7204 & 0.9933 \\
H17 & -1.7574 & 0.0000 & -1.9865 \\
H18 & 1.7574 & -0.0000 & -1.9865 \\
\hline
\end{tabular}


Table S.41.3. $\mathrm{B}_{9} \mathrm{H}_{9}{ }^{2-}$ : Vibrational frequencies, infrared integrated intensities and Raman activities (B3LYP-D2/cc-pVTZ results)

\begin{tabular}{|c|c|c|c|c|c|c|c|}
\hline \multirow[b]{2}{*}{ mode } & \multirow[b]{2}{*}{ symm. } & \multicolumn{3}{|c|}{ Harmonic } & \multicolumn{3}{|c|}{ Anharmonic } \\
\hline & & $\begin{array}{c}\omega \\
{\left[\mathrm{cm}^{-1}\right]}\end{array}$ & $\begin{array}{c}\mathrm{IR} \\
{\left[\mathrm{km} \mathrm{mol}^{-1}\right]}\end{array}$ & $\begin{array}{c}\text { Raman } \\
{\left[\AA^{6}\right]}\end{array}$ & $\begin{array}{c}\omega \\
{\left[\mathrm{cm}^{-1}\right]}\end{array}$ & $\begin{array}{c}\mathrm{IR} \\
{\left[\mathrm{km} \mathrm{mol}^{-1}\right]}\end{array}$ & $\begin{array}{c}\text { Raman } \\
{\left[\AA^{6}\right]}\end{array}$ \\
\hline 1 & $\mathrm{~B}_{1}$ & 168 & 0.003 & 0.000 & 175 & 0.014 & 0.000 \\
\hline 2 & $\mathrm{~B}_{1}$ & 343 & 0.000 & 0.032 & 331 & 0.000 & 0.031 \\
\hline 3 & $\mathrm{~A}_{2}$ & 343 & 0.000 & 0.032 & 329 & 0.000 & 0.030 \\
\hline 4 & $\mathrm{~A}_{1}$ & 435 & 0.038 & 0.181 & 415 & 0.076 & 0.165 \\
\hline 5 & $\mathrm{~B}_{2}$ & 435 & 0.038 & 0.180 & 414 & 0.103 & 0.163 \\
\hline 6 & $\mathrm{~A}_{1}$ & 526 & 0.000 & 0.560 & 527 & 0.000 & 0.541 \\
\hline 7 & $\mathrm{~A}_{2}$ & 558 & 0.000 & 0.000 & 537 & 0.000 & 0.000 \\
\hline 8 & $\mathrm{~A}_{1}$ & 590 & 0.246 & 0.074 & 577 & 0.302 & 0.075 \\
\hline 9 & $\mathrm{~B}_{2}$ & 591 & 0.247 & 0.074 & 576 & 0.296 & 0.071 \\
\hline 10 & $\mathrm{~B}_{1}$ & 650 & 1.860 & 0.000 & 630 & 1.723 & 0.000 \\
\hline 11 & $\mathrm{~A}_{2}$ & 662 & 0.000 & 0.047 & 647 & 0.000 & 0.028 \\
\hline 12 & $\mathrm{~B}_{1}$ & 662 & 0.001 & 0.047 & 647 & 0.002 & 0.030 \\
\hline 13 & $\mathrm{~B}_{2}$ & 722 & 0.000 & 0.000 & 691 & 0.008 & 0.000 \\
\hline 14 & $\mathrm{~B}_{2}$ & 729 & 1.948 & 0.057 & 713 & 2.153 & 0.080 \\
\hline 15 & $\mathrm{~A}_{1}$ & 729 & 1.946 & 0.057 & 714 & 2.216 & 0.083 \\
\hline 16 & $\mathrm{~A}_{2}$ & 761 & 0.000 & 0.106 & 734 & 0.000 & 0.058 \\
\hline 17 & $\mathrm{~B}_{1}$ & 761 & 0.000 & 0.106 & 735 & 0.000 & 0.059 \\
\hline 18 & $\mathrm{~A}_{1}$ & 762 & 0.000 & 0.222 & 745 & 0.000 & 0.217 \\
\hline 19 & $\mathrm{~A}_{1}$ & 785 & 0.000 & 0.917 & 770 & 0.000 & 1.043 \\
\hline 20 & $\mathrm{~A}_{1}$ & 789 & 0.233 & 0.001 & 765 & 0.322 & 0.001 \\
\hline 21 & $\mathrm{~B}_{2}$ & 789 & 0.224 & 0.001 & 764 & 0.283 & 0.000 \\
\hline 22 & $\mathrm{~B}_{1}$ & 839 & 0.000 & 0.022 & 819 & 0.004 & 0.027 \\
\hline 23 & $\mathrm{~A}_{2}$ & 840 & 0.000 & 0.022 & 817 & 0.000 & 0.027 \\
\hline
\end{tabular}

To be continued 
Table S.41.3 - Continued

\begin{tabular}{|c|c|c|c|c|c|c|c|}
\hline \multirow[b]{2}{*}{ mode } & \multirow[b]{2}{*}{ symm. } & \multicolumn{3}{|c|}{ Harmonic } & \multicolumn{3}{|c|}{ Anharmonic } \\
\hline & & $\begin{array}{c}\omega \\
{\left[\mathrm{cm}^{-1}\right]} \\
\end{array}$ & $\begin{array}{c}\mathrm{IR} \\
{\left[\mathrm{km} \mathrm{mol}^{-1}\right]} \\
\end{array}$ & $\begin{array}{c}\text { Raman } \\
{\left[\AA^{6}\right]}\end{array}$ & $\begin{array}{c}\omega \\
{\left[\mathrm{cm}^{-1}\right]} \\
\end{array}$ & $\begin{array}{c}\mathrm{IR} \\
{\left[\mathrm{km} \mathrm{mol}^{-1}\right]} \\
\end{array}$ & $\begin{array}{c}\text { Raman } \\
{\left[\AA^{6}\right]}\end{array}$ \\
\hline 24 & $\mathrm{~B}_{1}$ & 862 & 2.950 & 0.000 & 845 & 2.795 & 0.000 \\
\hline 25 & $\mathrm{~A}_{2}$ & 880 & 0.000 & 0.020 & 862 & 0.000 & 0.024 \\
\hline 26 & $\mathrm{~B}_{1}$ & 880 & 0.000 & 0.020 & 863 & 0.000 & 0.023 \\
\hline 27 & $A_{1}$ & 886 & 0.001 & 1.893 & 864 & 0.126 & 1.848 \\
\hline 28 & $A_{1}$ & 888 & 7.219 & 0.011 & 870 & 6.298 & 0.045 \\
\hline 29 & $\mathrm{~B}_{2}$ & 888 & 7.229 & 0.011 & 869 & 6.344 & 0.010 \\
\hline 30 & $\mathrm{~A}_{2}$ & 890 & 0.000 & 0.000 & 857 & 0.000 & 0.000 \\
\hline 31 & $\mathrm{~B}_{2}$ & 926 & 0.000 & 0.000 & 898 & 0.000 & 0.000 \\
\hline 32 & $\mathrm{~B}_{1}$ & 933 & 0.000 & 0.001 & 907 & 0.048 & 0.001 \\
\hline 33 & $\mathrm{~A}_{2}$ & 933 & 0.000 & 0.001 & 905 & 0.000 & 0.000 \\
\hline 34 & $\mathrm{~B}_{2}$ & 980 & 1.829 & 0.008 & 956 & 1.520 & 0.010 \\
\hline 35 & $\mathrm{~A}_{1}$ & 980 & 1.807 & 0.008 & 956 & 1.611 & 0.010 \\
\hline 36 & $\mathrm{~B}_{1}$ & 983 & 10.442 & 0.000 & 965 & 9.367 & 0.000 \\
\hline 37 & $\mathrm{~B}_{2}$ & 1000 & 0.000 & 0.000 & 961 & 0.003 & 0.000 \\
\hline 38 & $\mathrm{~B}_{2}$ & 1066 & 19.197 & 0.017 & 1038 & 16.800 & 0.012 \\
\hline 39 & $\mathrm{~A}_{1}$ & 1066 & 19.206 & 0.017 & 1038 & 16.000 & 0.013 \\
\hline 40 & $\mathrm{~A}_{2}$ & 2476 & 0.000 & 1.940 & 2353 & 0.000 & 2.085 \\
\hline 41 & $\mathrm{~B}_{1}$ & 2476 & 0.001 & 1.940 & 2347 & 279.076 & 1.586 \\
\hline 42 & $\mathrm{~B}_{2}$ & 2487 & 304.462 & 0.861 & 2363 & 137.705 & 1.213 \\
\hline 43 & $\mathrm{~A}_{1}$ & 2488 & 304.012 & 0.861 & 2359 & 260.905 & 0.852 \\
\hline 44 & $\mathrm{~B}_{1}$ & 2489 & 107.924 & 0.000 & 2373 & 846.885 & 0.520 \\
\hline 45 & $\mathrm{~A}_{1}$ & 2494 & 0.002 & 1.140 & 2363 & 2.632 & 1.350 \\
\hline 46 & $\mathrm{~A}_{1}$ & 2522 & 774.344 & 0.576 & 2403 & 747.204 & 0.994 \\
\hline 47 & $\mathrm{~B}_{2}$ & 2523 & 773.749 & 0.577 & 2399 & 972.342 & 0.347 \\
\hline To be & atinued & & & & & & \\
\hline
\end{tabular}


Table S.41.3 - Continued

\begin{tabular}{cccccccc}
\hline & & \multicolumn{3}{c}{ Harmonic } & \multicolumn{3}{c}{ Anharmonic } \\
mode & symm. & $\omega$ & IR & Raman & $\omega$ & IR & Raman \\
& & {$\left[\mathrm{cm}^{-1}\right]$} & {$\left[\mathrm{km} \mathrm{mol}^{-1}\right]$} & {$\left[\AA^{6}\right]$} & {$\left[\mathrm{cm}^{-1}\right]$} & {$\left[\mathrm{km} \mathrm{mol}^{-1}\right]$} & {$\left[\AA^{6}\right]$} \\
\hline 48 & $\mathrm{~A}_{1}$ & 2546 & 0.001 & 3.732 & 2432 & 106.355 & 3.791 \\
\hline
\end{tabular}


Figure S.41.2. Anharmonic IR spectrum of $\mathrm{B}_{9} \mathrm{H}_{9}{ }^{2-}$ obtained by convoluting the calculated intensities with Lorentzians having a FWHM of $4 \mathrm{~cm}^{-1}$ (B3LYP-D2/cc-pVTZ results): (top) full spectrum, (middle) contributions from fundamentals and combination bands, (bottom) contributions from overtones
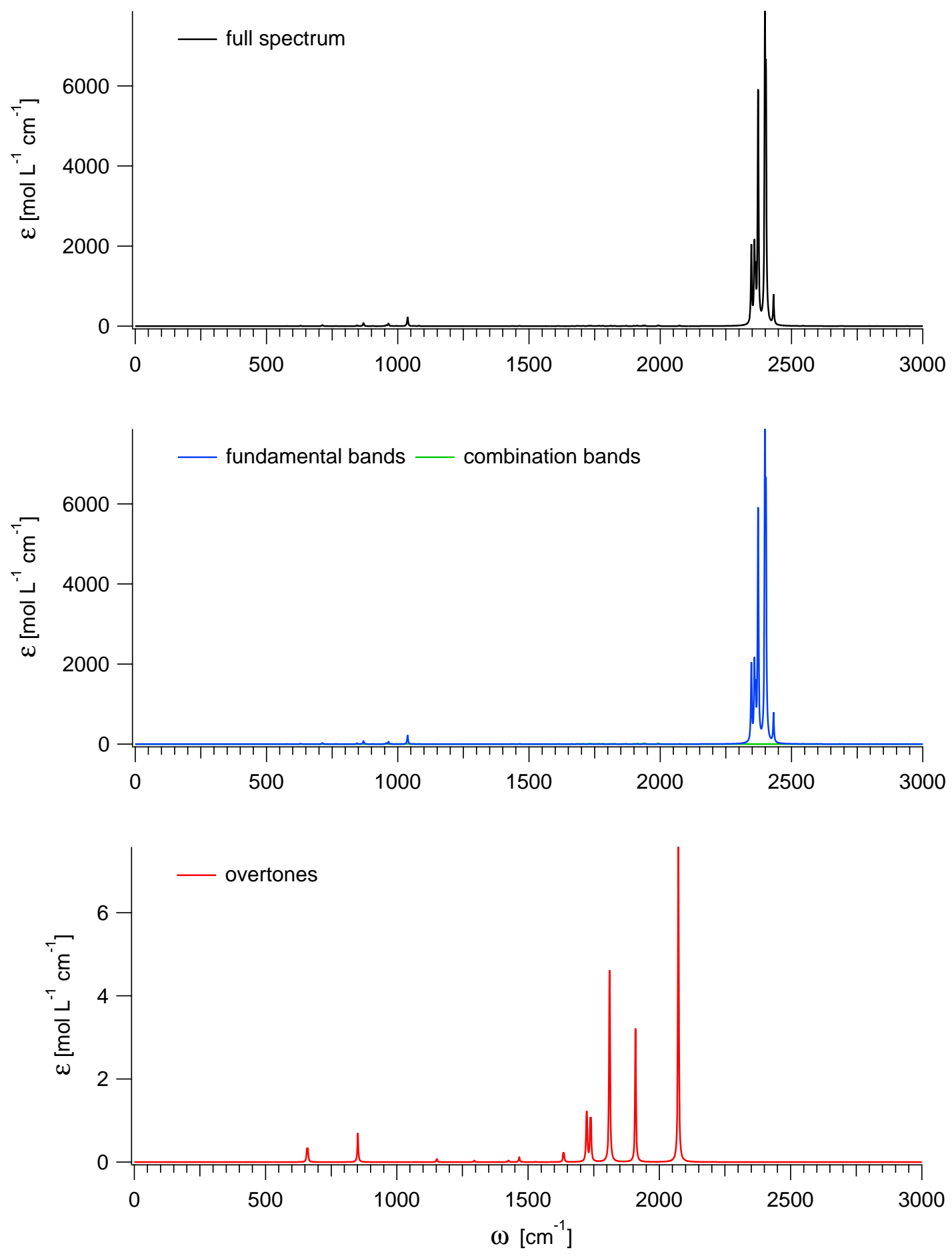
Figure S.41.3. Anharmonic Raman spectrum of $\mathrm{B}_{9} \mathrm{H}_{9}{ }^{2-}$ obtained by convoluting the calculated activities with Lorentzians having a FWHM of $4 \mathrm{~cm}^{-1}$ (B3LYP-D2/cc-pVTZ results): (top) full spectrum, (middle) contributions from fundamentals and combination bands, (bottom) contributions from overtones
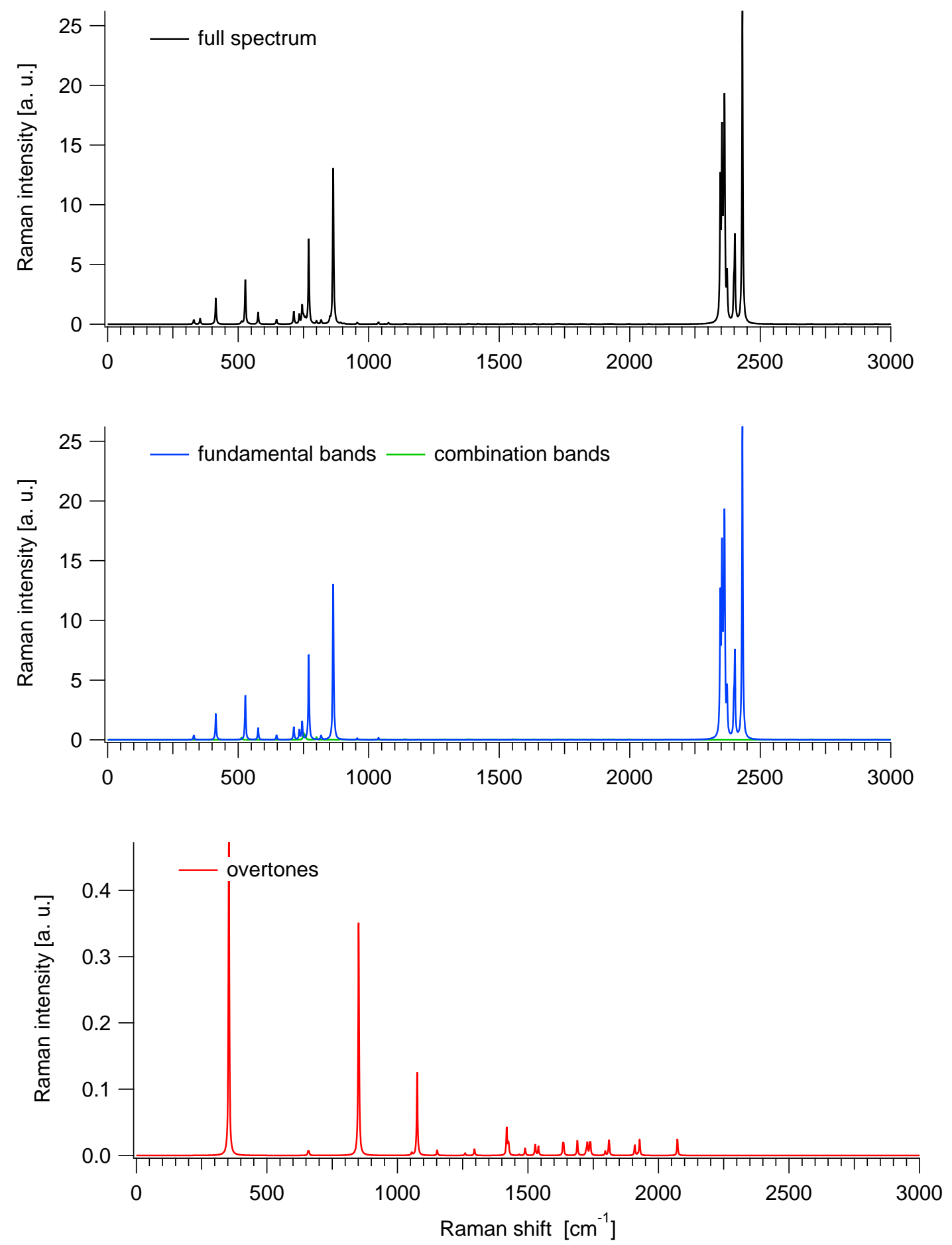
Table S.41.4. $\mathrm{B}_{9} \mathrm{H}_{9}{ }^{2-}$ : Ideal-gas thermodynamic functions at 1 bar (anharmonic B3LYPD2/cc-pVTZ results)

\begin{tabular}{cccc}
\hline$T$ & $C_{P}$ & $S$ & $H(T)-H(0 K)$ \\
{$[\mathrm{K}]$} & {$\left[\mathrm{J} \mathrm{mol}^{-1} \mathrm{~K}^{-1}\right]$} & {$\left[\mathrm{J} \mathrm{mol}^{-1} \mathrm{~K}^{-1}\right]$} & {$\left[\mathrm{kJ} \mathrm{mol}^{-1}\right]$} \\
\hline 100 & 44.362 & 243.367 & 3.618 \\
200 & 95.624 & 287.458 & 10.270 \\
300 & 165.007 & 339.504 & 23.342 \\
400 & 220.840 & 395.042 & 42.781 \\
500 & 261.049 & 448.884 & 66.985 \\
600 & 290.661 & 499.223 & 94.640 \\
700 & 313.393 & 545.808 & 124.889 \\
800 & 331.382 & 588.875 & 157.161 \\
900 & 345.887 & 628.773 & 191.050 \\
1000 & 357.724 & 665.849 & 226.250 \\
1100 & 367.473 & 700.415 & 262.526 \\
1200 & 375.564 & 732.747 & 299.690 \\
1300 & 382.328 & 763.084 & 337.594 \\
1400 & 388.021 & 791.631 & 376.120 \\
1500 & 392.844 & 818.571 & 415.169 \\
1600 & 396.958 & 844.059 & 454.665 \\
1700 & 400.488 & 868.234 & 494.542 \\
1800 & 403.536 & 891.213 & 534.746 \\
1900 & 406.181 & 913.104 & 575.235 \\
2000 & 408.489 & 933.998 & 615.971 \\
\hline
\end{tabular}

$T$ : Temperature. $C_{P}$ : Heat capacity at constant pressure. $S$ : Entropy. $H$ : Enthalpy. 
Figure S.41.4. $\mathrm{B}_{9} \mathrm{H}_{9}{ }^{2-}$ : Ideal-gas thermodynamic functions at 1 bar (anharmonic B3LYPD2/cc-pVTZ results)
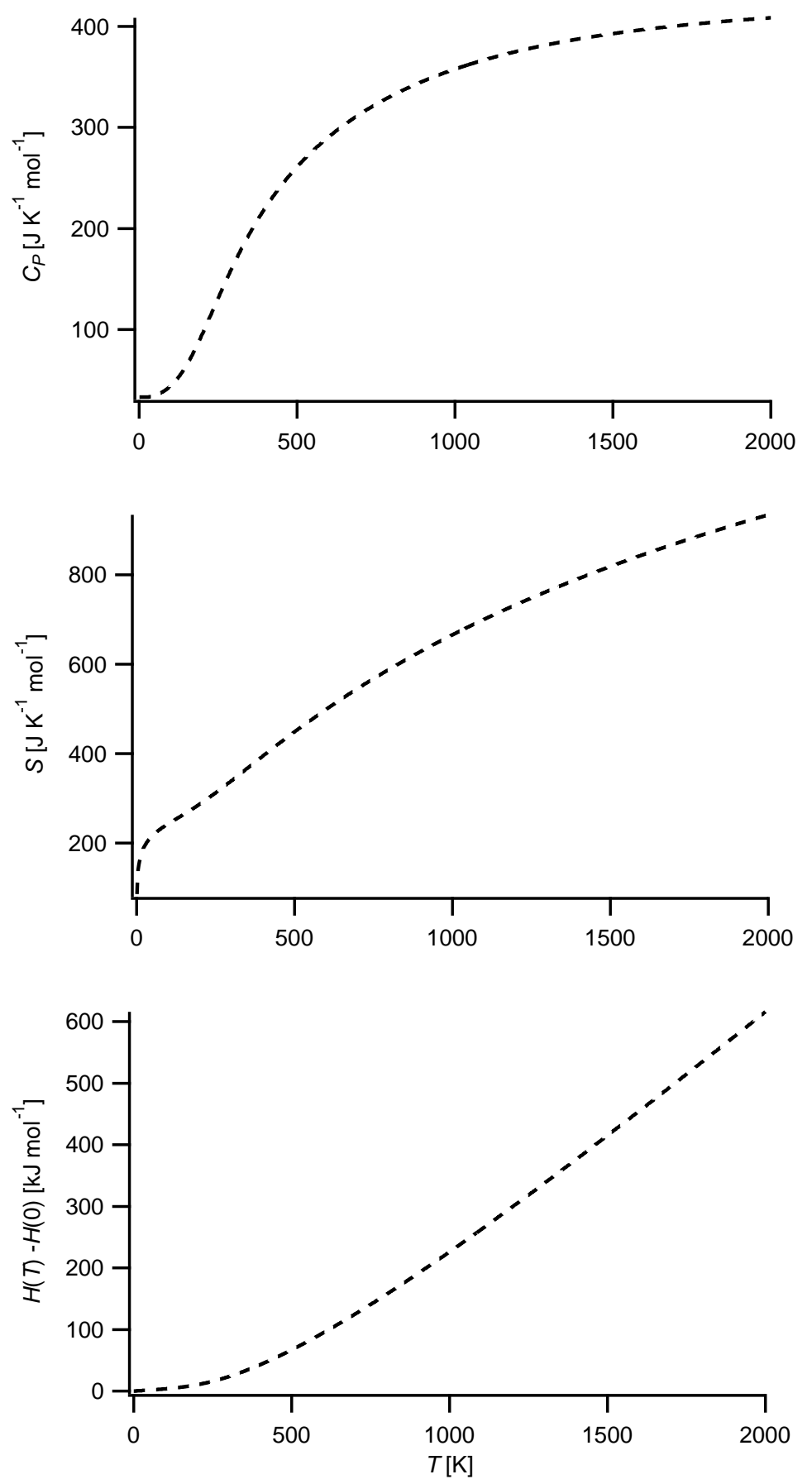

T: Temperature. $C_{P}$ : Heat capacity at constant pressure. $S$ : Entropy. $H$ : Enthalpy. 
Table S.41.5. $\mathrm{B}_{9} \mathrm{H}_{9}{ }^{2-}$ : Fits (red solid lines) of the computed thermodynamic functions (blue dashed lines) with NASA type functions (Equations (1) - (3)) in the $200-900 \mathrm{~K}$ temperature range (anharmonic B3LYP-D2/cc-pVTZ results). In each case, the difference curve between the thermodynamic and NASA functions is plotted in the upper graph

Fit parameters

\begin{tabular}{llllll}
\hline $\mathrm{a}_{1}$ & $-1.24015960 \mathrm{e}+01$ & $\mathrm{a}_{2}\left[\mathrm{~K}^{-1}\right]$ & $1.42231461 \mathrm{e}-01$ & $\mathrm{a}_{3}\left[\mathrm{~K}^{-2}\right]$ & $-1.22193332 \mathrm{e}-04$ \\
$\mathrm{a}_{4}\left[\mathrm{~K}^{-3}\right]$ & $1.55916164 \mathrm{e}-08$ & $\mathrm{a}_{5}\left[\mathrm{~K}^{-4}\right]$ & $2.10180513 \mathrm{e}-11$ & $\mathrm{a}_{6}[\mathrm{~K}]$ & $1.18905256 \mathrm{e}+03$ \\
$\mathrm{a}_{7}$ & $7.42280363 \mathrm{e}+01$ & & & &
\end{tabular}
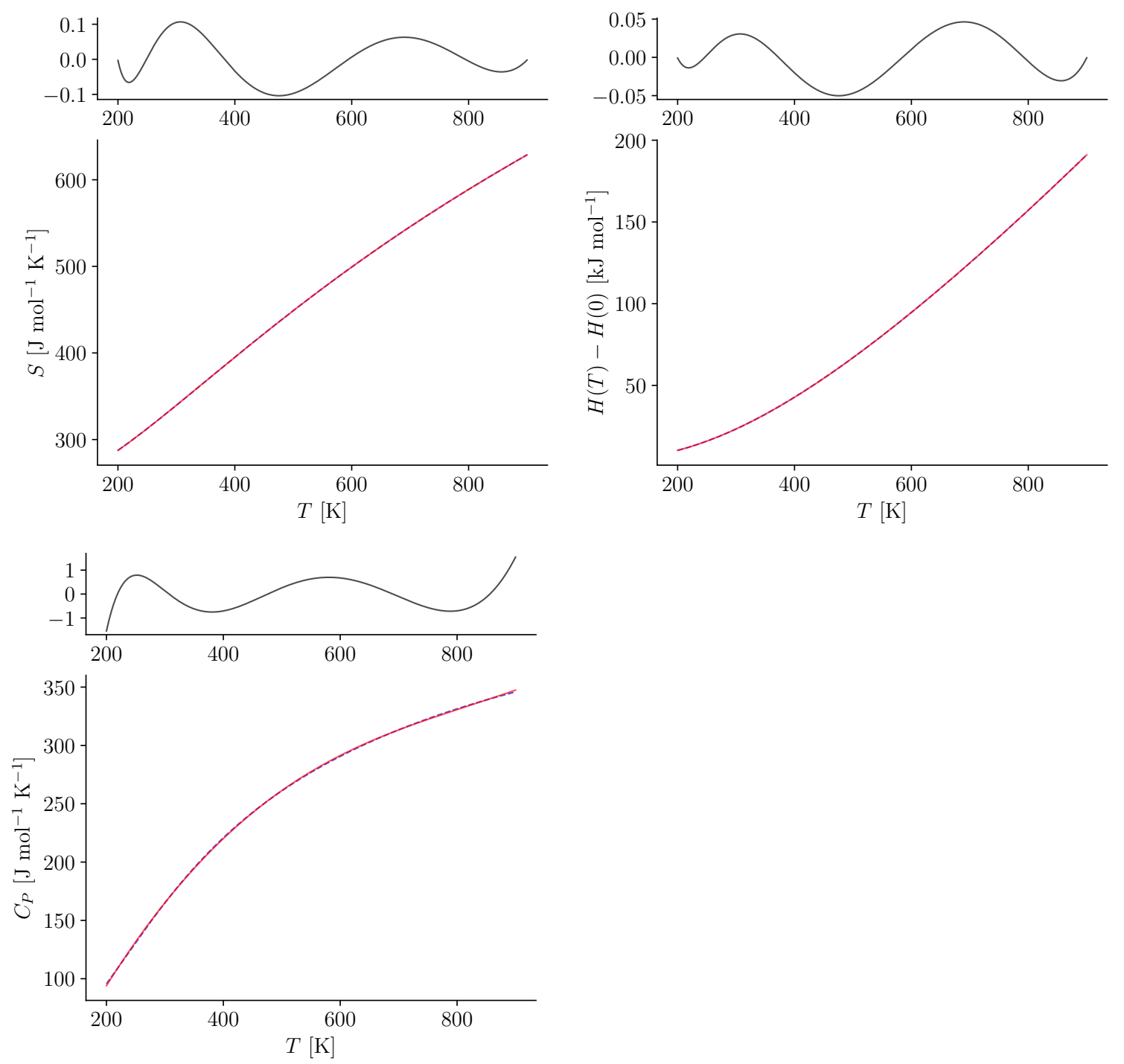


\section{$42 \quad \mathrm{~B}_{10} \mathrm{H}_{10}{ }^{2-}$}

Figure S.42.1. Structure of $\mathrm{B}_{10} \mathrm{H}_{10}{ }^{2-}$

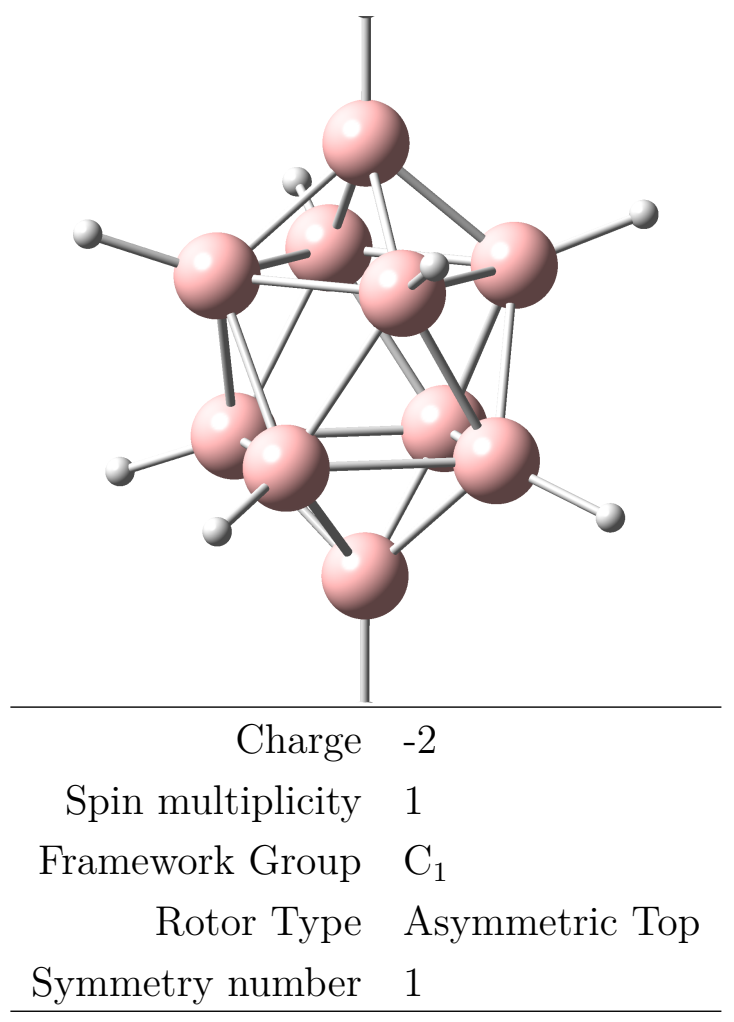

Table S.42.1. $\mathrm{B}_{10} \mathrm{H}_{10}{ }^{2-}$ : Selected anharmonic results obtained at the B3LYP-D2/cc-pVTZ level

\begin{tabular}{rll}
\hline $\mathrm{ZPE}_{\mathrm{v}}$ & 29273 & $\mathrm{~cm}^{-1}$ \\
$\mathrm{~A}$ & 0.08568 & $\mathrm{~cm}^{-1}$ \\
$\mathrm{~B}$ & 0.06588 & $\mathrm{~cm}^{-1}$ \\
$\mathrm{C}$ & 0.06588 & $\mathrm{~cm}^{-1}$ \\
\hline$C_{P}(300 \mathrm{~K})$ & 175.160 & $\mathrm{~J} \mathrm{~mol}^{-1} \mathrm{~K}-1$ \\
$S(300 \mathrm{~K})$ & 342.948 & $\mathrm{~J} \mathrm{~mol}^{-1} \mathrm{~K}-1$ \\
$H(300 \mathrm{~K})-H(0 \mathrm{~K})$ & 22.949 & $\mathrm{~kJ} \mathrm{~mol}^{-1}$ \\
\hline
\end{tabular}

$\mathrm{ZPE}_{\mathrm{v}}$ : vibrational contribution to the zero-point energy. A, B, C: Rotational constants. $C_{P}$ : Heat capacity at constant pressure. $S$ : Entropy. $H$ : Enthalpy. Ideal-gas calculations (1 bar). 
Table S.42.2. $\mathrm{B}_{10} \mathrm{H}_{10}{ }^{2-}$ : Cartesian coordinates $(\AA)$ of the B3LYP-D2/cc-pVTZ optimized structure

\begin{tabular}{cccc} 
atom & $\mathrm{x}$ & $\mathrm{y}$ & $\mathrm{z}$ \\
\hline B1 & 0.7644 & -0.3514 & 1.2523 \\
H2 & 1.1627 & -0.6576 & 2.3432 \\
B3 & 1.8613 & -0.0000 & 0.0000 \\
H4 & 3.0592 & -0.0000 & 0.0000 \\
B5 & 0.7644 & -1.2523 & -0.3514 \\
H6 & 1.1627 & -2.3432 & -0.6576 \\
B7 & -0.7644 & -1.1340 & 0.6371 \\
H8 & -1.1627 & -2.1218 & 1.1920 \\
B9 & 0.7644 & 1.2523 & 0.3514 \\
H10 & 1.1627 & 2.3432 & 0.6576 \\
B11 & -0.7644 & 0.6371 & 1.1340 \\
H12 & -1.1628 & 1.1920 & 2.1218 \\
B13 & -1.8613 & 0.0000 & -0.0000 \\
H14 & -3.0592 & 0.0000 & -0.0000 \\
B15 & -0.7644 & -0.6371 & -1.1340 \\
H16 & -1.1627 & -1.1920 & -2.1218 \\
B17 & 0.7644 & 0.3514 & -1.2523 \\
H18 & 1.1627 & 0.6576 & -2.3432 \\
B19 & -0.7644 & 1.1340 & -0.6371 \\
H20 & -1.1627 & 2.1218 & -1.1920 \\
\hline & & &
\end{tabular}


Table S.42.3. $\mathrm{B}_{10} \mathrm{H}_{10}{ }^{2-}$ : Vibrational frequencies, infrared integrated intensities and Raman activities (B3LYP-D2/cc-pVTZ results)

\begin{tabular}{|c|c|c|c|c|c|c|c|}
\hline \multirow[b]{2}{*}{ mode } & \multirow[b]{2}{*}{ symm. } & \multicolumn{3}{|c|}{ Harmonic } & \multicolumn{3}{|c|}{ Anharmonic } \\
\hline & & $\begin{array}{c}\omega \\
{\left[\mathrm{cm}^{-1}\right]}\end{array}$ & $\begin{array}{c}\mathrm{IR} \\
{\left[\mathrm{km} \mathrm{mol}^{-1}\right]}\end{array}$ & $\begin{array}{c}\text { Raman } \\
{\left[\AA^{6}\right]}\end{array}$ & $\begin{array}{c}\omega \\
{\left[\mathrm{cm}^{-1}\right]}\end{array}$ & $\begin{array}{c}\mathrm{IR} \\
{\left[\mathrm{km} \mathrm{mol}^{-1}\right]}\end{array}$ & $\begin{array}{c}\text { Raman } \\
{\left[\AA^{6}\right]}\end{array}$ \\
\hline 1 & $\mathrm{~A}$ & 426 & 0.000 & 0.000 & 408 & 0.000 & 0.000 \\
\hline 2 & $\mathrm{~A}$ & 454 & 0.008 & 0.000 & 440 & 0.003 & 0.000 \\
\hline 3 & $\mathrm{~A}$ & 454 & 0.008 & 0.000 & 440 & 0.003 & 0.000 \\
\hline 4 & $\mathrm{~A}$ & 491 & 0.000 & 0.013 & 477 & 0.001 & 0.013 \\
\hline 5 & $\mathrm{~A}$ & 491 & 0.000 & 0.013 & 477 & 0.001 & 0.013 \\
\hline 6 & $\mathrm{~A}$ & 565 & 0.000 & 0.100 & 553 & 0.000 & 0.079 \\
\hline 7 & $\mathrm{~A}$ & 565 & 0.000 & 0.100 & 553 & 0.000 & 0.079 \\
\hline 8 & $\mathrm{~A}$ & 601 & 0.000 & 0.171 & 583 & 0.000 & 0.186 \\
\hline 9 & $\mathrm{~A}$ & 601 & 0.000 & 0.171 & 583 & 0.000 & 0.206 \\
\hline 10 & $\mathrm{~A}$ & 610 & 0.000 & 0.393 & 594 & 0.010 & 0.384 \\
\hline 11 & $\mathrm{~A}$ & 624 & 0.000 & 0.001 & 603 & 0.000 & 0.000 \\
\hline 12 & $\mathrm{~A}$ & 624 & 0.000 & 0.001 & 601 & 0.000 & 0.000 \\
\hline 13 & $\mathrm{~A}$ & 656 & 2.067 & 0.000 & 640 & 1.898 & 0.000 \\
\hline 14 & A & 656 & 2.067 & 0.000 & 640 & 1.900 & 0.000 \\
\hline 15 & $\mathrm{~A}$ & 715 & 0.000 & 0.000 & 702 & 0.000 & 0.001 \\
\hline 16 & $\mathrm{~A}$ & 715 & 0.000 & 0.000 & 702 & 0.000 & 0.001 \\
\hline 17 & $\mathrm{~A}$ & 745 & 0.000 & 1.122 & 719 & 0.000 & 0.888 \\
\hline 18 & $\mathrm{~A}$ & 751 & 0.397 & 0.000 & 732 & 0.590 & 0.001 \\
\hline 19 & $\mathrm{~A}$ & 756 & 0.000 & 0.109 & 739 & 0.000 & 0.142 \\
\hline 20 & $\mathrm{~A}$ & 756 & 0.000 & 0.109 & 739 & 0.000 & 0.142 \\
\hline 21 & $\mathrm{~A}$ & 766 & 0.055 & 0.000 & 745 & 0.000 & 0.001 \\
\hline 22 & $\mathrm{~A}$ & 766 & 0.055 & 0.000 & 745 & 0.000 & 0.001 \\
\hline 23 & $\mathrm{~A}$ & 809 & 0.007 & 0.000 & 785 & 0.641 & 0.000 \\
\hline
\end{tabular}

To be continued 
Table S.42.3 - Continued

\begin{tabular}{|c|c|c|c|c|c|c|c|}
\hline \multirow[b]{2}{*}{ mode } & \multirow[b]{2}{*}{ symm. } & \multicolumn{3}{|c|}{ Harmonic } & \multicolumn{3}{|c|}{ Anharmonic } \\
\hline & & $\begin{array}{c}\omega \\
{\left[\mathrm{cm}^{-1}\right]} \\
\end{array}$ & $\begin{array}{c}\mathrm{IR} \\
{\left[\mathrm{km} \mathrm{mol}^{-1}\right]} \\
\end{array}$ & $\begin{array}{c}\text { Raman } \\
{\left[\AA^{6}\right]}\end{array}$ & $\begin{array}{c}\omega \\
{\left[\mathrm{cm}^{-1}\right]} \\
\end{array}$ & $\begin{array}{c}\mathrm{IR} \\
{\left[\mathrm{km} \mathrm{mol}^{-1}\right]} \\
\end{array}$ & $\begin{array}{c}\text { Raman } \\
{\left[\AA^{6}\right]}\end{array}$ \\
\hline 24 & $\mathrm{~A}$ & 843 & 0.000 & 1.479 & 820 & 0.009 & 1.637 \\
\hline 25 & $\mathrm{~A}$ & 854 & 0.000 & 0.078 & 837 & 0.000 & 0.102 \\
\hline 26 & $\mathrm{~A}$ & 855 & 0.000 & 0.078 & 837 & 0.000 & 0.098 \\
\hline 27 & $\mathrm{~A}$ & 870 & 1.698 & 0.000 & 837 & 1.044 & 0.001 \\
\hline 28 & $\mathrm{~A}$ & 870 & 1.698 & 0.000 & 837 & 1.034 & 0.001 \\
\hline 29 & $\mathrm{~A}$ & 885 & 0.000 & 0.033 & 869 & 0.001 & 0.024 \\
\hline 30 & $\mathrm{~A}$ & 885 & 0.000 & 0.033 & 869 & 0.001 & 0.024 \\
\hline 31 & $\mathrm{~A}$ & 901 & 0.000 & 0.000 & 887 & 0.000 & 0.000 \\
\hline 32 & $\mathrm{~A}$ & 910 & 0.000 & 0.000 & 891 & 0.000 & 0.000 \\
\hline 33 & $\mathrm{~A}$ & 925 & 2.933 & 0.000 & 902 & 3.095 & 0.000 \\
\hline 34 & $\mathrm{~A}$ & 925 & 2.933 & 0.000 & 902 & 3.089 & 0.000 \\
\hline 35 & $\mathrm{~A}$ & 932 & 0.000 & 0.001 & 907 & 0.000 & 0.005 \\
\hline 36 & $\mathrm{~A}$ & 932 & 0.000 & 0.001 & 905 & 0.000 & 0.003 \\
\hline 37 & $\mathrm{~A}$ & 934 & 0.000 & 0.001 & 907 & 0.003 & 0.007 \\
\hline 38 & $\mathrm{~A}$ & 934 & 0.000 & 0.001 & 907 & 0.002 & 0.007 \\
\hline 39 & $\mathrm{~A}$ & 958 & 0.000 & 0.020 & 929 & 0.023 & 0.023 \\
\hline 40 & $\mathrm{~A}$ & 958 & 0.000 & 0.020 & 929 & 0.025 & 0.023 \\
\hline 41 & $\mathrm{~A}$ & 1016 & 0.000 & 0.681 & 995 & 0.000 & 0.448 \\
\hline 42 & $\mathrm{~A}$ & 1025 & 28.941 & 0.000 & 998 & 26.857 & 0.000 \\
\hline 43 & $\mathrm{~A}$ & 1025 & 28.942 & 0.000 & 998 & 26.993 & 0.000 \\
\hline 44 & $\mathrm{~A}$ & 1092 & 12.882 & 0.000 & 1060 & 10.698 & 0.000 \\
\hline 45 & $\mathrm{~A}$ & 2506 & 0.000 & 1.748 & 2381 & 0.000 & 1.915 \\
\hline 46 & $\mathrm{~A}$ & 2506 & 0.000 & 1.748 & 2380 & 0.001 & 1.870 \\
\hline 47 & $\mathrm{~A}$ & 2508 & 0.000 & 1.102 & 2380 & 116.161 & 1.092 \\
\hline To be & ntinued & & & & & & \\
\hline
\end{tabular}


Table S.42.3 - Continued

\begin{tabular}{cccccccc}
\hline & & \multicolumn{3}{c}{ Harmonic } & \multicolumn{3}{c}{ Anharmonic } \\
mode & symm. & $\omega$ & IR & Raman & $\omega$ & IR & Raman \\
& & {$\left[\mathrm{cm}^{-1}\right]$} & {$\left[\mathrm{km} \mathrm{mol}^{-1}\right]$} & {$\left[\AA^{6}\right]$} & {$\left[\mathrm{cm}^{-1}\right]$} & {$\left[\mathrm{km} \mathrm{mol}^{-1}\right]$} & {$\left[\AA^{6}\right]$} \\
\hline 48 & $\mathrm{~A}$ & 2508 & 0.000 & 1.102 & 2380 & 115.806 & 1.092 \\
49 & $\mathrm{~A}$ & 2513 & 119.194 & 0.000 & 2385 & 20.026 & 0.000 \\
50 & $\mathrm{~A}$ & 2525 & 110.427 & 0.000 & 2401 & 11.616 & 0.121 \\
51 & $\mathrm{~A}$ & 2525 & 110.422 & 0.000 & 2401 & 11.322 & 0.120 \\
52 & $\mathrm{~A}$ & 2535 & 0.000 & 2.524 & 2402 & 0.013 & 2.292 \\
53 & $\mathrm{~A}$ & 2553 & 902.863 & 0.000 & 2435 & 65.168 & 0.004 \\
54 & $\mathrm{~A}$ & 2571 & 0.000 & 4.274 & 2458 & 1.516 & 5.321 \\
\hline
\end{tabular}


Figure S.42.2. Anharmonic IR spectrum of $\mathrm{B}_{10} \mathrm{H}_{10}{ }^{2-}$ obtained by convoluting the calculated intensities with Lorentzians having a FWHM of $4 \mathrm{~cm}^{-1}$ (B3LYP-D2/cc-pVTZ results): (top) full spectrum, (middle) contributions from fundamentals and combination bands, (bottom) contributions from overtones
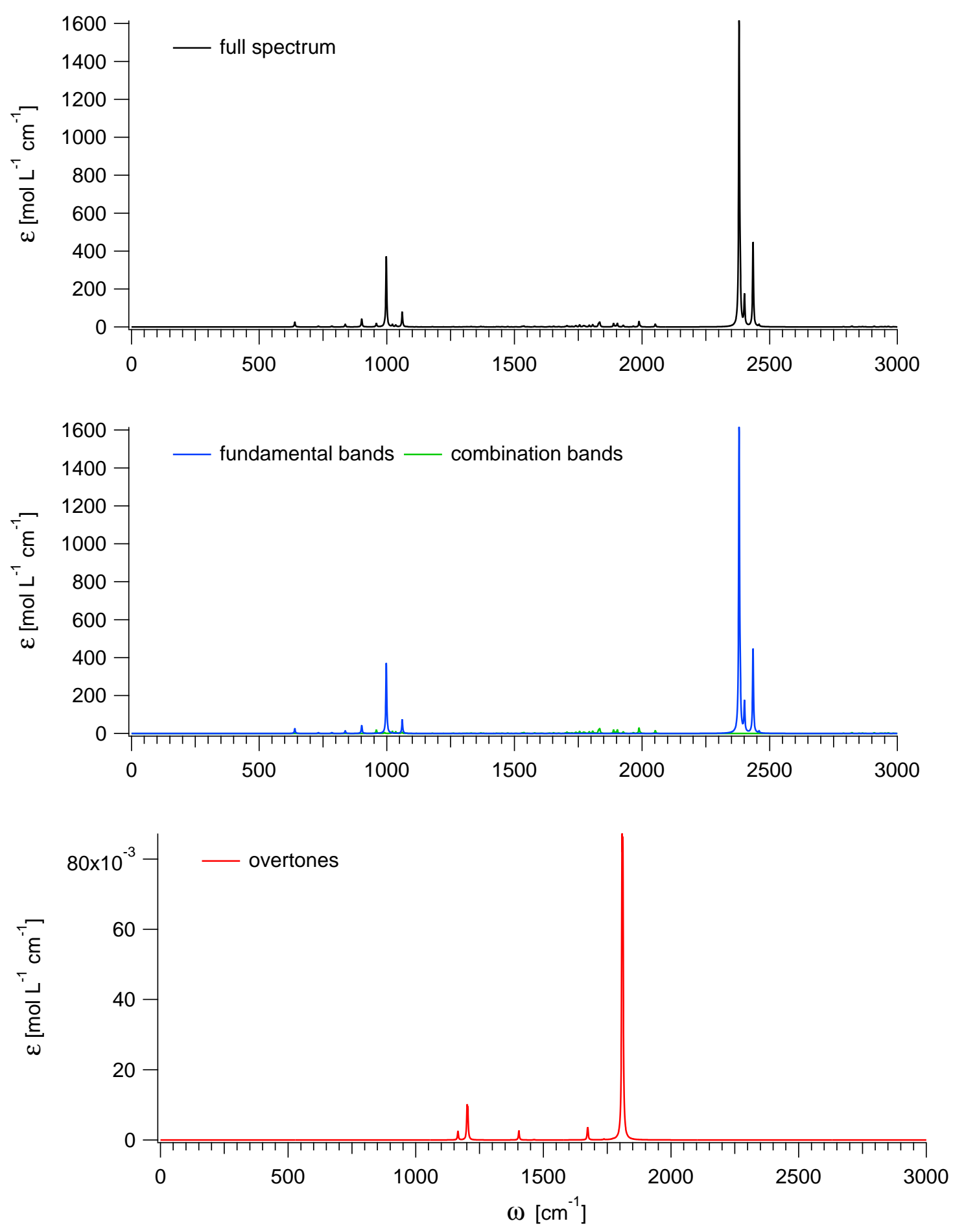
Figure S.42.3. Anharmonic Raman spectrum of $\mathrm{B}_{10} \mathrm{H}_{10}{ }^{2-}$ obtained by convoluting the calculated activities with Lorentzians having a FWHM of $4 \mathrm{~cm}^{-1}$ (B3LYP-D2/cc-pVTZ results): (top) full spectrum, (middle) contributions from fundamentals and combination bands, (bottom) contributions from overtones
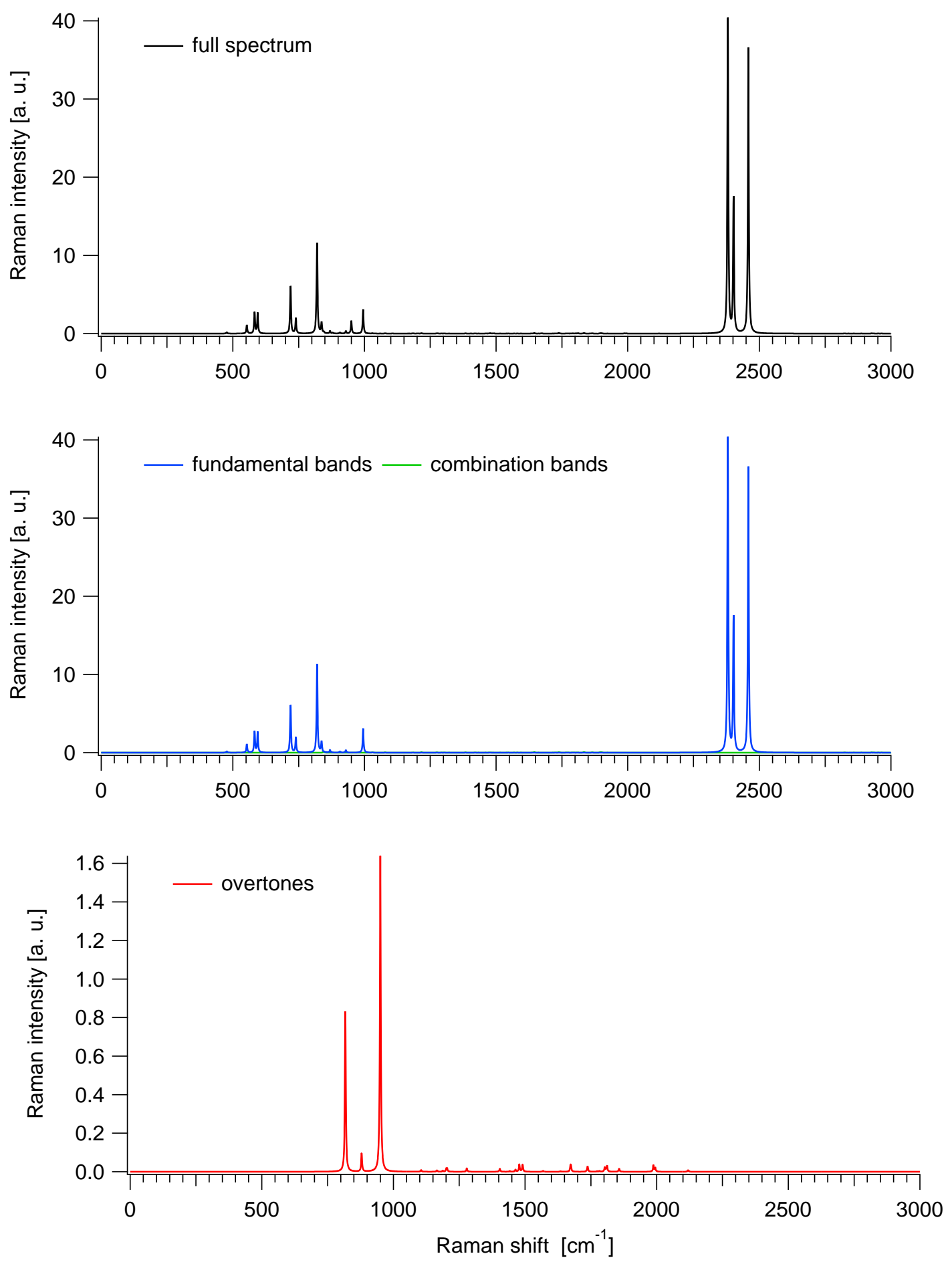
Table S.42.4. $\mathrm{B}_{10} \mathrm{H}_{10}{ }^{2-}$ : Ideal-gas thermodynamic functions at 1 bar (anharmonic B3LYPD2/cc-pVTZ results)

\begin{tabular}{cccc}
\hline$T$ & $C_{P}$ & $S$ & $H(T)-H(0 K)$ \\
{$[\mathrm{K}]$} & {$\left[\mathrm{J} \mathrm{mol}^{-1} \mathrm{~K}^{-1}\right]$} & {$\left[\mathrm{J} \mathrm{mol}^{-1} \mathrm{~K}^{-1}\right]$} & {$\left[\mathrm{kJ} \mathrm{mol}^{-1}\right]$} \\
\hline 100 & 37.504 & 249.527 & 3.386 \\
200 & 93.809 & 289.340 & 9.451 \\
300 & 175.160 & 342.948 & 22.949 \\
400 & 240.239 & 402.753 & 43.894 \\
500 & 286.777 & 461.648 & 70.373 \\
600 & 320.825 & 517.093 & 100.835 \\
700 & 346.831 & 568.585 & 134.273 \\
800 & 367.344 & 616.288 & 170.020 \\
900 & 383.853 & 660.542 & 207.608 \\
1000 & 397.315 & 701.705 & 246.689 \\
1100 & 408.397 & 740.110 & 286.992 \\
1200 & 417.593 & 776.051 & 328.306 \\
1300 & 425.282 & 809.789 & 370.461 \\
1400 & 431.754 & 841.550 & 413.322 \\
1500 & 437.240 & 871.530 & 456.779 \\
1600 & 441.920 & 899.902 & 500.743 \\
1700 & 445.936 & 926.817 & 545.141 \\
1800 & 449.404 & 952.406 & 589.912 \\
1900 & 452.415 & 976.787 & 635.006 \\
2000 & 455.043 & 1000.061 & 680.382 \\
\hline
\end{tabular}

$T$ : Temperature. $C_{P}$ : Heat capacity at constant pressure. $S$ : Entropy. $H$ : Enthalpy. 
Figure S.42.4. $\mathrm{B}_{10} \mathrm{H}_{10}{ }^{2-}$ : Ideal-gas thermodynamic functions at 1 bar (anharmonic B3LYPD2/cc-pVTZ results)
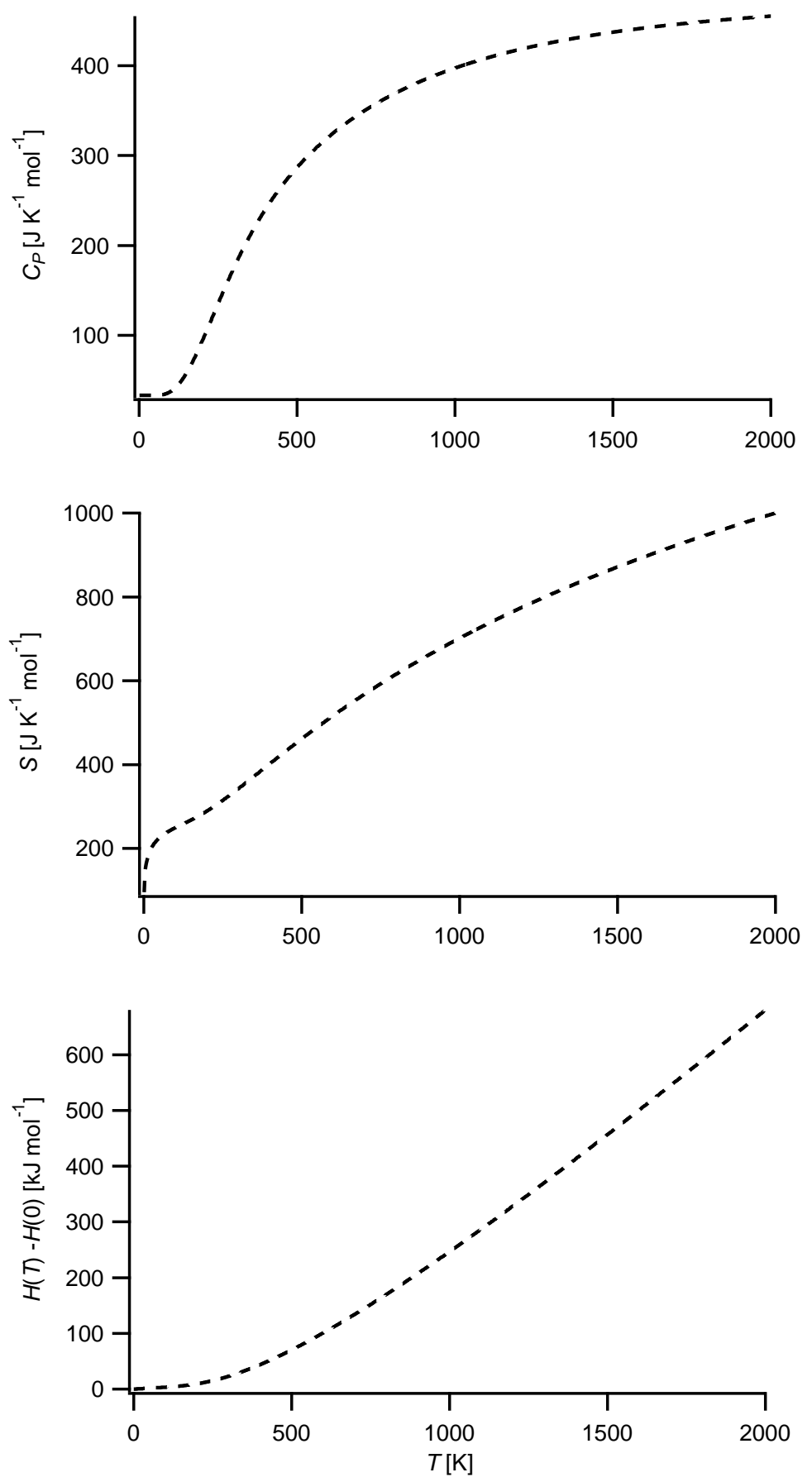

$T$ : Temperature. $C_{P}$ : Heat capacity at constant pressure. $S$ : Entropy. $H$ : Enthalpy. 
Table S.42.5. $\mathrm{B}_{10} \mathrm{H}_{10}{ }^{2-}$ : Fits (red solid lines) of the computed thermodynamic functions (blue dashed lines) with NASA type functions (Equations (1) - (3)) in the $200-900 \mathrm{~K}$ temperature range (anharmonic B3LYP-D2/cc-pVTZ results). In each case, the difference curve between the thermodynamic and NASA functions is plotted in the upper graph

Fit parameters

\begin{tabular}{llllll}
\hline $\mathrm{a}_{1}$ & $-1.67646723 \mathrm{e}+01$ & $\mathrm{a}_{2}\left[\mathrm{~K}^{-1}\right]$ & $1.67016990 \mathrm{e}-01$ & $\mathrm{a}_{3}\left[\mathrm{~K}^{-2}\right]$ & $-1.43328863 \mathrm{e}-04$ \\
$\mathrm{a}_{4}\left[\mathrm{~K}^{-3}\right]$ & $1.55760767 \mathrm{e}-08$ & $\mathrm{a}_{5}\left[\mathrm{~K}^{-4}\right]$ & $2.68122669 \mathrm{e}-11$ & $\mathrm{a}_{6}[\mathrm{~K}]$ & $1.52338766 \mathrm{e}+03$ \\
$\mathrm{a}_{7}$ & $9.30345994 \mathrm{e}+01$ & & & &
\end{tabular}
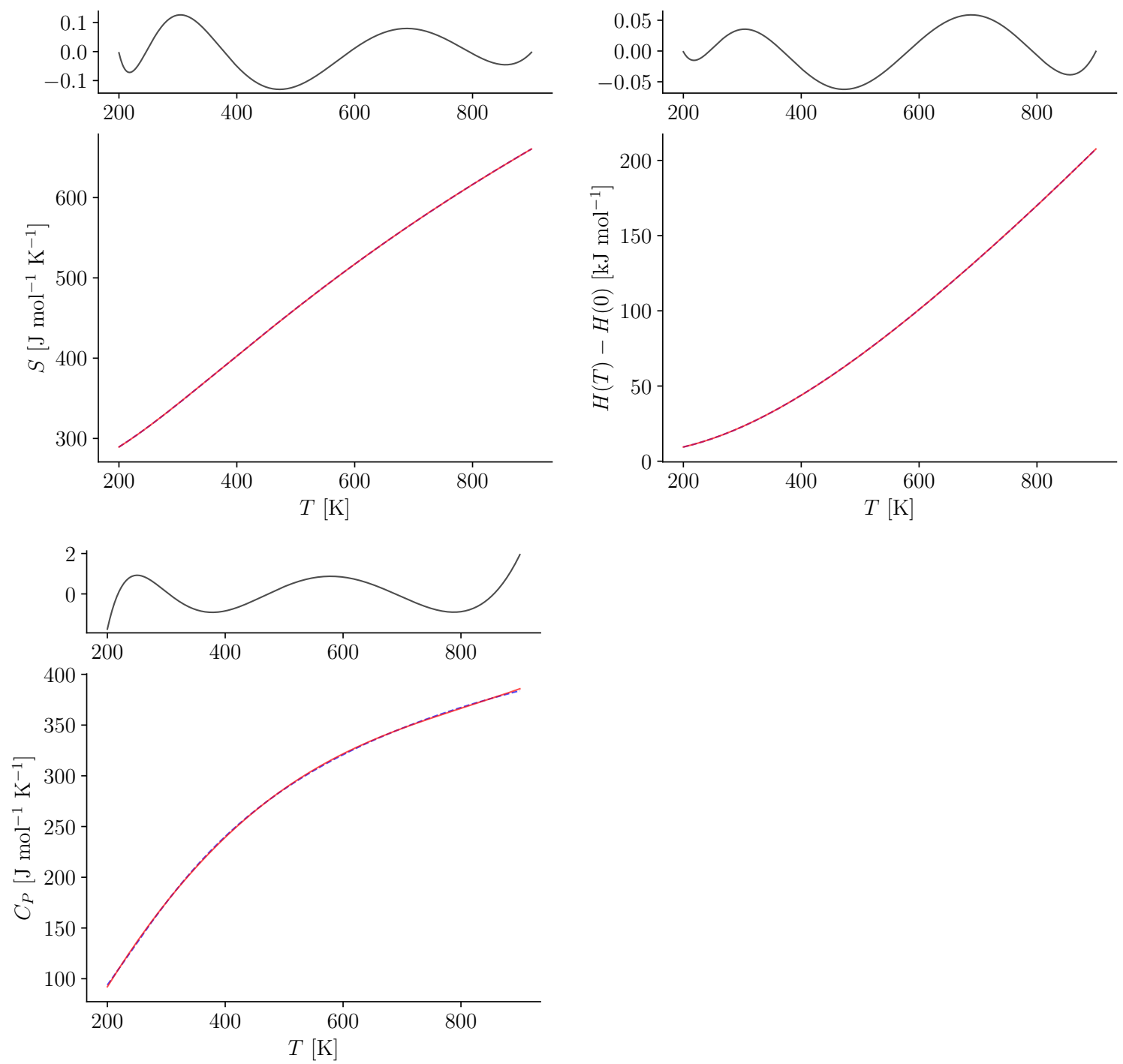


\section{$43 \quad \mathbf{B}_{11} \mathbf{H}_{14}$}

Figure S.43.1. Structure of $\mathrm{B}_{11} \mathrm{H}_{14}$

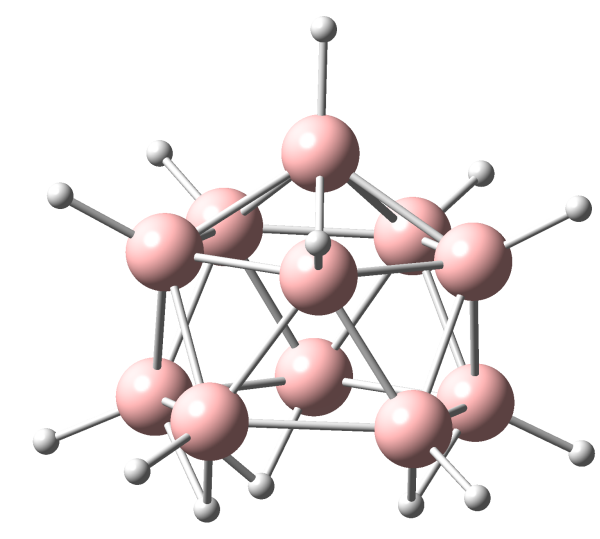

\begin{aligned} & \hline Charge -1 \\ & Spin multiplicity 1 \\ & Framework Group $\mathrm{C}_{1} \\ &$ Rotor Type Asymmetric Top \\ & Symmetry number 1 \\ & \hline\end{aligned}

Table S.43.1. $\mathrm{B}_{11} \mathrm{H}_{14}{ }^{-}$: Selected anharmonic results obtained at the B3LYP-D2/cc-pVTZ level

\begin{tabular}{rll}
\hline $\mathrm{ZPE}_{\mathrm{v}}$ & 39690 & $\mathrm{~cm}^{-1}$ \\
$\mathrm{~A}$ & 0.05753 & $\mathrm{~cm}^{-1}$ \\
$\mathrm{~B}$ & 0.05748 & $\mathrm{~cm}^{-1}$ \\
$\mathrm{C}$ & 0.04890 & $\mathrm{~cm}^{-1}$ \\
\hline$C_{P}(300 \mathrm{~K})$ & 208.922 & $\mathrm{~J} \mathrm{~mol}^{-1} \mathrm{~K}-1$ \\
$S(300 \mathrm{~K})$ & 369.391 & $\mathrm{~J} \mathrm{~mol}^{-1} \mathrm{~K}-1$ \\
$H(300 \mathrm{~K})-H(0 \mathrm{~K})$ & 27.101 & $\mathrm{~kJ} \mathrm{~mol}^{-1}$ \\
\hline
\end{tabular}

$\mathrm{ZPE}_{\mathrm{v}}$ : vibrational contribution to the zero-point energy. A, B, C: Rotational constants. $C_{P}$ : Heat capacity at constant pressure. $S$ : Entropy. $H$ : Enthalpy. Ideal-gas calculations (1 bar). 
Table S.43.2. $\mathrm{B}_{11} \mathrm{H}_{14}{ }^{-}$: Cartesian coordinates $(\AA)$ of the B3LYP-D2/cc-pVTZ optimized structure

\begin{tabular}{cccc} 
atom & $\mathrm{x}$ & $\mathrm{y}$ & $\mathrm{z}$ \\
\hline B1 & -1.5980 & 0.0015 & -0.8218 \\
B2 & 0.4997 & -1.4183 & 0.6110 \\
B3 & -0.5359 & -1.5692 & -0.8014 \\
B4 & 1.2835 & -0.9328 & -0.8782 \\
B5 & 1.5717 & -0.0015 & 0.6062 \\
B6 & 0.5024 & 1.4174 & 0.6110 \\
B7 & 0.0510 & -0.0000 & 1.5707 \\
B8 & -1.2054 & -0.8910 & 0.6942 \\
B9 & -0.5329 & 1.5702 & -0.8014 \\
B10 & -1.2037 & 0.8933 & 0.6942 \\
B11 & 1.2853 & 0.9304 & -0.8782 \\
H12 & -2.6823 & 0.0026 & -1.2977 \\
H13 & -0.8121 & -0.8180 & -1.7517 \\
H14 & 0.8373 & -2.4563 & 1.0814 \\
H15 & -0.9009 & -2.6469 & -1.1485 \\
H16 & 2.1306 & -1.5711 & -1.4114 \\
H17 & 0.9528 & -0.0009 & -1.7427 \\
H18 & 2.6230 & -0.0025 & 1.1580 \\
H19 & 0.8421 & 2.4547 & 1.0814 \\
H20 & 0.0845 & -0.0001 & 2.7569 \\
H21 & -2.0472 & -1.4924 & 1.2772 \\
H22 & -0.8104 & 0.8196 & -1.7517 \\
H23 & -0.8958 & 2.6486 & -1.1485 \\
\hline & -2.0443 & 1.4964 & 1.2772 \\
2.1336 & 1.5670 & -1.4114 \\
\hline & & & \\
\hline
\end{tabular}


Table S.43.3. $\mathrm{B}_{11} \mathrm{H}_{14}{ }^{-}$: Vibrational frequencies, infrared integrated intensities and Raman activities (B3LYP-D2/cc-pVTZ results)

\begin{tabular}{|c|c|c|c|c|c|c|c|}
\hline \multirow[b]{2}{*}{ mode } & \multirow[b]{2}{*}{ symm. } & \multicolumn{3}{|c|}{ Harmonic } & \multicolumn{3}{|c|}{ Anharmonic } \\
\hline & & $\begin{array}{c}\omega \\
{\left[\mathrm{cm}^{-1}\right]}\end{array}$ & $\begin{array}{c}\mathrm{IR} \\
{\left[\mathrm{km} \mathrm{mol}^{-1}\right]}\end{array}$ & $\begin{array}{c}\text { Raman } \\
{\left[\AA^{6}\right]}\end{array}$ & $\begin{array}{c}\omega \\
{\left[\mathrm{cm}^{-1}\right]}\end{array}$ & $\begin{array}{c}\mathrm{IR} \\
{\left[\mathrm{km} \mathrm{mol}^{-1}\right]}\end{array}$ & $\begin{array}{c}\text { Raman } \\
{\left[\AA^{6}\right]}\end{array}$ \\
\hline 1 & $\mathrm{~A}$ & 364 & 6.640 & 0.153 & 189 & 11.789 & 0.136 \\
\hline 2 & $\mathrm{~A}$ & 404 & 3.078 & 0.179 & 365 & 4.952 & 0.166 \\
\hline 3 & $\mathrm{~A}$ & 420 & 0.363 & 0.106 & 408 & 0.205 & 0.111 \\
\hline 4 & $\mathrm{~A}$ & 457 & 1.049 & 0.017 & 427 & 1.896 & 0.015 \\
\hline 5 & $\mathrm{~A}$ & 471 & 9.369 & 0.006 & 458 & 9.262 & 0.016 \\
\hline 6 & $\mathrm{~A}$ & 527 & 4.175 & 0.011 & 495 & 1.626 & 0.044 \\
\hline 7 & $\mathrm{~A}$ & 544 & 0.092 & 0.204 & 526 & 0.115 & 0.354 \\
\hline 8 & $\mathrm{~A}$ & 556 & 0.533 & 0.135 & 527 & 0.863 & 0.268 \\
\hline 9 & $\mathrm{~A}$ & 560 & 0.425 & 0.149 & 539 & 0.989 & 0.224 \\
\hline 10 & $\mathrm{~A}$ & 564 & 0.543 & 0.201 & 556 & 0.909 & 0.265 \\
\hline 11 & $\mathrm{~A}$ & 570 & 1.796 & 0.065 & 544 & 0.003 & 0.234 \\
\hline 12 & $\mathrm{~A}$ & 600 & 1.492 & 0.184 & 577 & 0.730 & 0.138 \\
\hline 13 & $\mathrm{~A}$ & 602 & 1.203 & 0.004 & 575 & 4.002 & 0.021 \\
\hline 14 & $\mathrm{~A}$ & 657 & 5.005 & 0.008 & 631 & 8.062 & 0.004 \\
\hline 15 & $\mathrm{~A}$ & 663 & 2.406 & 0.033 & 606 & 0.541 & 0.030 \\
\hline 16 & $\mathrm{~A}$ & 676 & 10.251 & 0.154 & 535 & 35.398 & 3.615 \\
\hline 17 & $\mathrm{~A}$ & 703 & 5.349 & 0.099 & 644 & 3.253 & 1.579 \\
\hline 18 & $\mathrm{~A}$ & 715 & 0.002 & 0.004 & 691 & 0.026 & 0.001 \\
\hline 19 & $\mathrm{~A}$ & 722 & 2.648 & 0.213 & 630 & 6.173 & 6.875 \\
\hline 20 & $\mathrm{~A}$ & 726 & 0.541 & 0.026 & 683 & 254.501 & 9.861 \\
\hline 21 & $\mathrm{~A}$ & 728 & 3.315 & 0.022 & 701 & 4.003 & 0.060 \\
\hline 22 & $\mathrm{~A}$ & 735 & 2.326 & 0.462 & 718 & 13.498 & 1.114 \\
\hline 23 & $\mathrm{~A}$ & 749 & 5.345 & 0.657 & 732 & 3.339 & 0.739 \\
\hline
\end{tabular}

To be continued 
Table S.43.3 - Continued

\begin{tabular}{|c|c|c|c|c|c|c|c|}
\hline \multirow[b]{2}{*}{ mode } & \multirow[b]{2}{*}{ symm. } & \multicolumn{3}{|c|}{ Harmonic } & \multicolumn{3}{|c|}{ Anharmonic } \\
\hline & & $\begin{array}{c}\omega \\
{\left[\mathrm{cm}^{-1}\right]} \\
\end{array}$ & $\begin{array}{c}\mathrm{IR} \\
{\left[\mathrm{km} \mathrm{mol}^{-1}\right]} \\
\end{array}$ & $\begin{array}{c}\text { Raman } \\
{\left[\AA^{6}\right]}\end{array}$ & $\begin{array}{c}\omega \\
{\left[\mathrm{cm}^{-1}\right]} \\
\end{array}$ & $\begin{array}{c}\mathrm{IR} \\
{\left[\mathrm{km} \mathrm{mol}^{-1}\right]} \\
\end{array}$ & $\begin{array}{c}\text { Raman } \\
{\left[\AA^{6}\right]}\end{array}$ \\
\hline 24 & $\mathrm{~A}$ & 755 & 6.882 & 0.054 & 732 & 1.367 & 0.054 \\
\hline 25 & $\mathrm{~A}$ & 787 & 1.327 & 0.066 & 763 & 5.283 & 0.084 \\
\hline 26 & $\mathrm{~A}$ & 793 & 0.581 & 0.018 & 763 & 1.389 & 0.021 \\
\hline 27 & $\mathrm{~A}$ & 798 & 0.783 & 0.052 & 777 & 0.672 & 0.029 \\
\hline 28 & $\mathrm{~A}$ & 802 & 0.885 & 0.046 & 778 & 1.327 & 0.052 \\
\hline 29 & $\mathrm{~A}$ & 812 & 0.941 & 0.060 & 786 & 0.056 & 0.103 \\
\hline 30 & $\mathrm{~A}$ & 823 & 0.821 & 0.045 & 799 & 2.478 & 0.050 \\
\hline 31 & $\mathrm{~A}$ & 825 & 1.915 & 0.013 & 803 & 3.872 & 0.000 \\
\hline 32 & $\mathrm{~A}$ & 835 & 0.942 & 0.606 & 827 & 3.363 & 0.304 \\
\hline 33 & $\mathrm{~A}$ & 849 & 1.174 & 0.001 & 765 & 4.909 & 0.064 \\
\hline 34 & $\mathrm{~A}$ & 880 & 1.191 & 0.206 & 856 & 1.751 & 0.393 \\
\hline 35 & $\mathrm{~A}$ & 907 & 0.531 & 0.089 & 887 & 0.484 & 0.077 \\
\hline 36 & $\mathrm{~A}$ & 924 & 1.573 & 0.033 & 881 & 0.415 & 0.027 \\
\hline 37 & $\mathrm{~A}$ & 939 & 0.111 & 0.022 & 911 & 0.005 & 0.045 \\
\hline 38 & $\mathrm{~A}$ & 951 & 0.160 & 0.022 & 921 & 0.364 & 0.051 \\
\hline 39 & $\mathrm{~A}$ & 953 & 4.073 & 0.003 & 927 & 3.170 & 0.006 \\
\hline 40 & $\mathrm{~A}$ & 957 & 1.236 & 0.035 & 926 & 3.910 & 0.028 \\
\hline 41 & $\mathrm{~A}$ & 966 & 0.041 & 0.020 & 941 & 0.040 & 0.001 \\
\hline 42 & $\mathrm{~A}$ & 974 & 1.205 & 0.021 & 946 & 0.934 & 0.036 \\
\hline 43 & $\mathrm{~A}$ & 976 & 0.771 & 0.005 & 946 & 0.843 & 0.001 \\
\hline 44 & $\mathrm{~A}$ & 980 & 0.437 & 0.006 & 950 & 0.551 & 0.004 \\
\hline 45 & $\mathrm{~A}$ & 986 & 4.895 & 0.008 & 952 & 7.419 & 0.035 \\
\hline 46 & $\mathrm{~A}$ & 997 & 0.582 & 0.005 & 963 & 0.017 & 0.009 \\
\hline 47 & $\mathrm{~A}$ & 1003 & 0.592 & 0.056 & 931 & 1.504 & 0.031 \\
\hline To be & ntinued & & & & & & \\
\hline
\end{tabular}


Table S.43.3 - Continued

\begin{tabular}{|c|c|c|c|c|c|c|c|}
\hline \multirow[b]{2}{*}{ mode } & \multirow[b]{2}{*}{ symm. } & \multicolumn{3}{|c|}{ Harmonic } & \multicolumn{3}{|c|}{ Anharmonic } \\
\hline & & $\begin{array}{c}\omega \\
{\left[\mathrm{cm}^{-1}\right]}\end{array}$ & $\begin{array}{c}\mathrm{IR} \\
{\left[\mathrm{km} \mathrm{mol}^{-1}\right]}\end{array}$ & $\begin{array}{c}\text { Raman } \\
{\left[\AA^{6}\right]}\end{array}$ & $\begin{array}{c}\omega \\
{\left[\mathrm{cm}^{-1}\right]}\end{array}$ & $\begin{array}{c}\mathrm{IR} \\
{\left[\mathrm{km} \mathrm{mol}^{-1}\right]}\end{array}$ & $\begin{array}{c}\text { Raman } \\
{\left[\AA^{6}\right]}\end{array}$ \\
\hline 48 & $\mathrm{~A}$ & 1037 & 7.809 & 0.050 & 972 & 26.519 & 0.194 \\
\hline 49 & $\mathrm{~A}$ & 1044 & 17.414 & 0.073 & 1014 & 29.140 & 0.140 \\
\hline 50 & $\mathrm{~A}$ & 1073 & 39.851 & 0.022 & 1037 & 13.134 & 0.006 \\
\hline 51 & $\mathrm{~A}$ & 1089 & 24.196 & 0.043 & 1042 & 29.217 & 0.017 \\
\hline 52 & $\mathrm{~A}$ & 1197 & 5.692 & 0.032 & 1182 & 1.087 & 0.016 \\
\hline 53 & $\mathrm{~A}$ & 1225 & 0.397 & 0.105 & 1138 & 0.118 & 0.011 \\
\hline 54 & $\mathrm{~A}$ & 1403 & 16.846 & 0.159 & 1381 & 5.840 & 0.028 \\
\hline 55 & $\mathrm{~A}$ & 1703 & 10.453 & 0.033 & 1528 & 0.002 & 0.001 \\
\hline 56 & $\mathrm{~A}$ & 2002 & 5.910 & 0.134 & 1882 & 0.280 & 0.005 \\
\hline 57 & $\mathrm{~A}$ & 2256 & 28.027 & 0.005 & 2197 & 9.596 & 0.000 \\
\hline 58 & $\mathrm{~A}$ & 2348 & 10.301 & 0.334 & 2230 & 5.464 & 0.088 \\
\hline 59 & $\mathrm{~A}$ & 2616 & 119.431 & 0.786 & 2504 & 205.361 & 0.719 \\
\hline 60 & $\mathrm{~A}$ & 2617 & 80.296 & 0.848 & 2504 & 4.712 & 1.050 \\
\hline 61 & $\mathrm{~A}$ & 2635 & 17.951 & 0.308 & 2519 & 3.481 & 0.434 \\
\hline 62 & $\mathrm{~A}$ & 2635 & 2.068 & 0.933 & 2524 & 133.101 & 0.801 \\
\hline 63 & $\mathrm{~A}$ & 2642 & 44.991 & 1.190 & 2521 & 0.472 & 1.254 \\
\hline 64 & $\mathrm{~A}$ & 2643 & 52.286 & 0.540 & 2531 & 2.710 & 0.573 \\
\hline 65 & $\mathrm{~A}$ & 2646 & 145.451 & 1.008 & 2519 & 72.255 & 1.486 \\
\hline 66 & $\mathrm{~A}$ & 2648 & 438.549 & 0.184 & 2540 & 406.725 & 0.364 \\
\hline 67 & $\mathrm{~A}$ & 2655 & 420.656 & 0.550 & 2544 & 535.847 & 0.098 \\
\hline 68 & $\mathrm{~A}$ & 2668 & 259.714 & 2.607 & 2585 & 45.944 & 5.546 \\
\hline 69 & $\mathrm{~A}$ & 2683 & 102.122 & 2.732 & 2512 & 220.731 & 0.487 \\
\hline
\end{tabular}


Figure S.43.2. Anharmonic IR spectrum of $\mathrm{B}_{11} \mathrm{H}_{14}{ }^{-}$obtained by convoluting the calculated intensities with Lorentzians having a FWHM of $4 \mathrm{~cm}^{-1}$ (B3LYP-D2/cc-pVTZ results): (top) full spectrum, (middle) contributions from fundamentals and combination bands, (bottom) contributions from overtones
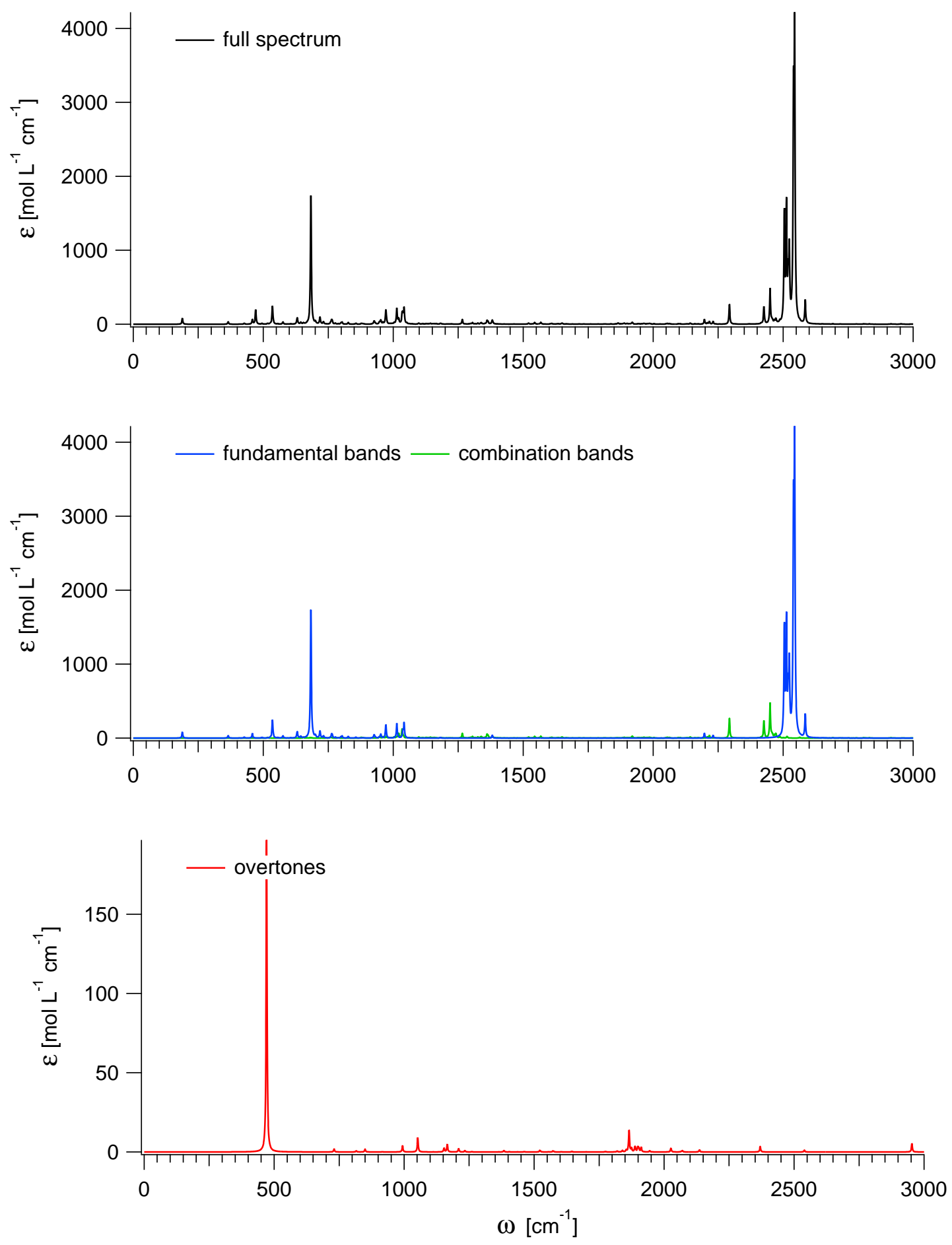
Figure S.43.3. Anharmonic Raman spectrum of $\mathrm{B}_{11} \mathrm{H}_{14}{ }^{-}$obtained by convoluting the calculated activities with Lorentzians having a FWHM of $4 \mathrm{~cm}^{-1}$ (B3LYP-D2/cc-pVTZ results): (top) full spectrum, (middle) contributions from fundamentals and combination bands, (bottom) contributions from overtones
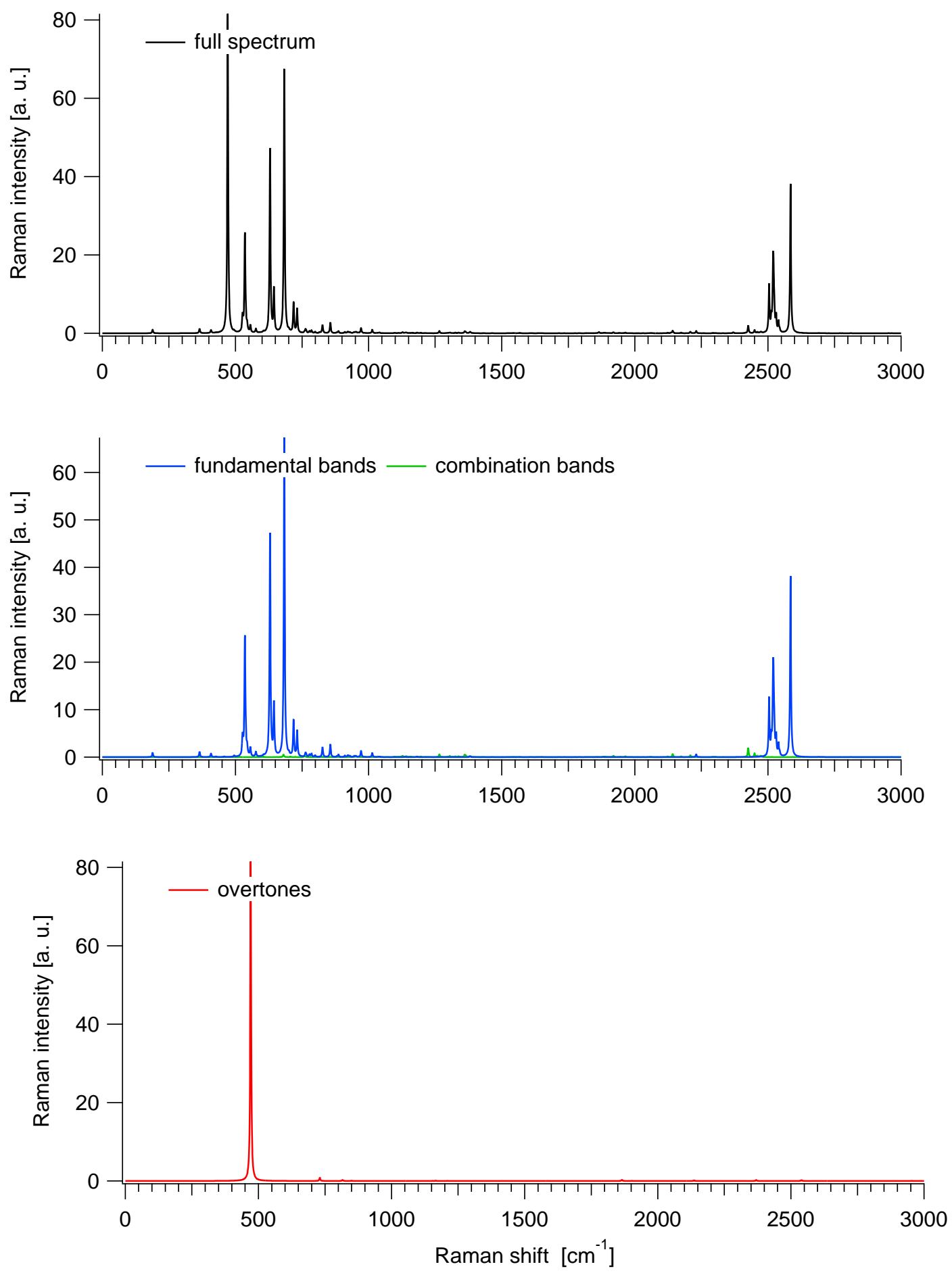
Table S.43.4. $\mathrm{B}_{11} \mathrm{H}_{14}{ }^{-}$: Ideal-gas thermodynamic functions at 1 bar (anharmonic B3LYP$\mathrm{D} 2 / \mathrm{cc}-\mathrm{pVTZ}$ results)

\begin{tabular}{cccc}
\hline$T$ & $C_{P}$ & $S$ & $H(T)-H(0 K)$ \\
{$[\mathrm{K}]$} & {$\left[\mathrm{J} \mathrm{mol}^{-1} \mathrm{~K}^{-1}\right]$} & {$\left[\mathrm{J} \mathrm{mol}^{-1} \mathrm{~K}^{-1}\right]$} & {$\left[\mathrm{kJ} \mathrm{mol}^{-1}\right]$} \\
\hline 100 & 43.731 & 256.941 & 3.573 \\
200 & 113.605 & 305.090 & 10.918 \\
300 & 208.922 & 369.391 & 27.101 \\
400 & 286.714 & 440.690 & 52.071 \\
500 & 344.174 & 511.162 & 83.760 \\
600 & 387.429 & 577.909 & 120.435 \\
700 & 421.203 & 640.269 & 160.932 \\
800 & 448.290 & 698.344 & 204.454 \\
900 & 470.371 & 752.463 & 250.423 \\
1000 & 488.557 & 802.993 & 298.399 \\
1100 & 503.650 & 850.287 & 348.032 \\
1200 & 516.258 & 894.667 & 399.046 \\
1300 & 526.855 & 936.420 & 451.217 \\
1400 & 535.817 & 975.802 & 504.363 \\
1500 & 543.440 & 1013.036 & 558.335 \\
1600 & 549.964 & 1048.323 & 613.014 \\
1700 & 555.579 & 1081.837 & 668.298 \\
1800 & 560.437 & 1113.733 & 724.105 \\
1900 & 564.664 & 1144.151 & 780.364 \\
2000 & 568.360 & 1173.210 & 837.020 \\
\hline & & & $S$ \\
\hline
\end{tabular}

$T$ : Temperature. $C_{P}$ : Heat capacity at constant pressure. $S$ : Entropy. $H$ : Enthalpy. 
Figure S.43.4. $\mathrm{B}_{11} \mathrm{H}_{14}{ }^{-}$: Ideal-gas thermodynamic functions at 1 bar (anharmonic B3LYPD2/cc-pVTZ results)
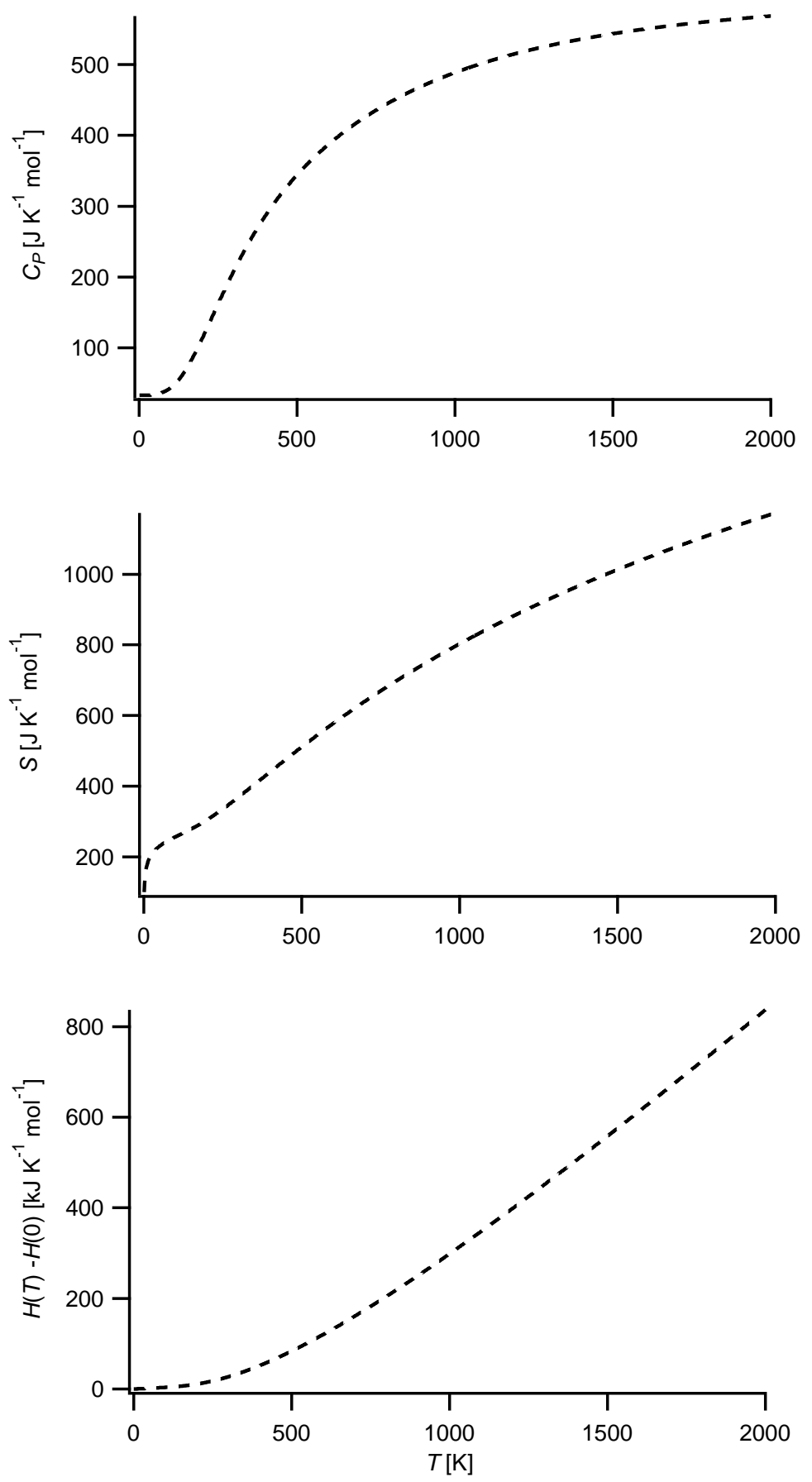

$T$ : Temperature. $C_{P}$ : Heat capacity at constant pressure. $S$ : Entropy. $H$ : Enthalpy. 
Table S.43.5. $\mathrm{B}_{11} \mathrm{H}_{14}{ }^{-}$: Fits (red solid lines) of the computed thermodynamic functions (blue dashed lines) with NASA type functions (Equations (1) - (3)) in the $200-900 \mathrm{~K}$ temperature range (anharmonic B3LYP-D2/cc-pVTZ results). In each case, the difference curve between the thermodynamic and NASA functions is plotted in the upper graph

Fit parameters

\begin{tabular}{llllll}
\hline $\mathrm{a}_{1}$ & $-1.80362672 \mathrm{e}+01$ & $\mathrm{a}_{2}\left[\mathrm{~K}^{-1}\right]$ & $1.85243695 \mathrm{e}-01$ & $\mathrm{a}_{3}\left[\mathrm{~K}^{-2}\right]$ & $-1.39851736 \mathrm{e}-04$ \\
$\mathrm{a}_{4}\left[\mathrm{~K}^{-3}\right]$ & $-4.04853619 \mathrm{e}-09$ & $\mathrm{a}_{5}\left[\mathrm{~K}^{-4}\right]$ & $3.71584656 \mathrm{e}-11$ & $\mathrm{a}_{6}[\mathrm{~K}]$ & $1.58759546 \mathrm{e}+03$ \\
$\mathrm{a}_{7}$ & $9.79994828 \mathrm{e}+01$ & & & &
\end{tabular}
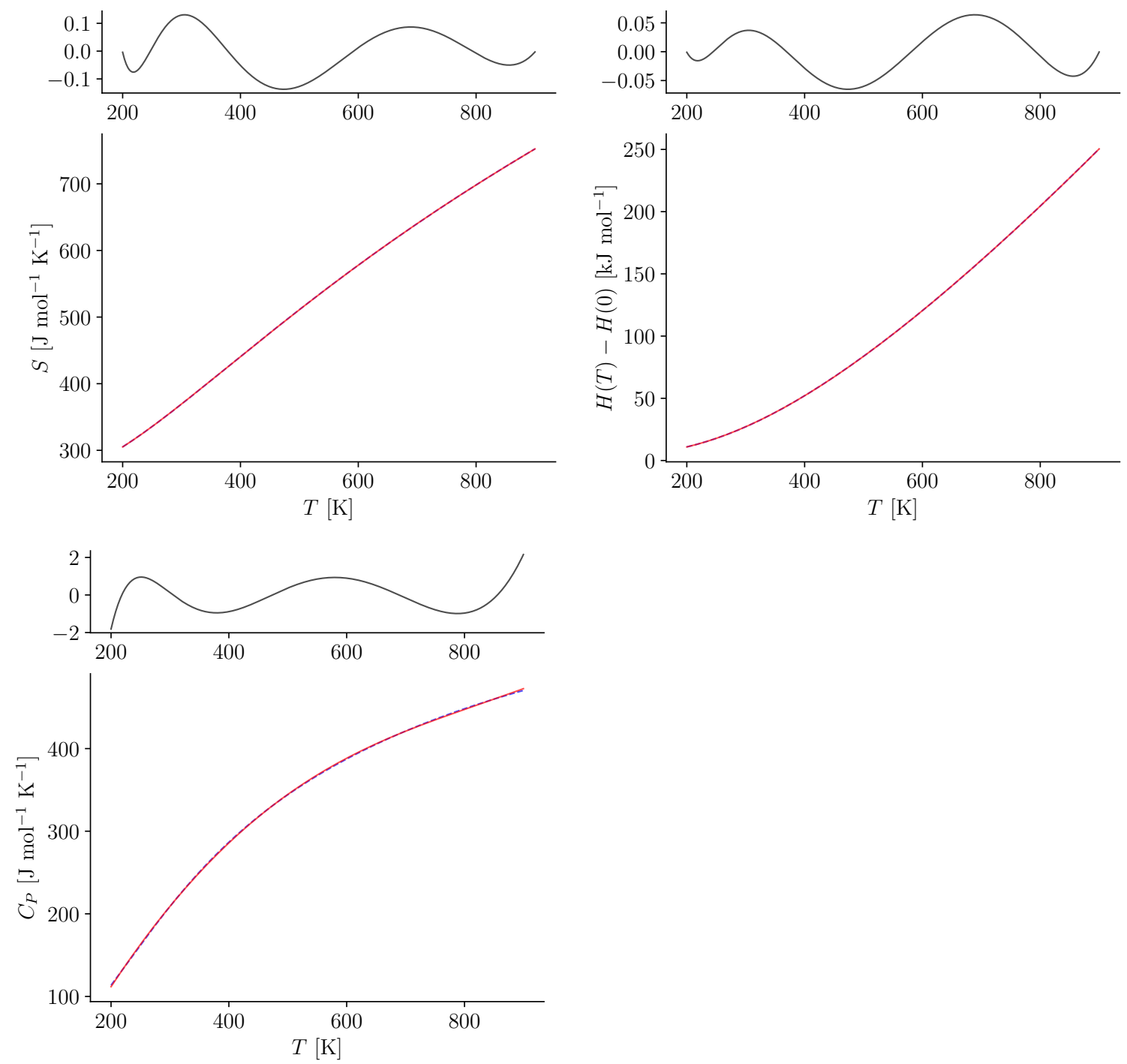


\section{$44 \mathrm{~B}_{12} \mathbf{H}_{12}{ }^{2-}$}

Figure S.44.1. Structure of $\mathrm{B}_{12} \mathrm{H}_{12}{ }^{2-}$

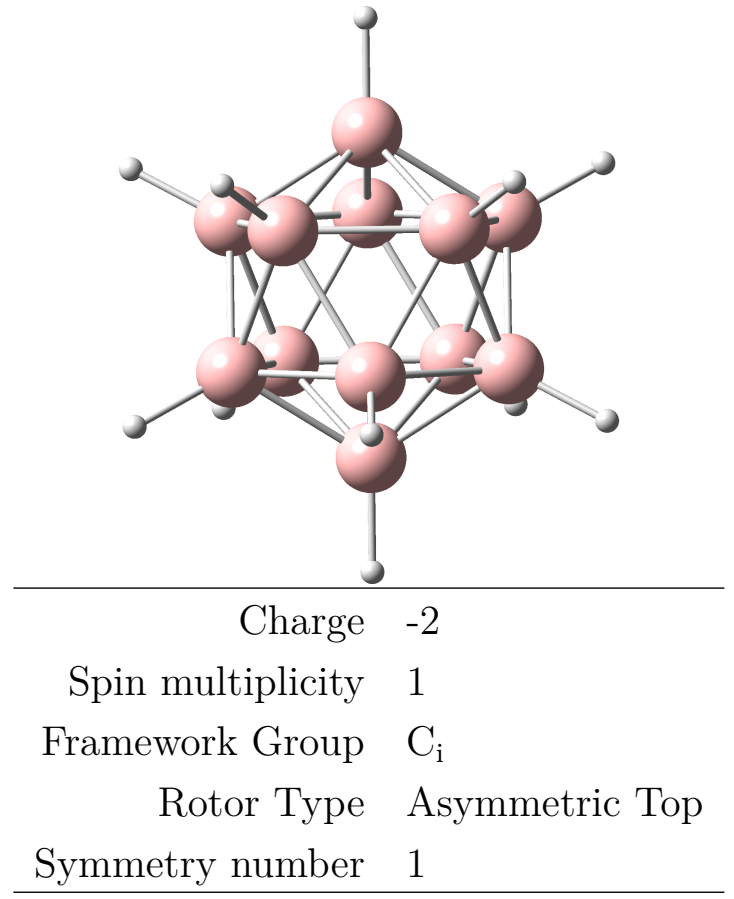

Table S.44.1. $\mathrm{B}_{12} \mathrm{H}_{12}{ }^{2-}$ : Selected anharmonic results obtained at the B3LYP-D2/cc-pVTZ level

\begin{tabular}{rll}
\hline $\mathrm{ZPE}_{\mathrm{v}}$ & 36515 & $\mathrm{~cm}^{-1}$ \\
$\mathrm{~A}$ & 0.05231 & $\mathrm{~cm}^{-1}$ \\
$\mathrm{~B}$ & 0.05230 & $\mathrm{~cm}^{-1}$ \\
$\mathrm{C}$ & 0.05230 & $\mathrm{~cm}^{-1}$ \\
\hline$C_{P}(300 \mathrm{~K})$ & 195.877 & $\mathrm{~J} \mathrm{~mol}^{-1} \mathrm{~K}-1$ \\
$S(300 \mathrm{~K})$ & 352.947 & $\mathrm{~J} \mathrm{~mol}^{-1} \mathrm{~K}-1$ \\
$H(300 \mathrm{~K})-H(0 \mathrm{~K})$ & 24.055 & $\mathrm{~kJ} \mathrm{~mol}^{-1}$ \\
\hline
\end{tabular}

$\mathrm{ZPE}_{\mathrm{v}}$ : vibrational contribution to the zero-point energy. A, B, C: Rotational constants. $C_{P}$ : Heat capacity at constant pressure. $S$ : Entropy. $H$ : Enthalpy. Ideal-gas calculations (1 bar). 
Table S.44.2. $\mathrm{B}_{12} \mathrm{H}_{12}{ }^{2-}$ : Cartesian coordinates $(\AA)$ of the B3LYP-D2/cc-pVTZ optimized structure

\begin{tabular}{cccc} 
atom & $\mathrm{x}$ & $\mathrm{y}$ & $\mathrm{z}$ \\
\hline B1 & -0.0000 & 0.0001 & -1.7003 \\
B2 & 1.4462 & -0.4698 & -0.7604 \\
B3 & 0.8938 & 1.2302 & -0.7604 \\
B4 & -0.8938 & 1.2303 & -0.7603 \\
B5 & -1.4462 & -0.4698 & -0.7604 \\
B6 & -0.8938 & -1.2302 & 0.7604 \\
B7 & -1.4462 & 0.4698 & 0.7604 \\
B8 & -0.0000 & 1.5206 & 0.7603 \\
B9 & 1.4462 & 0.4698 & 0.7604 \\
B10 & 0.0000 & -0.0001 & 1.7003 \\
B11 & 0.8938 & -1.2303 & 0.7603 \\
B12 & 0.0000 & -1.5206 & -0.7603 \\
H13 & 2.4652 & -0.8009 & -1.2961 \\
H14 & 1.5236 & -2.0971 & 1.2961 \\
H15 & 2.4652 & 0.8010 & 1.2961 \\
H16 & -1.5236 & -2.0970 & 1.2961 \\
H17 & -2.4652 & 0.8009 & 1.2961 \\
H18 & 0.0000 & 2.5921 & 1.2960 \\
H19 & -0.0000 & 0.0001 & 2.8982 \\
H20 & 0.0000 & -0.0001 & -2.8982 \\
H21 & 1.5236 & 2.0970 & -1.2961 \\
H22 & -1.5236 & 2.0971 & -1.2961 \\
H23 & -2.4652 & -0.8010 & -1.2961 \\
H24 & -0.0000 & -2.5921 & -1.2960 \\
\hline & & &
\end{tabular}


Table S.44.3. $\mathrm{B}_{12} \mathrm{H}_{12}{ }^{2-}$ : Vibrational frequencies, infrared integrated intensities and Raman activities (B3LYP-D2/cc-pVTZ results)

\begin{tabular}{|c|c|c|c|c|c|c|c|}
\hline \multirow[b]{2}{*}{ mode } & \multirow[b]{2}{*}{ symm. } & \multicolumn{3}{|c|}{ Harmonic } & \multicolumn{3}{|c|}{ Anharmonic } \\
\hline & & $\begin{array}{c}\omega \\
{\left[\mathrm{cm}^{-1}\right]}\end{array}$ & $\begin{array}{c}\mathrm{IR} \\
{\left[\mathrm{km} \mathrm{mol}^{-1}\right]}\end{array}$ & $\begin{array}{c}\text { Raman } \\
{\left[\AA^{6}\right]}\end{array}$ & $\begin{array}{c}\omega \\
{\left[\mathrm{cm}^{-1}\right]}\end{array}$ & $\begin{array}{c}\mathrm{IR} \\
{\left[\mathrm{km} \mathrm{mol}^{-1}\right]}\end{array}$ & $\begin{array}{c}\text { Raman } \\
{\left[\AA^{6}\right]}\end{array}$ \\
\hline 1 & $\mathrm{~A}_{\mathrm{u}}$ & 525 & 0.000 & 0.000 & 508 & 0.000 & 0.000 \\
\hline 2 & $\mathrm{~A}_{\mathrm{u}}$ & 525 & 0.000 & 0.000 & 508 & 0.000 & 0.000 \\
\hline 3 & $\mathrm{~A}_{\mathrm{u}}$ & 525 & 0.000 & 0.000 & 508 & 0.000 & 0.000 \\
\hline 4 & $\mathrm{~A}_{\mathrm{u}}$ & 525 & 0.000 & 0.000 & 507 & 0.000 & 0.000 \\
\hline 5 & $\mathrm{~A}_{\mathrm{u}}$ & 526 & 0.000 & 0.000 & 508 & 0.000 & 0.000 \\
\hline 6 & $\mathrm{~A}_{\mathrm{g}}$ & 578 & 0.000 & 0.141 & 569 & 0.000 & 0.145 \\
\hline 7 & $\mathrm{~A}_{\mathrm{g}}$ & 578 & 0.000 & 0.141 & 569 & 0.000 & 0.160 \\
\hline 8 & $\mathrm{~A}_{\mathrm{g}}$ & 579 & 0.000 & 0.141 & 569 & 0.000 & 0.147 \\
\hline 9 & $\mathrm{~A}_{\mathrm{g}}$ & 579 & 0.000 & 0.141 & 569 & 0.000 & 0.153 \\
\hline 10 & $\mathrm{~A}_{\mathrm{g}}$ & 579 & 0.000 & 0.141 & 568 & 0.000 & 0.152 \\
\hline 11 & $\mathrm{~A}_{\mathrm{g}}$ & 667 & 0.000 & 0.000 & 644 & 0.000 & 0.000 \\
\hline 12 & $\mathrm{~A}_{\mathrm{g}}$ & 667 & 0.000 & 0.000 & 644 & 0.000 & 0.000 \\
\hline 13 & $\mathrm{~A}_{\mathrm{g}}$ & 667 & 0.000 & 0.000 & 644 & 0.000 & 0.000 \\
\hline 14 & $\mathrm{~A}_{\mathrm{g}}$ & 668 & 0.000 & 0.000 & 643 & 0.000 & 0.000 \\
\hline 15 & $\mathrm{~A}_{\mathrm{u}}$ & 722 & 6.358 & 0.000 & 704 & 6.739 & 0.000 \\
\hline 16 & $\mathrm{~A}_{\mathrm{u}}$ & 723 & 6.372 & 0.000 & 703 & 6.801 & 0.000 \\
\hline 17 & $\mathrm{~A}_{\mathrm{u}}$ & 723 & 6.374 & 0.000 & 703 & 6.962 & 0.000 \\
\hline 18 & $\mathrm{~A}_{\mathrm{u}}$ & 756 & 0.001 & 0.000 & 728 & 0.000 & 0.000 \\
\hline 19 & $\mathrm{~A}_{\mathrm{u}}$ & 756 & 0.000 & 0.000 & 728 & 0.000 & 0.000 \\
\hline 20 & $\mathrm{~A}_{\mathrm{u}}$ & 756 & 0.000 & 0.000 & 728 & 0.000 & 0.000 \\
\hline 21 & $\mathrm{~A}_{\mathrm{u}}$ & 756 & 0.000 & 0.000 & 728 & 0.001 & 0.000 \\
\hline 22 & $\mathrm{~A}_{\mathrm{g}}$ & 757 & 0.000 & 2.918 & 741 & 0.000 & 3.199 \\
\hline 23 & $\mathrm{~A}_{\mathrm{u}}$ & 774 & 0.000 & 0.000 & 761 & 0.004 & 0.000 \\
\hline
\end{tabular}

To be continued 
Table S.44.3 - Continued

\begin{tabular}{|c|c|c|c|c|c|c|c|}
\hline \multirow[b]{2}{*}{ mode } & \multirow[b]{2}{*}{ symm. } & \multicolumn{3}{|c|}{ Harmonic } & \multicolumn{3}{|c|}{ Anharmonic } \\
\hline & & $\begin{array}{c}\omega \\
{\left[\mathrm{cm}^{-1}\right]}\end{array}$ & $\begin{array}{c}\mathrm{IR} \\
{\left[\mathrm{km} \mathrm{mol}^{-1}\right]} \\
\end{array}$ & $\begin{array}{c}\text { Raman } \\
{\left[\AA^{6}\right]}\end{array}$ & $\begin{array}{c}\omega \\
{\left[\mathrm{cm}^{-1}\right]} \\
\end{array}$ & $\begin{array}{c}\mathrm{IR} \\
{\left[\mathrm{km} \mathrm{mol}^{-1}\right]} \\
\end{array}$ & $\begin{array}{c}\text { Raman } \\
{\left[\AA^{6}\right]} \\
\end{array}$ \\
\hline 24 & $\mathrm{~A}_{\mathrm{u}}$ & 775 & 0.002 & 0.000 & 761 & 0.003 & 0.000 \\
\hline 25 & $\mathrm{~A}_{\mathrm{u}}$ & 775 & 0.000 & 0.000 & 760 & 0.000 & 0.000 \\
\hline 26 & $\mathrm{~A}_{\mathrm{g}}$ & 778 & 0.000 & 0.069 & 755 & 0.000 & 0.085 \\
\hline 27 & $\mathrm{~A}_{\mathrm{g}}$ & 778 & 0.000 & 0.069 & 756 & 0.000 & 0.088 \\
\hline 28 & $\mathrm{~A}_{\mathrm{g}}$ & 778 & 0.000 & 0.069 & 755 & 0.000 & 0.082 \\
\hline 29 & $\mathrm{~A}_{\mathrm{g}}$ & 779 & 0.000 & 0.069 & 755 & 0.000 & 0.085 \\
\hline 30 & $\mathrm{~A}_{\mathrm{g}}$ & 779 & 0.000 & 0.069 & 755 & 0.000 & 0.087 \\
\hline 31 & $\mathrm{~A}_{\mathrm{u}}$ & 865 & 0.000 & 0.000 & 847 & 0.001 & 0.000 \\
\hline 32 & $\mathrm{~A}_{\mathrm{u}}$ & 865 & 0.000 & 0.000 & 848 & 0.000 & 0.000 \\
\hline 33 & $\mathrm{~A}_{\mathrm{u}}$ & 865 & 0.000 & 0.000 & 848 & 0.001 & 0.000 \\
\hline 34 & $\mathrm{~A}_{\mathrm{u}}$ & 865 & 0.000 & 0.000 & 847 & 0.000 & 0.000 \\
\hline 35 & $\mathrm{~A}_{\mathrm{g}}$ & 951 & 0.000 & 0.106 & 928 & 0.000 & 0.181 \\
\hline 36 & $\mathrm{~A}_{\mathrm{g}}$ & 951 & 0.000 & 0.105 & 928 & 0.000 & 0.141 \\
\hline 37 & $\mathrm{~A}_{\mathrm{g}}$ & 952 & 0.000 & 0.106 & 927 & 0.000 & 0.189 \\
\hline 38 & $\mathrm{~A}_{\mathrm{g}}$ & 952 & 0.000 & 0.106 & 928 & 0.000 & 0.149 \\
\hline 39 & $A_{g}$ & 952 & 0.000 & 0.105 & 927 & 0.000 & 0.157 \\
\hline 40 & $\mathrm{~A}_{\mathrm{g}}$ & 956 & 0.000 & 0.000 & 933 & 0.000 & 0.000 \\
\hline 41 & $\mathrm{~A}_{\mathrm{g}}$ & 956 & 0.000 & 0.000 & 934 & 0.000 & 0.001 \\
\hline 42 & $\mathrm{~A}_{\mathrm{g}}$ & 956 & 0.000 & 0.000 & 933 & 0.000 & 0.002 \\
\hline 43 & $A_{g}$ & 957 & 0.000 & 0.000 & 933 & 0.000 & 0.000 \\
\hline 44 & $\mathrm{~A}_{\mathrm{u}}$ & 968 & 0.000 & 0.000 & 946 & 0.000 & 0.000 \\
\hline 45 & $\mathrm{~A}_{\mathrm{u}}$ & 968 & 0.000 & 0.000 & 945 & 0.004 & 0.000 \\
\hline 46 & $\mathrm{~A}_{\mathrm{u}}$ & 968 & 0.000 & 0.000 & 945 & 0.000 & 0.000 \\
\hline 47 & $\mathrm{~A}_{\mathrm{u}}$ & 968 & 0.000 & 0.000 & 944 & 0.002 & 0.000 \\
\hline
\end{tabular}

To be continued 
Table S.44.3 - Continued

\begin{tabular}{|c|c|c|c|c|c|c|c|}
\hline \multirow[b]{2}{*}{ mode } & \multirow[b]{2}{*}{ symm. } & \multicolumn{3}{|c|}{ Harmonic } & \multicolumn{3}{|c|}{ Anharmonic } \\
\hline & & $\begin{array}{c}\omega \\
{\left[\mathrm{cm}^{-1}\right]} \\
\end{array}$ & $\begin{array}{c}\mathrm{IR} \\
{\left[\mathrm{km} \mathrm{mol}^{-1}\right]} \\
\end{array}$ & $\begin{array}{c}\text { Raman } \\
{\left[\AA^{6}\right]}\end{array}$ & $\begin{array}{c}\omega \\
{\left[\mathrm{cm}^{-1}\right]} \\
\end{array}$ & $\begin{array}{c}\mathrm{IR} \\
{\left[\mathrm{km} \mathrm{mol}^{-1}\right]} \\
\end{array}$ & $\begin{array}{c}\text { Raman } \\
{\left[\AA^{6}\right]} \\
\end{array}$ \\
\hline 48 & $\mathrm{~A}_{\mathrm{u}}$ & 968 & 0.000 & 0.000 & 944 & 0.001 & 0.000 \\
\hline 49 & $\mathrm{~A}_{\mathrm{g}}$ & 989 & 0.000 & 0.000 & 963 & 0.000 & 0.000 \\
\hline 50 & $\mathrm{~A}_{\mathrm{g}}$ & 989 & 0.000 & 0.000 & 963 & 0.000 & 0.000 \\
\hline 51 & $\mathrm{~A}_{\mathrm{g}}$ & 989 & 0.000 & 0.000 & 963 & 0.000 & 0.000 \\
\hline 52 & $\mathrm{~A}_{\mathrm{u}}$ & 1086 & 43.790 & 0.000 & 1052 & 31.094 & 0.000 \\
\hline 53 & $\mathrm{~A}_{\mathrm{u}}$ & 1086 & 43.758 & 0.000 & 1052 & 30.822 & 0.000 \\
\hline 54 & $\mathrm{~A}_{\mathrm{u}}$ & 1086 & 43.773 & 0.000 & 1052 & 30.914 & 0.000 \\
\hline 55 & $\mathrm{~A}_{\mathrm{u}}$ & 2538 & 0.002 & 0.000 & 2407 & 155.087 & 0.000 \\
\hline 56 & $\mathrm{~A}_{\mathrm{u}}$ & 2539 & 0.001 & 0.000 & 2411 & 40.176 & 0.000 \\
\hline 57 & $\mathrm{~A}_{\mathrm{u}}$ & 2539 & 0.006 & 0.000 & 2406 & 162.202 & 0.000 \\
\hline 58 & $\mathrm{~A}_{\mathrm{g}}$ & 2544 & 0.000 & 1.599 & 2417 & 0.000 & 1.927 \\
\hline 59 & $\mathrm{~A}_{\mathrm{g}}$ & 2544 & 0.000 & 1.599 & 2413 & 0.000 & 1.903 \\
\hline 60 & $\mathrm{~A}_{\mathrm{g}}$ & 2544 & 0.000 & 1.599 & 2407 & 0.000 & 1.796 \\
\hline 61 & $\mathrm{~A}_{\mathrm{g}}$ & 2545 & 0.000 & 1.599 & 2416 & 0.000 & 1.838 \\
\hline 62 & $\mathrm{~A}_{\mathrm{g}}$ & 2545 & 0.000 & 1.599 & 2426 & 0.000 & 1.854 \\
\hline 63 & $\mathrm{~A}_{\mathrm{u}}$ & 2562 & 185.500 & 0.000 & 2441 & 51.273 & 0.000 \\
\hline 64 & $\mathrm{~A}_{\mathrm{u}}$ & 2562 & 185.519 & 0.000 & 2435 & 236.748 & 0.000 \\
\hline 65 & $\mathrm{~A}_{\mathrm{u}}$ & 2562 & 185.331 & 0.000 & 2438 & 70.154 & 0.000 \\
\hline 66 & $\mathrm{~A}_{\mathrm{g}}$ & 2597 & 0.000 & 6.042 & 2475 & 0.000 & 6.464 \\
\hline
\end{tabular}


Figure S.44.2. Anharmonic IR spectrum of $\mathrm{B}_{12} \mathrm{H}_{12}{ }^{2-}$ obtained by convoluting the calculated intensities with Lorentzians having a FWHM of $4 \mathrm{~cm}^{-1}$ (B3LYP-D2/cc-pVTZ results): (top) full spectrum, (bottom) contributions from fundamentals and combination bands
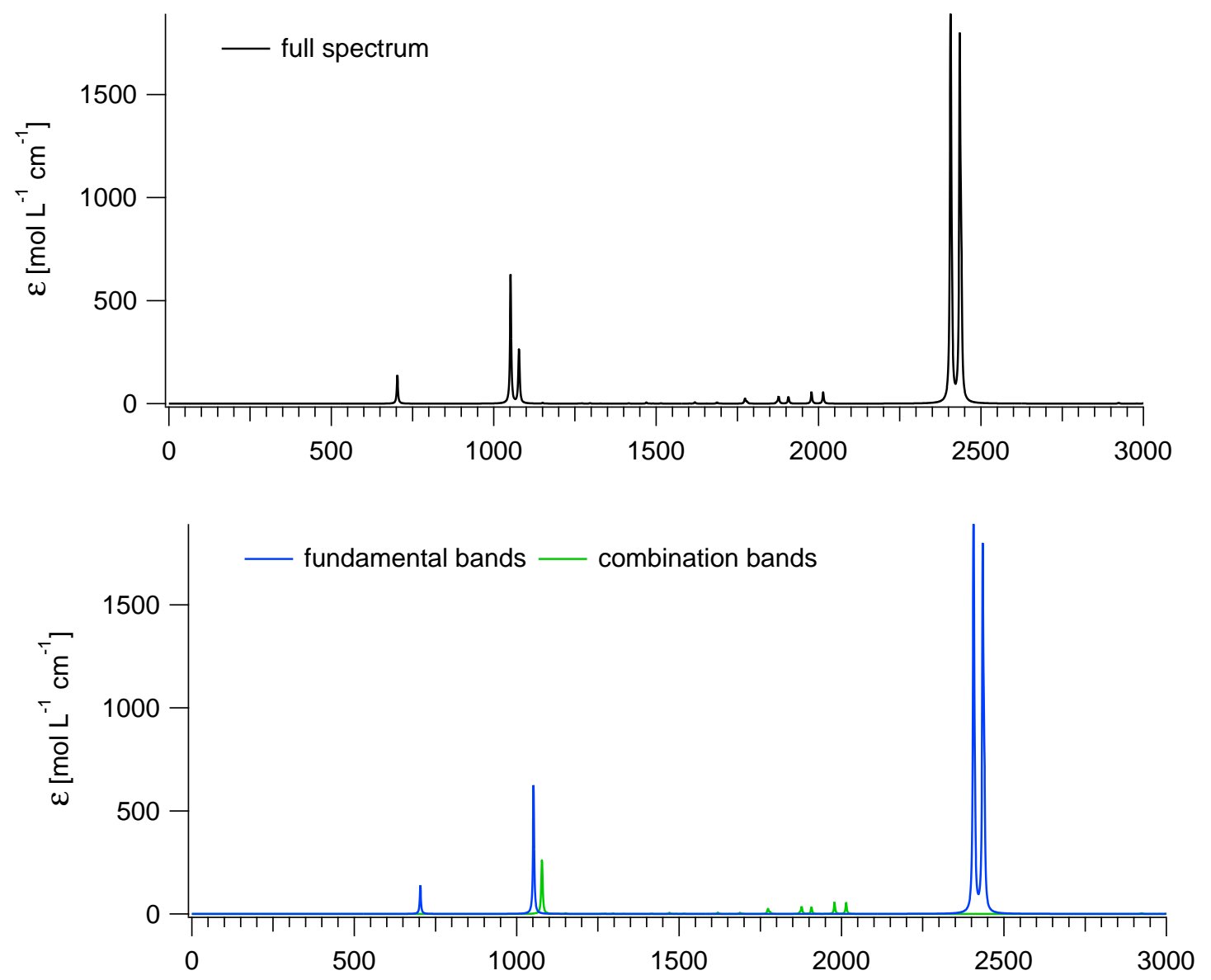
Figure S.44.3. Anharmonic Raman spectrum of $\mathrm{B}_{12} \mathrm{H}_{12}{ }^{2-}$ obtained by convoluting the calculated activities with Lorentzians having a FWHM of $4 \mathrm{~cm}^{-1}$ (B3LYP-D2/cc-pVTZ results): (top) full spectrum, (middle) contributions from fundamentals and combination bands, (bottom) contributions from overtones
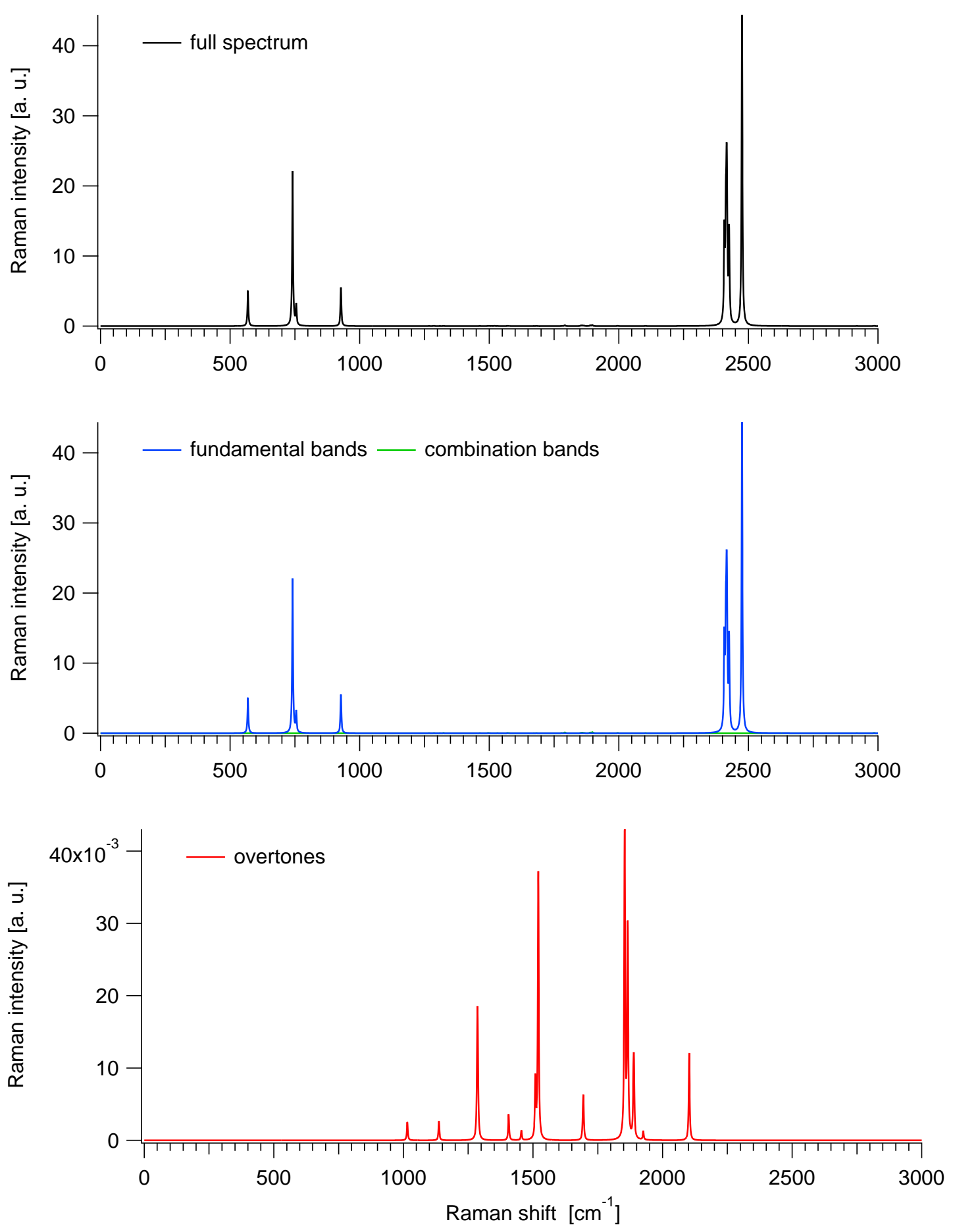
Table S.44.4. $\mathrm{B}_{12} \mathrm{H}_{12}{ }^{2-}$ : Ideal-gas thermodynamic functions at 1 bar (anharmonic B3LYPD2/cc-pVTZ results)

\begin{tabular}{|c|c|c|c|}
\hline $\begin{array}{c}T \\
{[\mathrm{~K}]} \\
\end{array}$ & $\begin{array}{c}C_{P} \\
{\left[\mathrm{~J} \mathrm{~mol}^{-1} \mathrm{~K}^{-1}\right]}\end{array}$ & $\begin{array}{c}S \\
{\left[\mathrm{~J} \mathrm{~mol}^{-1} \mathrm{~K}^{-1}\right]}\end{array}$ & $\begin{array}{c}H(T)-H(0 K) \\
{\left[\mathrm{kJ} \mathrm{mol}^{-1}\right.}\end{array}$ \\
\hline 100 & 36.238 & 255.491 & 3.363 \\
\hline 200 & 97.310 & 294.798 & 9.379 \\
\hline 300 & 195.877 & 352.947 & 24.055 \\
\hline 400 & 277.302 & 421.042 & 47.921 \\
\hline 500 & 336.175 & 489.603 & 78.756 \\
\hline 600 & 379.317 & 554.899 & 114.634 \\
\hline 700 & 412.199 & 615.947 & 154.280 \\
\hline 800 & 438.062 & 672.742 & 196.842 \\
\hline 900 & 458.827 & 725.580 & 241.723 \\
\hline 1000 & 475.727 & 774.827 & 288.479 \\
\hline 1100 & 489.623 & 820.841 & 336.768 \\
\hline 1200 & 501.144 & 863.953 & 386.324 \\
\hline 1300 & 510.771 & 904.458 & 436.934 \\
\hline 1400 & 518.872 & 942.615 & 488.428 \\
\hline 1500 & 525.736 & 978.654 & 540.667 \\
\hline 1600 & 531.590 & 1012.776 & 593.541 \\
\hline 1700 & 536.614 & 1045.158 & 646.958 \\
\hline 1800 & 540.951 & 1075.956 & 700.841 \\
\hline 1900 & 544.716 & 1105.306 & 755.129 \\
\hline 2000 & 548.002 & 1133.332 & 809.768 \\
\hline
\end{tabular}

$T$ : Temperature. $C_{P}$ : Heat capacity at constant pressure. $S$ : Entropy. $H$ : Enthalpy. 
Figure S.44.4. $\mathrm{B}_{12} \mathrm{H}_{12}{ }^{2-}$ : Ideal-gas thermodynamic functions at 1 bar (anharmonic B3LYPD2/cc-pVTZ results)
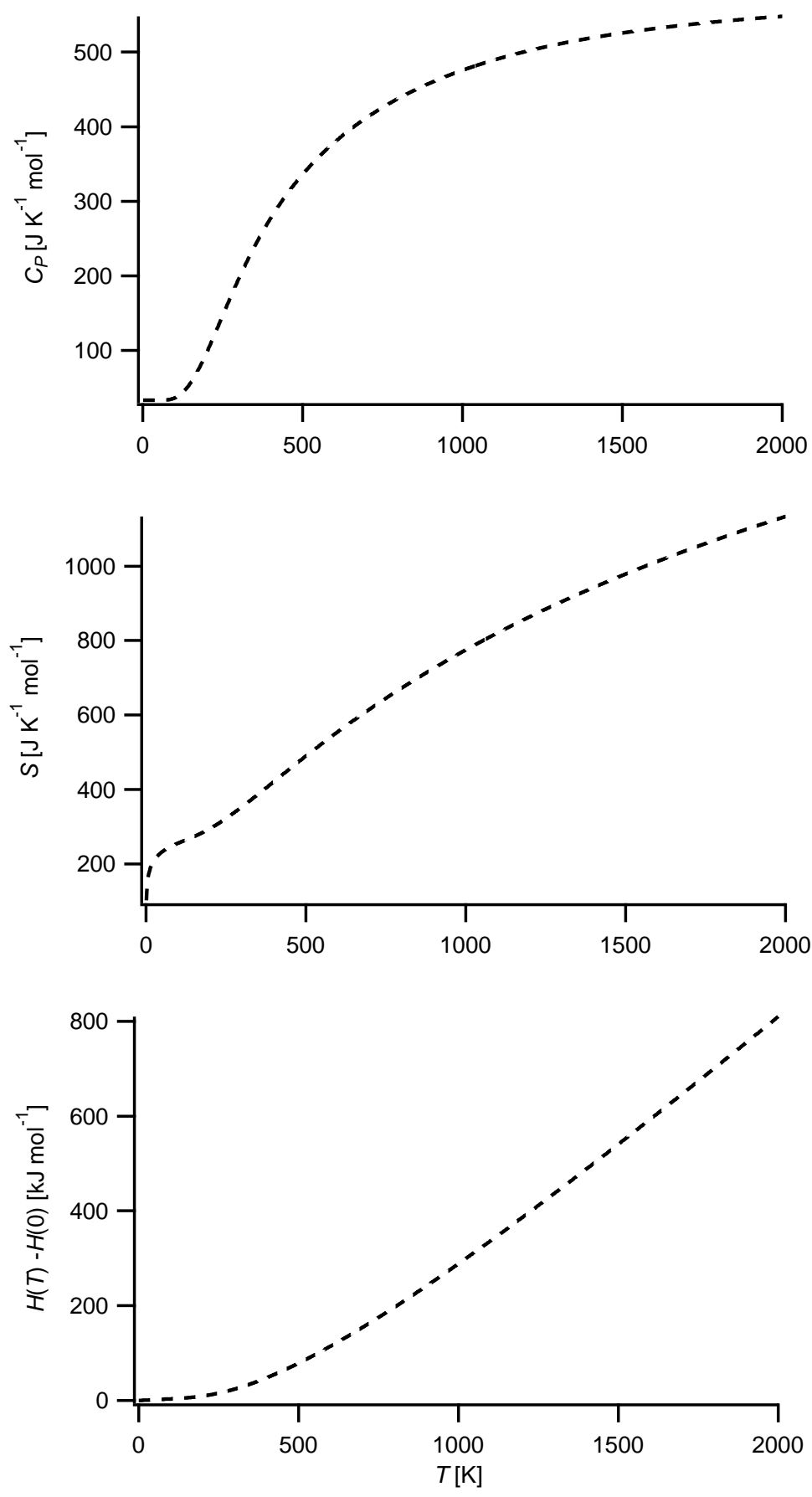

$T$ : Temperature. $C_{P}$ : Heat capacity at constant pressure. $S$ : Entropy. $H$ : Enthalpy. 
Table S.44.5. $\mathrm{B}_{12} \mathrm{H}_{12}{ }^{2-}$ : Fits (red solid lines) of the computed thermodynamic functions (blue dashed lines) with NASA type functions (Equations (1) - (3)) in the $200-900 \mathrm{~K}$ temperature range (anharmonic B3LYP-D2/cc-pVTZ results). In each case, the difference curve between the thermodynamic and NASA functions is plotted in the upper graph

Fit parameters

\begin{tabular}{llllll}
\hline $\mathrm{a}_{1}$ & $-2.00029105 \mathrm{e}+01$ & $\mathrm{a}_{2}\left[\mathrm{~K}^{-1}\right]$ & $1.78490914 \mathrm{e}-01$ & $\mathrm{a}_{3}\left[\mathrm{~K}^{-2}\right]$ & $-9.32270797 \mathrm{e}-05$ \\
$\mathrm{a}_{4}\left[\mathrm{~K}^{-3}\right]$ & $-8.14761823 \mathrm{e}-08$ & $\mathrm{a}_{5}\left[\mathrm{~K}^{-4}\right]$ & $7.59519574 \mathrm{e}-11$ & $\mathrm{a}_{6}[\mathrm{~K}]$ & $1.83484530 \mathrm{e}+03$ \\
$\mathrm{a}_{7}$ & $1.07790437 \mathrm{e}+02$ & & & &
\end{tabular}
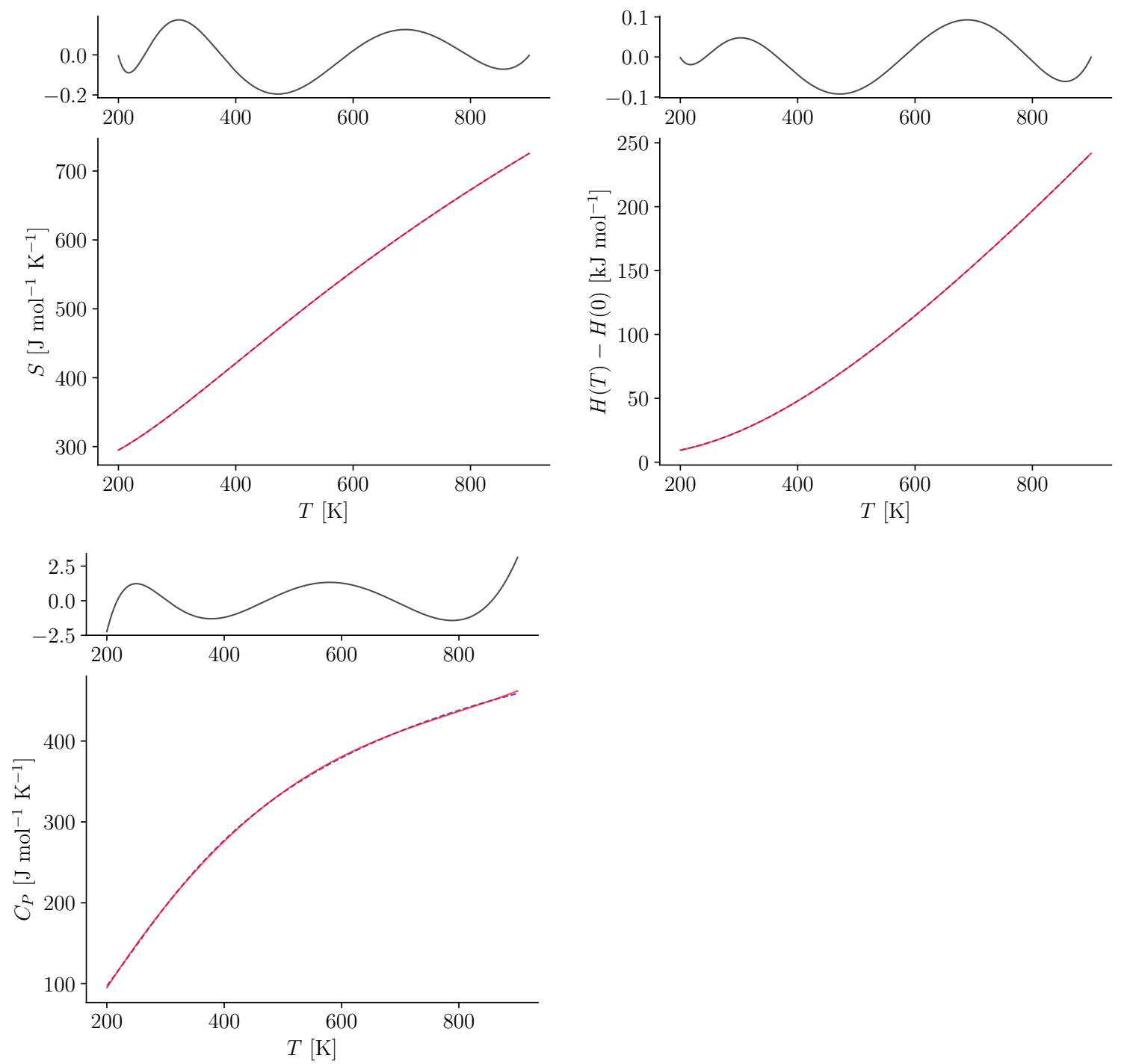